\title{
Aerodynamic Response of a Pitching Airfoil with Pulsed Circulation Control for Vertical Axis Wind Turbine Applications
}

Chad C. Panther

Follow this and additional works at: https://researchrepository.wvu.edu/etd

\section{Recommended Citation}

Panther, Chad C., "Aerodynamic Response of a Pitching Airfoil with Pulsed Circulation Control for Vertical Axis Wind Turbine Applications" (2015). Graduate Theses, Dissertations, and Problem Reports. 6374.

https://researchrepository.wvu.edu/etd/6374

This Dissertation is protected by copyright and/or related rights. It has been brought to you by the The Research Repository @ WVU with permission from the rights-holder(s). You are free to use this Dissertation in any way that is permitted by the copyright and related rights legislation that applies to your use. For other uses you must obtain permission from the rights-holder(s) directly, unless additional rights are indicated by a Creative Commons license in the record and/ or on the work itself. This Dissertation has been accepted for inclusion in WVU Graduate Theses, Dissertations, and Problem Reports collection by an authorized administrator of The Research Repository @ WVU.

For more information, please contact researchrepository@mail.wvu.edu. 


\title{
Aerodynamic Response of a Pitching Airfoil with Pulsed Circulation Control for Vertical Axis Wind Turbine Applications
}

\author{
Chad C. Panther \\ Dissertation submitted to the \\ Benjamin M. Statler College of Engineering and Mineral Resources \\ at West Virginia University
}

in partial fulfillment of the requirements for the degree of

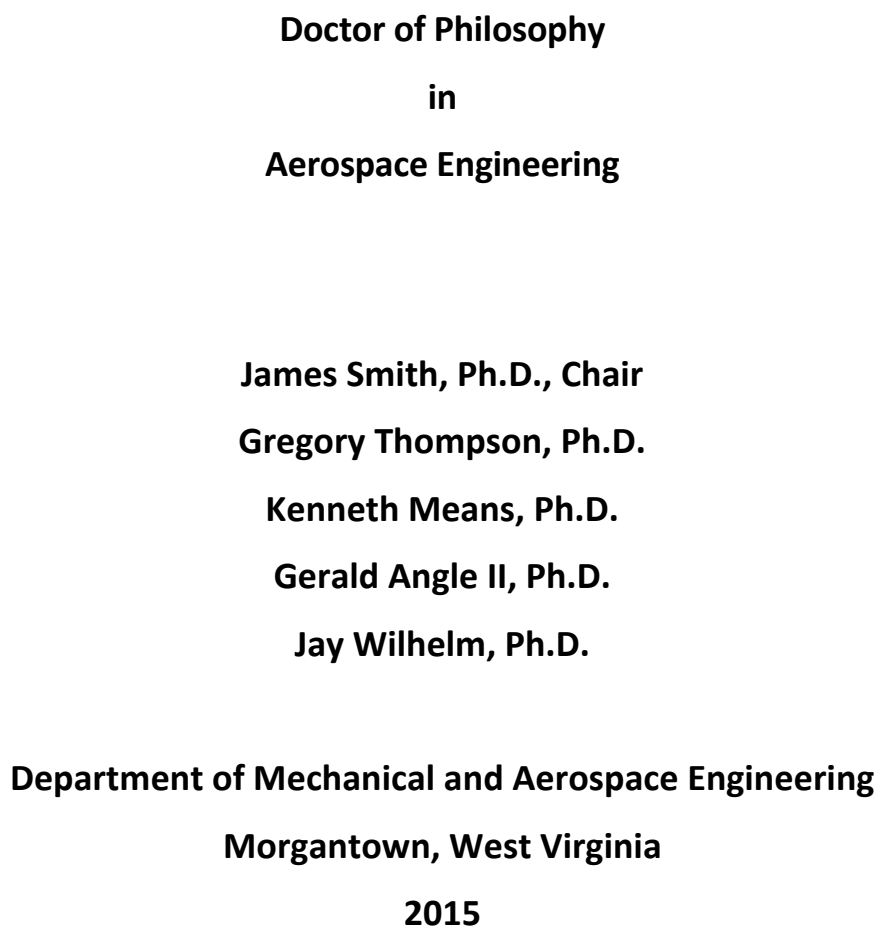

Keywords: Vertical Axis Wind Turbine, VAWT, Pulsed Circulation Control, Dynamic Stall Copyright $(0) 2015$ Chad C. Panther 


\title{
ABSTRACT
}

\section{Aerodynamic Response of a Pitching Airfoil with Pulsed Circulation Control for Vertical Axis Wind Turbine Applications}

\author{
Chad C. Panther
}

Vertical Axis Wind Turbines (VAWTs) have experienced a renewed interest in development for urban, remote, and offshore applications. Past research has shown that VAWTs cannot compete with Horizontals Axis Wind Turbines (HAWTs) in terms of energy capture efficiency. VAWT performance is plagued by dynamic stall (DS) effects at low tip-speed ratios $(\lambda)$, where each blade pitches beyond static stall multiple times per revolution. Furthermore, for $\lambda<2$, blades operate outside of stall during over $70 \%$ of rotation. However, VAWTs offer many advantages such as omnidirectional operation, ground proximity of generator, lower sound emission, and non-cantilevered blades with longer life. Thus, mitigating dynamic stall and improving VAWT blade aerodynamics for competitive power efficiency has been a popular research topic in recent years and the directive of this study.

Past research at $W V U$ focused on the addition of circulation control (CC) technology to improve VAWT aerodynamics and expand the operational envelope. A novel blade design was generated from the augmentation of a NACAOO18 airfoil to include CC capabilities. Static wind tunnel data was collected for a range of steady jet momentum coefficients $\left(0.01 \leq C_{\mu} \leq 0.10\right)$ for analytical vortex model performance projections. Control strategies were developed to optimize CC jet conditions throughout rotation, resulting in improved power output for $2 \leq \lambda \leq 5$. However, the pumping power required to produce steady CC jets reduced net power gains of the augmented turbine by approximately $15 \%$. The goal of this work was to investigate pulsed CC jet actuation to match steady jet performance with reduced mass flow requirements. To date, no experimental studies have been completed to analyze pulsed CC performance on a pitching airfoil.

The research described herein details the first study on the impact of steady and pulsed jet CC on pitching VAWT blade aerodynamics. Both numerical and experimental studies were implemented, varying $R e, k$, and $\pm \alpha$ to match a typical VAWT operating environment. A range of reduced jet frequencies $(0.25 \leq S t \leq 4)$ were analyzed with varying $C_{\mu}$, based on effective ranges from prior flow control airfoil studies. Airfoil pitch was found to increase the baseline lift-to-drag ratio $(L / D)$ by up to $50 \%$ due to dynamic stall effects. The influence of dynamic stall on steady CC airfoil performance was greater for $C_{\mu}=0.05$, increasing $L / D$ by $115 \%$ for positive angle-of-attack. Pulsed actuation was shown to match, or improve, steady jet lift performance while reducing required mass flow by up to $35 \%$. From numerical flow visualization, pulsed actuation was shown to reduce the size and strength of wake vorticity during DS, resulting in lower profile drag relative to baseline and steady actuation cases. A database of pitching airfoil test data, including overshoot and hysteresis of aerodynamic coefficients $\left(C_{l}, C_{d}\right)$, was compiled for improved analytical model inputs to update CCVAWT performance predictions, where the aforementioned $L / D$ improvements will be directly reflected.

Relative to a conventional VAWT with annual power output of $1 \mathrm{MW}$, previous work at WVU proved that the addition of steady jet CC could improve total output to $1.25 \mathrm{MW}$. However, the pumping cost to generate the continuous jet reduced yearly CCVAWT net gains to $1.15 \mathrm{MW}$. The current study has shown that pulsed CC jets can recover $4 \%$ of the pumping demands due to reduced mass flow requirements, increasing annual CCVAWT net power production to $1.19 \mathrm{MW}$, a $19 \%$ improvement relative to the conventional turbine. 
To my Parents. 


\section{ACKNOWLEDGMENTS}

I would first like to thank the members of my committee: Dr. Ken Means, Dr. Greg Thompson, Dr. Jay Wilhelm, Dr. Gerald Angle II, and Dr. Jim Smith for their assistance and comments. I would like to especially thank Dr. Smith for his continued support and confidence over the duration of this endeavor.

I would adamantly like to thank Mr. Cliff Judy, head of WVU's MAE Machine Shop, for his patience while teaching various tooling and machining techniques, while fabricating intricate parts for many wind tunnel models and test stand components. His dependability and skills are unmatched and have been greatly appreciated.

I would like to thank Dr. Wade Huebsch for crucial aid in allowing me access to his groups' supercomputing clusters and FLUENT licenses, saving me unknowable amounts of time and hassle. Special thanks goes to Dr. Chris Griffin for his CFD tutorial, use of his airfoil pitch mechanism during wind tunnel testing, and general friendly support during the completion of our degrees.

I would like to further thank Dr. Jay Wilhelm for his endless help in the wind tunnel, especially with all matters of electrical and computer integration into my experiment. Jay played an integral role in my experimental set-up, data acquisition, and data reduction procedures. My gratitude for his patience and willingness to help write MATLAB code along the way cannot be properly expressed.

An individual thanks to Dr. Drew Lowery is also due, for his persistent advice and support on signal processing and data reduction code.

I owe an additional debt of gratitude to Dr. Greg Thompson for the financial support he offered during my final semester. The worth of additional personal time this appointment allowed me to focus on the completion of this research is impossible to gauge. Thank You.

Dr. Pat Browning deserves many thanks for countless hours of discussion and help in the WVU Aero. Lab. Pat was a constant sounding board for numerous questions encountered during testing and his veteran knowledge of WVU's Subsonic Closed Loop Wind Tunnel was immensely helpful and his overall support was crucial for the completion of this work.

I want to extend a special and personal thanks to my closest friends, for understanding my two (plus) year sabbatical, and the unhesitant welcome on my return. You know who you are. I love and appreciate you all more than you will ever know.

I would like to thank my family for their support for the duration of this process, especially Uma, Pap, and Noni for their dependability and endless (and affordable) daycare services for Bode. I would particularly like to thank my Mom and Dad for unbounded support and encouragement to get me here. I would also like to especially thank Jess for her own selfless and enduring support, patience, and love while I completed this final degree. I could not have finished this without her behind me. There should be an honorary degree for such people. A small army of people made sacrifices to complete this project, but unfortunately only one person gets the credit. Finally, I would like to thank Bode Odie, the dinosaur boy, for unknowingly motivating me with constant laughter, innocence, curiosity, and love. Thank You. 


\section{TABLE OF CONTENTS}

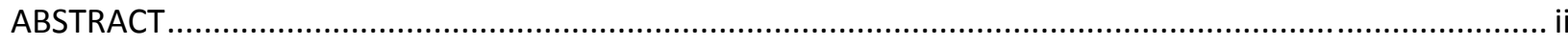

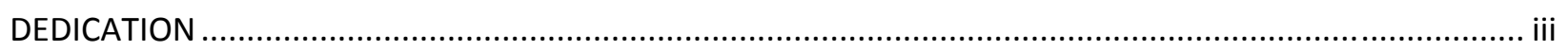

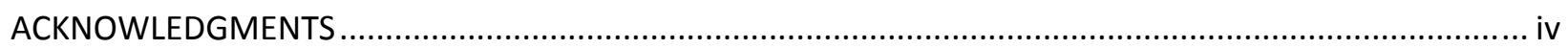

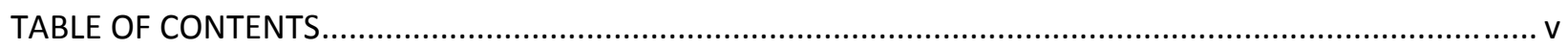

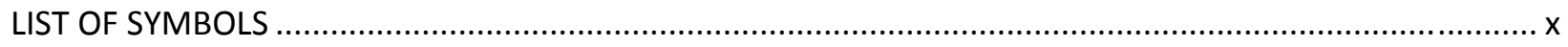

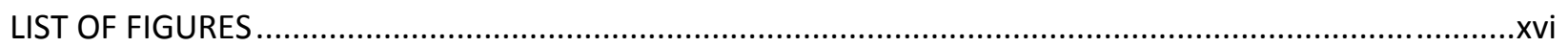

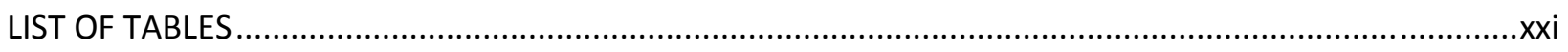

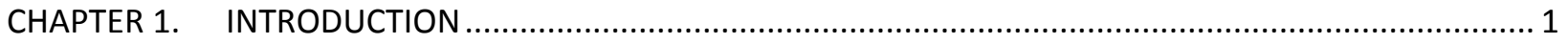

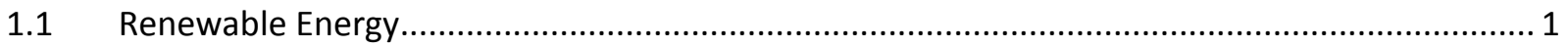

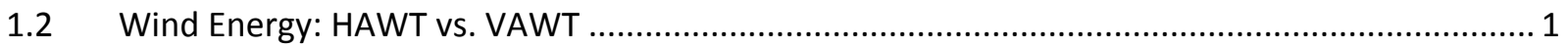

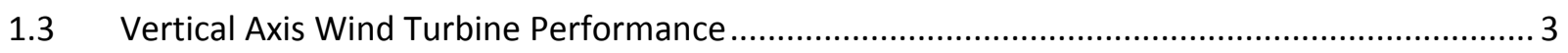

1.4 Circulation Control Vertical Axis Wind Turbine (CCVAWT) ..................................................... 10

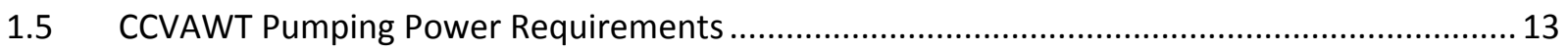

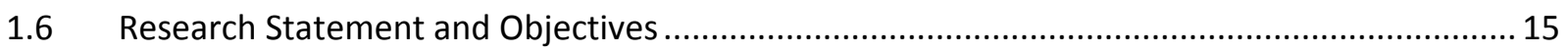

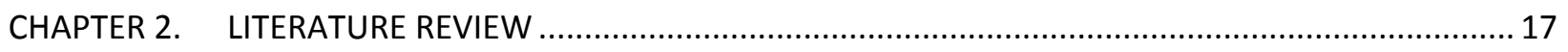

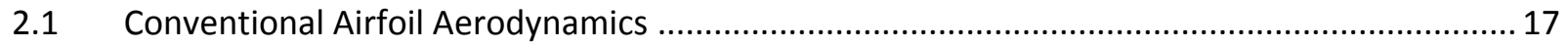

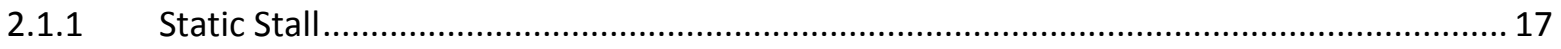

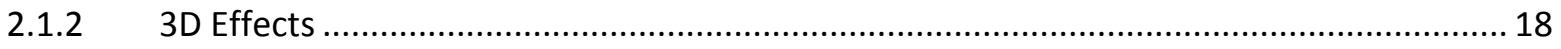

2.1.3 Traditional Aerodynamic Load Control ...................................................................... 19

2.2 Flow Control Airfoil Aerodynamics .................................................................................. 21

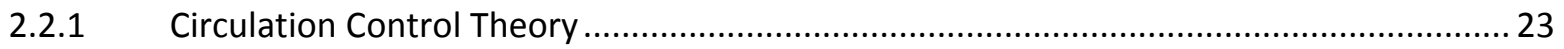

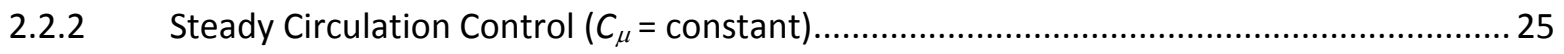

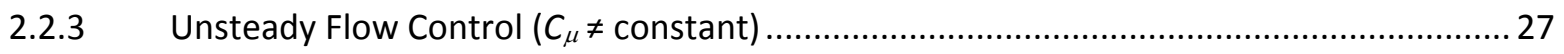

2.2.4 Previous Circulation Control Research at WVU …………............................................. 32

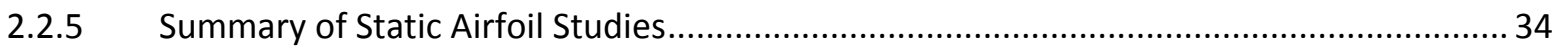

2.3 Pitching Airfoil Aerodynamics .......................................................................................... 35 


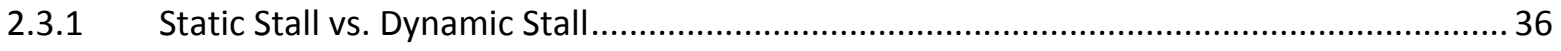

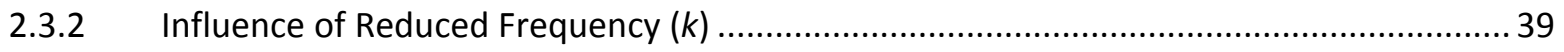

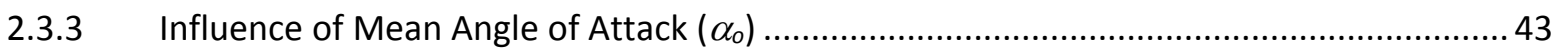

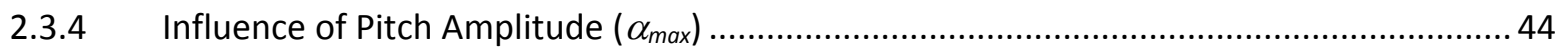

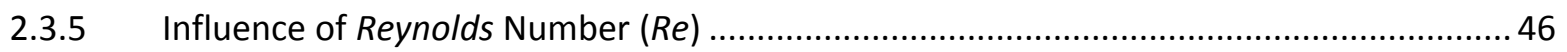

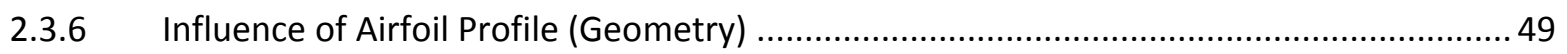

2.3.7 Influence of Flow Control on Dynamic Stall ............................................................. 51

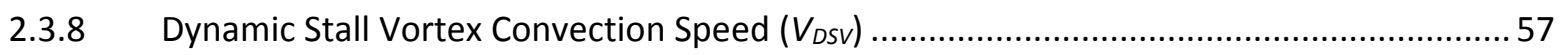

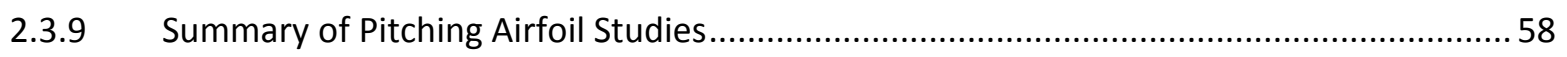

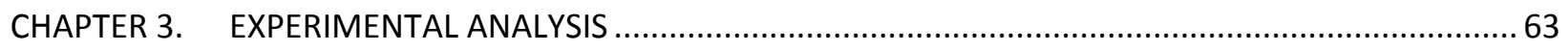

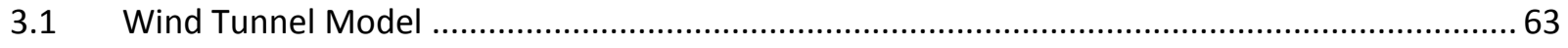

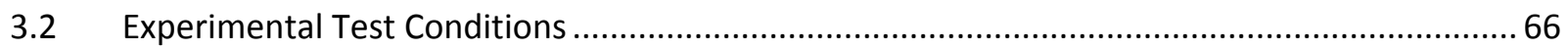

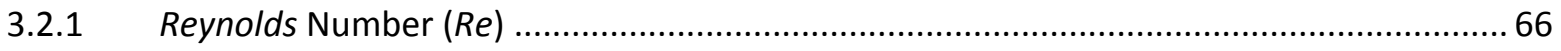

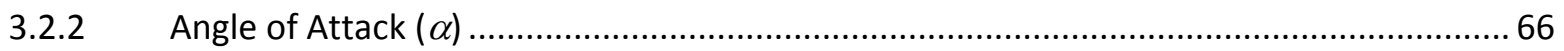

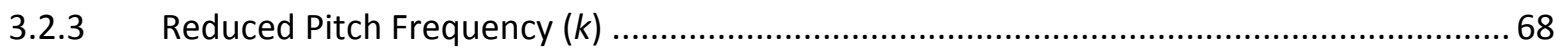

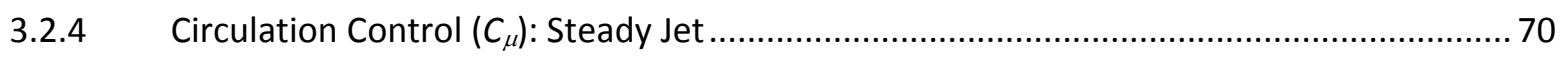

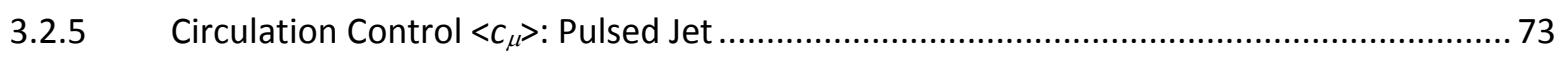

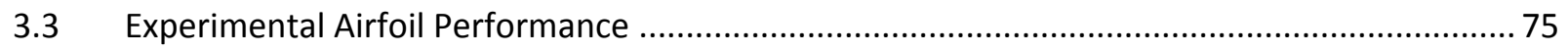

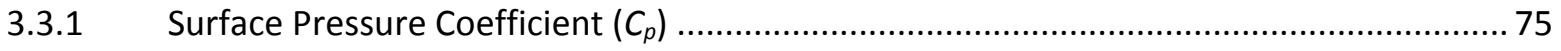

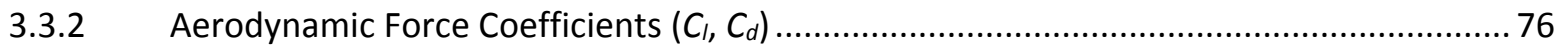

3.3.3 Frequency Response of Pressure Tubing …............................................................... 78

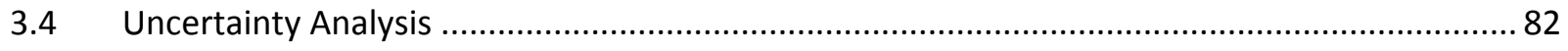

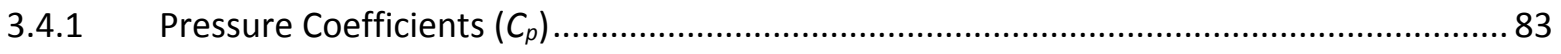

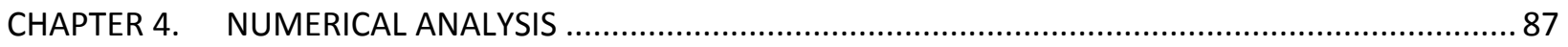

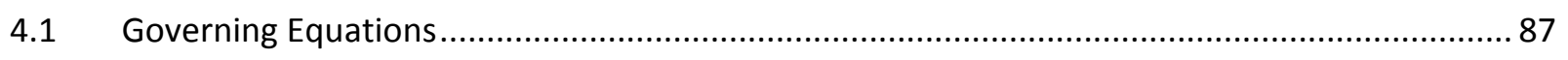

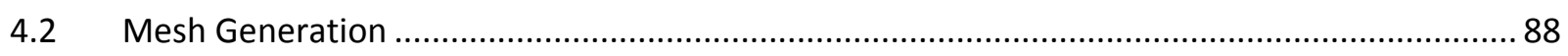




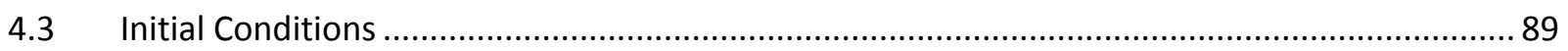

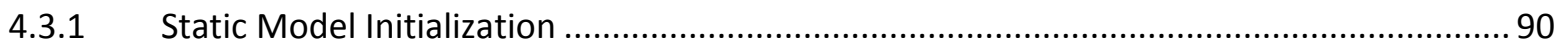

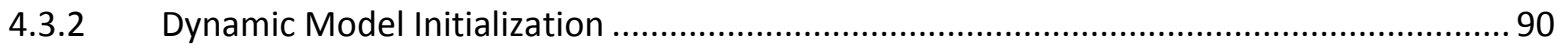

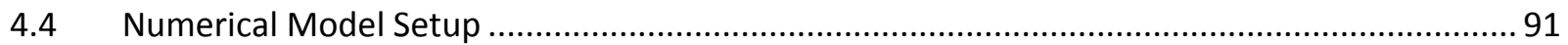

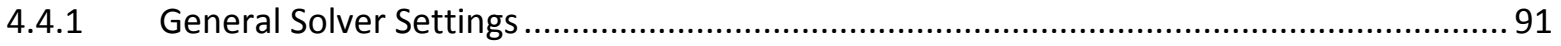

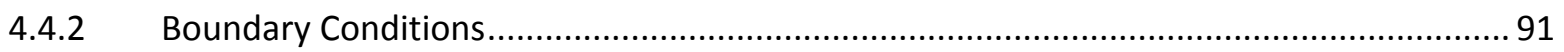

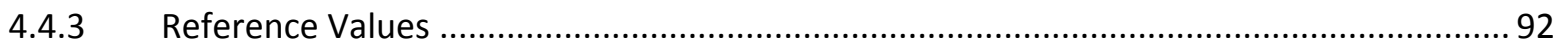

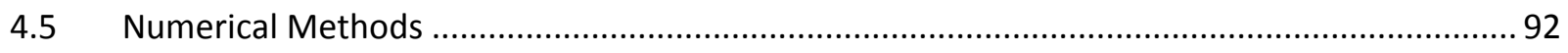

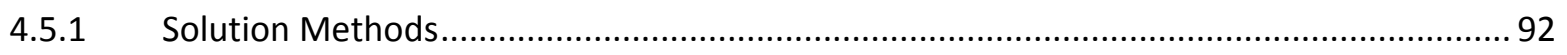

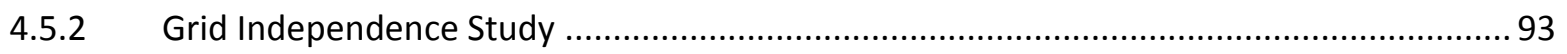

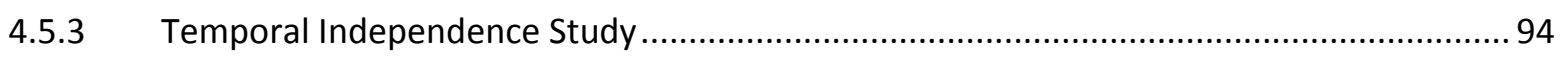

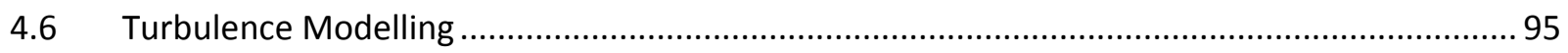

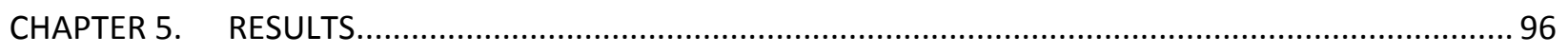

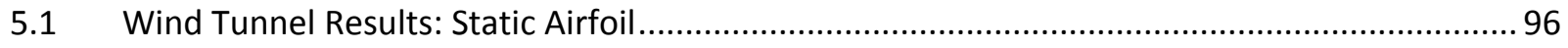

5.1.1 Static CCNACA0018 Pressure Data Validation ........................................................... 96

5.1.2 Static CCNACA0018 Lift Data: Baseline ................................................................... 99

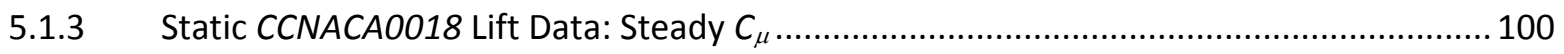

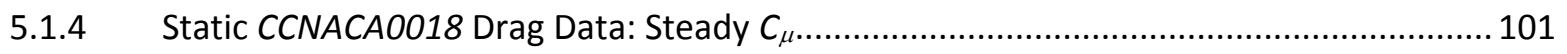

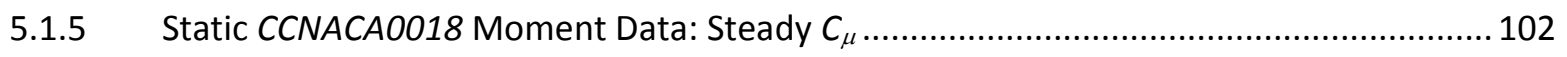

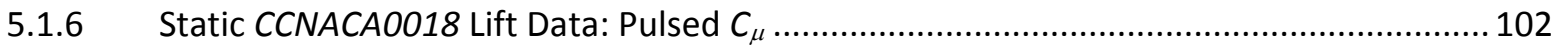

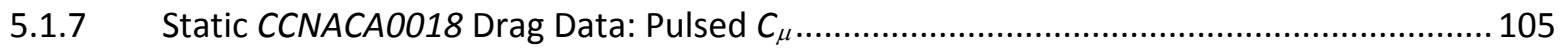

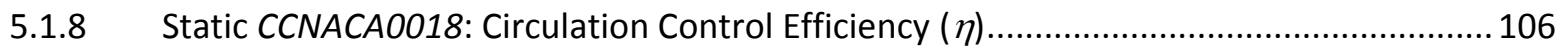

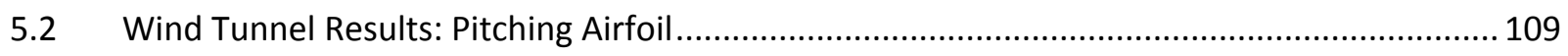

5.2.1 Baseline Validation with Historical Data ................................................................... 109

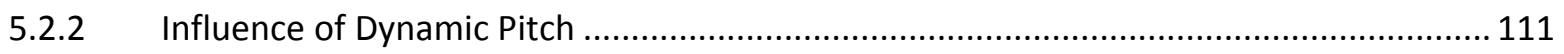




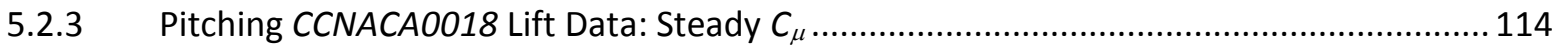

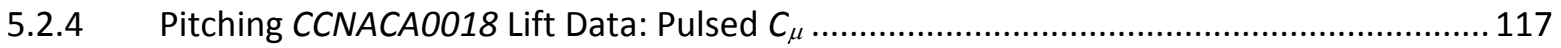

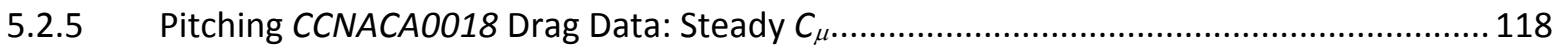

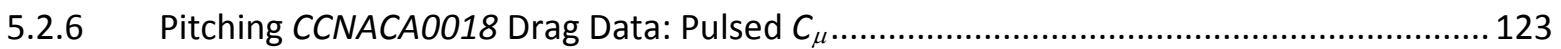

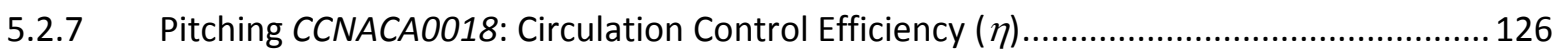

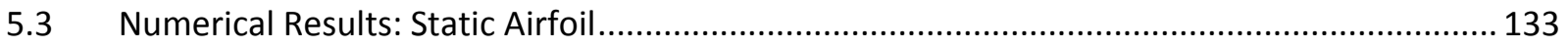

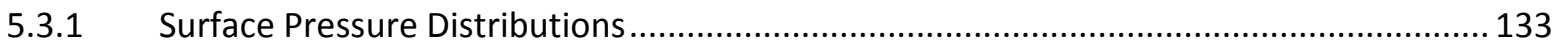

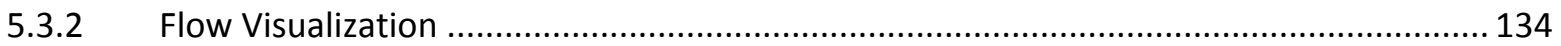

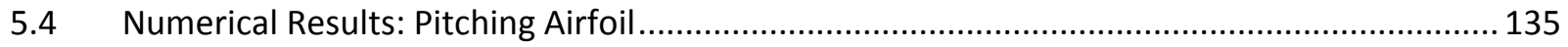

5.4.1 Turbulence Models \& Experimental Data Comparison ................................................ 135

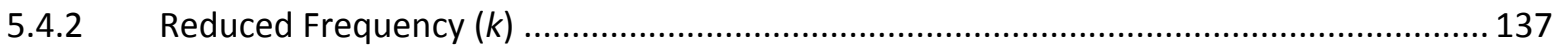

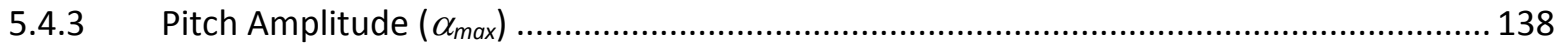

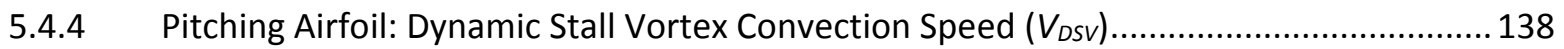

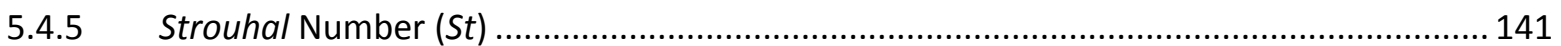

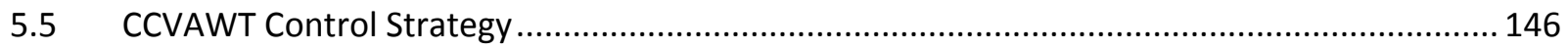

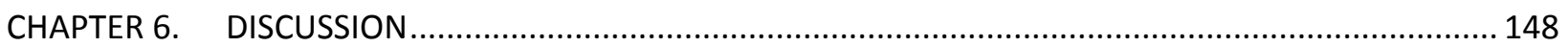

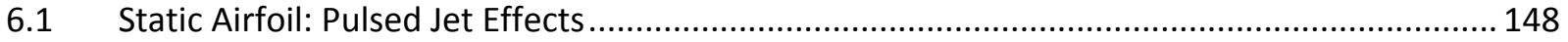

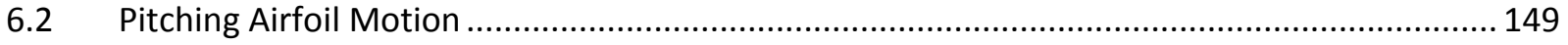

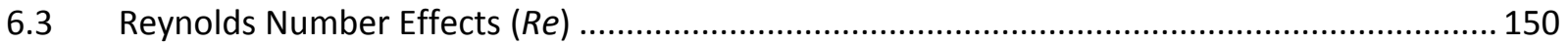

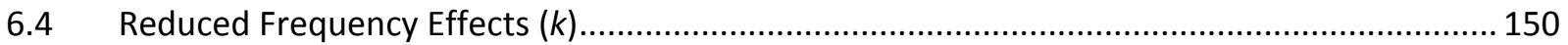

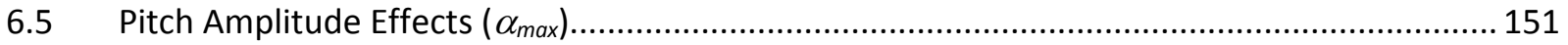

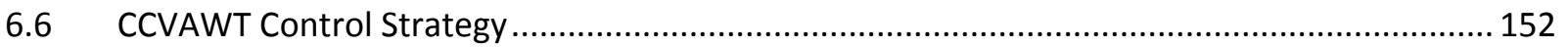

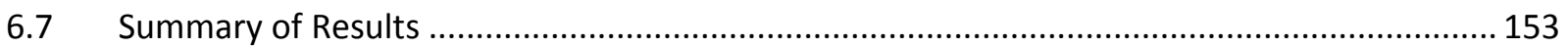

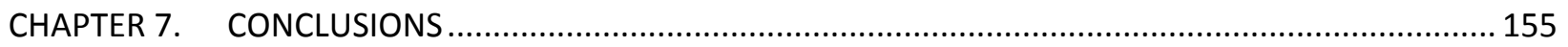

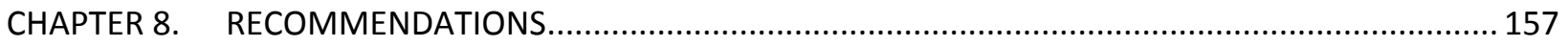

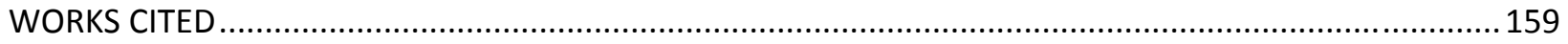


APPENDIX A: Experimental Error Analysis

APPENDIX B: CCNACA0018 Jet Velocity Measurements and Analysis Plots ........................................... 174

Appendix B.1 $R e=180 \times 10^{3}$ Jet Velocity Measurements and Spectral Analysis Plots ......................... 175

Appendix B.2 Re $=300 \times 10^{3}$ Jet Velocity Measurements and Spectral Analysis Plots .........................179

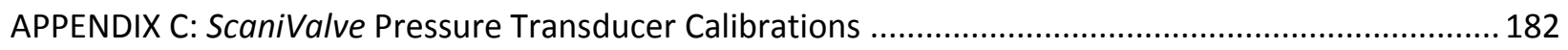

APPENDIX D: Unsteady Pressure Signal Attenuation in Cylindrical Tubes ............................................ 192

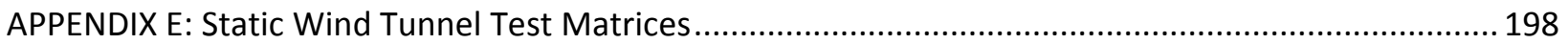

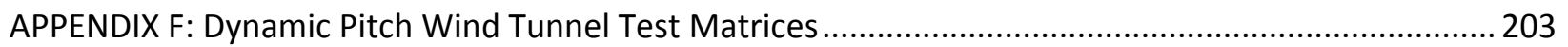

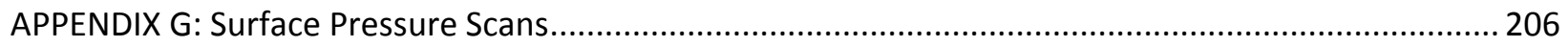

APPENDIX H: CCNACA0018 Pulsed CC Flow Actuation: Pneumatic Valves.............................................210

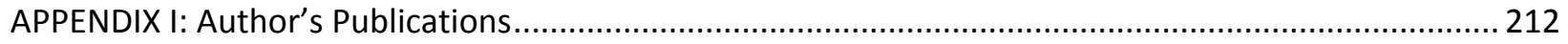

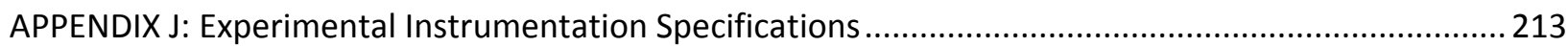

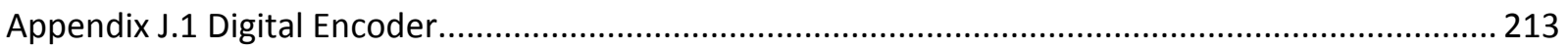

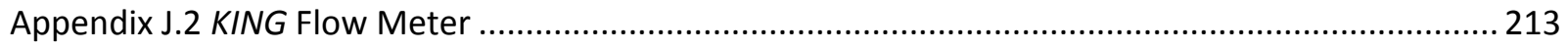

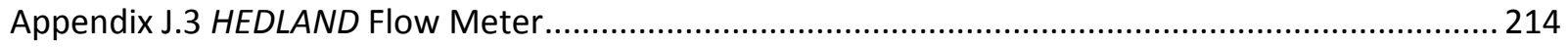

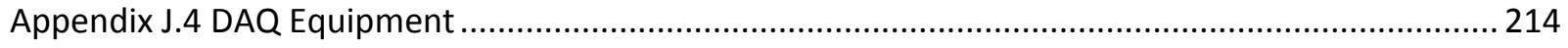

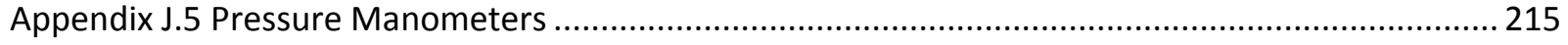

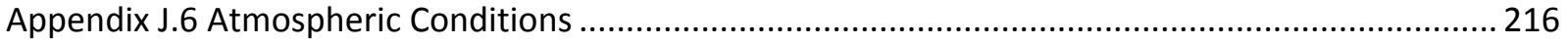

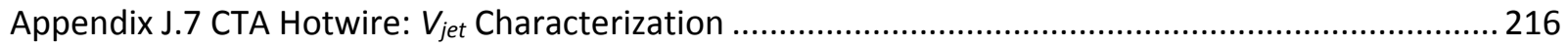

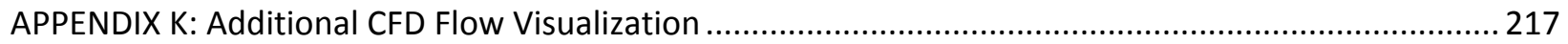




\section{LIST OF SYMBOLS}

\section{Arabic}

$A_{b l} \quad$ Airfoil planform area

$A_{j e t} \quad$ Cross-sectional area of blowing jet

AR VAWT aspect ratio

B Airfoil span

C Airfoil chord

$C_{a} \quad$ Section (2D) axial force coefficient

C Closed curve in a flow field

$C_{m(c / 4)} \quad$ Section (2D) moment coefficient about quarter-chord

$C_{m(L E)} \quad$ Section (2D) moment coefficient about leading edge

$C_{n} \quad$ Section (2D) normal force coefficient

$C_{p, i} \quad$ Pressure coefficient at port $i$

$C_{P} \quad$ VAWT power coefficient

$C_{\mu} \quad$ Steady momentum coefficient

$<c_{\mu}>\quad$ Unsteady momentum coefficient

$d_{\text {eff }} \quad$ Effective diameter of pneumatic pressure tube system

D VAWT diameter

Ds $\quad$ Directed line segment at a point $C$ 


\begin{tabular}{|c|c|}
\hline$D$ & Drag force \\
\hline$\Delta C_{V} / C_{\mu}$ & Lift augmentation factor \\
\hline$f_{a c t}$ & Actuator (jet) pulsing frequency $\left(f_{\text {act }}=f_{\text {jet }}\right)$ \\
\hline$f_{v}$ & Vortex shedding frequency \\
\hline$F^{+}$ & Natural Frequency $\left(F^{+}=S t\right)$ \\
\hline$h_{\text {slot }}$ & Blowing slot height \\
\hline$J_{j e t}$ & Momentum of jet \\
\hline$<J_{j e t}>$ & Oscillatory momentum of jet \\
\hline K & Reduced pitch frequency \\
\hline$L$ & Lift force \\
\hline$L^{\prime}$ & Lift per unit span \\
\hline$\dot{m}$ & Mass flow rate \\
\hline$\dot{m}_{\text {span }}$ & Mass flow rate per unit span \\
\hline$N$ & Number of VAWT blades, number of samples \\
\hline$p$ & Pressure \\
\hline$<p>$ & Fluctuating pressure \\
\hline$P$ & Power \\
\hline$q$ & Dynamic pressure \\
\hline$Q$ & Volumetric flowrate \\
\hline$R$ & Gas constant (air) \\
\hline $\operatorname{Re}$ & Reynolds number \\
\hline St & Strouhal number \\
\hline$t$ & Time, airfoil thickness \\
\hline$T$ & Temperature \\
\hline$T_{\text {conv }}$ & Airfoil convective time scale $\left(T_{\text {conv }}=V_{\infty} \cdot \mathrm{C}\right)$ \\
\hline
\end{tabular}


$V_{\infty} \quad$ Freestream air speed

$V_{D S V} \quad$ Convection speed of dynamic stall vortex

$V_{\text {rel }} \quad$ Relative velocity of VAWT blade

$V_{t} \quad$ Tangential speed of blade

$V_{\text {jet }} \quad$ Velocity of steady jet

$\left\langle V_{\text {jet }}>\quad\right.$ RMS velocity of pulsed jet

$[\mathrm{ft} / \mathrm{s}]$

$w_{i} \quad$ Instrumentation error

[based on equipment]

$W_{i} \quad$ Measurement error

$x_{T E} \quad$ Distance from actuator to TE

[in]

$y^{+} \quad$ Dimensionless distance from wall

\section{Greek}

$\alpha_{C l=0} \quad$ Zero lift angle of attack

$\alpha_{D S} \quad$ Dynamic stall angle of attack

$\alpha_{g} \quad$ Geometric angle of attack

$\alpha_{s s} \quad$ Static stall angle of attack

$\alpha_{o} \quad$ Mean angle of attack

$\delta \quad$ Flap deflection angle

$\Delta \bar{C}_{l} \quad$ Pitch cycle integrated lift

$\Delta t^{+} \quad$ Dimensionless time step $\left(=\Delta t \cdot c / V_{\infty}\right)$ 


$\begin{array}{llr}\mu & \text { Dynamic viscosity (of air) }\left[\mathrm{lb}_{\left.\mathrm{f}-\mathrm{s} / \mathrm{ft}^{2}\right]}\right. & \mu_{\mathrm{std}}=3.74 \times 10^{-7}\left[\mathrm{lb}_{\mathrm{f}}-\mathrm{S} / \mathrm{ft}^{2}\right] \\ \theta & \text { Orbital/Azimuthal position of blade } & \text { [deg] } \\ \rho & \text { Density (air) } & \rho_{\text {std }}=0.00238\left[\mathrm{slug} / \mathrm{ft}^{3}\right] \\ \Gamma & \text { Airfoil circulation } & {[-]} \\ \sigma & \text { VAWT solidity } & {[-]} \\ \tau & \text { Cycle period } & {[-]} \\ \omega & \text { Rotational frequency } & {[\mathrm{Hz}]}\end{array}$

\section{Subscripts}

act Actuator/Actuation

DS Dynamic stall

j Current location with respect to time or position

$j+1 \quad$ Future or upstream location, with respect to time or position

j-1 Previous or downstream location, with respect to time or position

jet Jet

$L E \quad$ Airfoil leading edge

pl Plenum

SS Static stall

TE $\quad$ Airfoil trailing edge 


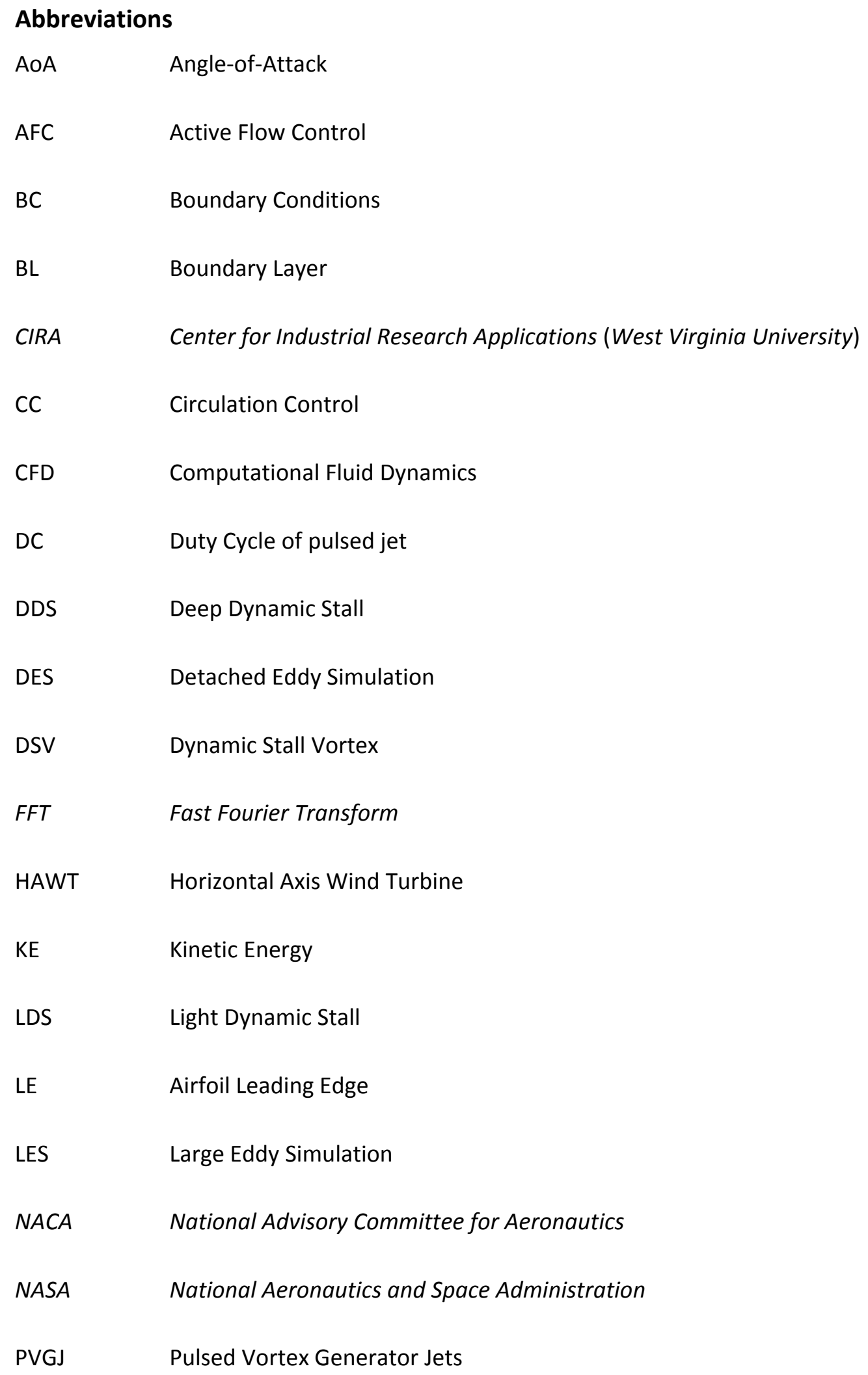




$\begin{array}{ll}\text { RANS } & \text { Reynolds Averaged Navier-Stokes equations } \\ \text { RMS } & \text { Root Mean Square } \\ \text { SA } & \text { Spalart-Allmaras Turbulence Model } \\ \text { SST } & \text { Shear-Stress Transport Turbulence Model } \\ \text { TE } & \text { Airfoil Trailing Edge } \\ \text { TF } & \text { Transfer Function } \\ \text { TSR } & \text { Tip Speed Ratio (also symbolized by } \lambda \text { ) } \\ \text { UDF } & \text { User-Defined Function } \\ \text { URANS } & \text { Unsteady Reynolds Averaged Navier-Stokes equations } \\ \text { VAWT } & \text { Vertical Axis Wind Turbine } \\ \text { ZNMF } & \text { Zero Net Mass Flux }\end{array}$

\section{Other Notation}

$\langle x\rangle \quad$ RMS value of variable of variable $x$

$\bar{x} \quad$ Time-Averaged value of variable $x$ 


\section{LIST OF FIGURES}

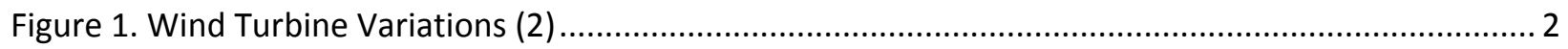

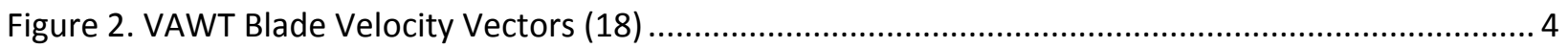

Figure 3. VAWT Blade AoA throughout Azimuth Rotation .............................................................. 5

Figure 4. VAWT Blade Velocity and Force Vectors (Image modified from Carrigan (2)) ........................... 6

Figure 5. Influence of Solidity $(\sigma)$ and Airfoil $L / D$ on VAWT Power Production (30) ................................ 9

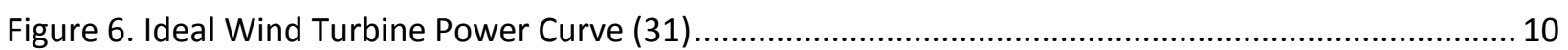

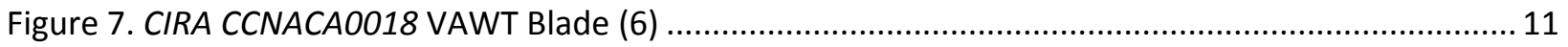

Figure 8. CAD Drawing of CCNACA0018 Wind Tunnel Model................................................................. 11

Figure 9. Measured Influence of $\alpha$ on CCNACA0018 Lift Augmentation ( $R e=300 \times 10^{3}, C_{\mu}=$ Steady) (38)....12

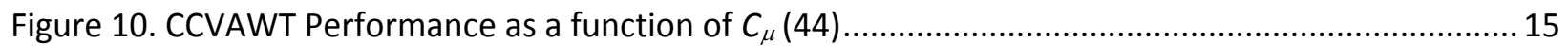

Figure 11. Schematic of Lift Variation with Angle of Attack (56) ........................................................ 18

Figure 12. 3D Effects on Wind Tunnel Measured Airfoil Performance: NACA0018 and NACA6418 (42)... 18

Figure 13. Influence of Camber on Lift-Curve Slope for NACA Airfoils with Thickness of $t / c=0.18 \ldots \ldots \ldots . . .19$

Figure 14. The Influence of LE and TE Flow Enhancement Devices on Airfoil Lift and Stall (57)............... 20

Figure 15. The Influence of TE Flap Deflection on Cambered Airfoil (NACA23012) Performance (59) ...... 21

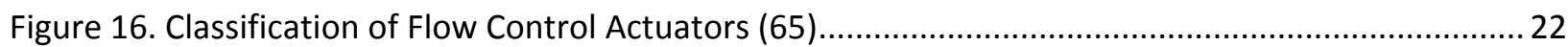

Figure 17. Kutta-Joukowski Theorem: Circulation $(\Pi$ ) around a Lifting Airfoil (56) .................................2 23

Figure 18. (a.) Sharp TE and the Kutta Condition (56) (b.) Tangential Blowing Over Rounded TE (46)....24

Figure 19. Influence of CC Jet on BL along a Coandă Surface (71).....................................................25

Figure 20. Comparison of NASA Flow Control Airfoil Performance $\left(\alpha=0^{\circ}\right)(45)$....................................... 26

Figure 21. Upper Surface vs. Lower Surface Blowing on CC Airfoil Performance: $R e=500 \times 10^{3}(46) \ldots \ldots \ldots . .26$

Figure 22. Jet Velocity Profiles From Literature (52) (73) (74) ........................................................... 28

Figure 23. Experimental Duty Cycle Investigation Results (45) (46) ..................................................... 28

Figure 24. a.) AFC Actuator Efficiency b.) L/D Ratio for Different Excitation Frequencies $\left(F^{+}=S t\right)(73) \ldots . .29$

Figure 25. Influence of $S t$ and $C_{\mu}$ on Oscillatory Blowing Lift Enhancement (52) (78) .............................31

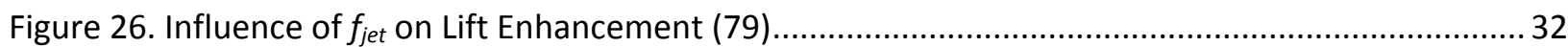

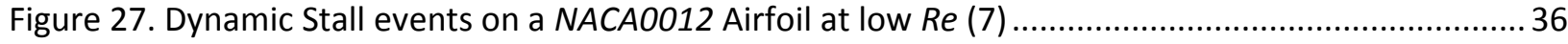

Figure 28. Common BL Characteristics of Light vs. Deep Dynamic Stall (90)........................................... 37

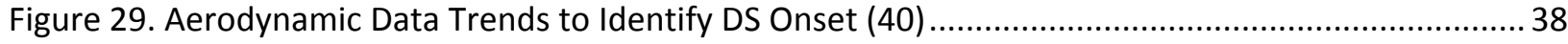

Figure 30. Influence of $k$ on Dynamic Stall AoA: NACA0012 vs. NACA0018 Airfoils (40)......................... 39

Figure 31. Influence of Pitch on $C_{p}$-Distribution of a Pitching NACA0018 Airfoil: $R e=240 \times 10^{3}(23) \ldots \ldots \ldots . . .40$

Figure 32. Influence of $k$ on Pitching NACA0012 Performance: $\alpha=15^{\circ}+10^{\circ} \cdot \sin (\omega \cdot t), \operatorname{Re}=2 \times 10^{6}(60)(96) . .41$

Figure 33. Influence of $k$ on DSV Formation on a Pitching NACA0012 Airfoil: $\operatorname{Re}=80 \times 10^{3}(55) \ldots \ldots \ldots \ldots \ldots . . . . .41$

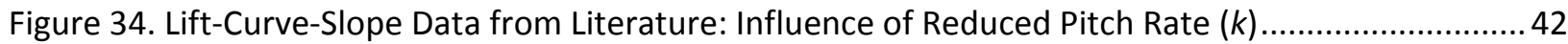

Figure 35. Influence of $k$ on Aerodynamic Performance of a Pitching NACA0012 Airfoil (101) ................ 43

Figure 36. Influence of $\alpha_{o}$ on Pitching NACA0012 Performance: $R e=2 \times 10^{6}, k=0.24$ (96) ......................44

Figure 37. Influence of $\alpha_{\max }$ on Aerodynamic Performance of a Pitching NACA0012 Airfoil (101)........... 45

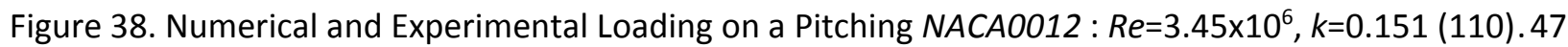

Figure 39. Influence of Fluctuating Freestream on VAWT Lift (26) ........................................................4 48

Figure 40. Measured Lift on 2-Bladed VAWT: NACA0022 Blades (113) .............................................. 48 
Figure 41. Formation and Evolution of DSV Vortex on Pitching NACA0018: $R e=250 \times 10^{3}, k=0.074(102) . .50$

Figure 42. Influence of $\alpha_{\text {mean }}$ on a Pitching NACA0012 with Oscillating TE Flap $\left(k_{\text {flap }}=0.042\right)(120) \ldots \ldots \ldots . . .52$

Figure 43. Reduced Frequency ( $k$ ) Effects on CC Airfoil Lift Hysteresis (109)........................................... 54

Figure 44. VAWT Blade DS and Resulting Normal $\left(C_{N}\right)$ and Tangential $\left(C_{T}\right)$ Forces: $\lambda=2.5(28)(117) \ldots \ldots \ldots 5$

Figure 45. Influence of $k$ on Force Hysteresis for a Pitching CC-Ellipse (110)............................................ 56

Figure 46. Influence of $k$ on NACA4415 Lift Enhancement due to LE Excitation (126).............................56

Figure 47. Influence of Pitch Rate on the Convection Speed of the Dynamic Stall Vortex (131) (132)......58

Figure 48. Summary of Dynamic Pitch Lift Data from Literature ........................................................... 61

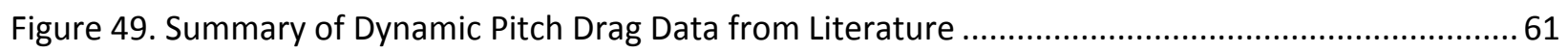

Figure 50. CCNACA0018 Wind Tunnel Model Static Pressure Port Locations ...........................................63

Figure 51. CCNACA0018 Wind Tunnel Model: Internal Plumbing and [14] DYNAMCO Pneumatic Valves 64

Figure 52. CCNACA0018 Wind Tunnel Model with Rapid-Prototyped Skin ............................................. 65

Figure 53. CCNACA0018 Wind Tunnel Model: Static Pressure Port Tubing at Airfoil Endplate .................65

Figure 54. Absolute Encoder Mounted Above Test Section .................................................................67

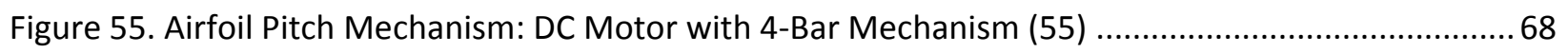

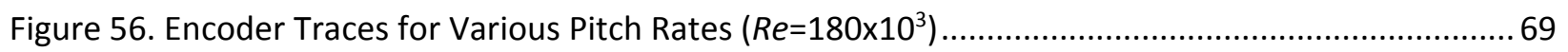

Figure 57. Experimental Conditions ( $\left.R e=180 \times 10^{3}, k=0.100\right)$ : Encoder Data vs. VAWT Theory .................. 70

Figure 58. Benchtop Measurements of $\mathrm{CC}$ Jet for $C_{\mu}$ Characterization: All Instrumentation...................... 71

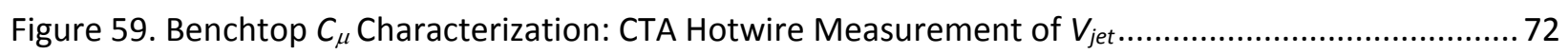

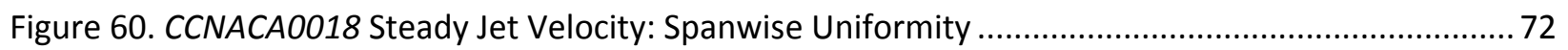

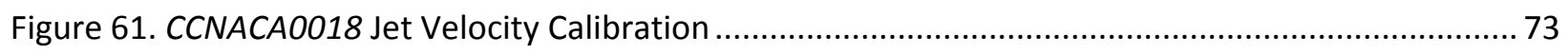

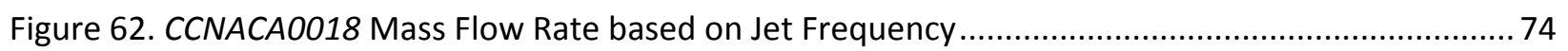

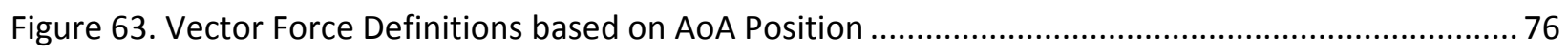

Figure 64. Schematic of Benchtop Experiment: Transient Signal Attenuation Analysis............................ 79

Figure 65. Pneumatic Tubing Response: Experimental vs. Analytical Model ........................................... 80

Figure 66. Analytical Model Validation with Experimental Data (140) (141) ............................................ 81

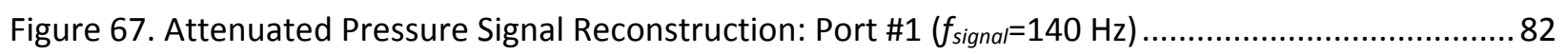

Figure 68. Experimental Measurement of CCNACA0018 Surface Pressure Distribution............................84

Figure 69. Grid Independence Study: Farfield Mesh for a.) Mesh 1, b.) Mesh 2, c.) Mesh 3, d.) Mesh 4 .. 89

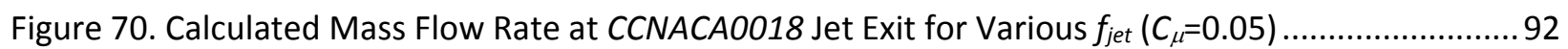

Figure 71. CFD Grid Independence Study: Static Airfoil $C_{p}$-Distributions (Baseline, $R e=180 \times 10^{3}$ ) ............ 94

Figure 72. CCNACA Model Surface Pressure Measurement Fidelity Testing..........................................97

Figure 73. Surface Pressure Instrumentation Check: Baseline CCNACA0018 $\left[\operatorname{Re}=300 \times 10^{3}, \alpha=0^{\circ}\right] \ldots \ldots \ldots . .98$

Figure 74. Surface Pressure Instrumentation Check: Baseline CCNACAO018 $\left[R e=300 \times 10^{3}, \alpha=10^{\circ}\right] \ldots \ldots \ldots . . .98$

Figure 75. Influence of Blunt TE on Lift Performance: a.) Smith, et al. (67) b.) Panther, et al. (38) ......... 99

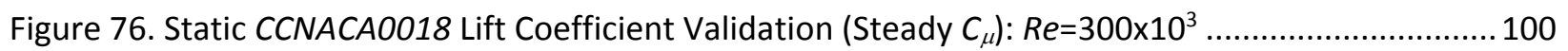

Figure 77. Influence of Pulsed CC Jet on Suction Surface $C p$-Distribution: $R e=300 \times 10^{3}, \alpha=10^{\circ} \ldots \ldots \ldots \ldots . . . . .101$

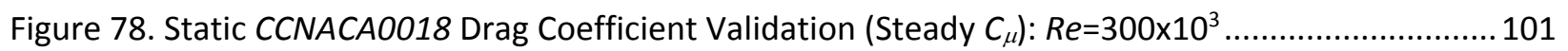

Figure 79. Static CCNACA0018 Moment Coefficient Validation (Baseline): $R e=300 \times 10^{3}(102) \ldots \ldots \ldots \ldots . . . . .102$

Figure 80. Static CCNACA0018 Lift Performance with Pulsed CC: $R e=180 \times 10^{3}$....................................... 104

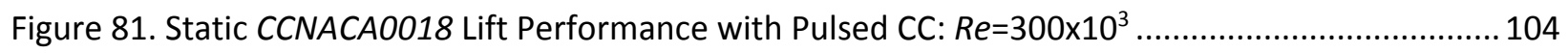




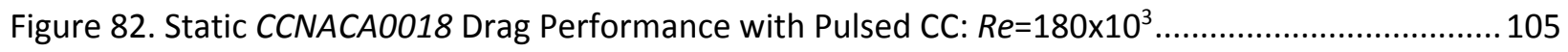

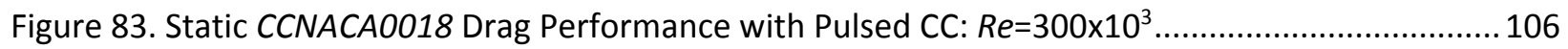

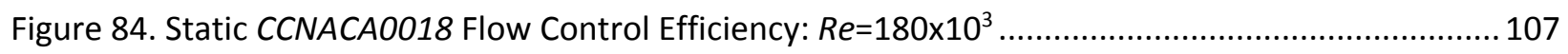

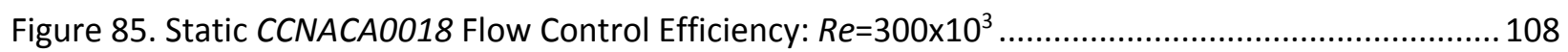

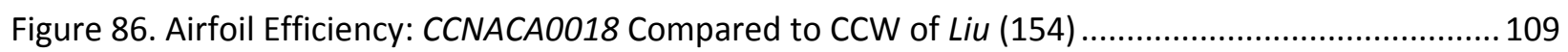

Figure 87. Baseline CCNACA0018 Lift Compared to Data from the Literature ......................................... 110

Figure 88. Baseline CCNACA0018 Drag Compared to Data from the Literature .................................... 110

Figure 89.Influence of Airfoil Pitch on Integrated Lift Coefficient: $R e=180 \times 103, k=0.100\left(\alpha_{\max }=30^{\circ}\right) \ldots . .111$

Figure 90. Influence of Dynamic Pitch on CCNACA0018 Total Airfoil Lift ............................................. 112

Figure 91. Influence of Dynamic Pitch on CCNACA0018 Total Airfoil Lift-to-Drag ................................. 112

Figure 92. Influence of Dynamic Pitch on CCNACA0018 Total Airfoil Efficiency.................................... 113

Figure 93. Pitching CCNACA0018 Lift Performance with Steady CC: $R e=180 \times 10^{3}, k=0.050\left(\alpha_{\max }=25^{\circ}\right) \ldots .114$

Figure 94. Pitching CCNACA0018 Lift Performance with Steady CC: $R e=180 \times 10^{3}, k=0.100\left(\alpha_{\max }=25^{\circ}\right) \ldots .115$

Figure 95. Pitching CCNACA0018 Lift Performance with Steady CC: $R e=300 \times 10^{3}, k=0.050\left(\alpha_{\max }=25^{\circ}\right) \ldots .116$

Figure 96. Pitching CCNACA0018 Lift Performance with Pulsed CC: $R e=180 \times 10^{3}, k=0.100\left(\alpha_{\max }=30^{\circ}\right) \ldots .117$

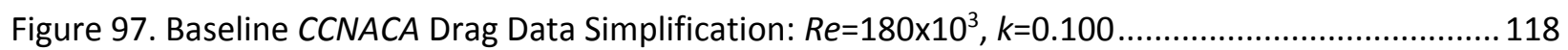

Figure 98. CCNACA0018 Drag Measurements: Static vs. Dynamic Pitch $\left(R e=300 \times 10^{3}\right)$.......................... 119

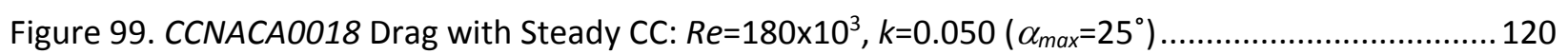

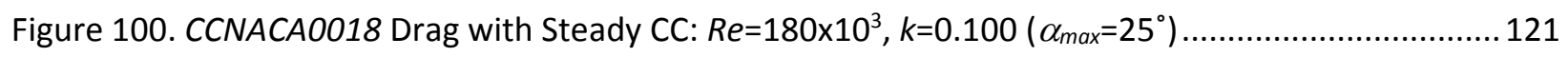

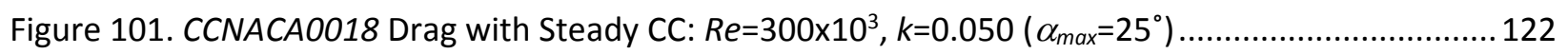

Figure 102. CCNACA0018 Drag with Pulsed CC $\left(C_{\mu}=0.01\right): R e=180 \times 10^{3}, k=0.100\left(\alpha_{\max }=25^{\circ}\right) \ldots \ldots \ldots \ldots \ldots . . .123$

Figure 103. $C C N A C A 0018$ Drag with Pulsed CC $\left(C_{\mu}=0.05\right): R e=180 \times 10^{3}, k=0.100\left(\alpha_{\max }=25^{\circ}\right) \ldots \ldots \ldots \ldots \ldots \ldots . .124$

Figure 104. CCNACA0018 Drag with Pulsed CC $\left(C_{\mu}=0.05\right): R e=180 \times 10^{3}, k=0.100\left(\alpha_{\max }=30^{\circ}\right) \ldots \ldots \ldots \ldots \ldots . . .125$

Figure 105. CCNACA0018 Efficiency with Steady CC: $R e=180 \times 10^{3}, k=0.100\left(\alpha_{\max }=25^{\circ}\right) \ldots \ldots \ldots \ldots \ldots \ldots \ldots \ldots . . . . . . . . . .126$

Figure 106. CCNACA0018 Efficiency with Pulsed CC $\left(C_{\mu}=0.01\right): R e=180 \times 10^{3}, k=0.050\left(\alpha_{\max }=25^{\circ}\right) \ldots \ldots \ldots . .127$

Figure 107. CCNACA0018 Efficiency with Pulsed CC $\left(C_{\mu}=0.05\right): R e=180 \times 10^{3}, k=0.050\left(\alpha_{\max }=25^{\circ}\right) \ldots \ldots \ldots . .128$

Figure 108. CCNACA0018 Efficiency with Pulsed CC $\left(C_{\mu}=0.01\right): R e=180 \times 10^{3}, k=0.100\left(\alpha_{\max }=25^{\circ}\right) \ldots \ldots \ldots . .129$

Figure 109. CCNACA0018 Efficiency with Pulsed CC $\left(C_{\mu}=0.05\right): R e=180 \times 10^{3}, k=0.100\left(\alpha_{\max }=25^{\circ}\right) \ldots \ldots \ldots . . .130$

Figure 110. CCNACA0018 Efficiency with Pulsed CC $\left(C_{\mu}=0.05\right): R e=180 \times 10^{3}, k=0.100\left(\alpha_{\max }=30^{\circ}\right) \ldots \ldots \ldots . . .131$

Figure 111. CCNACA0018 Efficiency with Steady and Pulsed CC: $R e=300 \times 10^{3}, k=0.050\left(\alpha_{\max }=25^{\circ}\right) \ldots \ldots .132$

Figure 112. Baseline $C_{p}$-Distributions $\left(R e=180 \times 10^{3} \alpha=10^{\circ}\right)$ : CFD vs. Experiment ..................................133

Figure 113. Influence of Jet Frequency on CCNACA0018 Lift Performance: $R e=180 \times 10^{3}, \alpha=0^{\circ} \ldots \ldots \ldots \ldots . . .134$

Figure 114. Velocity Magnitude of Baseline CCNACA0018: Laminar ( $\left.R e=180 \times 10^{3}, \alpha=0^{\circ}, C_{\mu}=0, S t=0\right) \ldots . .135$

Figure 115. Numerical Analysis of Pitching Baseline CCNACA0018: Comparison to Experiment..............136

Figure 116. Pitching CCNACA0018 Lift $\left(C_{\mu}=0.05, S t=0.00\right)$ : CFD vs Experiment ........................................ 137

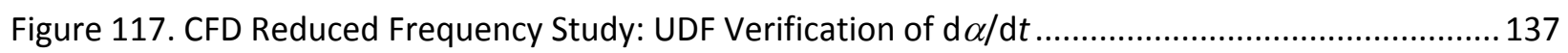

Figure 118. Static Pressure Contours for $V_{D S V}$ Calculation $\left(R e=180 \times 10^{3}, k=0.100, C_{\mu}=0.01(S t=0.00)\right) \ldots . .139$

Figure 119. Influence of CC on Vortex Shedding $\left(R e=180 \times 10^{3}, k=0.100, \alpha=28^{\circ} \uparrow\right)$............................. 140

Figure 120. Static Pressure Contours: Dynamic Stall Progression $\left(R e=180 \times 10^{3}, k=0.100, \alpha_{\max }=30^{\circ}\right) \ldots \ldots .141$

Figure 121. Influence of Various St on Pitching CCNACA0018 Lift $\left(C_{\mu}=0.01\right): \alpha(t)=0^{\circ}+20^{\circ} \sin (\omega \cdot t) \ldots \ldots \ldots .143$

Figure 122. Overlay of Normalized Lift and Mass Flow Curves: $R e=180 \times 10^{3}, C_{\mu}=0.01(S t=0.50) \ldots \ldots \ldots . . .143$ 
Figure 123. Influence of St on Pitching CCNACA0018 Lift $\left(C_{\mu}=0.05\right): \alpha(t)=0^{\circ}+20^{\circ} \sin (\omega \cdot t) \ldots \ldots \ldots \ldots \ldots \ldots \ldots \ldots \ldots \ldots \ldots . . .144$

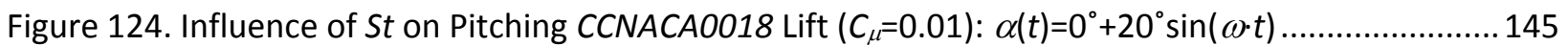

Figure 125. Influence of St on Pitching CCNACA0018 Lift $\left(C_{\mu}=0.05\right): \alpha(t)=0^{\circ}+20^{\circ} \sin (\omega \cdot t) \ldots \ldots \ldots \ldots \ldots \ldots . . . . . . .145$

Figure 126. Influence of $S t$ on Pitching CCNACA0018 Efficiency $\left(C_{\mu}=0.05\right): \alpha(t)=0^{\circ}+20^{\circ} \sin (\omega t) \ldots \ldots \ldots \ldots . . .146$

Figure 127. Baseline Lift Enhancement Pitching CCNACA0018 Airfoil: $R e=180 \times 10^{3}(k=0.100)$............... 147

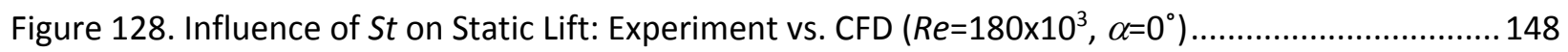

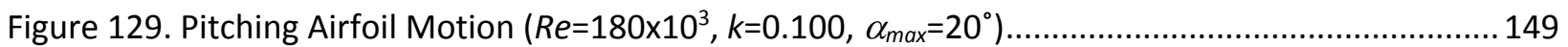

Figure 130. CCVAWT Lift Curves: Alternate Upper and Lower Slot Blowing: $\alpha(t)=0^{\circ}+25^{\circ} \sin (\omega \cdot t) \ldots \ldots \ldots . . .153$

Figure 131. Measured CCNACA0018 Jet Velocity: $R e=180 \times 10^{3} C_{\mu}=0.01 \mathrm{St}=0.00\left(f_{\text {act }}=0 \mathrm{~Hz}\right) \ldots \ldots \ldots \ldots \ldots \ldots . . . . . . .175$

Figure 132. Measured CCNACA0018 Jet Velocity: $R e=180 \times 10^{3}\left\langle c_{\mu}>=0.01 \mathrm{St}=0.25\left(f_{\text {jet }}=16.5 \mathrm{~Hz}\right) \ldots \ldots \ldots \ldots .175\right.$

Figure 133. Measured CCNACA0018 Jet Velocity: $R e=180 \times 10^{3}<c_{\mu}>=0.01 \mathrm{St}=0.50\left(f_{\text {jet }}=34 \mathrm{~Hz}\right) \ldots \ldots \ldots \ldots \ldots .176$

Figure 134. Measured CCNACA0018 Jet Velocity: $R e=180 \times 10^{3}\left\langle c_{\mu}>=0.01 S t=1.00\left(f_{j e t}=129 \mathrm{~Hz}\right) \ldots \ldots \ldots \ldots . . .176\right.$

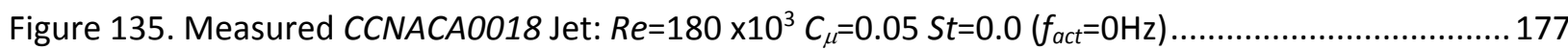

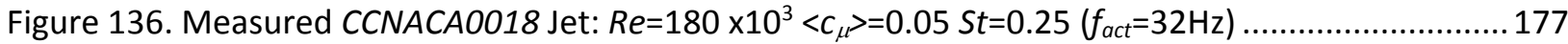

Figure 137. Measured CCNACA0018 Jet Velocity: $R e=180 \times 10^{3}<c_{\mu}>=0.05 \mathrm{St}=0.5\left(f_{\text {act }}=64 \mathrm{~Hz}\right) \ldots \ldots \ldots \ldots \ldots .178$

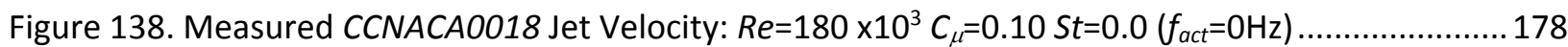

Figure 139. Measured CCNACA0018 Jet Velocity: $R e=300 \times 10^{3} \mathrm{C}_{\mu}=0.01 \mathrm{St}=0.0\left(f_{\text {act }}=0 \mathrm{~Hz}\right) \ldots \ldots \ldots \ldots \ldots \ldots \ldots \ldots \ldots \ldots \ldots \ldots . . .179$

Figure 140. Measured CCNACA0018 Jet Velocity: $R e=300 \times 10^{3}<c_{\mu}>0.01 \mathrm{St}=0.5\left(f_{\text {act }}=26 \mathrm{~Hz}\right)$.................179

Figure 141. Measured CCNACA0018 Jet Velocity: $R e=300 \times 10^{3} C_{\mu}=0.05 S t=0.0\left(f_{\text {act }}=0 \mathrm{~Hz}\right) \ldots \ldots \ldots \ldots \ldots \ldots . \ldots \ldots$

Figure 142. Measured CCNACA0018 Jet Velocity: $R e=300 \times 10^{3}\left\langle c_{\mu}\right\rangle=0.05 \mathrm{St}=0.5\left(f_{\text {act }}=54 \mathrm{~Hz}\right) \ldots \ldots \ldots \ldots \ldots . . . . .180$

Figure 143. Measured CCNACA0018 Jet Velocity: $R e=300 \times 10^{3} C_{\mu}=0.10 \mathrm{St}=0.0\left(f_{\text {act }}=\mathrm{OHz}\right) \ldots \ldots \ldots \ldots \ldots \ldots . . . \ldots 1$

Figure 144. ScaniValve Pressure Port Calibration Curves (Date: 05.01.13) ........................................ 184

Figure 145. ScaniValve Pressure Port Calibration Curves (Date: 05.02.2013) ...................................... 185

Figure 146. ScaniValve Pressure Port Calibration Curves (Date: 05.03.2013) ...................................... 186

Figure 147. ScaniValve Pressure Port Calibration Curves (05.04.2013) ............................................... 187

Figure 148. ScaniValve Pressure Port Calibration Curves (05.05.2013) ............................................. 188

Figure 149. ScaniValve Pressure Port Calibration Curves (05.06.2013) ............................................. 189

Figure 150. ScaniValve Pressure Port Calibration Curves (05.07.2013) ................................................. 190

Figure 151. Influence of Sampling Frequency on ScaniValve Pressure Measurements ......................... 191

Figure 152. Generalized Pneumatic Tube-Transducer System (138)........................................................ 192

Figure 153. ScaniValve ZOC33 Pressure Transducer Internal Volume (161) ........................................ 193

Figure 154. Analytical Frequency Response Model of CCNACA Pressure Port Tubing System ................ 195

Figure 155. Pneumatic Tubing Transfer Function Measurement: 2 Condenser Mic Signal Analysis ....... 196

Figure 156. DAQ Program to Measure Frequency Response Tubing ................................................ 197

Figure 157. WVU Aero. Lab Noise Floor during Frequency Response Testing .................................... 197

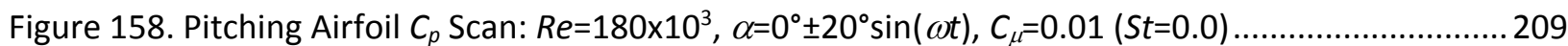

Figure 159. DYNAMCO DASH-1 Valve Specification Sheet (165) ..........................................................210

Figure 160. DYNAMCO Dash-1 Pneumatic Valve (CCNACA0018 Oscillatory Jet Driver) (165) ................ 211

Figure 161. CCNACA0018 Model Plenum Controls: MOSFET Power Control IC for Jet Actuation (166).. 211

Figure 162. US DIGITAL MAE3 Absolute Encoder Geometry (167) ..................................................2 213

Figure 163.KING 7520 Series Pneumatic Flow Meter: 0-42scfm (168).............................................. 214 
Figure 164. HEDLAND Flow Meter with Pressure Gage: 0.5-5 scfm (169) ........................................... 214

Figure 165. Pressure Instrumentation: (a.) HEISE PTE-1 (170), (b.) DWYER Series 475 (171) ................. 215

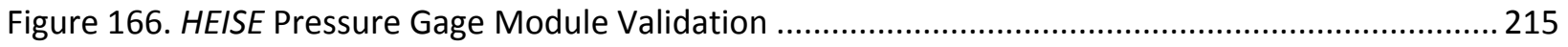

Figure 167. Hotwire Calibration generated by TSI THERMAL PRO Software ........................................216

Figure 168. Static Pressure Contours: Convection of Small Scale Vorticity for Various CC Modes .......... 217

Figure 169. Static Pressure Contours: Convection of DSV with Various CC Modes.................................218

Figure 170. Vorticity Magnitude Contours: DS Events on Pitching CCNACA0018 (Baseline) ...................219

Figure 171. Vorticity Magnitude Contours: DS Events on Pitching CCNACA0018 $\left(C_{\mu}=0.01, S t=0.00\right) \ldots . .220$

Figure 172. Vorticity Magnitude Contours: DS Events on Pitching CCNACA0018 $\left(C_{\mu}=0.01, S t=1.00\right) \ldots . .221$

Figure 173. Vorticity Magnitude Contours: DS Events on Pitching CCNACA0018 $\left(C_{\mu}=0.05, S t=0.00\right) \ldots . . .222$

Figure 174. Vorticity Magnitude Contours: DS Events on Pitching CCNACA0018 $\left(C_{\mu}=0.05, S t=1.00\right) \ldots . . .223$ 


\section{LIST OF TABLES}

Table 1. Advantages and Disadvantages of VAWT and HAWT ............................................................ 2

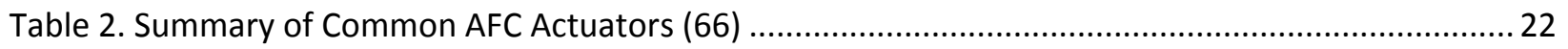

Table 3. CFD Literature Review: Static Airfoil Simulation Conditions ...................................................... 34

Table 4. Experimental Literature Review: Static Airfoil Tests .................................................................... 35

Table 5. Influence of $\alpha_{\max }$ on NACA0012 Stall Regimes: $R e=2 \times 10^{6}, k=0.1$ (62) .................................... 45

Table 6. Summary of Dynamic Stall Regimes based on AoA (105) ....................................................... 46

Table 7. Dynamic Stall Vortex Shedding Characteristics for Pitching CC Airfoil (109) .............................. 53

Table 8. Literature Review Summary: Experimental Pitching Airfoil Studies ............................................ 59

Table 9. Literature Review Summary: Numerical Pitching Airfoil Studies .................................................60

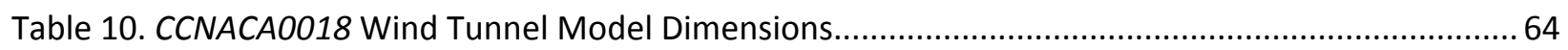

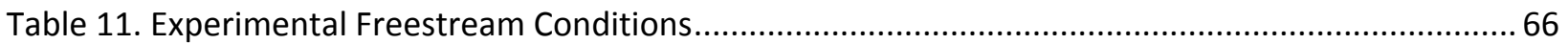

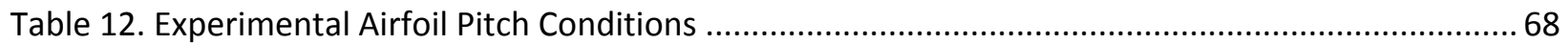

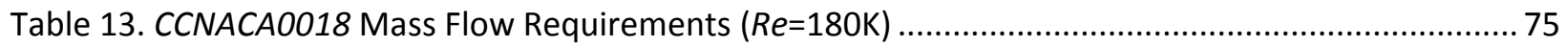

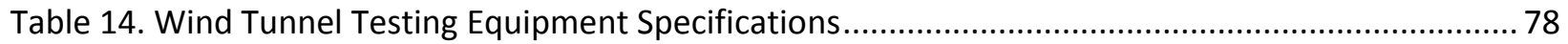

Table 15. Experimental Uncertainty of $C_{p}$ Measurements Compared to Literature ................................ 86

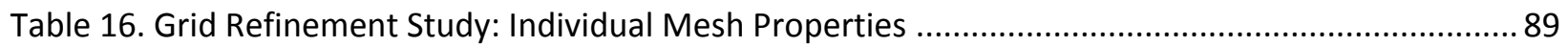

Table 17. Pneumatic Tube Geometry and Material for Static CCNACA0018 Testing ................................96

Table 18. CCNACAO018 Mass Flow Requirements for Various Experimental Blowing Conditions ...........103

Table 19. Baseline Numerical Model Results Compared to Experimental CCNACA0018 Data ................136

Table 20. Influence of Reduced Frequency on CCNACA Total Lift: $\alpha(t)=0^{\circ}+20^{\circ} \sin (\omega \cdot t)\left[R e=180 \times 10^{3}\right] \ldots .138$

Table 21. Influence of $\alpha_{\max }$ on CCNACA0018 Maximum Lift: $\alpha(t)=0^{\circ}+20^{\circ} \sin (\omega t)\left[R e=180 \times 10^{3}\right] \ldots \ldots \ldots . . .138$

Table 22. CCNACA0018 Mass Flow Requirements for Various CFD Blowing Conditions $\left(R e=180 \times 10^{3}\right) \ldots 139$

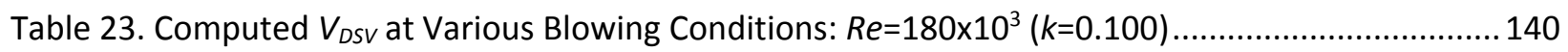

Table 24. Pulsed CC Jet Conditions from Numerical Analysis............................................................... 141

Table 25. Influence of Steady and Pulsed CC on Pitching CCNACA0018 Total Lift $\left(\Delta C_{1}\right) \ldots \ldots \ldots \ldots \ldots \ldots \ldots \ldots . . . . . . . . . . . .142$

Table 26. Experimental Trend Analysis: Influence of Re on Pitching CCNACA0018 Total Lift ..................150

Table 27. Experimental Trend Analysis: Influence of $k$ on Pitching CCNACA0018 Total Lift ...................150

Table 28. Numerical Trend Analysis: Influence of $k$ on Pitching CCNACA0018 Total Lift ........................151

Table 29. Experimental Trend Analysis: Influence of $\alpha_{\max }$ on Pitching CCNACA0018 Total Lift ................151

Table 30. Numerical Trend Analysis: Influence of $\alpha_{\max }$ on Pitching CCNACA0018 Total Lift ....................151

Table 31. Influence of Dynamic Pitch Variables on Baseline CCNACA0018 Performance ........................ 154

Table 32. Influence of Dynamic Pitch Variables on Steady and Pulsed Jet CCNACA0018 Performance .. 154

Table 33. Static Wind Tunnel Test Matrix: $R e=180 \times 10^{3}$ (All $C_{\mu}$ Conditions) .......................................... 198

Table 34. Static Wind Tunnel Test Matrix: $R e=300 \times 10^{3}$ (Baseline, $C_{\mu}=$ Steady Cases).............................. 199

Table 35. Static Wind Tunnel Test Matrix: $R e=300 \times 10^{3}$ (Pulsed $C_{\mu}=0.01,0.05$ Cases) .......................... 200

Table 36. Static Wind Tunnel Test Matrix: $R e=300 \times 10^{3}$ (Pulsed $C_{\mu}=0.10$ Pulsed Cases).......................... 201

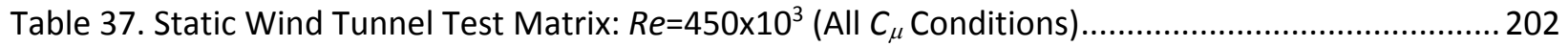

Table 38. Dynamic Pitch Wind Tunnel Test Matrix: $R e=180 \times 10^{3}, \alpha_{\max }=25^{\circ}$ (All $C_{\mu}$ Conditions) ............... 203

Table 39. Dynamic Pitch Wind Tunnel Test Matrix: $R e=180 \times 10^{3}, \alpha_{\max }=30^{\circ}$ (All $C_{\mu}$ Conditions) ............... 204

Table 40. Dynamic Pitch Wind Tunnel Test Matrix: $R e=300 \times 10^{3}, \alpha_{\max }=25^{\circ}$ (All $C_{\mu}$ Conditions) ...............204 
Table 41. Dynamic Pitch Wind Tunnel Test Matrix: $R e=450 \times 10^{3}, \alpha_{\max }=25^{\circ}$ (All $C_{\mu}$ Conditions) ........

Table 42. Dynamic Pitch Wind Tunnel Test Matrix: $R e=450 \times 10^{3}, \alpha_{\max }=30^{\circ}$ (All $C_{\mu}$ Conditions) ....... 


\section{CHAPTER 1. INTRODUCTION}

\subsection{Renewable Energy}

It would be hard to argue that a wholesale replacement of fossil fuel consumption is attainable in the foreseeable future due to ever increasing population growth and energy demands. Such demands have warranted the growing search for innovative improvements in renewable energy solutions. The improvement of renewable energy technologies is needed to meet growing rates of consumption and concerns over the environmental impact of the use of fossil fuels. Furthermore, one simply cannot argue that fossil fuels, particularly coal, oil and natural gas, are of infinite supply. Thus, pursuit of sustainable energy sources has merit due to both supply and emissions advantages.

Current forms of renewable energy capture include hydrodynamic, solar, wind, and geothermal technologies to name a few. Specific to this research effort, wind turbines are one such solution, which has the potential to provide inexpensive and localized renewable energy. The harnessing of wind energy is a promising technology able to provide a portion of the power requirements in many regions of the world.

\subsection{Wind Energy: HAWT vs. VAWT}

The growth and development of wind turbines has principally been driven by the projected increasing costs of fossil energy and the public mandate to improve environmental quality. Wind turbines can be categorized based on the axis the blades rotate. The two common orientations of wind machines are horizontal axis wind turbines (HAWTs) and vertical axis wind turbines (VAWTs). The most familiar, and commonly produced, orientation is the HAWT, which resembles an aircraft propeller with rotational axis parallel to the horizon. However, VAWTs have not yet benefited from the years of development undergone by HAWTs, and are reemerging for off-grid, urban-scale (1), and even offshore utility scale energy production. A typical VAWT bears resemblance to an eggbeater, revolving around a vertical axis as a carousel does.

Many variations of vertical axis turbines exist today, commonly classified as Darrieus- (lift-driven blades), Savonius- (drag-driven blades), or a hybrid-turbine that relies on both concepts for operation. Figure 1 includes common forms of HAWTs and VAWTs, along with some innovative vertical axis orientations designed for improved performance over a range of wind conditions. 


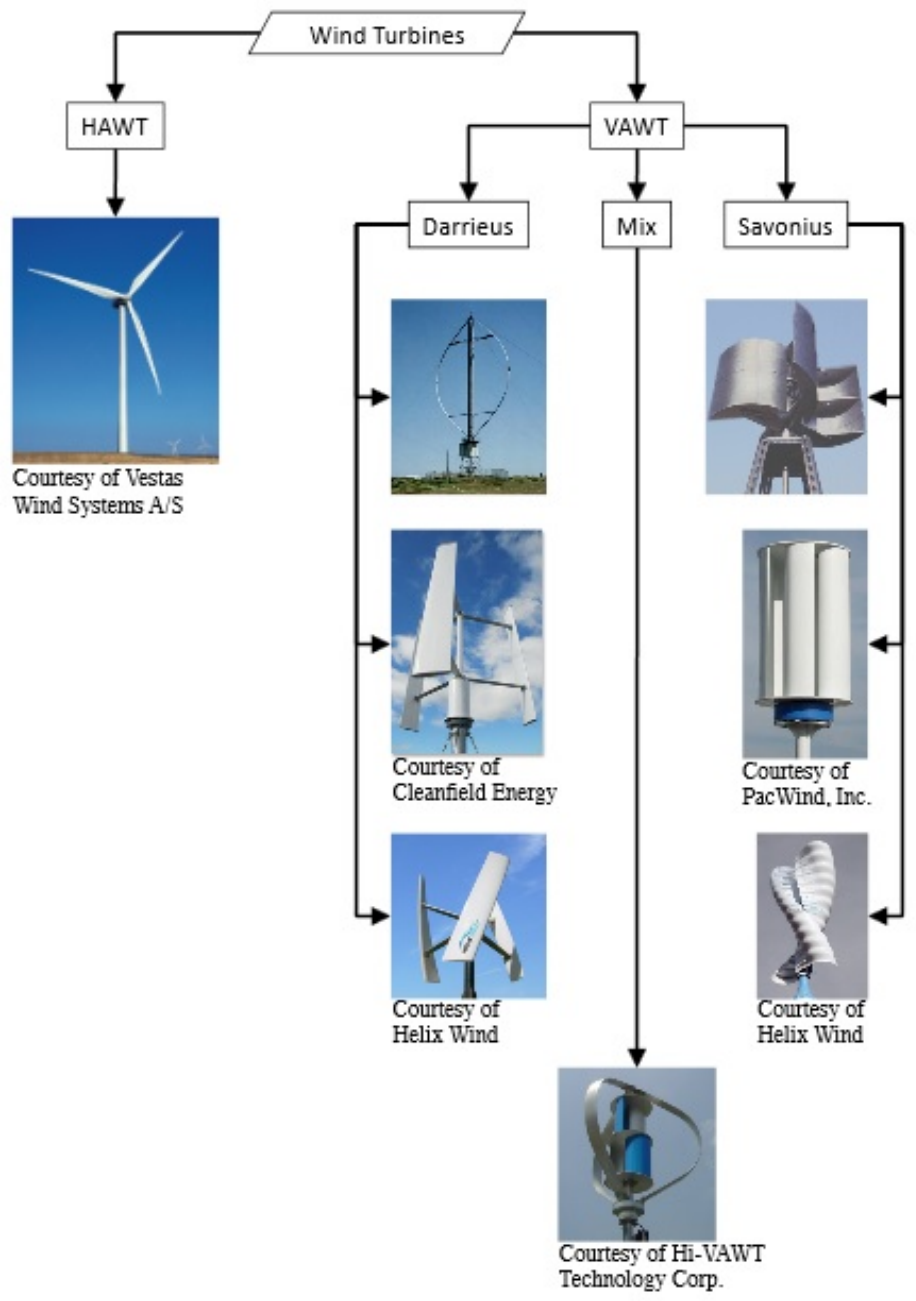

Figure 1. Wind Turbine Variations (2)

Comparing the VAWT and HAWT, crucial differences exist between structural design and blade aerodynamics. In general, the VAWT is advantageous in terms of structural integrity, fabrication, installation, and maintenance, while the HAWT currently maintains improved efficiency due to higher aerodynamic performance. For comparable units, VAWTs have a maximum average power efficiency of approximately 0.40 , while HAWTS operate at or near an efficiency of 0.49 (3). Principal differences between turbine orientations are summarized in Table 1.

Table 1. Advantages and Disadvantages of VAWT and HAWT

\begin{tabular}{|c|c|}
\hline VAWT & HAWT \\
\hline (+) omnidirectional operation (axisymmetric) & (-) yaw mechanism needed for variable wind \\
\hline (+) generator: ground proximity & (-) generator: located at top of tower \\
\hline (+) lower manufacturing cost (straight blades) & (-) higher manufaturing cost (twisted blades) \\
\hline (+) increased blade durability (dual mounting points) & (-) cantilevered blades subject to stress concentration \& failure \\
\hline (+) low noise due to smaller rotational speeds & (-) loud operation due to very high blade tip speeds \\
\hline$(-)$ variable blade AoA during rotation $( \pm \alpha)$ & $(+)$ constant blade AoA during operation ( $\alpha \approx$ constant) \\
\hline (-) currently does not have reliable blade pitch: DS effects at low TSR & (+) blade pitch capabilities: mitigates DS effects (larger operation envelope) \\
\hline$(-)$ currently does not have reliable self-start & $(+)$ self starting capabilities \\
\hline
\end{tabular}


Concerning structural integrity, large industrial cranes and even helicopters are necessary for HAWT installation which can be costly and often impossible due to the adverse terrain often common to locales where high energy winds are prevalent. Also, spanwise uniformity of straight VAWT blades can dramatically reduce manufacturing costs, particularly for large-scale turbines (4). Comparing the power train subsystems, VAWT turbine shaft assemblies carry axial and torque loads only, with minimal bending loads like those on a HAWT shaft (5). VAWTs also operate at lower rotational speeds, resulting in lower noise generation relative to HAWTs and a lesser threat to migratory birds (4).

Comparing turbine aerodynamics, VAWT blade operation is insensitive to wind direction. Both wind speed and direction are variable and unpredictable, making omnidirectional operation very attractive in terms of energy capture efficiency. HAWTs require continual closed loop control of turbine yaw mechanisms adapted to changing wind direction. However, HAWTs have blade pitch mechanisms that permit blade trimming for more effective energy collection over a larger range of wind speeds. In general, a HAWT blade section experiences constant angle of attack (AoA) during revolution for a given wind condition. VAWTs are subject to dynamic stall (DS) as each blade is pitched beyond static stall limits at low rotational speeds. The effects of dynamic stall on VAWT performance, are a sharp drop in blade lift that decreases power output while imparting unsteady loading on the central shaft, generator, and drive train (4) (6) (7). Furthermore, within the earth's boundary layer there is a gradient of wind speed with height, that can influence the performance of both large scale HAWTs and VAWTs (5).

To date, no technology to control or mitigate the effects of dynamic stall on VAWT blades has reached the built environment. Recent attempts to improve VAWT blade aerodynamics and performance output have included mechanical high lift augmentation such as TE flaps and multiple airfoil components (8), which introduces additional weight and mechanical complexity to the design (9). Some promise of performance enhancements have been shown in laboratory environments, including research efforts applying flow control to VAWT blades such as LE plasma actuation (10), synthetic jets (11), and various other techniques (12) (13) (14) (15) (16), but many are still early in development or have proven unfeasible.

\subsection{Vertical Axis Wind Turbine Performance}

This section includes a brief overview of the VAWT operational environment and the associated common language and nomenclature. In addition, parameters commonly used to analyze power output (i.e. performance) will be discussed. The unique characteristics of the VAWT flow field are of particular importance as they govern wind tunnel test conditions of the current study. 
VAWT operating speeds are classified by the ratio of blade speed to wind speed $\left(V_{\infty}\right)$, commonly known as the tip-speed ratio (TSR or $\lambda$ ); see Equation 1 . The tangential speed $\left(V_{t}\right)$ of the airfoil is determined by turbine radius $(R)$ and rotational speed $(\omega)$.

VAWT Tip Speed Ratio (TSR)

$$
\lambda=\frac{V_{t}}{V_{\infty}}=\frac{\omega \cdot R}{V_{\infty}}
$$

A plan view diagram of VAWT blade velocity and force vectors, as functions of $V_{\infty}$ and $V_{t}$, is included in Figure 2. An important feature of VAWT blade operation is that the retreating-side rotor, containing $180^{\circ}$ in Figure 2, experiences a lower relative wind speed than the advancing blade (17).

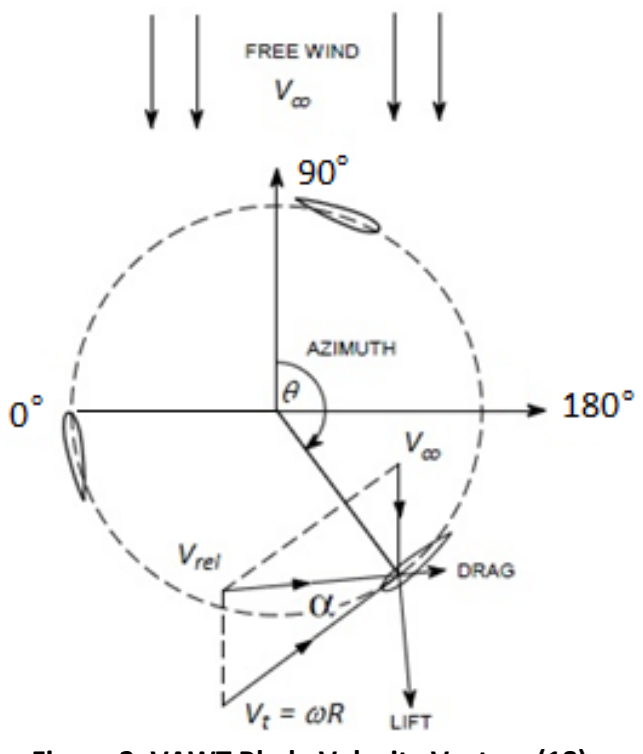

Figure 2. VAWT Blade Velocity Vectors (18)

The resultant flow $\left(V_{\text {rel }}\right)$ experienced by the blade is thus dependent upon the azimuth position $(\theta)$ and TSR $(\lambda)$, which are included in Equation 2.

VAWT Blade Relative Velocity

$$
V_{r e l}=V_{\infty} \sqrt{\left(1+\lambda^{2}\right)+2 \cdot \lambda \cdot \cos (\theta)}
$$

The angle of attack $(\alpha)$ is also governed by both $\theta$ and $\lambda$, as expressed in Equation 3 . It should be noted that Equation 3 is only an estimation as the influence of upwind airfoil wake disturbances (i.e. shed vorticity) on downwind velocity fields are not included.

VAWT Blade Angle of Attack

$$
\alpha=\tan ^{-1}\left(\frac{\cos (\theta)}{\lambda-\sin (\theta)}\right)
$$

The VAWT blade angle-of-attack function is plotted as a function of $\theta$ in Figure 3. Included in the diagram for reference is the static stall AoA $\left(\alpha_{s s}=15^{\circ}\right)$ from previous static wind tunnel tests of the 
baseline CCNACA0018 model. For pitching airfoil tests of this study, wind tunnel conditions related to $\lambda \leq 4$ will be of interest, where AoA excursions exceed $\alpha_{s s}$ and enter the DS regime. Dynamic stall causes a rapid loss in lift and subsequent drag increase that inhibit VAWT power production. From Figure 3, it should be noted that for $\lambda=2$, the blade operates outside of stall during more than $70 \%$ of rotation. It should also be noted that during the upwind portion of rotation, DS occurs on the airfoil inner surface, while during downwind operation stall events take place on the outside surface.

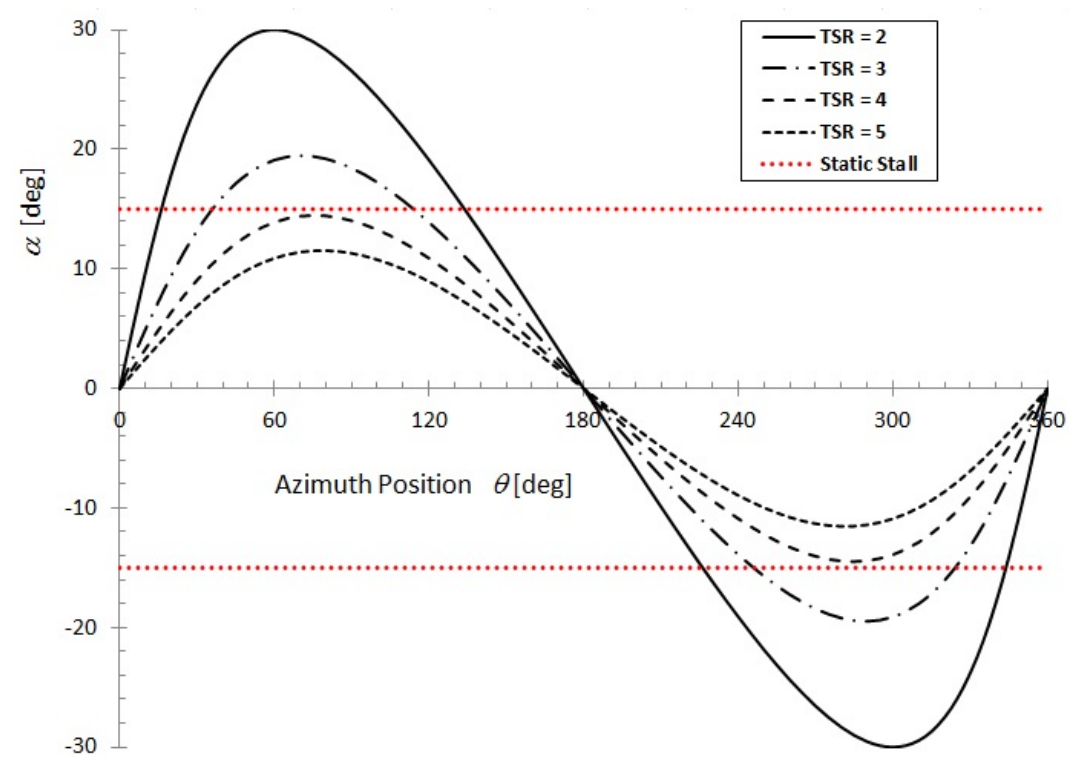

Figure 3. VAWT Blade AoA throughout Azimuth Rotation

The rotational speed of a pitching airfoil is commonly non-dimensionalized by airfoil chord $(c)$, angular speed $(\omega)$, and free stream conditions (Equation 4). It should be noted that although an operating VAWT blade experiences an unsteady $V_{\text {rel, }}$ common aerodynamic notation associated with wind tunnel testing utilizes $V_{\infty}$. Experimental testing was the core of this work, and thus $V_{\infty}$ will be used herein, based on the steady freestream velocity of the wind tunnel.

Reduced Frequency

$$
k=\frac{\omega \cdot c}{2 \cdot V_{\infty}}
$$

Equation 4

Liiva, et al. (19) and Crimi, et al. (20) describe $k$ as a measure of the degree of flowfield unsteadiness: $k=0$ represents steady flow, $k \leq 0.05$ indicates quasi-steady flow, and $k>0.05$ defines unsteady flow. Airfoil reduced frequency $(k)$ is the ratio of the convective time scale to the airfoil oscillation time scale $(1 / 2 \cdot \pi \cdot f$ in degrees, or $1 / \omega$ in radians) $(21)$.

When characterizing airfoil studies, the most common similarity parameter is the Reynolds number, a ratio of inertial to viscous forces on an airfoil (Equation 5). 


$$
\operatorname{Re}=\frac{\rho_{\infty} \cdot V_{\infty} \cdot c}{\mu}
$$

A typical VAWT blade operating within the TSR range of $(1 \leq \lambda \leq 3)$ experiences conditions in the range of $250 \times 10^{3} \leq \operatorname{Re} \leq 500 \times 10^{3}$ (22) (23). Aerodynamic forces on a VAWT blade dictate the output of the turbine. Throughout a single blade rotation, blade incidence variations influence the aerodynamic lift $(L)$ and drag $(D)$ forces on the airfoil. The lift and drag forces are more commonly presented as nondimensional lift- $\left(C_{l}\right)$ and drag-coefficients $\left(C_{d}\right)$ :

Coefficient of Lift

$$
C_{l}=\frac{2 \cdot L}{\rho_{\infty} \cdot A \cdot V_{\infty}^{2}}
$$

Equation 6

Coefficient of Drag

$$
C_{d}=\frac{2 \cdot D}{\rho_{\infty} \cdot A \cdot V_{\infty}^{2}}
$$

Each aerodynamic force has unique normal and axial components. The normal vector $\left(F_{n}\right)$ is orthogonal to the mean chord of the airfoil, and has little influence on VAWT energy generation during azimuthal rotation; the normal force is a key factor of structural design considerations, however (24). The axial force is oriented along the chord of the airfoil, regardless of $\alpha$, and is also referred to as the tangential component $\left(F_{t}\right)$. Individual force components are further detailed in Figure 4. Lift $(L)$ and drag $(D)$ forces are normal and parallel to the relative velocity component, respectively.
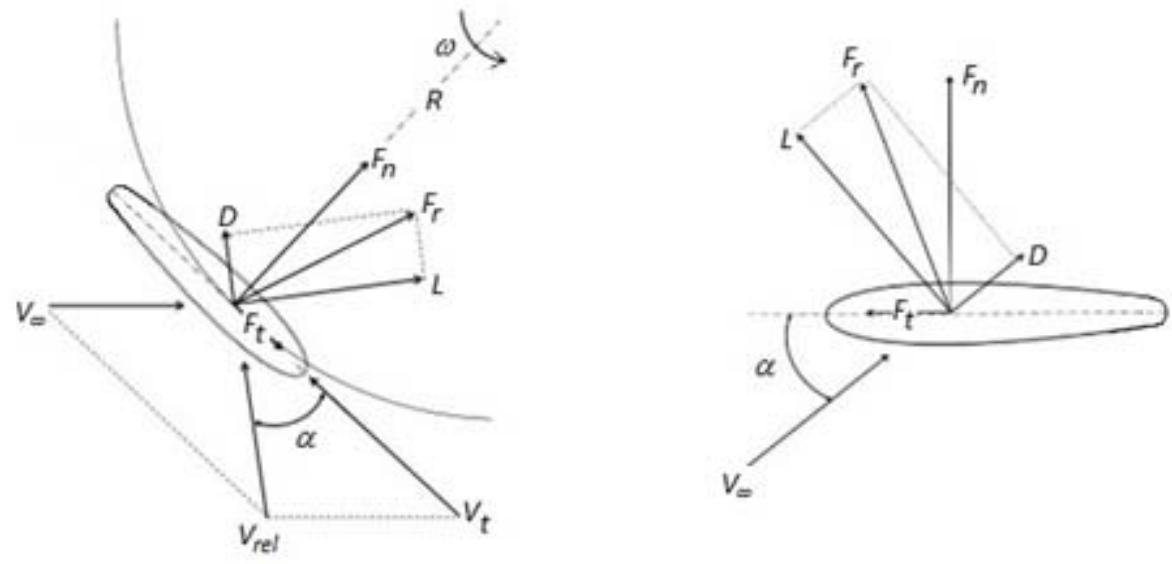

Figure 4. VAWT Blade Velocity and Force Vectors (Image modified from Carrigan (2))

A Darrieus VAWT blade utilizes aerodynamic lift to generate torque. When broken down into components, the thrust component contributes to the turbine rotation, whereas the radial component can lead to turbine vibration and blade fatigue (25). The tangential force imparted on a conventional airfoil in freestream air is given by Equation 8. The tangential force $\left(F_{t}\right)$, or torque, that drives VAWT 
rotation for power production is defined in Equation 9; the dimensionless coefficient variation is included in Equation 10. Tangential forces are also commonly referred to as thrust components.

Airfoil Tangential Force

$$
F_{t}=1 / 2 \cdot \rho_{\infty} \cdot A \cdot V_{\infty}^{2} \cdot C_{t}
$$

Equation 8

VAWT Blade Tangential Force

$$
F_{t}=(L \cdot \sin \alpha+D \cdot \cos \alpha) \cdot R_{V A W T}
$$

Equation 9

VAWT Blade Tangential Force Coefficient

$$
C_{t}=C_{l} \cdot \sin \alpha-C_{d} \cdot \cos \alpha
$$

Equation 10

The normal force experienced by a conventional airfoil in freestream air is given by Equation 11 . The normal force $\left(F_{n}\right)$ on a VAWT blade (Equation 12) acts toward the central shaft and does not produce any effect on rotor torque. The contributions of the lift force $(L)$ and drag force $(D)$ on VAWT blade normal force are evident in Equation 12. However, $F_{n}$ oscillations cause structural loading on the turbine. The normal force and coefficient form are included in Equation 12 and Equation 13, respectively.

Airfoil Normal Force

$$
F_{n}=1 / 2 \cdot \rho_{\infty} \cdot A \cdot V_{\infty}^{2} \cdot C_{n}
$$

Equation 11

VAWT Blade Normal Force

$$
F_{n}=(L \cdot \cos \alpha-D \cdot \sin \alpha) \cdot R_{V A W T}
$$

VAWT Blade Normal Force Coefficient

$$
C_{n}=C_{l} \cdot \cos \alpha+C_{d} \cdot \sin \alpha
$$

Equation 13

The aerodynamic moment about the airfoil also contributes to VAWT torque. The instantaneous power resulting from individual blade torque $\left(F_{t}\right)$, factoring the number of blades $(N)$ and rotational speed $\left(\omega_{b}\right)$, can be calculated via Equation 14.

Instantaneous VAWT Power

$$
P_{V A W T}=N \cdot F_{t} \cdot \omega_{b l}
$$

Equation 14

The available power in the wind $\left(P_{\text {WIND }}\right)$ is a function of VAWT geometry (turbine swept area, $\left.A_{V A W T}\right)$, ambient air conditions $\left(\rho_{\infty}\right)$, and wind speed $\left(V_{\infty}\right)$, included in Equation 15.

Available Wind Power

$$
P_{\text {WIND }}=1 / 2 \rho_{\infty} \cdot A_{V A W T} \cdot V_{\infty}^{3}
$$

The swept area for a double-bladed VAWT is defined by Equation 16, factoring turbine radius (RVAWT $)$ and the length $\left(I_{b l}\right)$, or span, of each blade. 
Finally, the common metric for wind turbine power classification, the turbine power coefficient $\left(C_{P, V A W T}\right)$, can be determined from the ratio of Equation 17.

VAWT Power Coefficient

$$
C_{P, V A W T}=\frac{P_{V A W T}}{P_{\text {WIND }}}=\frac{4 \cdot N \cdot F_{t} \cdot \omega_{b l}}{\rho_{\infty} \cdot A_{V A W T} \cdot V_{\infty}^{3}}
$$

For steady wind conditions, VAWT $C_{P}$ is generally computed by averaging $F_{t}$ over one rotor cycle, making $C_{P}$ independent of azimuth position, resulting in a single-valued metric characterizing VAWT performance (24). For analysis with unsteady wind conditions, performance is typically computed by averaging $P_{\text {VAWT }}$ and $P_{\text {WIND }}$ over one rotation cycle before taking the ratio (26).

The overall performance of a vertical axis rotor is mainly influenced by: rotor geometry (turbine radius and height), rotational speed $(\omega)$, airfoil shape, mean angle of attack $\left(\alpha_{o}\right)$, amplitude $\left(\alpha_{\max }\right)$, and $\operatorname{Re}(6)$ (9) (18) (27). The power produced by a VAWT at its regulated wind speed is much higher than would be anticipated from an analysis based on steady airfoil data; the additional power output is a direct consequence of an unsteady flow phenomenon known as dynamic stall (5) (28) (29).

Soraghan, et al. (30) completed analytical VAWT performance predictions with airfoils of various lift-to-drag $(L / D)$ characteristics to highlight the relationship between airfoil characteristics and optimum turbine performance (see Figure 5). Improving $L / D$ from 10 to 20 can improve VAWT power output by $100-150 \%$ for all solidity and TSR modelled. Further increasing $L / D$ continues to improve power output, with less dramatic increments. Thus, optimizing airfoil $L / D$ is a key design parameter that directly impacts VAWT performance. 


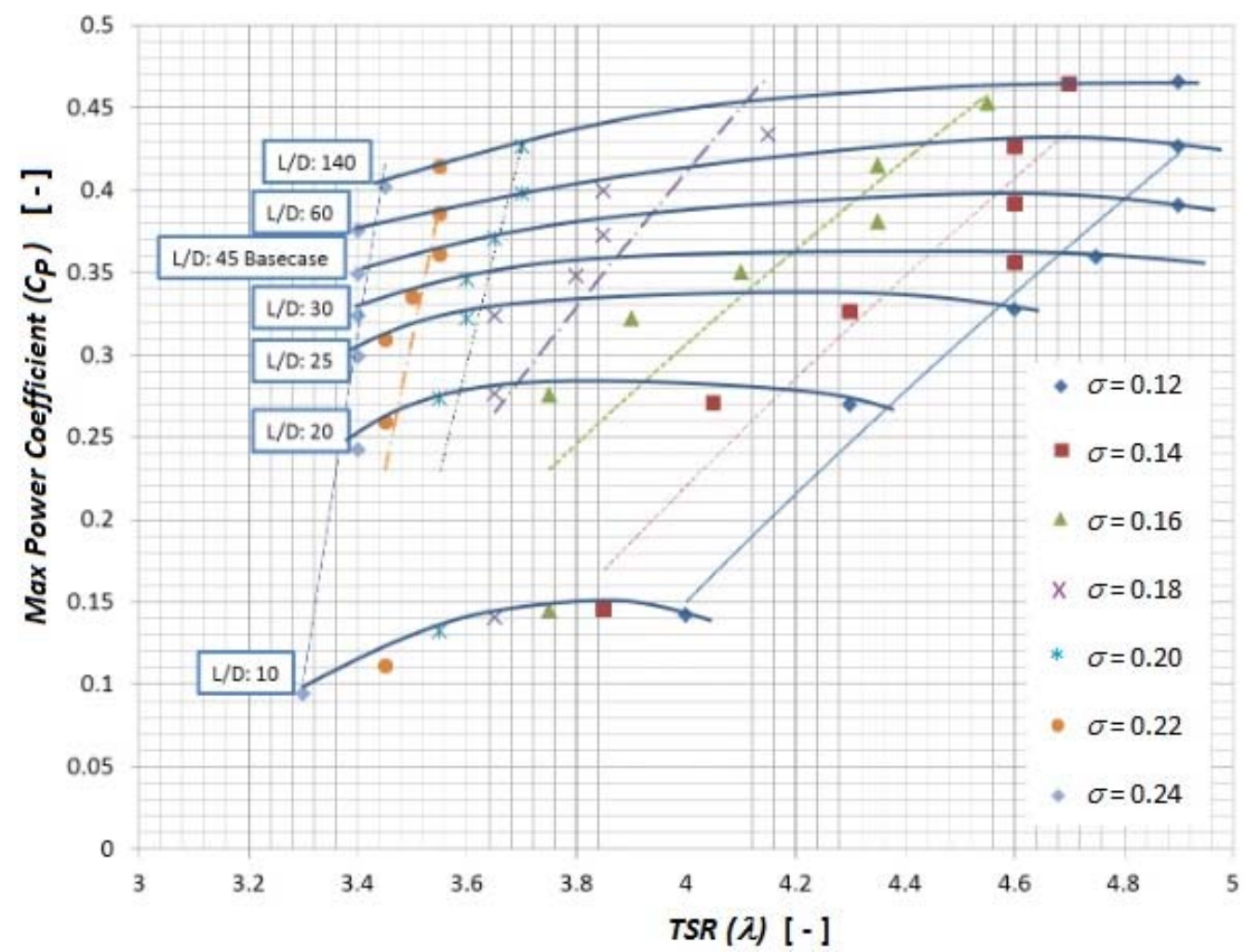

Figure 5. Influence of Solidity $(\sigma$ ) and Airfoil $L / D$ on VAWT Power Production (30)

For a given wind turbine, a minimum wind speed governs the cut-in point of operation, where minimal energy is extracted from the wind (region I of Figure 6). Power output $\left(C_{P}\right)$ increases with wind speed until, ideally, the rated wind speed is reached where maximum power output is generated. Typical HAWT blades can be feathered to produce a flat power curve as wind speed is further increased (blue line within region III of Figure 6). At maximum operating speed, or cut-out speed, the turbine shuts down until wind speeds drop to acceptable ranges to avoid excessive loading and failure. Cut-out speed is determined by the blade stall angle and structural capacity of the turbine. Standard fixed bladed VAWTs do not have the capabilities to follow the same power curve (18). Instead of the ideal, constant power curve above the rated wind speed, VAWTs show a sharp decrease in $C_{P}$ revealing a narrow operational envelope (black line within region III of Figure 6). Some form of aerodynamic load control is necessary for a VAWT to capture more energy outside its rated wind speed (31). 


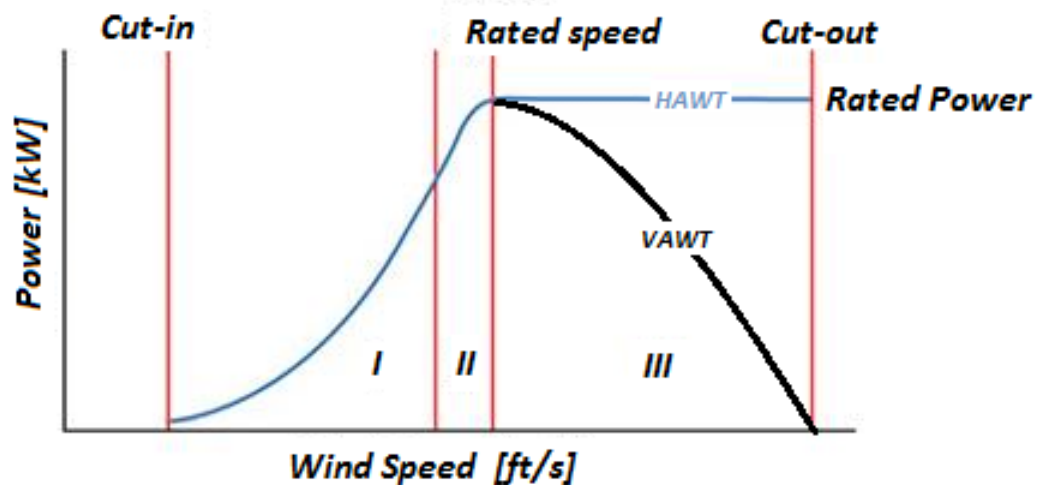

Figure 6. Ideal Wind Turbine Power Curve (31)

\subsection{Circulation Control Vertical Axis Wind Turbine (CCVAWT)}

The ongoing need for improved extraction methods to harvest renewable resources, including wind power, have prompted research by the Center for Industrial Research Applications at West Virginia University (herein "CIRA" and "WVU", respectively). Recent work at CIRA and WVU revealed proof of concept that circulation control (CC) can be applied to VAWT blades to expand the operational envelope and performance (31) (32). Large aerodynamic improvements were proven for a conventional NACA0018, modified to include TE circulation control (herein described as "CCNACA0018"), with computational (33) (34), analytical (31) (35) (36) (37), and experimental methods (38). Analytical turbine models revealed the augmented blades increased both VAWT performance and operational envelope. However, energy penalties to produce the pressurized CC jet reduced the relative VAWT performance enhancements to $15 \%$. The objective of this work is to reduce the mass flow requirements (pumping power) for a given lift performance with pulsed circulation control.

VAWTs generally have symmetrical blade profiles and common blade designs rely on the traditional NACA0018 airfoil (23) (39) (40) as a balance between aerodynamic performance and structural integrity, due primarily to the moderate $t / c$ ratio (22). The final blade shape selection, based on results from extensive CFD simulations and wind tunnel testing, was a conventional NACA0018 profile, augmented to include CC capabilities. More specifically, the sharp trailing edge (TE) of the traditional airfoil was replaced by a rounded $T E$ (also known as a Coandă surface, $r_{T E}=0.25 \mathrm{in}$ ). The chord length $(c)$ for the conventional and augmented profiles was 9in and 8.12in, respectively. Tangential blowing slots, each having a height $\left(h_{\text {slot }}\right)$ of 0.015 in, were located adjacent to the Coandă surface on both the upper and lower TE surfaces. A comparison of the conventional profile to the recently developed blade can be seen in Figure 7. 


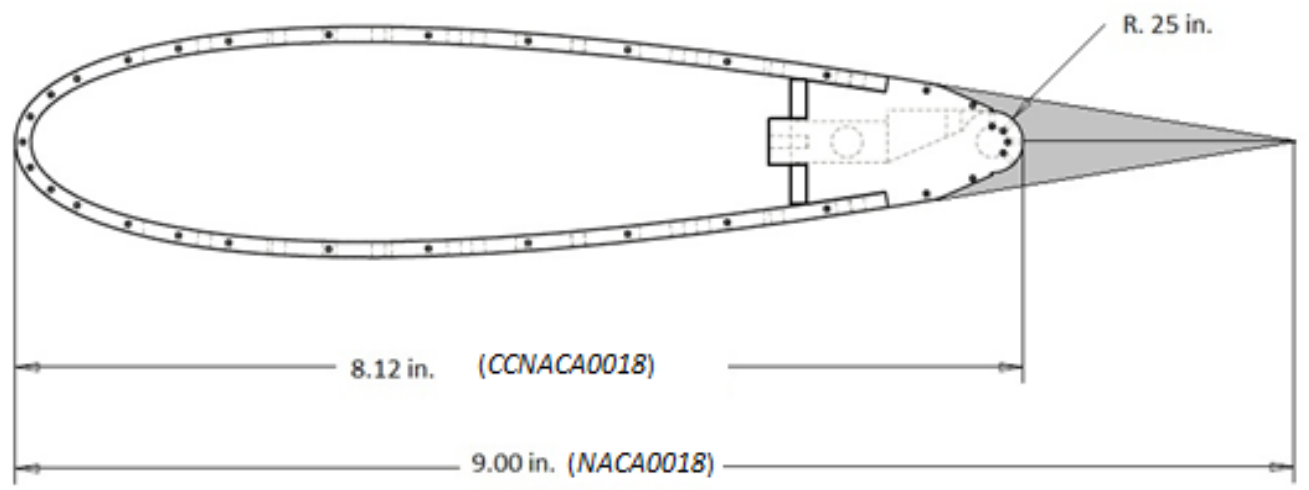

Figure 7. CIRA CCNACA0018 VAWT Blade (6)

Specific details of the profile selection process, airfoil geometry, and computational results, can be found in work from Graham, et al. (33) (34) and Panther, et al. (38). This novel blade design was experimentally validated in extensive wind tunnel experiments. Static, two-dimensional tests were conducted in WVU's Subsonic Closed Loop Wind Tunnel. Sectional aerodynamic forces $\left(C_{1}, C_{d}\right)$ were measured using both a force balance and surface pressure integrations, and validated with data from the literature for a conventional NACA0018 profile (38) (41) (42). The newly developed VAWT blade showed increased lift performance, achieving a $C_{I, \max }$ of 2.3 at a momentum coefficient of $10 \%$. Lift augmentation factors $\left(\Delta C_{l} / C_{\mu}\right)$ of nearly 30 were measured at $C_{\mu}=.02$.

The CCNACA0018 model, used during previous static testing with constant blowing, is capable of providing near surface actuation of pulsed CC flow. Pneumatic valves were included to have local control of the jet to measure the response time required for lift enhancement after control actuation. The CCNACA0018 wind tunnel CAD model, with internal pneumatic valving, can be seen below in Figure 8 .

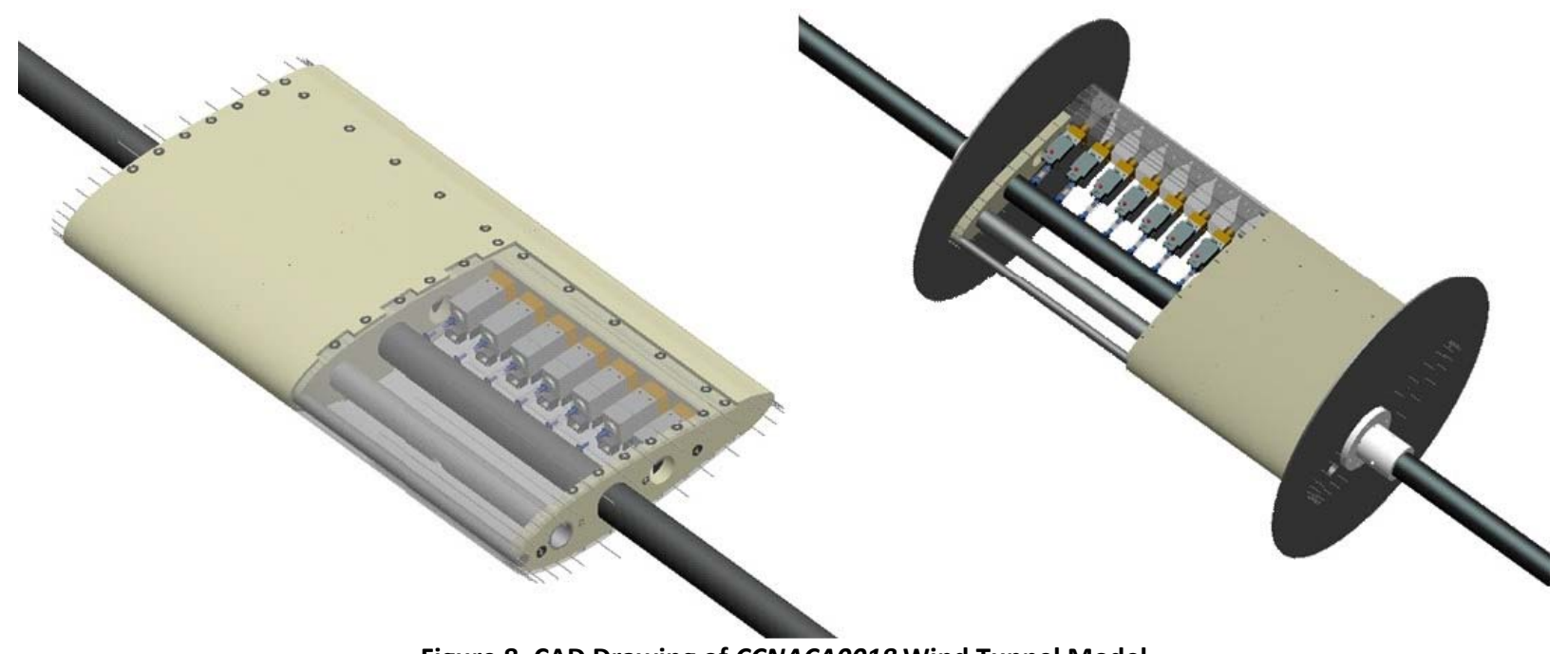

Figure 8. CAD Drawing of CCNACA0018 Wind Tunnel Model 
Static wind tunnel testing proved performance enhancements such as lift augmentation factor $\left(\Delta C_{/} / C_{\mu}\right)$, a metric used to compare the lift improvement due to $\mathrm{CC}$ relative to the baseline model, is included in Figure 9 for various $\alpha$.

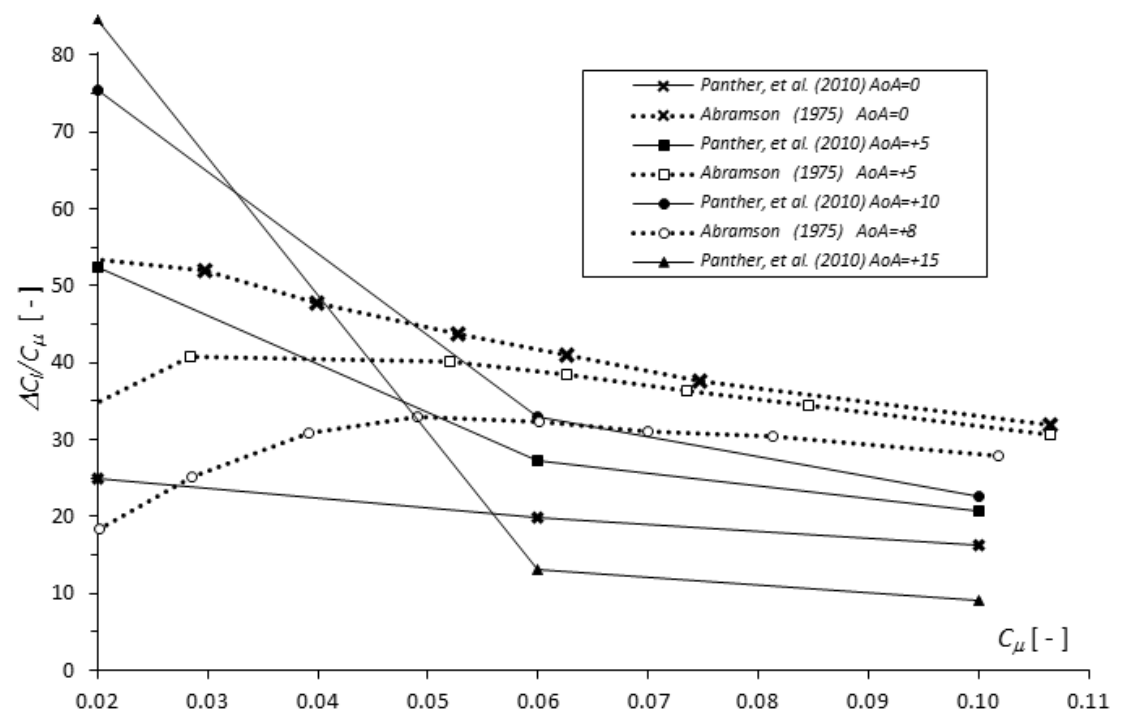

Figure 9. Measured Influence of $\alpha$ on CCNACA0018 Lift Augmentation (Re=300x10,$\left.C_{\mu}=S t e a d y\right)$ (38)

From Figure 9, the largest benefits of flow control for the CCNACA0018 airfoil, relative to baseline conditions, are for low levels of CC blowing $\left(C_{\mu}=0.02\right)$. Also included is the experimental data of a $20 \%$ t/c CC-Ellipse at similar $\alpha$ and $C_{\mu}$ conditions (43). The CC-Ellipse contains a more consistent lift enhancement due to flow control, primarily due to much larger $h_{\text {slot }}$ and $r_{T E}$. However, larger TE geometries will not be beneficial for VAWT applications, due to the large drag penalty of the baseline airfoil. The sectional aerodynamic coefficients from static wind tunnel testing were compiled into data tables and used as inputs for CIRA's recently developed analytical vortex model to calculate CCVAWT performance predictions (35) (36) (44). The analytical model was used to develop a control scheme to increase a VAWT's operational performance envelope (31). Using the optimal blowing schedule, with static wind tunnel data inputs, it was projected that the CCVAWT produced a net increase of $22 \%$ in overall power capture, including the power required to produce the CC jet (31).

Past work has shown that actual power produced by a VAWT at its rated wind speed is higher than analytical predictions based on static airfoil data. Significant differences between test behavior and calculations are attributed to dynamic effects, which have a measurable effect on HAWTs but appears to be even more important to VAWT aerodynamics (5) (28) (29) (45). Thus, dynamic wind tunnel data is needed to update CCVAWT performance projections to more accurate and realistic values. Analytical models, using unsteady input data, more accurately predict blade forces and power output of an augmented VAWT, transforming the vortex model into a powerful and effective CCVAWT design tool. 
Thus, the need for pitching CCNACA0018 airfoil data, under all blowing conditions, was recognized as the next step to progress CCVAWT research and development

An inherent concern, common to the use of pneumatic lift augmentation, is the quantity of energy required to supply the CC air. Past research has proven the ability of pulsed jets to produce competitive lift enhancement, relative to steady blowing, while drastically reducing air flow rates $(\dot{m})$ (29) (45) (46) (47). Thus, the objective of this work is to employ modulated jet blowing into a novel VAWT blade design, to reduce $\dot{m}$ while still achieving steady jet lift enhancement. Previous CIRA research will be extended to include experimental wind tunnel testing of a pitching CCNACAO018 VAWT blade with pulsed CC jets. It is thought that energy conservation due to the oscillating jet, along with dynamic stall lift overshoot, could further increase the $22 \%$ improvement in overall power capture observed from the original addition of circulation control to a VAWT blade.

\subsection{CCVAWT Pumping Power Requirements}

The approach for calculating the effect of blowing on VAWT performance is to calculate the gross power produced using flow control minus the pumping system power consumed by the jet, as outlined by Sasson, et al. (48) for a NACA0012 VAWT blade, equipped with LE blowing augmentation. The power required to produce the jet for the CCNACA0018 VAWT blade will herein be referred to as the blowing power, defined by Equation 18.

cC Blowing Power $\quad P_{C C}=N \cdot P_{p l} \cdot \dot{V}_{j}=N \cdot\left(\frac{1}{2} \rho \cdot V_{j}^{2}+\rho \cdot g \sum h_{l}\right) \cdot A_{j} \cdot V_{j}$

Equation 18

The number of blades $(N)$, plenum pressure $\left(P_{p l}\right)$, and CC jet-airflow rate $\left(\dot{V}_{j}\right)$ are all considered to calculate the pumping power penalty associated with creating the CC jet. The $\Sigma h$, term of Equation 18 is a sum of all secondary losses inherent within any real system; for an ideal system, the loss term would equal zero. Furthermore, a dimensionless pumping power coefficient can be determined by Equation 19.

Blowing Power Coefficient $\quad C_{P_{C C}}=\frac{P_{C C}}{\frac{1}{2} \rho \cdot V_{\infty}^{3} \cdot A_{V A W T}}=\frac{N \cdot h_{j e t}}{2 R}\left(\frac{V_{j}}{V_{\infty}}\right)^{3}\left[1+\frac{2 g \sum h_{l}}{V_{j}^{2}}\right]$

Equation 19

Sasson, et al. (48) nondimensionalize the secondary losses as $\Phi=\left(4 \cdot h_{j}\right) /\left(c \cdot C_{\mu}\right) \cdot\left[g \cdot \Sigma h_{1} / V_{\infty}^{2}\right]$, and substitute turbine solidity $(\sigma)$ and momentum coefficient $\left(C_{\mu}\right)$ into Equation 19 to further simplify the input pumping power coefficient (Equation 20). 
Blowing Power Coefficient

$$
C_{P_{C C}}=\frac{\sigma}{2}\left(\frac{C_{\mu}^{3}}{8\left(h_{j} / c\right)}\right)^{1 / 2}(1+\Phi)
$$

Finally, the blowing power coefficient can be subtracted from the gross turbine performance to calculate the net power production of a CCVAWT (Equation 21).

cCVAWT Net Power Coefficient $\quad C_{P, N E T}=C_{P, C C V A W T}=C_{P, V A W T}-C_{P_{C C}}$

Equation 21

Maximum energy and power can be generated by a wind turbine at the edge of DS (49). Above the regulation wind speed, the power output typically drops off abruptly. To control the peak power output of a VAWT without adversely affecting its performance at low and medium wind speeds, it is necessary to tailor the DS characteristics of its blades; this study will use the provision for active boundary layer control to achieve the desired aerodynamic characteristics to optimize power output through a wide range of wind conditions. The research herein concerns the development of unsteady CC schemes to optimize energy capture, and resulting power production, of a pitching VAWT blade.

Positive results from past work by Wilhelm (31) show the improved power curve of a CCVAWT relative to conventional turbine output. This proves the aerodynamic enhancement of VAWT blades due to the addition of CC. The power output of a conventional dual-bladed VAWT $(\sigma=0.1)$ with NACA0018 profiles is included in Figure 10 (blue line with square symbols). The power predictions were computed with an analytical vortex model. Also included in Figure 10 , are the $C_{P}$-curves employing various magnitudes of CC. For steady $C_{\mu}=0.01$ (red line with " + "-symbols), a $29 \%$ increase in baseline performance was found for TSR $<5$. For steady CC input of $C_{\mu}=0.10$, baseline $C_{P}$ was increased by $253 \%$, not including pumping power requirements. Considering the entire range of operating conditions $(1 \leq \lambda \leq 11)$, using a control strategy to optimize $C_{\mu}$ (grey line of Figure 10) based on wind speed, CC improved VAWT performance by $23 \%$, with significant gains for low TSR $(<4)$. However, after factoring CC jet pumping requirements, the net CCVAWT performance gains were reduced to $<15 \%$. It should be noted that DS occurs on VAWT blades during operation at $\lambda \leq 4$, the region where CC was proven most effective. 

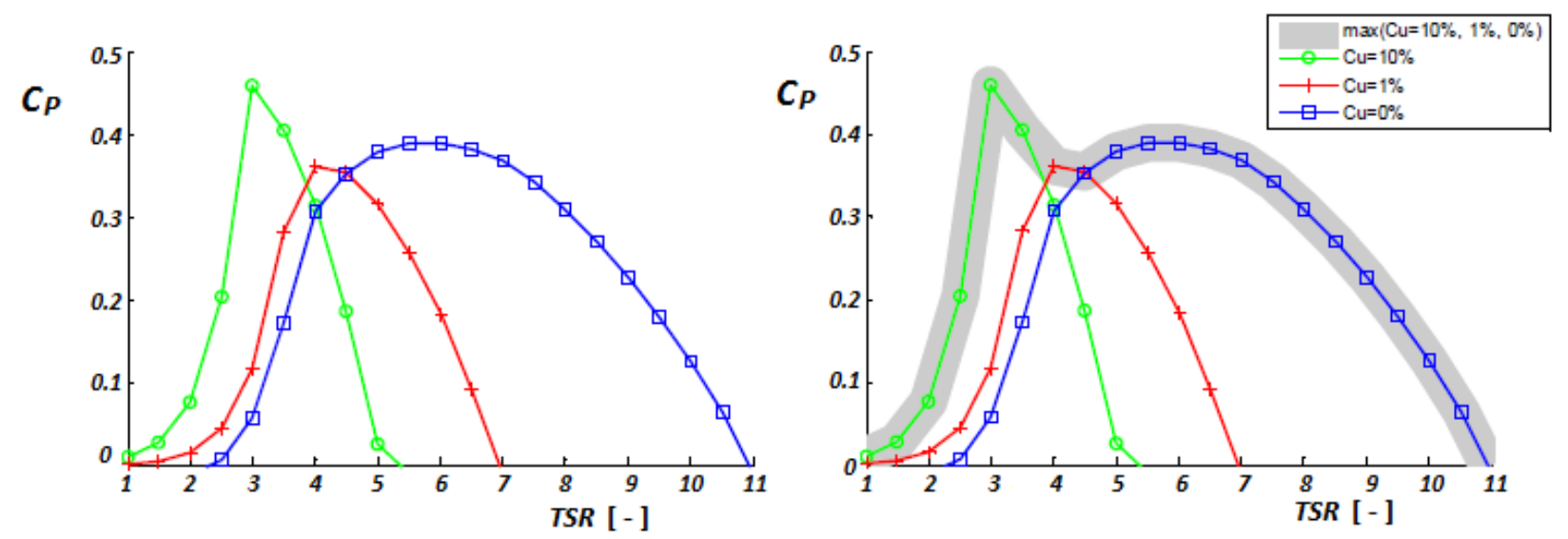

Figure 10. CCVAWT Performance as a function of $C_{\mu}(44)$

\subsection{Research Statement and Objectives}

Modern active flow control (AFC) offers the advantage of lift-enhancement with reduced mass flow requirements. However, past studies of such flow control have been nearly exclusively limited to static testing conditions. Successful application of an augmented CC blade to a conventional VAWT requires an understanding of the performance of both the actuator and the flow over the airfoil in unsteady flow conditions. Large $\alpha$-variations of VAWT blades create periodic blade loading and structural force oscillations which can lead to fatigue-associated problems (39) (48). Therefore, accurate knowledge [measured data] of the dynamic loading is essential for modified VAWT design. To achieve maximum efficiency, the energy expenditure penalty cannot overshadow any performance augmentations of the pneumatic system. In past studies, to avoid jet mass flow rate penalties, pulsed blowing jets, synthetic jets, and closed loop feedback systems were developed from static airfoil tests (29) (45) (46) (47) (50) (51) (52). A limited number of studies have considered leading edge flow control techniques in dynamic environments, including vortex generators (53), plasma actuation (54), and dynamic roughness elements (55).

The goal of this research is to extend recent CIRA research by analyzing unsteady CC jet performance on a pitching CCNACA0018 airfoil. Experimental and computational analysis will focus on the ability of a pulsed jet to enhance lift with reduced mass flow requirements to conserve energy expenditure. Specific goals include a parametric analysis of the relationship between the jet pulsing frequency $\left(f_{\text {jet }}\right)$ and $\mathrm{CC}$ magnitude $\left(C_{\mu}\right)$, for both static and pitching airfoil conditions.

To the author's knowledge, no comparable studies incorporating unsteady circulation control with VAWT blade dynamic stall effects have been piloted. Thus, the objective of this document is to 
detail the research completed to present the aerodynamic performance of a novel airfoil design within conditions derived from common VAWT operation.

The principal objective of this research was to examine the benefits of pulsed blowing to reduce mass flow requirements for desired CCVAWT blade performance. Furthermore, the effects of dynamic stall on both steady and unsteady flow control for the CCNACA0018 profile were previously unknown. In pursuit of this primary goal, a few other aspects of dynamic stall and unsteady flow control were explored to better understand the aerodynamics of a modified VAWT blade with active flow control. Thus, the objectives of this research were to answer the following questions:

I. Can experimental and CFD analyses prove a pulsed CC jet can match steady jet performance enhancements for a static CCNACA0018 airfoil, at reduced mass flow requirements?

II. What is the dependency of flow control parameters $\left(C_{\mu}, S t\right)$ to achieve desired aerodynamic enhancements?

III. How does dynamic pitch influence CCNACA0018 performance under simulated VAWT conditions?

IV. What is the relationship between effective flow control parameters $\left(C_{\mu}, S t\right)$ and flow conditions $\left(\operatorname{Re}, k, \alpha_{\max }\right)$ ? 


\section{CHAPTER 2. LITERATURE REVIEW}

This chapter provides historical background of pertinent topics of this research effort. Previous research efforts from the literature are summarized to formulate the founding work that preceded the current work. Germane areas of review include steady airfoil aerodynamics, various flow control technologies, pitching airfoil aerodynamics and dynamic stall. Experimental, computational, and analytical methods were all considered, including sufficient detail to support each study.

\subsection{Conventional Airfoil Aerodynamics}

This section reviews the fundamental concepts of flow separation and stall of standard airfoils to introduce the foundation of flow control research. Traditional forms of airfoil load enhancements are included to investigate the advantages and disadvantages of VAWT implementation, and provide metrics for flow control airfoil performance.

\subsubsection{Static Stall}

The lift performance of 2D airfoils, with respect to angle-of-attack, is generally characterized by two distinct regions, representative of boundary layer $(B L)$ state. When the $B L$ is stable, flow remains attached to the airfoil upper surface, where lift increases linearly with $\alpha$. As AoA is increased, BL instabilities lead to separation, where the lift curve slope $\left(\mathrm{d} C_{1} / \mathrm{d} \alpha\right)$ decreases until a point of maximum lift $\left(C_{l, \text { max }}\right)$. When $\alpha$ becomes too large, adverse pressure gradients on the airfoil upper surface cause the $\mathrm{BL}$ to fully separate, developing a wide turbulent wake region and a complete loss of lift. This condition is commonly known as airfoil "stall," and is a fundamental concept the current research revolves about. The static stall AoA $\left(\alpha_{S S}\right)$ in a given $C_{1}-\alpha$ curve is easily identified as the inflection point about $C_{1, \text { max }}$ (Figure 11). Static stall effects on conventional airfoils are well documented and easily accessed in the literature, including sharp $C_{\text {/ }}$ drop-off with rapid rise in $C_{d}$, and will not be discussed further. 


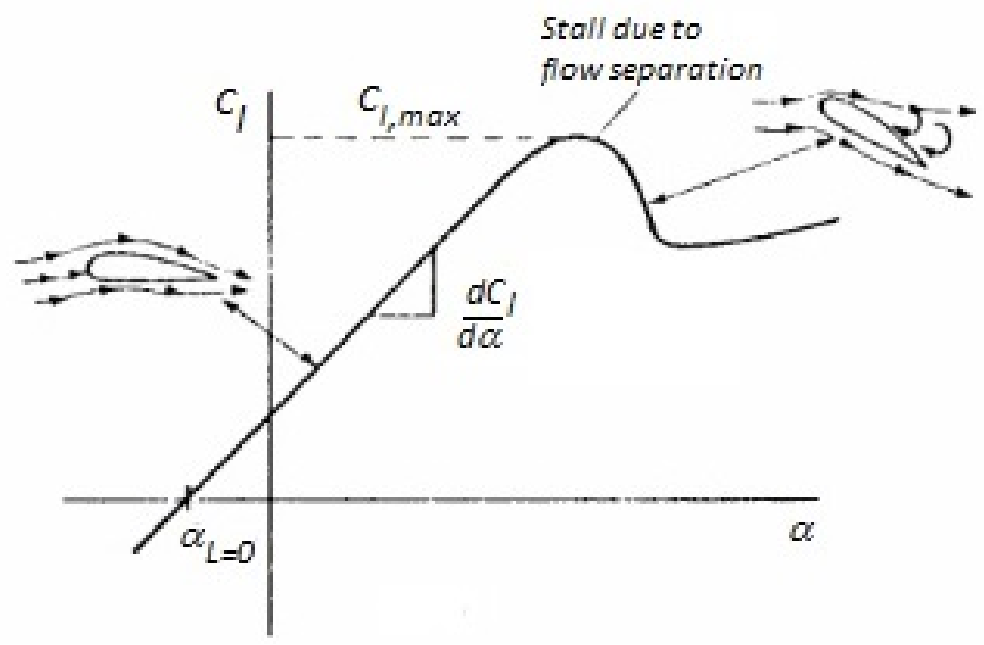

Figure 11. Schematic of Lift Variation with Angle of Attack (56)

\subsubsection{D Effects}

The influence of 3-dimensional effects on the aerodynamic performance of a symmetric (NACA0018) and cambered airfoil (NACA 6418) were determined through extensive wind tunnel testing by Jacobs, et al. (42). The 3D effects on force measurements of $C_{l}$ and $C_{d}$ are included in Figure 12; the legend included within the drag plot of Figure 12 (right hand side) applies to the lift plot as well.
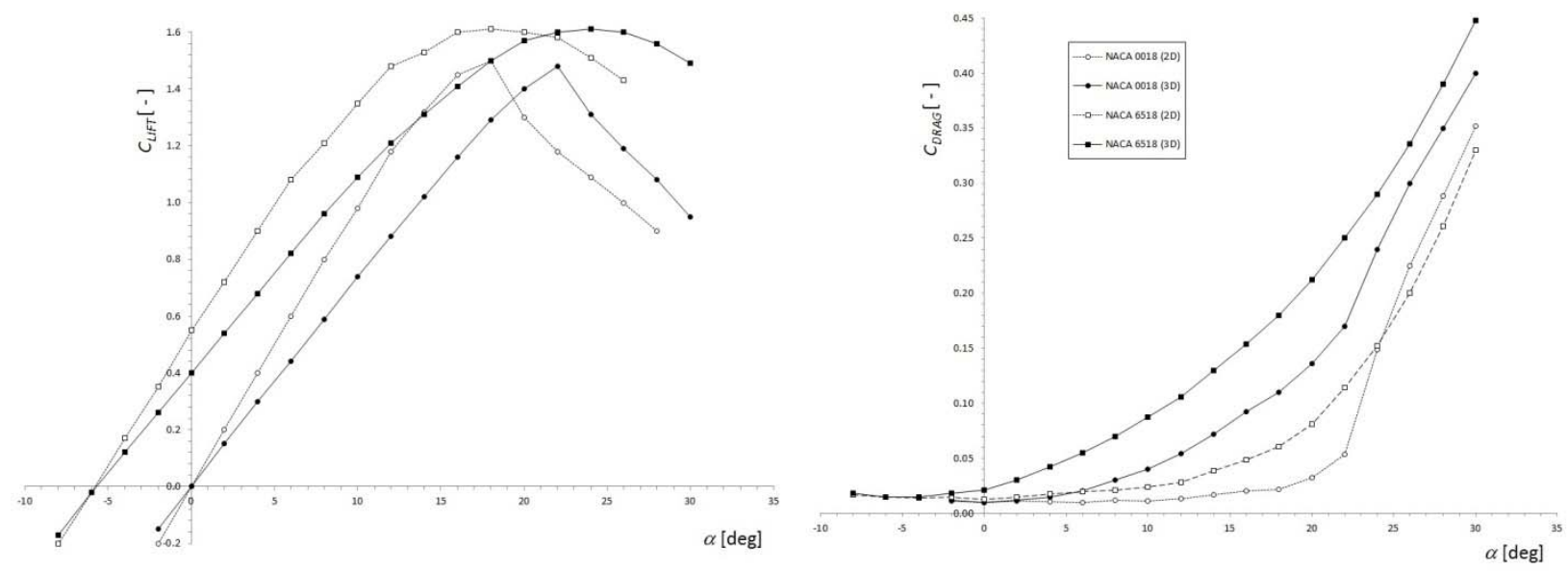

Figure 12. 3D Effects on Wind Tunnel Measured Airfoil Performance: NACA0018 and NACA6418 (42)

It can be seen that tip losses of 3D airfoils reduce $\mathrm{d} C_{1} / \mathrm{d} \alpha$ and increase $C_{d}$ for both the symmetric and cambered profiles. The 3D effects also influence maximum lift $\left(C_{l, \text { max }}\right)$ and $\alpha_{S s}$. The 3D effects are crucial to consider during VAWT blade design. For a rectangular wing, the aspect ratio compares the airfoil span to chord $(A R=b / c)$. A component of airfoil, known as induced drag, is inversely proportional to $A R$. The drag is a result of tip-vorticity losses. Thus, airfoil drag decreases with increasing $A R$. 


\subsubsection{Traditional Aerodynamic Load Control}

The most elementary way to tailor airfoil characteristics to suit a given application is by changing the physical shape. To increase $C_{\text {I }}$ at low $\alpha$, for example, the airfoil chord line is bent or curved to create camber. The influence of camber on the lift performance of several NACA profiles was measured by

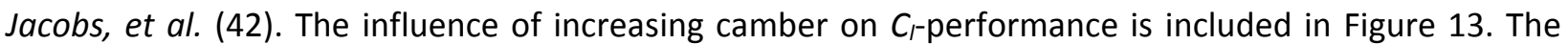
profile of each airfoil is situated between the representative $C_{1}$-curve data points for $2 \mathrm{D}$ and 3D measurements. It can be seen that increasing camber has little impact on 3D experiments. However, increasing the camber of 2D airfoils shows a linear decrease in $d C_{l} / d \alpha$. This result is pertinent to the current study as increasing the magnitude of blowing on a CC airfoil has been proven analogous to such $C_{1}$-enhancements due to increasing camber at low $\alpha$. CC is often said to create a "virtual camber" for a given airfoil, with effects increasing with $C_{\mu}$, as will be discussed in further detail in a later section.

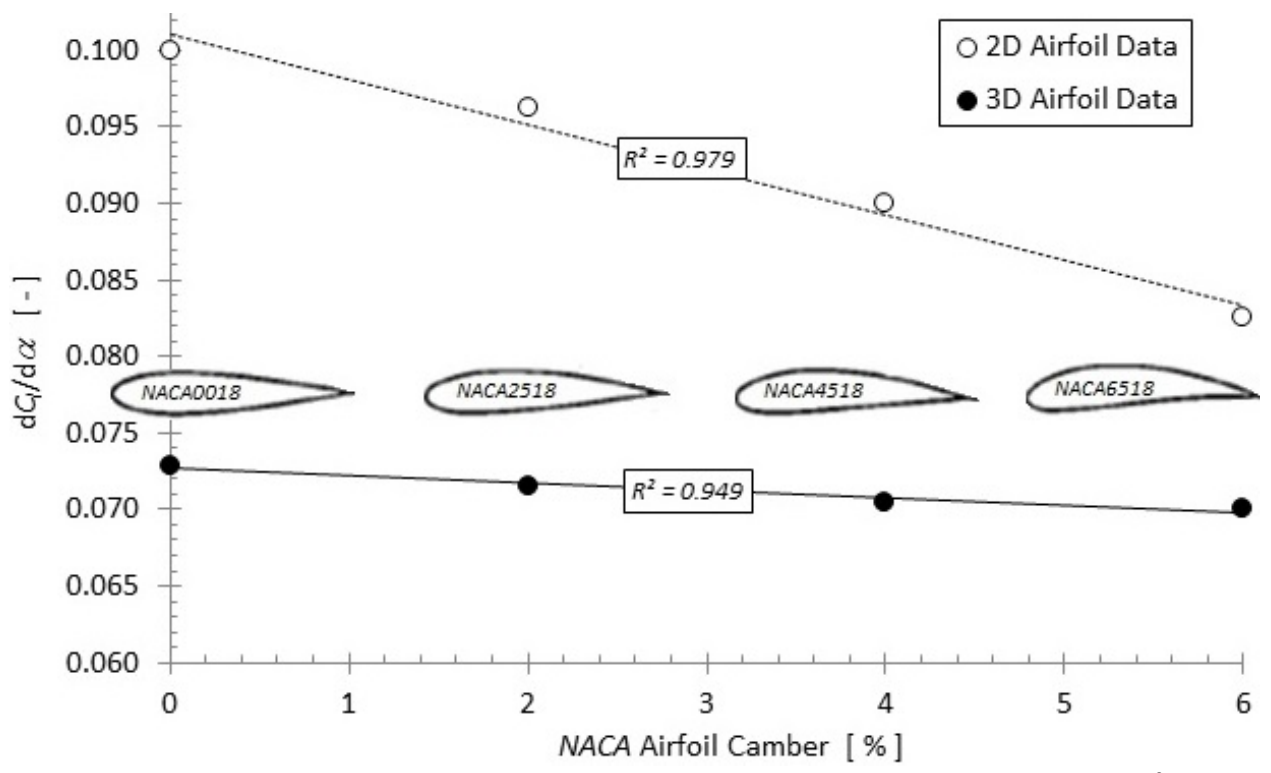

Figure 13. Influence of Camber on Lift-Curve Slope for NACA Airfoils with Thickness of $t / c=0.18$

Furthermore, increasing camber improves the maximum lift coefficient $\left(C_{l, \max }\right)$ of an airfoil, but decreases the stall angle ( $\left.\alpha_{\text {stall }}\right)$. Thus, cambered airfoils are dismissed from VAWT applications since lift improvement would only be noticed for a narrow band of operating conditions, while increased drag would prevail in all conditions (relative to a symmetric profile).

For applications with broader operating conditions, mechanical actuation is a standard technique that offers comparable $C_{1}$-enhancements of increased camber, but has the bonus option of returning to a symmetric profile for low drag operation. The most commonly used mechanical devices for load control are LE flaps or slats and TE flaps (57). The influences of mechanical enhancement on 
airfoil lift and stall characteristics are viewed in Figure 14. LE devices extend the lift curve, increasing $C_{l, \max }$ and $\alpha_{\text {stall. }}$ LE control methods cannot be used to decrease lift, however, and are not considered an option for turbine blade load mitigation (58). TE control changes the effective camber of the airfoil and can shift the zero-lift angle of attack to positive or negative values, depending on the direction of flap deflection $\left( \pm \delta_{\text {flap }}\right.$ ) (57). Flap deflection toward the pressure surface of an airfoil increases $C_{\text {I }}$ while flap deflection toward the suction surface reduces lift. The aerodynamic characteristics of a CC airfoil are thus analogous to a TE flap, where upper and lower slot blowing reflects positive and negative flap deflections, and the magnitude of $C_{\mu}$ is comparable to that of $\delta_{\text {flap }}$.

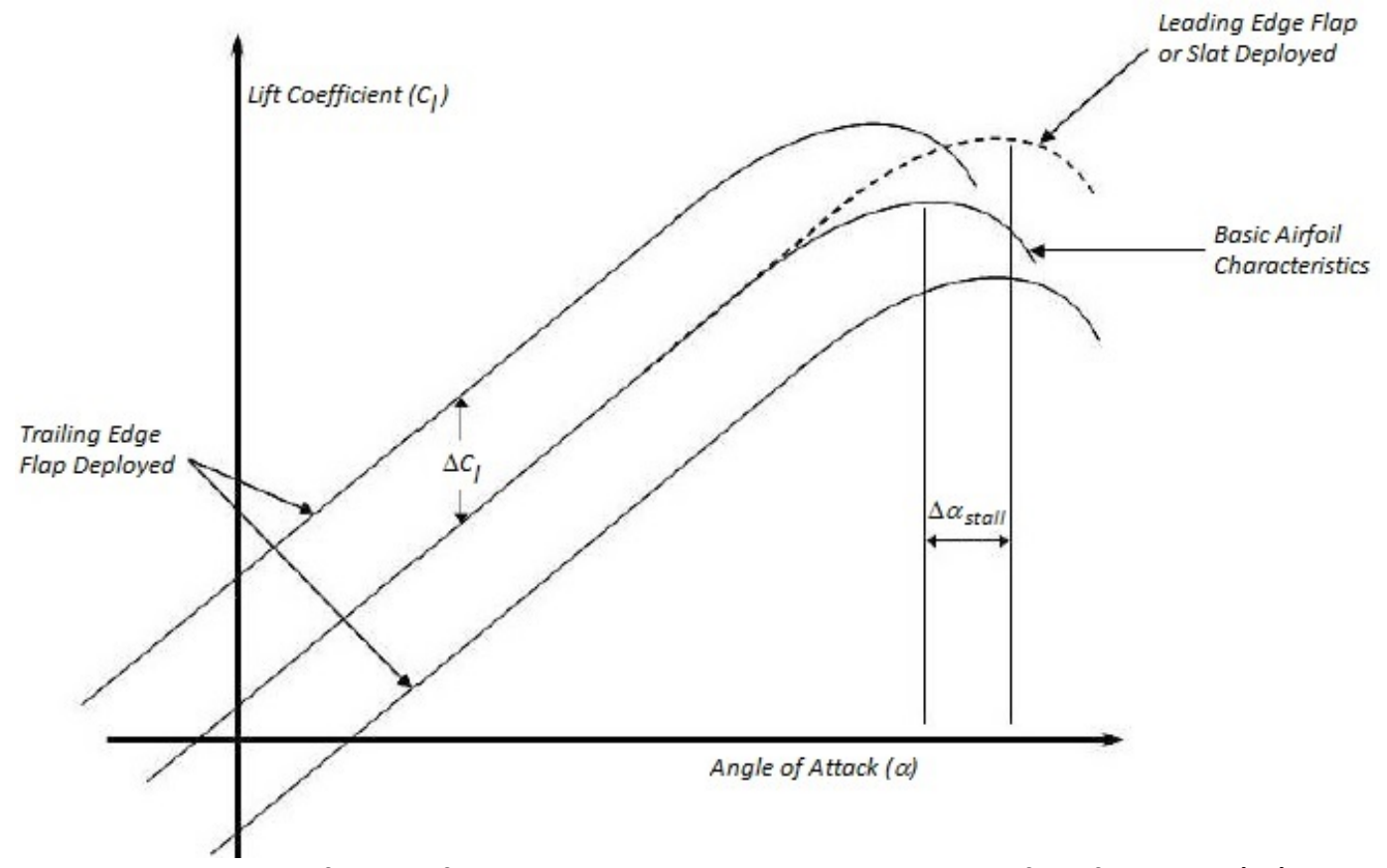

Figure 14. The Influence of LE and TE Flow Enhancement Devices on Airfoil Lift and Stall (57)

TE flaps have been examined for wind turbine control applications and have been proven effective in controlling power output, but disadvantages resulted from their complexity, added structural weight (e.g. linkages, support structure, actuators), and additional maintenance (57). Such disadvantages of TE flaps could be avoided with the use of pneumatic flow control.

Wang \& Lu (59) completed static CFD modeling of a flapped NACA23012, with various deflection angles, for helicopter applications. The effects of TE flap deflection on a cambered airfoil can be seen in Figure 15 . The baseline case $\left(\delta_{\text {flap }}=0^{\circ}\right)$ is represented by dashed lines, positive $\delta_{\text {flap }}$ by solid lines with hollow symbols, and negative $\delta_{\text {flap }}$ with solid lines and symbols. Positive $\delta_{\text {flap }}$ were shown to increase $C_{I, \max }$ but decrease $\alpha_{\text {stall, }}$ while negative $\delta_{\text {flap }}$ had the opposite effect. 

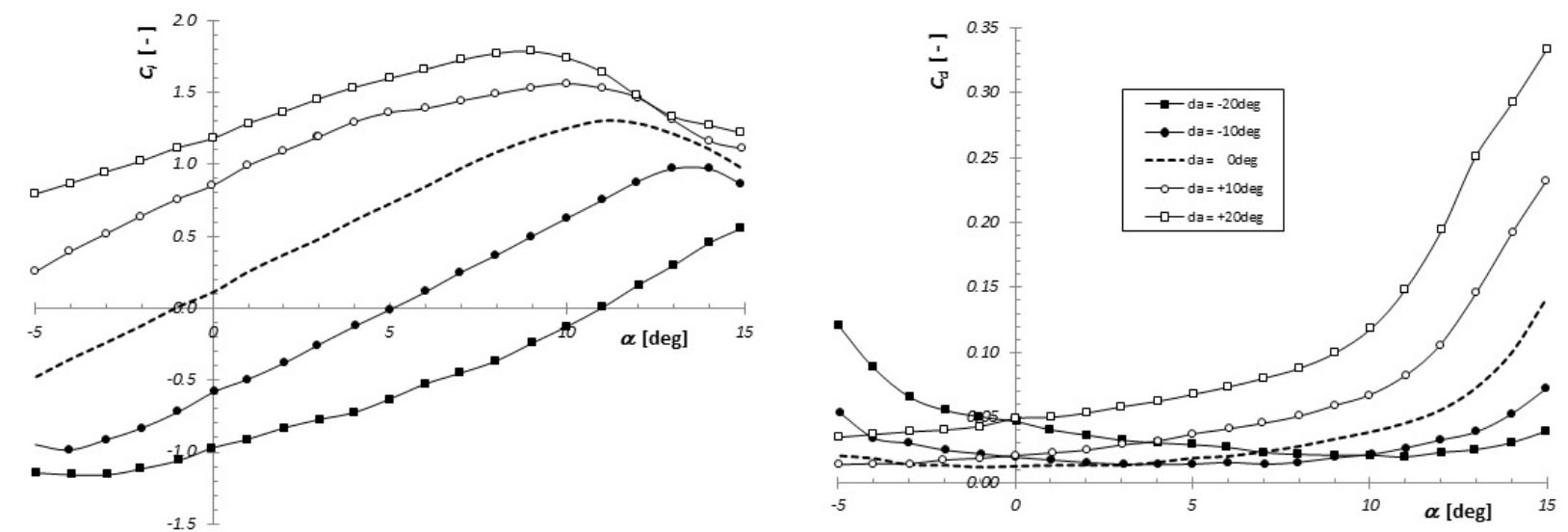

Figure 15. The Influence of TE Flap Deflection on Cambered Airfoil (NACA23012) Performance (59)

The influence of variable camber on $C_{d}$ was of particular interest from this study. The negative camber $\left(\delta_{\text {flap }}=-10^{\circ},-20^{\circ}\right)$, analogous to lower slot CC blowing, is shown to reduce drag at higher angles of attack $\left(\alpha \geq 5^{\circ}\right)$ compared to baseline and positive $\delta_{\text {flap }}$ cases. The benefits of conventional load enhancement are proven for fixed wing flight where operating $\alpha$-ranges are minimal. However, cambered airfoils improve VAWT performance over a very limited range of conditions, proving them undesirable. Flapped airfoils offer flexibility and an expanded range of improvement, but the weight penalties of additional flap actuation mechanisms have negated such benefits. Thus, conventional airfoil load enhancement via physical augmentation has been found undesirable for improving VAWT performance.

\subsection{Flow Control Airfoil Aerodynamics}

This section reviews various forms of pneumatic and mechanical flow control techniques implemented in past studies. Flow control methods can be classified into passive and active methods. Passive methods utilize geometric modifications while active control methods require external energy to influence flow. Active control techniques include various pneumatic (e.g. CC, synthetic jets), plasma, and combustion forms. Passive techniques have included slats (60), vortex generators (61), drooped LE (62), combined nose droop-Gurney flap (63), TE flap (64), and moving surfaces. This study focuses on circulation control, and thus will include an in-depth background on this technology, while other techniques will be briefly reviewed. Popular flow control methods from past research, classified by actuation type, are included in Figure 16. The common goal of all reviewed methods is the mitigation of airfoil separation (stall). To date, the majority of flow control airfoil research has been founded in steady flow environments to better understand the impact of control parameters on static lift and drag. The 
review focuses on previous studies of airfoils with comparable characteristics to the CCNACAOO18 (e.g. $t / c \approx 0.18$, high camber, TE flap, flow control) at similar test conditions (e.g. $R e, \pm \alpha$ ).

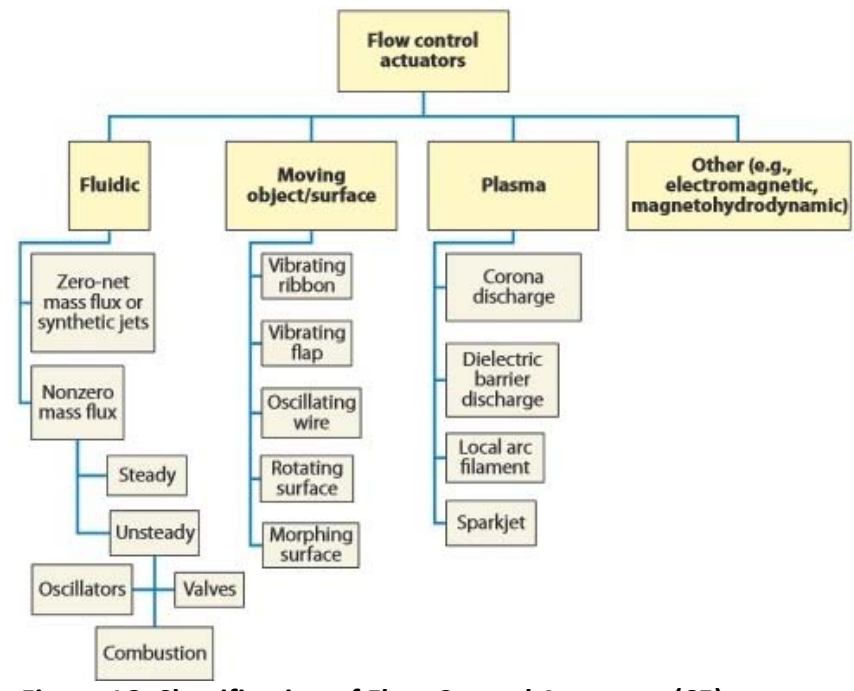

Figure 16. Classification of Flow Control Actuators (65)

Various passive and active dynamic stall flow control methods have been previously studied with the goal of increasing $L / D(11)$. A comparison summary of common AFC actuators, including advantages and disadvantages, is included in Table 2.

Table 2. Summary of Common AFC Actuators (66)

\begin{tabular}{|c|c|c|}
\hline Type & Advantages & Disadvantages \\
\hline Flaps & $\begin{array}{l}\text { - Simple design amenable to different } \\
\text { frequency ranges of interest } \\
\text { - Can produce spanwise or stream- } \\
\text { wise vorticity }\end{array}$ & $\begin{array}{l}\text { - Product of max deflection and } \\
\text { bandwidth is constant } \\
\text { - Susceptible to fluid loading }\end{array}$ \\
\hline ZNMF & $\begin{array}{l}\text { - Requires no external flow source } \\
\text { - Amenable to various types of } \\
\text { drivers and sizes }\end{array}$ & $\begin{array}{l}\text { - Peak velocities typically } \\
\text { limited to low to moderate } \\
\text { subsonic speeds }\end{array}$ \\
\hline Pulsed jets & $\begin{array}{l}\text { - Capable of high velocities with } \\
\text { either fast time response OR } \\
\text { high-frequency response but } \\
\text { generally not both }\end{array}$ & $\begin{array}{l}\text { - May not be amenable to } \\
\text { feedback control due to either } \\
\text { frequency-or time-response } \\
\text { limitations } \\
\text { - Requires an external flow } \\
\text { source }\end{array}$ \\
\hline VGJ & $\begin{array}{l}\text { - Good control authority similar to } \\
\text { mechanical vortex generators } \\
\text { - Amenable to pulsing } \\
\text { implementations }\end{array}$ & $\begin{array}{l}\text { - Many adjustable parameters } \\
\text { (mom. ratio, pitch and yaw } \\
\text { angles, etc.) make it non- } \\
\text { trivial to optimize } \\
\text { - Requires an external flow } \\
\text { source }\end{array}$ \\
\hline PRT & $\begin{array}{l}\text { - Capable of producing large } \\
\text { perturbations in high-speed flows }\end{array}$ & $\begin{array}{l}\text { - Not currently amenable to } \\
\text { feedback control } \\
\text { - Requires an external flow } \\
\text { source }\end{array}$ \\
\hline SDBD plasma & $\begin{array}{l}\text { - Easily installed on models (arrays) } \\
\text { - Can produce spanwise or stream- } \\
\text { wise vorticity } \\
\text { - No moving parts }\end{array}$ & $\begin{array}{l}\text { - Limited velocity output } \\
\text { - Requires high voltage } \\
\text { (kilovolts) }\end{array}$ \\
\hline Combustion & $\begin{array}{l}\text { - Capable of producing large } \\
\text { perturbations in high-speed flows }\end{array}$ & $\begin{array}{l}\text { - Currently limited to relatively } \\
\text { low frequencies - a few } \\
\text { hundred hertz } \\
\text { - Requires combustion }\end{array}$ \\
\hline SparkJet & $\begin{array}{l}\text { - All solid-state device capable of } \\
\text { producing large perturbations in } \\
\text { high-speed flows }\end{array}$ & $\begin{array}{l}\text { - Currently limited to relatively } \\
\text { low frequencies - a few } \\
\text { hundred hertz }\end{array}$ \\
\hline
\end{tabular}




\subsubsection{Circulation Control Theory}

$\mathrm{CC}$, as the name suggests, is rooted on a mathematical concept known as circulation. Airfoil circulation $(\Pi)$, by definition, is simply the negative of the line integral of velocity around a closed curve (Equation 22), and is a kinematic property depending only on the velocity field $\left(V_{\infty}\right)$ and the choice of the path (in this case governed by airfoil profile) (56).

Airfoil Circulation

$$
\Gamma=-\oint_{C} V \cdot d s
$$

A quantitative theory relating $\Gamma$ to the aerodynamic lift $(L)$ of an infinite wing is commonly known today as the Kutta-Joukowski theorem. The relation states that lift per unit span ( $\left.L^{\prime}\right)$ on a 2D body is directly proportional to $\Gamma$, as seen by Equation 23; in other words, the generated lift is thus the result of a bound vortex (67).

Kutta-Joukowski Theorem $\quad L^{\prime}=\rho_{\infty} \cdot V_{\infty} \cdot \Gamma$

Equation 23

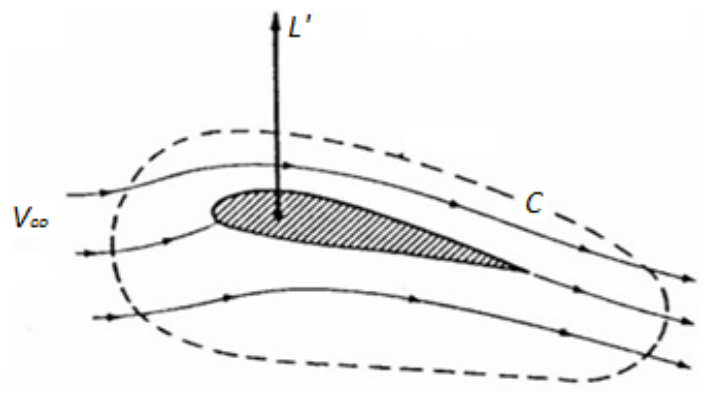

Figure 17. Kutta-Joukowski Theorem: Circulation ( $\Pi$ ) around a Lifting Airfoil (56)

Line integrals, by mathematical convention, have a positive sense when the path is counterclockwise. However, in aerodynamics, it is expedient to consider a clockwise circulation to be positive (hence the minus sign in Equation 22).

In practice, the most effective way to maintain lift at low airspeeds is to increase the circulation (66). If the circulation can be controlled by means of blowing high-energy air tangentially into the boundary layer near the TE of an airfoil, it can be concluded from the Kutta-Joukowski theorem that a variety of lift values can be obtained at a constant angle of attack $(\alpha)$. The previous statement is the foundation of of circulation control (CC) technology.

Conventional airfoils have a sharp TE, which governs the amount of circulation about the airfoil according to the Kutta condition. The Kutta condition, a potential flow theory provision, limits the 
magnitude of circulation about a given airfoil. The principle states that if the sharp TE of a conventional airfoil is finite, then the aft location where the upper and lower airfoil surfaces meet becomes a fixed stagnation point $(V=0 \mathrm{ft} / \mathrm{s})$.

a.)

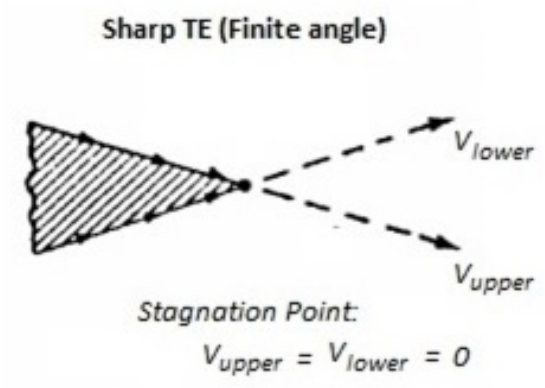

b.)

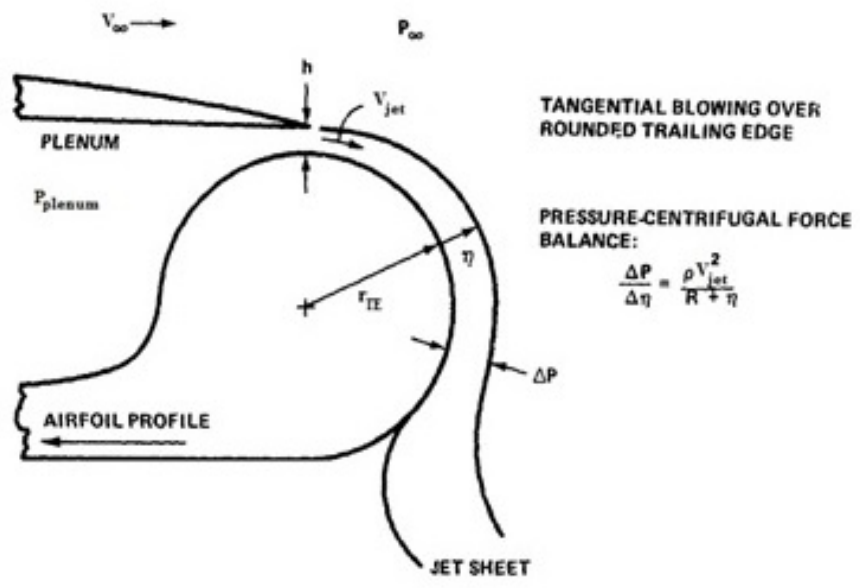

Figure 18. (a.) Sharp TE and the Kutta Condition (56) (b.) Tangential Blowing Over Rounded TE (46)

In contrast, CC airfoils have a blunt TE (Coandă surface) which relaxes the Kutta condition, allowing the location of the rear stagnation point (and resulting circulation) to be controlled by varying the magnitude of $\mathrm{CC}$ blowing $\left(C_{\mu}\right)$, allowing lift forces to be manipulated for a fixed position.

The use of blowing air to increase airfoil lift has been studied as far back as the late 1920s. CC is commonly defined as the use of tangential blowing jets located adjacent to a rounded Coandă surface. The fundamental principle governing CC flow physics is the Coandă effect. This phenomenon allows a jet of air to remain attached to a rounded surface due to a balance of centrifugal force and the sub-ambient pressure of the jet sheet (see Figure 18). The addition of high momentum flow into the boundary layer $(B L)$ has been researched in many diverse applications, including fixed wing aircraft, rotorcraft, hydrodynamic applications, propellers, and even modern ground vehicles. A more comprehensive and in-depth look into the history of CC and its applications can be found is provided by Kweder \& Panther (68).

The governing parameter of $\mathrm{CC}$ is the momentum coefficient $\left(C_{\mu}\right)$ of the blowing jet. The blowing coefficient is simply the jet thrust divided by the product of free stream dynamic pressure $\left(q_{\infty}\right)$ and the planform area of the airfoil $\left(A_{b l}\right)$, as seen in Equation 24 . The mass flow of the jet $\left(\dot{m}_{j e t}\right)$ is generally determined by flow meters in the supply line, while the jet velocity is commonly calculated assuming isentropic expansion from measured internal plenum pressure $\left(P_{p l}\right)$ and temperature $\left(T_{p l}\right)$, to free stream static pressure (see Equation 25) (43). 


$$
C_{\mu}=\frac{(\dot{m} V)_{j e t}}{q_{\infty} \cdot A_{b l}}
$$

CC Jet Velocity

$$
V_{j e t}=\left[\frac{2 \cdot \gamma \cdot R \cdot T_{p l}}{\gamma-1}\left(1-\left(\frac{P_{\infty}}{P_{p l}}\right)^{\gamma-1 / \gamma}\right)\right]^{1 / 2}
$$

Boundary layer $(\mathrm{BL})$ formation begins at the LE of the airfoil and continues to grow, due to viscous effects, as it flows over the airfoil towards the TE. A persistent adverse pressure gradient $(\mathrm{d} p / \mathrm{d} x>0)$ reduces the wall shear to zero (separation point) and causes backflow, while the BL thickens. Laminar flows have poor resistance to adverse pressure gradients and separate easily, while turbulent BLs contain more energy and can prolong separation (69). The interaction between the wall jet and BL flow can be seen in Figure 19 (70) (71). Figure 19 also illustrates the aforementioned use of CC jets to shift the stagnation point on a Coandă surface (71).

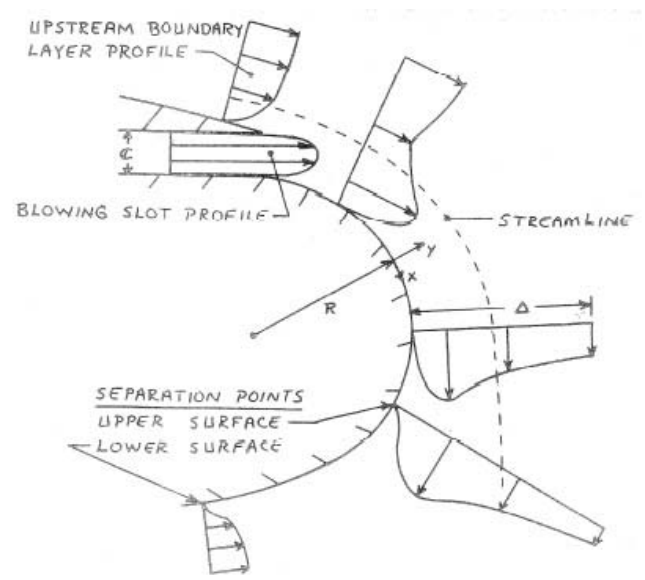

Figure 19. Influence of CC Jet on BL along a Coandă Surface (71)

\subsubsection{Steady Circulation Control ( $C_{\mu}=$ constant $)$}

Now that the underlying fundamentals have been covered, this section will include past research involving experimental and numerical investigations of CC airfoils. Jones (45) compiled data from various NASA studies, comparing $L / D$ performance of multiple flow control airfoils. The variation of airfoil thickness and camber, TE geometry (rounded, elliptical), and flap style are realized to offer a wide range of performance to suit a desired application. The $L / D$ curves were generated at $\alpha=0^{\circ}$, revealing the vast range of attainable aerodynamic performance by simply varying $C_{\mu}$. 


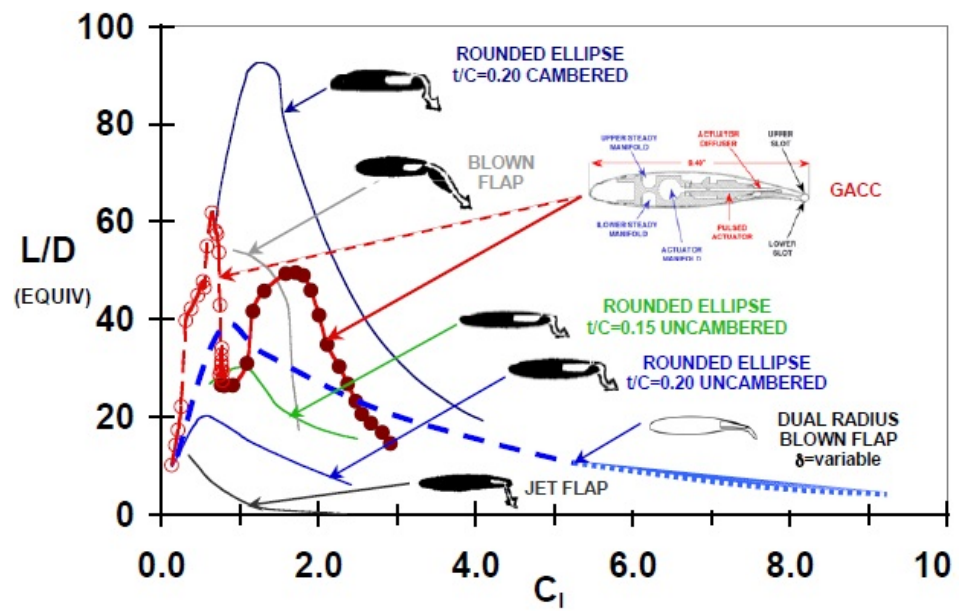

Figure 20. Comparison of NASA Flow Control Airfoil Performance $\left(\alpha=0^{\circ}\right)(45)$

Jones \& Englar (46) tested the influence of lower surface blowing on the lift performance of the GACC airfoil (profile included in Figure 20), with the lift performance included in Figure 21.

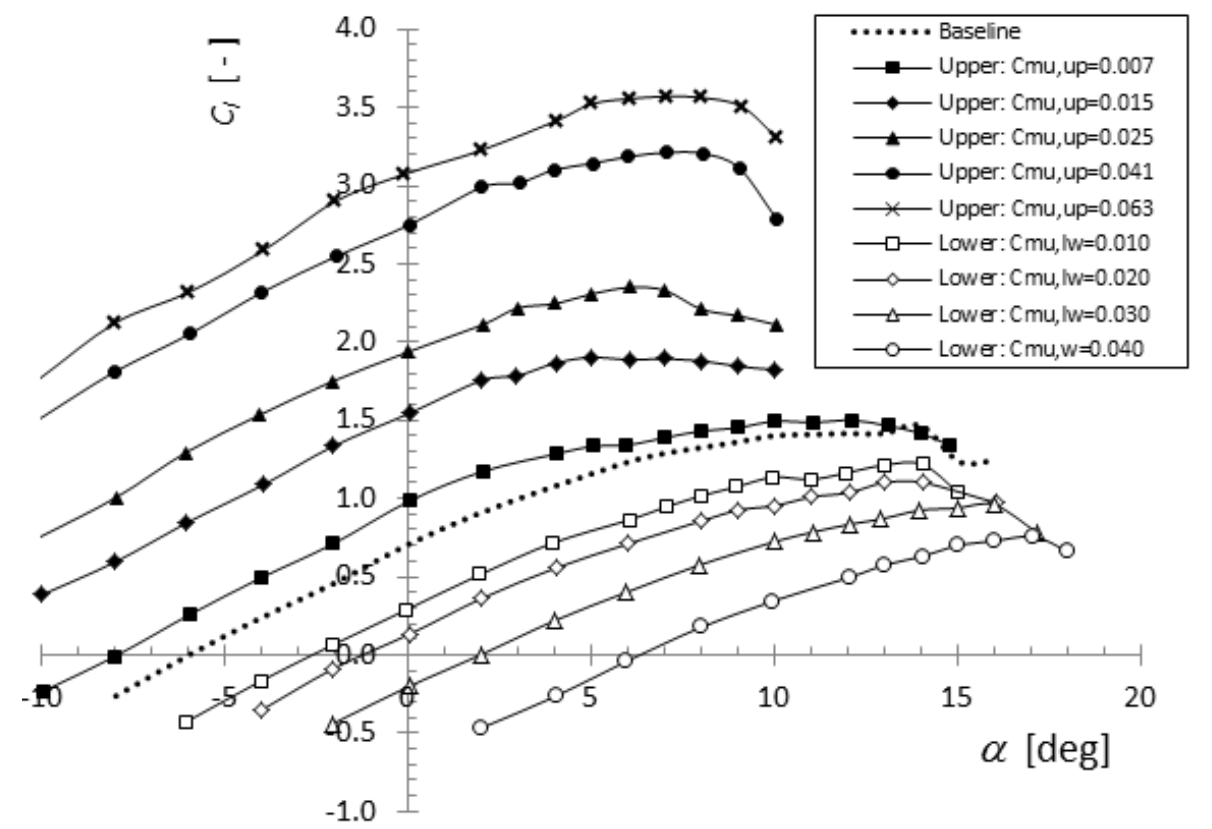

Figure 21. Upper Surface vs. Lower Surface Blowing on CC Airfoil Performance: $R e=500 \times 10^{3}(46)$

As seen in Figure 21, upper surface blowing (filled symbols) produced common results of CC airfoils: $C_{l, \max }$ increases and $\alpha_{\text {stall }}$ decreases with increasing $C_{\mu}$. However, the opposite was found while increasing $C_{\mu}$ from the lower surface slot (open symbols): $C_{l, \max }$ decreased but $\alpha_{\text {stall }}$ was extended by as much as $3^{\circ}$. This result appears to be the result of an inverse camber created by lower slot blowing, as similar effects were observed by Wang \& Lu (59) (see Figure 15). 


\subsubsection{Unsteady Flow Control ( $C_{\mu} \neq$ constant $)$}

This section reviews past research from the literature of static airfoils with various forms of active flow control (AFC). A myriad of factors influence the performance of pitching airfoils. Thus, past work of flow control airfoils in a static environment are summarized to better isolate variables that influence control authority, including actuation frequency $(S t)$, amplitude $\left(C_{\mu}\right)$, duty cycle $(D C)$, and waveform (e.g. square, sinusoid). This section attempts to summarize past work to ascertain trends for optimal control methods applicable to the current CCNACA0018 study.

A concept to increase the effectiveness of CC blowing is to blow intermittently, where the chief purpose is the reduction of mass flow compared to steady blowing. The pulsed jet acts to increase mixing in the BL relative to the steady jet (47). In 1970, theoretical and experimental groundwork was completed by Verhoff (72) on a flat plate with no pressure gradient $\left(V_{\infty}=0 \mathrm{ft} / \mathrm{s}\right)$. Seminal conclusions were that the rate of mixing with a pulsing jet was an order of magnitude greater than that of a steady jet. To minimize redundancy throughout this review and the remainder of the paper, it should be noted that any reference to "reductions of mass flow or blowing-power requirements" due to pulsed actuation are relative to steady flow control conditions.

Four similarity parameters are known to characterize the operating conditions of pulsed jets: velocity ratio $(V R)$, duty cycle $(D C)$, Strouhal number $(S t)$, and momentum coefficient $\left(C_{\mu}\right)(53)(61)$. From this point forward, to decipher between a steady and pulsed CC jet, an oscillatory momentum coefficient will be defined by $\left\langle c_{\mu}\right\rangle$, following the common notation. The $V R$ is the ratio of jet exit velocity to the free stream velocity, included in Equation 26. Lift enhancement generally saturates when $V R>4$.

Jet Velocity Ratio

$$
V R=\frac{V_{\text {jet }}}{V_{\infty}}
$$

Equation 26

Figure 22 compares normalized jet velocity performance from various experimental airfoil models. The influence of flow control component geometry (e.g. pneumatic valve bodies, plenum, jet slot) of each model is apparent as each pulsed jet has a unique waveform and characteristic behavior. The various jet characteristics (under similar test conditions), unique to each airfoil model, likely contributes to the discrepancy of effective actuation frequency ranges found in the literature $(0.3 \leq S t$ $\leq 4.0)$. 


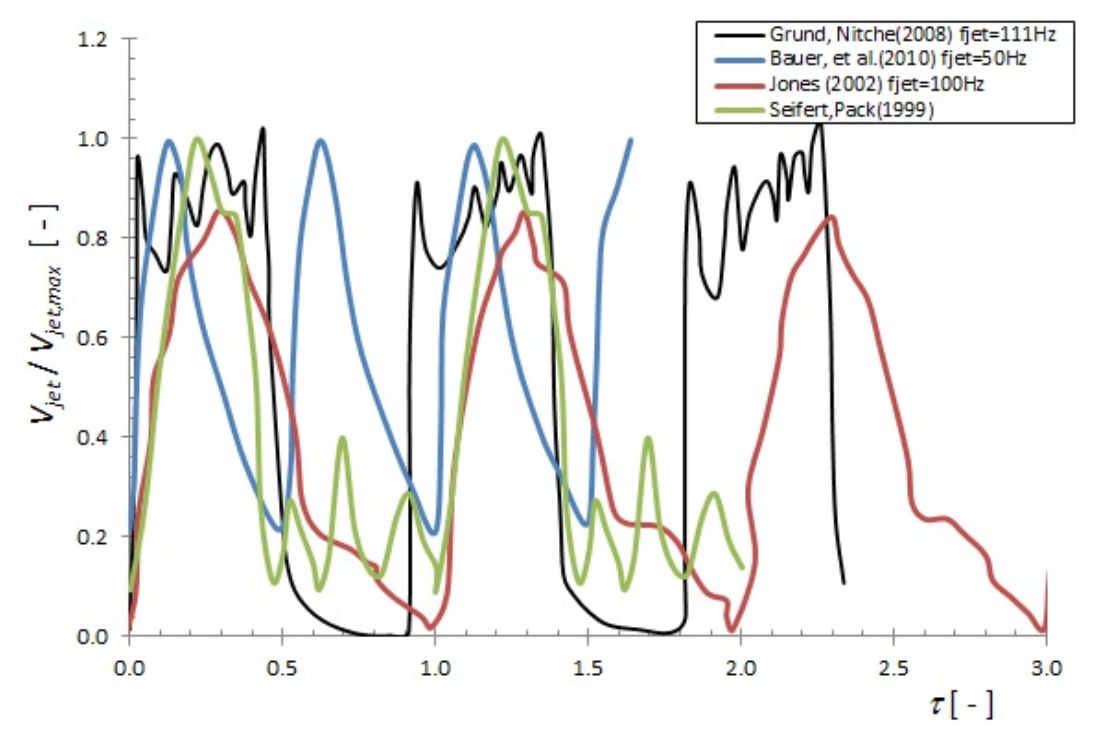

Figure 22. Jet Velocity Profiles From Literature (52) (73) (74)

Duty cycle, $D C$ (Equation 27 ), is the fraction an entire pulse cycle $(\tau)$ during which the jet is actuated (i.e. "on").

Duty Cycle

$$
D C=\frac{t_{a c t}}{\tau}
$$

Equation 27

The duty cycle of pulsed actuation has been shown to influence flow control authority for a given airfoil. Jones \& Englar (45) (46) tested pulsed pneumatic high-lift airfoils in an effort to reduce mass flow requirements and cruise drag penalties associated with CC wings. Static wind tunnel testing (2D) of two supercritical airfoils was completed at low $\alpha$, primarily focused on the effects of $D C$ on lift generation. The general aviation CC (GACC) airfoil profile and $C_{/}$performance as a function of $D C$ $(20 \% \leq D C \leq 80 \%)$ are included in Figure 23 (note: circles represent steady jet CC, diamonds refer to pulsed CC jet CC). A pulsed CC jet of $C_{\mu}=0.0075(D C=40 \%)$ generated the same lift as a steady jet $\left(C_{\mu}=0.0175\right)$ with a $55 \%$ reduction in mass flow requirement.
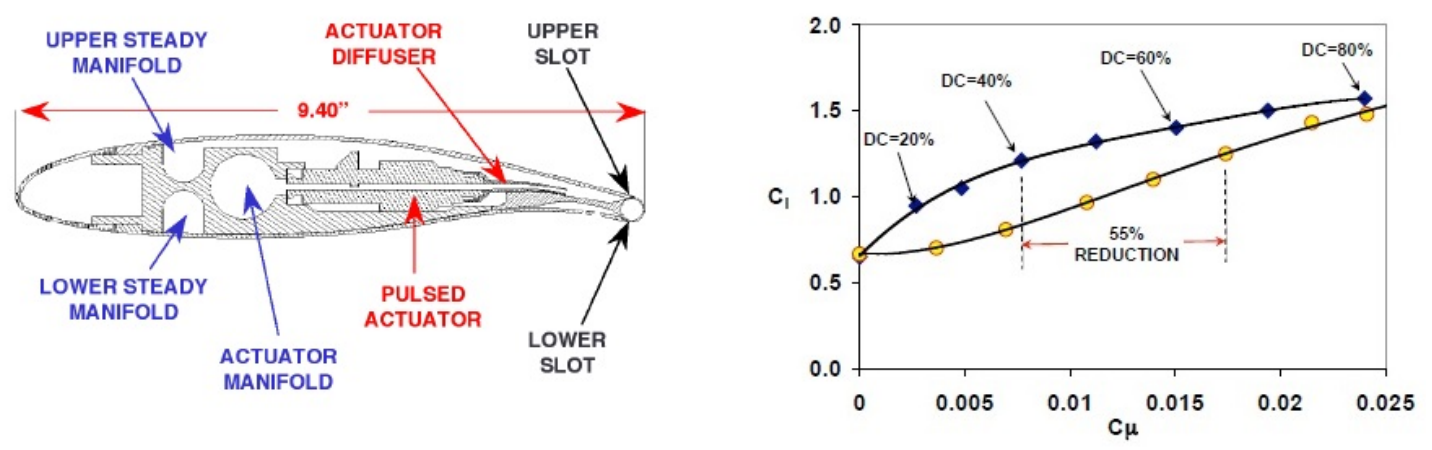

Figure 23. Experimental Duty Cycle Investigation Results (45) (46) 
The Strouhal number (Equation 28), a ratio of the local inertial force to convective force, is a common dimensionless parameter used for unsteady flows with a characteristic frequency of oscillation. The Strouhal equation includes frequency $(f)$, airfoil chord $(c)$, and freestream velocity $\left(V_{\infty}\right)$.

Strouhal Number

$$
S t=\frac{f \cdot c}{V_{\infty}}
$$

Magill, et al. (53) (61) reported peak lift enhancement for an AFC airfoil to occur near $S t=0.5$, for low-to-moderate $\alpha$. Seifert, et al. (52) claimed that separation control using periodic addition of momentum, at a St slightly higher than the airfoil natural vortex shedding frequency, can save up to $90 \%$ of the momentum required to obtain similar gains using steady blowing. The natural vortex shedding frequency is estimated by the inverse of the convective time scale, based on $c$ and $V_{\infty}$. Seifert, et al. (52) reported optimal actuation frequencies of $(0.5 \leq S t \leq 1.5)$ for $C_{1}$-enhancement, regardless of $R e$.

Grund \& Nitche (73) completed wind tunnel tests of a Stemme S10 glider configuration (HQ41profile), examining periodic excitation on a plain TE flap. A pulsating jet was introduced from the suction side of the flap near the separation area, which delayed flow separation for a flap-deflection angle $(\delta)$ greater than $16^{\circ}$. The effective control of the detached flow (unexcited wing) on the flap led to an increase in $L / D$ of up to $25 \%$. The efficiency of the AFC HQ41 airfoil is shown by the lift augmentation factor $\left(\Delta C_{l} / C_{\mu}\right)$, as seen in Figure 24a. The highest values for actuator efficiency were obtained for low AoA $\left(2^{\circ} \leq \alpha \leq 6^{\circ}\right)$ and small magnitudes of blowing $\left(C_{\mu}\right)$. An optimum frequency range was identified as $S t=$ $0.5\left(f_{a c t}=135 \mathrm{~Hz}\right)$, based on the $L / D$ ratio (Figure $\left.24 \mathrm{~b}\right)$. The effective $S t$ for a static airfoil validates the previous claims of Magill, et al. (53) (61).

a.)

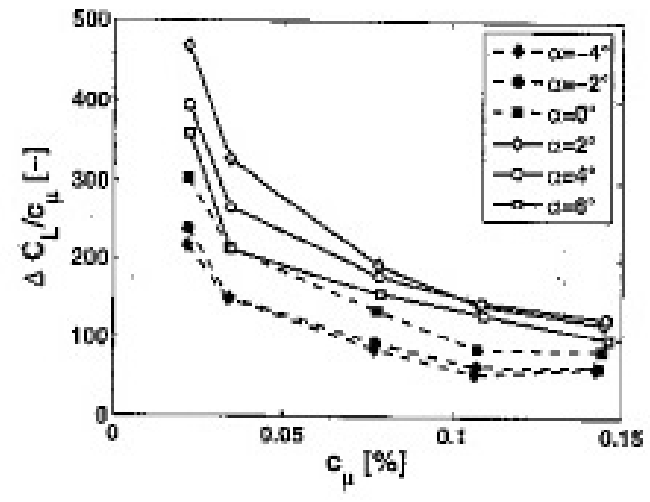

b.)

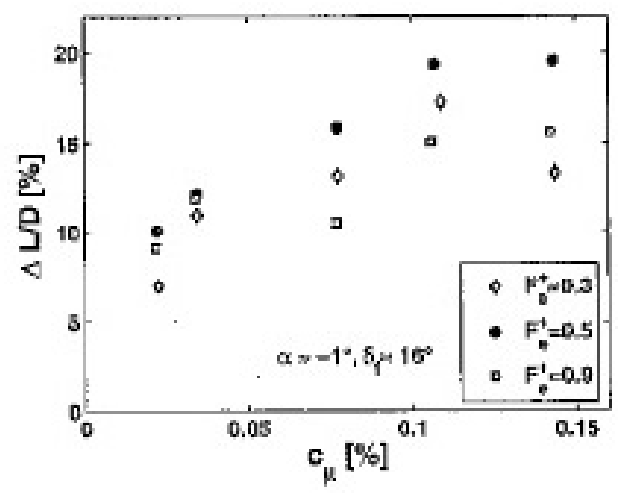

Figure 24. a.) AFC Actuator Efficiency b.) L/D Ratio for Different Excitation Frequencies $\left(F^{+}=S t\right)(73)$

Experiments by Seifert, et al. (75) reported actuation at reduced jet frequencies of $S t=0.3,0.6$ to be more effective at altering the LSB than steady suction, while steady blowing was found entirely 
ineffective. Experimental work by Timor, et al. (76) reported that high actuation frequency (St=7.5) of low momentum TE jets $\left(\left\langle c_{\mu}\right\rangle=0.04\right)$ increased $C_{1, \text { max }}$ by $20 \%$ and extended $\alpha_{\text {stall }}$ by $6^{\circ}$. According to Post \& Corke (77), when $f_{\text {jet }}$ is much larger than the fluid response frequency, the airfoil senses a constant body force and control authority is obtained.

Experiments by Oyler \& Palmer (47) were conducted on a $17 \%$ thick airfoil with a TE flap shoulder jet with both steady and pulsed blowing. Intermittent jet blowing was able to reduce airflow rates, compared with steady blowing, while delaying flow separation on a TE flap deflected at $40^{\circ}$. Lift

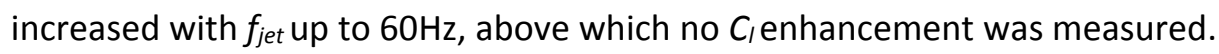

Seifert, et al. (52) completed experimental testing of a flapped NACA0015 airfoil, equipped with AFC capabilities at the LE $(x / c=0.10)$ and TE $(x / c=0.70)$. Various blowing schemes were tested, first investigating the individual effects of steady and pulsed blowing, and also simultaneously employing both. Thus, a combined momentum coefficient $\left(C_{\mu}\right)$ was defined (Equation 29), where $C_{\mu}$ represents the steady jet coefficient and $\left\langle c_{\mu}\right\rangle$ defines the oscillatory blowing momentum coefficient, determined from the RMS value of measured jet velocity $\left(\left\langle V_{j}\right\rangle\right)$. The same method to calculate $\left\langle c_{\mu}\right\rangle$, defined by Equation 30 , will be used in the present study.

Combined Momentum Coefficient

Pulsed Momentum Coefficient

$$
\begin{gathered}
\left(C_{\mu}\right)=\left(C_{\mu}:<c_{\mu}>\right)=(\text { steady: oscillatory }) \\
<c_{\mu}>=\frac{h_{\text {slot }}\left(\rho_{j}+\rho_{\infty}\right)<V_{j}>^{2}}{2}
\end{gathered}
$$

Equation 29

Equation 30

The influences of St and $C_{\mu}$ amplitude on NACA0015 lift are included in Figure 25. It should be noted that some authors use the symbol $F^{+}$(Reduced Frequency) to define an oscillatory jet, while others label the equivalent variable Strouhal number $(S t)$. For congruity through the remainder of this work, St will be used, but the reader should be aware of figures from literature that use the coequal variable form. 
(a)

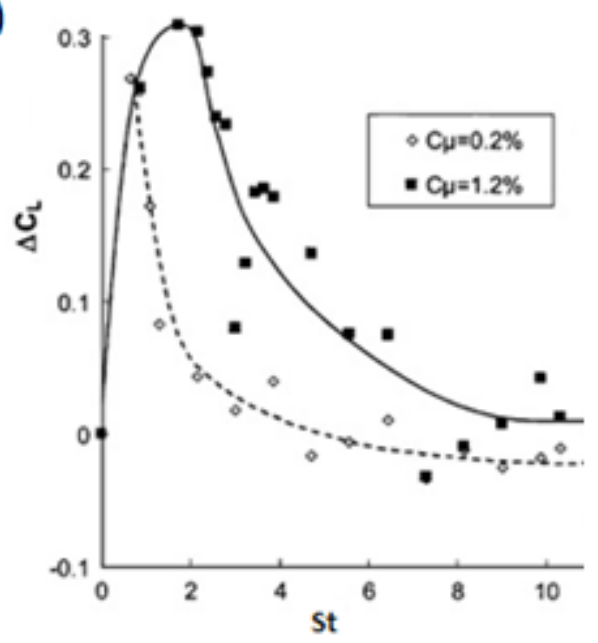

(b)

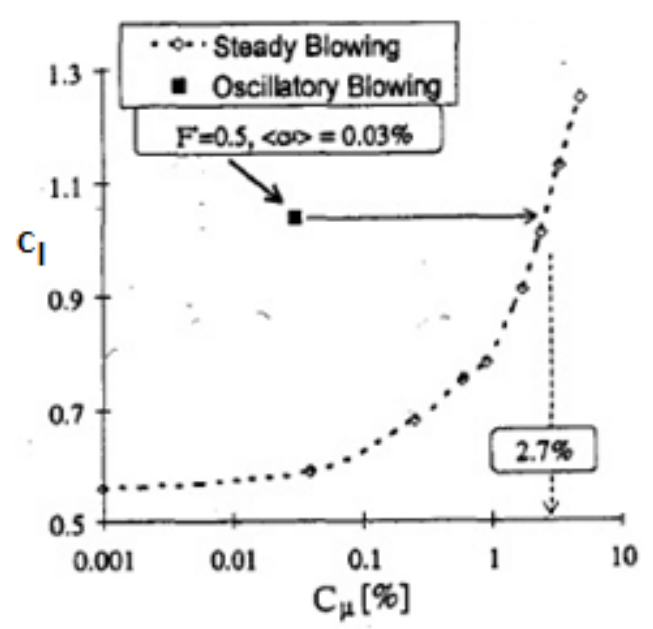

Figure 25. Influence of $S t$ and $C_{\mu}$ on Oscillatory Blowing Lift Enhancement (52) (78)

Results demonstrated that oscillatory blowing can effectively delay separation prior to stall, and reattach airfoil flow post-stall. Figure 25(a) shows the effect of reduced frequency on lift enhancement, indicating a relationship between $C_{\mu}$ and St for the modified NACA0015 airfoil. Figure 25(b) compares the magnitude of $C_{\mu}$ required for steady and pulsed blowing to achieve the same lift performance on a NACA0015 airfoil with flap-shoulder jets. Unsteady control achieved steady blowing lift with a $150 \%$ reduction in required mass flow.

Liu, et al. (79) completed 2D URANS studies of a CC wing with a $30^{\circ}$ dual-radius CC flap, to investigate the performance of pulsed jets relative to steady jets, with specific interest of the effects of jet frequency and optimum wave shape. The pulsed jet characteristics were user defined by the instantaneous momentum coefficient $\left(C_{\mu}(t)\right)$, which varies with time as shown in Equation 31.

Instantaneous Momentum Coefficient

$$
C_{\mu}(t)=C_{\mu, 0}+C_{\mu, 0} \cdot F(f, t)
$$

Equation 31

A set of preliminary calculations were completed using a sinusoidal function in the form described by Equation 32 , where the jet frequency if defined by $f_{\text {jet }}$.

Sinusoidal Jet Function

$$
F(t)=\cos \left(2 \cdot \pi \cdot f_{j e t} \cdot t\right)
$$

Equation 32

Numerical results concluded this shape to be ineffective as the computed $C_{l}$ values simply oscillated about the mean value, resulting in a time-averaged $C_{l}$ value no higher than the steady state value achieved with a fixed jet operating at the mean $C_{\mu, 0}$ value (79). Improved results were obtained when the function $F(t)$ was chosen to be a square shape wave form with a $50 \% D C$. The effects of actuation frequency and $C_{\mu}$ magnitude on $\Delta C_{l}$ of the CCW airfoil are included in Figure 26 , showing $f_{\text {jet }}=400 \mathrm{~Hz}$ to be optimal for matching steady CC jet lift enhancement. 


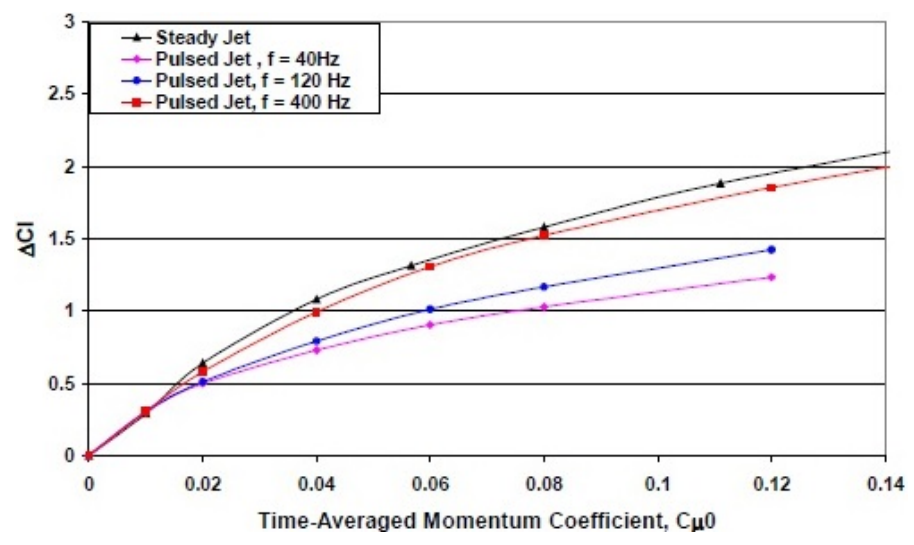

Figure 26. Influence of $f_{\text {jet }}$ on Lift Enhancement (79)

Ultimately, through various airfoil profiles and blowing strategies, oscillatory excitation has been proven to generate similar separation control and lift enhancement established with steady blowing, while drastically reducing mass flow and energy expenditure. A review (78) of a wide variety of airfoil data indicates that, for the vast majority of cases reviewed in the literature, optimum normalized jet actuation frequency $(S t)$ for separation control are in the range $(0.3 \leq S t \leq 4)$. Excitation at even higher frequencies $(2 \leq S t \leq 11)$ appears to have very little effect on lift. Similar results were recorded in an independent study by Zhou, et al. (80).

\subsubsection{Previous Circulation Control Research at WVU}

To date, all wind tunnel testing of circulation control models at $W V U$ has been conducted on static airfoils. Harness (81) tested a cambered CC elliptical airfoil. Results showed that adding camber produced a shift in the rear stagnation point, further increasing CC augmented lift. Also, TE geometry was determined to be an important factor in CC, with a rounded TE yielding better lift enhancement compared to various elliptical Coandă surfaces.

Myer (71) tested a $20 \%$ CC-Ellipse for comparison to potential flow solutions (82). Static airfoil tests revealed increased lift at all AoA, with better performance at low AoA $\left(\alpha<10^{\circ}\right)$. A maximum section lift coefficient of 4.58 using $C_{\mu}=0.17$ was measured, but desired lift augmentation was also found at low blowing rates. Myer (71) also concluded that the addition of camber resulted in an increase in lift augmentation ratio over that of a pure elliptical airfoil.

Holt (29) completed static tests with the same 20\% CC-Ellipse as Myer (71), but extended the research to include pulsed blowing. Based on the actuation capabilities of the wind tunnel model jet 
actuation below $50 \mathrm{~Hz}$ ( $S t=0.33$ ) was reported to reduce mass flow by $25 \%$ while increasing steady CC jet lift by $15 \%$.

Walters, et al. (83) tested a cambered elliptical airfoil section with a rounded TE, expanding the pulsed CC investigate to include higher AoA. Comparable results reported by Holt (29) for $\alpha=0^{\circ}$ were recorded by Walters, et al. (83) for higher AoA.

The applicability of CC high lift generation for STOL aircraft was first demonstrated at West Virginia University in 1974 with the WVU CC Technology Demonstrator STOL Aircraft by Loth \& Boasson (84). They noted that CC airfoil performance and blowing power requirements depend on both the jet momentum and jet to free stream velocity ratio as a significant portion of jet momentum is lost to shear over the Coandă surface (84). The research was expanded to include the first in-flight tests of CC applied to fixed wing aircraft.

Gibbs, et al. (70) developed a self-contained analysis method for arbitrary circulation controlled airfoils in incompressible flow. The analytical model predicted optimal blowing slot conditions on a given airfoil profile for desired lift-performance $\left(C_{l}\right)$ at specified free stream conditions.

Angle (85) completed his Ph.D. research of a 10\% CC-Ellipse (Circulation Controlled Helicopter Blade, "CCHB"), examining the aerodynamic benefits of LE and TE actuation for rotorcraft applications. More specifically, the research investigated the plausibility of utilizing CC for lift augmentation on the retreating rotor blade of a helicopter to replace the complex mechanical swash-plate. A pivotal result was the response time for CC lift augmentation, measured from initiation of the blowing jet to be 56 msec at $\alpha=0.68^{\circ}$ (85). From the measured and numerical data, it was concluded that CC can effectively be used on a helicopter main rotor for rotor speeds $\omega<275 \mathrm{rpm}$, with four defined azimuth-actuation points per blade revolution. Kweder (86) completed tests on the same 10:1 CC-ellipse to determine the effect of CC blowing on $\alpha_{\text {stall, }}$ concluding that $C_{\mu}=0.0127$ increased lift by $75 \%$ but decreased stall AoA by $2^{\circ}$.

Wilhelm (31) completed his dissertation research on the feasibility of applying circulation control to each airfoil of a 2-bladed H-rotor wind turbine, to increase energy capture over that of a traditional VAWT with fixed blades. He incorporated the effects of CC into existing VAWT analytical models (momentum model (35), vortex model (36)), resulting in power projections for baseline (NACA0018 blades and CCNACA0018 blades with $\left.C_{\mu}=0\right)$ and augmented VAWTs $\left(C_{\mu}=0.02,0.05,0.10\right)$ over a large range of wind conditions $(1 \leq \lambda \leq 6)$. Static wind tunnel data with steady CC, compiled from the aforementioned 2D CCNACA0018 model, were then used as updated aerodynamic coefficient inputs to determine VAWT performance enhancements due to various magnitudes of steady CC. 


\subsubsection{Summary of Static Airfoil Studies}

Table 3 summarizes pertinent aspects of CFD studies of static flow control airfoils previously discussed in this work. The numerical methodology included in Table 3 was used to guide the preliminary CFD analysis of the current study. Solution techniques involving turbulence modelling, jet boundary conditions (BCs), time step $\left(\Delta t^{+}\right)$, grid topology, and farfield boundaries are known parameters that influence numerical analysis of flow control airfoils, and are thus summarized here for reference to the current work.

Table 3. CFD Literature Review: Static Airfoil Simulation Conditions

\begin{tabular}{|c|c|c|c|c|c|c|c|c|c|c|c|c|c|}
\hline Author[s] & Solver & Airfoil & Airfoil Profile & $\operatorname{Re}[-]$ & AoA & Code & Model & Control & Actuation & $\Delta t^{+}$ & Discretization & Grid Topology & Farfield \\
\hline $\begin{array}{l}\text { Liu, et al. } \\
\text { (2001) }\end{array}$ & URANS & $\begin{array}{c}C C \text { Wing } \\
30^{\circ} \mathrm{CC} \text { Jet Flap } \\
\text { (2D) }\end{array}$ & & $3.95 \times 10^{5}$ & $\boldsymbol{\alpha}=0^{\circ}$ & - & $\begin{array}{l}1 . B L \\
2 . S A\end{array}$ & \begin{tabular}{|l} 
Steady CC \\
Pulsed CC
\end{tabular} & $\begin{array}{l}S t=0.00 \\
S t=0.28 \\
S t=0.85 \\
S t=2.83\end{array}$ & - & - & $\begin{array}{l}\text { (2D)C-Grid } \\
\text { (3D) C.H Grid }\end{array}$ & - \\
\hline $\begin{array}{l}\text { Jones, et al. } \\
\text { (2002) }\end{array}$ & URANS & $\begin{array}{l}(2 D) G A C C \\
1796 \text { profile }\end{array}$ & & $533 \times 10^{3}$ & $0^{\circ} \leq \alpha \leq 16^{\circ}$ & FUN3D & 1. $S A$ & $\begin{array}{l}\text { Steady CC } \\
\text { Pulsed CC }\end{array}$ & $25 \mathrm{~Hz} \leq f_{i \in \leq} \leq 200 \mathrm{~Hz}$ & - & - & $\begin{array}{l}\text { unstructured } \\
\text { tringular cells }\end{array}$ & all : $20 \mathrm{c}$ \\
\hline $\begin{array}{l}\text { Liu } \\
\text { (2003) }\end{array}$ & URANS & $\begin{array}{c}\text { (3D) NACAO012 } \\
\\
(2 D / 3 D) \text { cC Wing } \\
30^{\circ} \text { Jet Flap } \\
\text { (2D/3D) }\end{array}$ & & $\begin{array}{l}1.5 \times 10^{6} \\
3.95 \times 10^{6}\end{array}$ & $\alpha=8^{\circ}$ & $\begin{array}{l}\text { CCW3D } \\
\text { (G. Tech) }\end{array}$ & $\begin{array}{l}\text { 1. BL } \\
\text { 2. SA }\end{array}$ & $\begin{array}{l}\text { TE Jet Flap } \\
(x / c=0.89) \\
\text { Steady CC } \\
\text { Pulsed CC }\end{array}$ & $S t=0.00$ & Implicit & $\begin{array}{l}\text { space: }: 2^{\text {sd }} / 4^{\text {s }} \text { Ord. } \\
\text { time: } 1^{1 n} \text { Ord. }\end{array}$ & $\begin{array}{l}\text { (2D)C-Grid } \\
\text { structured } \\
\text { (3D) C-H Grid } \\
\text { hyperbolic }\end{array}$ & all $: 6 c$ \\
\hline $\begin{array}{c}\text { Zha, et al. } \\
(2006)\end{array}$ & RANS & $\begin{array}{l}\text { CFJO025 } \\
\text { (2D/3D) }\end{array}$ & & $380 \times 10^{3}$ & $\left.-10^{\circ}\right) \leq \alpha \leq 45$ & FLUENT & 1. $k-\varepsilon$ & $\begin{array}{l}\text { LE Blowing } \\
(x / c=0.071)\end{array}$ & $\begin{array}{l}C_{\mu}=\frac{\dot{m}_{j e t} \cdot V_{j e}}{q_{\infty} \cdot S} \\
\left(0.18 \leq C_{\mu u} \leq 0.32\right)\end{array}$ & $N A$ & $\begin{array}{l}\text { space: } \\
\text { inviscid : } 2^{\text {ed }} \text { Ord. Up. } \\
\text { viscous : } 2^{\text {sd }} \text { Ord. Cent. }\end{array}$ & $\begin{array}{l}\text { C-Grid } \\
\text { structured }\end{array}$ & all : 20c \\
\hline $\begin{array}{c}\text { Rumsey, et } \\
\text { al. } \\
\text { (2011) }\end{array}$ & $\begin{array}{l}\text { RANS } \\
\text { LES }\end{array}$ & $\begin{array}{c}30 \% \text { CC-Ellipse } \\
\text { (2D) }\end{array}$ & & $489 \times 10^{3}$ & $\alpha=0^{\circ}$ & $\begin{array}{l}\text { FUN3D } \\
\text { CFL3D }\end{array}$ & $\begin{array}{l}\text { RANS: } \\
1 . S A \\
\text { 2. SARC } \\
\text { 3. EASM } k-w \\
\text { 4. SSTRC } \\
\text { LES: } \\
\text { 5. Smagor. }\end{array}$ & Steady Cc & $\begin{array}{l}C_{\mu}=\frac{\dot{m}_{j \mu} \cdot V_{j e}}{q_{w} \cdot S} \\
\left(0.04 \leq C_{\mu} \leq 0.23\right)\end{array}$ & Implicit & 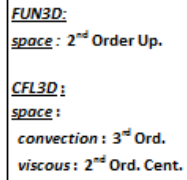 & $\begin{array}{l}\text { FUN3D: } \\
\text { unstructured } \\
\text { CFL3D: } \\
\text { structured }\end{array}$ & - \\
\hline $\begin{array}{c}\text { Nishino, et } \\
\text { al. } \\
\text { (2012) }\end{array}$ & LES & $\begin{array}{l}30 \% \text { CC-Ellipse } \\
\text { (2D) }\end{array}$ & & $490 \times 10^{3}$ & $\alpha=0^{\circ}$ & $C D P$ & \begin{tabular}{|l} 
LES: \\
1. Smargor.
\end{tabular} & SteadyCC & $\begin{array}{c}C_{\mu}=\frac{\rho \int V(n)^{2} c h}{q_{\infty} \cdot c} \\
\left(0.04 \leq C_{\mu} \leq 0.05\right)\end{array}$ & $\begin{array}{l}\Delta t^{+}=5 \times 10^{-5} \\
\Delta t^{+}=2 \times 10^{-4} \\
\text { Implicit }\end{array}$ & space: $2^{\text {ed }}$ Ord. & $\begin{array}{l}\text { structured } \\
\text { hex cells }\end{array}$ & $\begin{array}{l}\text { inlet : } 3.62 \mathrm{c} \\
\text { above : } 2.33 \mathrm{c} \\
\text { below : } 2.33 \mathrm{c} \\
\text { outlet : } 9 \mathrm{c}\end{array}$ \\
\hline $\begin{array}{r}\text { Li, et al. } \\
\text { (2013) }\end{array}$ & $\begin{array}{c}\text { URANS } \\
\text { LES }\end{array}$ & $\begin{array}{c}\text { NACA0018 } \\
\text { (rounded TE) } \\
\text { (2D/2.5D) }\end{array}$ & & $300 \times 10^{3}$ & $0^{\circ} \leq \alpha \leq 45^{\circ}$ & FLUENT & \begin{tabular}{|l} 
RANS: \\
$1 . S S T$ \\
LES: \\
2. Smagor.- \\
Lilly
\end{tabular} & $N A$ & $N A$ & $\begin{array}{l}\Delta t^{+}=1 \times 10^{-2} \\
\text { Implicit }\end{array}$ & 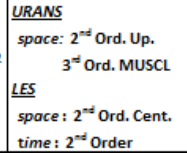 & $\begin{array}{l}\text { 0-Grid } \\
\text { structured } \\
\text { quad cells }\end{array}$ & all : 30c \\
\hline
\end{tabular}

Table 4 summarizes experimental studies of static flow control airfoils. The modified airfoils are detailed along with freestream conditions, angle-of-attack, and flow control method. Also, the measurement techniques and resulting performance quantities $\left(C_{p}, C_{l}, C_{d}\right)$ are summarized. The experimental studies were reviewed for comparison to the results of the current study, with emphasis on effective flow control actuation with pulsed jets. Specific results will be discussed further, but the summary table is presented to efficiently provide pertinent testing details. As previously discussed, effective pulsed flow control was most consistently achieved with low amplitude control $\left(C_{\mu} \leq 0.05\right)$ 
within the reduced jet frequencies in the range $0.3 \leq S t \leq 4.0$. The range of effective jet frequencies is due to the variety of unique airfoil profiles and test conditions $(R e, \alpha)$ of each study.

Table 4. Experimental Literature Review: Static Airfoil Tests

\begin{tabular}{|c|c|c|c|c|c|c|}
\hline Author(s) & Airfoil & $\operatorname{Re}[-]$ & AoA & Flow Control & Actuation & Reported Quantities [Uncertainty \pm 96 ] \\
\hline $\begin{array}{l}\text { Harness } \\
(1970) \\
W v U\end{array}$ & Symmetric CC-Ellipse (2D) & $4.4 \times 10^{5}$ & $\alpha=0^{\circ}$ & $\begin{array}{l}C C \text { Jet } \\
x / c=0.9445\end{array}$ & Steady Jet & $\begin{array}{l}- \text { Measured: static surface pressure: } P_{i} \\
- \text { Calculated: } C_{p}, C_{1}, C_{m}\end{array}$ \\
\hline $\begin{array}{l}\text { Myer } \\
(1972) \\
W V U\end{array}$ & 2096 Cambered CC-Ellipse (2D) & $\begin{array}{l}4.4 \times 10^{5} \text { to } \\
6.7 \times 10^{5}\end{array}$ & $\left(-10^{\circ}\right) \leq \alpha \leq 10^{\circ}$ & $\begin{array}{l}C C \text { Jet } \\
x / c=0.9445\end{array}$ & $\frac{\text { Steady Jet }}{0.02 \leq C_{\mu \mu}} \leq 0.17$ & $\begin{array}{l}\text { - Measured: static surface pressure: } P_{i} \\
- \text { Calculated: } C_{p}, C_{1}, C_{m}\end{array}$ \\
\hline $\begin{array}{l}\text { Holt } \\
(1972) \\
w V U\end{array}$ & 2096 Cambered CC-Ellipse (2D) & $4.3 \times 10^{5}$ & $a=0^{\circ}$ & $\begin{array}{l}C C \text { Jet } \\
x / c=0.9445\end{array}$ & $\begin{array}{l}\text { Pulsed Jet } \\
C_{\mu \mu}=0.05 \\
0.27 \leq \text { St } \leqslant 0.67\end{array}$ & $\begin{array}{l}- \text { Measured: static surface pressure: } P_{i} \\
- \text { Calculated: } C_{p}, C_{1}, C_{m}\end{array}$ \\
\hline $\begin{array}{l}\text { Walters, et al. } \\
\text { (1972) } \\
\text { WVu }\end{array}$ & $20 \%$ Cambered CC-Ellipse (2D) & $\begin{array}{l}4.4 \times 10^{5} \text { to } \\
5 \times 10^{5}\end{array}$ & $0^{\circ} \leq \alpha \leq 10^{\circ}$ & $\begin{array}{l}\text { CC Jet } \\
x / c=0.9445\end{array}$ & $\begin{array}{l}\text { Steady Jet } \\
\text { Pulsed Jet }\end{array}$ & $\begin{array}{l}- \text { Measured: static surface pressure: } P_{i} \\
- \text { Calculated: } C_{p}, C_{1}, C_{m}\end{array}$ \\
\hline $\begin{array}{l}\text { Oyler, et al. } \\
\text { (1972) }\end{array}$ & 17\% Flap Jet (2D) & $3.55 \times 10^{6}$ & $a=0^{\circ}, 20^{\circ}$ & $\begin{array}{l}\text { Steady Flap Jet } \\
\text { Pulsed Flap Jet } \\
\delta_{\text {flop }}=0^{\circ}, 40^{\circ}\end{array}$ & $\begin{array}{l}0.01 \leq C_{\mu z} \leq 0.165 \\
15 \mathrm{~Hz} \leq f_{j e t} \leq 80 \mathrm{~Hz}\end{array}$ & $\begin{array}{l}\text { - Measured: static surface pressure: } P_{i} \\
\text { - Measured: BL (hot-wire) } \\
\text { - Measured: airfoil forces }\left(F_{n}, F_{a}, F_{m}\right) \\
\text { - Calculated: } C_{p}, C_{1}, C_{m}\end{array}$ \\
\hline $\begin{array}{l}\text { Greenblatt, } \\
\text { et al. (2000) }\end{array}$ & Cambered CC-Ellipse (2D) & $2 \times 10^{5}$ & $\left(-4^{5}\right) \leq \alpha \leq 16^{\circ}$ & $\begin{array}{l}\text { LE Tangential Jet } \\
\text { Flap Shoulder Jet }\end{array}$ & $\begin{array}{l}\text { Steady: } 0.017 \leq C_{\mu \mu} \leq 0.075 \\
\text { Pulsed: } 0.002 \leq C_{\mu} \leq 0.012 \\
0 \leq 5 \mathrm{st} \leq 10\end{array}$ & $\begin{array}{l}\text { - Measured: static surface pressure: } P_{i} \\
\text { - Measured: airfoil forces }\left(F_{n}, F_{a}, F_{m}\right) \\
\text { - Calculated: } C_{p}, C_{1}, C_{m}\end{array}$ \\
\hline $\begin{array}{l}\text { Jones } \\
\text { (2006) }\end{array}$ & Gen. Aviation Circulation Control Wing (2D) & $1.13 \times 10^{6}$ & $\left(-18^{\circ}\right) \leq \alpha \leq 20^{\circ}$ & $\begin{array}{l}\text { Steady CC Jet } \\
\text { Pulsed CC Jet }\end{array}$ & $\begin{array}{l}\text { Upper Jet: } \\
0.017 \leq C_{\mu} \leq 0.075 \\
\text { Lower Jet: } \\
0.024 \leq C_{\mu} \leq 0.054 \\
\end{array}$ & $\begin{array}{l}\text { - Measured: static surface pressure: } P_{i} \\
\text { - Measured: wake rake pressure } \\
\text { - Measured: airfoil forces }\left(F_{n}, F_{q}, F_{m}\right) \\
\text { - Calculated: } C_{p}, C_{l}, C_{l} \quad\left(\delta C_{d}= \pm 0.0005\right)\end{array}$ \\
\hline $\begin{array}{l}\text { Angle } \\
(2008) \\
W v U\end{array}$ & TE 1096 CC-Ellipse Helicopter Blade (2D) & $\begin{array}{l}3.08 \times 10^{5} \text { to } \\
9.24 \times 10^{5}\end{array}$ & $\left(-4^{\circ}\right) \leq \alpha \leq 16^{\circ}$ & $\begin{array}{l}\text { SteadyCC } \\
\text { TE Jet } \\
\text { LE Jet }\end{array}$ & $\begin{array}{l}\text { Upper Jet: } \\
0.04 \leq C_{\mu \mu} \leq 0.645 \\
\text { Lower Jet: } \\
0.04 \leq C_{\mu \mu} \leq 0.645\end{array}$ & $\begin{array}{l}\text { - Measured: static surface pressure: } P_{i} \\
\text { - Measured: airfoil forces }\left(F_{n}, F_{a}\right) \\
\text { - Calculated: } C_{p}, C_{l}, C_{d} \quad\left(\delta C_{d}= \pm 0.0126\right)\end{array}$ \\
\hline $\begin{array}{l}\text { Kweder } \\
\text { (2008) } \\
\text { WvU }\end{array}$ & TE $10 \%$ CC-Ellipse Helicopter Blade (2D) & & $0^{\circ} \leq \alpha \leq 16^{\circ}$ & $\begin{array}{l}\text { SteadyCC } \\
\text { TE Jet } \\
\text { LE Jet }\end{array}$ & $\begin{array}{l}\text { Upper Jet: } 0.04 \leq C_{\mu} \leq 0.20 \\
\text { Lower Jet: } \\
0.04 \leq C_{\mu} \leq 0.20\end{array}$ & $\begin{array}{l}\text { - Measured: airfoil forces }\left(F_{n}, F_{a}\right) \\
\text { - Calculated: } C_{1}, C_{d} \quad\left(\delta C_{d}= \pm 0.05\right) \\
\text { - } \delta C_{\mu z}= \pm 0.03\end{array}$ \\
\hline $\begin{array}{l}\text { Seifert, et al. } \\
\text { (1996) } \\
\text { Seifert, et al. } \\
\text { (1999) }\end{array}$ & modifed NACA0015 with TE Flap (2D) & $\begin{array}{l}17 \times 10^{6} \text { to } \\
38 \times 10^{6}\end{array}$ & $\left(-9^{\circ}\right) \leq \alpha \leq 15^{\circ}$ & $\begin{array}{l}\text { LE Jet } \\
x / c=0.10 \\
\text { Flap Shoulder Jet } \\
x / c=0.70\end{array}$ & $\begin{array}{l}\text { Steady Jet } \\
C_{\mu \mu}=0.02 \\
\text { Pulsed Jet } \\
0.03 \leq C_{\mu \mu} \leq 0.06 \\
0.5 \leq S t \leq 1.5 \\
0.5 \leq 5\end{array}$ & $\begin{array}{l}\text { - Measured: static surface pressure: } P_{i} \\
- \text { Calculated: } C_{p}, C_{1}, C_{i}\end{array}$ \\
\hline $\begin{array}{l}\text { Seifert, et al. } \\
(2000)\end{array}$ & 2096 Glauert-Goldschmied Airfoil (2D/3D) & $\begin{array}{l}2 \times 10^{6} \text { to } \\
40 \times 10^{6}\end{array}$ & $\alpha=0^{\circ}$ & $\begin{array}{l}\text { Tangential Jets } \\
\text { Steady, Pulsed } \\
x / c=0.25 \\
x / c=0.59\end{array}$ & $\begin{array}{l}\text { Periodic Excitation } \\
0.3 \leq S t \leq 1.0 \\
0.001 \leq<c_{\mu}>\leq 0.0055\end{array}$ & $\begin{array}{l}\text { - Measured: static surface pressure: } P_{i} \\
\text { - Calculated: } C_{p}, C_{d}, C_{m}, C_{n} \\
\left.\text { - } \delta<c_{\mu}\right\rangle= \pm 25 \mathrm{FS} \%, \delta\left[C_{p}\right]= \pm 5 \mathrm{FS} \%\end{array}$ \\
\hline $\begin{array}{l}\text { Timor, et al. } \\
\text { (2007) }\end{array}$ & cropped NACA0018 (3D) & $\begin{array}{l}1.6 \times 10^{5} \text { to } \\
3 \times 10^{5}\end{array}$ & $0^{\circ} \leq \alpha \leq 20^{\circ}$ & $\begin{array}{l}\text { Tangential Jets: } \\
\text { Steady, Pulsed } \\
x / c=0.70\end{array}$ & $\begin{array}{l}\text { Periodic Excitation } \\
3.04 \leq s t \leq 9.5 \\
0.02 \leq<c_{\mu}>\leq 0.04\end{array}$ & $\begin{array}{l}\text { - Measured: static surface pressure: } P_{i} \\
\text { - Calculated: } C_{p}, C_{1}, C_{m}, C_{n} \\
\text { - } \delta\left[C_{p}\right)= \pm 2 \%= \pm 0.05, \delta\left[C_{l, \max }\right)= \pm 0.03\end{array}$ \\
\hline
\end{tabular}

\subsection{Pitching Airfoil Aerodynamics}

This section reviews past research from the literature of pitching airfoil studies related to the current research work. Dynamic stall of pitching airfoils results in unique aerodynamic performance as a function of airfoil profile, freestream conditions, pitch rate, average AoA, and pitch amplitude. Thus, this review focuses on previous studies of airfoils with comparable characteristics to the CCNACA0018 and/or similar test conditions (e.g. $R e, k, \pm \alpha$ ) using equivalent measurement techniques. Concerning airfoil profiles, symmetric NACA profiles were considered for baseline comparisons of the current work, while pitching studies of airfoils with camber, TE flaps, and/or flow control were considered to 
benchmark CCNACA flow control cases. This myriad of factors combines and contributes to the severity of aerodynamic force coefficient hysteresis loops. With so many parameters influencing dynamic stall, a sound review of past research is necessary to correlate a myriad of results. The review includes dynamic stall analysis from both numerical and experimental methods.

\subsubsection{Static Stall vs. Dynamic Stall}

Before reviewing past research of dynamic stall, a brief comparison of static and dynamic stall characteristics is warranted. Carr (7) created an excellent visual representation of dynamic stall (DS) events, with correlation to airfoil $C_{l}$ and $C_{m}$, for a pitching NACA0012 airfoil (Figure 27). A sound description of boundary layer state with sketches is included. The dotted line of Figure 27 represents the static coefficient curve while solid lines detail dynamic results.

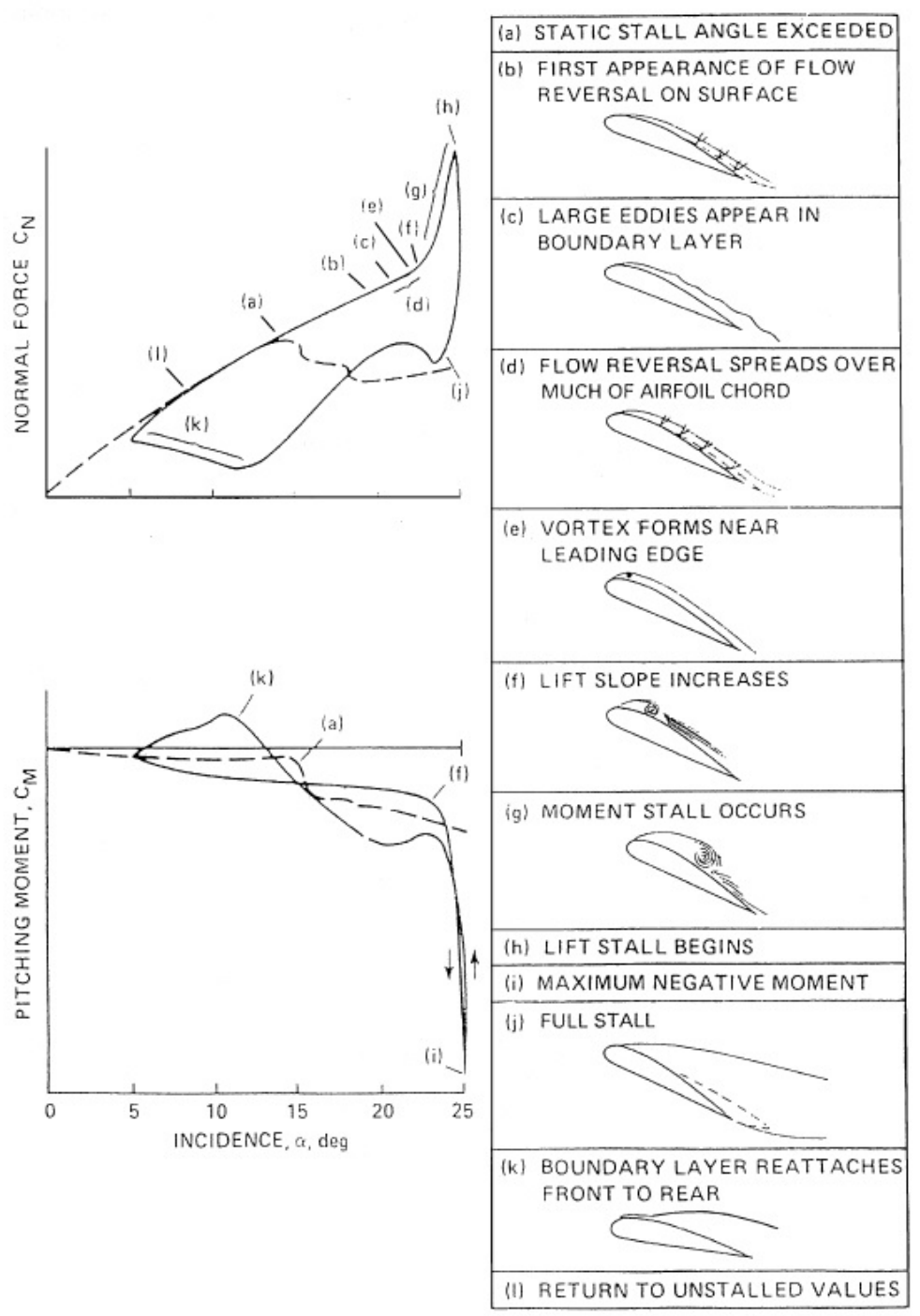

Figure 27. Dynamic Stall events on a NACA0012 Airfoil at low $\operatorname{Re}(7)$ 
Past experiments show that a pitching airfoil tolerates large regions of reversed flow on its surface before succumbing to large-scale, boundary layer separation (60). The tolerance allows the airfoil to pitch well beyond the quasi-steady stall $\alpha$ and attain a much higher $C_{\text {I }}$ than would normally occur (87). The enhanced lift experienced during pitching motions can be attributed to the shedding of a concentrated vortex from the LE of the airfoil. While the vortex resides over the suction surface of the airfoil, enhanced lift is produced (54).

Past work has identified sub-regimes of dynamic stall, indicating the severity of DS characteristics. Such sub-groups are commonly known as light (LDS) and deep dynamic stall (DDS). LDS, characterized by a weak stall vortex and a viscous zone on the order of magnitude of the airfoil thickness, is found to be sensitive to geometrical parameters (e.g. LE radius, camber, $t / c$ ) that affect quasi-steady stall (60) (88). LDS commonly occurs when the oscillation rate is low and $\alpha_{\text {max }}$ remains close to static stall (87). A $C_{1}-\alpha$ hysteresis loop with gradual drop-off is often observed under such conditions, signifying a slow and gradual process; the airfoil generally experiences a quicker lift-recovery. McAlister \& Tung (89) provide a supporting description of light dynamic stall (LDS) for a pitching NACA0012 airfoil at low $\operatorname{Re}\left(2 \times 10^{5}\right)$ under the following conditions: $\alpha(t)=15^{\circ}+10^{\circ} \sin (\omega \cdot t)$ [k=0.100]. Wind tunnel smoke flow visualization was used to correlate measured $C_{l}$ and $C_{m}$ stall events.

(a) LIGHT STALL

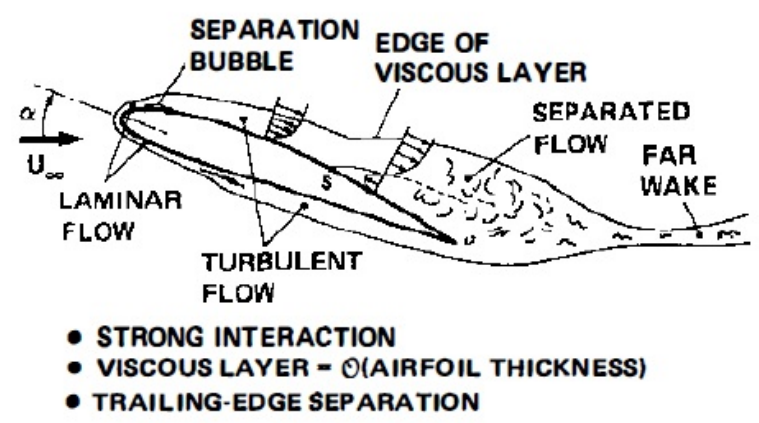

\section{(b) DEEP STALL}

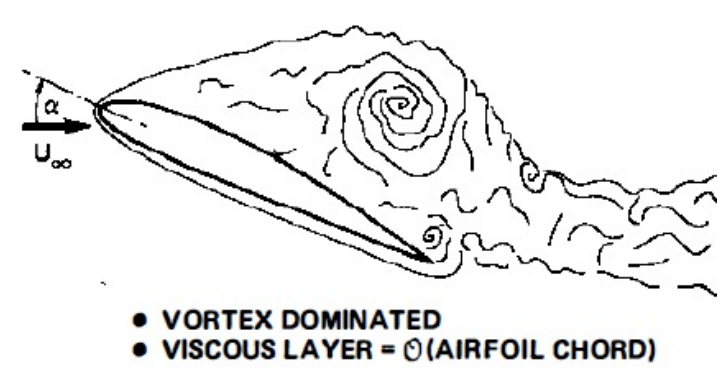

Figure 28. Common BL Characteristics of Light vs. Deep Dynamic Stall (90)

DDS, however, is relatively uninfluenced by the geometrical parameters of the airfoil and exhibits large-scale vortex shedding (Figure 28(b)), larger force-coefficient overshoots, and hysteresis amplitudes. The scale of the viscous zone is on the order of magnitude of the airfoil chord; major influences of this flow phenomenon are the amount of time the airfoil spends above the $\alpha_{S S}$, along with pitch amplitude $\left(\alpha_{\max }\right)$ (87). Leishman (91) published results for a pitching NACA0015 under deep dynamic stall (DDS) conditions. 
Examination of aerodynamic loads, surface pressure distributions, and flow visualization techniques have resulted in the identification of stall onset criteria (40), visualized in Figure 29. Of relevance to the current study, the normal force (Figure 29a) and drag force (Figure 29c) deviation trends will be used to determine the AoA of DS onset. The force-curve deviations are determined by sharp increases in slope. Also, $C_{p}$ deviation (Figure 29e) and collapse (Figure 29f) will be used to analyze DSV movement along the airfoil upper surface.

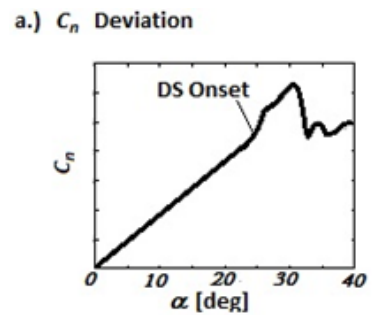

b.) $C_{m}$ Break $\left(\Delta C_{m}=0.05\right)$

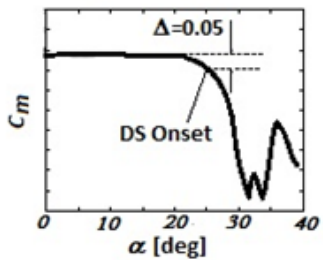

e.) $0.25 c \quad c_{p}$ Deviation

d.) $C_{a}$ Maximum

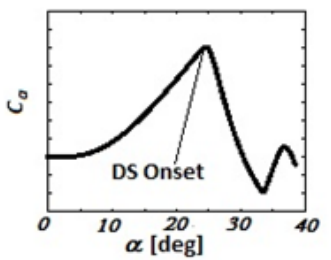

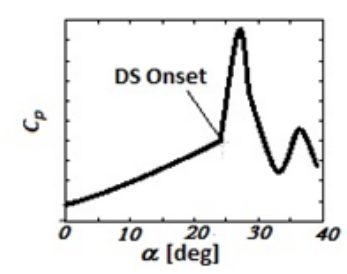

c.) $C_{d}$ Deviation

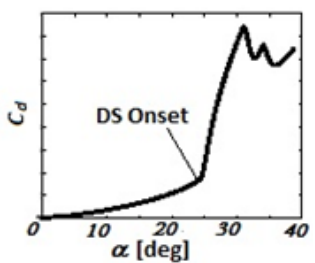

f.) LE $C_{p}$ Collapse

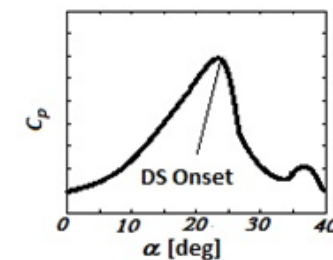

Figure 29. Aerodynamic Data Trends to Identify DS Onset (40)

Surface pressure scans can reveal trend departures at critical points on the airfoil to identify early stall behavior: $C_{p}$ deviations (Figure 29e) at the airfoil quarter-chord $(x / c=0.25)$, as well as suction pressure collapse at the LE (Figure 29f), both represent preliminary stages of the DS process (40). Dominant features or small-scale remnants of each of the six criteria can be observed for any dynamic stall occurrence. The degree of each, however, is unique to each pitching airfoil scenario and one, or many, characteristic(s) may be more prevalent than others. The use of surface flow anomalies for DS detection is the precursor to closed-loop stall control.

Dynamic stall analyses are most commonly conducted on oscillating airfoils with a sinusoidalpitching motion, defined by Equation 33, where $\alpha_{0}$ is the mean angle of oscillation, $\alpha_{1}$ is the pitch amplitude angle, $\omega$ is the pitch frequency $[\mathrm{rad} / \mathrm{s}]$, and $t$ represents time.

$$
\alpha=\alpha_{o}+\alpha_{1} \cdot \sin (\omega \cdot t)
$$

Equation 33

The power produced by a VAWT at its regulated wind speed is much higher than would be anticipated from an analysis based on steady airfoil data. The additional power output is a direct consequence of an unsteady flow phenomenon known as dynamic stall (5) (28) (29). The flow over 
airfoils undergoing constant pitching or oscillating motions exhibit lift coefficients of up to five times the static values (60).

\subsubsection{Influence of Reduced Frequency $(k)$}

The influence of pitch-rate on aerodynamics has been a popular topic of debate from a variety of sources. Many sources concur that this parameter is the dominating parameter influencing aerodynamics during DS. Sharma, et al. (92) reported that $k$ dominates the influence of $R e$, and increasing pitch rate enlarges hysteresis loops and increases the dynamic stall AoA $\left(\alpha_{D S}\right)$ increases due to the movement of separation point towards the TE. Soltani, et al. (93) used hot-film and surface pressure measurements on a pitching HAWT section to conclude that the width and shape of hysteresis loops was strongly impacted by reduced frequency, but was only slightly influenced by Re. As $k$ was increased, $(x / c)_{\text {transition }}$ shifted toward the airfoil LE.

Sheng, et al. (40) compiled experimental data from 13 airfoils tested at the University of Glasgow, primarily symmetric and cambered NACA profiles of various thicknesses. The data revealed a pitch rate of $k=0.01$ to mark the boundary of quasi-steady and dynamic stall regimes. For $k>0.01, \alpha_{D S}$ was shown to increase linearly with pitch rate. Figure 30 compares the influence of reduced pitch frequency on dynamic airfoil stall for NACA0012 (open circles) and NACA0018 (filled circles) airfoils. Both profiles reveal a linear relationship between $k$ and $\alpha_{D S}$, with the thicker NACAO018 profile having a higher stall angle for $k>0.020$.

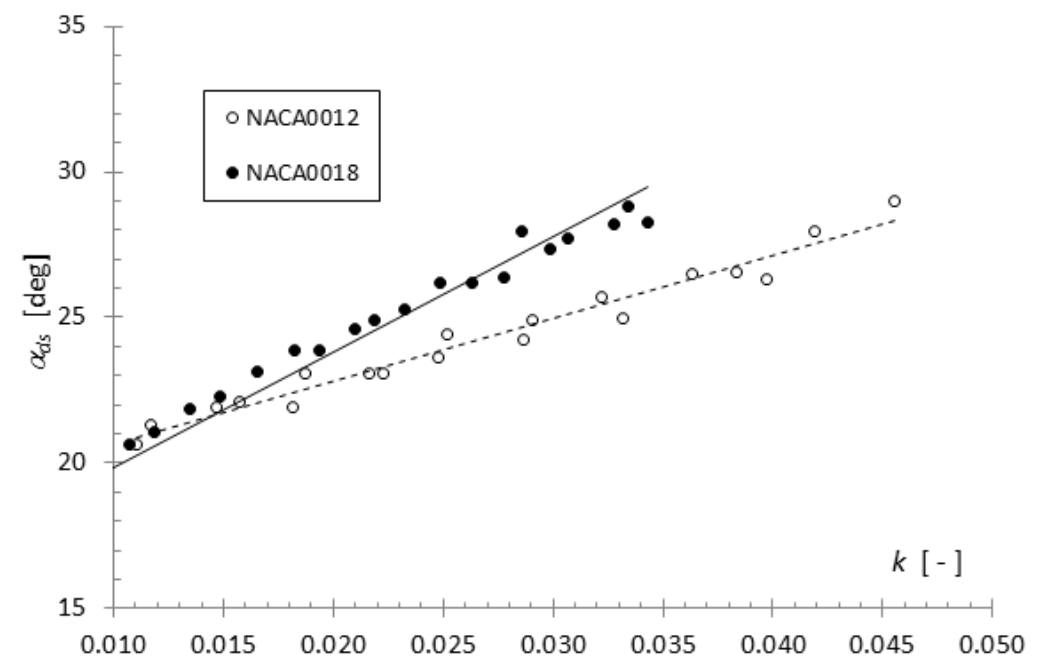

Figure 30. Influence of $k$ on Dynamic Stall AoA: NACA0012 vs. NACA0018 Airfoils (40)

Raghunathan, et al. (23) conducted wind tunnel investigations on a NACA0018 airfoil oscillating about $\alpha_{0}=0^{\circ}$ for performance estimates of the Wells self-rectifying air turbine for wave energy 
applications. For a range of low reduced pitch frequencies, increasing $k$ was shown to increase $C_{l, \text { max }}$ $C_{d, \max }$, and $\alpha_{\text {stall. }}$. The influence of $k$ on surface pressure distributions is evident in Figure 31, comparing upper surface separation for near static stall.
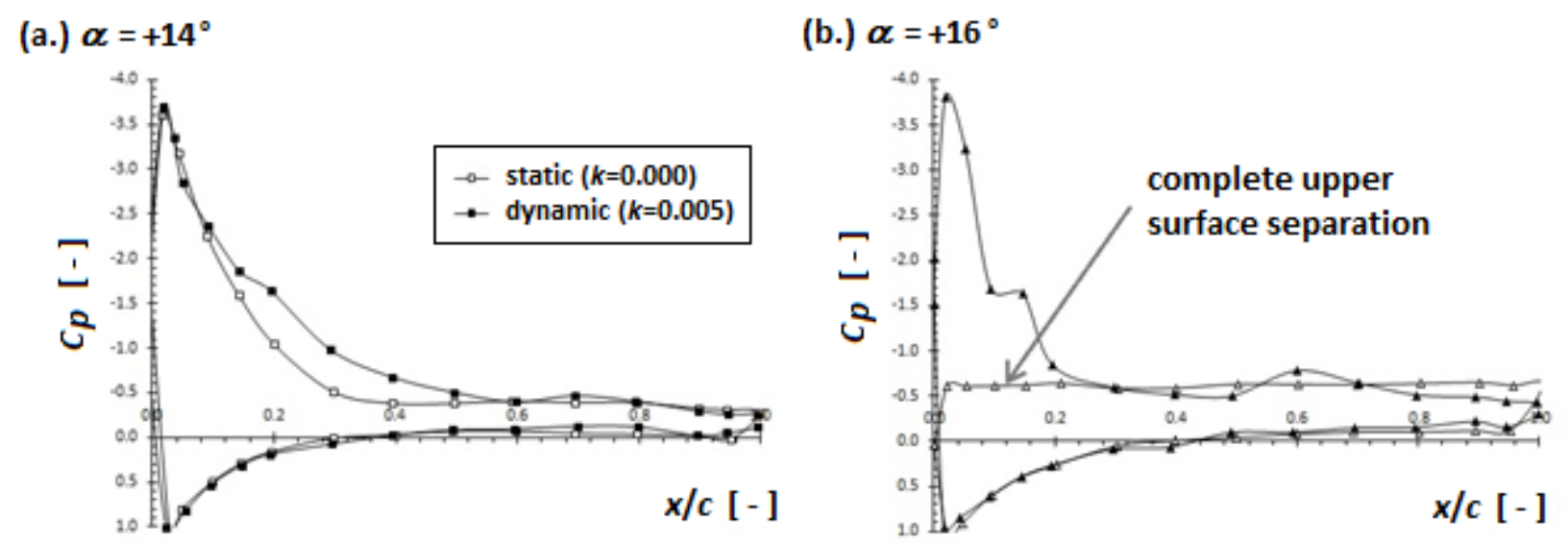

Figure 31. Influence of Pitch on $C_{p}$-Distribution of a Pitching NACA0018 Airfoil: $R e=240 \times 10^{3}$ (23)

Prior to static stall $\left(\alpha=14^{\circ}\right)$, a difference between separation point is apparent as the static airfoil separates at $x / c=0.3$ while the oscillating case reveals separation at $x / c=0.5$. Reduced separation is exacerbated post static stall $\left(\alpha=16^{\circ}\right)$ as the fixed airfoil reveals complete upper surface stall, while the pitching case shows flow attachment up to about $(x / c) \approx 0.3$.

Lee, et al. (94) used multiple hot-film sensor arrays on a NACA0012 airfoil, oscillating within and beyond $\alpha_{S S}$, to monitor spatial-temporal progressions of the LE stagnation, separation, and reattachment points, and the state of the unsteady boundary layer on the upper surface. Key results showed that increasing $k$ delayed the turbulent $\mathrm{BL}$ breakdown and the onset of flow reversal to higher AoA, increasing $\alpha_{\text {stall. }}$

Tsang, et al. (95) converted direct force measurements via piezoelectric load cells to investigate a NACA0012 airfoil undergoing dynamic stall. Test results showed increasing $\alpha_{D S}$ with pitch rate. Meanwhile, the angle at which the unsteady lift coefficient returned to its pre-stall value was delayed (i.e. increased $C_{l}$ hysteresis). Pitch frequency had little to no effect on $C_{l, \text { max }}$ when the airfoil oscillated within $\alpha_{s s}$. When the airfoil pitched past $\alpha_{S S}$, DS occurred and increasing $k$ resulted in $C_{l, \max }$ rises.

The aforementioned work of McCalister, et al. (60) (96) reported reduced frequency to be the most important parameter, finding it common to find $100 \%$ increases in force and moment coefficients as $k$ was varied from 0.004 to 0.25 . Quasi-steady conditions, where coefficients do not deviate from steady values, was found to be $k=0.004$ for cases without endplates; Sheng, et al. (40) stated $k=0.01$ as the demarcation point between regimes. McAlister, et al. (96) reported endplates to have an effect on 
the stall onset and the strength of the shed vortex only at low $k$, although they appear to offer some improvement in the stability and performance characteristics over the entire frequency range $(0.004-$ $0.25)$, explaining the different values of $k$ representing quasi-steady conditions. An overall summary of test results from Carr, et al. (60) (96) revealed suction surface flow reversal to be delayed with increasing $k$ and the subsequent development of DS events were correspondingly delayed to higher AoA. The influence of increasing $k$ on NACA0012 lift and drag is included in Figure 32.

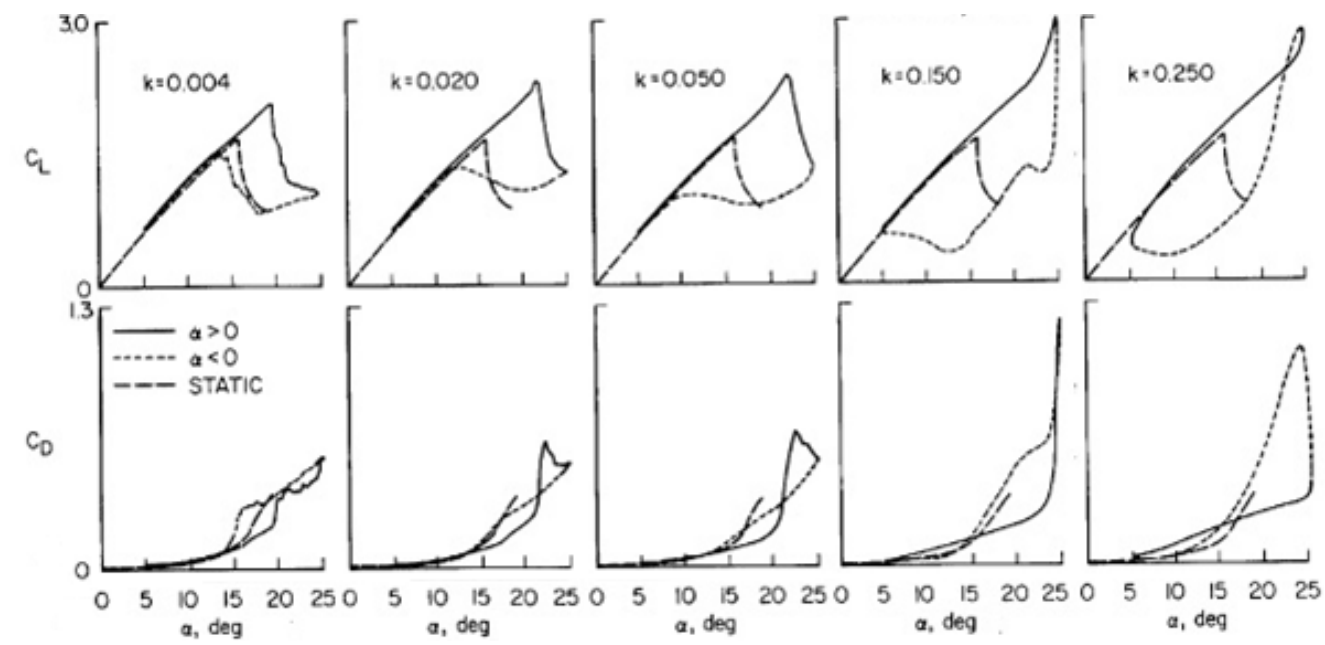

Figure 32. Influence of $k$ on Pitching NACA0012 Performance: $\alpha=15^{\circ}+10^{\circ} \cdot \sin (\omega t), \operatorname{Re}=2 \times 10^{6}(60)(96)$

Wind tunnel testing, with PIV measurements, completed by Griffin, et al. (55) (97) on a pitching NACA0012 airfoil with LE dynamic roughness elements, concluded that $k$ significantly impacted the development of the DSV, delaying formation to higher AoA. Flow visualization of such results, for $\alpha=26^{\circ}$ and $\alpha=28^{\circ}$ are included in Figure 33; the study included actuation frequencies in the range $0.8 \leq S t \leq 2.0$.
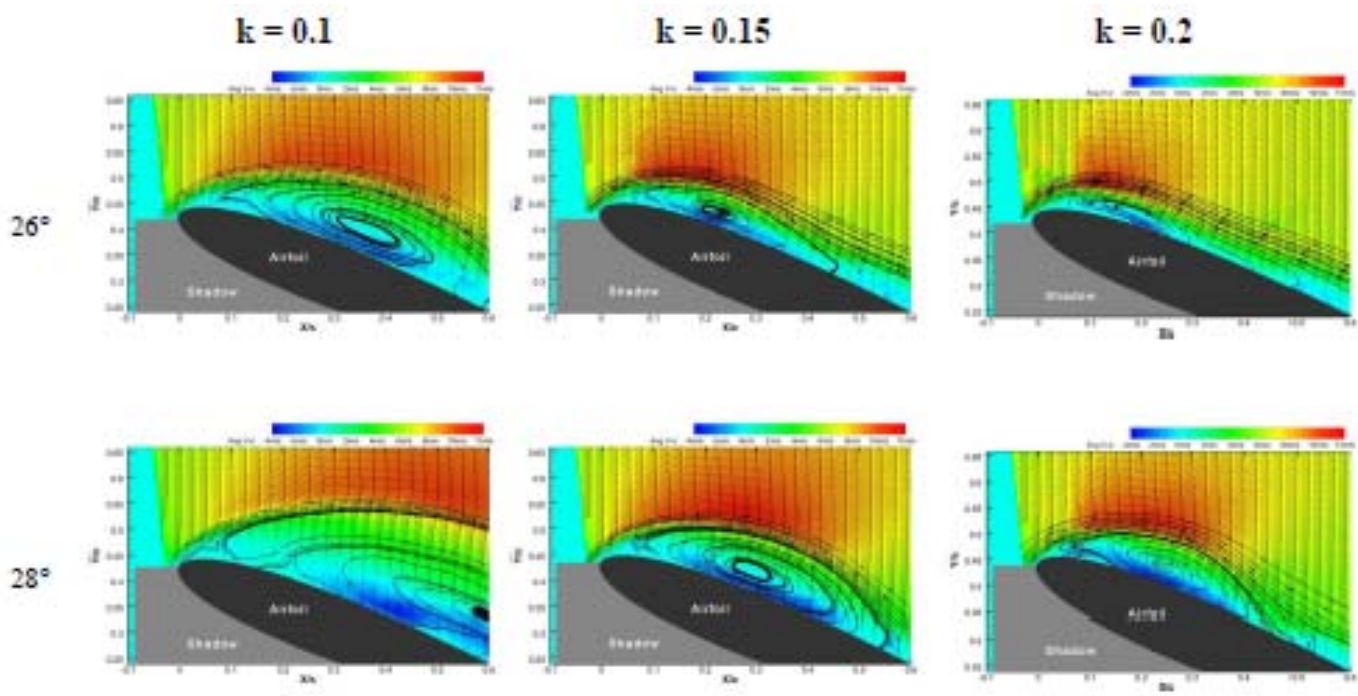

Figure 33. Influence of $k$ on DSV Formation on a Pitching NACA0012 Airfoil: $\operatorname{Re}=80 \times 10^{3}(55)$ 
Lorber \& Carta (98) completed experimental tests on a Sikorsky SSC-AO9 airfoil, concluding that an increased pitch rate causes stall events to be delayed, strengthens the stall vortex, and increases in airfoil loading. A collection of data from the literature revealed a linear relation between $k$ and the liftcurve-slope $\left(\mathrm{d} C_{1} / \mathrm{d} \alpha\right)$ of pitching airfoils; the data can be seen in Figure 34. Compiling such results helps to solidify parametric trends as unique errors of individual studies is filtered out (e.g. wind tunnel blockage and 3D effects).

\begin{tabular}{|c|c|c|c|}
\hline Reference & $R e$ & $k$ & $\mathrm{~d} C_{l} / \mathrm{d} \alpha$ \\
\hline Angelo, et al. (1988) & 1100000 & 0.050 & 0.0887 \\
\hline Amet, et al. (2009) & NA & 0.105 & 0.1054 \\
\hline Amiralaei, et al. (1988) & 1000 & 0.100 & 0.063 \\
\hline Danao, et al. (2013) & NA & 0.050 & 0.0697 \\
\hline Laneville, et al. (1986) & 38000 & 0.161 & 0.1333 \\
\hline Liggitt PhD Thesis (2012) Exper. & 1630000 & 0.021 & 0.0451 \\
\hline Liggitt PhD Thesis (2012) CFD & 1630000 & 0.021 & 0.0619 \\
\hline McCroskey (1982) & 3450000 & 0.151 & 0.1054 \\
\hline Mueller-Vahl, et al. (2014) & 250000 & 0.007 & 0.0663 \\
\hline Shrewsbury, et al. (1990) Exper. & 3060000 & 0.100 & 0.0777 \\
\hline Shrewsbury, et al. (1990) CFD & 3450000 & 0.151 & 0.0934 \\
\hline Shrewsbury, et al. (1990) Exper. & 3450000 & 0.151 & 0.1034 \\
\hline Woo, Glezer (2010) Exper. & NA & 0.000 & 0.0417 \\
\hline Woo, Glezer (2010) Exper. & NA & 0.072 & 0.0719 \\
\hline
\end{tabular}

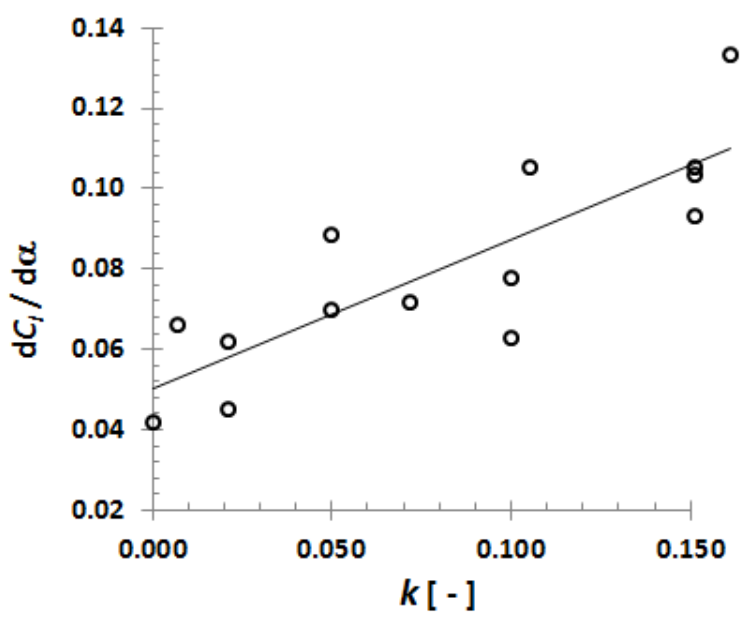

Figure 34. Lift-Curve-Slope Data from Literature: Influence of Reduced Pitch Rate ( $k$ )

Koochesfahani (99) measured the vortical flow patterns (PIV) in the wake of an oscillating NACA0012. Estimates of the magnitude of axial flow in the cores of upper surface vortices showed a linear dependence on $k$.

Kaplan, et al. (100) investigated the effects of pitching motion on a 2D SD7003 airfoil, using both CFD and wind tunnel tests at $R e=10 \times 10^{3}$. Detailed flowfield visualizations, focusing on suction surface vorticity and aerodynamic loading, were compared for sinusoidal, "trapezoidal," and "triangular" pitching motions with identical nominal angle of attack $(\alpha)$ and $k$ limits. Results were in contrast to the common assertion that $k, R e$, and $\alpha$ limits are the governing parameters for aerodynamic loads time history and evolution of vortex shedding (100). Instead, Kaplan, et al. showed that all cases of the pitching motion exhibited markedly different $C_{l}$ time history, and that matching of $\alpha$-limits, $k$, and $R e$ is insufficient to obtain the same wake geometry or aerodynamic performance. The implication was the need for a more general criterion of $\alpha$-time history, to better understand and predict airfoil performance for a given $\alpha$-path of travel. The work of Kaplan, et al. (100) indicates a need to closely match operational VAWT $\alpha$-time histories to wind tunnel test conditions for the current CCNACA airfoil 
analysis. The results also stress the importance of fully detailing airfoil pitch-motions when reporting experimental or numerical results of any kind.

Amiralaei, et al. (101) numerically studied the low Re aerodynamics of a harmonically pitching NACA0012, focusing on the influence of unsteady parameters $\left(\alpha_{\max }, k\right.$, and $\left.R e\right)$ on airfoil performance. Seminal results showed the parameters to be of great importance to pitching airfoil performance, affecting $C_{l, \text { max }}$, hysteresis loops, strength and number of generated vortices, and the extent of the socalled "figure-eight phenomenon region" (101). The group concluded that achieving optimum lift demands a careful selection of these parameters. The influence of reduced pitching rate on the lift and drag of the oscillating NACA0012 airfoil can be seen in Figure 35. For $k \leq 0.170$, the pitching rate does not appear to influence $\mathrm{d} C_{l} / \mathrm{d} \alpha$ at the $R e$ considered. For $k=0.250$, lift recovery is affected and the $C_{I^{-}}$ hysteresis loop is significantly larger than the slower pitch cases.
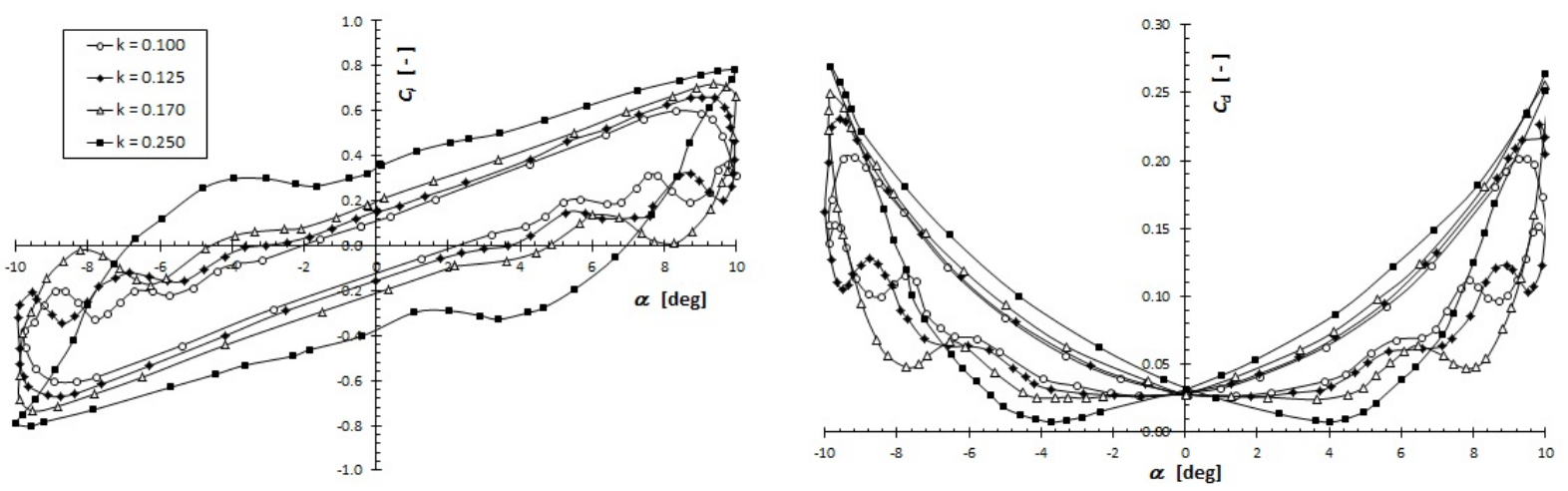

Figure 35. Influence of $k$ on Aerodynamic Performance of a Pitching NACA0012 Airfoil (101)

Mueller-Vahl, et al. (102) experimentally studied the dynamic stall mechanism on a NACA0018 airfoil for VAWT applications. PIV measurements revealed the strength of the DSV to be dependent upon the reduced pitching frequency $(k)$, having a large impact on $C_{p}$ at $k=0.074$, becoming less pronounced at $k=0.037$, and undetectable for $k=0.018$ (102).

\subsubsection{Influence of Mean Angle of Attack $\left(\alpha_{0}\right)$}

The effects of mean AoA $\left(\alpha_{0}\right)$ on pitching airfoil performance has been accounted by a variety of studies. Comprehensive experimental testing by Piziali (103) of a NACA0015 wing undergoing pitching motions representative of helicopter rotor blades showed $C_{l, \text { max }}$ to be a direct function of $\alpha_{0}$. Increasing mean AoA from $4^{\circ}$ to $13^{\circ}$ resulted in a maximum lift increase of over $100 \%$. In addition, $\alpha_{o}$ was not shown to influence lift and drag hysteresis loops until the value approached the static stall AoA ( $\left.\alpha_{s s}\right)$. 
Soltani, et al. (93) completed extensive experiments to study the effect of various parameters on surface pressure distribution and transition point of a cambered HAWT airfoil section. Mean AoA was reported to have a significant influence on the location of airfoil BL transition, and varied the shape and width of hysteresis loops.

The aforementioned tests of Tsang, et al. (95) indicated that $\alpha_{0}$ had a slight effect on dynamic stall angle $\left(\alpha_{0} \uparrow: \alpha_{D S} \downarrow\right)$, and little effect on $C_{1, \max }$. Carr, et al. (60) (96) commented on the effect of changing $\alpha_{o}$ while maintaining a constant pitch amplitude, concluding that oscillation within the stall regime (attached flow) results in negligible force coefficient hysteresis, while such effects become more significant the further pitch amplitude exceeds stall conditions. McAlister, et al. (96) determined oscillation amplitude as an important parameter affecting the DSV. A range of mean angle of attack were analyzed $\left(\alpha_{o}=6^{\circ}, 11^{\circ}, 15^{\circ}\right)$ at a constant pitching rate of $k=0.24$; results are included in Figure 36 .
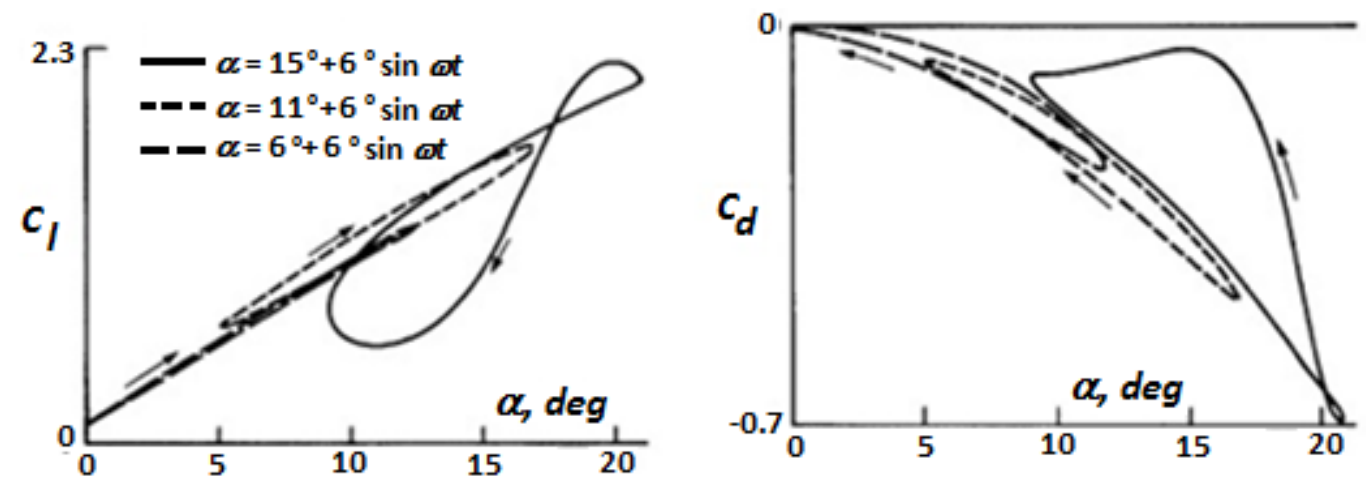

Figure 36. Influence of $\alpha_{0}$ on Pitching NACA0012 Performance: $R e=2 \times 10^{6}, k=0.24$ (96)

\subsubsection{Influence of Pitch Amplitude $\left(\alpha_{\max }\right)$}

The effects of pitch amplitude $\left(\alpha_{\max }\right)$ have also been proven to impact airfoil aerodynamics. The aforementioned tests by Piziali (103) directly linked $\alpha_{\max }$ to force coefficient hysteresis loops. When $\alpha_{\max }<\alpha_{s s}$, no performance hysteresis was noticed between pitch-up and pitch-down motions, as expected. However, the severity of hysteresis showed incremental increase for every degree $\alpha_{\max }$ exceeded $\alpha_{s s}$. The aforementioned tests of Lee, et al. (94) showed that BL transition and separation were delayed with increasing $\alpha_{\max }$, while reattachment of the unsteady $\mathrm{BL}$ was promoted as $\alpha_{\max }$ decreased.

Carr, et al. (60) (96) concluded oscillation amplitude to impact stall behavior for a constant pitch rate, influencing the strength and timing of the DSV. For low amplitude pitch, the DSV was always shed at $\alpha_{\max }$, even if actual stall would not have occurred until later if the amplitude has been larger (i.e. the change in pitch direction precipitates stall). This earlier vortex shedding resulted in a milder stall due to 
reduced vortex strength. When amplitude was increased, a fully developed vortex was found to naturally develop due to the breakdown of the $\mathrm{BL}$, before $\alpha_{\max }$ was reached.

Lombardi (104) completed wind tunnel tests on a modified NACA0015 profile, concluding stall regime to be dependent on amplitude. The severity of stall increased as amplitude exceeded static stall conditions. To illustrate this point, light stall characteristics were noted for $\alpha_{\max }=18^{\circ}$, mild stall for a $\alpha_{\max }=20^{\circ}$, and deep DS for $\alpha_{\max }=25^{\circ}$ (static stall was $\alpha_{s s}=14^{\circ}$ )

Koochesfahani (99) measured the vortical flow patterns, via PIV imaging, in the wake of a NACA0012 (2D) pitching $(0.02 \leq k \leq 0.14)$ at small amplitudes at $R e=12 \times 10^{3}$. Estimates of the magnitude of axial flow in the cores of upper surface vortices showed a linear dependence on pitch amplitude $\left(\alpha_{\max }\right)$.

The previously described studies of Amiralaei, et al. (101) considered the influence of pitch amplitude on pitch NACA0012 aerodynamics. The influence of $\alpha_{\max }$ on aerodynamic loading is included in Figure 37, where force hysteresis loops are broadened with increasing $\alpha_{\max }$. Ultimately, it was reported that $\alpha_{\max }$ has a strong influence on $C_{l, \max }$, force hysteresis loops, and lift enhancing vortical structures, but no noticeable effect on the lift curve slopes $\left(d C_{1} / d \alpha\right)$. The predicted drag coefficients show increased hysteresis loops and $C_{d, \max }$ with increasing $\alpha_{\max }$. Furthermore, and of interest to the present CCNACA0018 airfoil, it was observed that $\alpha_{\max }, k$, and $R e$ are effective in changing the number, strength, and development angle of generated vorticity on the suction surface of the airfoil.
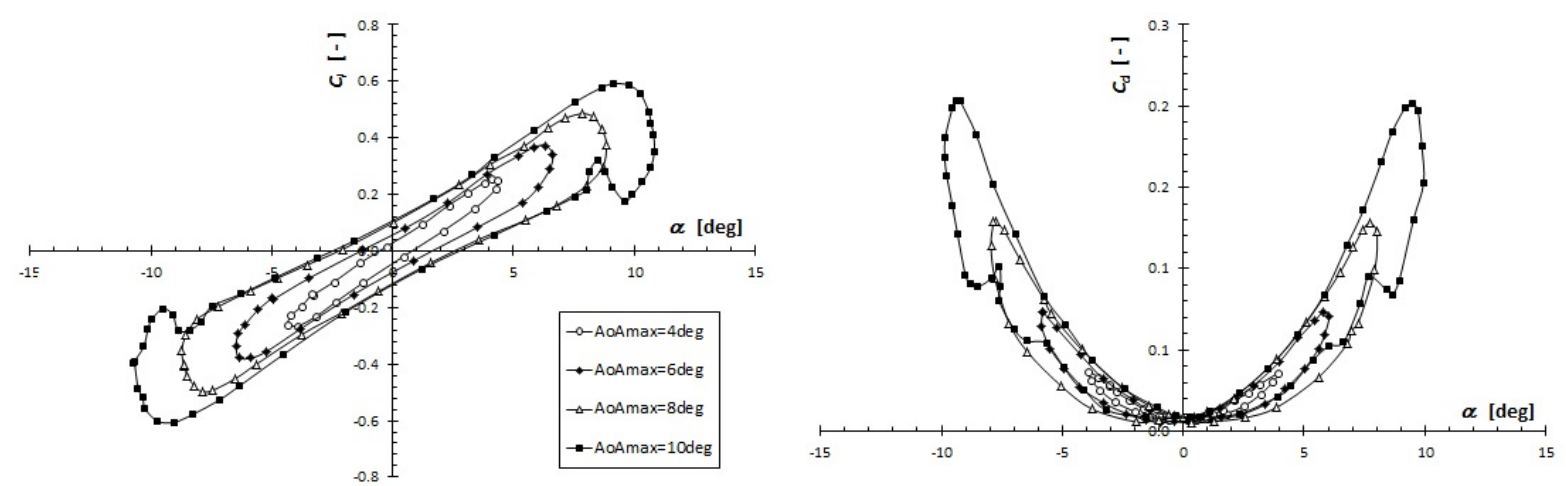

Figure 37. Influence of $\alpha_{\max }$ on Aerodynamic Performance of a Pitching NACA0012 Airfoil (101)

Sankar, et al. (62) completed numerical studies of a conventional NACA0012 to determine dynamic stall characteristics for rotorcraft applications. The influence of $\alpha_{\max }$, under constant $R e$, was found to directly influence the stall regime encountered during pitch, as summarized in Table 5 .

Table 5. Influence of $\alpha_{\max }$ on NACA0012 Stall Regimes: $R e=2 \times 10^{6}, k=0.1$ (62)

\begin{tabular}{|c|c|c|}
\hline Stall Regime & Pitching Motion & AoA Range \\
\hline None - Light Stall & $\alpha=11^{\circ} \pm 4^{\circ} \sin (\omega \mathrm{t})$ & $\left(9^{\circ} \leq \alpha \leq 15^{\circ}\right)$ \\
\hline Light - Medium Stall & $\alpha=14^{\circ} \pm 4^{\circ} \sin (\omega \mathrm{t})$ & $\left(10^{\circ} \leq \alpha \leq 18^{\circ}\right)$ \\
\hline Deep Stall & $\alpha=15^{\circ} \pm 10^{\circ} \sin (\omega \mathrm{t})$ & $\left(5^{\circ} \leq \alpha \leq 25^{\circ}\right)$ \\
\hline
\end{tabular}


McCroskey, et al. (105) reported similar results, based on extensive experimental investigations of BL transition, separation, and unsteady stall on eight different oscillating airfoil profiles. Table 6 summarizes the data, highlighting the type of stall encountered by varying $\alpha_{\max }$ in relation to $\alpha_{s s}$.

\begin{tabular}{|c|c|c|}
\multicolumn{2}{c}{ Table 6. Summary of Dynamic Stall Regimes based on AoA (105) } \\
\hline Stall Regime & $\alpha_{\max }$ & Stall Characteristics \\
\hline No Stall & $\alpha_{\max } \leq \alpha_{S S}$ & No flow separation, No hysteresis loops \\
\hline Stall Onset & $\alpha_{\max } \approx \alpha_{S S}$ & Small amount of separation, Minimal hysteresis \\
\hline Light Stall & $\alpha_{S S} \leq \alpha_{\max } \leq\left(\alpha_{S S}+2^{\circ}\right)$ & Distinct hysteresis and gradual $C_{m}$ stall \\
\hline Deep Stall & $\alpha_{\max }>\left(\alpha_{S S}+2^{\circ}\right)$ & Vortex shedding, large hysteresis, intense $C_{m}$ stall \\
\hline
\end{tabular}

\subsubsection{Influence of Reynolds Number (Re)}

The influence of freestream conditions on pitching airfoil performance has been the subject of numerous reports. Experiments by Sharma, et al. (92) (106) reported that Re effects increase at low pitch rates (approaching quasi-steady conditions), causing substantial rise in hysteresis behavior and stall AoA $\left(\alpha_{\text {stall }}\right)$ shift. As Re $\left(0.7 \times 10^{6}\right)$ increases, such hysteresis effects become negligible and Re effects become limited to the weakening in strength of DSV. Also, the reattachment process during the pitchdown maneuver was reported independent of $R e$.

Water-tunnel tests by Conger, et al. (107) were completed to study unsteady vortex dynamics of a pitching NACA0015. Unsteady surface $C_{p}$ distributions and near surface vorticity measurements (PIV) showed no significant effect of Reynolds number in the range of $50 \times 10^{3} \leq R e \leq 220 \times 10^{3}$ (the lower range of interest for the current study). Furthermore, $C_{l, \max }$ and $\alpha_{\text {stall }}$ were found independent of $R e$.

Of all the parameters studied by Carr, et al. (60) (96), Re was reported to have the least dramatic influence of DS characteristics. Re effects were detectable for low $k$ where a delay in reattachment was reported during pitch-down, though the dependence was weak. Concerning flow reversal, a more gradual progression of rear-to-front $(T E \rightarrow L E)$ flow breakdown was found at lower $R e$. The aforementioned tests completed by Griffin, et al. (55) (97) concluded that Re did not influence measured velocity fields, with particular emphasis on development of the DSV.

Martin, et al. (108) completed experimental studies of DS on a pitching NACA0012 airfoil under actual helicopter operating and conditions $(R e, k)$. Unlike the majority of literature reviewed thus far, the study concluded a strong connection between Re and dynamic stall, including airfoil force coefficients.

Reynolds number $(R e)$ has been proven to strongly influence the shear layer separation characteristics of an oscillating airfoil. Shrewsbury, et al. (109) has shown that vortex convection speed, 
on the suction surface of a pitching airfoil, is related to the freestream velocity. Shrewsbury \& Sankar (110) reported both experimental (Figure 38: filled symbols) and numerical data (Figure 38: open symbols) for a conventional (2D) NACA0012 airfoil pitching at $k=0.151$ and $R e=3.45 \times 10^{6}$ to investigate deep dynamic stall $\left(\alpha_{\max }=25^{\circ}\right)$. The $k-\varepsilon$ turbulence model ( 2 additional turbulence equations) was used for computations, resulting in fairly good agreement to experimental data.
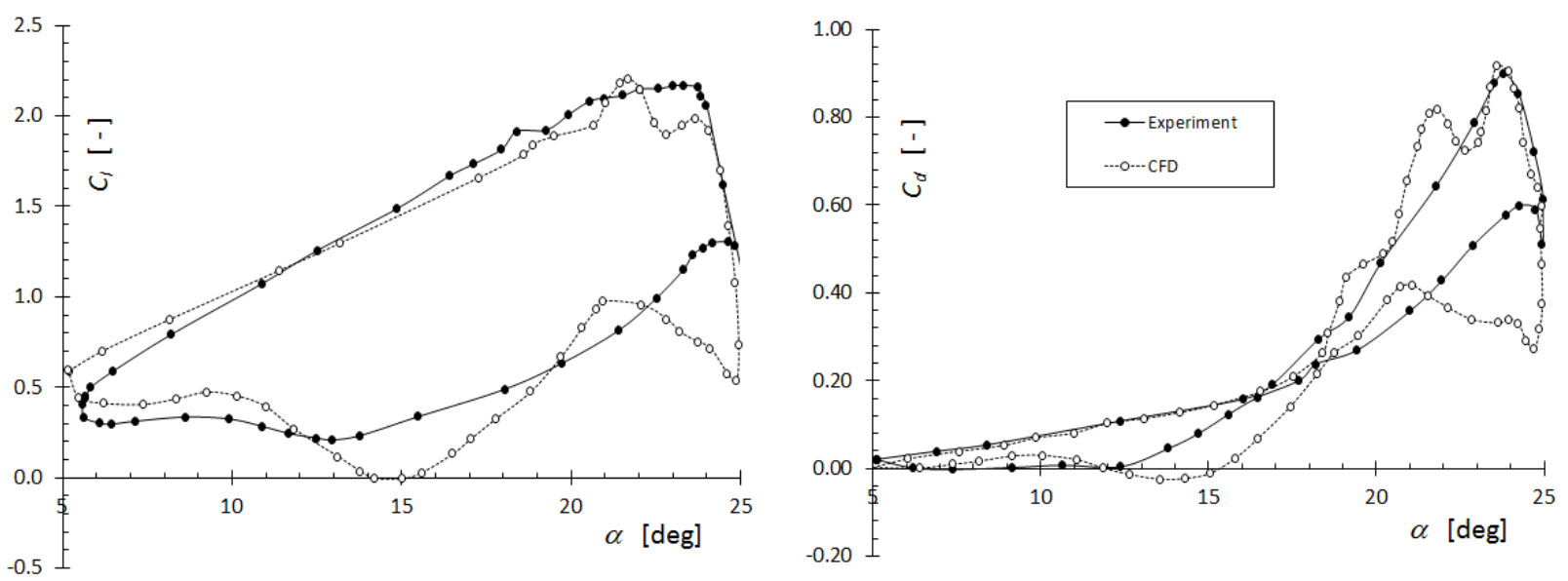

Figure 38. Numerical and Experimental Loading on a Pitching NACA0012 : $R e=3.45 \times 10^{6}, k=0.151(110)$

According to Claessens (27), the angle at which stall ( $\left.\alpha_{D S}\right)$ occurs is dependent on $R e$ and the nose radius of the airfoil. Timmer, et al. (22) compiled dynamic stall test data for a range of airfoils, extrapolating an analytical expression to estimate $\alpha_{D S}$, based on airfoil thickness near the LE $(x / c=0.0125)$, included in Equation 34.

Deep Stall AoA

$$
\alpha_{D S}=1114\left(\frac{y}{c}\right)_{x / c=0.0125}
$$

For the CCNACA0018 model of the current study, having a non-dimensional thickness of 0.0281 at $x / c=0.0125$, the onset of deep dynamic stall is predicted to occur at approximately $\alpha_{D S} \approx 31^{\circ}$.

Tests from Lorber, et al. (98) concluded Re to have a strong effect on integrated loads on a pitching Sikorsky SSC-AO9 rotor section. In particular, increasing Re diminished the vortex-induced lift peak $\left(C_{l, \max }\right)$, and its position retreated from $\alpha=16^{\circ}$ to $\alpha=12^{\circ}$, suggesting minor compressibility prevents the development of a strong suction peak, reducing the strength of the stall vortex. These results supported pressure measurement results of St. Hilaire, et al. (111) for a pitching NACA0012 profile.

Danao, et al. (26) completed a 2D numerical study of 3-bladed VAWT to determine performance under fluctuating wind conditions. The CFD simulations were validated with experimental data of a wind tunnel scale rotor with fluctuating $V_{\infty}\left(V_{\infty(A V G)} \pm 12 \%\right.$ variations $)$. A follow-up study considered varying 
the mean freestream flow by $\pm 7 \%$ (112). Results showed a dependency on Re, increasing $d C_{I} / d \alpha$ with $V_{\infty}$, generating higher rotor output torque. The influence of variable $R e$ on VAWT blade lift is included in Figure 39 , where $C_{l, \max }$ varied by as much as $15 \%$. On a different note, Figure 39 highlights the dramatic disparity between static and pitching airfoil performance, indicating the need to use the latter as input for analytical performance calculations.

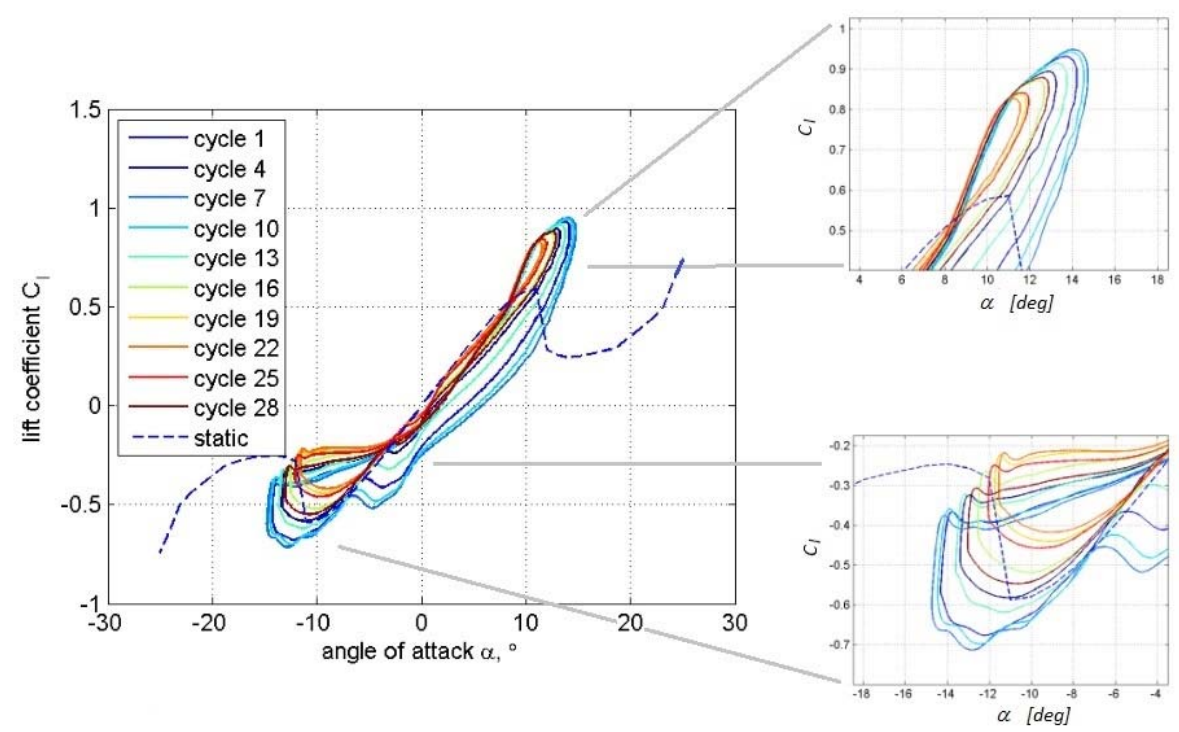

Figure 39. Influence of Fluctuating Freestream on VAWT Lift (26)

Howell, et al. (113) studied a dual-bladed VAWT to determine dynamic stall flow features and blade-wake interactions. For this analysis, two forms of $C_{l}$ were calculated, using the instantaneous relative velocity of the blade throughout rotation and the mean relative velocity, averaged over a rotation cycle. The resulting lift curves for a NACA0022 airfoil can be found in Figure 40, where dashed lines (open symbols) represents instantaneous lift $\left(V_{\text {rel }}\right)$ and solid lines (filled symbols) shows the mean $C_{l}$ result $\left(V_{\text {mean }}\right)$.

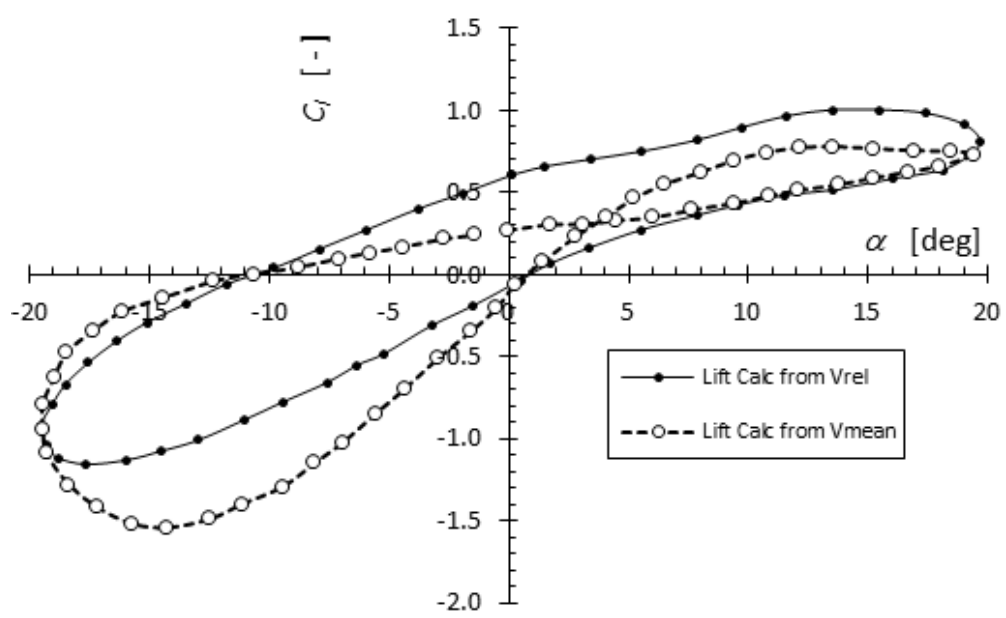

Figure 40. Measured Lift on 2-Bladed VAWT: NACA0022 Blades (113) 
The resulting lift hysteresis loops are significantly different based on the form of freestream velocity considered. The dynamic stall behavior of a rotating VAWT blade was concluded to be different from that of a pitching airfoil due to the variation (direction and magnitude) of the relative incoming velocity vector (113).

Lovato's (114) experimental analysis of an oscillating NACA0015 airfoil reported that Re significantly influences dynamic airfoil performance. More specifically, increasing Re caused a decrease in surface $C_{p}, C_{l}, C_{d}, \alpha_{D S}$. However, Akbari \& Price (115) conducted numerical analysis of a pitching NACA0012, concluding that Re had negligible effects on dynamic stall. Bousman (116) led a series of wind tunnel tests of a pitching NACA0012, under rotorcraft flow conditions, also concluding DS characteristics to be independent of $R e$.

Tchon, et al. (117) addressed the differences between the dynamic stall on an airfoil in rotational VAWT motion and the dynamic stall of an airfoil performing an equivalent pitching motion based only on the variation of the effective angle of attack $\left(\alpha_{e}\right)$. A pure pitching motion is not able to take into account the variation of the effective flow velocity and $R e$ that results in a much higher peak in the normal force $\left(C_{N}\right)$, indicating a stronger stall vortex during the upwind rotation than the downwind half (117).

\subsubsection{Influence of Airfoil Profile (Geometry)}

The influence of profile contour has been shown to govern unique airfoil performance characteristics. Initiation of the DS process is known to be influenced by airfoil geometry, beginning at either the LE or TE of airfoil suction surface. Experimental work by Sharma, et al. (92) (106) concluded for a variety of conditions, that the stall process, with laminar separation bubble, is initiated at the TE of the NACA0015 profile. Considering the TE CC capabilities of the CCNACA0018 airfoil, this is thought to be a favorable result as flow control authority increases as actuation is nearer the point of airfoil surface separation. Experimental work by Piziali (103) corroborated the TE type dynamic stall process for the NACA0015 airfoil at similar test conditions. Sharma, et al. (92) also noted that the effects of LE contamination, independent of $R e$ and $k$, cause a shift in $\alpha_{D S}$ (this conclusion is of particular interest to VAWTs, where dirt, bugs, and ice could significantly impact performance).

Extensive experimental results by Carr, et al. (60) (96) for a NACA0012 airfoil, with several LE modifications, determined stall initiated from abrupt turbulent LE separation, as opposed to the TE disturbances that dominate the stall process of the thicker NACA0015 airfoil. LE bubble bursting was 
determined to be a characteristic of sharp LE airfoils, in this case due to the thin profile (12\% $t / c)$. Furthermore, flow visualization revealed the TE stall characteristics of cambered airfoils, where stall developed as a relatively gradual forward progression of flow reversal from the aft region of the airfoil, without the formation of a LE vortex. Carr, et al. (60) concluded the type of BL separation, stall, and resultant force behavior to be primarily dependent on airfoil geometry.

Greenblatt, et al. (118) measured vorticity (PIV) on a rotating VAWT blade (NACA0018 profile), reporting a large region of vortical flow near the TE. The same observation was made by Mueller-Vahl, et al. (102) on a NACA0018 undergoing sinusoidal pitch, indicating the stalled TE region to originate from TE separation on thick airfoils, particularly at low Re.

Motivated by the scarcity of data, Mueller-Vahl, et al. (102) experimentally studied the dynamic stall mechanism on a NACA0018 airfoil for VAWT applications. PIV measurements revealed the discovery of a new DSV, not previously described in the literature, which the team termed the aft dynamic stall vortex (ADSV). In contrast to the well documented leading edge vortex associated with thin airfoils, the ADSV forms across the aft region of a thick airfoil near the region $(0.5 \leq x / c \leq 0.6)$. ADSV formation and movement during pitch-up can be seen in the $C_{p}$-distributions (overlaid with vorticity field vectors) included in Figure 41. For $\alpha=20^{\circ}$ during pitch-up, the upper surface flow begins showing separation near $0.6 \leq x / c \leq 1.0$. As the airfoil pitches further to $\alpha=25.5^{\circ}$, separation moves forward to $0.30 \leq x / c \leq 0.6$ and the formation of the ADSV appears aft of this region. This description parallels previous reports of thick airfoil TE stall by Sharma, et al. (92) (106) and Carr, et al. (60) (96) for a NACA0015 profile. The TE vortex activity included in Figure 41 will be of interest to the CFD portion of this study.

(a) $\alpha=20^{\circ}$ (pitch-up)

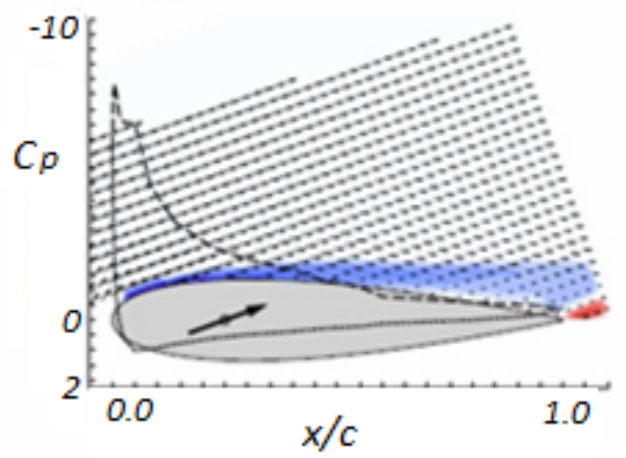

(b) $\alpha=25.5^{\circ}$ (pitch-up)

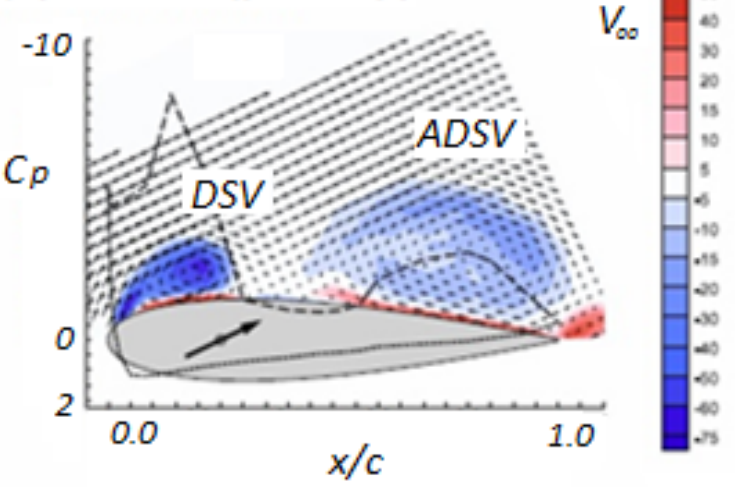

Figure 41. Formation and Evolution of DSV Vortex on Pitching NACA0018: $R e=250 \times 10^{3}, k=0.074(102)$ 


\subsubsection{Influence of Flow Control on Dynamic Stall}

The effect of flow control on pitching airfoil performance has been a popular topic of research in recent years, primarily directed at helicopter applications. Shrewsbury, et al. (109) (119) completed URANS simulations to evaluate the dynamic airloads of a CC airfoil for application to rotorcraft. The research objective was the increase the dynamic performance of airfoil sections with CC and to alleviate the dynamic stall problem common to rotorcraft. Steady jet $\left(C_{\mu}=0.05\right)$ stall characteristics differed from conventional DS in that the minimum lift produced was well below the baseline value. However, lift recovery after the dramatic DS was almost immediate, helping to mitigate the hysteresis effects; $C_{d}$ and $C_{m}$ showed similar characteristics. Furthermore, as $k$ increased, DS produced a complex bimodal characteristic for the aerodynamic load histories over a range of $\alpha_{\max }$, a result not generated by the baseline or NACA0015 simulations.

Post \& Corke (77) completed wind tunnel tests of a pitching NACA0015 airfoil with LE plasma actuation for control the DS vortex encountered on retreating helicopter blades. Compared to the baseline airfoil, unsteady actuation generated higher lift at low AoA and during the entire pitch-down cycle. It was concluded that optimal frequency scaling for the control of flow separation is based on having two spanwise vortices in the length of the separation bubble (wing chord) and the penalty for being outside this range was more severe for frequencies below optimal than those exceeding the optimum. For lift cycle enhancement, $S t=0.25$ outperformed both $S t=0$ (steady) and $S t=1$ cases.

Griffin, et al. (55) (97) completed wind tunnel tests, including stereoscopic PIV flow measurements, on a pitching NACA0012 airfoil with LE dynamic roughness elements. The spanwise roughness elements covered the LE over the region of $0.05 \leq x / c \leq 0.20$ and remained flush to the airfoil surface when actuation was off. When activated, the roughness elements became an array of oscillating bumps with variable amplitude and frequency control. A parametric study was completed, varying amplitude and actuation frequency in the range $1.1 \leq S t \leq 3.2\left(30 \mathrm{~Hz} \leq f_{a c t} \leq 90 \mathrm{~Hz}\right)$. A strict dependence of actuation frequency was established for fixed freestream conditions ( $R e=150 \times 10^{3}, k=0.1$ ), where actuation of St=2.1 provided the most substantial delay of DSV development, the primary goal of the study. Actuation frequencies of $S t=1.1$ and $S t=3.2$ still delayed development relative to the clean airfoil condition.

Greenblatt, et al. (118) conducted wind tunnel tests of a scaled VAWT, with modified NACA0015 airfoils having LE plasma actuators. To measure the effects of plasma pulsations on DS control. The actuators were located on a single side of the blade, limiting flow control to the upwind half of the turbine. High frequency plasma actuation of $S t=10.7\left(f_{a c t}=500 \mathrm{~Hz}\right.$, constant amplitude) increased 
measured turbine torque by $8 \%$ for $\lambda=1.48$, and $16.4 \%$ during $\lambda=1.37$ operation, relative to baseline conditions, indicating a possible connection between pitch frequency and effective actuation frequency. Simultaneous PIV measurements of local vorticity fields revealed DSV characteristics. The LE control method was able to modify the DSV and delayed shedding, but stall elimination was not achieved. For the small-scale VAWT, the power increases resulting from actuation never exceeded $1.6 \mathrm{~W}$, while the required power to drive the continuous actuation (20\% DC) was $8.4 \mathrm{~W}$ per blade, significantly higher than turbine power increases due to flow control. Reductions in actuator DC and implementation of closed-loop control were identified as techniques with potential to improve modified VAWT power efficiency.

Liggitt (120) completed numerical analysis of a pitching NACA0012 with a TE flap that oscillated at twice the airfoil pitch rate. The influence of the mean angle of attack $\left(\alpha_{\text {mean }}\right)$ on $C_{I}$ and $C_{m}$ can be seen in Figure 42. Compared to static test results, the pitching airfoil exhibits significantly different performance for both $\alpha_{0}$ cases. The $C_{1}$-curves show diminished lift for the pitching airfoil, and a bimodal hysteresis loop for $\alpha_{o}=4^{\circ}$. The $C_{m}$-curves show the maxima to be more than twice the magnitude of steady measurements.
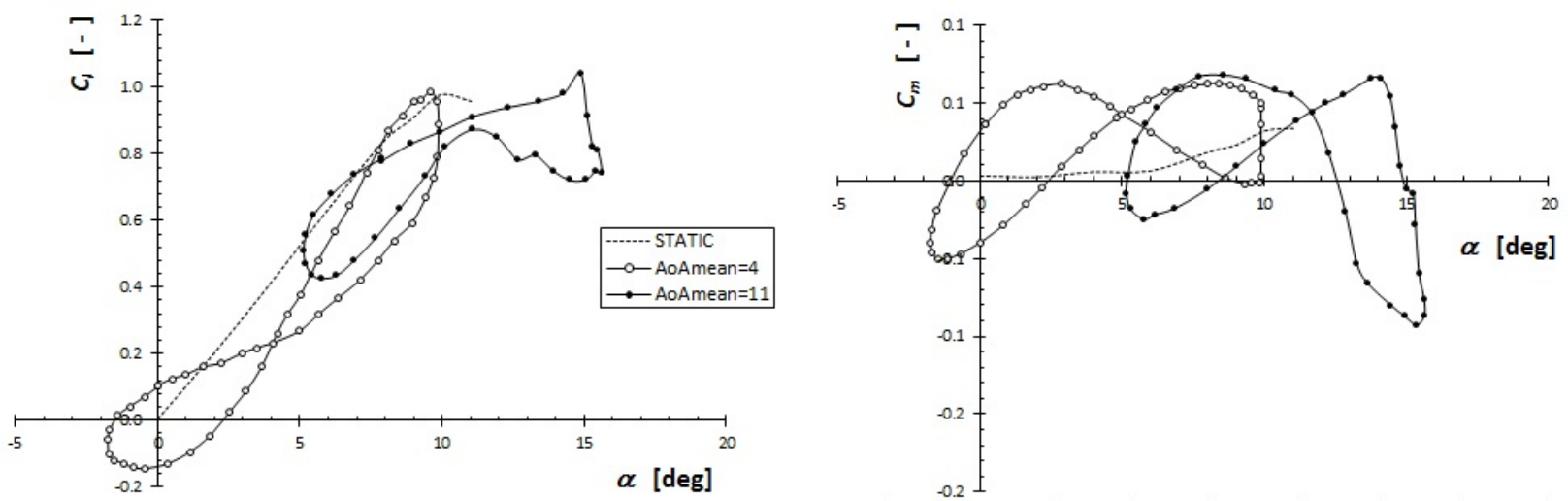

Figure 42. Influence of $\alpha_{\text {mean }}$ on a Pitching NACA0012 with Oscillating TE Flap $\left(k_{f l a p}=0.042\right)(120)$

The closest effort of dynamic stall research of a CC airfoil, a sub-topic of the present study, came from a numerical investigation of an oscillating airfoil with steady circulation control (109). The research focused on a numerical study to evaluate the dynamic stall characteristics of an oscillating airfoil with constant CC blowing. The study, aimed at rotorcraft applications, analyzed a XW103 airfoil using timeaccurate Navier-Stokes analysis method was used to evaluate the dynamic airloads of a CC airfoil. Dynamic (pitching airfoil, steady $C_{\mu}$ ) simulations were completed using the following conditions: $\left(0^{\circ} \leq \alpha \leq 8^{\circ}\right), \alpha_{0}=4^{\circ}, \alpha_{1}=4^{\circ}$, reduced frequency range of $(0.1 \leq k \leq 1.0), C_{\mu}=0.05$ and $R e=3.06 \times 10^{6}$. Results indicated that CC is a feasible method to pneumatically modulate the lift of retreating helicopter blades, 
eliminating the need for mechanical pitching to maintain a uniform lift distribution across the rotor disk (109) (110). It was observed that the CC airfoil, unlike conventional airfoils, began to lose lift as soon as the separation vortex appeared on the upper surface. As the vortex develops and increases in size, the $C_{I}$ loss continues until the vortex is convected off the TE; this corresponds to the minimum lift condition. The loss in lift was contributed to an interference with the global circulation caused by the jet exhaust sheet; once the vortex was clear of the airfoil surface, global circulation was observed to re-establish (109) (110). It was also concluded that the time interval associated with circulation recovery, after the vortex has been convected off the TE was independent of airfoil pitching frequency $\left(\omega_{b l}\right)$; a value of jet response time in the range of 3.0-3.8 chords of travel were observed. The jet response timing was comparable to experimental and computational values observed by (85); Shrewsbury (109) examined a $10 \% \mathrm{t} / \mathrm{c}$ CC ellipse, with both LE and TE slots, also motivated by rotorcraft applications. However, the time required for the formation and subsequent convection of the upper surface vortex varied slightly with $k$. A summary of the convection times obtained from simulations can be seen in Table 7 , where vortex convection velocities $\left(V_{D S V}\right)$ were found ranging from 0.13-0.24. Conventional airfoils have demonstrated vortex convection velocities $\left(V_{D S V}\right)$ which are nearly constant at about $0.25-0.30$ of the freestream velocity $\left(V_{\infty}\right)(121)$, proving that CC slows down the formation and convection of suction surface vortices. Chandrasekhara et al. (122) (123) completed experimental testing of an oscillating NACA0012 airfoil to study the effects of compressibility on dynamic stall, also aimed at rotorcraft applications. This work further supported magnitudes of shed vorticity velocity, reporting $V_{D S V}=0.30 V_{\infty}$ for the entire range of $R e$ and $k$ test conditions.

Table 7. Dynamic Stall Vortex Shedding Characteristics for Pitching CC Airfoil (109)

\begin{tabular}{|c|c|}
\hline$S t_{D S V}$ & $V_{\text {DSV }} / V_{\infty}$ \\
\hline$[-]$ & {$[-]$} \\
\hline 0.1 & 0.13 \\
\hline 0.2 & 0.19 \\
\hline 0.3 & 0.21 \\
\hline 0.4 & 0.24 \\
\hline
\end{tabular}

The common range of vortex convection velocity, nondimensionalized by freestream test conditions specific to each case, correlates St range of $(0.20 \leq S t \leq 0.40)$. This indicates that achieving effective usage of AFC, to have authority over unsteady local flow, the actuation frequency should correspond to $S t \geq 0.4$.

A bimodal hysteresis loop at high reduced frequencies $(k \geq 0.30)$ was also identified during pitch excursions (109). The observed behavior of CC airfoils experiencing oscillatory pitching is the result of a 
complex relationship between the airfoil pitching frequency and the vortex convection/jet response cycle, which represents the time required to re-establish global circulation, once it has been disrupted from LE separation (109). The influence of $k$ on the $C_{1}$-hysteresis loops can be seen in Figure 43; the absence of the bimodal behavior for $k=0.5$ occurs because the periods of the pitching cycle and the jet/circulation response cycle are the same. Similar modal instabilities of separation bubbles were also observed on flat plates by (124).
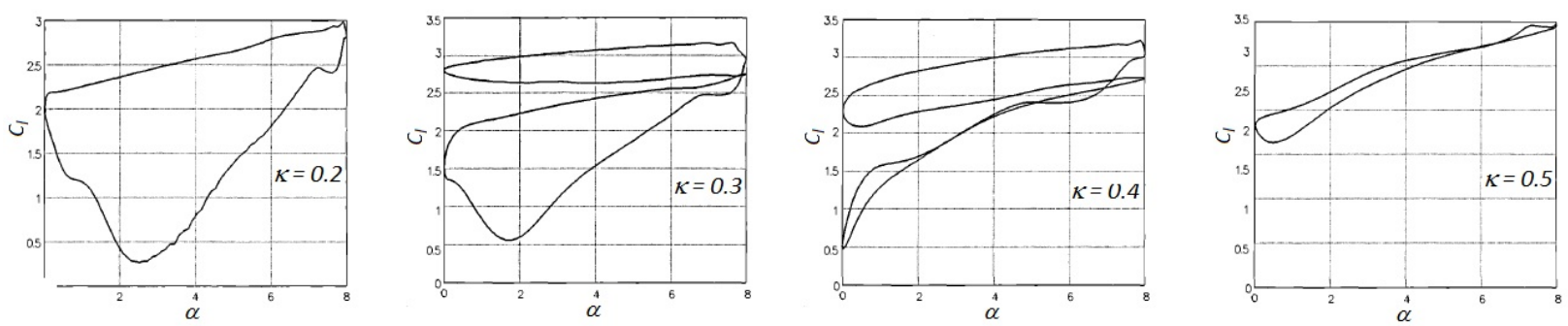

Figure 43. Reduced Frequency (k) Effects on CC Airfoil Lift Hysteresis (109)

Post (54) studied the influence of LE plasma actuators for separation control on both stationary and oscillating airfoils, to mitigate retreating blade stall on helicopter rotors. Experimental studies of the modified NACA0015 airfoil characterized the plasma actuation effectiveness (e.g. actuator configuration, $x / c$, actuation input) on stationary and oscillating airfoils at high $\alpha$. Dynamic wind tunnel testing showed LE plasma actuation could prevent separation up to $\alpha=22^{\circ}$, exceeding $\alpha_{\text {stall }}$ by $8^{\circ}$. This resulted in a $300 \%$ improvement in the cycle integrated $L / D(54)$. Aiken, et al. (125) also studied the influence of plasma actuation on transient separation control of a helicopter blade. Aiken, et al. (125) proposed that if $\alpha_{\text {stall }}$ could be shifted to an angle beyond the rotorcraft's physical pitching capabilities, the eminent stall feature could be eradicated, producing the highly-sought stall-free rotor. The same idea can be realized for a VAWT blade operating at low TSR, where stall limits the power output

Figure 44 presents the dynamic stall intervals of a VAWT operating at a TSR of 2.5. During the first upwind half rotation $\left(25^{\circ} \leq \theta \leq 195^{\circ}\right)$, corresponding to a blade effective angle of attack $\left(\alpha_{\text {eff }}\right)$ of $\alpha_{\text {eff }} \rightarrow$ $-7^{\circ} \rightarrow-23^{\circ} \rightarrow 9^{\circ}$, dynamic stall occurs on the inner surface of the blade, i.e., the surface located inside the rotation disk. During the second, or downwind, half rotation $\left(230^{\circ} \leq \theta \leq 360^{\circ}\right)$ dynamic stall occurs on the outer surface of the blade, i.e., the surface located outside the rotation disk (117). This also signifies the necessity of a VAWT blade to have dual slot CC blowing capabilities, as the dynamic stall bubble appears on both surfaces of the blade depending on orbital posiiton. This dynamic stall generates multiple LE and TE vortices, causing peaks in the normal and tangential forces exerted on the VAWT blade (Figure 44). More precisely, the result is the generation of only one pair of contrarotating LE-TE vortices during each half rotation, as observed by experiments (28). 

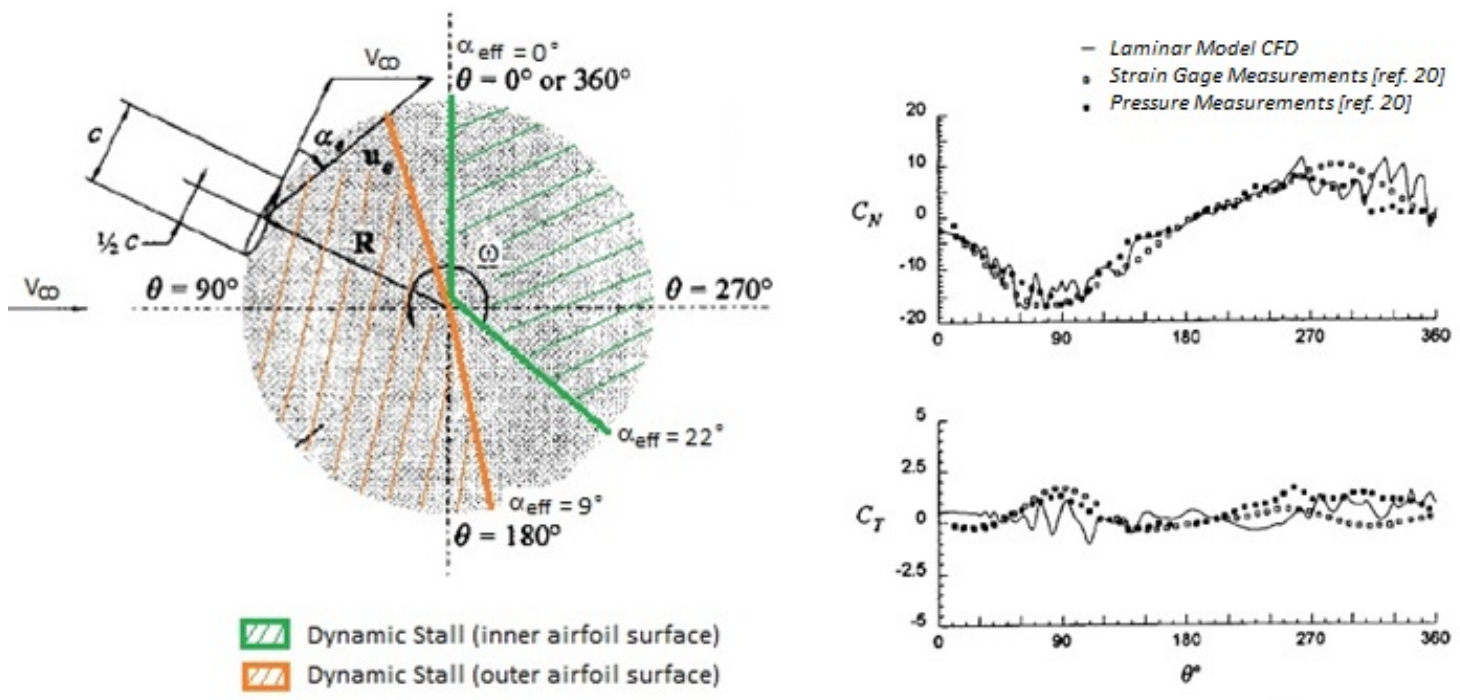

Figure 44. VAWT Blade DS and Resulting Normal $\left(C_{N}\right)$ and Tangential $\left(C_{T}\right)$ Forces: $\lambda=2.5$ (28) (117)

CC airfoil performance studies have all been limited to static conditions, with the exception of computational work completed by Shrewsbury, et al. (109) (110). To date, no experimental record of CC performance on a pitching airfoil could be found. Shrewsbury, et al. (109) (110) completed numerical simulations of a 2D CC-Ellipse $(t / c=0.16)$ using time-accurate URANS solutions to evaluate the dynamic airloads of a circulation control airfoil for helicopter rotor applications. Simulations were completed for $R e=3.6 \times 10^{6}$ and $\alpha_{o}=4^{\circ}$, varying reduced oscillating frequency $(k=0.1,0.3)$ and pitch amplitude $\left(\alpha_{\max }=6^{\circ}\right.$, $8^{\circ}$ ). For higher levels of reduced frequency, dynamic stall produced a complex bimodal characteristic for aerodynamic force coefficient histories at both values of $k$. For $k=0.1$ and $\alpha_{\max }=8^{\circ}$, the pitch cycle produced a single hysteresis loop, similar to that observed for conventional airfoils, except that $C_{1}$-stall is not nearly as abrupt, occurring over a much greater portion of the pitch cycle (110). The aerodynamic force hysteresis loops for both reduced frequency cases can be seen in Figure 45 ( $k=0.1$ : filled circles, $k=0.3$ : open circles). The CC-Ellipse shows a sharper $C_{1}$-stall for $k=0.1$, but displays a more rapid liftrecovery during the pitch-down portion of the cycle. 


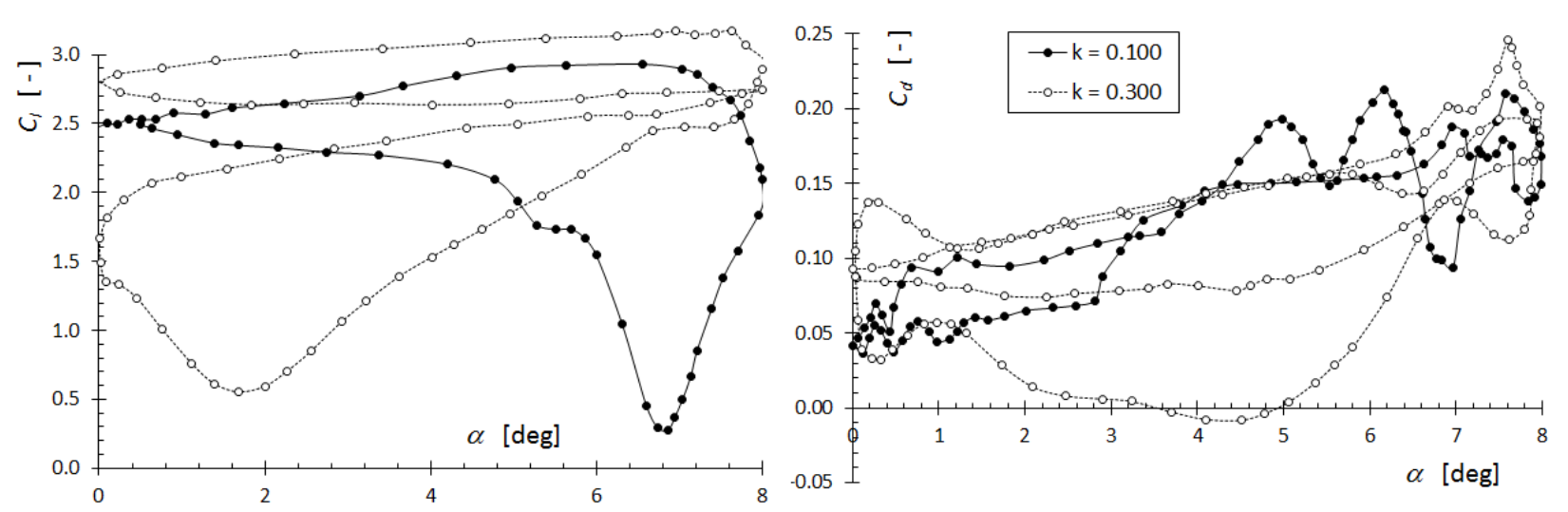

Figure 45. Influence of $k$ on Force Hysteresis for a Pitching CC-Ellipse (110)

Woo \& Gleezer (126) studied the actuation timing of a NACA4415 airfoil with LE combustionbased actuator jets, undergoing various pitch and plunge motions. They also looked into time delays, using "bursts" of 5 pulses separated by various lengths of delay periods (no actuation); their intent was to exploit the "memory" of the flow, based on jet-pulsing frequency, to further reduce mass flow rates and actuation power requirements. Significant alterations of the lift curve, at various $k$ and blowing schemes can be seen in Figure 46.

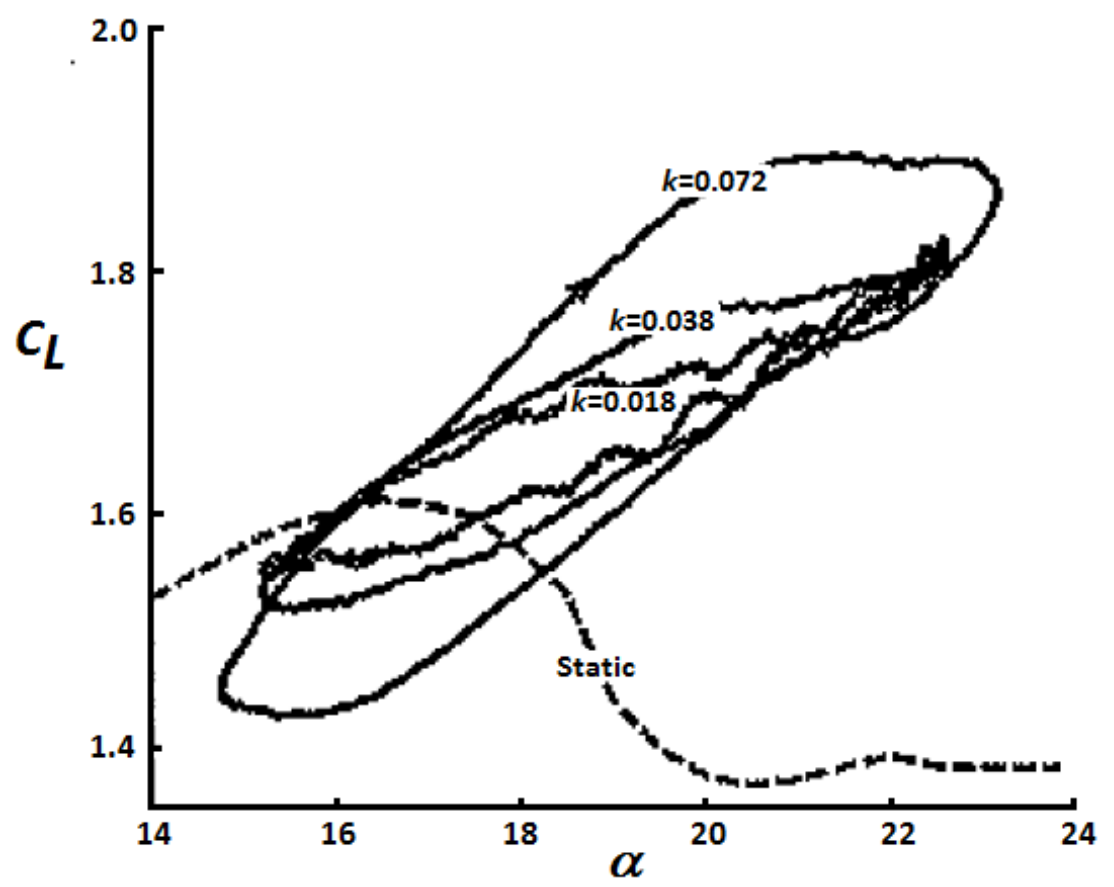

Figure 46. Influence of $k$ on NACA4415 Lift Enhancement due to LE Excitation (126)

Greenblatt, et al. (127) studied dynamic stall control on a pitching NACA0015 airfoil equipped with LE excitation (ZNMF). The baseline characteristics of the NACA0015 airfoil were significantly influenced by reduced pitch frequency $(0.05 \leq k \leq 0.3)$. However, two cases of excitation $(S t=0.6,1.1)$ were found to significantly reduce the dependence of aerodynamic coefficients on $k$, with essentially the 
same hysteresis loops for all aerodynamic coefficients $\left(C_{l}, C_{d}, C_{m}\right)$. Ultimately, Greenblatt, et al. (127) (128) outlined the parameters affecting separation control and demonstrated that they are similar under dynamic and static conditions, and TE flap-shoulder excitation was found to be superior to LE actuation under rotorcraft $R e$ and $k$ conditions.

Within the past couple years, several independent research efforts have emerged to investigate potential benefits of adding flow control actuation to VAWT blades. Greenblatt, et al. (10) built a small, high-solidity VAWT with dielectric barrier discharge (DBD) plasma actuators for low-speed wind tunnel testing at the Israel Institute of Technology. The objective was to assess turbine performance enhancements and the ability of LE plasma actuation to control dynamic stall on the upwind portion of rotation $\left(0^{\circ} \leq \theta \leq 180^{\circ}\right.$ ) (previous work by Post, et al. (54) (77) demonstrated the effective application of DBD actuators on both stationary and pitching airfoils). A parametric study revealed the actuator duty cycle $(D C)$ dependence, for effective control of LE separation, was independent of static and dynamic pitch conditions; $D C$ of $1 \%$ and $50 \%$ produced comparable aerodynamic performance (129). On the other hand, optimum actuation frequency $(S t)$ showed substantially different dependence, with an important link traced to the actuation frequency relative to the turbine reduced frequency $(k)(10)$. Actuation has been identified as the most difficult technical barrier to overcome to implement blade stall control into a full scale rotorcraft (130).

\subsubsection{Dynamic Stall Vortex Convection Speed ( $\left.V_{D S V}\right)$}

The convection speed of the dynamic stall vortex (DSV), over the upper surface of a pitching airfoil has been the subject of past studies. Lorber \& Carta (131) studied a pitching Sikorsky SSC-AO9 airfoil (see previous section for details), measuring unsteady surface pressure measurements to assess the speed of the DSV $\left(V_{D S V}\right)$. The airfoil was undergoing ramp-up maneuvers, meaning pitch-up from a resting neutral position $\left(\alpha=0^{\circ}\right)$, at $R e=2 \times 10^{6}$. It should be noted that the region of constant vortex speed, dependent upon airfoil profile, generally begins near $x / c=0.10$ and ends at $x / c=0.80$ (98). Vortex propagation speed was reported to increase almost linearly with pitch rate, from $0.13 V_{\infty}(k=0.001)$ to $0.33 V_{\infty}(k=0.020)(98)$, consistent with previous investigations by St. Hilaire, et al. (111) for a NACA0012 airfoil undergoing sinusoidal pitch.

Green, et al. (132) experimentally examined the DSV convection speed on a pitching NACA23012 airfoil, undergoing both ramp-up maneuver and sinusoidal pitch oscillations, at $R e=1.5 \times 10^{6}$. The results of both studies can be seen in Figure 47, where the velocity is normalized by freestream conditions $\left(V_{\infty}\right)$ 
to remove $R e$ influences. Results indicate that for quasi-steady and very low reduced frequency conditions $(k \leq 0.020), k$ has a strong, linear influence on $V_{D S V}$. However, at higher pitch frequencies $(k \geq 0.040)$, the influence of $k$ diminishes.

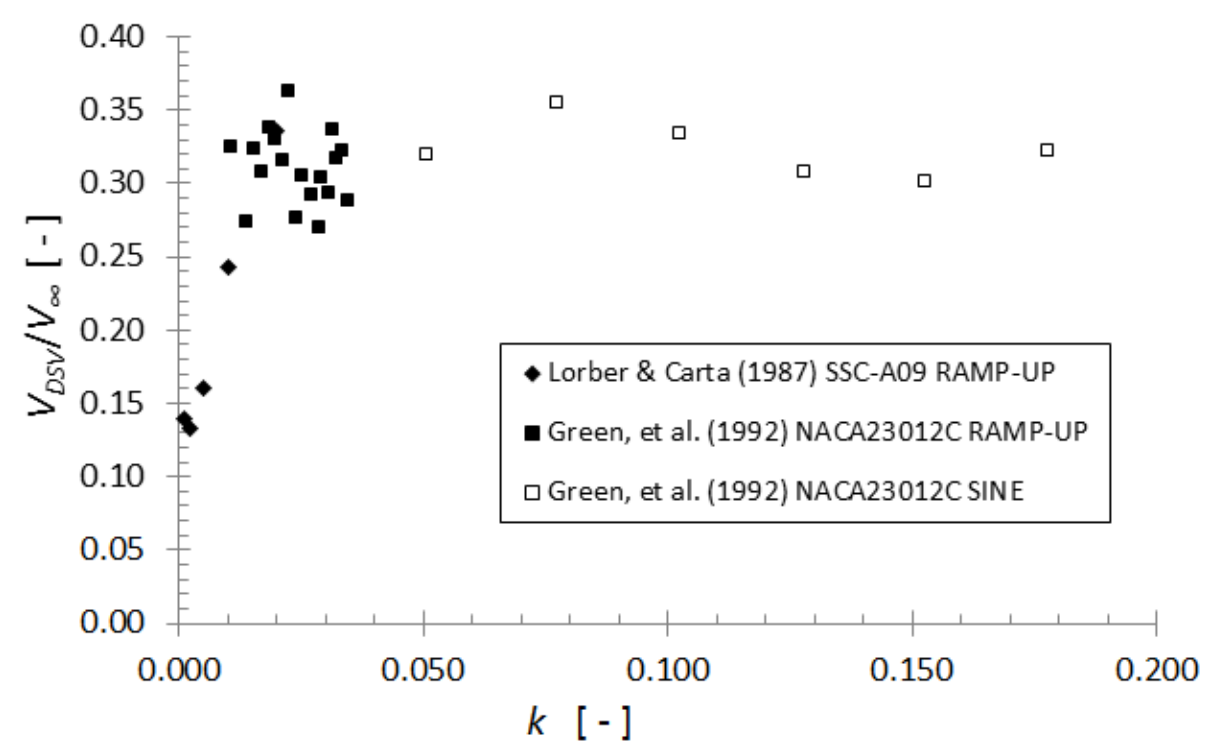

Figure 47. Influence of Pitch Rate on the Convection Speed of the Dynamic Stall Vortex (131) (132)

Jung, et al. (133) experimentally examined the DSV convection speed on a pitching NACAO012 airfoil, at reduced frequencies of $k=0.1,0.2,0.3$, and 0.4 ; mean incidence and oscillation amplitude were $0^{\circ}$ and $3^{\circ}$, respectively. Jung, et al. (133) found that the shedding frequency in the wake of a pitching airfoil was much different from that for the case of a steady airfoil at a given $\alpha$, implying that the state of the boundary layer dictated the vortex-shedding characteristics (133). This conclusion could prove critical to future CCVAWT flow control methodology, indicating that as TSR $(\lambda)$ increases, the pulsed jet St for effective control authority would increase as well.

\subsubsection{Summary of Pitching Airfoil Studies}

The in-depth review of literature studies on pitching airfoil analysis was completed to establish expected trends for the current study. Influential parameters on pitching airfoil performance have been established, including reduced airfoil pitch rate $(k)$, mean angle-of-attack $\left(\alpha_{0}\right)$, and pitch amplitude $\left(\alpha_{\max }\right)$ relative to the static stall AoA $\left(\alpha_{s s}\right)$. Re has been shown to also influence the dynamic stall characteristics of a given airfoil, but to a lesser degree. Table 8 includes the test conditions of the reviewed pitching airfoil studies, along with a summary of results from each parametric analysis. A summary of conditions pertaining to each piece of reviewed literature is ultimately included here to offer details of each study in an organized fashion. 
Table 8. Literature Review Summary: Experimental Pitching Airfoil Studies

\begin{tabular}{|c|c|c|c|c|c|c|c|}
\hline Author $(5)$ & Airfoil Profile & $\operatorname{Re}[-]$ & $k[-]$ & Pitch Motion & Amplitude & Measurements/Calcuations & Key Results \\
\hline $\begin{array}{l}\text { Martin, et al. } \\
\text { (1974] }\end{array}$ & $\begin{array}{l}\text { NACA0012 } \\
\text { (2D) }\end{array}$ & $1 \times 10^{6}$ & $\begin{array}{c}\text { helicopter } \\
\text { condtions }\end{array}$ & $\alpha(t)=10^{\circ}+5^{2} \sin (\omega \cdot t)$ & $a_{\max }=15^{\circ}$ & $\begin{array}{l}\text { - Measured: static surface pressure: } P_{i} \\
\text { - Measured:flow viz (smoke) }\end{array}$ & $\begin{array}{l}-\operatorname{Re} \uparrow: \text { no significant effect on DS } \\
-k \uparrow:\end{array}$ \\
\hline $\begin{array}{c}\text { Carr, et al. } \\
\text { (1977) } \\
\text { McAlister, et al. } \\
\text { (1978) }\end{array}$ & $\begin{array}{l}\text { NACA0012 } \\
\text { various LE modif. } \\
\text { (2D) }\end{array}$ & $2.5 \times 10^{6}$ & $0.05-0.25$ & $\begin{array}{l}\alpha(t)=15^{\circ}+10^{\circ} \sin (\boldsymbol{\omega} \cdot t) \\
\boldsymbol{\alpha}(t)=15^{\circ}+6^{\circ} \sin (\boldsymbol{\omega} \cdot t) \\
\boldsymbol{\alpha}(t)=11^{\circ}+6^{\circ} \sin (\boldsymbol{\omega} \cdot t) \\
\boldsymbol{\alpha}(t)=6^{\circ}+6^{\circ} \sin (\boldsymbol{\omega} \cdot t)\end{array}$ & $\begin{array}{l}a_{\max }=25^{\circ} \\
a_{\max }=21^{\circ} \\
a_{\max }=17^{\circ} \\
a_{\max }=12^{\circ}\end{array}$ & $\begin{array}{l}\text { - Measured: static surface pressure: } P_{i} \\
\text { - Measured: flow viz (smoke) } \\
\text { - Measured: BL (hot-wire) }\end{array}$ & 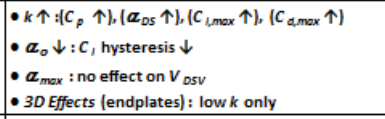 \\
\hline $\begin{array}{l}\text { St. Hilaire, et al. } \\
\text { (1983) }\end{array}$ & $\begin{array}{l}\text { NACA0012 } \\
\text { (2D) }\end{array}$ & $2.77 \times 10^{6}$ & 0.124 & $\alpha(t)=10^{\circ}+10^{\circ} \sin (\omega \cdot t)$ & $\alpha_{\max }=20^{\circ}$ & $\begin{array}{l}\text { - Measured: static surface pressure: } P_{i} \\
- \text { Calculated: } C_{p}, C_{l}, C_{d}, C_{m}\end{array}$ & $\begin{array}{l}\text { - } k \uparrow:(D S V \text { strength } \uparrow),\left(V_{D S V} \uparrow\right) \\
\text { - } k \uparrow:\left(\boldsymbol{a}_{D S} \uparrow\right)\end{array}$ \\
\hline $\begin{array}{l}\text { Raghunathan, } \\
\text { et al. (1986) }\end{array}$ & $\begin{array}{l}\text { NACA0018 } \\
\text { (2D) }\end{array}$ & $2.4 \times 10^{5}$ & 0.01 & $\alpha(t)=0^{\circ}+16^{\circ} \sin (\omega \cdot t)$ & $\boldsymbol{\alpha}_{\max }=16^{\circ}$ & $\begin{array}{l}\text { - Measured: static surface pressure: } P_{i} \\
\text { - Calculated: } C_{p}, C_{l}, C_{d}\end{array}$ & - $k \uparrow:\left(C_{l, \max } \uparrow\right),\left(C_{d, \max } \uparrow\right),\left(\alpha_{a s} \uparrow\right)$ \\
\hline $\begin{array}{l}\text { Angell, et al. } \\
\quad \text { (1988) }\end{array}$ & $\begin{array}{l}\text { NACA0021 } \\
\text { (2D) }\end{array}$ & $1.1 \times 10^{6}$ & 0.05 & $\begin{array}{l}\boldsymbol{\alpha}(t)=0^{\circ}+30^{\circ} \sin (\boldsymbol{\omega} \cdot t) \\
\boldsymbol{\alpha}(t)=0^{\circ}+20^{\circ} \sin (\boldsymbol{\omega} \cdot t) \\
\text { VAWT Function } \boldsymbol{\alpha} \text {-Path }\end{array}$ & $\begin{array}{l}\boldsymbol{\alpha}_{\max }=30^{\circ} \\
\boldsymbol{\alpha}_{\max }=20^{\circ}\end{array}$ & - Measured: static surface pressure: $P_{i}$ & $\begin{array}{l}\text { - } R e \uparrow: \text { min. influence on } C_{p}, C_{l} \\
-k \uparrow: \text { : (hysteresis } \uparrow),\left(C_{p} \uparrow\right) \\
\left.-\boldsymbol{a}_{\max } \uparrow: \text { : (hysteresis } \uparrow\right),\left(C_{l, \max } \uparrow\right) \\
\end{array}$ \\
\hline $\begin{array}{l}\text { Lorber, et al. } \\
\quad \text { (1989) }\end{array}$ & $\begin{array}{l}\text { Sikorsky SSC-AO9 } \\
\text { (2D) }\end{array}$ & $\begin{array}{l}2 \times 10^{6} \\
4 \times 10^{6}\end{array}$ & $0.001-0.10$ & $\begin{array}{l}\boldsymbol{\alpha}(t)=\boldsymbol{\alpha}_{0}+\boldsymbol{\alpha}_{m} \sin (\boldsymbol{\omega} \cdot t) \\
\boldsymbol{\alpha}_{0}=5^{\circ}, 10^{\circ}, 12^{2}, 20^{\circ}\end{array}$ & $\begin{array}{l}a_{\max }=10^{\circ} \\
a_{\max }=20^{\circ} \\
a_{\max }=30^{\circ}\end{array}$ & $\begin{array}{l}\text { - Measured: static surface pressure: } P_{i} \\
\text { - Measured: BL (hot-wire) } \\
\text { - Calculated: } C_{p}, c_{1}, c_{d}, c_{m}\end{array}$ & $\begin{array}{l}\text { - } k \uparrow:(D S V \text { strength } \uparrow),\left(V_{D S V} \uparrow\right),\left(\alpha_{D S} \uparrow\right) \\
\text { - } k \uparrow:\left(C_{l, \max } \uparrow\right),\left(C_{d, \max } \uparrow\right),\left(\mathrm{d} C_{l} / \mathrm{d} \alpha \downarrow\right) \text { pre-stall } \\
\text { - } R e \uparrow:\left(C_{l, \max } \downarrow\right),(\mathrm{DSV} \text { strength } \downarrow)\end{array}$ \\
\hline $\begin{array}{l}\text { Koochesfahani } \\
\text { (1989) }\end{array}$ & $\begin{array}{l}\text { NACAO012 } \\
\text { (2D) }\end{array}$ & $1.2 \times 10^{4}$ & $0.02-0.14$ & $\begin{array}{l}\boldsymbol{\alpha}(t)=0^{\circ}+2^{\circ} \sin (\boldsymbol{\omega} \cdot t) \\
\boldsymbol{\alpha}(\boldsymbol{t})=0^{\circ}+4^{\circ} \sin (\boldsymbol{\omega} \cdot t)\end{array}$ & $\begin{array}{l}a_{\max }=2^{\circ} \\
a_{\max }=4^{\circ}\end{array}$ & $\begin{array}{l}\text { - Measured: wake velocity profile }\left(v / V_{\mathrm{eo}}\right) \\
\text { - Measured: flow viz (dye injection) } \\
\text { - Calculated: } C_{F} \quad \text { (mean streamwise force) }\end{array}$ & $\begin{array}{l}\text { - } k \uparrow:(D S V \text { strength } \uparrow),\left(V_{D S V} \uparrow\right) \\
\text { - } k \uparrow:\left(C_{L_{\text {max }}} \uparrow\right),\left(C_{d, \max } \uparrow\right),\left(\boldsymbol{C}_{D S} \uparrow\right) \\
\text { - } R e \uparrow:\left(C_{\text {Lmax }} \downarrow\right),(D S V \text { strength } \downarrow)\end{array}$ \\
\hline $\begin{array}{c}\text { Conger, et al. } \\
\text { (1992] }\end{array}$ & $\begin{array}{l}\text { NACA0015 } \\
\text { (2D) }\end{array}$ & $\begin{array}{l}0.5 \times 10^{5} \\
2.2 \times 10^{5}\end{array}$ & $0.03-0.20$ & Ramp-Up (constant $k$ ) & $a_{\max }=40^{\circ}$ & $\begin{array}{l}\text { - Measured: surface pressure: } P_{i} \text { Flow-Viz (PIV) } \\
\text { - Calculated: } c_{p}, c_{1}\end{array}$ & - $R e \uparrow:$ no influence on $C_{p}, C_{1}$ \\
\hline $\begin{array}{l}\text { Piziali } \\
\text { (1994) }\end{array}$ & $\begin{array}{c}\text { NACAOOO15 } \\
\text { (2D/3D) }\end{array}$ & $\begin{array}{c}1.4 \times 10^{6} \text { to } \\
2.4 \times 10^{6}\end{array}$ & $0.04-0.20$ & $\begin{array}{l}\alpha(t)=\alpha_{o}+\alpha_{m} \sin (\omega \cdot t) \\
\alpha_{o}=4^{\circ}, 9^{\circ}, 11^{\circ}, 13^{\circ}, 15^{\circ}, 17^{\circ}\end{array}$ & $\begin{array}{l}\alpha_{\max }=6^{\circ}, 11^{\circ} \\
\alpha_{\max }=13^{\circ}, 15^{\circ}\end{array}$ & $\begin{array}{l}\text { - Measured: static surface pressure: } P_{i} \\
\text { - Measured: Flow-Viz (Tufts) } \\
\text { - Calculated: } C_{p}, C_{l}, C_{m} \quad \delta\left[C_{l}\right)= \pm 5 \%\end{array}$ & $\begin{array}{l}-\boldsymbol{a}_{o} \uparrow:\left(C_{l, \max } \uparrow\right),\left(\boldsymbol{a}_{a s} \uparrow\right) \\
-\boldsymbol{a}_{\max } \uparrow:\left(C_{l}, C_{d} \text { Hysteresis }\right) \uparrow\end{array}$ \\
\hline $\begin{array}{l}\text { Lee, et al. } \\
\text { (1998) }\end{array}$ & $\begin{array}{l}\text { NACAO012 } \\
\text { (2D) }\end{array}$ & $1.69 \times 10^{5}$ & $0.05-0.11$ & $\begin{array}{l}\boldsymbol{\alpha}(t)=0^{\circ}+7.5^{\circ} \sin (\boldsymbol{\omega} \cdot t) \\
\boldsymbol{\alpha}(t)=7.5^{\circ}+7^{\circ} \sin (\boldsymbol{\omega} \cdot t)\end{array}$ & $\begin{array}{l}\alpha_{\max }=7.5^{\circ} \\
\alpha_{\max }=14.5^{\circ}\end{array}$ & $\begin{array}{l}\text { - Measured: static surface pressure: } P_{i} \\
\text { - Measured: }(x / c)_{\text {transition }} \text { (hot-film) }\end{array}$ & $\begin{array}{l}-k \uparrow: \text { delayed turb. BL breakdown, }\left(\boldsymbol{\alpha}_{a s} \uparrow\right) \\
-\boldsymbol{\alpha}_{\max } \uparrow: \text { BL transition delayed }\left(\boldsymbol{\alpha}_{\operatorname{san} \mid} \uparrow\right) \\
-\boldsymbol{\alpha}_{\max } \downarrow: \text { BL reattachment improved }\end{array}$ \\
\hline $\begin{array}{l}\text { Schreck, et al. } \\
\quad \text { (2002) }\end{array}$ & $\begin{array}{l}\text { NACA0015 } \\
\text { (2D) }\end{array}$ & $\begin{array}{l}2.4 \times 10^{4}- \\
1.92 \times 10^{5}\end{array}$ & $0.01-0.64$ & Ramp-Up (constant $k$ ) & $\begin{array}{l}\boldsymbol{a}_{\max }=30^{\circ} \\
\boldsymbol{a}_{\max }=40^{\circ}\end{array}$ & $\begin{array}{l}\text { - Measured: static surface pressure: } \boldsymbol{P}_{i} \\
\text { - Measured: LE shear stess } \\
\text { - Calculated: } C_{p}, C_{1}, C_{\alpha_{1}} C_{m}\end{array}$ & $\begin{array}{l}\text { - } k \uparrow: \text { delay of BL reversal to higher } \boldsymbol{\alpha} \\
\text { - } k \uparrow:\left(C_{b \max } \uparrow\right),\left(C_{d, \max } \uparrow\right),\left(\boldsymbol{\alpha}_{D S} \uparrow\right) \\
\text { - } k \text { : strong, orderly influence on BL processes } \\
\text { - Re: secondary influence on BL processes }\end{array}$ \\
\hline $\begin{array}{l}\text { Soltani, et al. } \\
\quad \text { (2008) }\end{array}$ & \begin{tabular}{|c|} 
HAWT Airfoil \\
$1796(t / c)$ w/ camber \\
(2D)
\end{tabular} & $\begin{array}{c}0.43 \times 10^{6}- \\
0.84 \times 10^{6}\end{array}$ & $0.03-0.10$ & $\begin{array}{l}\boldsymbol{\alpha}(\boldsymbol{t})=8^{8}+2^{\circ} \sin (\boldsymbol{\omega} \cdot t) \\
\boldsymbol{\alpha}(\boldsymbol{t})=5^{2}+5^{\circ} \sin (\boldsymbol{\omega} \cdot t)\end{array}$ & $a_{\max }=10^{\circ}$ & $\begin{array}{l}\text { - Measured: static surface pressure: } P_{i} \\
\text { - Measured: }(x / c)_{\text {transition }} \text { (hot-film) }\end{array}$ & $\begin{array}{l}\text { - } R e \uparrow: \text { (hysteresis } \downarrow),(x / c)_{\text {trons }} \text { shift } \\
\text { - } k \uparrow: \text { : (hysteresis } \uparrow),(x / c)_{\text {trons }} \text { shift } \\
\left.\text { - } \boldsymbol{\alpha}_{\mathrm{o}} \uparrow: \text { : (hysteresis } \uparrow\right),(x / c)_{\text {trons }} \text { shift }\end{array}$ \\
\hline $\begin{array}{l}\text { Tsang, et al. } \\
\text { (2008) }\end{array}$ & $\begin{array}{l}\text { NACAO012 } \\
\text { (2D) }\end{array}$ & $7.7 \times 10^{4}$ & $0.005-0.04$ & $\begin{array}{l}\alpha(t)=\alpha_{0}+\alpha_{m} \sin (\omega \cdot t) \\
\alpha_{0}=0^{\circ}, 5^{\circ}, 10^{\circ}, 15^{\circ}\end{array}$ & 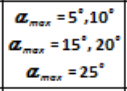 & $\begin{array}{l}\text { - Measured: airfoil forces (piezo load cells) } \\
\text { - Calculated: } C_{1}, \text { Cd, Spectral Analysis }\left(C_{1}, C_{d}\right)\end{array}$ & $\begin{array}{l}-k \uparrow:\left(C_{,} \text {hysteresis } \uparrow\right),\left(\boldsymbol{a}_{D S} \uparrow\right) \\
-\boldsymbol{a}_{\max } \uparrow:\left(\boldsymbol{a}_{D S} \uparrow\right),\left(\boldsymbol{C}_{l, \max } \uparrow\right) \\
-\boldsymbol{a}_{o}: \text { small effect on } \boldsymbol{a}_{D S}, \text { no effect on } C_{l, \operatorname{mox}}\end{array}$ \\
\hline $\begin{array}{l}\text { Sharma, et al. } \\
\quad(2009)\end{array}$ & $\begin{array}{l}\text { NACA0015 } \\
\text { (2D) }\end{array}$ & $\begin{array}{l}0.2 \times 10^{6} \\
0.7 \times 10^{6}\end{array}$ & $0.001-0.5$ & $\alpha(t)=10^{\circ}+15^{\circ} \sin (\omega \cdot t)$ & $a_{\max }=25^{\circ}$ & $\begin{array}{l}\text { - Measured: static surface pressure: } P_{i} \\
\text { - Calculated: } c_{p}, c_{n}, C_{m}\end{array}$ & $\begin{array}{l}\text { - } k \downarrow: \text { Re effects } \uparrow \\
\text { - } k \uparrow: \text { (hysteresis } \uparrow),\left(\boldsymbol{\alpha}_{D S} \uparrow\right), \\
\text { - LE Contamination } \uparrow:\left(\boldsymbol{\alpha}_{D S} \text { shift) }\right.\end{array}$ \\
\hline $\begin{array}{l}\text { Mueller-Vahl, et al. } \\
\quad(2009)\end{array}$ & $\begin{array}{l}\text { NACAO018 } \\
\text { (2D) }\end{array}$ & $5 \times 10^{5}$ & $0.018-0.074$ & $\alpha(t)=0^{\circ}+30^{\circ} \sin (\omega \cdot t)$ & $\boldsymbol{\alpha}_{\max }=30^{\circ}$ & $\begin{array}{l}- \text { Measured: static surface pressure: } P_{i} \\
- \text { Calculated: } c_{p}, c_{i}, c_{m}\end{array}$ & $\begin{array}{l}\text { - Re: influence large for light/medium DS } \\
\text { - } \boldsymbol{a}_{\max } \uparrow:(C, \uparrow),\left(c_{d} \uparrow\right),\left(c_{p} \uparrow\right),\left(\boldsymbol{a}_{D S} \uparrow\right)\end{array}$ \\
\hline
\end{tabular}

The CFD studies from the literature include dynamic performance of both conventional airfoils and those modified to include flow control. Table 9 summarizes past numerical research pertinent to the current study, including descriptions of airfoil profile and motion, along with specific modeling techniques of interest. Numerical methodology from past dynamic stall studies were used to guide the CFD work of the current study. Based on the reviewed literature, the one-equation Spalart-Allmaras turbulence model (with curvature correction) was found to offer an acceptable balance of accuracy and computational time for airfoil studies with comparable conditions. Furthermore, small time steps $\left(\Delta t^{+} \leq 0.001\right)$ and higher-order discretization schemes ( $\geq 2^{\text {nd }}$ Order) were reported necessary for reliable dynamic stall computations, considering BL development and separation, and the resulting aerodynamic loading. 
Table 9. Literature Review Summary: Numerical Pitching Airfoil Studies

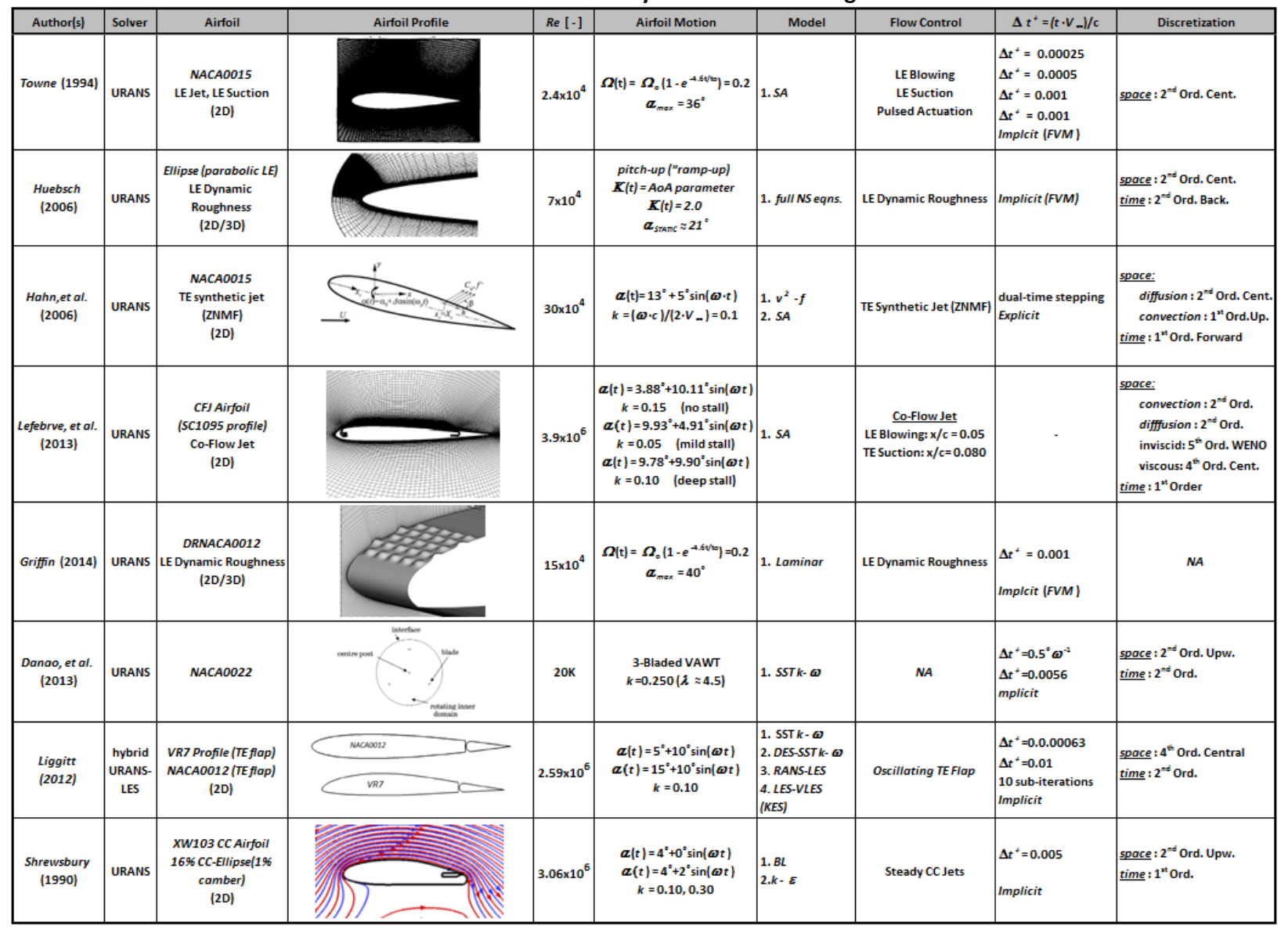

The resulting airfoil data from all reviewed literature are summarized here, including both numerical and experimental research, to estimate limits of acceptable $C_{l}$ and $C_{d}$ ranges for the current study. A similar method to synthesize airfoil performance from collected experimental data was completed by Gangwani (134) to predict dynamic stall and unsteady airloads for a given airfoil under desired conditions. Despite many applications involving the NACA0018 profile, aerodynamic performance at the appropriate combination of $R e$ and $\pm \alpha$ for VAWT applications is non-existent, or at the very least extremely limited (22).

The summary of pitching airfoil lift performance from pertinent literature studies is included in Figure 48. A solid green line was estimated to encompass average range of repeated trends, factoring $\mathrm{d} C_{1} / \mathrm{d} \alpha, C_{l, \max }$, and hysteresis loops. The same process was completed to establish a general range of dynamic pitch influence on airfoil drag, as seen in Figure 49, where a solid red line was estimated to establish an acceptable range of results. Airfoil data for a pitching CCNACA0018 does not currently exist. The estimated limits established from related literature provide a suitable range of expected results for comparison to measured and computed airfoil performance of this research. 


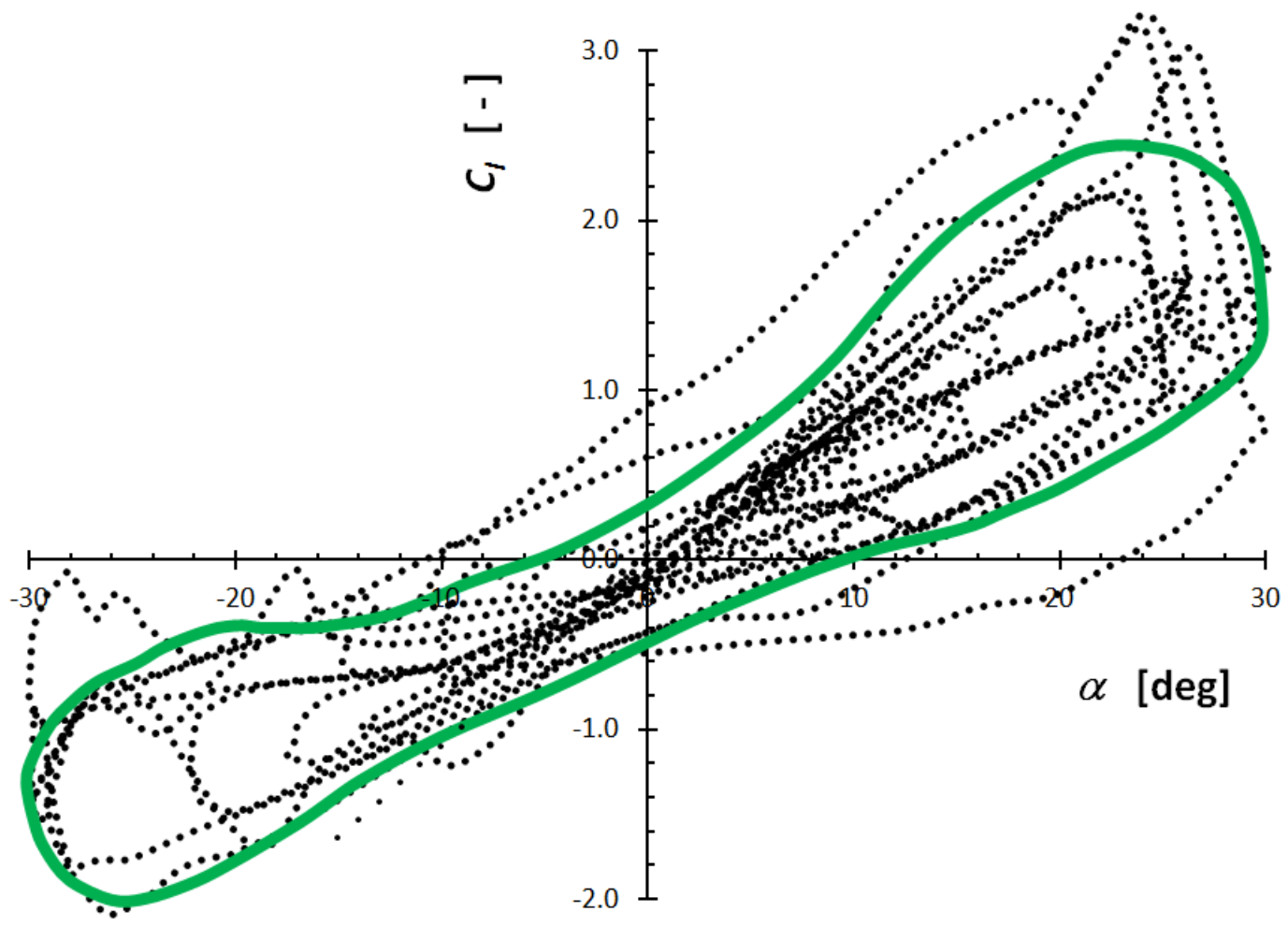

Figure 48. Summary of Dynamic Pitch Lift Data from Literature

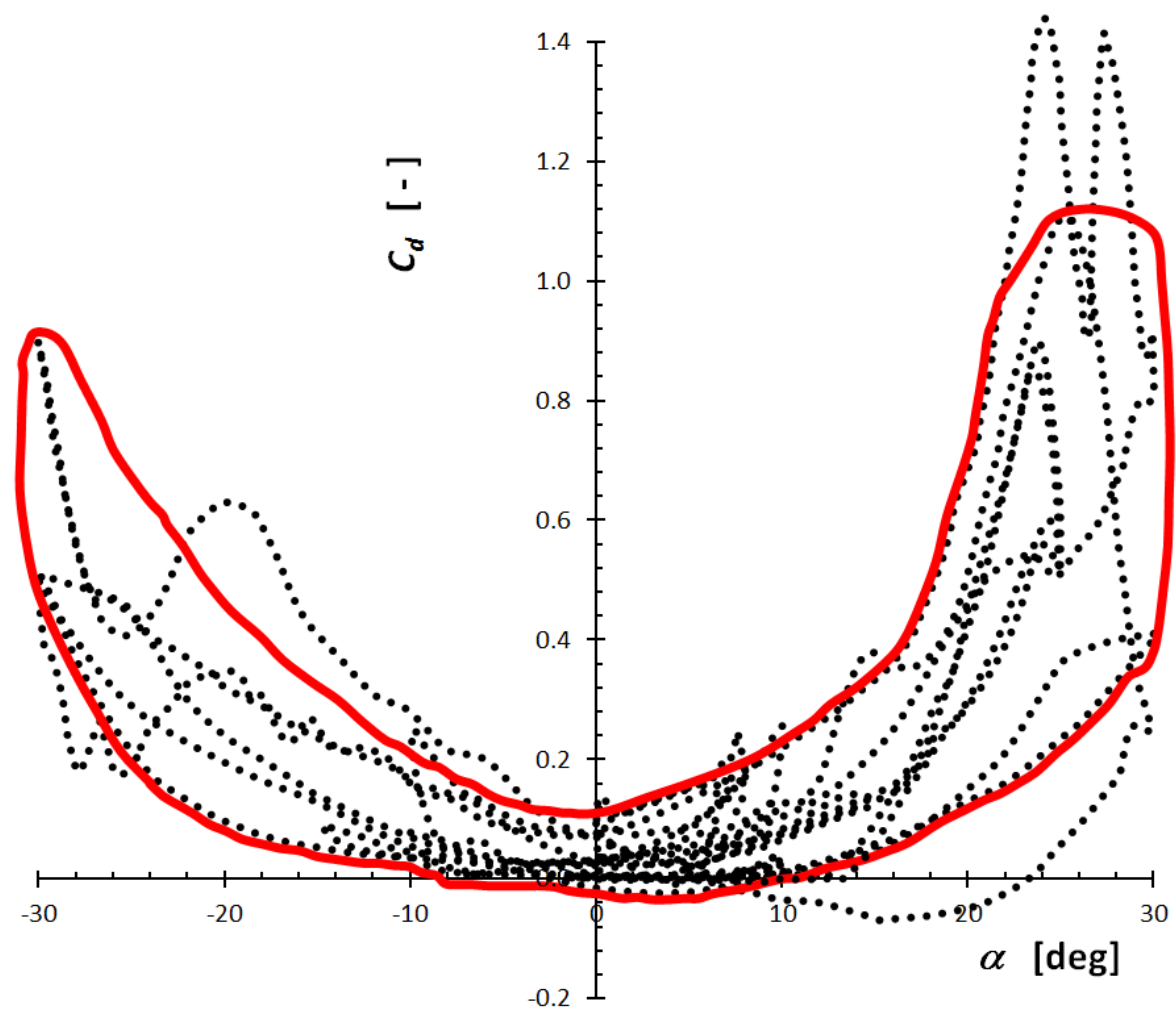

Figure 49. Summary of Dynamic Pitch Drag Data from Literature 
It should be noted that the qualitative details of dynamic stall, whether in the incipient or deep flow regimes, are common to most airfoils. However, the magnitudes of the quantitative features of transient stall are strongly dependent to each airfoil and operating environment. The high demand of dynamic stall research, primarily within the past two decades, has generated a more profound understanding of the qualitative aspects of the flow phenomenon. Thus, to attain the precise, quantitative dynamic stall properties of a novel airfoil design, thorough experimental and computational testing was completed. 


\section{CHAPTER 3. EXPERIMENTAL ANALYSIS}

This chapter details the wind tunnel testing completed with the CCNACA0018 model. The goal of this experiment was to measure the aerodynamic performance $\left(C_{l}\right.$ and $\left.C_{d}\right)$ of the CCNACAO018 airfoil under simulated VAWT conditions, investigating both steady and pulsed jet CC actuation. Aerodynamic coefficients were calculated from measured surface pressure distributions in both static and dynamic environments. Static tests were first completed to isolate the effects of blowing magnitude $\left(C_{\mu}\right)$ and unsteady jet frequency $\left(f_{\text {jet }}\right)$. Next, the influence of induced circulation and dynamic stall effects due to airfoil pitch were targeted, varying oscillation angle $\left(\alpha_{\max }\right)$ and reduced pitch rate $(k)$. Free stream effects were considered for both flow regimes through multiple Reynolds number $(R e)$ test conditions. All tests were completed in the West Virginia University Closed Loop Subsonic Wind Tunnel.

\subsection{Wind Tunnel Model}

The CCNACA0018 wind tunnel model used in this study was used in research by CIRA at WVU (31) (38). The enhanced NACA0018 profile, included in Figure 50, was equipped with a total of 33 static pressure taps. The coordinates of each pressure port are included for reference. There was a concentration of ports located near the LE and TE of the model, improving spatial resolution of surface pressure scans near regions of unsteady and transitional flow.
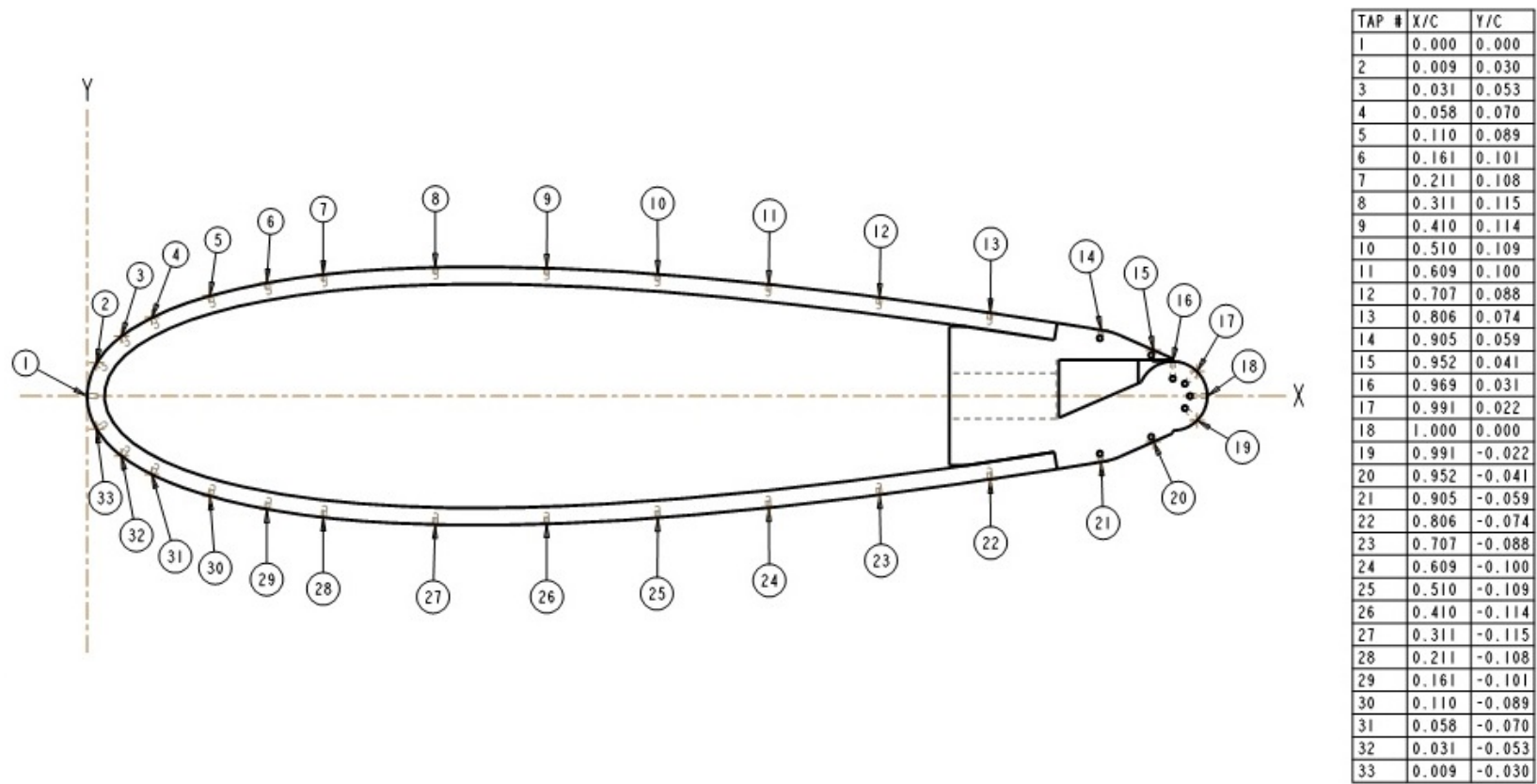

Figure 50. CCNACA0018 Wind Tunnel Model Static Pressure Port Locations 
The wind tunnel model dimensions are summarized in Table 10, including TE radius $\left(r_{T E}\right)$, blowing slot height $\left(h_{\text {slot }}\right)$, chord $(c)$, span $(b)$, location of blowing slot $\left((x / c)_{\text {slot }}\right)$, and various ratios of TE geometry for easy comparison to CC airfoils from the literature.

Table 10. CCNACA0018 Wind Tunnel Model Dimensions

\begin{tabular}{|c|c|c|}
\hline$r_{T E}$ & 0.25 & [in.] \\
\hline$h_{\text {slot }}$ & 0.015 & [in.] \\
\hline$c$ & 8.13 & [in.] \\
\hline$b$ & 16.5 & [in.] \\
\hline$(x / c)_{\text {slot }}$ & 0.969 & {$[-]$} \\
\hline$h_{\text {slot }} / r_{T E}$ & 0.06 & {$[-]$} \\
\hline$h_{\text {slot }} / c$ & 0.002 & {$[-]$} \\
\hline$r_{T E} / c$ & 0.031 & {$[-]$} \\
\hline
\end{tabular}

At maximum angle of incidence $\left(\alpha_{\max }=30^{\circ}\right)$, the physical airfoil blockage ratio with respect to wind tunnel dimensions $\left(A_{\text {model }} / A_{\text {tunnel }}\right)$ falls below the historic recommended value of 0.10 for negligible interference effects (135). The internal components for flow control actuation are included in Figure 51.
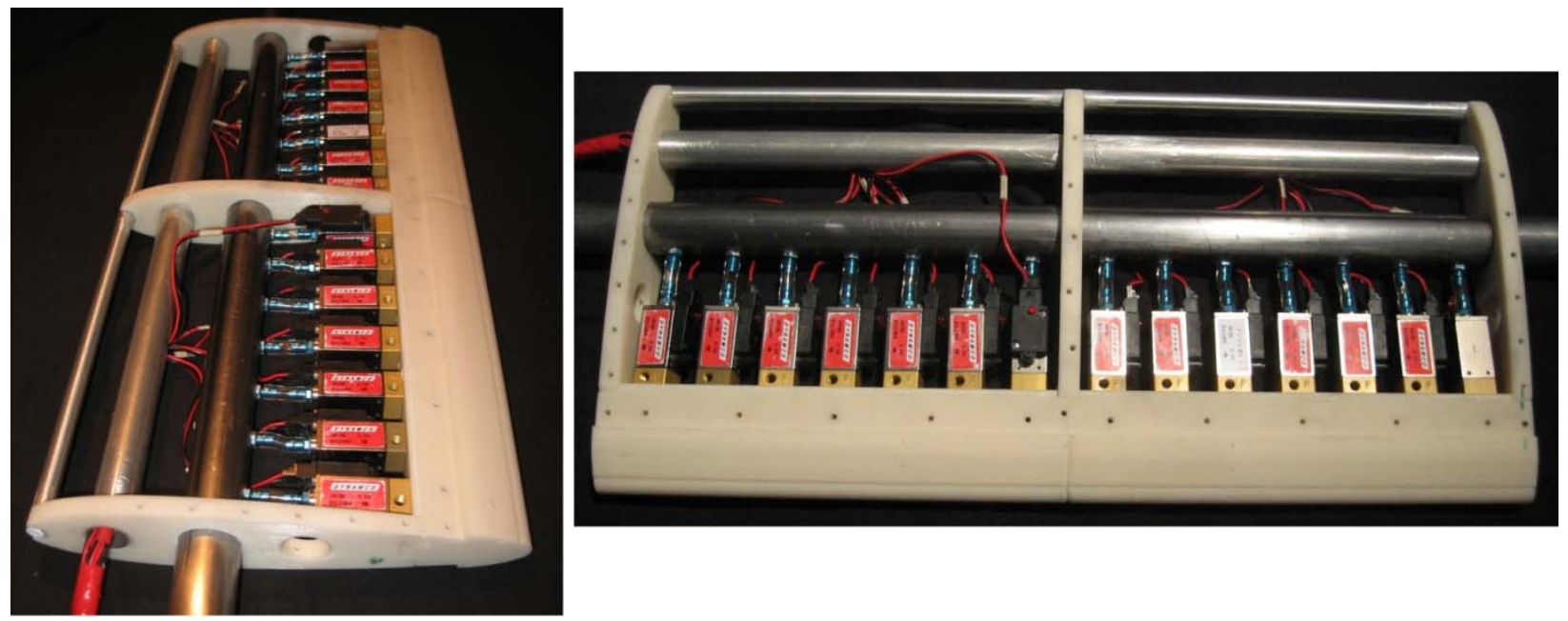

Figure 51. CCNACA0018 Wind Tunnel Model: Internal Plumbing and [14] DYNAMCO Pneumatic Valves

Once all electrical and pneumatic connections were tested and secured, the prototyped skin sections were attached to the ribs and TE diffuser section with flanged screws (see Figure 52). All joints and connectors were filled and sealed for a uniform, smooth surface before painting. An advantage of using prototyped skin was the inclusion of static pressure taps formed into the skin. The creation of such ports on wind tunnel models, regardless of the machining technique used, is otherwise arduous and unreliable. The printed material was ABS plastic. 


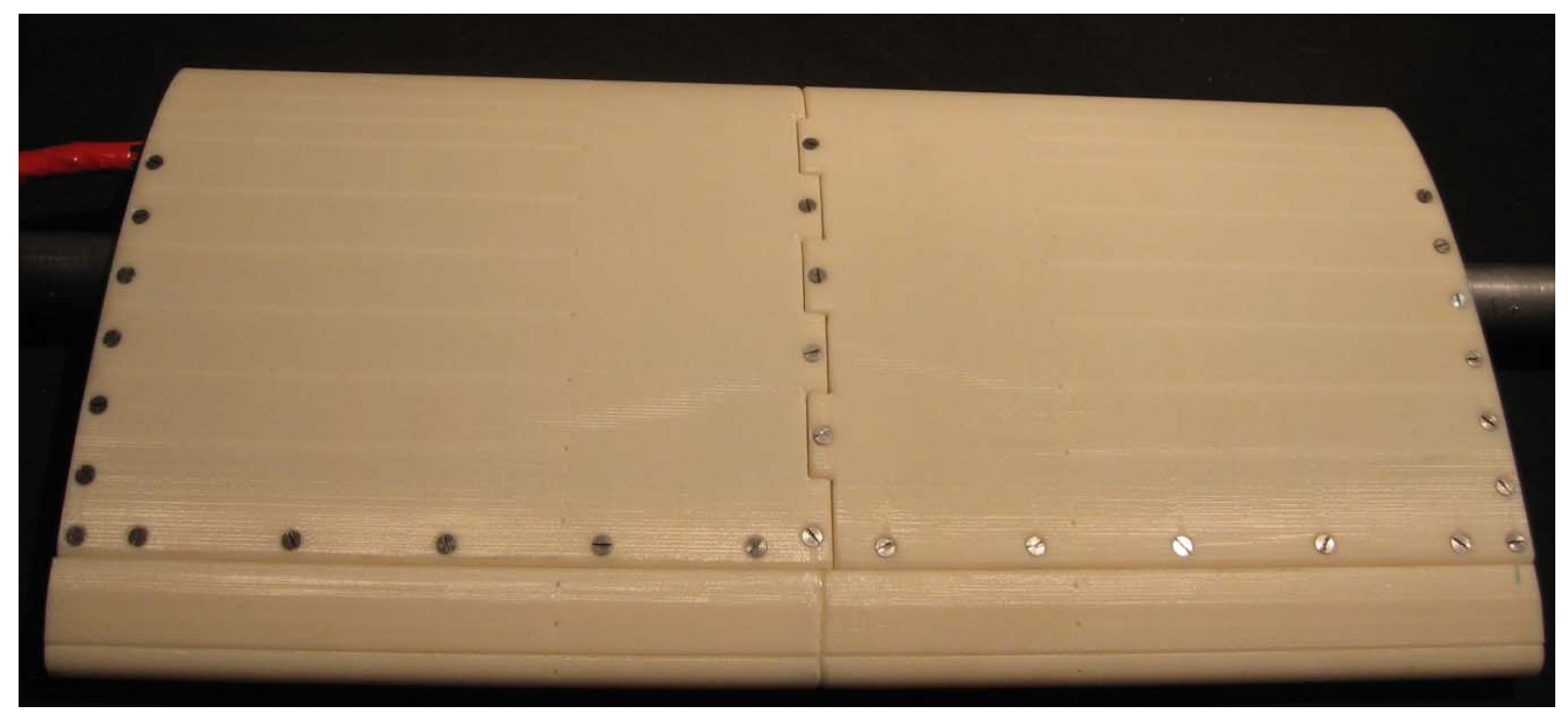

Figure 52. CCNACA0018 Wind Tunnel Model with Rapid-Prototyped Skin

Aluminum tubes (ID=0.053") were then inserted into the printed channels to eliminate outside signal contamination (e.g. between adjacent ports or from the pressurized plenum). The endplates (Figure 53) on the model were machined to allow passage of the aluminum tubes for connection to the pneumatic tubing and pressure transducers. A more detailed description of the wind tunnel model is provided by Panther, et al (38).

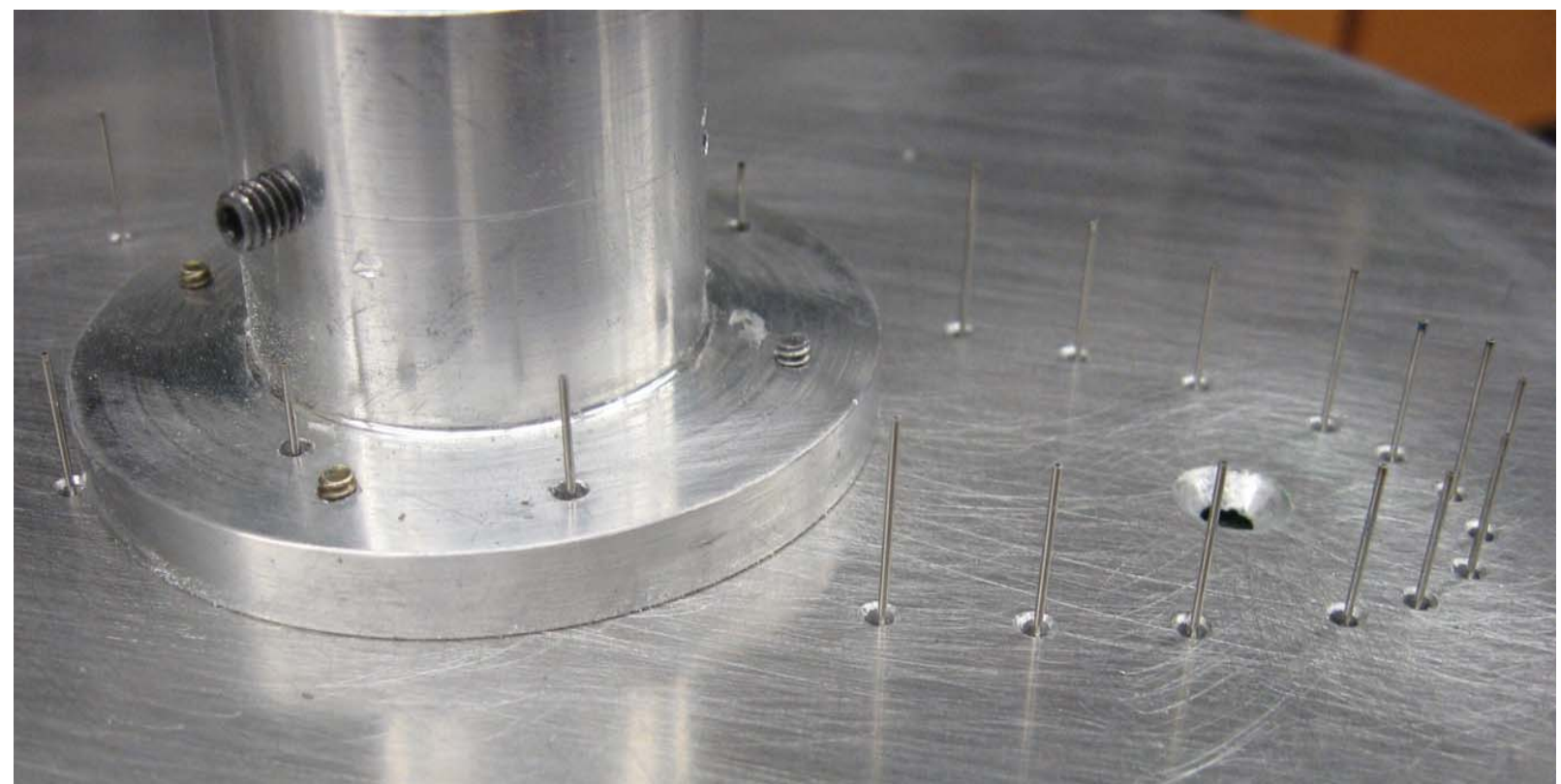

Figure 53. CCNACA0018 Wind Tunnel Model: Static Pressure Port Tubing at Airfoil Endplate 


\subsection{Experimental Test Conditions}

\subsubsection{Reynolds Number (Re)}

The Reynolds number was the similitude parameter used to define the freestream conditions $(\infty)$, using airfoil chord (c) as the characteristic length (Equation 35 ).

Reynolds Number

$$
\operatorname{Re}=\frac{\rho_{\infty} \cdot V_{\infty} \cdot c}{\mu_{\infty}}
$$

Equation 35

The ideal gas law was used to calculate air density, based on measured air temperature $\left(T_{\infty}\right)$ and pressure $\left(P_{\infty}\right)$. Sutherland's formula (Equation 36$)$ was used to calculate the dynamic viscosity of air based on $T_{\infty}$. The reference values in Equation 36 are $T_{o}=518.7 \mathrm{R}$ and $\mu_{0}=3.62 \times 10^{-7} \mathrm{Ib}_{\mathrm{f}}-\mathrm{s} / \mathrm{ft}^{2}$.

Sutherland's Formula

$$
\mu=\mu_{o}\left(\frac{T_{\infty}}{T_{o}}\right)^{1.5} \frac{\left(T_{o}+198.72\right)}{\left(T_{\infty}+198.72\right)}
$$

Equation 36

To determine flow velocity $\left(V_{\infty}\right)$, the freestream dynamic pressure $\left(\Delta P_{\infty}\right)$ of the wind tunnel was measured with multiple pitot-static probes and a HEISE handheld digital pressure calibrator. The freestream velocity calculation from measured values is included in Equation 37.

Freestream Velocity

$$
V_{\infty}=\sqrt{\frac{2 \cdot \Delta P_{\infty}}{\rho_{\infty}}}
$$

Equation 37

For the current experimental work, the test Re range matched previous CIRA work (18) (22) (38), including $180 \times 10^{3} \leq \operatorname{Re} \leq 300 \times 10^{3}$. The target Re range, along with average values of freestream velocity from testing, is included in Table 11.

\section{Table 11. Experimental Freestream Conditions}

\begin{tabular}{|c|c|}
\hline$R e$ & $V_{\infty}$ \\
\hline$[-]$ & {$[\mathrm{ft} / \mathrm{s}]$} \\
\hline $180 \times 10^{3}$ & 45 \\
\hline $300 \times 10^{3}$ & 75 \\
\hline
\end{tabular}

\subsubsection{Angle of Attack $(\alpha)$}

The $\alpha$-position of the airfoil, for both static and dynamic test conditions, was monitored by a US DIGITAL MAE3 Absolute Magnetic Encoder. The components (base, press-mount magnetic hub, and 
encoder body) can be found in the left side of Figure 54 . The sensor provided digital feedback over $360^{\circ}$ of rotation, without stops or gaps. The encoder magnet was mounted directly onto a 1/4in.(OD) rod, extending from the central shaft used to mount the CCNACA0018 model (aligned with the airfoil $0.25 c$ pitch-axis). The sensor housing was hard mounted to a 0.5in thick aluminum plate, attached to an independent, steel frame located outside on the wind tunnel walls. The DAQ program simultaneously recorded real-time $\alpha$ and pressure signals. Based on manufacturer specifications, the absolute encoder provided measurement resolution of $\pm 0.25^{\circ}$.
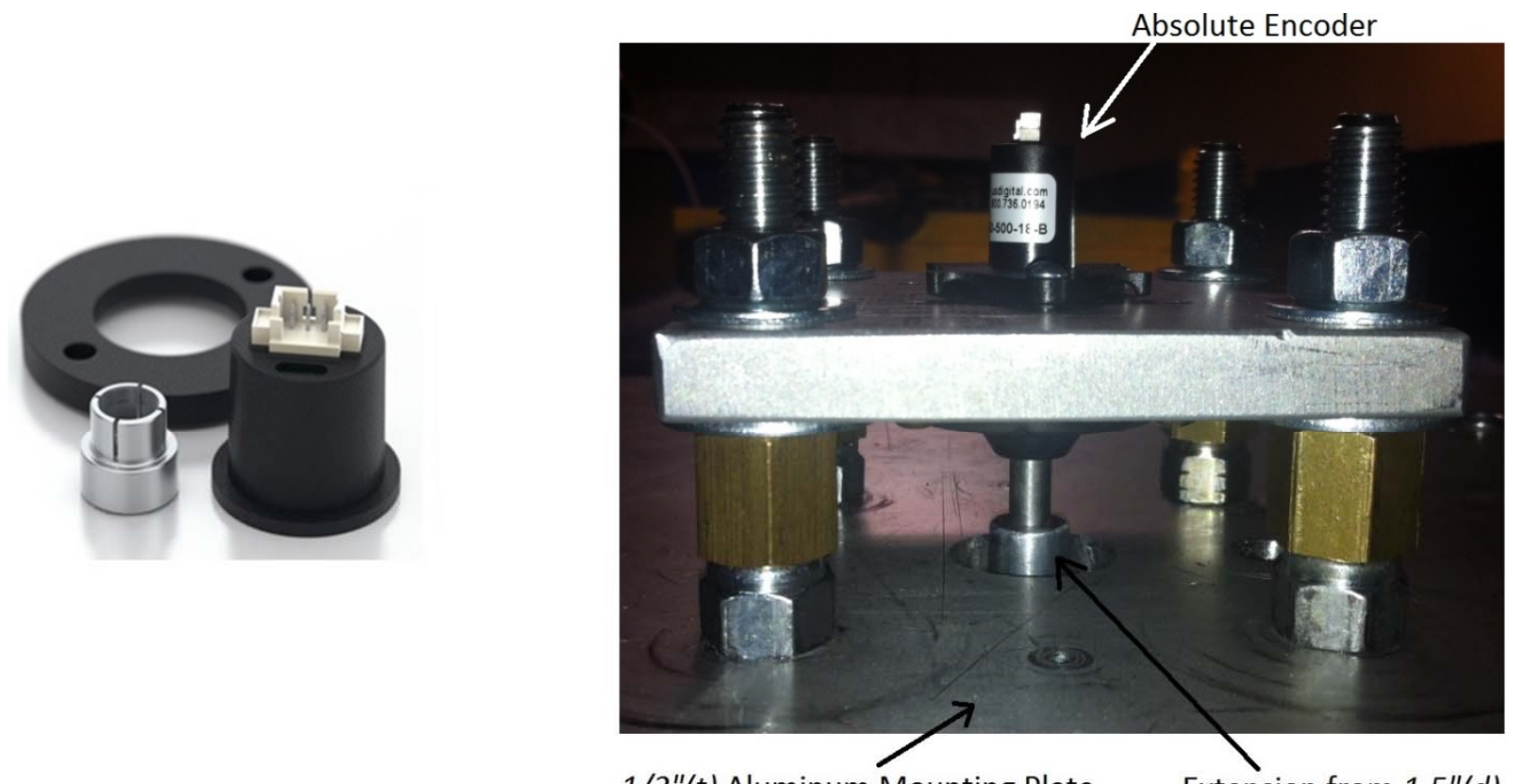

1/2"(t) Aluminum Mounting Plate

Extension from $1.5^{\prime \prime}(d)$

Central Shaft

Figure 54. Absolute Encoder Mounted Above Test Section

The bearings that connected the central airfoil shaft to the surrounding wind tunnel structure had set screws for position adjustment (see Figure 55). The zero position of the airfoil, with respect to the freestream flow, was determined using both geometric measurements (i.e. model LE and TE equidistant to wind tunnel side walls) and LE stagnation point readings (i.e. model was adjusted until the pressure tap \#1 reached a maximum pressure reading, per wind speed setting, revealing it to be the stagnation point). The stagnation reading at port \#1 ensured the airfoil was directly aligned with the oncoming flow. The latter method was used during testing so that any skewed flow at a given wind speed setting could be accounted for and removed as a tare value. Once the zero position was determined each day, the set screws were securely tightened before testing. This method was verified by physical measurement of the airfoil LE and TE to wind tunnel sidewalls. Comparing methods showed close agreement, with less than $2 \%$ difference between zero AoA position, for each day of testing. 


\subsubsection{Reduced Pitch Frequency $(k)$}

Oscillating airfoil studies are governed by the reduced pitch rate $(k)$, defined by Equation 38 .

Reduced Pitch Frequency

$$
k=\frac{\omega \cdot c}{2 \cdot V_{\infty}}
$$

Equation 38

The airfoil pitch conditions for each $R e$ are included in Table 12, along with motor speed ( $\left.\omega_{\text {motor }}\right)$ and the nominal airfoil oscillation frequencies ( $\omega_{\text {airfoil }}[\mathrm{rad} / \mathrm{s}]$ and $\left.f_{\text {airfoil }}[\mathrm{Hz}]\right)$.

Table 12. Experimental Airfoil Pitch Conditions

\begin{tabular}{|c|c|c|c|c|}
\hline$R e$ & $k$ & $\sigma_{\text {motor }}$ & $\sigma_{\text {airfoil }}$ & $f_{\text {airfoil }}$ \\
\hline$[-]$ & {$[-]$} & {$[\mathrm{rpm}]$} & {$[\mathrm{rad} / \mathrm{s}]$} & {$[\mathrm{Hz}]$} \\
\hline \multirow{3}{*}{$180 \times 10^{3}$} & 0.025 & 32 & 0.61 & 0.53 \\
\cline { 2 - 5 } & 0.05 & 65 & 1.24 & 1.08 \\
\cline { 2 - 5 } & 0.1 & 130 & 2.48 & 2.17 \\
\hline \multirow{3}{*}{$300 \times 10^{3}$} & 0.025 & 53 & 1.01 & 0.88 \\
\cline { 2 - 5 } & 0.05 & 110 & 2.1 & 1.83 \\
\cline { 2 - 5 } & 0.1 & - & - & - \\
\hline
\end{tabular}

The pitching motion of the wind tunnel model was facilitated by a basic four bar mechanism, designed and fabricated to oscillate with a $40^{\circ}$ range of rotation; see Figure 55.

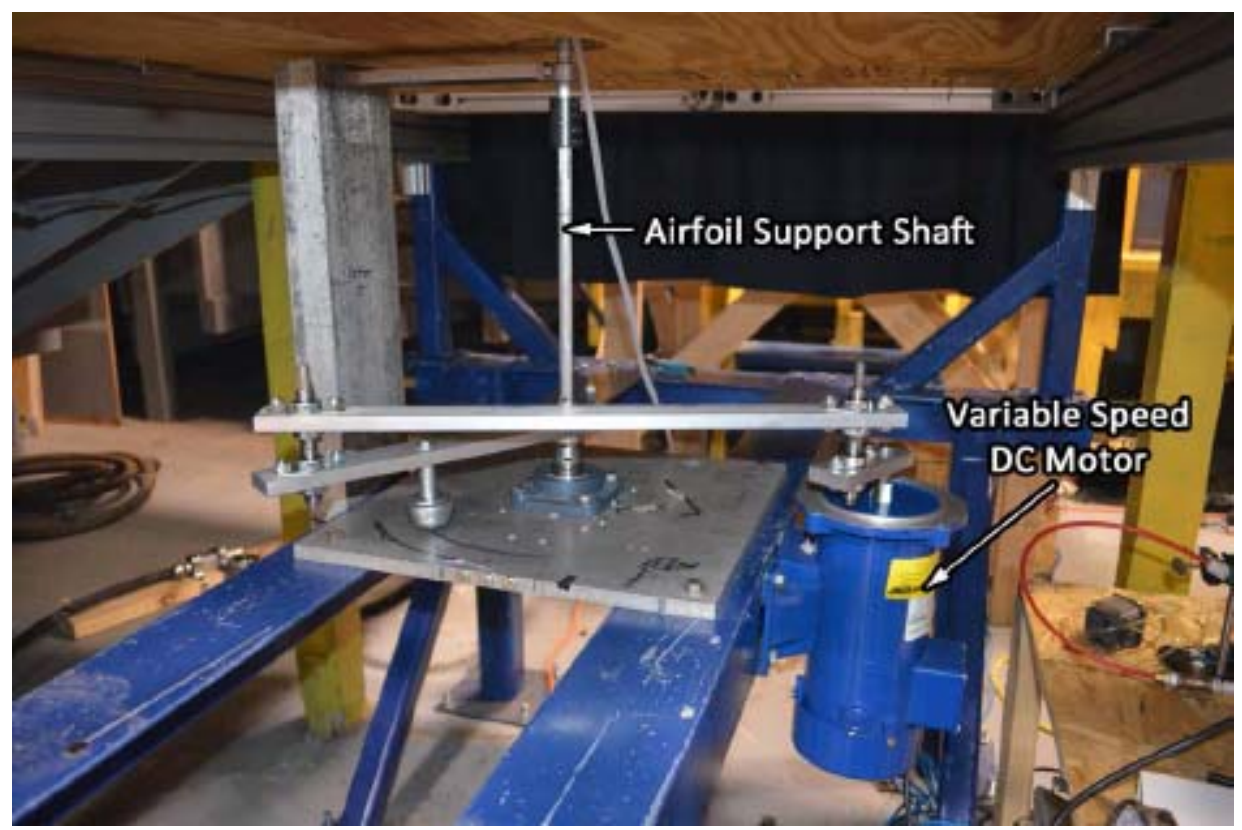

Figure 55. Airfoil Pitch Mechanism: DC Motor with 4-Bar Mechanism (55)

A 0.5 HP variable speed DC motor drove the system and pitch rate was adjusted by varying motor speed. A dialed motor controller was used to adjust $\omega_{\text {motor. }}$ A tachometer (non-contact) with a 
digital display was used to monitor $\omega_{\text {motor; }}$ the tachometer resolution was $0.1 \mathrm{rpm}$. A handheld laser stroboscope was used to validate the tachometer measurements.

The AoA range was adjustable by changing the lengths of system links. Airfoil pitch was limited to the range of $\Delta \alpha=40^{\circ}$ for this study. The maximum pitch AoA was changed by adjusting the model shaft with a series of set screws in the mounting bearings. When geometrically centered, the model would pitch between $\pm 20^{\circ}$, with $\alpha_{\max }=20^{\circ}$. To increase $\alpha_{\max }$ to $30^{\circ}$, for example, the airfoil shaft was rotated clock-wise $10^{\circ}$ and the set-screws secured the shaft and model in place, now ready to pitch between $-10^{\circ} \leq \alpha \leq+30^{\circ}$. Wind tunnel conditions were designed to reflect a VAWT operational environment; $\pm \alpha$-excursions for pitch oscillations were based on low TSR in the range $(1 \leq \lambda \leq 3)$, focusing on $\pm 25^{\circ}$ and $\pm 30^{\circ}$ to examine moderate and deep stall regime flow, respectively.

Encoder traces at all reduced pitching frequencies tested for $R e=180 \times 10^{3}$ are included in Figure 56. As reduced frequency is increased, the pitching motor operates with more rotational speed and torque, resulting in smoother and more uniform pitch curves (Figure 56). The increase in $k$ is shown by the increase in pitch-up acceleration $(\mathrm{d} \alpha / \mathrm{d} t=\dot{\alpha})$ for a given condition.

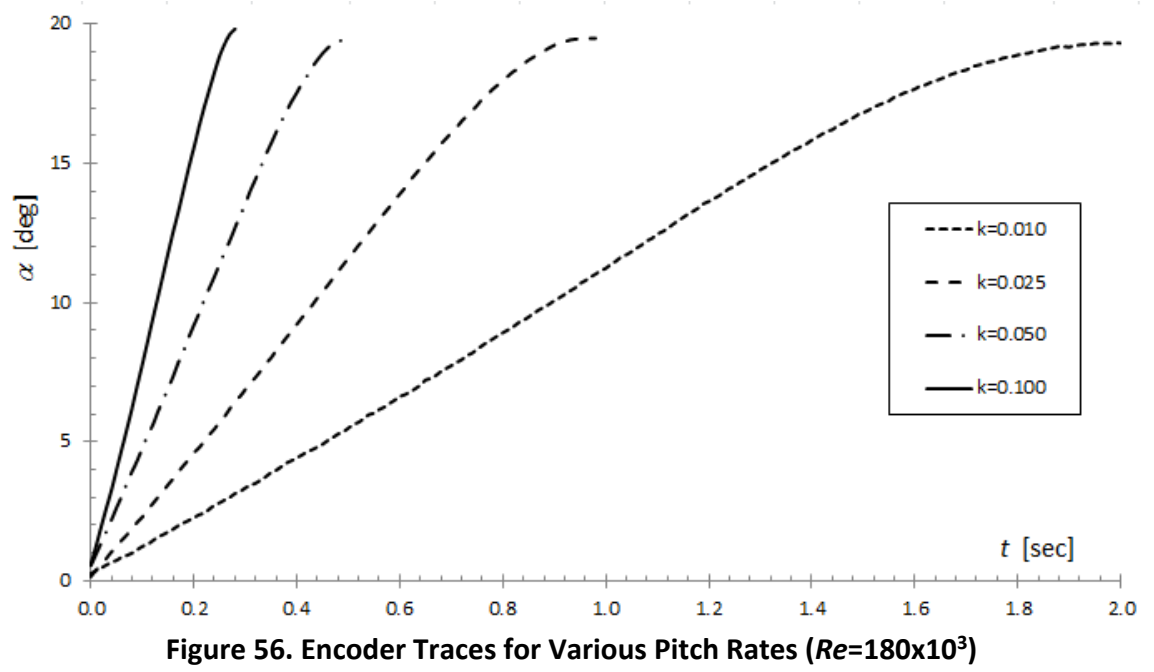

Figure 57 compares measured encoder traces $\left(R e=180 \times 10^{3}, k=0.100, \alpha_{\max }=20^{\circ}\right)$ from wind tunnel testing to the estimated VAWT blade AoA as a function of azimuthal position. The conditions show reasonable agreement during the first half of the pitch cycle (max $\alpha$-difference of $15 \%$ ), but shows a phase distortion during the last half of the cycle. The disagreement is a result of the 4-bar mechanism creating a sinusoidal pitch motion, while VAWT theory results show the path to be a distorted, quasisine waveform. The objective of testing was to simulate operational VAWT conditions, and thus the pitching motions generated during wind tunnel tests were considered acceptable as comparable dynamic stall conditions $\left(k, \alpha_{\max }\right)$ were created. 


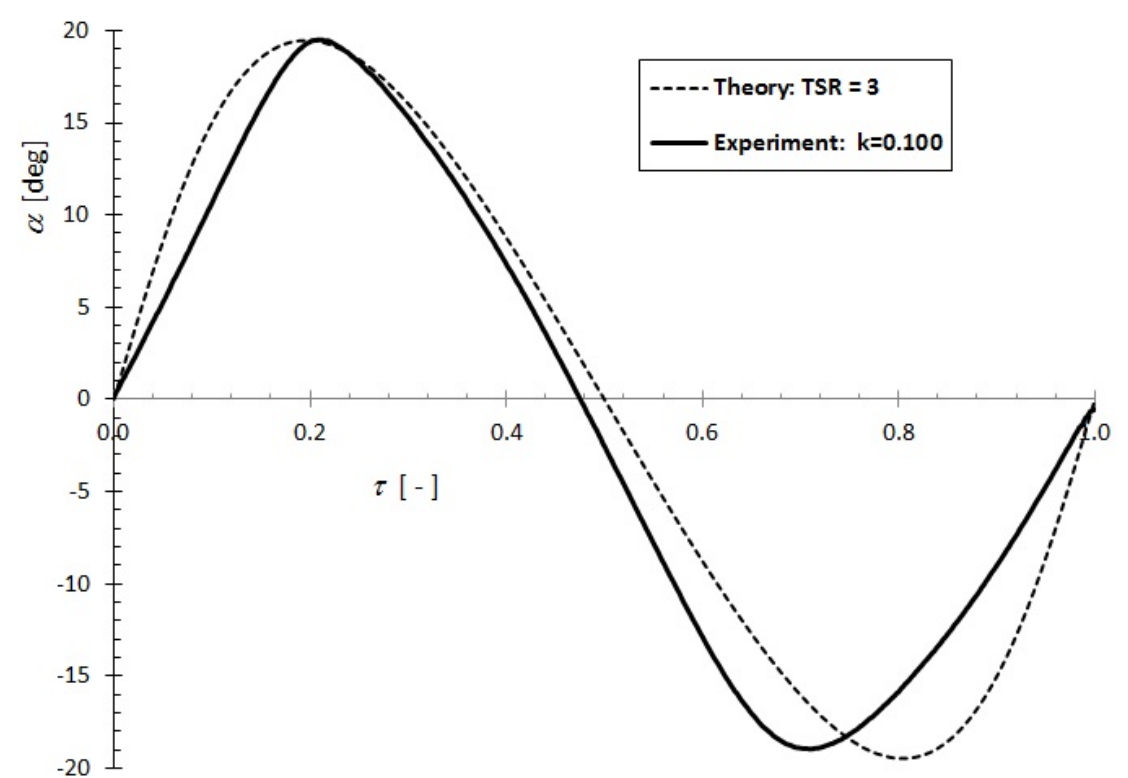

Figure 57. Experimental Conditions $\left(R e=180 \times 10^{3}, k=0.100\right)$ : Encoder Data vs. VAWT Theory

\subsubsection{Circulation Control $\left(C_{\mu}\right)$ : Steady Jet}

Circulation control magnitude was characterized by the momentum coefficient $\left(C_{\mu}\right)$, defined by Equation 39.

Steady Momentum Coefficient

$$
C_{\mu}=\frac{(\dot{m} \cdot V)_{j e t}}{q_{\infty} \cdot A_{j e t}}
$$

Equation 39

Compressed air stored in a large holding tank was used as the CC flow source. A series of regulators, with both line pressure and temperature measurements, were used to adjust the flow to desired conditions. Hotwire calibration of the CC jets was completed during benchtop tests for the frequency range $\left(0 \leq f_{j e t} \leq 125\right) \mathrm{Hz}$, at 0.5 inch intervals along the airfoil span. This calibration was used to determine the steady $C_{\mu}$ during wind tunnel testing, based on plenum pressure and atmospheric conditions. The benchtop test set-up is pictured in Figure 58. Measurement instrumentation includes a HEDLAND flow meter with pressure gage, a KING flow meter with HEISE gage pressure measurement, and a TSI constant temperature hotwire anemometer (CTA). The supply flow temperature and pressure measurements were necessary to determine the air density entering each device, to properly adjust volumetric flow rate readings from standard conditions $(\mathrm{scfm})$ to laboratory environment values. A HEDLAND variable area type pneumatic flow meter (range: $0.5-5 \mathrm{scfm}$ ) was used to monitor air supply for lower levels of $\mathrm{CC}\left(C_{\mu} \leq 0.05\right)$. A second HEDLAND flow meter with a larger range (10-100 scfm) was used to measure air supply for high levels of $\mathrm{CC}\left(C_{\mu} \geq 0.05\right)$. The flow meters were calibrated in a 
controlled laboratory at standard conditions by $\operatorname{HEDLAND}\left(70^{\circ} \mathrm{F}\right.$ at $\left.100 \mathrm{psi}\right)$. The meters had built in pressure gages so the indicated flow rate $\left(Q_{\text {ind }}\right)$, measured in standard cubic feet per minute (scfm), could be converted to an actual flow rate $\left(Q_{a c t}\right)$ based on WVU laboratory conditions (Equation 40$)$. Furthermore, air density must be known for volumetric flow measurements to determine mass flow $(\dot{m}=\rho Q)$.

Actual Volumetric Flow Rate

$$
Q_{a c t}=Q_{\text {indic }}\left[\frac{\rho_{\text {stnd }}}{\rho_{\text {act }}}\right]^{1 / 2}
$$

Equation $\mathbf{4 0}$

The supply temperature was measured with an OMEGA CL3512A handheld temperature calibrator (not pictured) with T-Type thermocouple (accuracy of $\pm 0.5^{\circ} \mathrm{C}$ or $0.4 \%$ of the measurement, whichever value is larger). A closer look at the hotwire measurement locations along the span of the CCNACA0018 model is included in Figure 59.

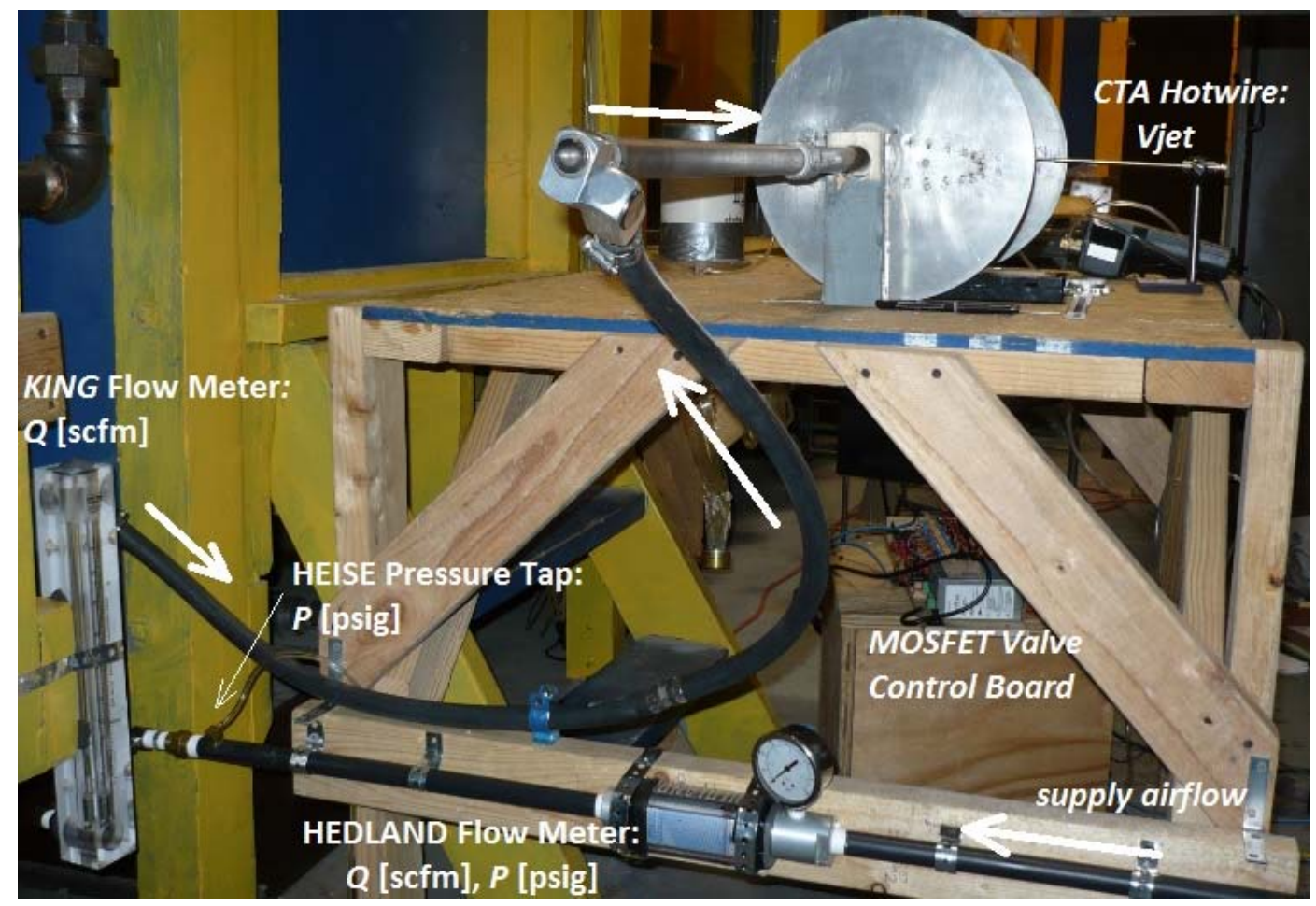

Figure 58. Benchtop Measurements of $\mathrm{CC}$ Jet for $\boldsymbol{C}_{\mu}$ Characterization: All Instrumentation 

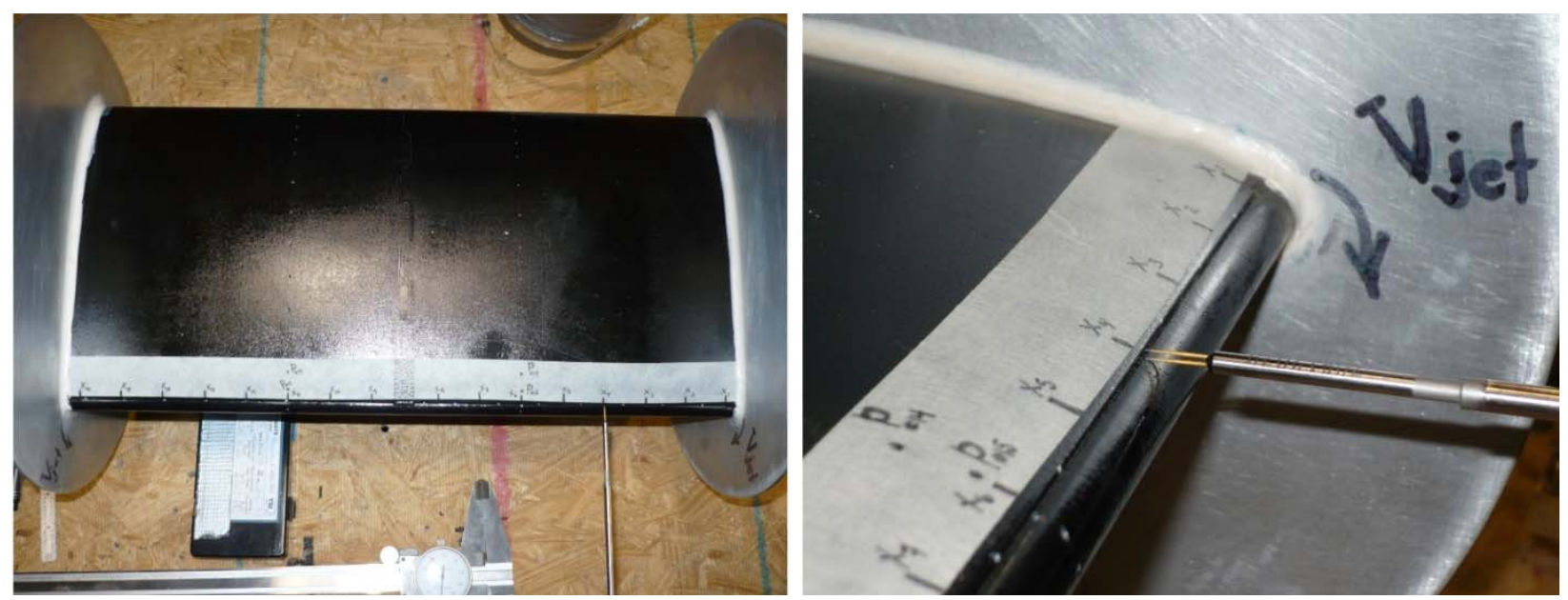

Figure 59. Benchtop $C_{\mu}$ Characterization: CTA Hotwire Measurement of $\boldsymbol{V}_{\text {jet }}$

The performance of the CC jet can be seen in Figure 60, relative to calculated ideal values assuming isentropic expansion at the jet exit from plenum pressure. Measurements show less than $5 \%$ variation along the span of the airfoil, validating the two-dimensionality of the flow control jet. The average discrepancy between measured and ideal conditions was $4 \%$, while the maximum disagreement for an individual measurement was $9 \%$. This validates the $2 \mathrm{D}$ jet assumption, resulting in a maximum measured uncertainty of $\delta C_{\mu}= \pm 5 \%$ along the entire model span.

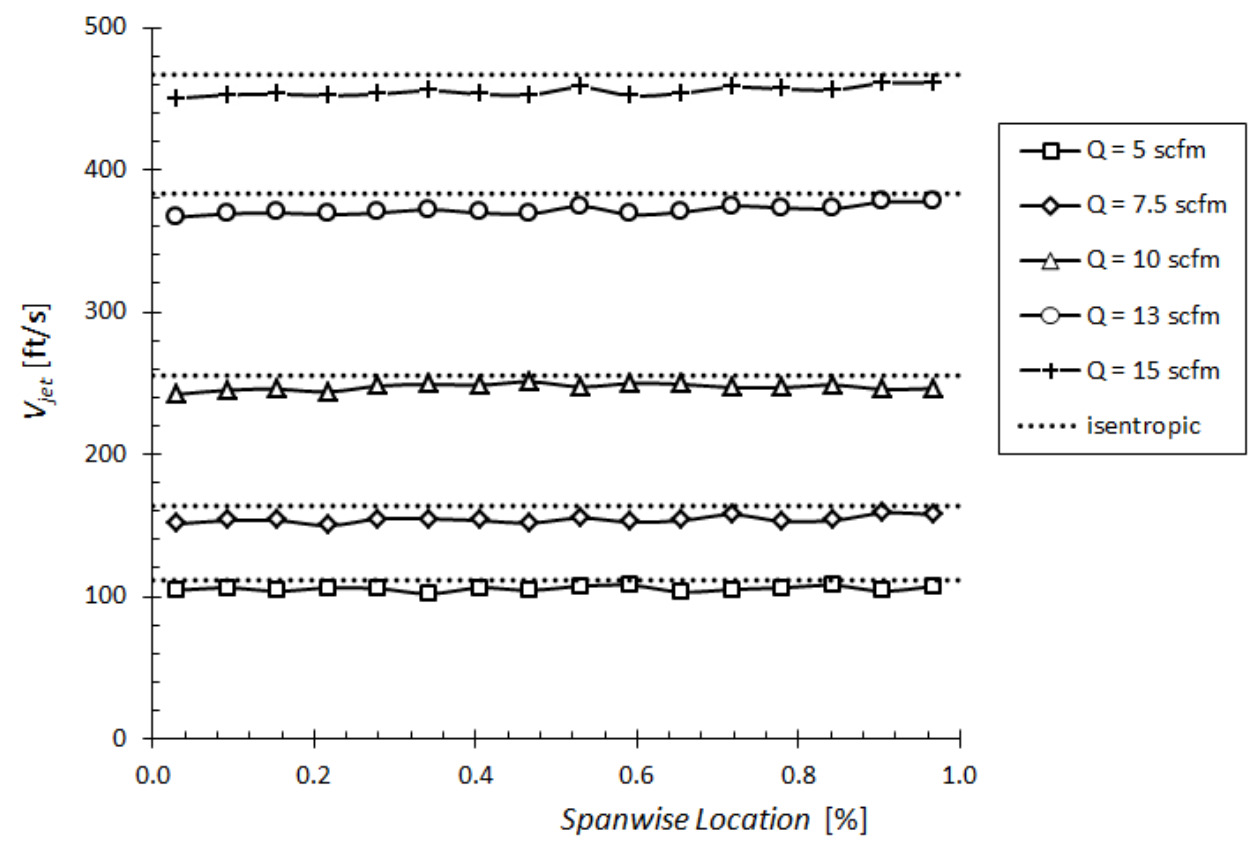

Figure 60. CCNACA0018 Steady Jet Velocity: Spanwise Uniformity 
A linear relationship exists between the airfoil plenum pressure $\left(P_{\text {plenum }}\right)$, the volumetric flow $(Q)$, and the resulting jet velocity $\left(V_{\text {jet }}\right)$, included in Figure 61.

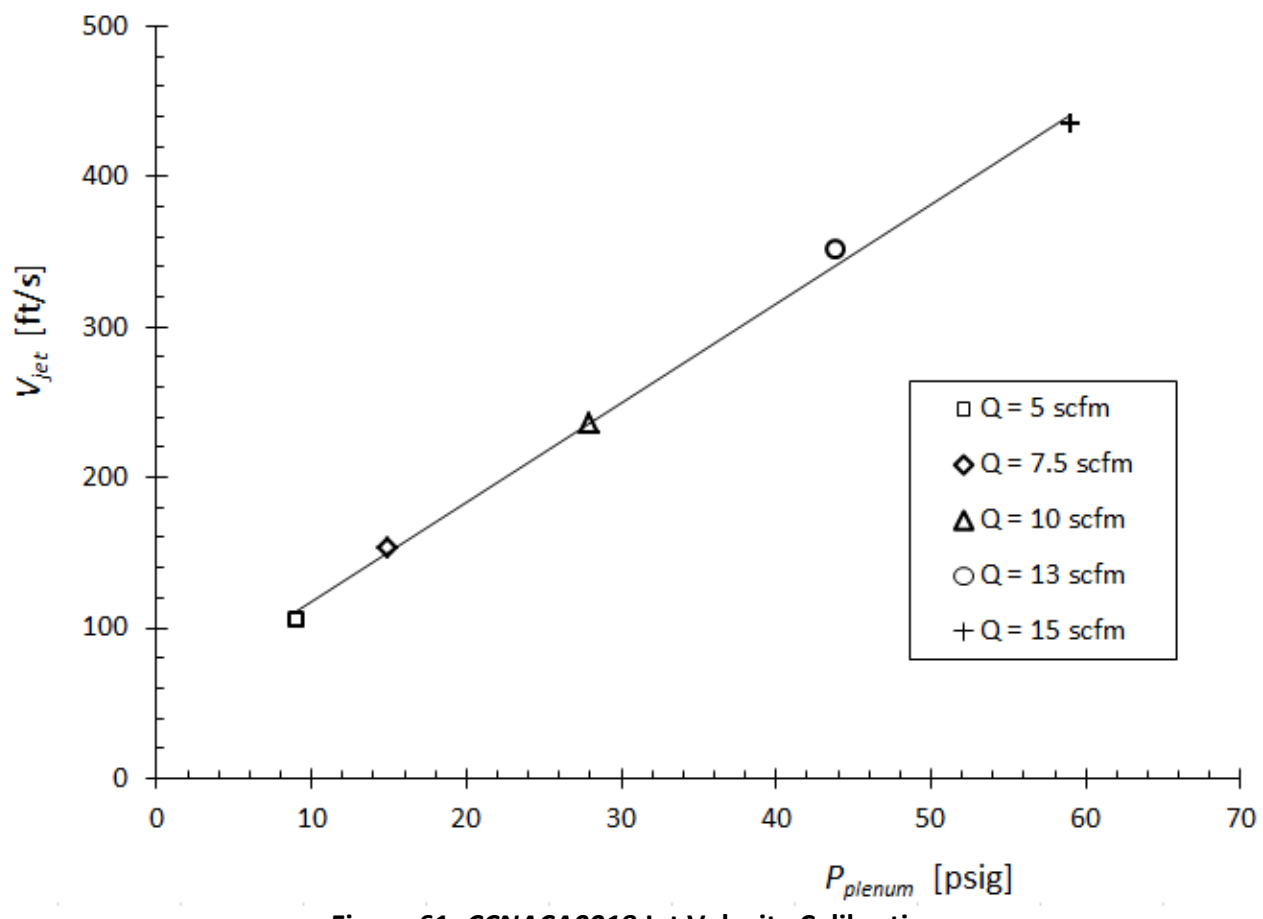

Figure 61. CCNACA0018 Jet Velocity Calibration

\subsubsection{Circulation Control $\left\langle c_{\mu}\right\rangle$ : Pulsed Jet}

The CCNACA0018 wind tunnel model, as previously detailed, was not originally designed for oscillatory control testing, but for the response time of the jet to influence the global flowfield (31). Thus, the pulsed CC capabilities were primarily constrained by the internal DYNAMCO pneumatic valves and the diffuser volume connecting said valves to the TE slot exit plane. The momentum coefficient $\left(C_{\mu}\right)$ is essential to define the efficiency of aerodynamic flow control systems such as the CCNACA0018 airfoil. The pulsed jet momentum coefficient (Equation 41) was defined by calculating <RMS $>$ values of jet mass flow rate and velocity over time.

Pulsed Momentum Coefficient

$$
\left\langle c_{\mu}\right\rangle=\frac{\left\langle\dot{m}_{j}\right\rangle\left\langle V_{j}\right\rangle}{q_{\infty} S}
$$

A series of benchtop calibrations were performed for the desired $C_{\mu}$ conditions of the test matrix. Results are included in Appendix B, including spatial $V_{\text {jet }}$ measurements and additional spectral analysis to confirm the dominant frequency of the unsteady jet under various conditions. The pulsed actuation frequency for testing was defined by the St, using the airfoil chord as the characteristic length as seen in Equation 42. 


$$
S t=\frac{f_{j e t} \cdot c}{V_{\infty}}
$$

A NI LabVIEW DAQ program with a graphical user interface (GUI) was used to input the desired $f_{j e t}$ to control the DYNAMCO valves. When pulsed jet conditions were desired, steady jet conditions were first obtained by adjusting the plenum pressure based on the aforementioned calibration. The desired value of $f_{j e t}$ was then input into the GUI to achieve a pulsed jet before measurements were recorded. Steady $C_{\mu}$ conditions were used as a starting point to directly facilitate mass flow comparisons with a pulsed jet.

Figure 62 compares the mass flow rate expenditure for each blowing condition tested at $R e=180 \times 10^{3}$. As the control input frequency was increased, diminished performance was noticed. Ideally, jet mass flow would decrease as $f_{\text {jet }}$ increased. Valve performance degraded with increasing plenum pressure, limiting test conditions to $S t=0.25,0.50$. Higher actuation frequencies $(0.5 \leq S t \leq 4.0)$ were considered during numerical CCNACA0018 analysis.

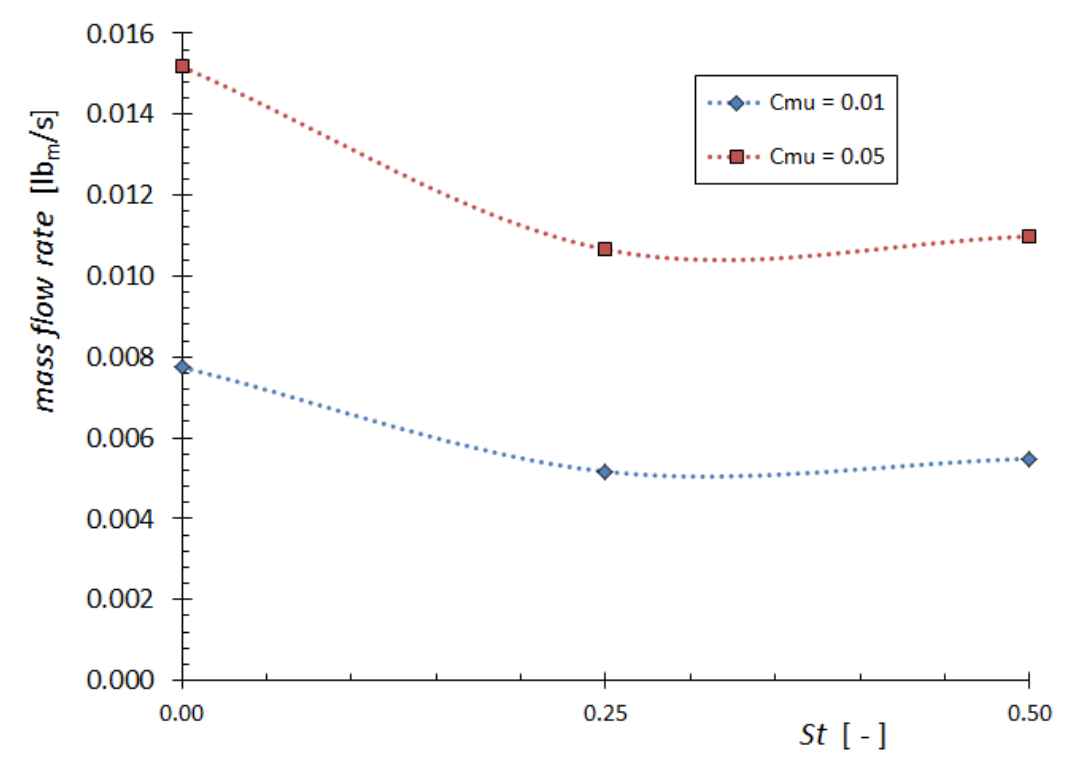

Figure 62. CCNACA0018 Mass Flow Rate based on Jet Frequency

Upstream pressure and input frequency strongly influenced the fidelity of the pneumatic valves. Table 13 includes the measured jet characteristics for all blowing conditions, comparing the RMS values of $\left\langle V_{\text {jet }}>\right.$ and $<\dot{m}>$ between steady $(S t=0)$ and pulsed $(S t \neq 0)$ jet modes, and the resulting mass flow savings each unsteady jet configuration. 
Table 13. CCNACA0018 Mass Flow Requirements ( $R e=180 \mathrm{~K})$

\begin{tabular}{|c|c|c|c|c|c|}
\hline \multirow{2}{*}{$\begin{array}{c}\text { Blowing } \\
\text { Condition }\end{array}$} & $S t$ & $f_{j e t}$ & $\left\langle V_{j e t}\right\rangle$ & $\langle\dot{m}\rangle$ & $\Delta \dot{m}$ \\
\cline { 2 - 6 } & {$[-]$} & {$[\mathrm{Hz}]$} & {$[\mathrm{ft} / \mathrm{s}]$} & {$\left[\mathrm{Ib}_{\mathrm{m}} / \mathrm{s}\right]$} & {$[\%]$} \\
\hline$C_{\mu}=0.01$ & 0.00 & 0 & 58.4 & 0.0078 & - \\
\hline$\left\langle c_{\mu}\right\rangle=0.01$ & 0.25 & 17 & 59.7 & 0.0052 & $-33 \%$ \\
\hline$\left\langle c_{\mu}\right\rangle=0.01$ & 0.50 & 34 & 68.4 & 0.0055 & $-29 \%$ \\
\hline$C_{\mu}=0.05$ & 0.00 & 0 & 96.1 & 0.0152 & - \\
\hline$\left\langle c_{\mu}\right\rangle=0.05$ & 0.25 & 17 & 95.9 & 0.0107 & $-30 \%$ \\
\hline$\left\langle c_{\mu}\right\rangle=0.05$ & 0.50 & 34 & 100.9 & 0.0110 & $-28 \%$ \\
\hline
\end{tabular}

\subsection{Experimental Airfoil Performance}

The parameters to evaluate CCNACA0018 performance, derived from measured values, are the surface pressure coefficient $\left(C_{p}\right)$, sectional lift coefficient $\left(C_{l}\right)$, and the pressure drag coefficient $\left(C_{d, p}\right)$. Surface pressure scans were completed for both static and dynamic wind tunnel testing of the 2D CCNACA0018 model.

\subsubsection{Surface Pressure Coefficient $\left(C_{p}\right)$}

Airfoil surface pressure measurements were made via a ScaniValve ZOC33 miniature pressure scanner with transducers having a differential pressure range of $\pm 2.5 \mathrm{psi}$. The length of all pneumatic tubing was minimized to reduce attenuation and maximize dynamic response. Data acquisition was completed with customized LabView software. The data acquisition program followed the included procedures for each pitching airfoil test case: (1) a pressure port was selected (starting with LE port \#1) (2) a time delay allowed for ScaniValve response (3) a record trigger was established for $\alpha=0^{\circ}$ during the following pitch-up cycle (4) a pressure-time series data was recorded in a continuous record for a userdefined number of pitch cycles (5) the data series were stored (6) the subsequent pressure port was selected and the DAQ steps were completed until all 32 ports were sampled for the desired test condition. Due to unavoidable freestream unsteadiness of the closed loop wind tunnel, each cycle of pressure measurements was found to have minor irregularities, primarily during stall conditions. Thus, to facilitate parametric comparisons, the randomness was removed by ensemble averaging the pressure data over a number of cycles (minimum of 10 pitch cycles), as is a common experimental data reduction technique (96) (132). 
The dimensionless pressure coefficient $\left(C_{p}\right)$ was calculated at each pressure tap location, using the input measurements for port pressure $\left(P_{i}\right)$, ambient air pressure $\left(P_{\infty}\right)$, and tunnel speed $\left(\Delta P_{\infty}\right)$, as listed in Equation 43.

Pressure Coefficient

$$
C_{p, i}=\frac{P_{i}-P_{\infty}}{q_{\infty}}=\frac{P_{i}-P_{\infty}}{\Delta P_{\infty}}
$$

Equation 43

Two pitot-static probes, located at different points near the airfoil model within the test section, were connected at a single junction and ported to a ScaniValve transducer to record the free stream dynamic pressure. Measuring $\Delta P_{\infty}$ at multiple locations in this way allows a natural average of the flow velocity to be monitored, avoiding skewed readings from poor positioning of a single probe.

\subsubsection{Aerodynamic Force Coefficients $\left(C_{l}, C_{d}\right)$}

Airfoil normal and axial force coefficients were calculated from measured surface pressure distributions. Integration of the surface pressure distributions results in vector force coefficients, which can be resolved into lift and drag components based on angle-of-attack. Figure 63 includes a schematic of an airfoil with the aerodynamic load vectors, using $0.25 c$ as a reference point.

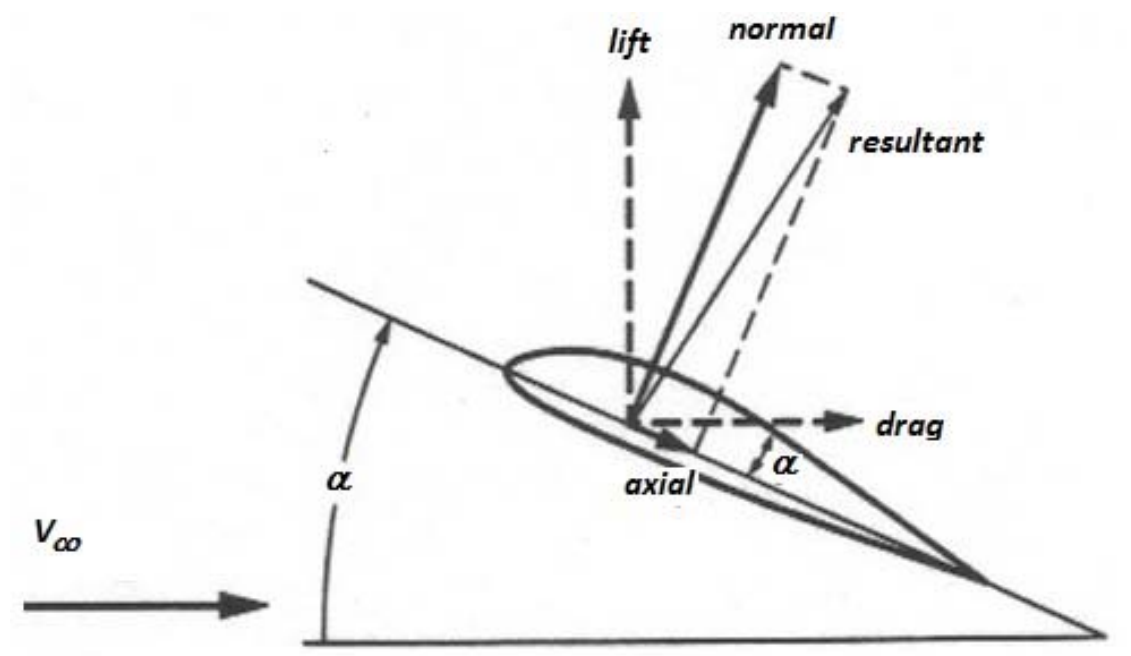

Figure 63. Vector Force Definitions based on AoA Position

The normal force coefficient $\left(C_{n}\right)$ was calculated from surface pressure measurements based on the area enclosed by the suction surface ("upper" surface: $C_{p, \mathrm{u}}$ ) and pressure surface ("lower" surface: $C_{p, L}$ ) $C_{p^{-}}$ distributions along the chord of the airfoil $(x / c)$, as stated in Equation 44.

Normal Force Coefficient

$$
C_{n}=\int_{x / c=0}^{x / c=1}\left(C_{p, L}-C_{p, U}\right) \cdot d(x / C)
$$


The axial force coefficient $\left(C_{a}\right)$ was calculated in a similar manner, but instead integrating with respect to the thickness profile of the airfoil $(y / c)$, as outlined by Equation 45.

Axial Force Coefficient

$$
C_{a}=\int_{y / c=0}^{y / c=1}\left[C_{p, L} \cdot\left(\frac{d y}{d x}\right)_{L}-C_{p, U} \cdot\left(\frac{d y}{d x}\right)_{U}\right] \cdot d(x / c)
$$

Trigonometric relations between the load vectors were manipulated, using $\alpha$ as a reference position, to calculate the section lift coefficient $\left(C_{1}\right)$, as seen in Equation 46.

\section{D Lift Coefficient}

$$
C_{l}=C_{n} \cdot \cos \alpha-C_{a} \cdot \sin \alpha
$$

Equation 46

Similarly, the pressure induced drag coefficient $\left(C_{d, p}\right)$ was found using Equation 47. $C_{d, p}$ is often referred to as the "lift-induced pressure drag".

2D Drag Coefficient

$$
C_{d, p}=C_{n} \cdot \sin \alpha+C_{a} \cdot \cos \alpha
$$

Equation 47

As a measure of the improved lift over an entire pitch-cycle, a $\int C_{l}$ metric (Equation 48) was used to compare the change in average lift enhancement for each flow control condition relative to the baseline airfoil, as defined by (136).

Pitch-Cycle Integrated Lift

$$
\int C_{l}=\oint_{\alpha} C_{l(C C)} \cdot d \alpha / \oint_{\alpha} C_{l(\text { Baseline })} \cdot d \alpha
$$

This parameter indicates the net effect of the control scheme on the lift produced over the entire pitch cycle, factoring both lift enhancement and recovery. For light stall, the integrated lift factor generally does not vary from case to case (104).

The instrumentation equipment specifications for all equipment used during wind tunnel testing are included in Table 14. 
Table 14. Wind Tunnel Testing Equipment Specifications

\begin{tabular}{|c|c|c|c|c|c|}
\hline Instrument & Manufacturer & Model & Measurand & Range & Accuracy \\
\hline ESP (Pressure Scanner) & ScaniValve & $Z O C 33 / 64 P x$ & $\boldsymbol{P}_{i}$ & $\pm 2.5 \mathrm{psid}$ & $\pm 0.15 \% \mathrm{FS}( \pm 0.0075 \mathrm{psi})$ \\
\hline Pressure Gage & HEISE (Module I) & PTE-1 (HQS-2) & $P_{\infty}$ & $(0-15)$ psi & $\pm 0.05 \% \mathrm{FS}( \pm 0.0075 \mathrm{psi})$ \\
\hline Pressure Gage & HEISE (Module II) & PTE-1 (HQS-2) & $\boldsymbol{P}_{\text {rot }}$ & $(0-100) \mathrm{psi}$ & $\pm 0.05 \% \mathrm{FS}( \pm 0.050 \mathrm{psi})$ \\
\hline Pressure Gage & HEISE (Module III) & PTE-1 (HQS-1) & $\Delta P_{\infty}$ & $( \pm 0.50)$ in. $\mathrm{H}_{2} \mathrm{O}$ & $\pm 0.07 \% \mathrm{FS}\left( \pm 0.0007\right.$ in. $\mathrm{H}_{2} \mathrm{O}$ \\
\hline Pressure Manometer & DWYER & Series 475 Mark II & $\Delta P_{\infty}$ & $(0-20)$ in. $\mathrm{H}_{2} \mathrm{O}$ & $\pm 1.0 \% \mathrm{FS}\left( \pm 0.005\right.$ in. $\left.\mathrm{H}_{2} \mathrm{O}\right)$ \\
\hline Flow Meter & KING & 7520 Series & $Q_{j e t}$ & $(0-42) \mathrm{scfm}$ & $\pm 2 \% \mathrm{FS}( \pm 0.84 \mathrm{scfm})$ \\
\hline \multirow{2}{*}{ Flow Meter } & HEDLAND & H271A-010-EG & $Q_{j e t}$ & $(0.5-5) \mathrm{scfm}$ & $\pm 2 \% \mathrm{FS}$ ( $\pm 0.1 \mathrm{scfm})$ \\
\hline & ASHCROFT & $Q-8956$ & $\boldsymbol{P}_{\text {jet }}$ & $(0-30) p s i g$ & $\pm 0.5 p s i g$ \\
\hline \multirow{2}{*}{ Flow Meter } & HEDLAND & $H 671 A-100-E G$ & $Q_{j e t}$ & $(0-100 \mathrm{scfm})$ & $\pm 2 \% \mathrm{FS}( \pm 0.1 \mathrm{scfm})$ \\
\hline & HEDLAND & $N A$ & $\boldsymbol{P}_{\text {jet }}$ & $(0-50) p s i g$ & $\pm 0.5 p s i g$ \\
\hline Hot-Wire Anemometer & TSI & IFA-300 & $v_{\text {jet }}$ & $(0-42) \mathrm{scfm}$ & $\pm 2 \% \mathrm{FS}( \pm 0.84 \mathrm{scfm})$ \\
\hline Thermometer & R.E. White Instrum., INC. & NA & $P_{\infty}$ & $(27.50-31.50)$ in. $\mathrm{Hg}$ & \pm 0.003 psia \\
\hline Hygrometer & ABBEON CAL, INC & HTAB-176 & $T_{\infty}$ & $(0-120)^{\circ} \mathrm{F}$ & $\pm 0.5^{\circ} \mathrm{F}$ \\
\hline Digital Encoder & US DIGITAL & MAE3 Absolute & $\alpha$ & $(0-360)^{\circ}$ & $\pm 0.5^{\circ}$ \\
\hline
\end{tabular}

\subsubsection{Frequency Response of Pressure Tubing}

Pressure signal attenuation due to viscous effects in tubing must be considered when taking dynamic pressure measurements. Specifically, frictional damping and wave reflection are predominant sources of error. Pressure magnitude response is a function of tubing (length $(/)$ and inner diameter $(d)$ ), and signal frequency $\left(f_{\text {sig }}\right)$. Phase lag distortions must also be considered for unsteady pressure measurements. Pressure waves propagate at the speed of sound, from the airfoil surface through connective tubing until reaching the pressure transducer. Therefore, best practice is to minimize tube length to reduce this distortion.

Past experiments of Whitmore, et al. (137) revealed the influence of small variations in tubing geometry (ID) on magnitude response of sinusoidal pressure signals. Small diameter tubing ( $d=0.02 \mathrm{in}$.) attenuated oscillating waves $\left(0.5 \mathrm{~Hz} \leq f_{\text {sig }} \leq 200 \mathrm{~Hz}\right.$ ) while larger tubing ( $d=0.06 \mathrm{in}$.) amplified low frequency signals $\left(20 \mathrm{~Hz} \leq f_{\text {sig }} \leq 70 \mathrm{~Hz}\right)$ and reduced high frequency magnitudes $\left(75 \mathrm{~Hz} \leq f_{\text {sig }} \leq 120 \mathrm{~Hz}\right)$. Such results are typical, and illustrate the importance of experimental characterization of any pneumatic tube system to be used for transmission of unsteady pressure measurements.

A model which corrects for attenuation and phase lag associated with pneumatic tubing was originally developed by Bergh \& Tijdeman (138), and has been reproduced and refined many times (139). The model characterizes the frequency response as a function of tubing and transducer dimensions, at ambient air conditions. The result is a frequency-dependent transfer function that relates an unsteady input pressure on the airfoil model surface $\left(P_{i n}\right)$ to the measured pressure sensed at the transducer $\left(P_{\text {out }}\right)$, in the frequency domain (Equation 49). 
Transfer Function $\quad \frac{P_{\text {out }}(\omega)}{P_{\text {in }}(\omega)}=\frac{1}{\cosh \left[\omega \cdot \Gamma_{p} \cdot \frac{l_{t}}{c}\right]+V \frac{\omega \cdot \Gamma_{p} \cdot l_{t}}{A_{t} \cdot c} \cdot \sinh \left[\omega \cdot \Gamma_{p} \cdot \frac{l_{t}}{c}\right]}=\psi(\omega)$

In Equation 49, $\omega$ is the signal frequency [rad/s], $\Gamma_{p}$ is the wave propagation factor [-], $c$ is the sonic velocity at ambient conditions [ft/s], $A_{t}$ is the cross-sectional area of the tube, and $\Psi(\omega)$ is the complex transfer function which characterizes the system. A theoretical transfer function was calculated based on the exact pressure system geometry used during experimental testing.

A series of experimental measurements with high frequency condenser mics and sinusoidal wave sweeps through each pressure port and connective tube was also completed for validation. Each of the 33 static pressure ports was connected by lengths of $57 \mathrm{in}$. vinyl rubber tubes $(0.063 \mathrm{in} . \mathrm{ID}, 0.125$ in. $O D$ ) to a ScaniValve ZOC33 transducer (internal reference volume of $0.01 \mathrm{in}^{3}$ ). Further details of the calibration process, including calibration curves from each day of testing, can be found in Figure 64. The controlled sinusoidal wave source was generated by a speaker tube, controlled by a function generator capable of varying signal amplitude and frequency.

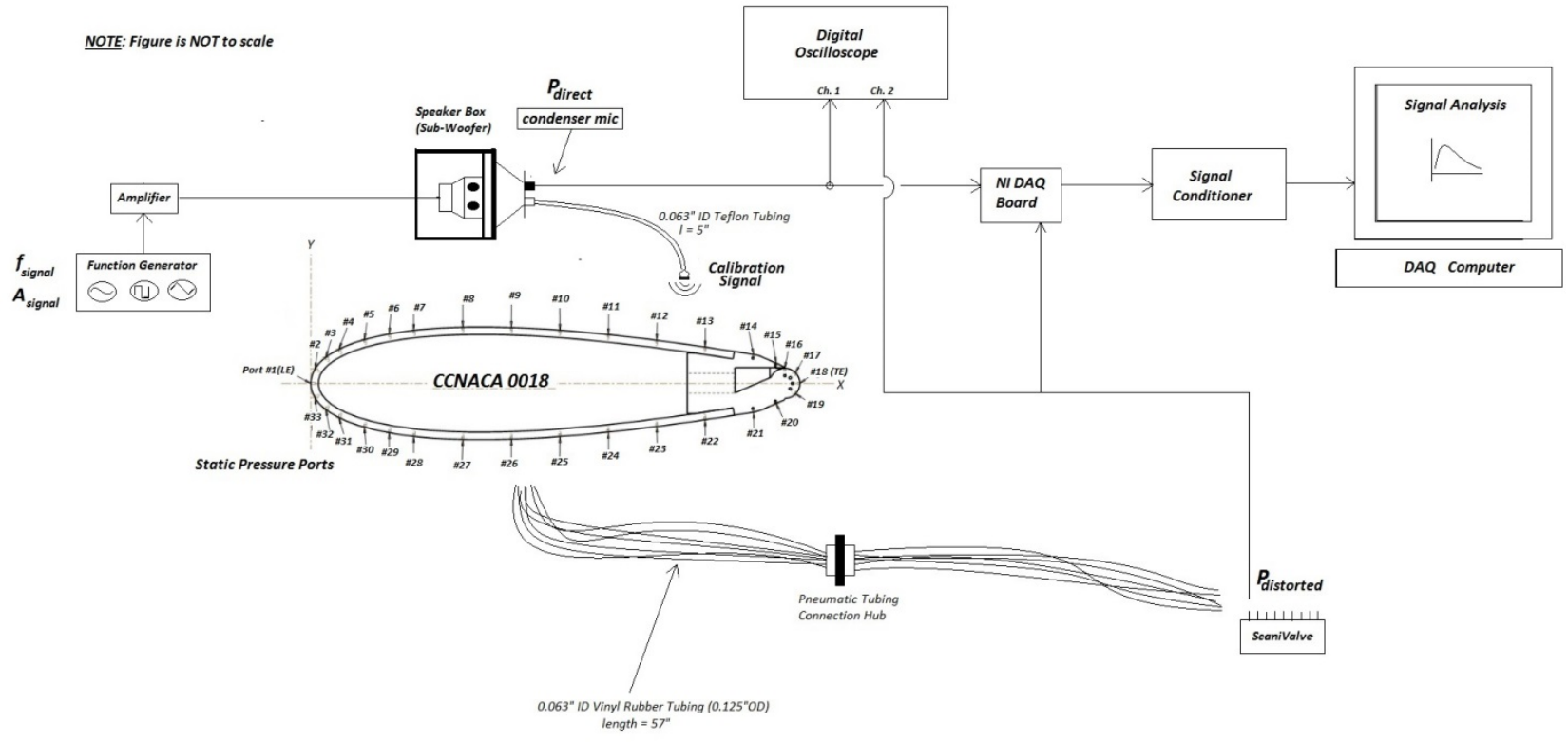

Figure 64. Schematic of Benchtop Experiment: Transient Signal Attenuation Analysis

Frequency response data recorded in the laboratory for each part is compared to results predicted by the analytical model in Figure 65; the experimental data represents an average response from all 33 ports. The two methods show close agreement for the range of $\left(f_{\text {sig }} \leq 50 \mathrm{~Hz}\right)$ and $\left(90 \mathrm{~Hz} \leq f_{\text {sig }} \leq 200 \mathrm{~Hz}\right)$. A maximum difference of $16 \%$ was observed for $f_{\text {sig }} \approx 65 \mathrm{~Hz}$, likely caused by electrical interference present during experimental testing but not simulated in the analytical model. 


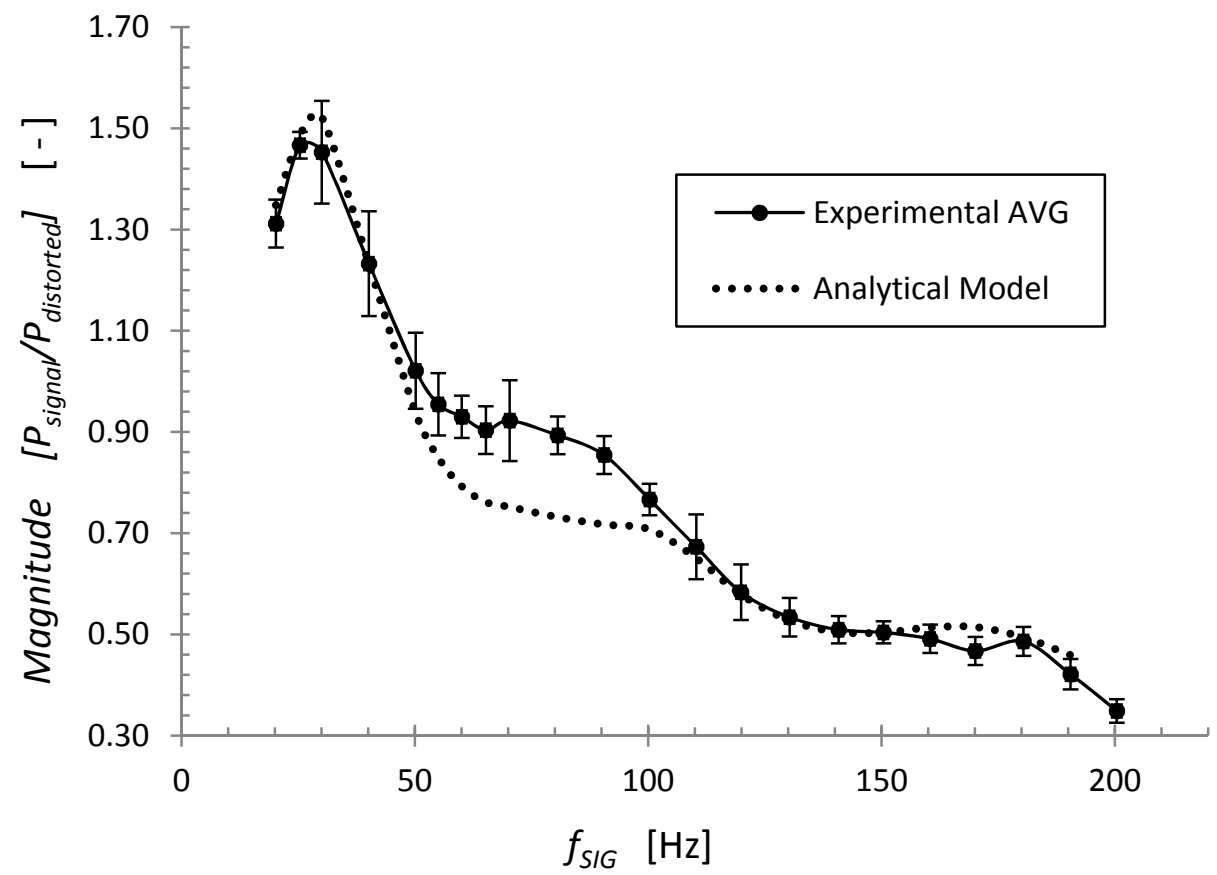

Figure 65. Pneumatic Tubing Response: Experimental vs. Analytical Model

The experimental uncertainty, whose range is represented by variation bars in Figure 65, was determined based on correlation and variance analysis at each input frequency signal, for all 33 ports. The largest range of uncertainty occurs for $f_{s i g}<40 \mathrm{~Hz}$, where the total harmonic distortion (THD) of the input signal was large (THD $\approx 20 \%$ ) near the lower limit of speaker design operation. A spike in the uncertainty is also noticed in the $\left(60 \mathrm{~Hz} \leq f_{\text {sig }} \leq 70 \mathrm{~Hz}\right)$, most likely a cause of the aforementioned electrical noise (laboratory lighting, exhaust fans, additional computers and equipment in operation at various times during testing).

The analytical method was validated by modeling a range of experimental test conditions reported by Rofail, et al. (140) and Mousley (141); a comparison of measured and computed results are included in Figure 66. The computed results show excellent agreement to experimental data, with a maximum disagreement $\leq 10 \%$ (within the experimental error limits of the measurement equipment). 


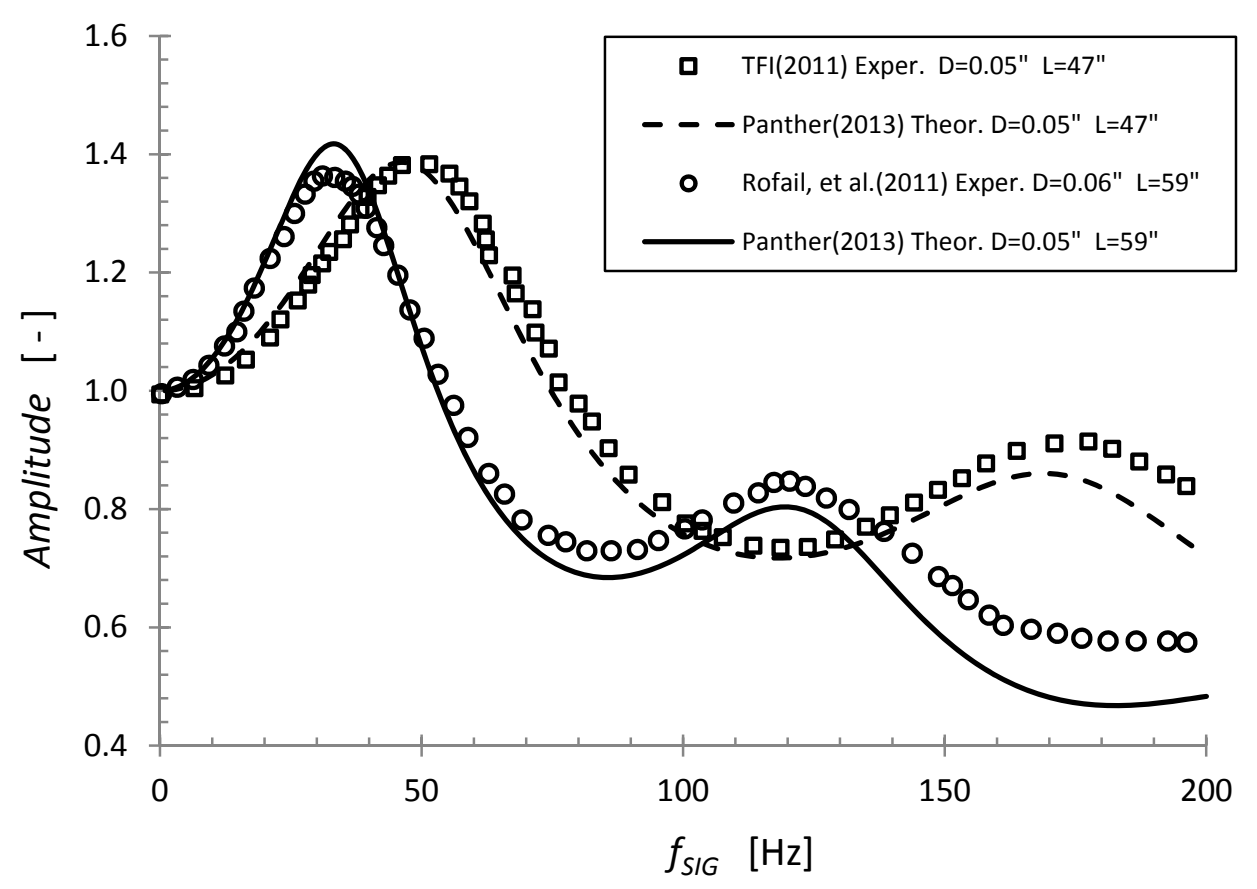

Figure 66. Analytical Model Validation with Experimental Data (140) (141)

During testing, continuous pressure traces were recorded for each port during the duration of a full pitch cycle. To correct this data for distortion effects from pneumatic tubing, a Fourier transform was used to translate the measured data from a time based domain to a frequency-based domain. The appropriate transfer function (TF) was then applied to the frequency-domain data. Finally, inverse FFT was used to convert the data back into the time domain to reduce transducer outputs to actual pressures at the surface of the model. Essentially, all experimental surface pressures were transformed to represent measurements at the surface of the airfoil, from recorded values at the sensor. The corrected pressure data was then used for data reduction to calculate $C_{p}, C_{1}$, and $C_{d}$. An example of signal reconstruction, using the measured TF from port \#1, and an input signal of $140 \mathrm{~Hz}$ (sine wave), is included in Figure 67. The input signal, measured directly with a condenser microphone, is represented by a solid black line, while the distorted signal measured at the location of the ScaniValve sensor is displayed by the red line; the difference between RMS values is $18 \%$. The distorted signal was then corrected by the aforementioned TF process, and is included in Figure 67 as a dashed green line; the input and corrected signals show a disagreement of $1 \%$. 


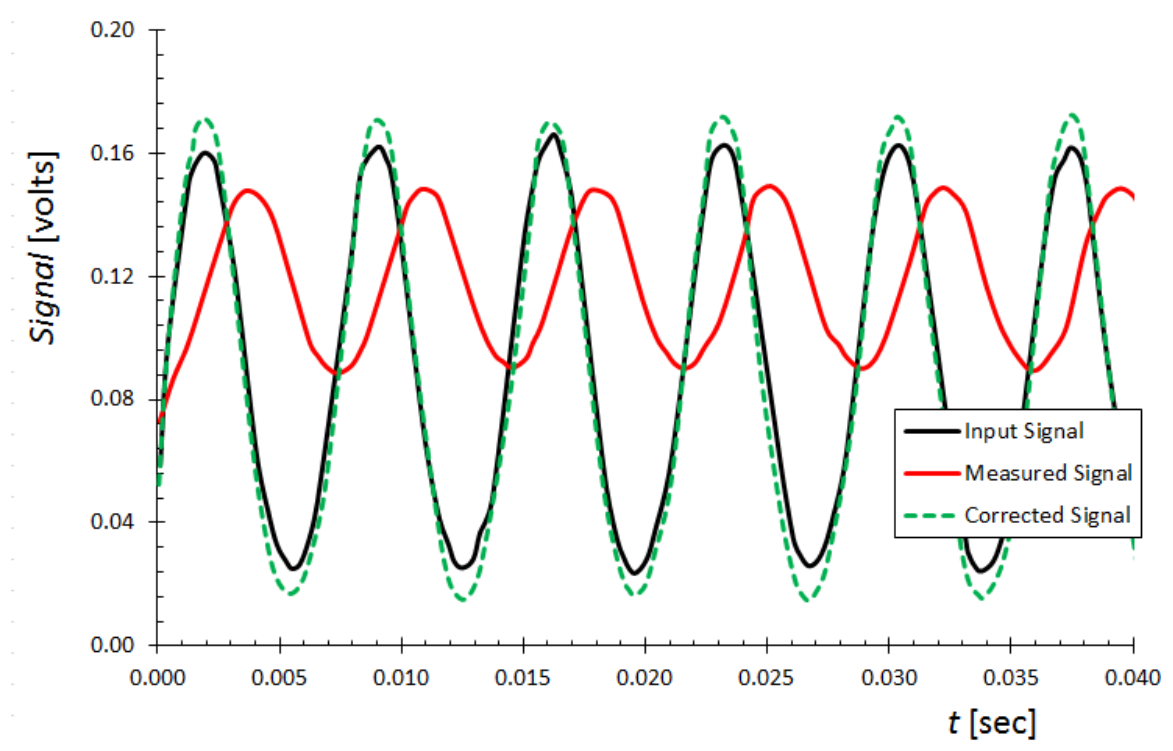

Figure 67. Attenuated Pressure Signal Reconstruction: Port \#1 $\left(f_{\text {signal }}=140 \mathrm{~Hz}\right)$

\subsection{Uncertainty Analysis}

This section details the experimental uncertainty methodology considered while reducing wind tunnel data. Every measurement includes some level of error, and this error can never be known exactly. However, a probable bound on the error can usually be estimated, known as uncertainty. The American Institute of Aeronautics and Astronautics (AIAA) in conjunction with Working Group 15 of the Advisory Group for Aerospace Research and Development (AGARD) Fluid Dynamics Panel developed a standard for assessment of wind tunnel data uncertainty (142). Thus, the AIAA Standard (142) for experimental uncertainty assessment methodology was selected and followed as a guideline to assess the experimental data presented in this research. In the methodology discussed below, the $95 \%$ confidence large-sample uncertainty assessment approach is used.

When estimating uncertainty, two types of error are of general interest: precision and bias error. Total error $\left(\delta_{X}\right)$ of a measured quantity $(X)$ combines individual uncertainty contributions from the aforementioned sources, bias error $\left(\beta_{x}\right)$ and precision error $\left(P_{X}\right)$, as an RMS quantity as listed in Equation 50.

Total Experimental Error

$$
\delta_{X}=\sum\left(\beta_{X}^{2}+P_{X}^{2}\right)^{1 / 2}
$$

Equation 50

Bias errors are constant and dependent upon instrumentation (e.g. scale resolution, manufacturer specifications). Precision errors are commonly due to limitations on repeatability for the measurement system and environmental effects unique to a given test facility (143). $P_{x}$ is estimated using statistical 
analysis (i.e. $P_{x}$ is assumed proportional to the sample standard deviation of $N$ measurements of value $X)$. The precision limit for single tests can be estimated by Equation 51, where $t$ is the coverage factor and $\sigma_{X}$ is the standard deviation of the sample of $N$ readings (of result $X$ ). For $N \geq 10$, a $95 \%$ confidence interval is achieved with $t=2$.

Single Test

Precision Error

$$
P_{X}=t \sigma_{X}
$$

Equation 51

The pressure distribution was collected per single test and used to calculate $C_{l}$ and $C_{d}$ through integration. $C_{l}$ and $C_{d}$ values, at each AoA, were then averaged from a minimum of $M=10$ repeat tests. The precision limit on a multiple test basis is given by Equation 52 .

Multiple Test

$$
P_{X}=\frac{t \sigma_{X}}{\sqrt{M}}
$$

Equation 52

Bias limits are based on instrumentation specifications and sensitivity coefficients based on the data reduction equations. The bias limits of the results for single and multiple tests are determined in the same manner (Equation 53).

Bias Error

$$
\beta_{X}^{2}=\sum \theta_{i}^{2} \omega_{i}^{2}
$$

Equation 53

The instrumentation bias $\left(\omega_{i}\right)$ is preferably manufacturer specified, but can alternatively be taken as half the smallest measurement increment for a rough estimate.

\subsubsection{Pressure Coefficients $\left(C_{p}\right)$}

Differential pressure measurements could have been obtained by referencing the upper surface to the lower surface taps at the same chord location, to derive the airfoil normal force and pitching moment. However, single measurements at each tap location were preferred because (1) they provided a more definitive observation of the formation and convection of the stall vortex and (2) they enabled the calculation of the chord force (due to pressure only) and enabled the construction of lift and drag forces, as pointed out by McCalister, et al. (144).

The static accuracy of the ScaniValve ESP module is $\omega_{s v}= \pm 0.15 \% \mathrm{FS}$, including hysteresis, nonlinearity, and non-repeatability effects. The HEISE differential pressure transducer was used to measure the dynamic pressure of the tunnel, the difference between the freestream total pressure $\left(P_{o}\right)$ and static pressure $\left(P_{\infty}\right)$; the accuracy of HEISE modules is listed as $\omega_{\text {HEISE }} \pm 0.05 \% \mathrm{FS}$. From Equation 43 , $C_{p}$ is dependent on $P_{\#}$ and $P_{\text {ref }}$ measured by the ScaniValve ESP, along with $\Delta P_{\infty}$ measured by the HEISE digital manometer (see Figure 68). 


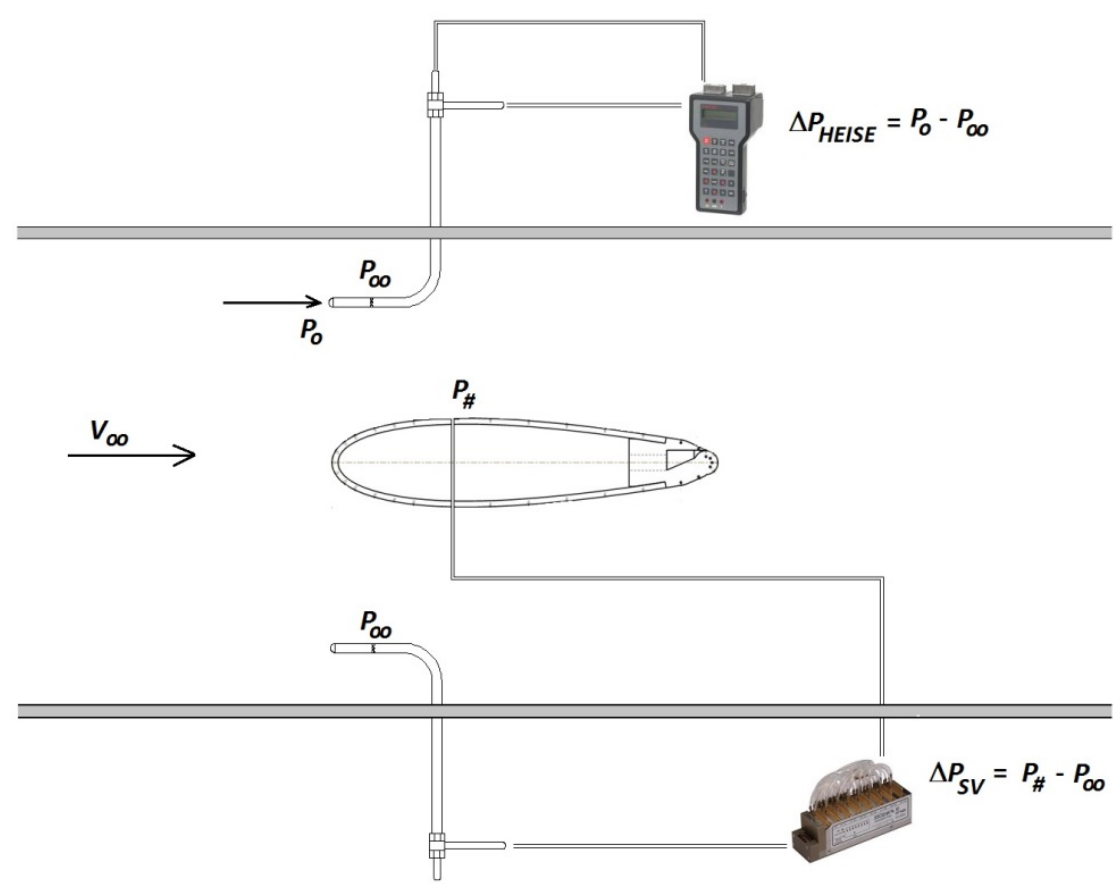

Figure 68. Experimental Measurement of CCNACA0018 Surface Pressure Distribution

Thus, the uncertainty of surface pressure coefficients is governed by differential pressure measurements from the ScaniValve transducers $\left(\Delta P_{S V}\right)$ and HEISE digital manometer $\left(\Delta P_{\text {HEISE }}\right)$; the data reduction equation is included in Equation 54.

Pressure Coefficient

$$
C_{p}=\frac{\Delta P_{S V}}{\Delta P_{H E I S E}}
$$

Kline \& McClintock (145) reported a method for error propagation in calculated quantities. The procedures determine the uncertainty of a calculation based on measurements and the tolerances on those measurements. The methodology for $N$ measured quantities $x_{1}, x_{2}, \ldots, x_{N}$, with errors $\delta x_{1}, \delta x_{2}, \ldots, \delta x_{N}$, then the uncertainty associated with a calculated function $y=f\left(x_{1}, x_{2}, \ldots, x_{N}\right)$ is given by Equation 55 .

Kline-McClintock Uncertainty Method

$$
\delta y=\left\{\left[\left(\frac{\partial y}{\partial x_{1}}\right) \delta x_{1}\right]^{2}+\left[\left(\frac{\partial y}{\partial x_{2}}\right) \delta x_{2}\right]^{2}+\ldots+\left[\left(\frac{\partial y}{\partial x_{N}}\right) \delta x_{N}\right]^{2}\right\}^{1 / 2}
$$

Equation 55

Applying the Kline-McClintock method the surface pressure coefficient calculation (Equation 56), based on measurements of airfoil surface differential pressure and freestream dynamic pressure, results in

Kline-McClintock Method: $C_{p}$ Calculation

$$
\delta C_{p}=\left\{\left[\left(\frac{\partial C_{p}}{\partial\left(\Delta P_{S V}\right)}\right) \delta\left(\Delta P_{S V}\right)\right]^{2}+\left[\left(\frac{\partial C_{p}}{\partial\left(q_{\infty}\right)}\right) \delta\left(q_{\infty}\right)\right]^{2}\right\}^{1 / 2}
$$

Equation 56 
The sensitivity coefficients, partial derivatives of the original data reduction equation, are calculated for each measured quantity, included in Equation 57 and Equation 58.

Sensitivity Coefficient:

Airfoil $\Delta P$

$$
\frac{\partial C_{p}}{\partial\left(\Delta P_{S V}\right)}=\frac{1}{q_{\infty}}
$$

Equation 57

Sensitivity Coefficient:

$$
\frac{\partial C_{p}}{\partial\left(q_{\infty}\right)}=\frac{-\Delta P_{S V}}{q_{\infty}^{2}}
$$

Equation 58

Plugging the sensitivity coefficients back into Equation 56 results in Equation 59.

Kline-McClintock

Method: $C_{p}$ Calculation

$$
\delta C_{p}=\left[\left(\frac{\delta\left(\Delta P_{S V}\right)}{q_{\infty}}\right)^{2}+\left(-\frac{\delta\left(q_{\infty}\right) \Delta P_{S V}}{\left(q_{\infty}\right)^{2}}\right)^{2}\right]^{1 / 2}
$$

Equation 59

Since $\Delta P_{S V}$ and $q_{\infty}$ are measured by the same piece of equipment, they will have the same level of uncertainty: $\delta\left(\Delta P_{S V}\right)=\delta\left(q_{\infty}\right)=\delta P$. Substituting these error quantities into Equation 59 and simplifying leaves Equation 60:

Kline-McClintock

Method: $C_{p}$ Calculation

$$
\delta C_{p}=\left[\frac{\delta P^{2}\left[q_{\infty}^{2}+\left(\Delta P_{S V}\right)^{2}\right]}{q_{\infty}^{4}}\right]^{1 / 2}
$$

Equation 60

For a given experimental $R e$, the maximum value of surface pressure uncertainty $\left(\delta C_{p}\right)$ would result when $\Delta P_{S V}$ reached a maximum. For the experimental test conditions reported, the relationship of $\Delta P_{S V}$ and $q_{\infty}$ for the CCNACA0018 was such that $C_{p, \max } \leq-5$ (i.e. $\Delta P_{S V}=-5 q_{\infty}$ represents a worst-case scenario for this evaluation). A final substitution and simplification step gives the uncertainty in the calculated airfoil pressure coefficient as a function of possible error in measured airfoil differential pressure and freestream dynamic pressure from wind tunnel tests (Equation 61).

Kline-McClintock Method: $C_{p}$ Calculation

$$
\delta C_{p}=\frac{\sqrt{26} \cdot \delta P}{q_{\infty}}
$$

Equation 61

To determine $\delta P$, three contributing error sources must be considered (146): (1) the specified accuracy of the ScaniValve ZOC-33 transducers, $\delta P_{1}(2)$ the bit error associated with DAQ A/D conversion of pressure signals, $\delta P_{2}(3)$ calibration uncertainty from the HEISE PTE-1 digital pressure calibrator.

The manufacturer specified static accuracy of the ScaniValve transducers is $\pm 0.1 \% \mathrm{FS}$, which corresponds to $\delta P_{1}= \pm 0.0025$ psi for a full scale range of 2.5 psid. The ScaniValve unit signal output was $\pm 2.5 V_{D C}$ (5 volt absolute), giving a signal resolution of $1 \mathrm{psi} /$ volt. The data acquisition board used to acquire pressure data had 16 bit resolution and was manufactured by National Instruments (NI PCleEX 6361 DAQ Card). A $\pm 5 V_{D C}$ input range (10 volt absolute) resulted in $(10 \mathrm{volt}) /\left(2^{16} \mathrm{bit}\right)=153 \mu \mathrm{V} / \mathrm{bit}$ 
resolution. Multiplying the ScaniValve and DAQ card resolution values results in $\delta P_{2}= \pm 0.000153$ psi. Finally, a HEISE PTE-1 digital pressure calibrator was used as a standard for all calibration, having a specified accuracy of $\pm 0.05 \%$ FS for the HQS-2 module with range $0-15$ psia $\left(\delta P_{3}= \pm 0.0075 p s i\right)$. It is clear that the HEISE pressure calibrator was the largest contributor of measured pressure uncertainty. Adding the 3 sources of pressure error in quadrature results in $\delta P= \pm 0.0000563 p s i$. Plugging the total measured pressure uncertainty $\delta P$ in Equation 61 results in $\delta C_{p}= \pm 0.10$ for $R e=300 \times 10^{3}$ and $\delta C_{p}= \pm 0.20$ for $R e=180 \times 10^{3}$. These values agree well with experimental uncertainty ranges of $\left( \pm 0.02 \leq \delta C_{p} \leq \pm 0.1\right)$, previously determined by Zeiger (146) following the Kline-McClintock methodology surface pressure measurements on a wind tunnel model with a comparable pressure scanner manufactured by Pressure Systems Inc. (PSI ESP pressure scanner, static accuracy=0.10\% FS). The $C_{p}$ error reported here falls on the high side of uncertainty detailed by Zeiger (146) due to differences in calibration equipment; Zeiger employed a Barocel precision pressure transducer for standard calibration, having a smaller range (0$1.934 \mathrm{psi}$ ) with improved accuracy of $\pm 0.0001 \mathrm{psi}( \pm 0.05 \%$ of the reading plus $\pm 0.001 \% \mathrm{FS})$.

The experimental uncertainty of the current study is compared to Zeiger (146) and McCroskey, et al. (88) from extensive dynamic tests of various airfoil profiles at NASA Ames Research Center and the U.S. Army Aeromechanics Laboratory (AVRADCOM); the results are summarized in Table 15.

Table 15. Experimental Uncertainty of $C_{p}$ Measurements Compared to Literature

\begin{tabular}{|c|c|c|c|}
\hline Author & Instrumentation & Uncertainty & $\%$ Maximum \\
\hline Zeiger (146) & PSI ESP Transducers & $\pm 0.02 \leq \delta C_{p} \leq \pm 0.1$ & $\pm 5 \% C_{p, \max }$ \\
\hline McCroskey, et al. (88) & Kulite Differ. Transducers & $\pm 0.07 \leq \delta C_{p} \leq \pm 0.4$ & $\pm 4.5 \% C_{p, \max }$ \\
\hline Panther & ScaniValve ESP Transducers & $\pm 0.02 \leq \delta C_{p} \leq \pm 0.2$ & $\pm 4 \% C_{p, \max }$ \\
\hline
\end{tabular}

The data of McCroskey, et al. (88) was collected from a series of (26) Kulite differential pressure transducers, surface mounted to each airfoil model. The benefit of surface mounted transducers is direct measurement of pressure signals, without distortion effects of pneumatic tubing. The calculated uncertainty of surface pressure distributions agrees well with trusted data reported in the literature. To further increase the confidence of pressure measurements during wind tunnel testing, a minimum of 5 runs was completed for a given condition. A detailed description matrix of completed test conditions is included in APPENDIX E: Static Wind Tunnel Test Matrices and APPENDIX F: Dynamic Pitch Wind Tunnel Test Matrices. Overall, this chapter provided a summary of the experimental testing apparati utilized for this study. The wind tunnel model was detailed, followed by descriptions of test conditions and measurement equipment. The calculation of aerodynamic force coefficients from measured surface pressure data was outlined, followed by a sample error calculation for surface pressure coefficient. 


\section{CHAPTER 4. NUMERICAL ANALYSIS}

This chapter outlines the numerical methodology for both static and pitching airfoil analyses of the current study. The computational simulations were completed for both experimental data validation and flow visualization purposes. Transient simulations of both stationary and pitching airfoils were created to predict the influence of steady and pulsed jet $C C$ on $C_{p}, C_{l}$, and $C_{d}$. A brief summary of governing theory will be presented, followed by model details and solution procedures.

\subsection{Governing Equations}

Numerical Modeling using RANS and URANS methods is fundamentally centered on the NavierStokes (N-S) equations. The governing equations, for a 2D Cartesian coordinate system, are included in Equation 62 and Equation 63.

$\begin{array}{lll}\begin{array}{ll}\text { Navier-Stokes: } \\ \text {-coordinate }\end{array} & \rho \frac{\partial u}{\partial t}+\rho \cdot u \frac{\partial u}{\partial x}+\rho \cdot v \frac{\partial u}{\partial y}=-\frac{\partial \hat{p}}{\partial x}+\mu\left[\frac{\partial^{2} u}{\partial x^{2}}+\frac{\partial^{2} u}{\partial y^{2}}\right] & \text { Equation } 62 \\ \begin{array}{ll}\text { Navier-Stokes: } \\ \text { y-coordinate }\end{array} & \rho \frac{\partial v}{\partial t}+\rho \cdot u \frac{\partial v}{\partial x}+\rho \cdot v \frac{\partial v}{\partial y}=-\frac{\partial \hat{p}}{\partial y}+\mu\left[\frac{\partial^{2} v}{\partial x^{2}}+\frac{\partial^{2} v}{\partial y^{2}}\right] & \text { Equation } 63\end{array}$

The terms $u$ and $v$ are velocity vector terms in the $x$ - and $y$-coordinate planes, respectively. The first term of the $N-S$ equations, $\rho \frac{\partial(\text { velocity })}{\partial t}$, represents local flow acceleration in each respective coordinate vector. The remaining terms on the left-hand-side of the equations, $\rho \cdot($ velocity $) \frac{\partial(\text { velocity })}{\partial(\text { coordinate })}$, describes the convection properties of the flow. The first term on the right-hand-side of the $\mathrm{N}-\mathrm{S}$ equations, $-\frac{\partial \hat{p}}{\partial(\text { coordinate })}$, is known as the piezometric pressure gradient, while the bracketed terms define the viscous terms.

Also included in the governing equations are the equation of state for an ideal gas (Equation 64), the continuity equation (Equation 65), and the Rayleigh Equation (Equation 66).

Equation of State

Continuity Equation

$$
P=\rho \cdot R \cdot T
$$

$$
\frac{\partial \rho}{\partial t}+\frac{\partial(\rho \cdot u)}{\partial x}+\frac{\partial(\rho \cdot v)}{\partial y}+\frac{\partial(\rho \cdot w)}{\partial z}=0
$$

Equation 65 


$$
R \frac{D^{2} R}{D t^{2}}+\frac{3}{2}\left(\frac{D R}{D t}\right)^{2}=\frac{p_{v}-p}{\rho_{L}}
$$

The current research involves complex, time-dependent flow structures, incompressible jets, and a dynamically pitched airfoil, resulting in the necessity to employ a viscid Navier-Stokes simulation to more accurately predict losses, secondary flows and separations. Transient simulations, versus steady state models, are necessary when some kind of time-dependent flow behavior has a strong influence on the global flowfield, as is the case in this research. Periodic vortex shedding is expected to be one of the dominant flow structures during present simulations.

In this CFD effort, ANSYS FLUENT 15.0 was used for all problem setups, computations, and postprocessing of results. FLUENT's density-based implicit solver was used to predict the time-dependent flow of a pitching airfoil equipped with both steady and pulsed circulation control jets. Unsteady boundary conditions (BC) will be defined via user-defined functions (UDF). Dynamic mesh adaption methods will be employed for both steady-state and transient flows. Transient solutions were calculated using the second-order implicit unsteady solution animation feature; animations of unsteady flows will be created using FLUENT's unsteady solution animation feature (147).

Due to the high computational cost of the full Reynolds-stress model, owed to the additional five equations for turbulence formulation, the one-equation Spalart-Allmaras turbulence model, with curvature correction (SARC), along with the zero-equation laminar model were chosen for this investigation. Past research has proven such models reliable for circulation control airfoil flows as well as pitching airfoil simulations (148) (149) (150).

\subsection{Mesh Generation}

All simulations reported were computed on a 2D airfoil to reflect experimental conditions, although some influence of 3D effects are inherent to experimental testing. Furthermore, 2D models were used to minimize the severe computational expenses of 3D numerical analysis. Four meshes of varying size and density were generated to establish grid independent solutions. ANSYS ICEM software was used to create each grid. A hybrid (structured-unstructured) O-grid topology was used to balance numerical stability with computational cost. The details of each grid are included in Table 16. Grid properties include the total number of mesh nodes $\left(N_{\text {total }}\right)$, the node count along the airfoil surface $\left(N_{\text {airfoil }}\right)$, with specific node concentrations at the airfoil leading edge $\left(N_{L E}\right)$, mid-section of the airfoil $\left(N_{\text {body }}\right)$, rounded trailing edge $\left(N_{T E}\right)$, and blowing jets $\left(N_{\text {slot }}\right)$. Also included are specifications of minimum grid spacing along the airfoil surface $\left((\Delta x / c)_{\min }\right)$ and normal to the airfoil surface $\left((\Delta y / c)_{\min }\right)$. Grid spacing 
along the airfoil surface were concentrated at the LE and TE sections of the airfoil, with relaxed spacing around the mid-chord. Hyperbolic grid stretching was used to achieve strategic spacing and heavy node concentrations to improve numerical stability in areas with complex flow structures.

Table 16. Grid Refinement Study: Individual Mesh Properties

\begin{tabular}{|l|c|c|c|c|c|c|c|c|}
\hline Grid & $N_{\text {total }}$ & $N_{\text {airfoil }}$ & $N_{L E}$ & $N_{\text {body }}$ & $N_{\text {TE }}$ & $N_{\text {slot }}$ & $(\Delta x / c)_{\min }$ & $(\Delta y / c)_{\min }$ \\
\hline Mesh 1 & 15,273 & 228 & 20 & 120 & 50 & 10 & $1.5 \times 10^{-4}$ & $1.6 \times 10^{-4}$ \\
\hline Mesh 2 & 43,806 & 420 & 40 & 228 & 120 & 15 & $1.0 \times 10^{-4}$ & $1.0 \times 10^{-4}$ \\
\hline Mesh 3 & 71,561 & 546 & 60 & 366 & 150 & 15 & $1.0 \times 10^{-4}$ & $1.0 \times 10^{-4}$ \\
\hline Mesh 4 & 125,194 & 704 & 80 & 384 & 200 & 20 & $7.0 \times 10^{-5}$ & $7.0 \times 10^{-5}$ \\
\hline
\end{tabular}

The initial grid (Mesh 1) was coarse, containing 228 total nodes along the surface of the airfoil and totaling just over $15 \mathrm{~K}$ nodes. The final grid (Mesh 4) was refined to have over 700 airfoil surface nodes and $125 \mathrm{~K}$ total grid points. The increase in grid density during the refinement process can be seen in the farfield view included in Figure 69.
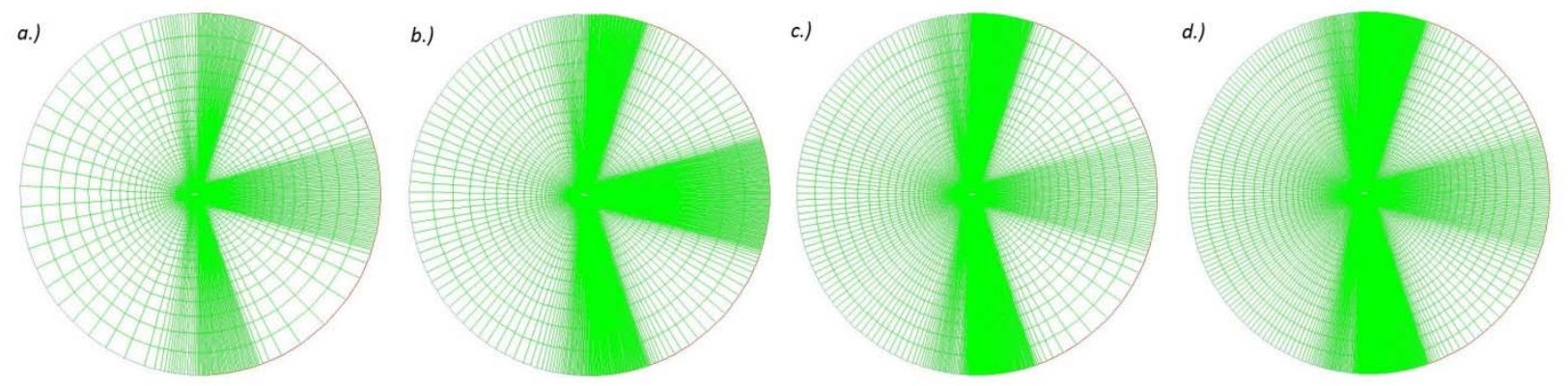

Figure 69. Grid Independence Study: Farfield Mesh for a.) Mesh 1, b.) Mesh 2, c.) Mesh 3, d.) Mesh 4

\subsection{Initial Conditions}

Initial conditions (ICS) should not influence the final simulation results. Instead, ICs should only affect the convergence path (i.e. the iteration count, $N_{\text {iter, }}$ for steady flowfields or the number of timesteps, $\Delta t$, for an unsteady solution procedure). Proper knowledge of governing flow characteristics guides appropriate selection on such conditions, improving numerical stability and accelerating solution convergence. Steady state results were first computed to provide more robust initial conditions for the transient solutions reported herein. 


\subsubsection{Static Model Initialization}

For stationary airfoil calculations, hybrid initialization was used for the baseline cases at $\alpha=0^{\circ}$, $10^{\circ}$. After suitable convergence was established (stabilized aerodynamic coefficients), the baseline solution was used as the starting point for the minimum case of steady blowing $C_{\mu}=0.01$. This result was in turn used to begin the simulation for an increased magnitude of blowing of $C_{\mu}=0.05$. This tiered initialization approach, with respect to the magnitude of blowing, helped to speed convergence by minimizing the changes in flowfield characteristics between each flow control case. In addition, the steady blowing solutions were used to initialize the pulsed jet simulations in a similar manner.

Furthermore, when turbulent solutions were computed for comparison, preliminary solutions were computed using the laminar solver model. The converged laminar solution was then used to initialize the more complex turbulence model. This procedure was found to significantly reduce CPU time to reach a converged solution, when compared to initializing a turbulent simulation from rest.

\subsubsection{Dynamic Model Initialization}

Pitching airfoil simulations for each blowing condition were initialized from transient, static (airfoil) solutions $\left(\alpha=0^{\circ}\right)$ having a minimum of 10 domain flow though periods, the time it takes a single particle to traverse the computational domain (55). For low Re flow, the solution about a NACA airfoil at zero- $\alpha$ is periodic due to TE edge vorticity and resultant stagnation point oscillations (151). However, although the processes that characterize DS are known to be independent of airfoil starting position $(\alpha)$ (152), the timing of dynamic stall development depends on the initial position of the TE wake and thus has an impact on the aerodynamic loading variation with AoA (151). Thus, for each condition, a minimum of three pitch cycles were completed to establish a time-periodic solution, and the solutions reported reflect the fourth pitch cycle airfoil characteristics (from rest).

For dynamic pitch simulations of the baseline CCNACA airfoil $\left(C_{\mu}=0\right)$, and steady blowing conditions $\left(C_{\mu} \neq 0, S t=0\right)$, the pitch rate was the governing parameter for time-step selection. When modeling a pitching airfoil with pulsed circulation control, the actuation frequency $\left(f_{\text {act }} \gg f_{\text {pitch }}\right)$ dictated the time-step; a significant reduction in $\Delta t$ was needed for proper resolution of the unsteady jet.

Also, as the complexity of unsteady flow problems increases, solutions can be initialized by first solving the problem under steady conditions for a number of iterations. Following the same principle, higher order turbulence models can be initialized from lower order laminar models ( 0 - and 1-Equation) to speed convergence. 
The sinusoidal pitching motions used for simulations did not perfectly match experimental conditions in terms of AoA amplitude. As previously mentioned, baseline pitch computations were initialized from a fully converged static airfoil solution at $\alpha=0^{\circ}$. The clean airfoil then completed five full pitch cycles before a time-periodic solution was reached. For flow control cases, actuation was initiated during pitch-up as the airfoil reached $0^{\circ}$, completing the fifth cycle of the baseline case. It was found that only one additional pitch cycle was needed to report CC results; additional oscillation cycles did not show significant change to airfoil coefficients. Thus, for proper time management, when $\alpha_{\max }=30^{\circ}$ was computed, calculations followed pitch-up from $0^{\circ}$ to $30^{\circ}$ and back down to $0^{\circ}$. Experimental conditions included measurements following the path: $\alpha(t)=10^{\circ}+20^{\circ} \sin (\omega t)$, reaching a minimum AoA of $-10^{\circ}$ compared to $\alpha_{\min }=0^{\circ}$ for CFD modelling.

\subsection{Numerical Model Setup}

\subsubsection{General Solver Settings}

All CFD simulations utilized the time-dependent Reynolds-Averaged Navier-Stokes (URANS) equations, using a pressure-based solver with absolute velocity formulation. All models were twodimensional to reflect experimental wind tunnel conditions.

\subsubsection{Boundary Conditions}

A velocity-inlet boundary condition $(\mathrm{BC})$ was used to define the velocity and scalar properties of the flow at the inlet boundary (upwind half of the O-grid farfield boundary). Velocity magnitude components were prescribed in the $x$ - and $y$-directions based on the desired angle of attack. A pressureoutlet $B C$ was utilized to define the static pressure (gauge) at the outflow boundary (aft portion of the O-grid farfield boundary), along with scalar flow properties in the event of backflow. The use of a pressure outlet BC, instead of an outflow condition, often results in a better convergence rate when backflow occurs during the iteration process (147).

The airfoil surface was defined as a smooth, stationary wall with the no-slip BC enforced. For baseline simulations, the jet slots were also modeled as solid walls. For blowing cases, a velocity inlet condition was defined, normal to the slot plane, based on the desired $C_{\mu}$ to match experimental conditions. The mass flow was monitored and recorded for each simulation in order to calculate and verify the simulated $C_{\mu}$ condition matched the corresponding experiment. The pulsed jet was created by a user defined function (UDF), written in C-code and implemented in FLUENT software. The function 
generated a sinusoidal jet velocity normal to the jet exit, with adjustable frequency and amplitude. A monitor was set up to calculate the mass flow rate at the jet exit to verify that the desired $C_{\mu}$ setting was achieved, relative to experimental test conditions. An example of the sinusoidal behavior of the pulsed jet $\left(C_{\mu}=0.05\right)$ is included in Figure 70 , where the black line represents a steady jet $(S t=0.0)$, blue line signifies pulsed jet behavior at $S t=0.5$, and the red line describes an unsteady jet of $S t=2.0$.

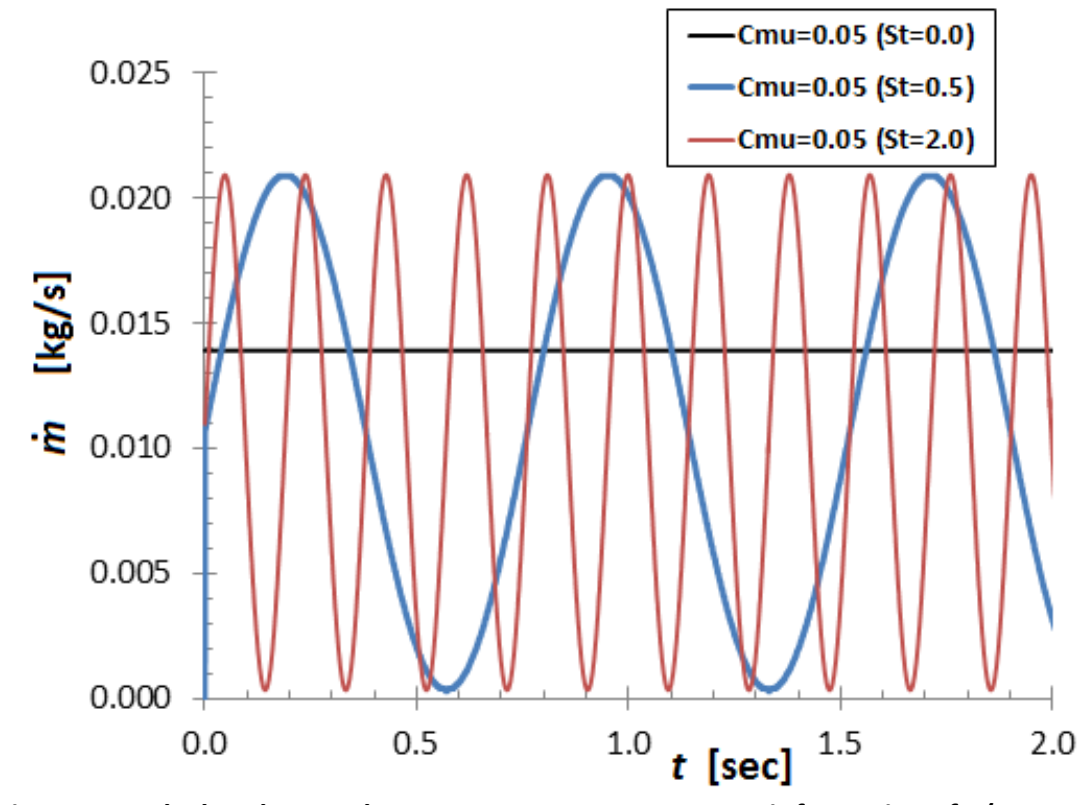

Figure 70. Calculated Mass Flow Rate at CCNACA0018 Jet Exit for Various $f_{j e t}\left(C_{\mu}=0.05\right)$

\subsubsection{Reference Values}

All solutions were computed from the farfield inlet discussed in the previous section. The simulation model was given a unit chord of $1 \mathrm{~m}$ to allow for easy scaling if desired for future work. The unit chord allowed simple calculation of non-dimensional scaling parameters such as $R e$ and St, along with performance coefficients of lift and drag. To achieve the desired condition $R e=180 \times 10^{3}$, the farfield velocity was defined to be $V_{\infty}=2.629 \mathrm{~m} / \mathrm{s}$, along with the default properties for air: $T_{\infty}=288.16 \mathrm{~K}$, $\mu_{\infty}=1.789 \times 10-5 \mathrm{~kg} / \mathrm{m} \cdot \mathrm{s}$.

\subsection{Numerical Methods}

\subsubsection{Solution Methods}

FLUENT uses a control-volume (CV) approach to convert the governing PDEs into algebraic form to be solved numerically. The CV technique involves integrating the governing equations about each cell, 
yielding discrete equations that conserve each flow quantity on a control-volume basis (147). The pressure-based coupled solver (PBCS) was implemented to improve convergence by solving the momentum and pressure-based continuity equations in a coupled manner. Although there is a slightly higher computational cost due to associated memory requirements, the benefits far outweigh the drawbacks, allowing the use of skewed meshes and larger time steps for robust convergence (147). Pressure-velocity coupling was achieved with the PISO scheme (Pressure Implicit solution by Split Operator method). PISO has been found to improve convergence speeds when compared to FLUENT's SIMPLE scheme (Semi Implicit Method for Pressure Linked Equations) (147). In addition, PISO is useful for unsteady flow problems and grids containing increased skewness. The PISO algorithm correction terms (neighbor, skewness) were maintained at default values of unity.

For spatial discretization, a least squares cell based method was used in conjunction with the PRESTO! (Pressure Staggering Option) scheme for pressure terms and MUSCL discretization for momentum. The PRESTO! Interpolation method is advantageous for high swirling flows and steep pressure gradients (common to CC airfoils) while MUSCL is a locally $3^{\text {rd }}$ order convection discretization scheme for unstructured meshes, more accurate in predicting secondary flows and vorticity (147). For turbulent simulations with the SARC model, $3^{\text {rd }}$ Order MUSCL scheme was used to discretize the modified turbulent viscosity.

\subsubsection{Grid Independence Study}

To remove numerical bias due to mesh geometry, four meshes were created for static airfoil simulations at $\alpha=0^{\circ}$ and $\alpha=10^{\circ}$. Aerodynamic force coefficients $\left(C_{l}, C_{d}\right.$, and $\left.C_{m}\right)$ and surface pressure distributions $\left(C_{p}\right)$ were considered to determine sufficient grid density while minimizing computational costs. The aforementioned flow variables were considered to help identify solutions that numerically converge, but reveal non-physical flow physics (e.g. negative drag, or thrust, for a baseline airfoil case). Figure 71 includes the calculated $C_{p}$-distribution results for each of the four meshes; the URANS simulations were computed for a static, baseline airfoil at $\alpha=0^{\circ}$ (Figure 71a) and $\alpha=10^{\circ}$ (Figure 71b). The rounded TE $(x / c \geq 0.95)$ represented the region of highest flow complexity, and thus compared to establish grid independent solutions. 
(a.) $\alpha=0^{\circ}$

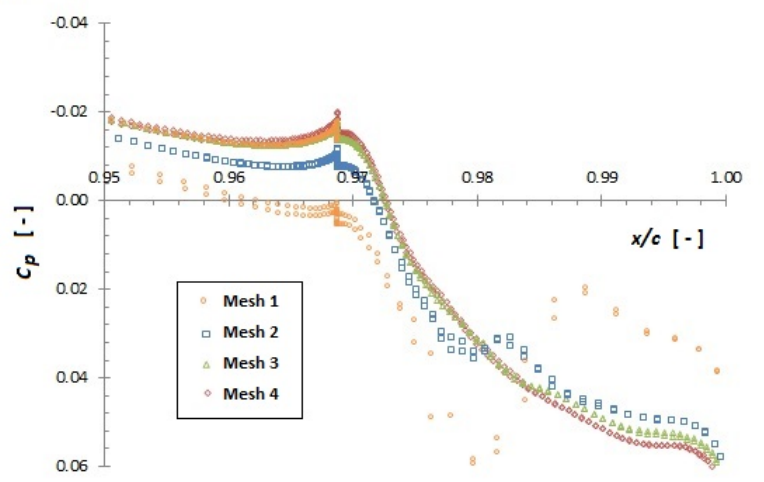

(b.) $\alpha=10^{\circ}$

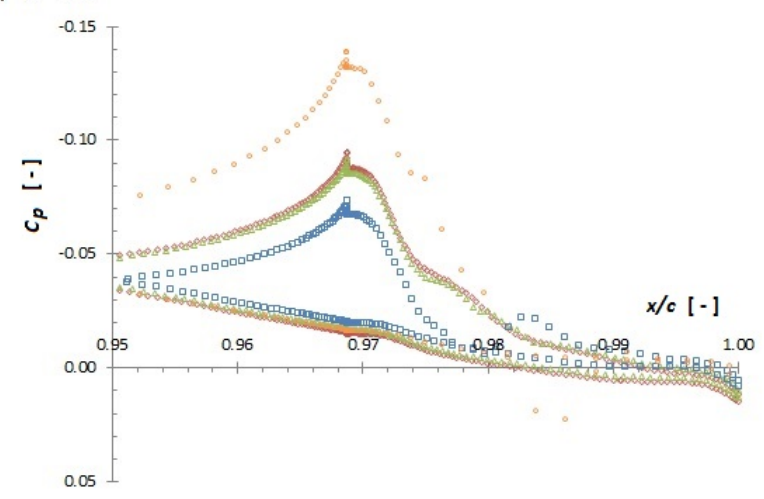

Figure 71. CFD Grid Independence Study: Static Airfoil $C_{p}$-Distributions (Baseline, $R e=180 \times 10^{3}$ )

As seen in Figure 71, large discrepancy between Mesh 1 (orange circles) and Mesh 2 (blue squares) solutions are evident, for both $\alpha$. The grid was then refined and results for Mesh 3 (green triangles) again showed discrepancy, but to a lesser degree. After a final grid refinement, Mesh 4 (red diamonds) showed excellent agreement to Mesh 3, with maximum difference of approximately 3\%. Thus, Mesh 3 was determined to have sufficient refinement to produce grid-independent solutions.

\subsubsection{Temporal Independence Study}

All solutions reported were calculated using $2^{\text {nd }}$ Order Implicit transient formulation. Higher order term relaxation (HOTR) was used to improve the general solution behavior due to the use of higher order spatial discretization (i.e. $>1^{\text {st }}$ Order) (147). Time independent solutions were determined by trial and error, testing time-solutions in the range $0.00001 \mathrm{~s} \leq \Delta t \leq 0.0015 \mathrm{~s}$. Also of significance is the number of sub-iterations computed for each physical time step. For each time step simulated, the number of sub-iterations $\left(N_{\text {sub }}\right)$ from zero, increased until variation in integrated aerodynamic coefficients was $\leq 1 \%$, following the numerical work of Ligitt (120). In general, as the time step is decreased, the number of sub-iterations required for convergence of all flow coefficients is decreased. For all reported results, a physical time step of $\Delta t=0.0001 \mathrm{~s}\left(\Delta t^{+}=0.0003\right)$ was used, with a maximum of 20 sub-iterations per physical time step. The nondimensional time-step $\left(\Delta t^{+}\right)$is defined by Equation 67 .

Non-dimensional Time Step

$$
\Delta t^{+}=\frac{\Delta t \cdot V_{\infty}}{c}
$$

Equation 67

Ligitt (120) found that integrated quantities $\left(C_{l}, C_{d}\right.$, and $\left.C_{m}\right)$ all predict the same behaviors using URANS models, any of which can be used to formally verify solution accuracy. Furthermore, a minimum non-dimensional time step of $\Delta t^{+}=0.0025$ is necessary for $2^{\text {nd }}$ Order temporal accuracy, making the timestep considered a suitable selection based on model settings and grid fineness. 


\subsection{Turbulence Modelling}

The influence of turbulence modelling was examined during preliminary static airfoil simulations. The low Reynolds' number condition $\left(R e=180 \times 10^{3}\right)$ of experimental testing was chosen for computational replication to better suit the simplified Laminar solver model. The goal here, as is present in the majority of current numerical research, was to find a compromise between solution accuracy and CPU time. Laminar airfoil simulations offer expedited convergence but can suffer accuracy at higher AoA when separation (and resulting turbulent flow) is present. On the contrary, turbulent models improve separated flow predictions at the expense of significantly increased computational time. Discrepancy between laminar solver computations and turbulent experimental data is common, however. In laminar computations, the boundary layer has less energy and separates at lower angles of attack (117). The vorticity is then shed in succession of small vortices over a long period of time instead of a big vortex over a small period of time as in the turbulent experiments (117). This qualitative comparison confirms that turbulence changes the flow structure and stresses the need for turbulence modeling to validate airfoil calculations using fully laminar flow. Thus, three models were compared on a static airfoil at a neutral position $\left(\alpha=0^{\circ}\right)$ and an elevated position with separation $\left(\alpha=10^{\circ}\right)$ :

1.) Laminar Model (0-equation)

2.) Spalart-Allmaras Model with Rotation Curvature Corrections (1-equation)

3.) $k-\varepsilon$ Model with Curvature Correction (2-equation) 


\section{CHAPTER 5. RESULTS}

\subsection{Wind Tunnel Results: Static Airfoil}

All wind tunnel data included in this section was collected using the same (2D) CCNACAO018 airfoil model. More in depth details of the wind tunnel model are provided by Panther, et al. (38). Results will include surface pressure measurements and the resulting integrated aerodynamic coefficients. Measured data from baseline and steady blowing conditions will first be compared to previous CIRA experiments to validate the experimental test set-up. Experimental results for pulsed CC on a steady airfoil will then be compared to constant blowing results to evaluate airfoil performance.

\subsubsection{Static CCNACA0018 Pressure Data Validation}

A series of surface pressure scans were measured with various equipment to validate the ScaniValve calibration and measurement procedure. Furthermore, such fidelity tests were completed to verify that all pressure ports and tubing returned reliable signals, independent of measurement equipment. If a given set of pressure measurements could be matched by secondary equipment for each of the 32 ports, the model could be considered reliable for current wind tunnel testing. For the validation study, a $\mathrm{H}_{2} \mathrm{O}$ manometer bank and HEISE handheld pressure calibrator were used to supplement ScaniValve measurements. A schematic to help visualize the validation can be found in Figure 72. Furthermore, each of the three instruments were connected to the wind tunnel model with different forms of pneumatic tubing, to identify any possible distortion effects caused by length, inner diameter, wall-thickness, and material. The specifications for each length of tubing are summarized in Table 17.

Table 17. Pneumatic Tube Geometry and Material for Static CCNACA0018 Testing

\begin{tabular}{|l|c|c|c|c|c|}
\hline EQUIPMENT & TUBE & LENGTH & MATERIAL & ID & OD \\
\hline HEISE Gage & $\mathrm{L}_{1}$ & $24 \mathrm{in.}$ & Nylon (Rigid) & $0.057 \mathrm{in.}$ & $0.089 \mathrm{in}$. \\
\hline $\mathrm{H}_{2} \mathrm{O}$ Manometer & $\mathrm{L}_{2}$ & $120 \mathrm{in}$. & Silicon (Flexible) & $0.106 \mathrm{in}$. & $0.138 \mathrm{in}$. \\
\hline ScaniValve ESP & $\mathrm{L}_{3}$ & $58 \mathrm{in}$. & Urethane (Flexible) & $0.054 \mathrm{in}$. & $0.086 \mathrm{in}$. \\
\hline
\end{tabular}




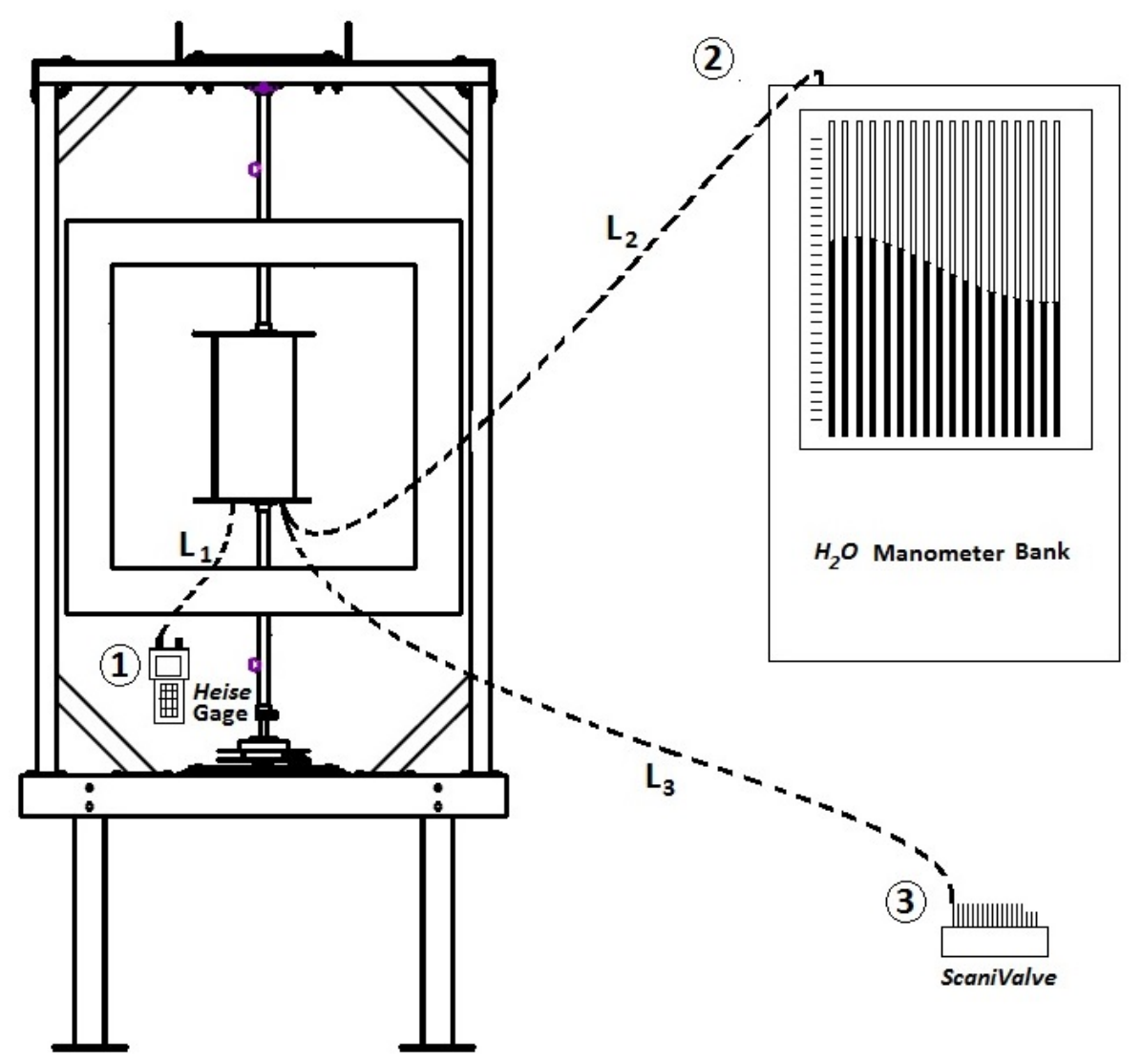

Figure 72. CCNACA Model Surface Pressure Measurement Fidelity Testing

The dotted lines in Figure 72 represent the tubing connections between the wind tunnel model and each of the three pressure instruments. For method 1 , a single tube had to be manually switched between the HEISE Gage and each of the 32 airfoil ports to record the chordwise pressure distribution. However, for methods 2 and 3, each port on the model had its own connection to either the ScaniValve transducer or the manometer bank. The measured results for each device are included in Figure 73 for $\alpha=0^{\circ}$ $\left(R e=300 \times 10^{3}\right)$, compared to theoretical calculations from DesignFOIL software. Measured values show quantitative agreement for the LE $(x / c \leq 0.20)$, but reduce to qualitative agreement aft of the quarter chord. It should be noted that all symbols represent an average of five runs recorded for each pressure port. The three methods show close agreement, with the $\mathrm{H}_{2} \mathrm{O}$ manometer bank under predicting upper surface pressure values (open triangles) by an average of $4.9 \%$ relative to the averaged value (solid lines) for a given $x / c$ location.

Figure 73 , compares the measured $C_{p}$-distributions for each device, compared to theory, for $\alpha=10^{\circ}$. All three instruments show close agreement for each tap location, within $5 \%$ difference. Both the upper and lower taps at $x / c=0.707$ (upper tap \#12 and lower tap \#23) were found be unreliable by all equipment, for both AoA. The measurements at these taps showed abnormalities for all equipment, 
indicating the internal tubing to be the problem. These particular ports are located directly above the pneumatic valves inside the high-pressure plenum of the model. It is thought that high pressure used during past testing of flow control cases $\left(P_{\text {plenum }} \geq 25 p \operatorname{sig}\right.$ for $\left.C_{\mu}=0.05,0.10\right)$ could have damaged the taps within the ABS plastic skin of the model. Whatever the cause of malfunction, measurements recorded from these pressure taps were deemed unreliable and discarded for all data presented hereafter.

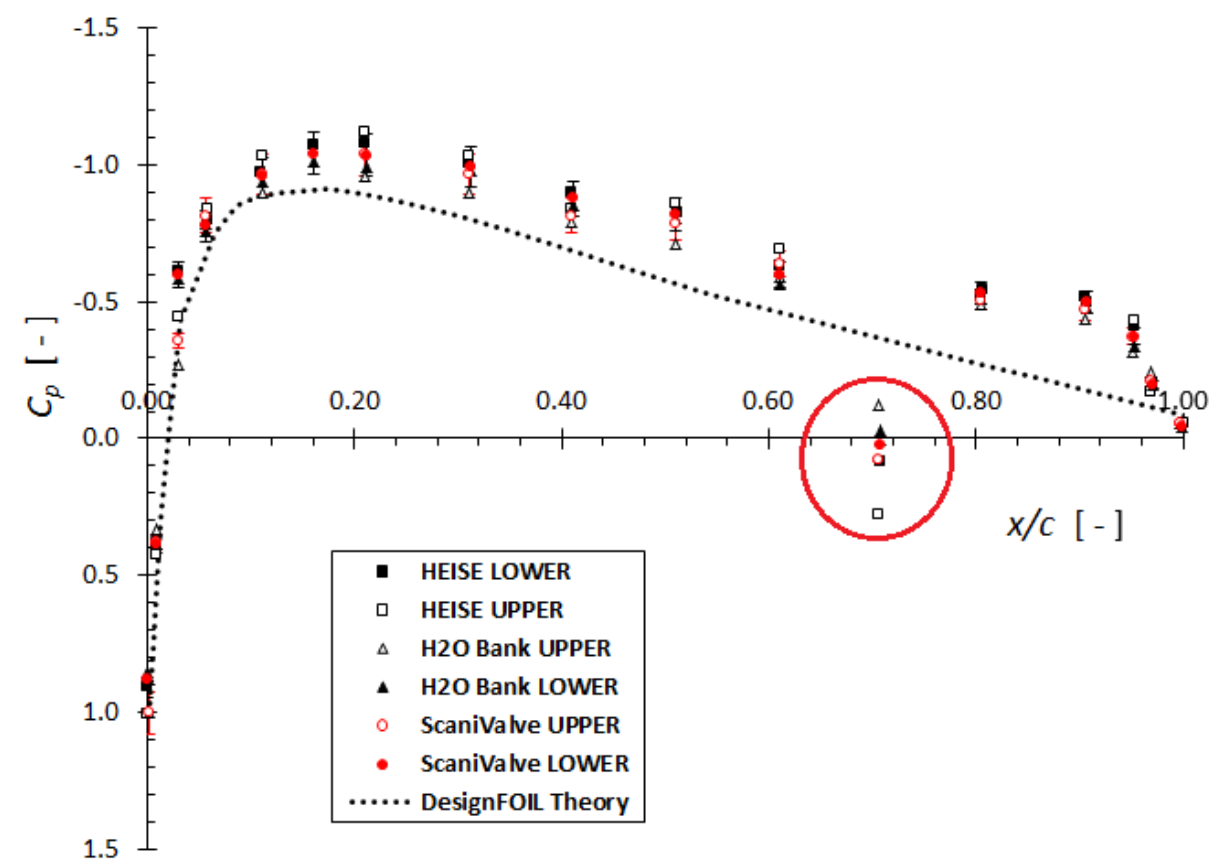

Figure 73. Surface Pressure Instrumentation Check: Baseline CCNACAO018 $\left[R e=300 \times 10^{3}, \alpha=0^{\circ}\right]$

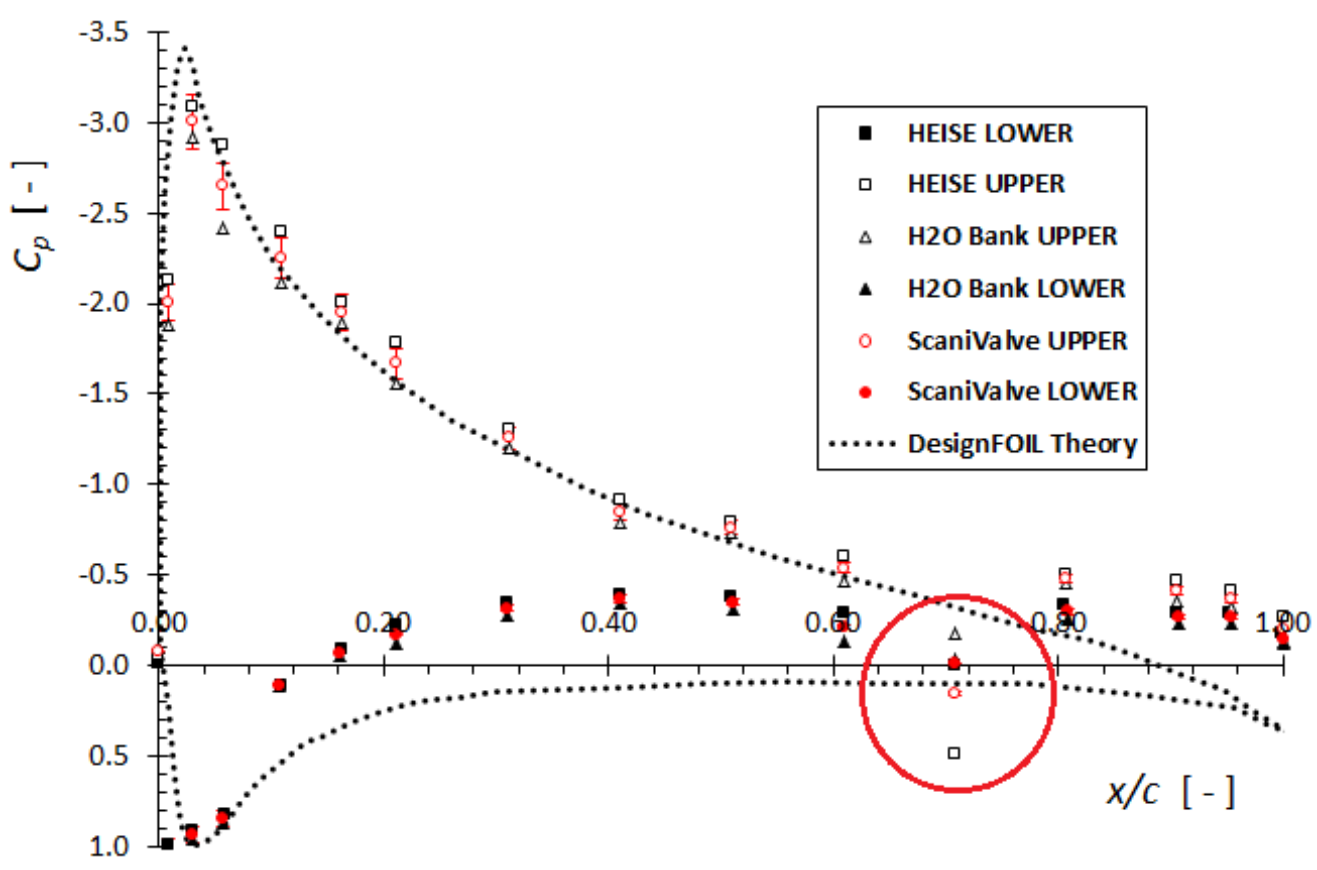

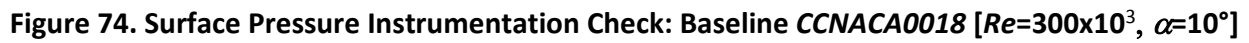


The pressure measurements at each port showed a maximum discrepancy of $<5 \%$ between equipment. Based on the results of the set-up validation testing, it was determined that the ScaniValve multiport pressure transducer, in conjunction with $58^{\prime \prime}\left(0.054^{\prime \prime} I D\right)$ tubing was credible to be used for static testing of the CCNACA0018 model with varying modes of circulation control.

\subsubsection{Static CCNACA0018 Lift Data: Baseline}

The static lift performance of the clean airfoil was compared to experimental results provided by Hoerner, et al. (67) for a NACA0018, comparing the lift-curve slope of the profile with a conventional, sharp TE to the results from an augmented, blunt TE (Figure 75)

a.) Smith, et al. (1936)
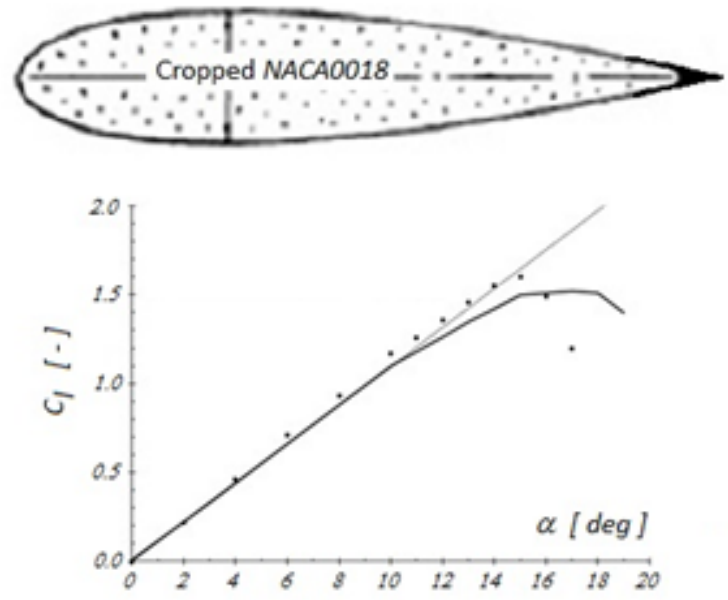

- Thin Airfoil THEORY

- Smith, et al. (1936) NACA0018

- Smith, et al. (1936) NACAOO18 (Blunte TE) b.) Panther, et al. (2010)
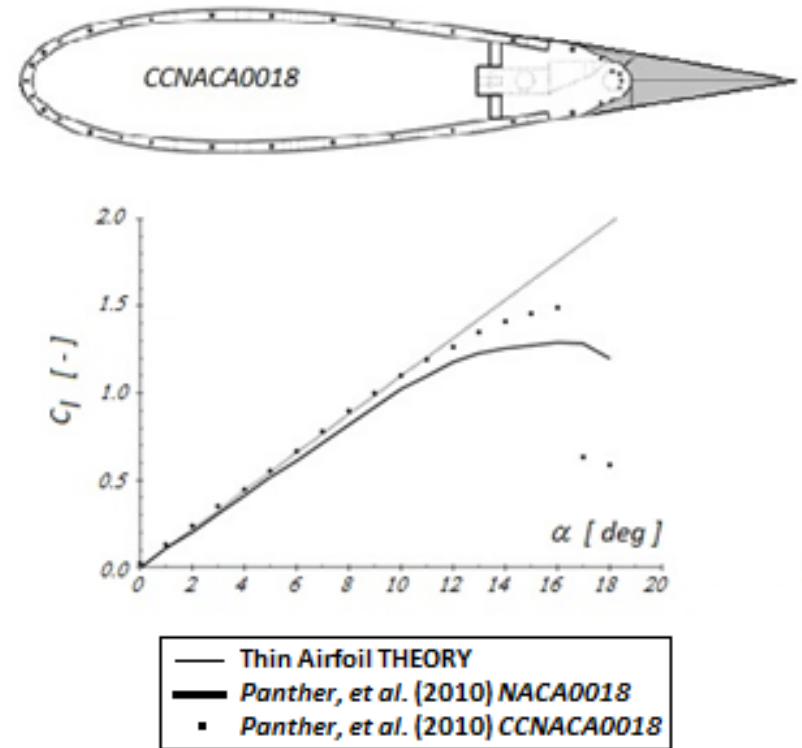

Figure 75. Influence of Blunt TE on Lift Performance: a.) Smith, et al. (67) b.) Panther, et al. (38)

As seen in Figure 75 , the blunt TE causes a slight decrease in $d C_{l} / d \alpha$ and $C_{l, \text { max }}$, but does not affect $\alpha_{\text {stall; }}$ similar trends were observed from the CCNACA0018 airfoil during baseline testing $\left(C_{\mu}=0\right)$. A similar data set was established for three variations of the conventional NACA0012 profile, formed by removing various amounts from the rear section to examine the aerodynamic influence of a blunt TE by Smith, et al. (153). The 2D aerodynamic characteristics of airfoils with $1.5 \%, 4.0 \%$, and $12.5 \%$ of the original chord removed from the TE were compared, concluding that thickening the TE (sharp $\rightarrow$ blunt) results in an increase in $C_{d}$ for most $C_{l}$. However, $C_{l, \max }$ was noticed to progressively increase (slightly) as $t_{T E}$ increased, 
for the three variations tested (153). The CCNACA0018 ( $c=8.13 \mathrm{in})$ was created from a NACA0018 profile with chord of 9in, equating to $a 9.7 \%$ removal of the original chord.

\subsubsection{Static CCNACA0018 Lift Data: Steady $C_{\mu}$}

The steady sectional lift coefficient $\left(C_{l}\right)$ results for $R e=300 \times 10^{3}$, including baseline and various steady blowing conditions, are included in Figure 76. The current data shows excellent agreement to previous CIRA data (38) for all blowing conditions. Earlier data was recorded on various test set-ups with different CCNACA models; the close agreement improves confidence of the measured data of this study. Low levels of steady blowing enhanced lift for all AoA tested.

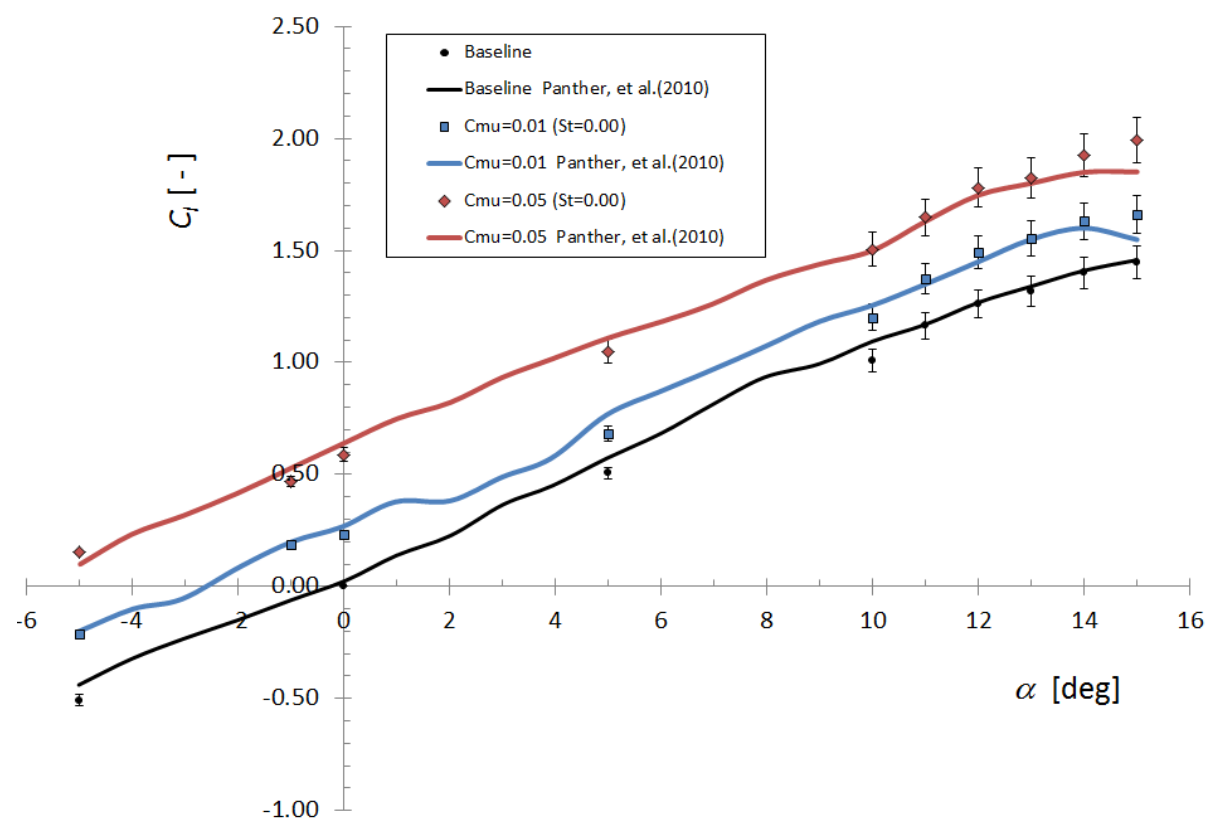

Figure 76. Static CCNACA0018 Lift Coefficient Validation (Steady $C_{\mu}$ ): $R e=300 \times 10^{3}$

The influence of pulsed jet CC on CCNACA0018 suction surface pressure distribution are included in Figure 77. The unsteady jet is able to produce similar suction peaks as steady jet conditions, for both magnitudes of blowing $\left(C_{\mu}=0.01,0.05\right)$. 


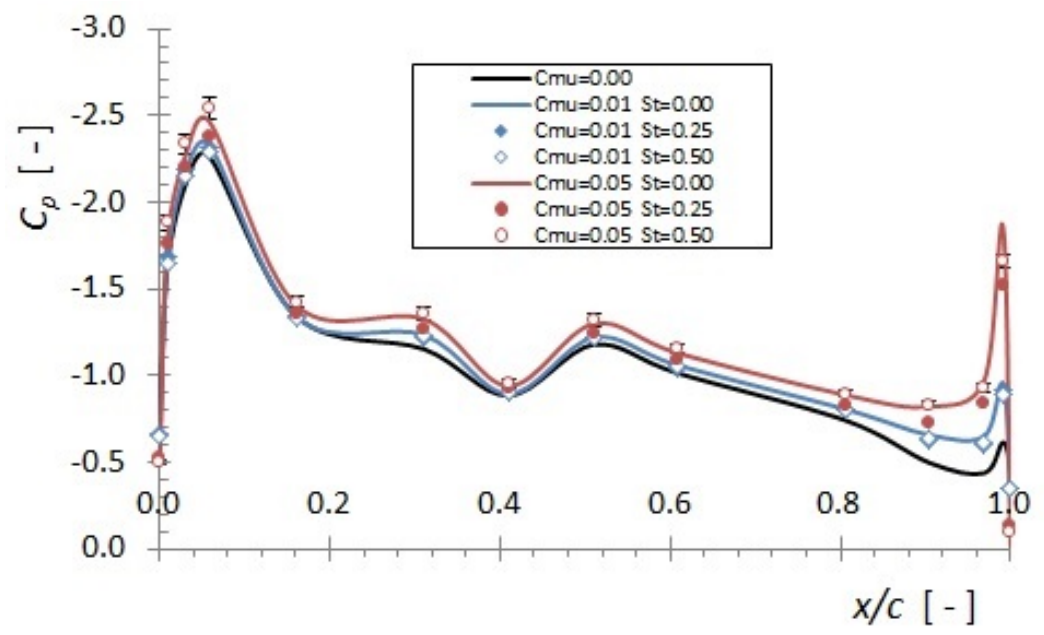

Figure 77. Influence of Pulsed CC Jet on Suction Surface $C p$-Distribution: $R e=300 \times 10^{3}, \alpha=10^{\circ}$

\subsubsection{Static CCNACA0018 Drag Data: Steady $C_{\mu}$}

The baseline $\left(C_{\mu}=0.00\right)$ steady drag coefficient $\left(C_{d}\right)$ results for $R e=300 \times 10^{3}$ are plotted in Figure 78, showing close agreement to previous experiments. Also included is the measured drag for an unmodified NACA0018 airfoil, measured at $R e=360 \times 10^{3}$. The effect of the rounded TE on drag is clear, as the sharp TE of the conventional airfoil significantly reduces drag relative to the baseline CCNACA profile. The drag decreases with increasing $C_{\mu}$ for the steady conditions tested.

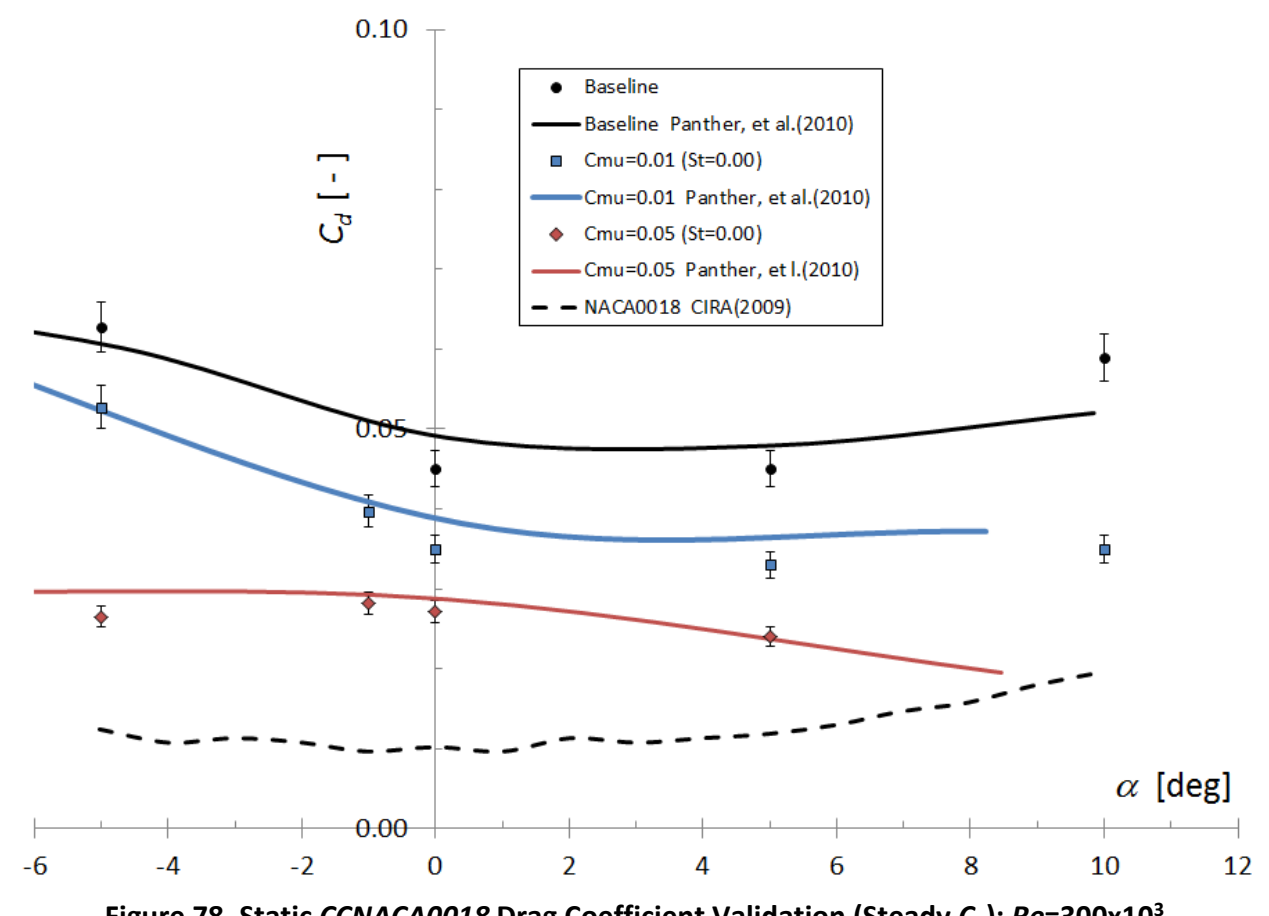

Figure 78. Static CCNACA0018 Drag Coefficient Validation (Steady $C_{\mu}$ ): $\operatorname{Re}=300 \times 10^{3}$ 


\subsubsection{Static CCNACA0018 Moment Data: Steady $C_{\mu}$}

The baseline $\left(C_{\mu}=0.00\right)$ static moment coefficient $\left(C_{m}\right)$ results for $R e=300 \times 10^{3}$ are plotted in Figure 79. Such $C_{m}$-data was not recorded during previous CIRA investigations and thus, experimental data from Mueller-Vahl, et al. (102) of a static NACA0018 airfoil was used as a benchmark comparison. Theoretical uncertainty bars, as outlined in Chapter 3, are included on data points from the current study. The disagreement between data sets above $15^{\circ}$, where a sharp drop-off appears due to complete airfoil stall, is a discrepancy between $\alpha_{\text {stall; }}$ the magnitude for $C_{m}$ otherwise agrees well with past experiments.

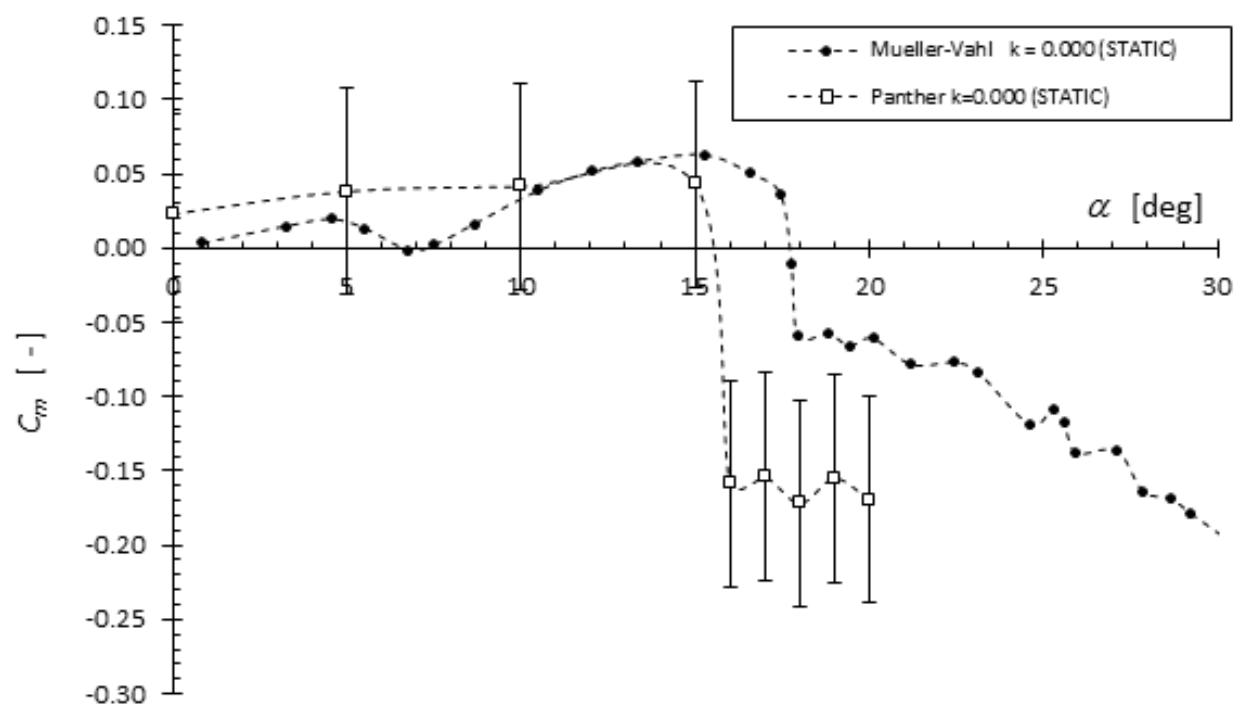

Figure 79. Static CCNACA0018 Moment Coefficient Validation (Baseline): $R e=300 \times 10^{3}$ (102)

\subsubsection{Static CCNACA0018 Lift Data: Pulsed $C_{\mu}$}

The mass flow requirements for each blowing condition, governed by valve operation capabilities at higher upstream pressure, are included in Table 18 . The mass flow rate savings $(\Delta \dot{m})$ due to pulsed CC are listed in the right hand column. 
Table 18. CCNACA0018 Mass Flow Requirements for Various Experimental Blowing Conditions

\begin{tabular}{|c|c|c|c|c|}
\hline$R e$ & $C_{\mu}$ & St & $\dot{m}$ & $\Delta \dot{m}$ \\
\hline [- ] & [-] & [-] & {$\left[\mathrm{lb} \mathrm{b}_{\mathrm{m}} / \mathrm{s}\right]$} & [\%] \\
\hline \multirow{6}{*}{$\begin{array}{l}\stackrel{m}{0} \\
\underset{+}{+} \\
\underset{0}{0} \\
\underset{+1}{0}\end{array}$} & \multirow{3}{*}{0.01} & 0.00 & 0.0104 & - \\
\hline & & 0.25 & 0.0080 & $-29 \%$ \\
\hline & & 0.50 & 0.0090 & $-22 \%$ \\
\hline & \multirow{3}{*}{0.05} & 0.00 & 0.0230 & - \\
\hline & & 0.25 & 0.0210 & $-12 \%$ \\
\hline & & 0.50 & 0.0220 & $-4 \%$ \\
\hline \multirow{6}{*}{$\begin{array}{l}m_{0} \\
\underset{-1}{x} \\
\stackrel{8}{8}\end{array}$} & \multirow{3}{*}{0.01} & 0.00 & 0.0120 & - \\
\hline & & 0.25 & 0.0090 & $-28 \%$ \\
\hline & & 0.50 & 0.0110 & $-6 \%$ \\
\hline & \multirow{3}{*}{0.05} & 0.00 & 0.0470 & - \\
\hline & & 0.25 & 0.0440 & $-6 \%$ \\
\hline & & 0.50 & 0.0450 & $-4 \%$ \\
\hline
\end{tabular}

For all plots of static aerodynamic performance data included herein, it should be noted that solid lines were used to represent baseline (black) and steady jet $\left(C_{\mu}=0.01\right.$ : blue, $C_{\mu}=0.05$, red) airfoil data. Symbols describe pulsed jet airfoil data (squares: $S t=0.25$, circles: $S t=0.50$ ), with the fill color matching the solid line color of the representative steady jet case; theoretical uncertainty bars were included based on the procedures outlined in Section 3.4. For all solid lines, data points exist at the same AoA locations as symbols. The steady $C_{/}$performance for pulsed CC blowing at a $R e=180 \times 10^{3}$ is plotted in Figure 80. For $C_{\mu}=0.01$, both pulsing frequencies $(S t=0.25,0.50)$ produce similar results, generating $95 \%$ of steady blowing lift. Mass flow savings of $29 \%$ and $22 \%$ were realized for $S t=0.25$ and St=0.50, respectively. For higher blowing conditions $\left(C_{\mu}=0.05\right)$, a higher jet frequency $(S t=0.50)$ was found beneficial, matching $96 \%$ of steady blowing lift for $\alpha \geq 0^{\circ}$. It is interesting to note that pulsed blowing at $5 \%$ showed decreased performance for negative AoA, while an oscillating jet of $1 \%$ maintained desirable performance at all airfoil positions. 


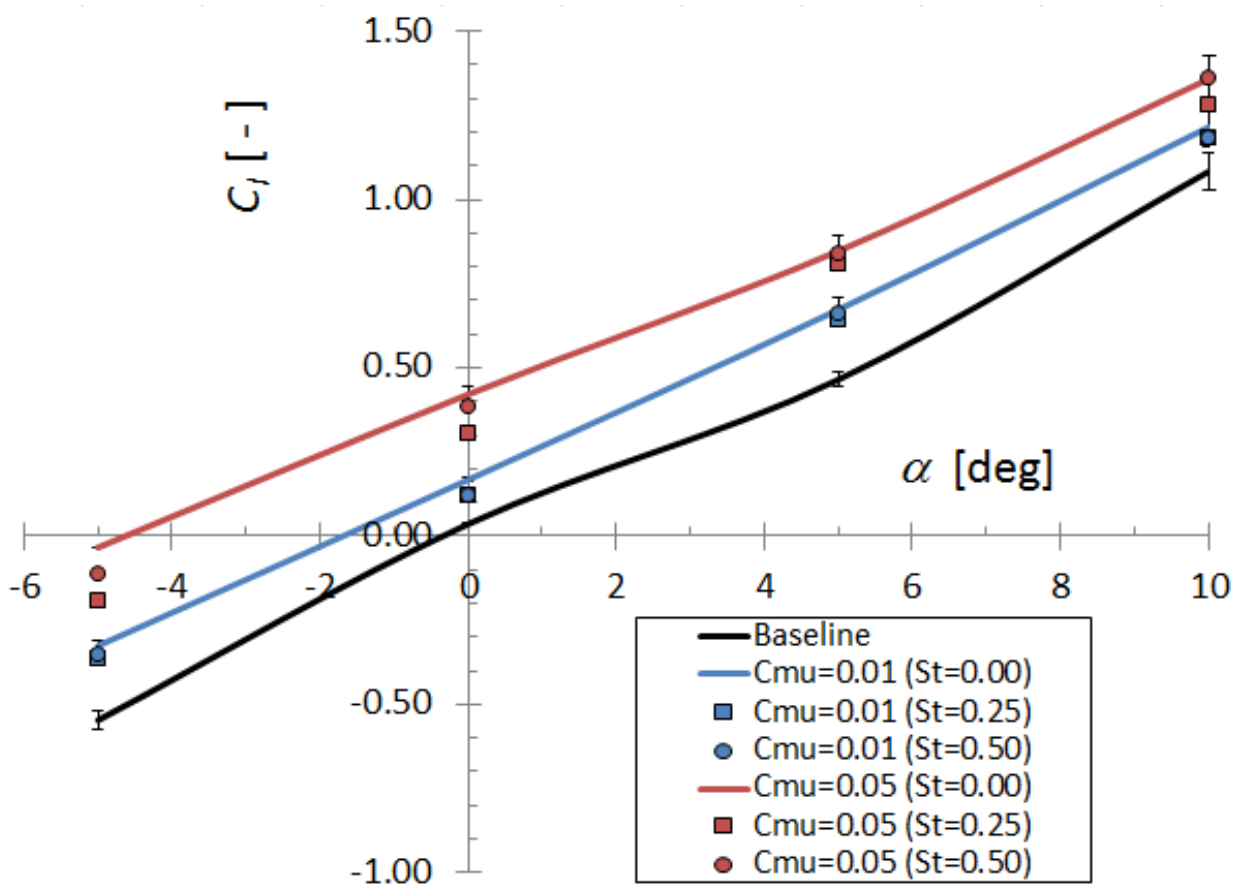

Figure 80. Static CCNACA0018 Lift Performance with Pulsed CC: $R e=180 \times 10^{3}$

The steady $\left(C_{l}\right)$ for pulsed CC blowing of $1 \%(S t=0.25)$, at a Reynolds number of $300 \times 10^{3}$, is plotted in Figure 81 . The pulsed CC jet provides up to $95 \%$ of the lift generated by steady blowing of the same magnitude, with a reduced mass flow rate of $28 \%$. Unfortunately, the pulsed blowing data for $C_{\mu}=0.05\left(R e=300 \times 10^{3}\right)$ was corrupt from unknown causes and the data was lost.

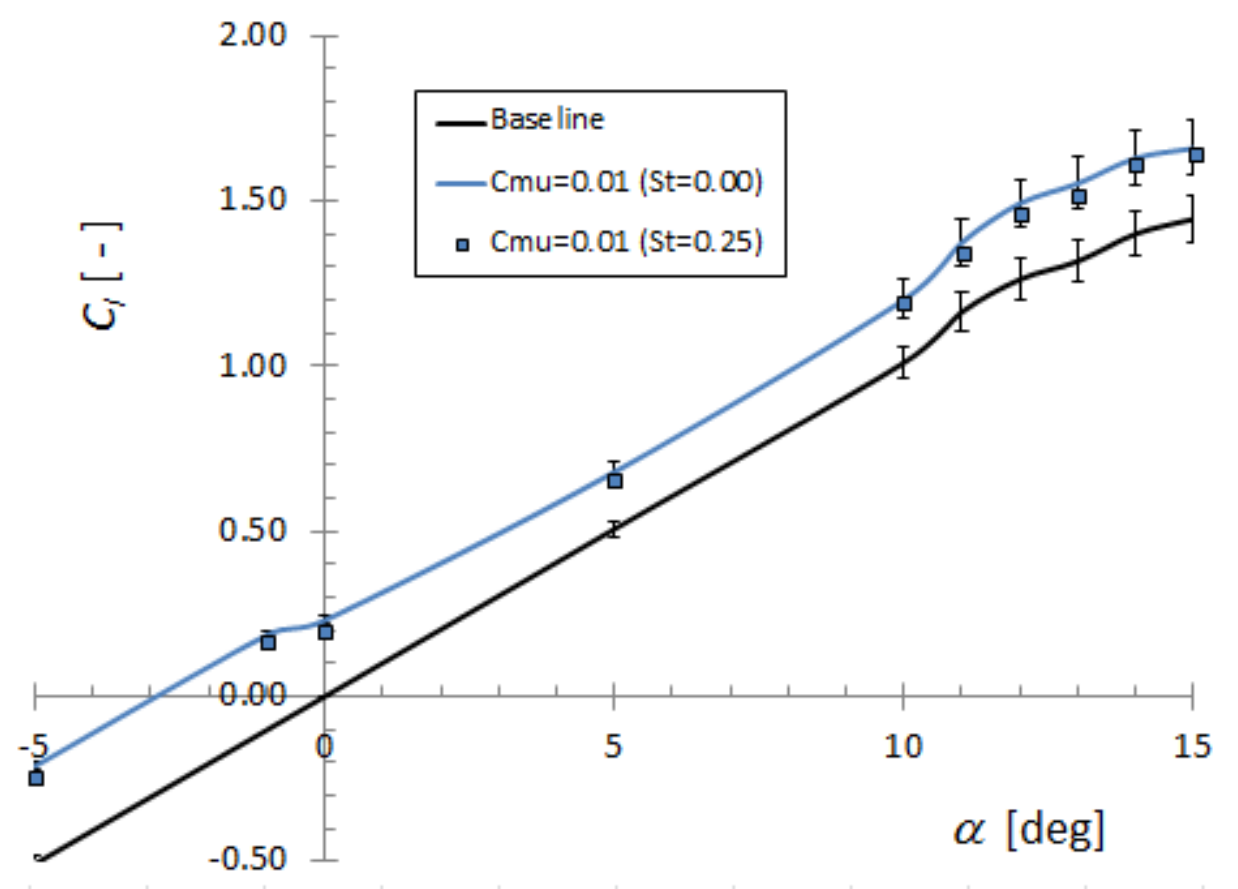

Figure 81. Static CCNACA0018 Lift Performance with Pulsed CC: $R e=300 \times 10^{3}$ 


\subsubsection{Static CCNACA0018 Drag Data: Pulsed $C_{\mu}$}

Static $C_{d}$ measurements on the static CCNACA airfoil with pulsed blowing are included in Figure 82. For both magnitudes of $\mathrm{CC}$, the common trend of decreasing drag with increasing $C_{\mu}$ is observed, for both steady and pulsed jet control.

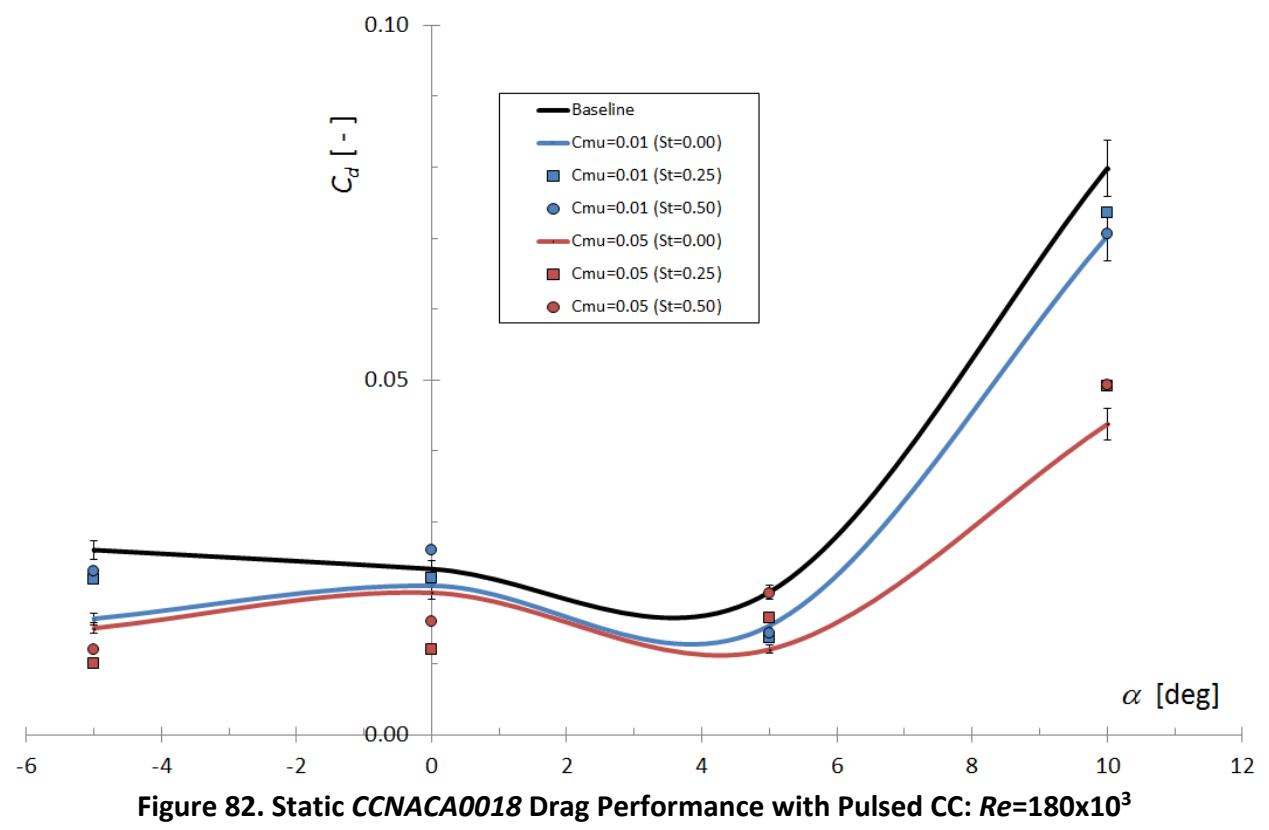

Static $C_{d}$ measurements on the static $C C N A C A\left(R e=300 \times 10^{3}\right)$ airfoil with pulsed blowing are included in

Figure 83. Compared to the baseline airfoil, steady blowing reduces sectional drag for all AoA. Pulsed blowing, with $S t=0.25$, further reduces $C_{d}$ by up to $67 \%$ at $\alpha=10^{\circ}$ compared to steady control. 


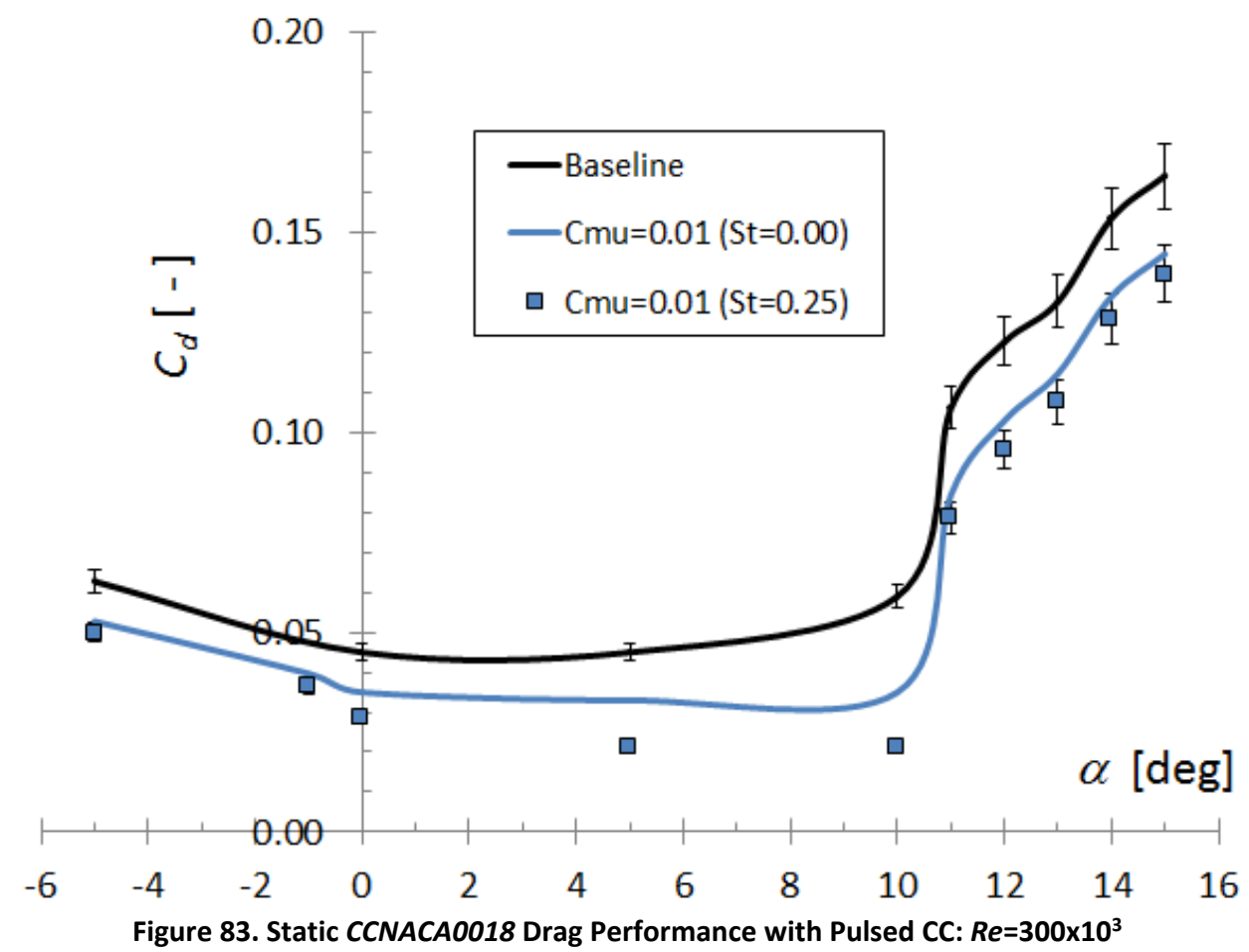

\subsubsection{Static CCNACA0018: Circulation Control Efficiency $(\eta)$}

A common metric for modified airfoil performance is the efficiency rating $(\eta)$, a modified lift-todrag ratio $(L / D)$ that compensates for thrust created by the CC jet (45) (46). To make direct comparisons of different blown systems (e.g. CC airfoils, jet flaps), it is necessary to define an equivalent lift-to-drag ratio $(L / D)$. For $C C$ airfoils, the efficiency (Equation 68 ) must factor the required energy to obtain improved performance (74); this also avoids the infinite efficiency that would occur when drag goes to zero as a result of blowing.

Steady CC Airfoil Efficiency

$$
\eta=\frac{C_{l}}{\left(C_{d}+C_{\mu}\right)}
$$

As previously mentioned, pulsed blowing was achieved by first setting the model up for steady blowing conditions at the desired $C_{\mu}$. Next, a user defined frequency input was applied to create a pulsed flow from the steady jet conditions. If the steady jet was $C_{\mu}=0.01$, for example, then pulsed cases for this steady condition were also labeled as $1 \%$ blowing (with various $\mathrm{St}$ ) for simplicity and comparison purposes. In reality, pulsing the jet lowered the <RMS> values of both $\dot{m}_{j e t}$ and $V_{j e t}$, in turn lowering the time averaged momentum coefficient, as described by Liu, et al. (79) (154), among others. For efficiency calculations of pulsed CC cases (Equation 69), a time averaged momentum coefficient was calculated 
based on RMS averaging $\left(\left\langle c_{\mu}\right\rangle\right)$ was calculated to compensate for mass flow savings. This updated version of $\eta$ now includes compensation for reduced flow rates to better reflect the efficiency of unsteady flow control airfoils.

Pulsed CC Airfoil Efficiency

$$
\langle\eta\rangle=\frac{C_{l}}{\left(C_{d}+\left\langle c_{\mu}\right\rangle\right)}
$$

Equation 69

The efficiency rating is beneficial to VAWT analysis, as maximizing $L / D$ increases turbine torque and power output. Furthermore, the metric offers a more concise and direct description of airfoil performance by combining force and momentum coefficients.

Figure 84 includes static CCNACA0018 efficiency for $R e=180 \times 10^{3}$, at various flow control modes. The worth of $\eta$ is apparent in Figure 84 , considering $5 \%$ blowing at $\alpha=5^{\circ}$. Although the largest lift enhancements were measured for $C_{\mu}=0.05$, the efficiency value at $5^{\circ}$ was less than half that of the baseline airfoil and lower blowing cases $\left(C_{\mu}=0.01\right)$.

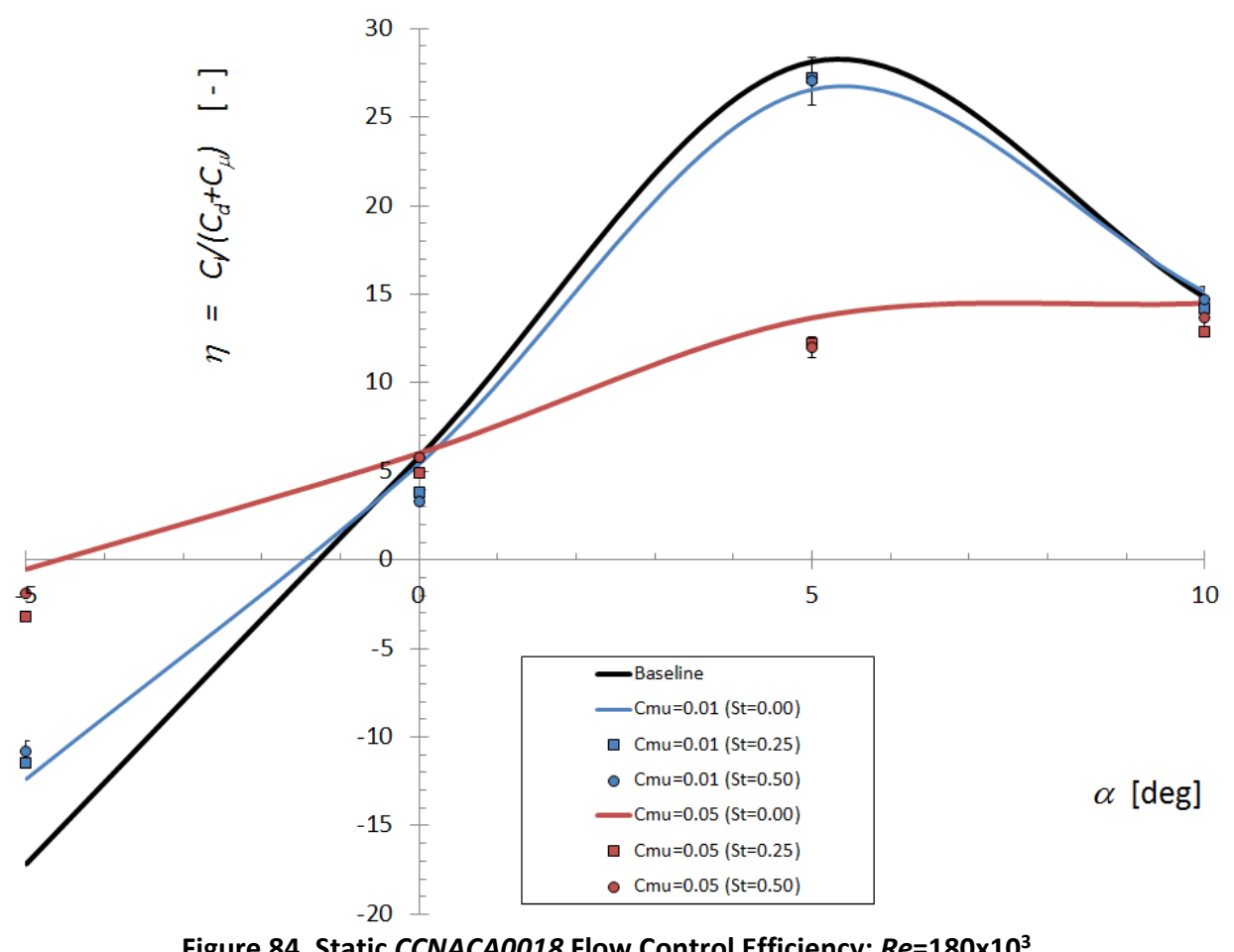

Figure 84. Static CCNACA0018 Flow Control Efficiency: $R e=180 \times 10^{3}$

However, at every other AoA tested, 5\% blowing conditions matched, or exceeded, those of baseline and low $C_{\mu}$ cases. This disparity might indicate a need to vary flow control conditions, based on specific AoA, to optimize VAWT blade performance.

The static airfoil efficiency for $R e=300 \times 10^{3}$ is plotted in Figure 85 . For positive AoA, unsteady $C_{\mu}=0.01(S t=0.25)$ provides optimal performance over steady flow control of $1 \%$ and $5 \%$. The higher 
efficiency is achieved at mass flow reductions of $30 \%$ and $188 \%$, when compared to steady CC of $C_{\mu}=0.01$ and $C_{\mu}=0.05$, respectively. For $\alpha \leq 0^{\circ}$, steady $5 \%$ blowing results showed maximum efficiency, in agreement with outcomes for $R e=180 \mathrm{~K}$.

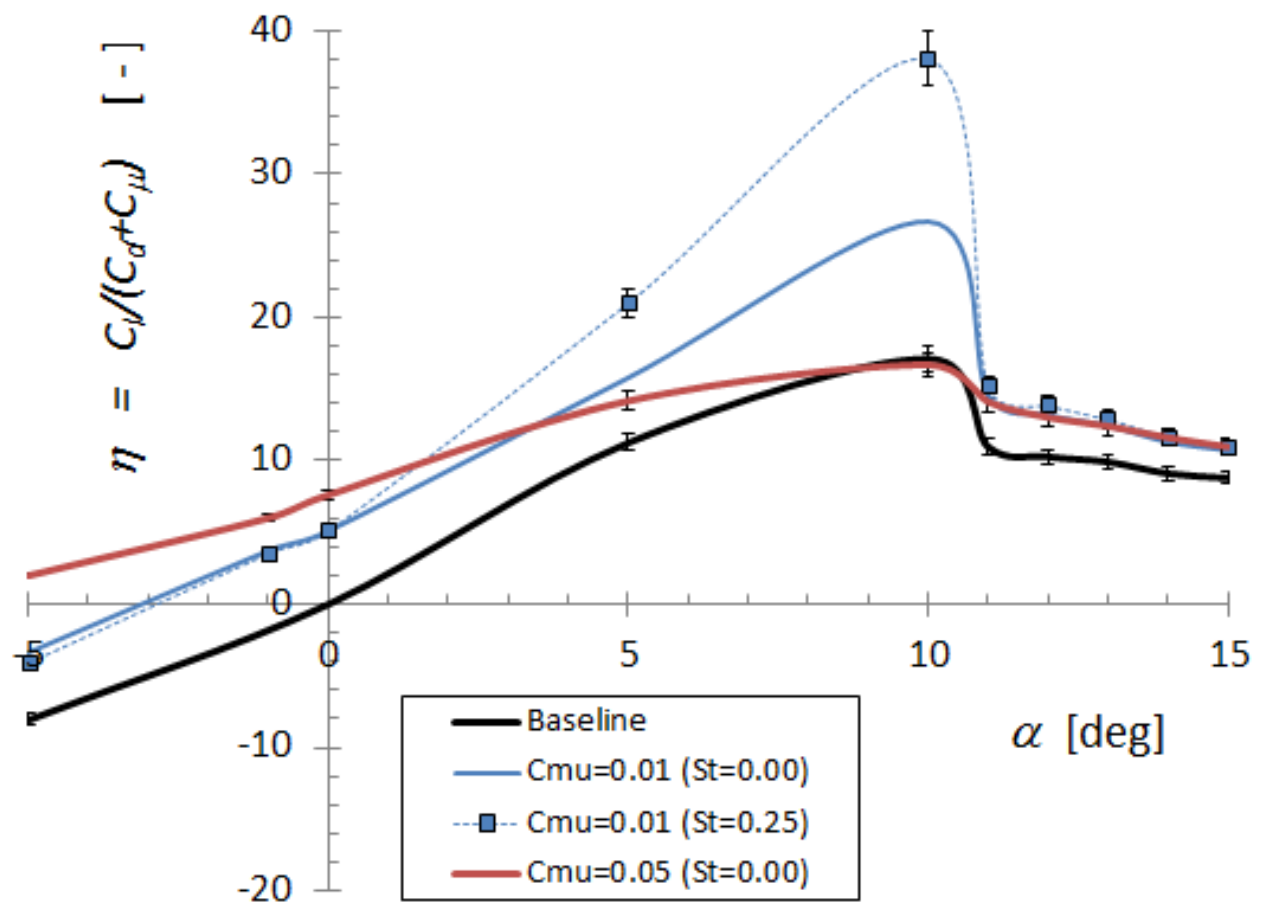

Figure 85. Static CCNACA0018 Flow Control Efficiency: $R e=300 \times 10^{3}$

Measured CCNACA0018 efficiency with pulsed blowing is compared to numerical results from Liu (154) for a $18 \% t / c$ CC wing (Figure 86). The two airfoils show qualitative agreement, with optimal performance found for low momentum blowing $\left(C_{\mu}<0.02\right)$. Also, both airfoils show comparable performance trends for pulsed blowing, generating over $90 \%$ of steady CC lift enhancement with $30-50 \%$ mass flow reduction. 


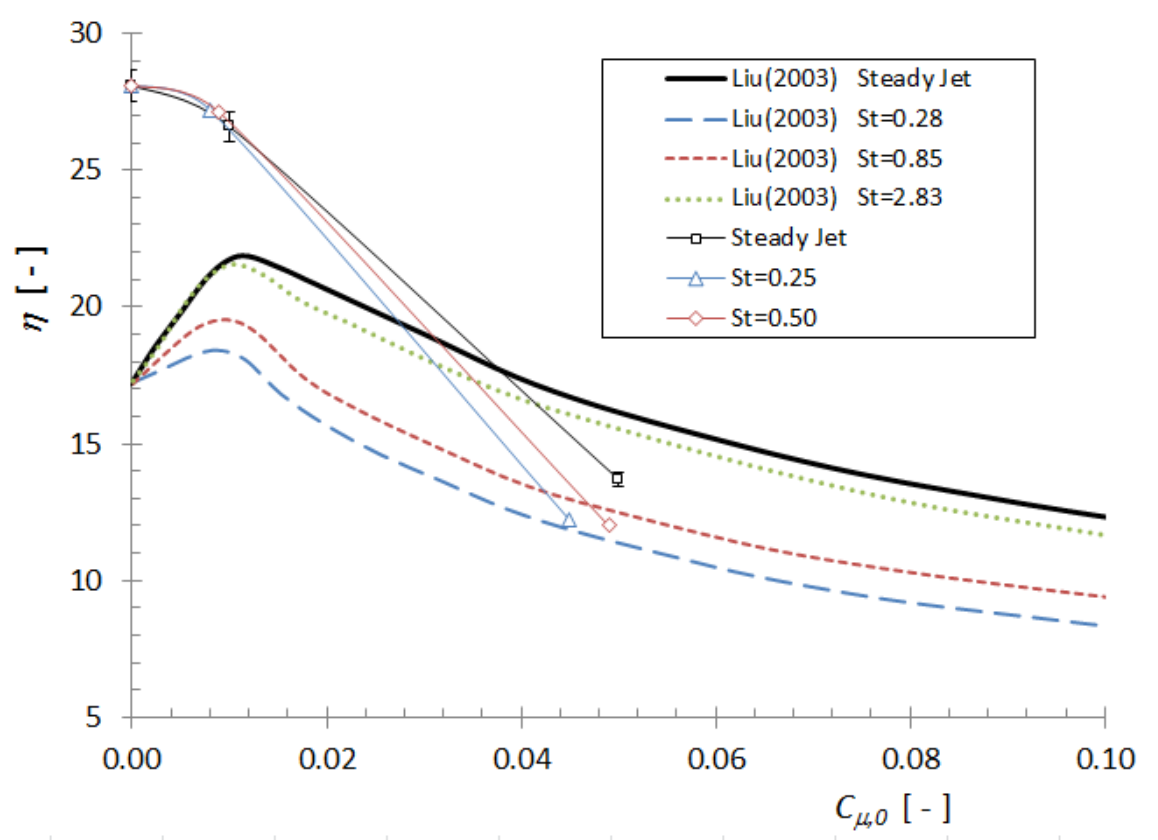

Figure 86. Airfoil Efficiency: CCNACA0018 Compared to CCW of Liu (154)

\subsection{Wind Tunnel Results: Pitching Airfoil}

All wind tunnel data included in this section was measured with the (2D) CCNACA0018 airfoil model, undergoing sinusoidal pitch oscillations, in the WVU Closed Loop Subsonic Wind Tunnel. The data was collected to expand CIRA's database of static CCVAWT blade performance to include the influence of dynamic pitch and stall on airfoil performance. Measured data from baseline and steady blowing conditions will first be compared to previous CIRA experiments to validate the experimental test set-up. For steady blowing conditions, both lift and drag results measured for the CCNACA will be reported to update previous CIRA data. Next, to evaluate the performance of pulsed CC, airfoil efficiency will be compared against steady blowing. This form of data presentation was chosen to reduce the number of plots needed for sufficient evaluation of results.

\subsubsection{Baseline Validation with Historical Data}

To validate the pitching airfoil data measured for the baseline CCNACA0018 model, the aforementioned limits based on compiled data from the literature (see Figure 48 and Figure 49 Section 2.3.9) will be used for comparison. The baseline lift measured for the CCNACAO018 under various $R e, k$, and $\alpha_{\max }$ are included in Figure 87 . It can be seen that the measured lift shows excellent agreement compared to data from the literature. 


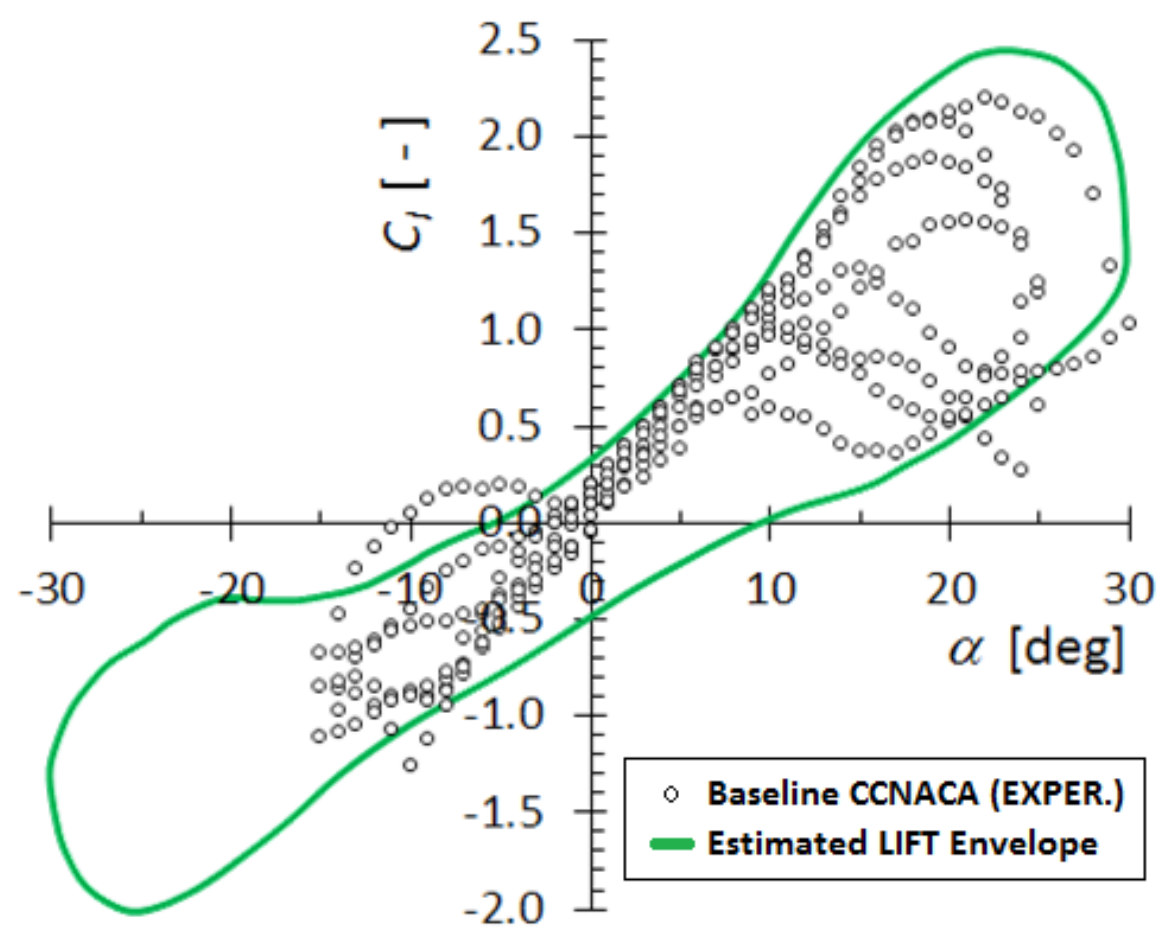

Figure 87. Baseline CCNACA0018 Lift Compared to Data from the Literature

The measured CCNACA0018 drag is compared to the estimated limit range from historic data in Figure 88. The baseline drag data from this study shows close agreement to that of conventional airfoil data from the literature.

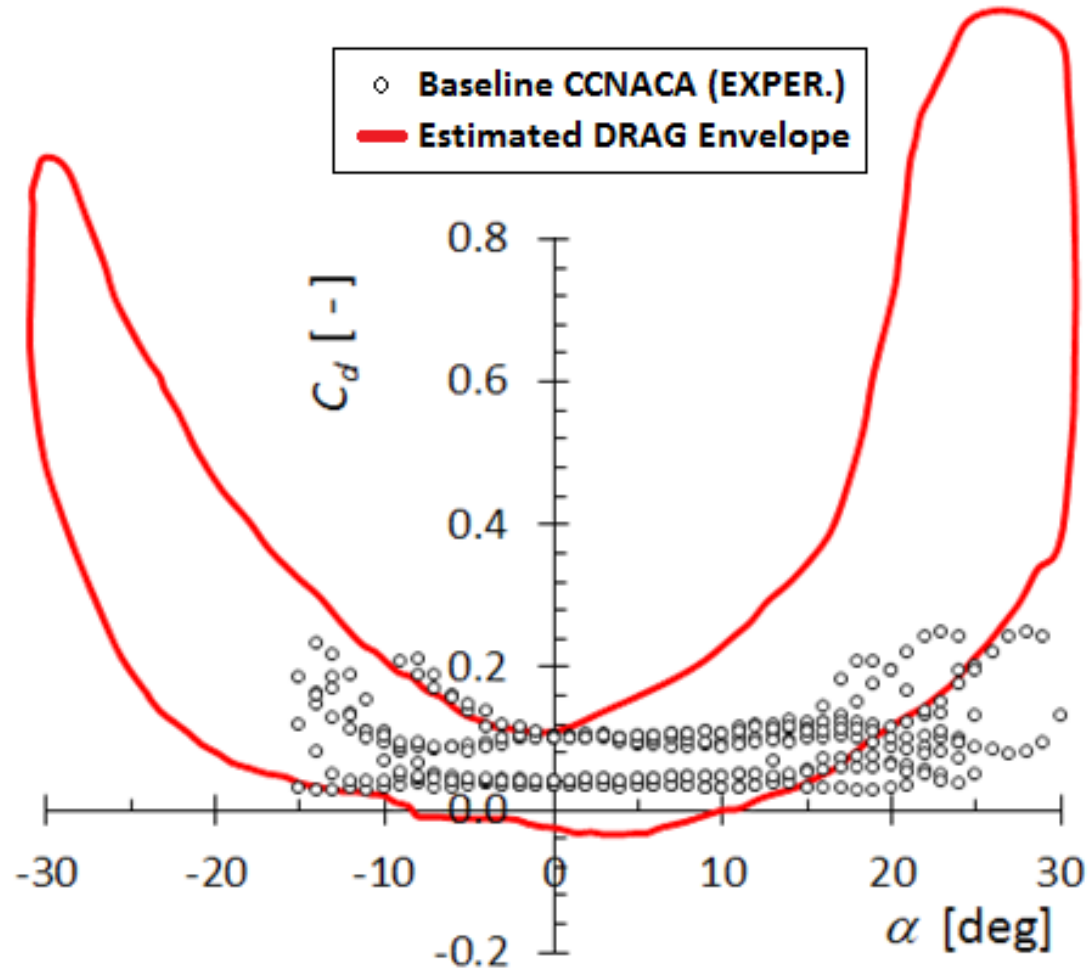

Figure 88. Baseline CCNACA0018 Drag Compared to Data from the Literature 


\subsubsection{Influence of Dynamic Pitch}

The influence of pitching motion and dynamic stall on baseline lift, relative to static wind tunnel measurements, can be seen in Figure 89. For the static case, stall was reached at $\alpha_{s s}=16^{\circ}$. Typical dynamic stall characteristics are realized, including an increase in $\mathrm{d} C_{1} / \mathrm{d} \alpha$ near static stall, along with lift overshoot and $\alpha_{\text {stall }}$ extension. The increased lift-curve slope occurs due to the formation and strengthening of the DSV.

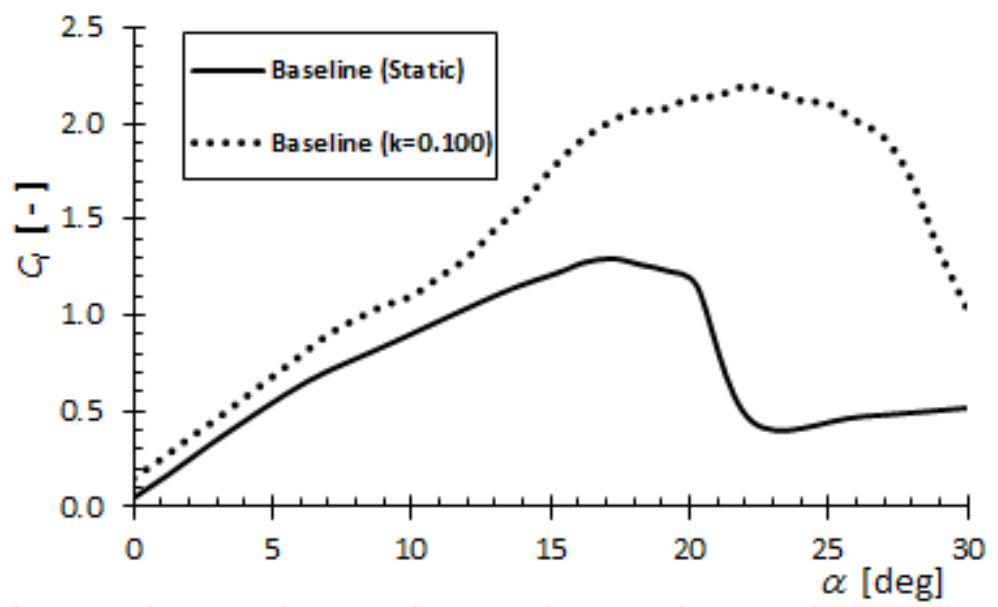

Figure 89.Influence of Airfoil Pitch on Integrated Lift Coefficient: $R e=180 \times 103, k=0.100\left(\alpha_{\max }=30^{\circ}\right)$

Dynamic pitch prolonged stall by approximately $6^{\circ}\left(\alpha_{D S}=22^{\circ}\right)$, where maximum lift was increased by $70 \%$ $\left(C_{l, \max }=2.194\right)$. The total baseline lift during pitch-up, calculated by the total area under the $C_{1}-\alpha$ curve, was increased by $87 \%$ due to the influence of dynamic stall. A comparison of total lift for all baseline and blowing conditions is included in Figure 90, compared to static data $(k=0.000)$. For each baseline and blowing condition, an increase in reduced pitch frequency increases total lift between $0^{\circ} \leq \alpha \leq 30^{\circ}$. The figure proves the need to use pitching airfoil data for analytical model inputs, as the maximum pitch rate increased baseline, steady $C_{\mu}=0.01$, and $C_{\mu}=0.05$ lift by $65 \%, 71 \%$, and $47 \%$, respectively. 


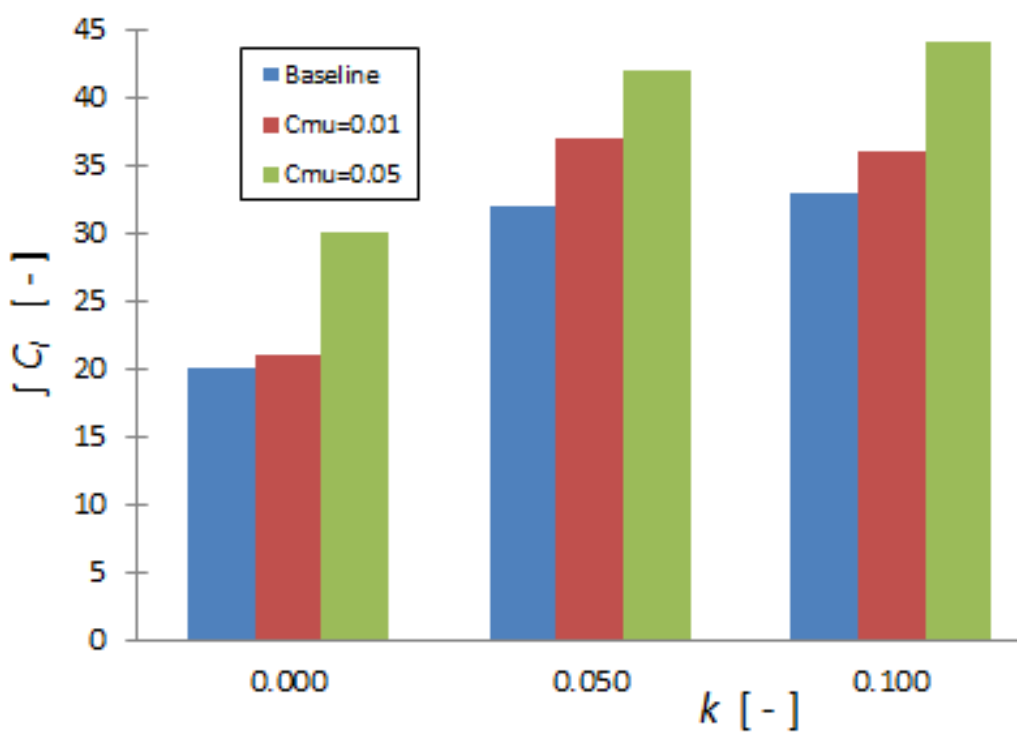

Figure 90. Influence of Dynamic Pitch on CCNACA0018 Total Airfoil Lift

A similar comparison of airfoil lift to drag, as a function of reduced pitch frequency, is included in Figure 91. For the static case, all CC conditions reveal similar total L/D values between $0^{\circ} \leq \alpha \leq 30^{\circ}$. As $k$ increases, airfoil lift increases while drag shows negligible changes, resulting in significant L/D improvements for all cases.

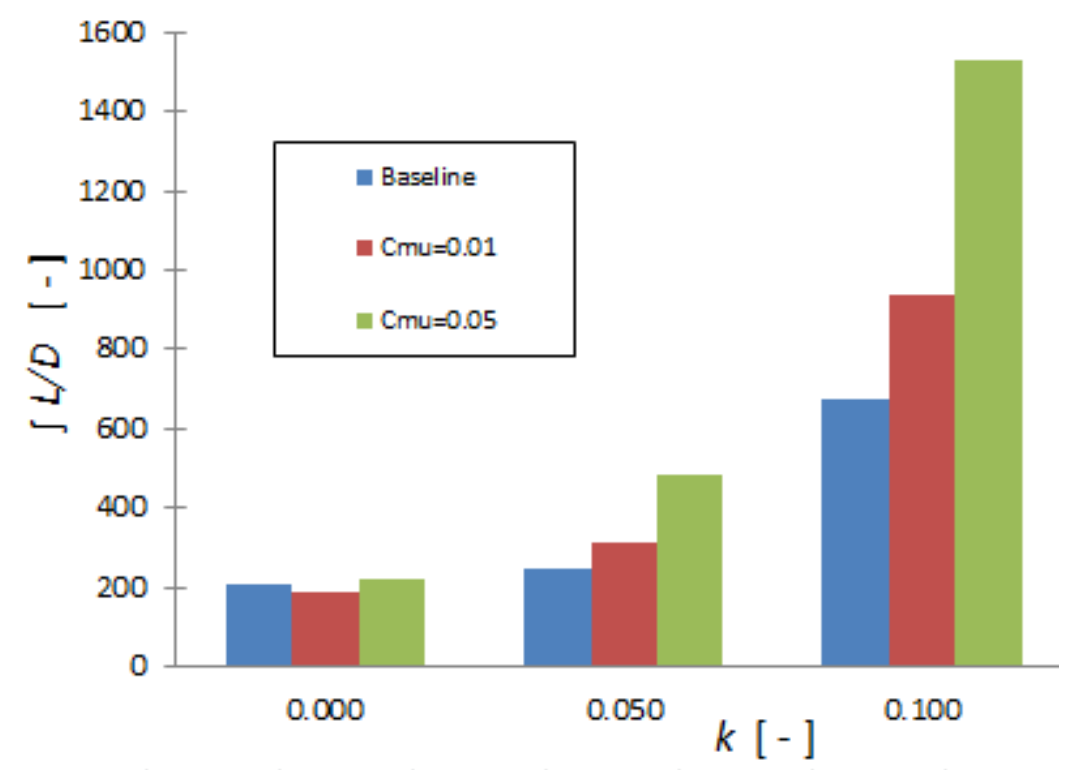

Figure 91. Influence of Dynamic Pitch on CCNACA0018 Total Airfoil Lift-to-Drag

Finally, the airfoil efficiency $(\eta)$ is compared for baseline and steady CC conditions based on the rate of airfoil pitch (Figure 92). The efficiency metric is essential to estimate CCVAWT performance, due to the consideration of momentum addition to create the CC jet. For mild pitch rotation $(k=0.050)$, a 
steady jet of $C_{\mu}=0.01$ reveals an increased efficiency of $17 \%$ relative to baseline performance, while $C_{\mu}=0.05$ resulted in a baseline improvement of $22 \%$. For the higher pitch rate $(k=0.100)$, low levels of $C C$ showed the best efficiency, outperforming baseline and high CC $\left(C_{\mu}=0.05\right)$ by $8 \%$ and $26 \%$, respectively. For reference, the range of pitch rates examined during wind tunnel testing reflects VAWT operation in the range $1 \leq \lambda \leq 4$.

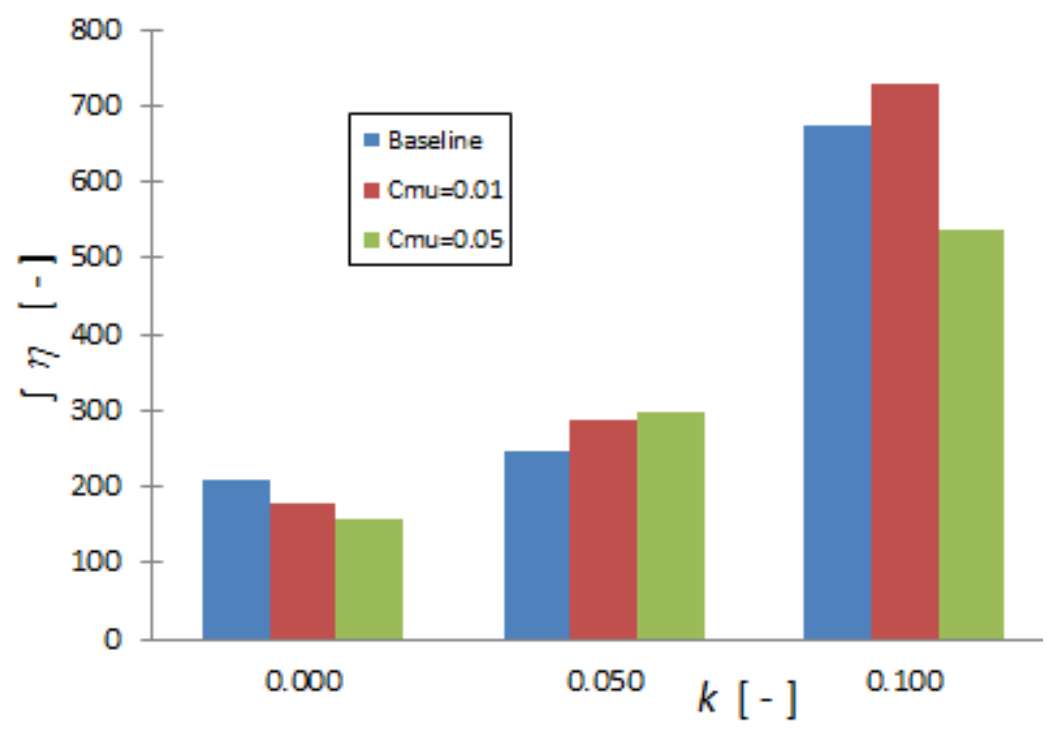

Figure 92. Influence of Dynamic Pitch on CCNACA0018 Total Airfoil Efficiency 


\subsubsection{Pitching CCNACA0018 Lift Data: Steady $C_{\mu}$}

The influence of dynamic pitch $(k=0.050)$ on low Reynolds number aerodynamics of the CCNACA with steady circulation control is depicted in Figure 93, compared to static airfoil characteristics. It should be noted that dynamic measurements are reported in $1^{\circ}$ increments, but solid lines were chosen to represent each blowing condition to lessen graphical clutter and simplify comparison. In addition, uncertainty bars are included on static data points only to reduce clutter and improve clarity for each plot.

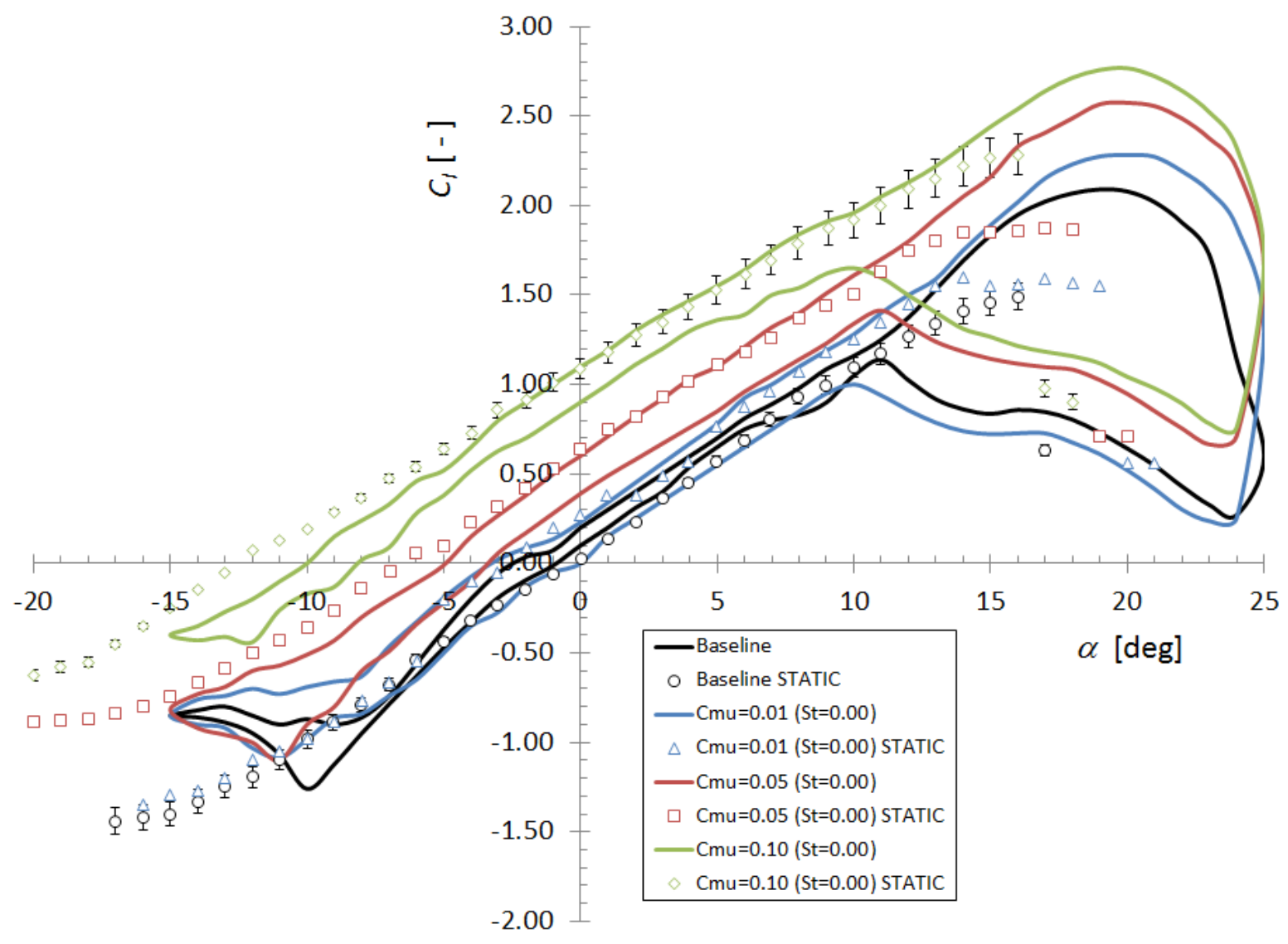

Figure 93. Pitching CCNACA0018 Lift Performance with Steady CC: $R e=180 \times 10^{3}, k=0.050\left(\alpha_{\max }=25^{\circ}\right)$ 
The influence of dynamic pitch, at a higher rotation rate $(k=0.100)$, of the CCNACA with steady circulation control is depicted in Figure 94, compared to static airfoil characteristics. The most noticeable difference between pitch rates is the improved post-stall lift recovery for $k=0.100$. Also, the slower pitch causes a more dramatic lift drop-off at stall at the end of the pitch-up maneuver $\left(23^{\circ} \leq \alpha \leq 25^{\circ}\right)$. For $k=0.100$, the stall process is more gradual up to $\alpha_{\max }=25^{\circ}$ and lift drop-off occurs due to the physical pitch-down motion of the airfoil.

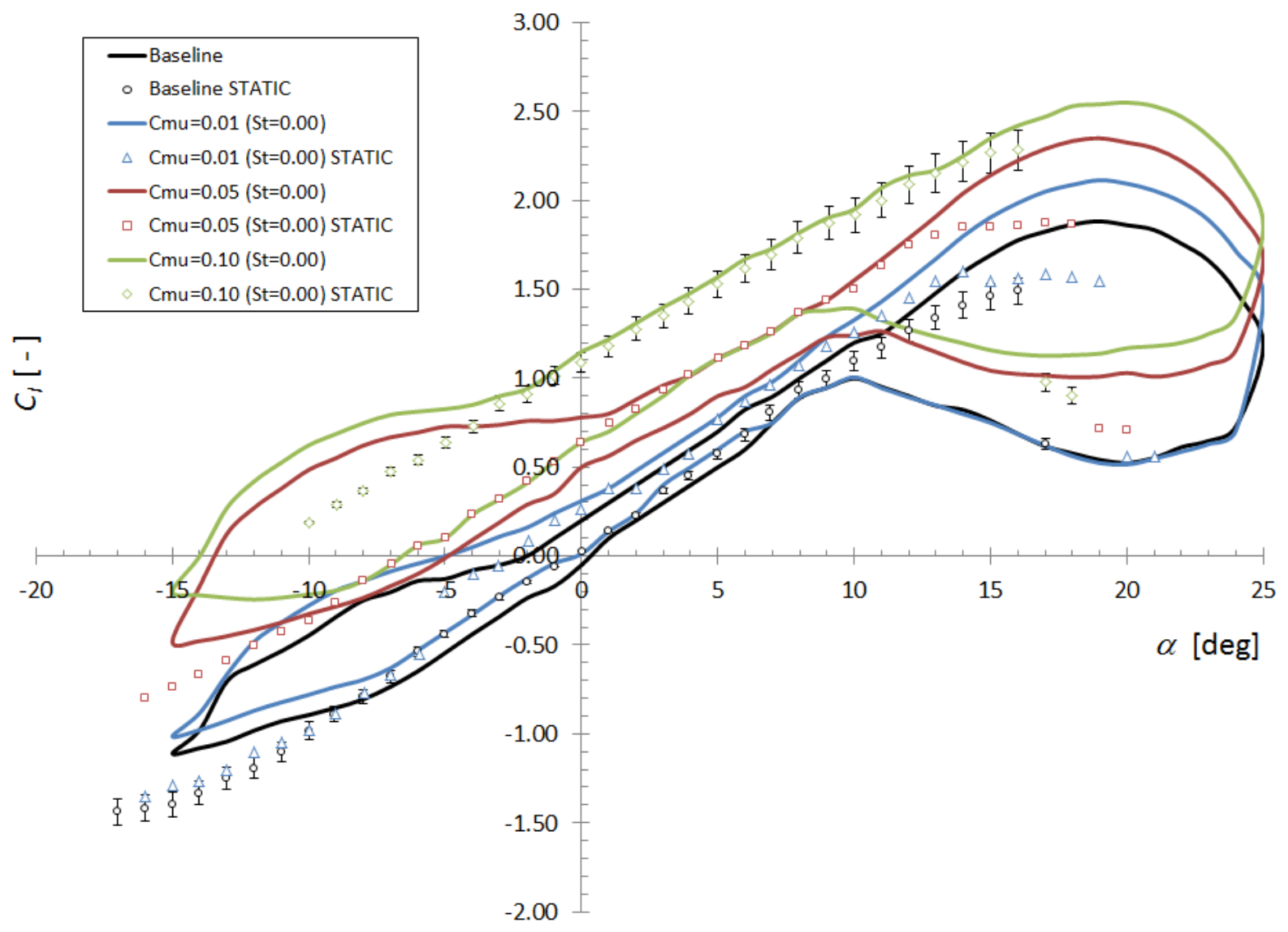

Figure 94. Pitching CCNACA0018 Lift Performance with Steady CC: $R e=180 \times 10^{3}, k=0.100\left(\alpha_{\max }=25^{\circ}\right)$ 
The lift enhancement due to steady momentum input for higher Reynolds number flow $\left(\operatorname{Re}=300 \times 10^{3}\right)$ is plotted in Figure 95. For this flow condition, similar lift enhancements between each steady $C_{\mu}$ increment are comparable to previous flow conditions for $\alpha \geq 0^{\circ}$. However, for negative AoA, tighter hysteresis loops are noticed for higher magnitudes of blowing $\left(C_{\mu}=0.05,0.10\right)$, whereas baseline and $C_{\mu}=0.01$ show greater discrepancy between pitch-up and pitch-down motions.

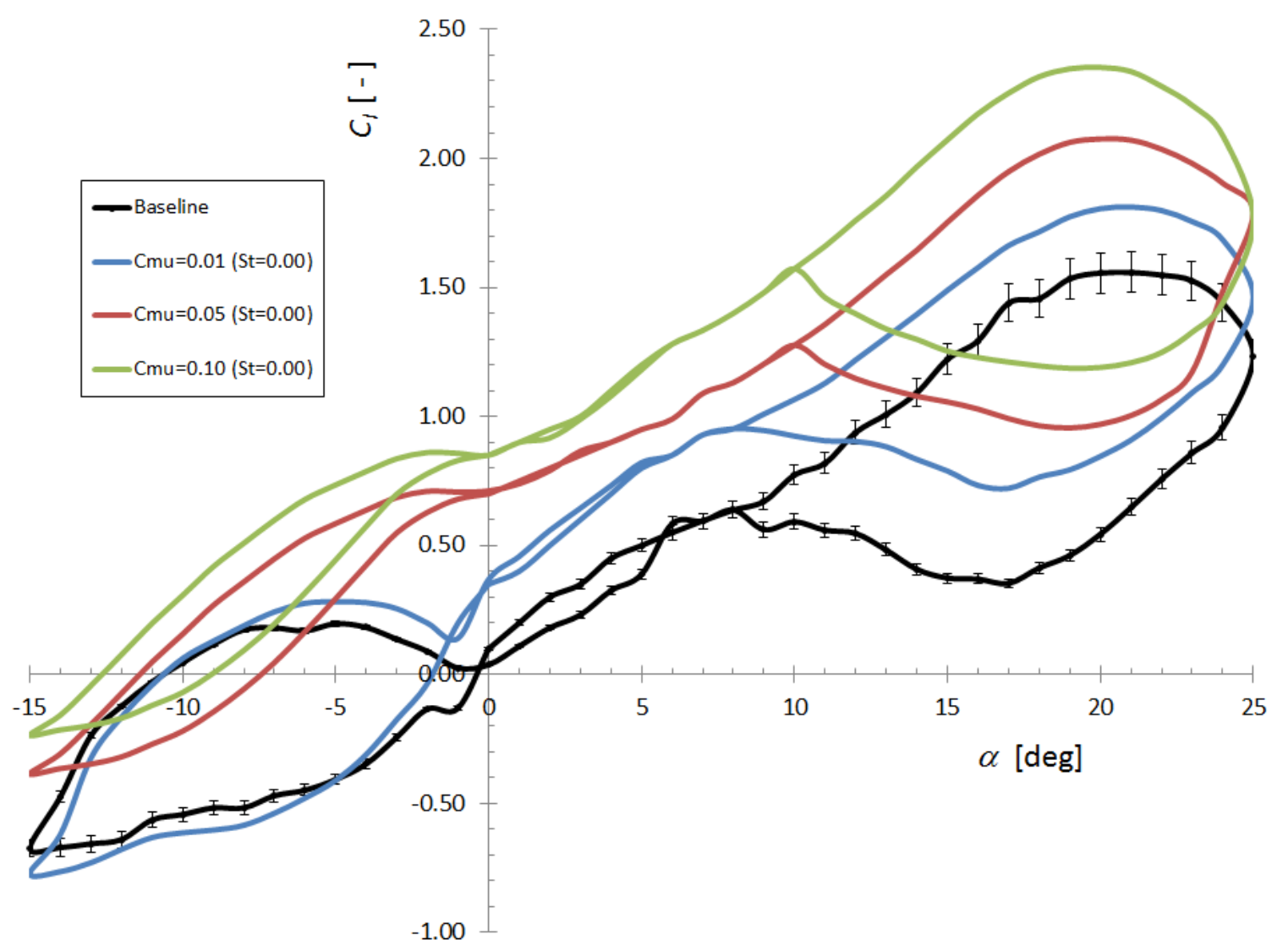

Figure 95. Pitching CCNACA0018 Lift Performance with Steady CC: $R e=300 \times 10^{3}, k=0.050\left(\alpha_{\max }=25^{\circ}\right)$ 


\subsubsection{Pitching CCNACA0018 Lift Data: Pulsed $C_{\mu}$}

The influence of pitching motion on CCNACA lift, both steady and pulsed CC blowing of $C_{\mu}=0.05$, are shown in Figure 96 . Steady blowing shows optimal lift enhancement during the pitch cycle, while pulse blowing of $S t=0.50$ offers comparable performance with $5 \%$ reduced mass flow. Pulsed blowing of $S t=0.25$ also shows comparable lift enhancements for $\alpha \geq 13^{\circ}$, with $12 \%$ reduced mass flow compared to steady blowing.

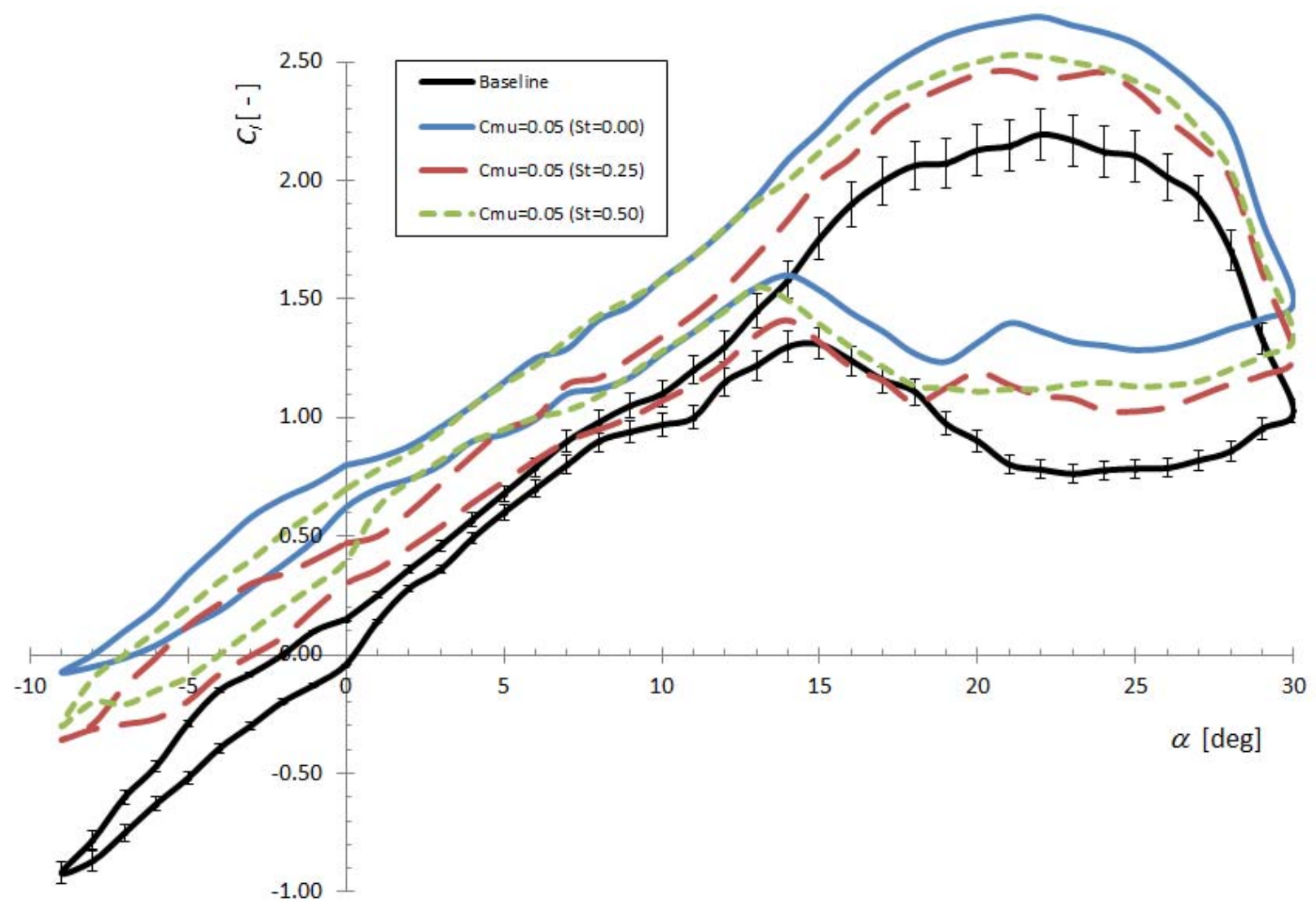

Figure 96. Pitching CCNACA0018 Lift Performance with Pulsed CC: $R e=180 \times 10^{3}, k=0.100\left(\alpha_{\max }=30^{\circ}\right)$ 


\subsubsection{Pitching CCNACA0018 Drag Data: Steady $C_{\mu}$}

The influence of airfoil pitch on the baseline drag is included in Figure 97. For all drag measurements from the pitching CCNACA experiments, the values corresponding to pitch-up and pitchdown maneuvers were very close at small AoA (Figure 97).
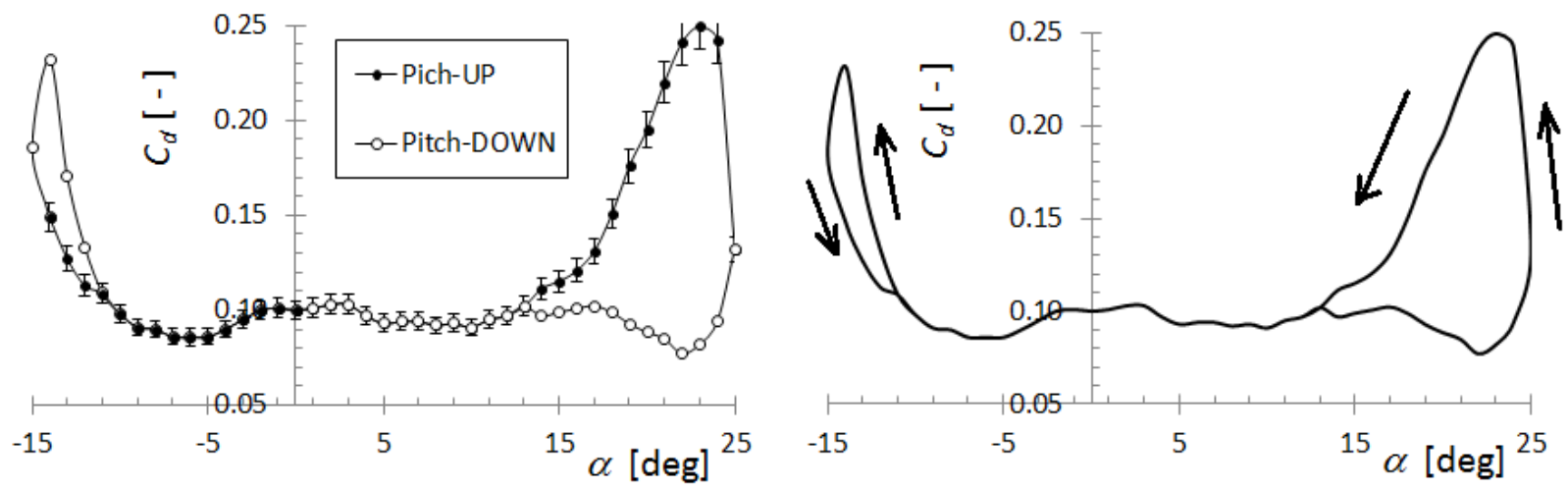

Figure 97. Baseline CCNACA Drag Data Simplification: $R e=180 \times 10^{3}, k=0.100$

The plot on the left in Figure 97 includes each data point throughout the pitch cycle (filled symbols represent data recorded while the airfoil increases AoA while open symbols reflect data points from the pitch-down portion of pitch). For a given angle of attack, when data points fell within the range of experimental uncertainty, the two points were averaged for simplification. This process was followed to improve clarity when comparing multiple data sets on a single plot; the salient features of the force hysteresis loops are still preserved for evaluation between varying test conditions. Thus, it is evident how the updated force curves for the pitching CCNACA will provide more realistic inputs to analytical models for more accurate power predictions of a CCVAWT. 
Figure 98 reveals the influence of sinusoidal pitch $(k=0.100)$ on measured sectional drag of the baseline CCNACA0018 at $R e=300 \times 10^{3}$. The static drag curve closely compares to the dynamic curve for pre-stall AoA $\left(0^{\circ} \leq \alpha \leq+10^{\circ}\right)$, falling nearly within the experimental error limits of each other. Since $\alpha_{S S}<\alpha_{D S}$, the rapid change in $\mathrm{d} C_{d} / \mathrm{d} \alpha$ occurs at the corresponding $A \circ A$; the magnitude and slope otherwise show agreement for the static curve and the pitch-up portion of the dynamic curve.

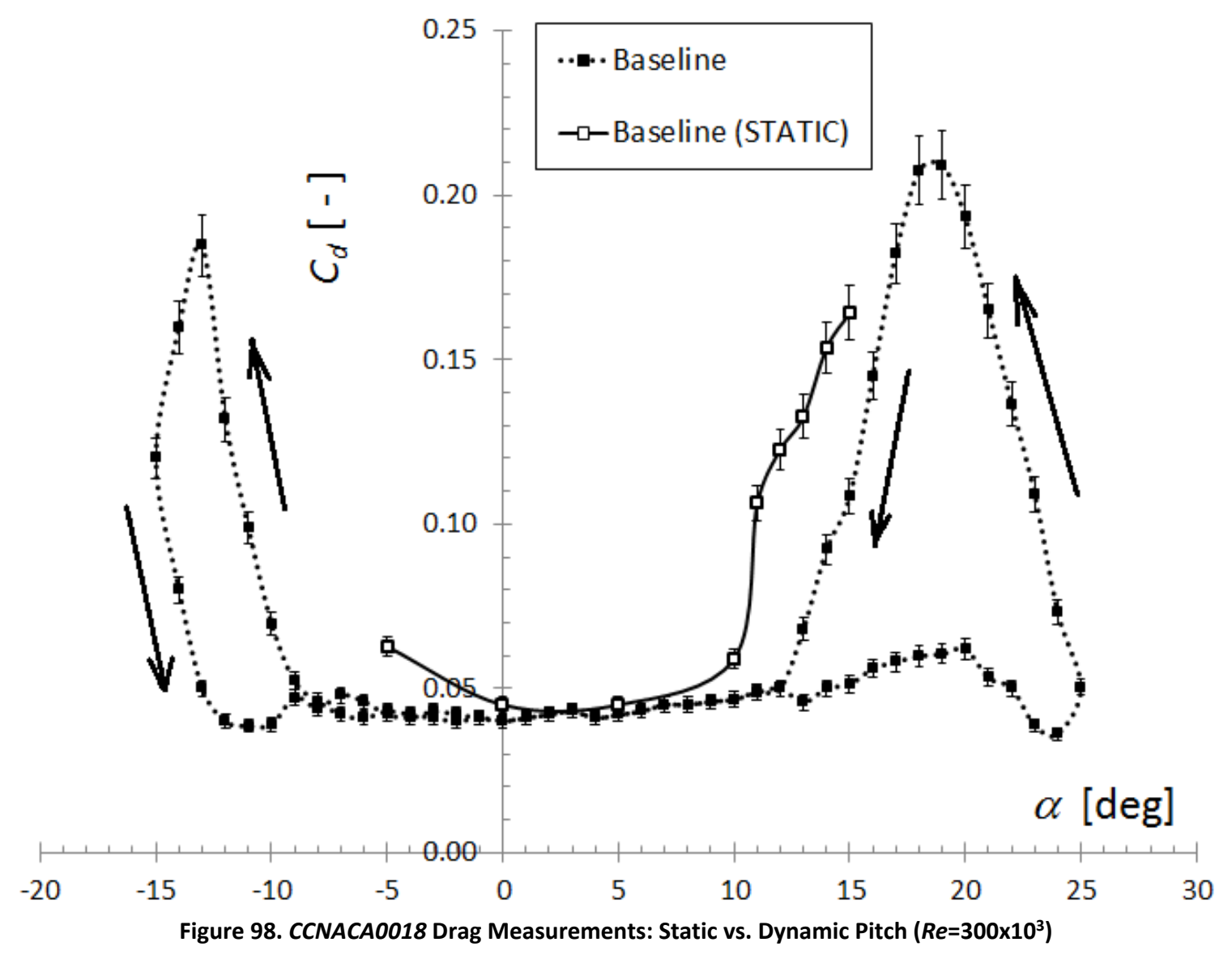


Figure 99 shows the influence of steady blowing CC on a pitching CCNACA airfoil at $\operatorname{Re}=180 \times 10^{3}$ at the slower pitch rate of $k=0.050$. A "figure-8" hysteresis loop is created for positive AoA. During pitchup, the DSV formation (LE) begins around $\alpha=15^{\circ}$ as marked by an increase in drag. As the vortex travels toward the TE $(x / c \approx 0.50)$, the drag is temporarily decreased as the vortex generates large recirculation on the airfoil upper surface, decreasing profile drag $\left(\alpha=18^{\circ}\right)$. As the vortex reaches the TE and is eventually shed, $C_{d}$ increases rapidly as the airfoil experiences complete upper surface separation (dynamic stall, $\alpha=22^{\circ}$ ).

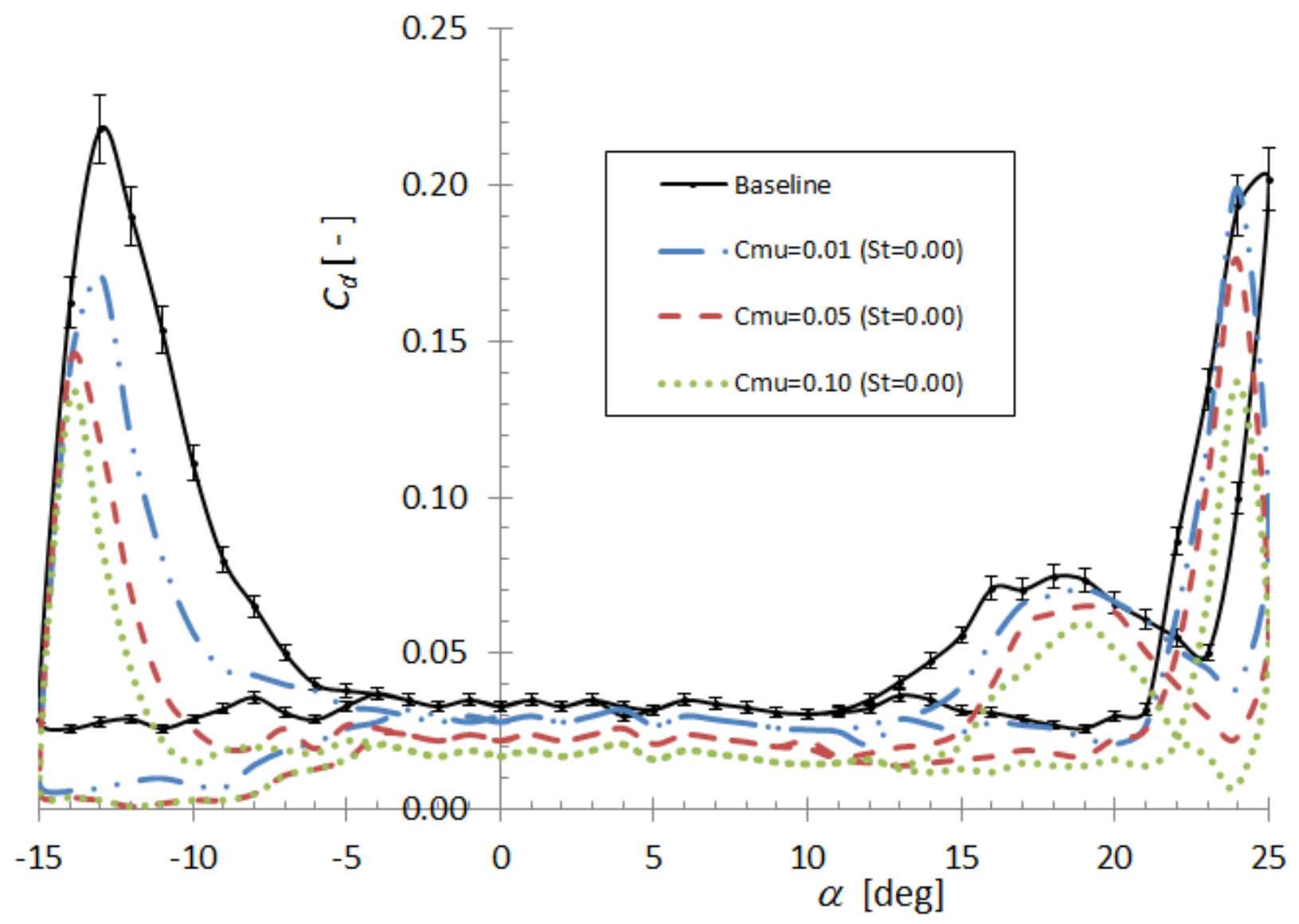

Figure 99. CCNACA0018 Drag with Steady CC: $R e=180 \times 10^{3}, k=0.050\left(\alpha_{\max }=25^{\circ}\right)$ 
Figure 100 shows the influence of steady blowing CC on a pitching CCNACA airfoil at $\operatorname{Re}=180 \times 10^{3}$ for an increased pitch rate of $k=0.100$. Arrows are included to decipher measurements from pitch-up and pitch-down motions. Compared to $k=0.050$, a single counter-clockwise (CCW) hysteresis loop forms for positive AoA. The increased rotation rate (and inertial forces) creates a stronger upper surface vortex, increasing airfoil circulation during pitch up and mitigating profile drag increases. The mild $C_{d}$ increase from the $k=0.050$ case $\left(15^{\circ} \leq \alpha \leq 20^{\circ}\right)$, due to DSV formation, is shifted to $21^{\circ} \leq \alpha \leq 25^{\circ}$ for $k=0.100$, where full stall appears to be prematurely imparted by the airfoil stopping at $\alpha=25^{\circ}$ and beginning pitchdown motion. The DSV looks to form naturally, but is prematurely convected and shed due to a rapid change in airfoil motion caused by the physical pitch mechanism, versus large pressure gradients and full-scale separation.

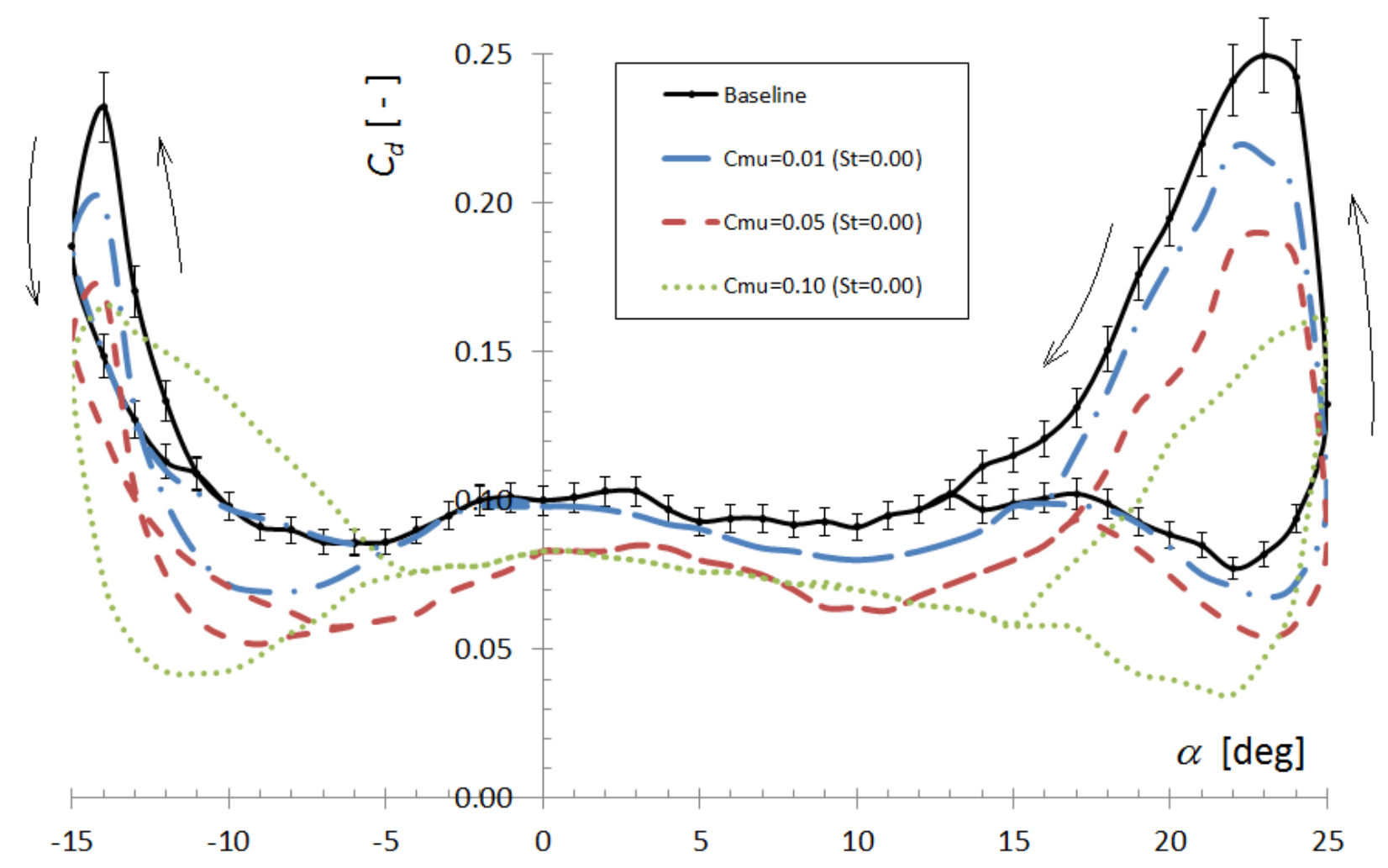

Figure 100. CCNACA0018 Drag with Steady CC: $\operatorname{Re}=180 \times 10^{3}, k=0.100\left(\alpha_{\max }=25^{\circ}\right)$ 
The drag measurements from a pitching CCNACA airfoil $(k=0.050)$ at the higher $\operatorname{Re}=300 \times 10^{3}$ are included in Figure 101, for steady CC actuation. Overall, increasing $C_{\mu}$ reduces $C_{d}$ at nearly all AoA positions throughout the pitch cycle. As in previous cases, higher levels of steady blowing $\left(C_{\mu}=0.05,0.10\right)$ show a reduced reattachment process, marked by a flatter slope during pitch-down, likely a cause of the increased camber created by CC.

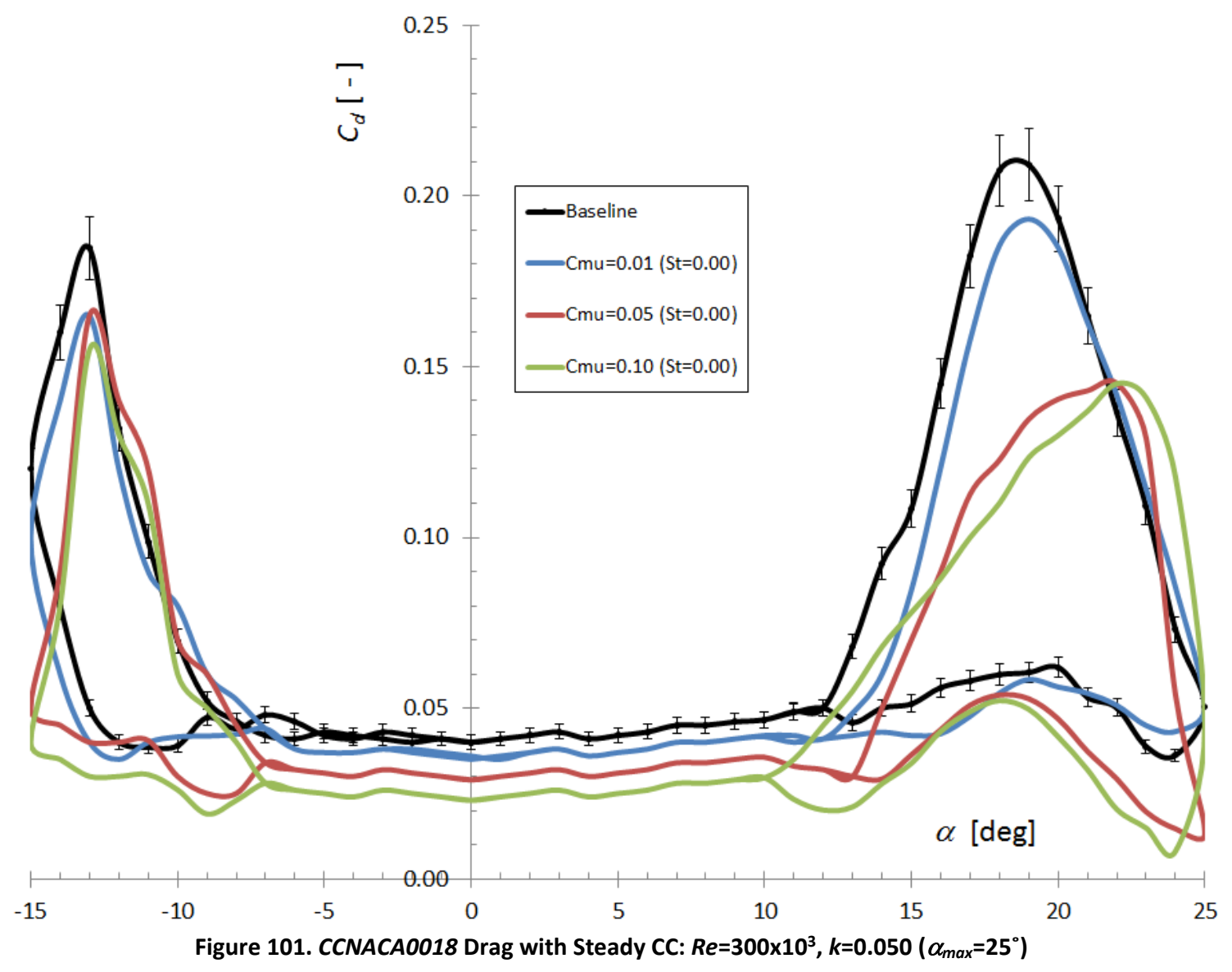




\subsubsection{Pitching CCNACA0018 Drag Data: Pulsed $C_{\mu}$}

Figure 102 includes CCNACA0018 section drag results for a variety of pulsed blowing conditions $\left(C_{\mu}=0.01\right)$ at $\operatorname{Re}=180 \times 10^{3} \quad(k=0.100)$ following the following sinusoidal pitch: $\alpha(t)=5^{\circ}+20^{\circ} \sin (\omega t)$. Compared to the baseline airfoil, all $1 \%$ blowing conditions show reduced drag at nearly every AoA. All blowing cases are very comparable in performance, with $S t=0.25$ showing slightly better overall results.

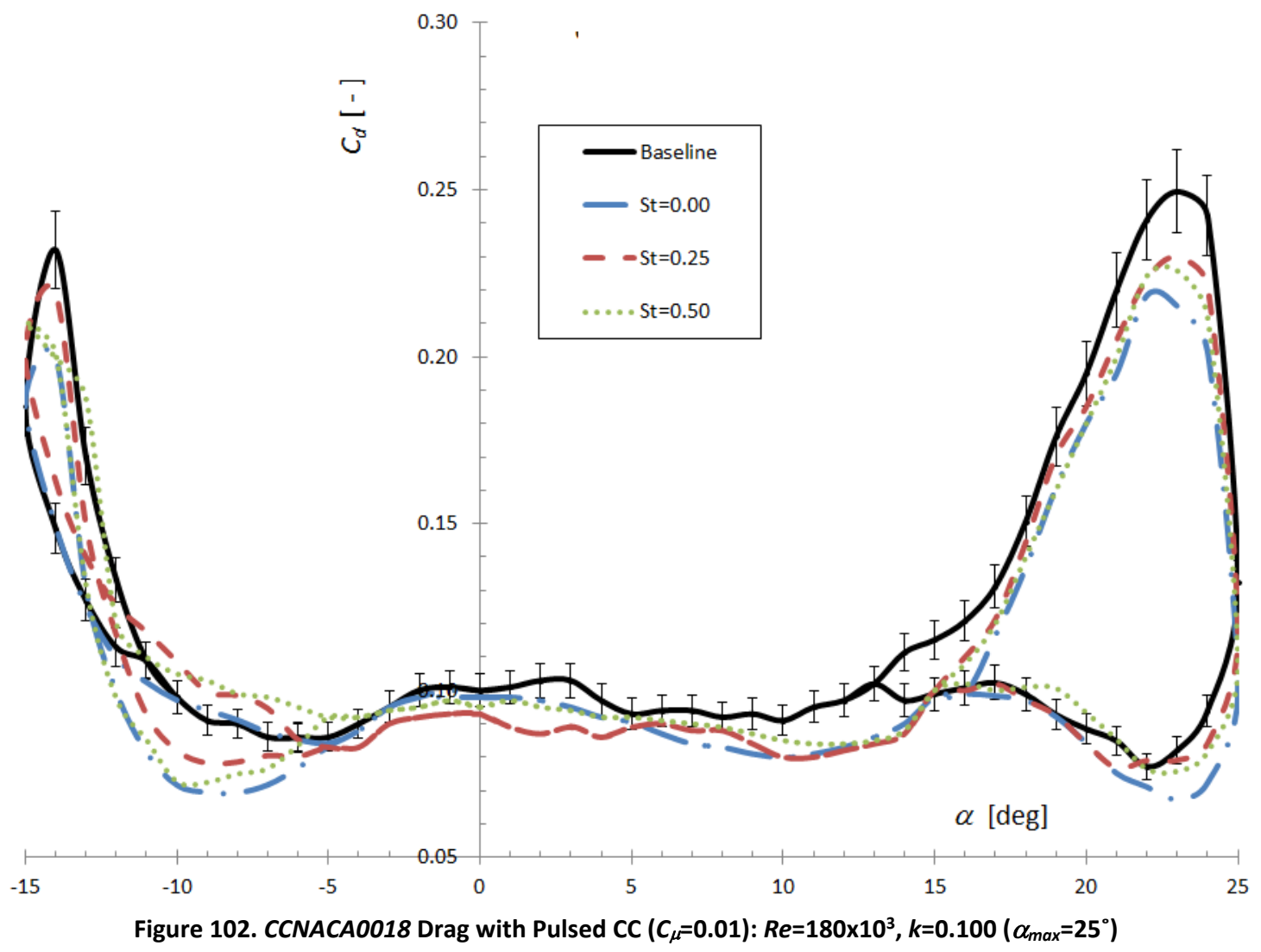


Figure 103 includes CCNACA0018 section drag results for a variety of pulsed blowing conditions $\left(C_{\mu}=0.05\right)$ at $\operatorname{Re}=180 \times 10^{3}(k=0.100)$ following the following sinusoidal pitch: $\alpha(t)=5^{\circ}+20^{\circ} \sin (\omega t)$. Again, all cases of blowing show a reduction in drag relative to the baseline airfoil. Compared to $1 \%$ blowing, $C_{d}$ is further reduced, showing improved results for all cases considered. All cases of $5 \%$ blowing show comparable drag measurements, similar to the previously described $1 \%$ blowing cases.

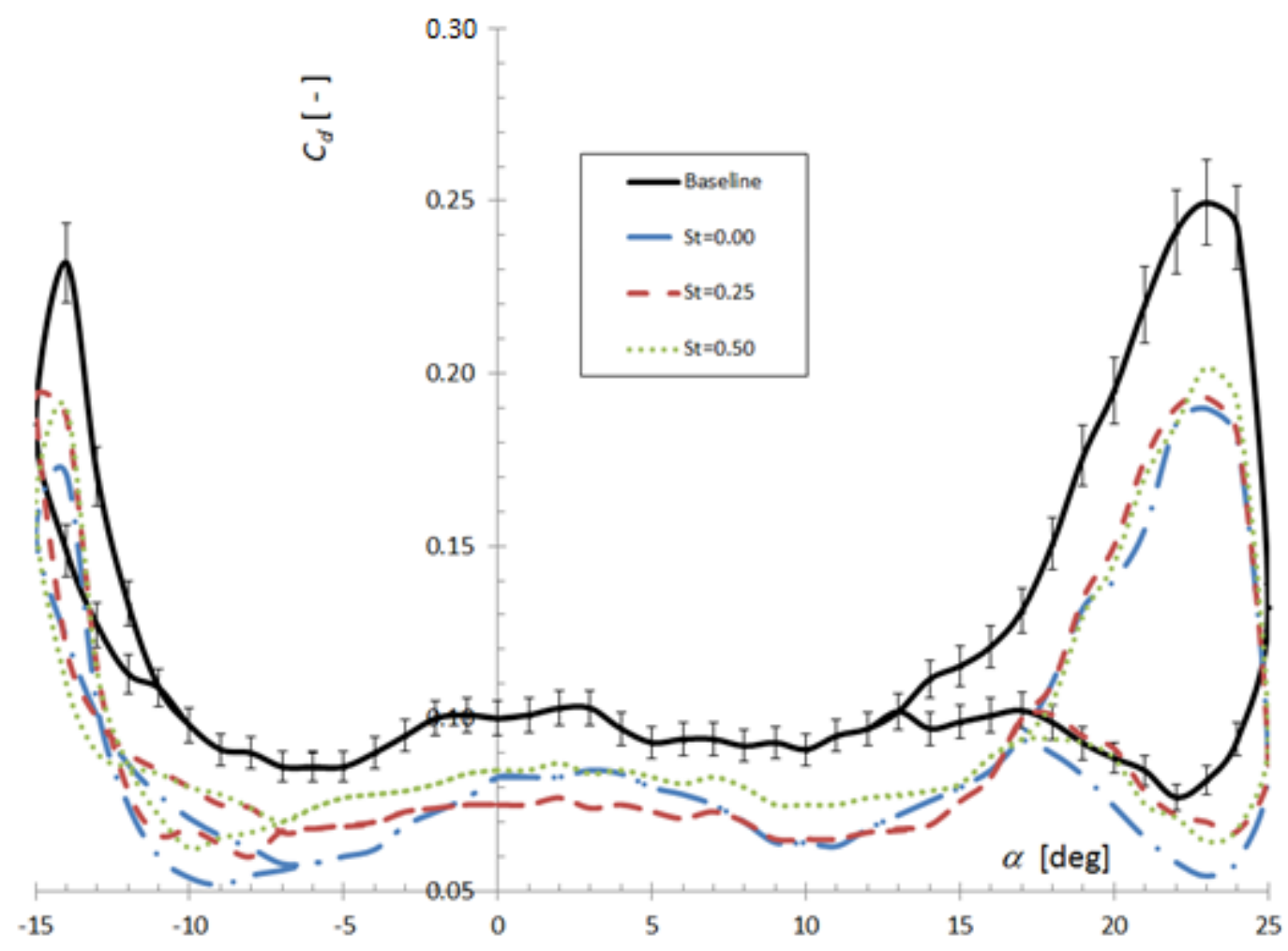

Figure 103. CCNACA0018 Drag with Pulsed CC $\left(C_{\mu}=0.05\right): R e=180 \times 10^{3}, k=0.100\left(\alpha_{\max }=25^{\circ}\right)$ 
The effect of increasing the pitch amplitude $\left(\alpha_{\max }=30^{\circ}\right)$ on drag is shown in Figure 104. Positive AoA shows similar trends to the smaller pitch amplitude of $\alpha_{\max }=25^{\circ}$. Negative AoA, on the other hand, show smaller hysteresis loops due to a smaller portion of the pitch cycle travelling into this region. The state of the boundary layer is thus more stable, resulting in reduced flow separation and lower magnitudes of drag for all cases of actuation. All cases of pulsed CC blowing show more effective flow reattachment during pitch-down, compared to steady CC.

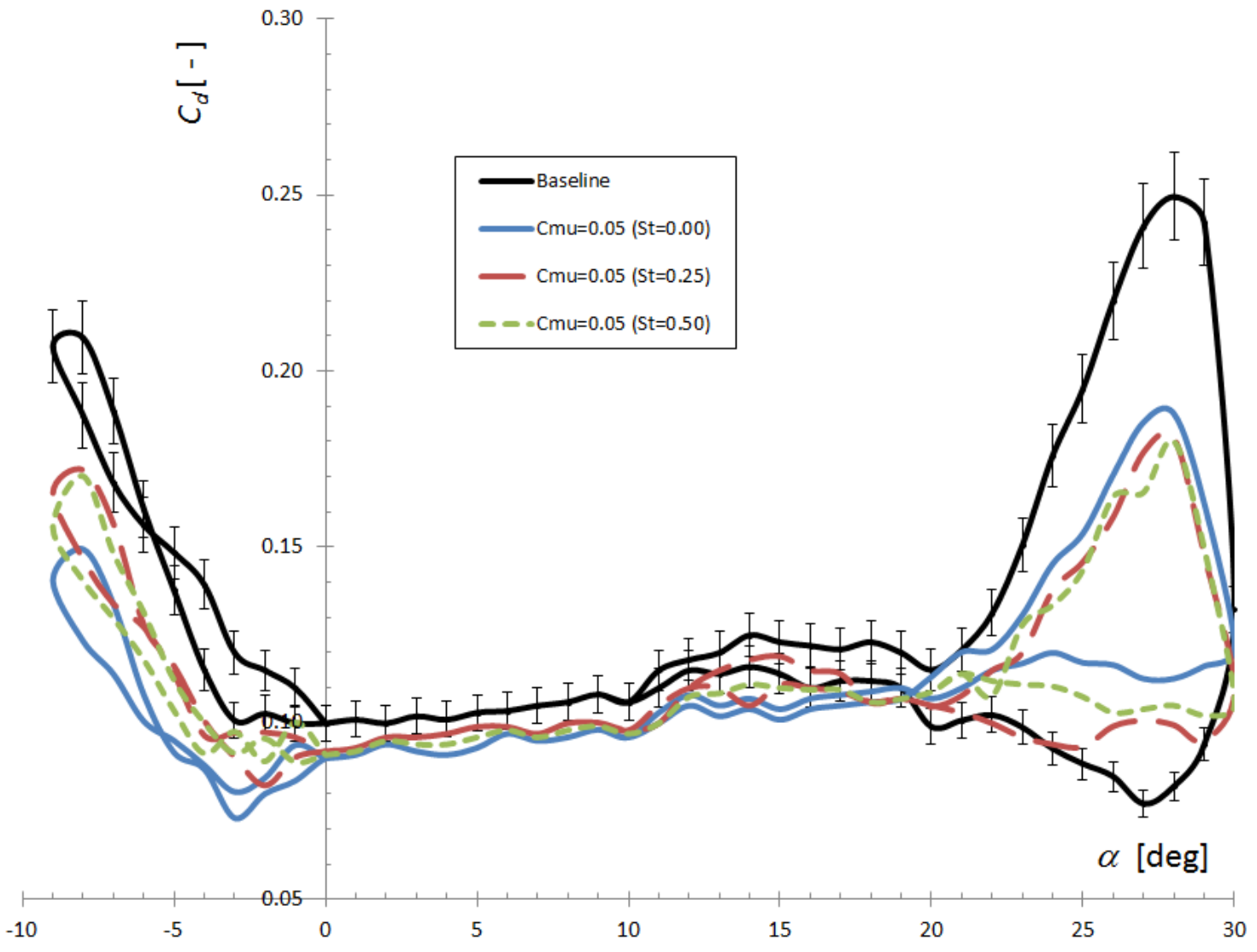

Figure 104. CCNACA0018 Drag with Pulsed CC $\left(C_{\mu}=0.05\right): R e=180 \times 10^{3}, k=0.100\left(\alpha_{\max }=30^{\circ}\right)$ 


\subsubsection{Pitching CCNACA0018: Circulation Control Efficiency $(\eta)$}

The efficiency metric was also calculated from pitching CCNACA0018 airfoil data to compare various flow control conditions. Figure 105 includes CCNACA0018 efficiency for a variety of steady blowing conditions at $R e=180 \times 10^{3}(k=0.100)$ following the following sinusoidal pitch: $\alpha(t)=5^{\circ}+20^{\circ} \sin (\omega t)$. A steady blowing condition of $C_{\mu}=0.01$ outperforms higher $C_{\mu}$ and baseline airfoil conditions for positive AoA, while higher levels of blowing $\left(C_{\mu}=0.05,0.10\right)$ show better performance for negative AoA, despite the jet-thrust component being removed from drag measurements.

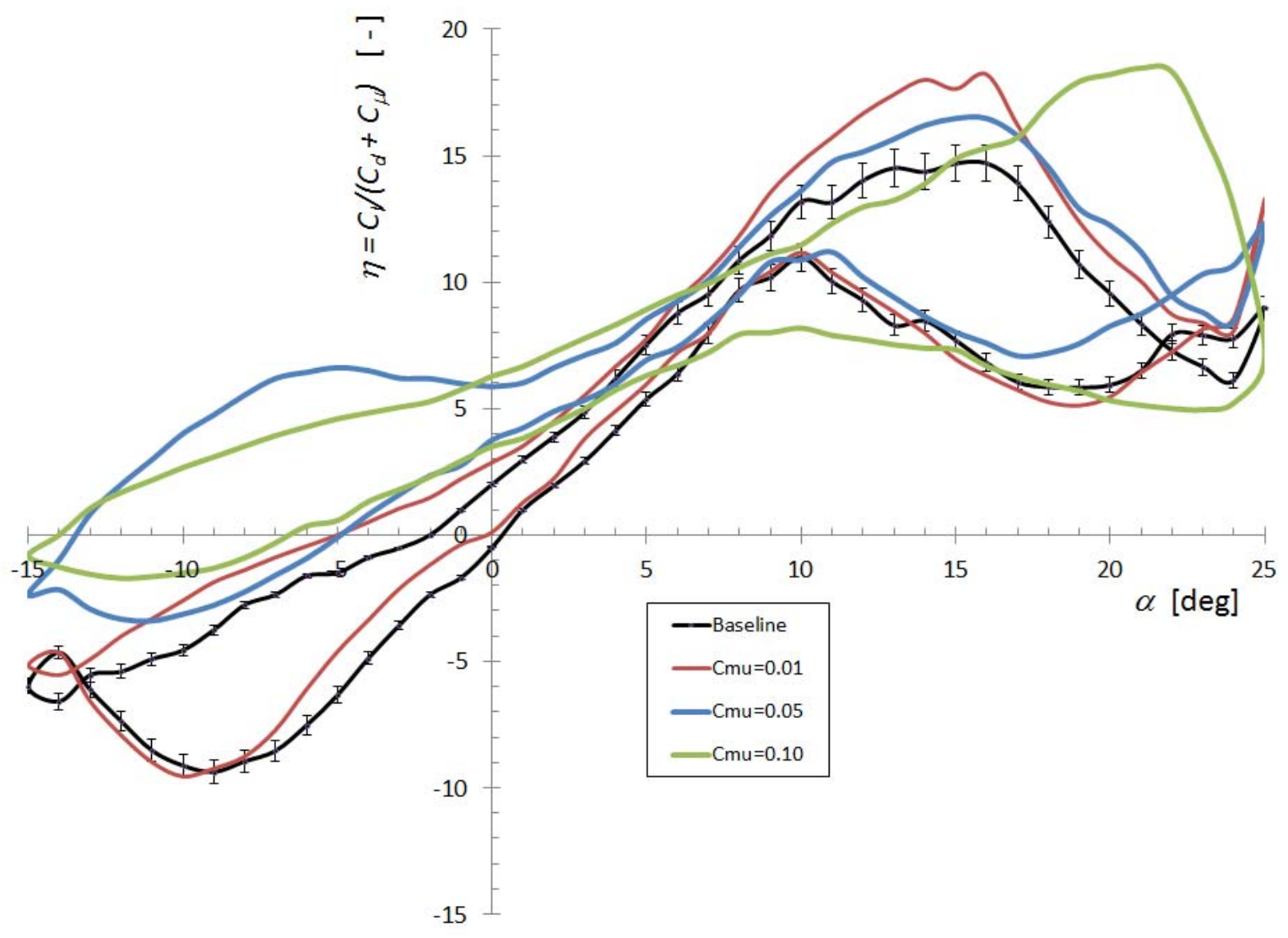

Figure 105. CCNACA0018 Efficiency with Steady CC: $R e=180 \times 10^{3}, k=0.100\left(\alpha_{\max }=25^{\circ}\right)$ 
Figure 106 includes pulsed blowing airfoil efficiency for the same wind tunnel conditions, for various actuation cases of $1 \%$ blowing. CCNACA0018 efficiency enhancements due to steady blowing are also realized for pulsed blowing of $S t=0.25\left(f_{\text {jet }}=17 \mathrm{~Hz}\right)$ and $S t=0.50\left(f_{\text {jet }}=34 \mathrm{~Hz}\right)$, with mass flow reductions of $29 \%$ and $22 \%$, respectively. Steady blowing and pulsed blowing of $S t=0.50$ have produced maximum dimensionless airfoil efficiency values of nearly 50 under these conditions.

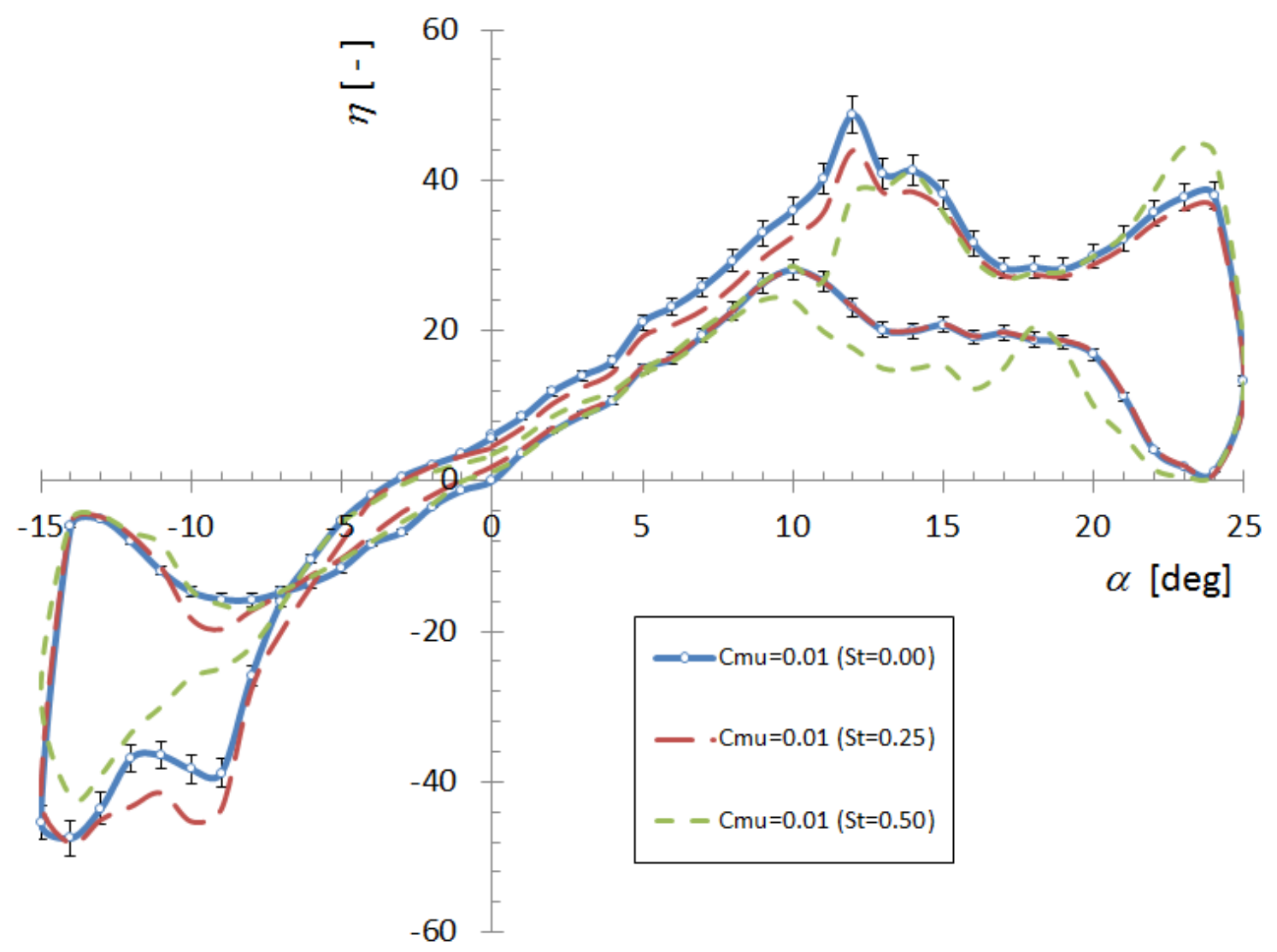

Figure 106. CCNACA0018 Efficiency with Pulsed CC $\left(C_{\mu}=0.01\right): \operatorname{Re}=180 \times 10^{3}, k=0.050\left(\alpha_{\max }=25^{\circ}\right)$ 
Pulsed CC airfoil efficiency, under the same wind tunnel conditions, is plotted in Figure 107 for various modes of $5 \%$ blowing. Pulsed blowing cases of $S t=0.25\left(f_{\text {jet }}=17 \mathrm{~Hz}\right)$ and $S t=0.50\left(f_{\text {jet }}=34 \mathrm{~Hz}\right)$ again match $\geq 90 \%$ of steady CC efficiency, with required flow reductions of $12 \%$, and $4 \%$, respectively. All cases reveal a maximum nondimensional efficiency values approaching 30.

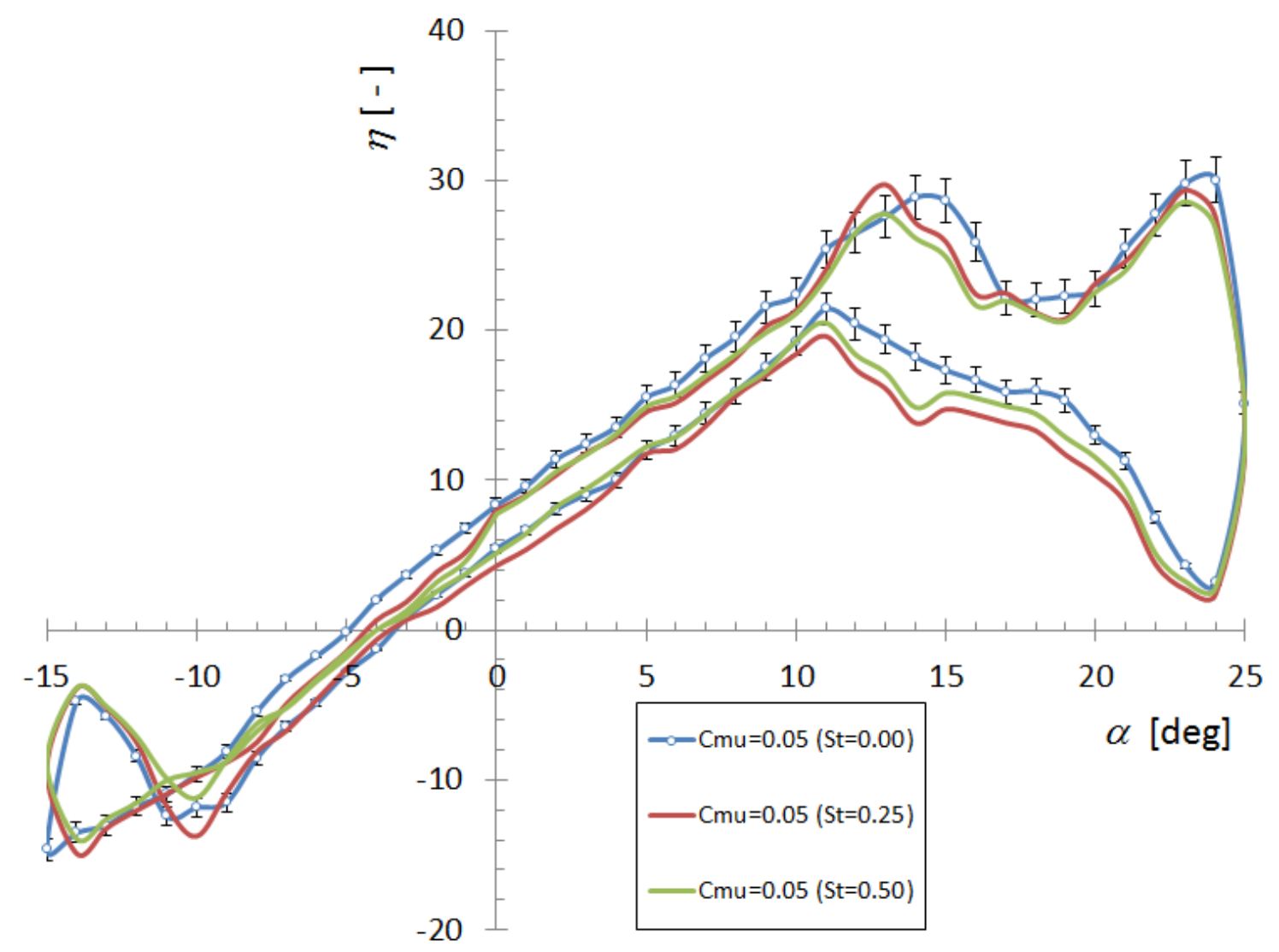

Figure 107. CCNACA0018 Efficiency with Pulsed CC $\left(C_{\mu}=0.05\right): \operatorname{Re}=180 \times 10^{3}, k=0.050\left(\alpha_{\max }=25^{\circ}\right)$ 
Figure 108 includes CCNACA0018 efficiency for a variety of pulsed blowing conditions $\left(C_{\mu}=0.01\right)$ at a higher pitch rate of $k=0.100\left(\operatorname{Re}=180 \times 10^{3}\right)$ following the following the same sinusoidal path of travel: $\alpha(t)=5^{\circ}+20^{\circ} \sin (\omega t)$. Steady blowing experiences a "jet stall" around $\alpha=16^{\circ}$, while the pulsed jet of $S t=0.25$ shows superior performance above $\alpha_{S S}$ (at a reduced mass flow rate $29 \%$ ), as efficiency approaches $\eta=25$. For negative AoA, a higher pulsing frequency $(S t=0.50)$ results in improved performance for $1 \%$ blowing.

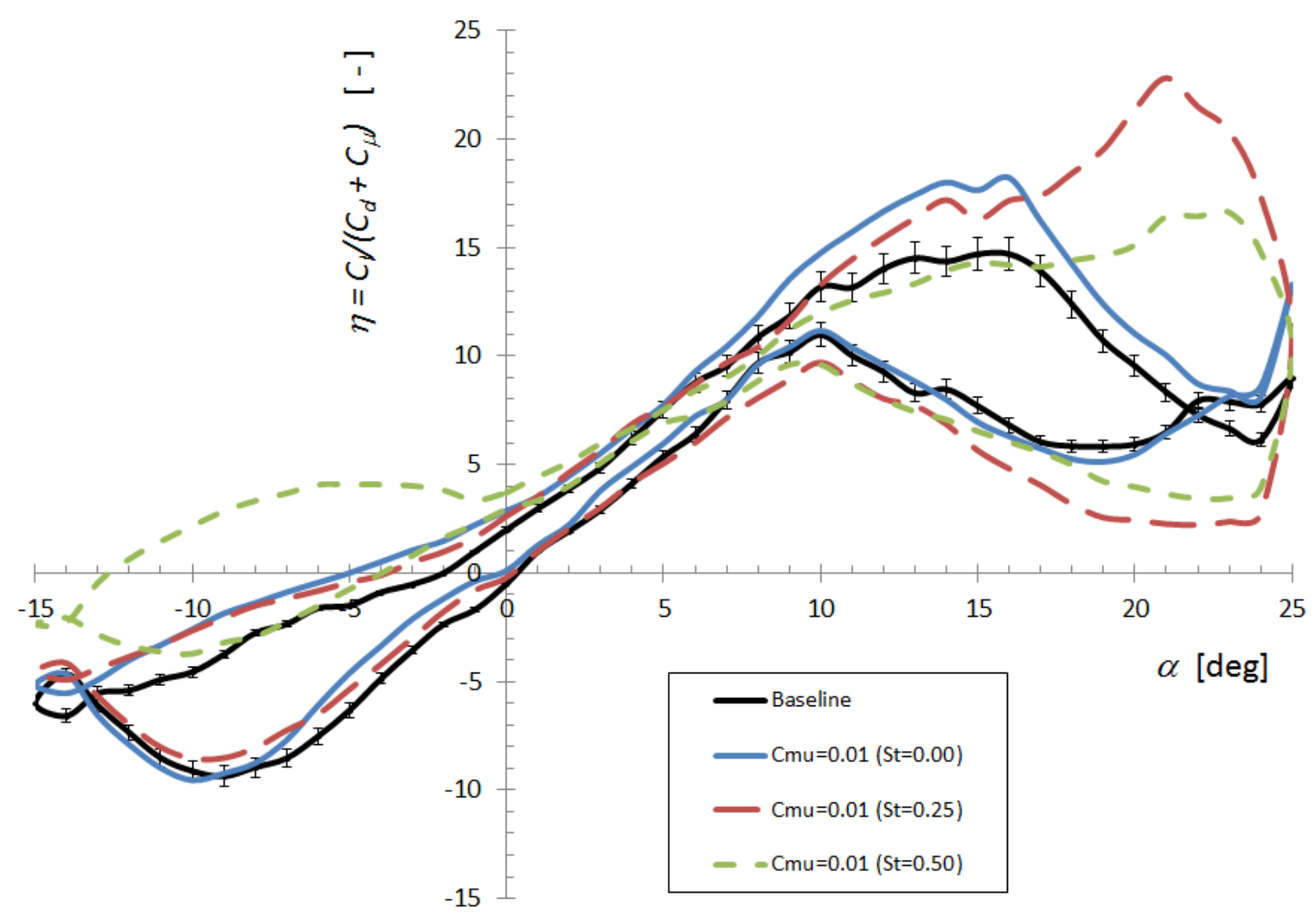

Figure 108. CCNACA0018 Efficiency with Pulsed CC $\left(C_{\mu}=0.01\right): \operatorname{Re}=180 \times 10^{3}, k=0.100\left(\alpha_{\max }=25^{\circ}\right)$ 
Figure 109 includes CCNACA0018 efficiency for a variety of pulsed blowing conditions $\left(C_{\mu}=0.05\right)$ at $\operatorname{Re}=180 \times 10^{3} \quad(k=0.100)$ following the following sinusoidal pitch: $\alpha(t)=5^{\circ}+20^{\circ} \sin (\omega t)$. For $5 \%$ blowing, steady "jet stall" is noticed earlier at $\alpha=15^{\circ}$, due to increased camber with higher $C_{\mu}$. Both conditions of pulsed blowing $(S t=0.25,0.50)$ offer comparable dimensionless efficiencies near 20 for positive AoA, while the steady jet condition gives better performance for negative $\alpha$. Baseline and steady jet experience $\eta$-stall around $15^{\circ}$, while pulsed jet efficiency creates advanced values up until $\alpha=25^{\circ}$.

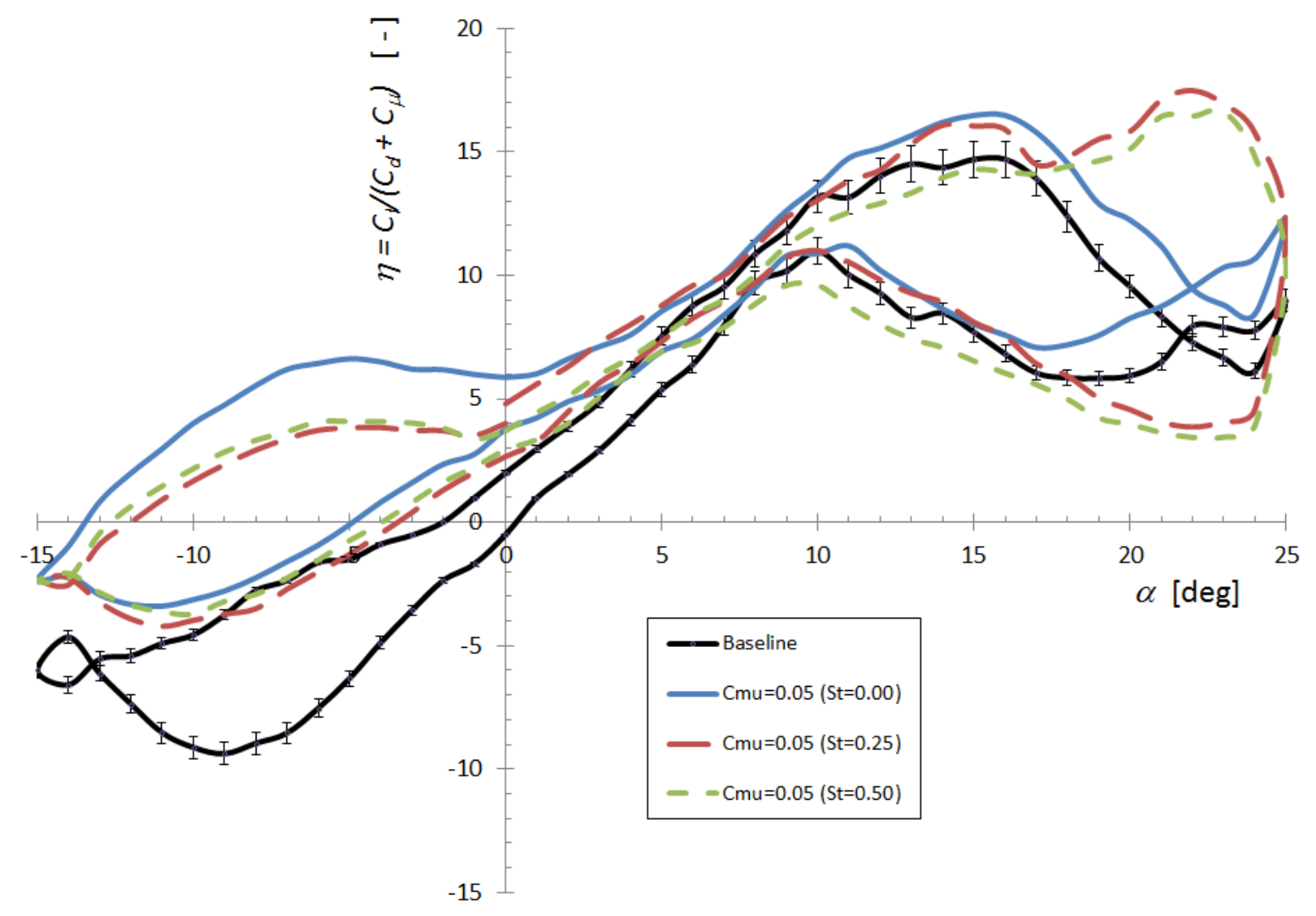

Figure 109. CCNACA0018 Efficiency with Pulsed CC $\left(C_{\mu}=0.05\right): \operatorname{Re}=180 \times 10^{3}, k=0.100\left(\alpha_{\max }=25^{\circ}\right)$ 
Figure 110 compares pulsed blowing efficiency $\left(C_{\mu}=0.05\right)$ at an increased pitch amplitude of $30^{\circ}$. Both steady and pulsed actuation show diminished performance relative to a clean airfoil for $\alpha \geq 15^{\circ}$. However, an interesting result is enhanced performance for $\alpha \leq 5^{\circ}$, including positive efficiency for almost all negative AoA positions. Steady actuation increases baseline efficiency by up to $85 \%$ for negative AoA, while pulsed blowing shows $\eta$-improvements $\geq 75 \%(\Delta \dot{m}=5 \%)$.

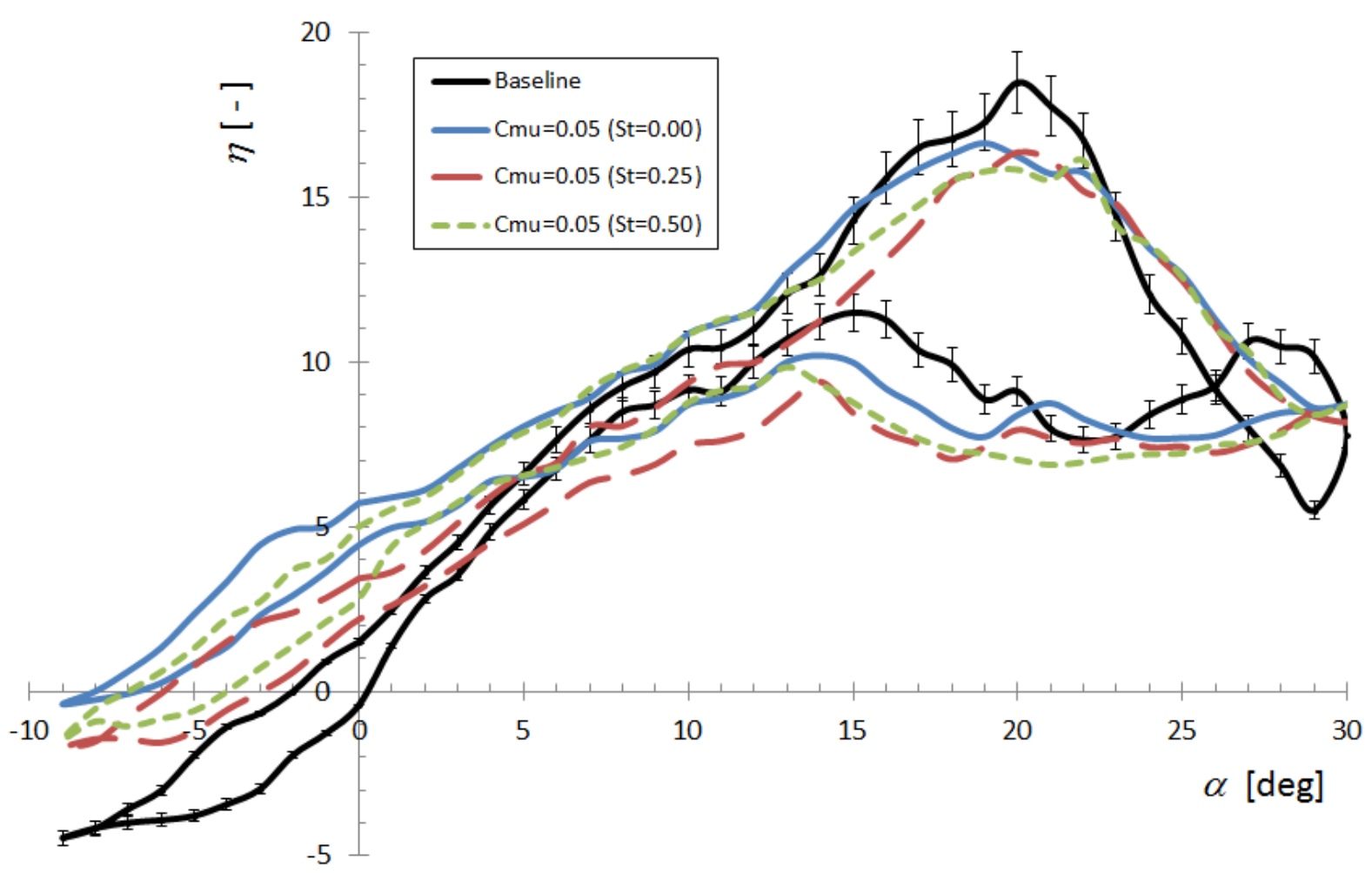

Figure 110. CCNACA0018 Efficiency with Pulsed CC $\left(C_{\mu}=0.05\right): \operatorname{Re}=180 \times 10^{3}, k=0.100\left(\alpha_{\max }=30^{\circ}\right)$ 
Figure 111 compares steady and pulsed CC efficiency for St=0.50, at an increased freestream flow of $R e=300 \times 10^{3}$. Approaching airfoil static stall during pitch-up, steady blowing of $C_{\mu}=0.01$ reveals optimal efficiency $\left(0^{\circ} \leq \alpha \leq 15^{\circ}\right)$, while pulsed actuation shows top performance, with $\eta$-values near 50 prior to stall. Furthermore, pulsed actuation reveals competitive performance, relative to steady CC and clean airfoil cases, for negative AoA; efficiency values are positive for nearly every AoA of airfoil pitch.

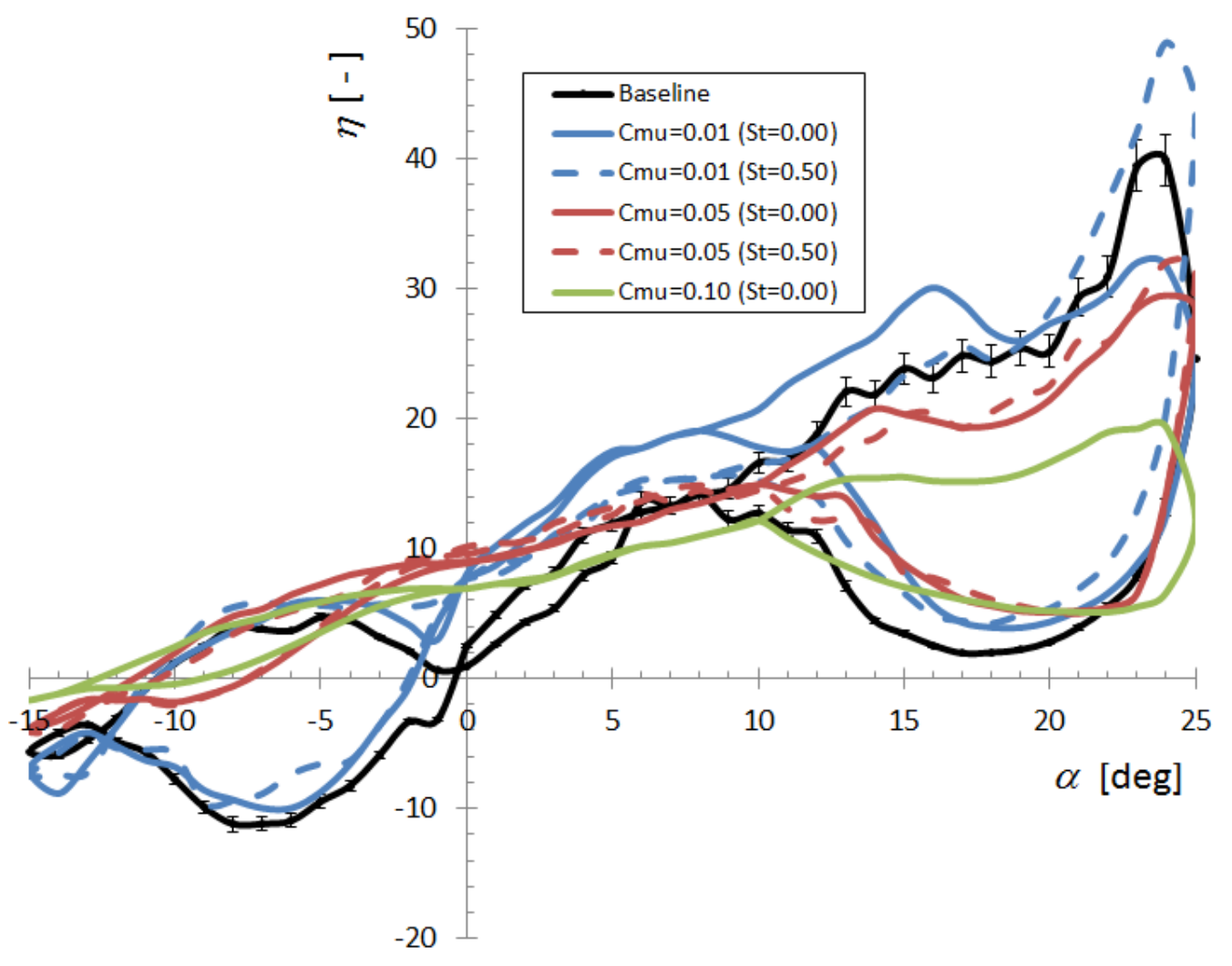

Figure 111. CCNACA0018 Efficiency with Steady and Pulsed CC: $R e=300 \times 10^{3}, k=0.050\left(\alpha_{\max }=25^{\circ}\right)$ 


\subsection{Numerical Results: Static Airfoil}

\subsubsection{Surface Pressure Distributions}

Static pressure distributions of the baseline CCNACA0018 are included, comparing computational results of Laminar, SARC, and realizable $k-\varepsilon$ turbulence models to experimental data of this study. All three models show good agreement to experimental data. The laminar model results show an oscillating $C_{p}$ trace along the airfoil upper surface due to instantaneous vorticity. The turbulence models average instantaneous fluctuations of this nature, appearing more stable when analyzing surface pressure distributions. If the laminar prediction of upper surface pressure were averaged, the results would agree to the more complex turbulence models. Thus, the laminar model was chosen as sufficient for airfoil surface pressure predictions while significantly reducing computational expenses. Static CCNACA0018 lift performance at zero AoA, with various modes of CC, are included in Figure 112.

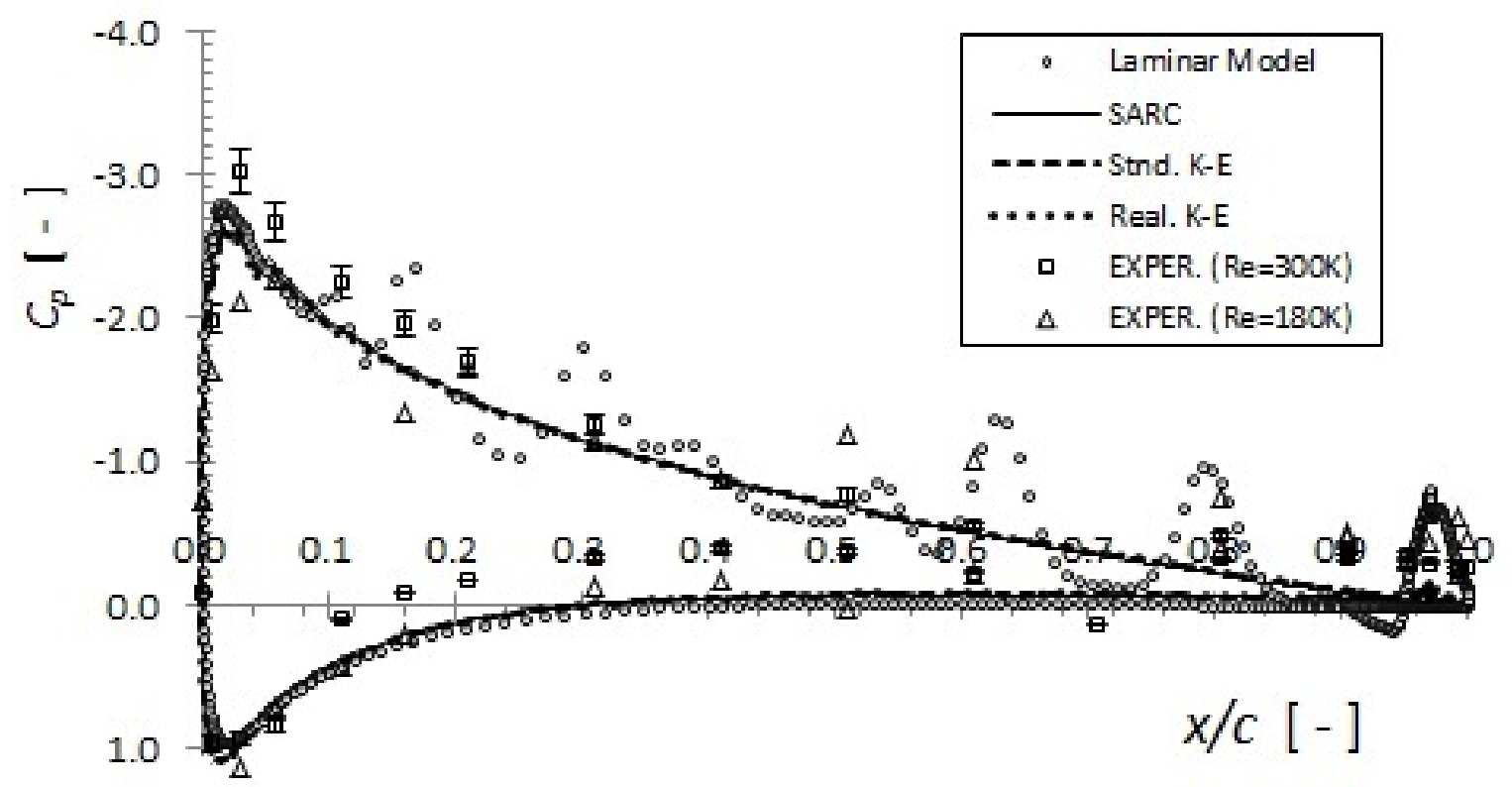

Figure 112. Baseline $C_{p}$-Distributions $\left(\operatorname{Re}=180 \times 10^{3} \alpha=10^{\circ}\right)$ : CFD vs. Experiment 


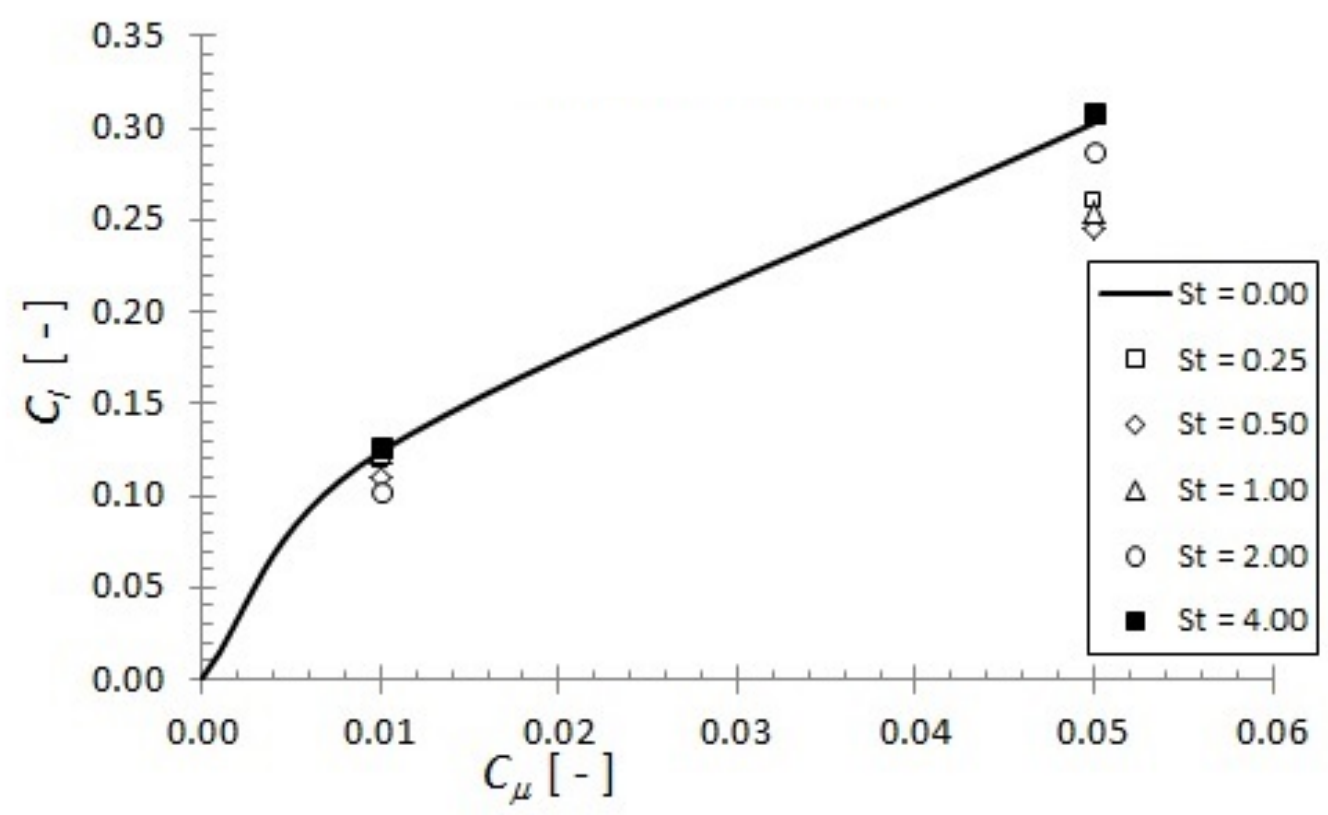

Figure 113. Influence of Jet Frequency on CCNACA0018 Lift Performance: $\operatorname{Re}=180 \times 10^{3}, \alpha=0^{\circ}$

Optimal performance, in terms of both lift enhancement and mass flow reduction, was found for the highest jet frequency $(S t=4.00)$ for both levels of CC magnitude. Required source flow was reduced by up to $30 \%$ relative to steady jet conditions.

\subsubsection{Flow Visualization}

Contours of the velocity magnitude around the stationary (baseline) CCNACA0018 airfoil can be found in Figure 114. Small pockets of reversed flow can be seen on the suction surface of the airfoil, beginning at approximately $x / c=0.5$. Also, Coandă turning of the freestream flow around the upper portion of the rounded TE is resolved. Static airfoil flow visualizations of this nature were important to verify proper flow physics were established, with special attention payed to the complex interaction of the high pressure CC jet and freestream flow. The visualization study showed improved jet attachment to the rounded TE, and subsequent freestream flow entrainment with increasing $C_{\mu}$, as expected. No unnatural flow phenomenon, such as jet attachment along the TE and further upstream along the lower airfoil surface, were observed. Such studies help validate that sufficient mesh refinement and model B.C.s were initially implemented, before the additional complexity of pitching airfoil aerodynamics are introduced. 


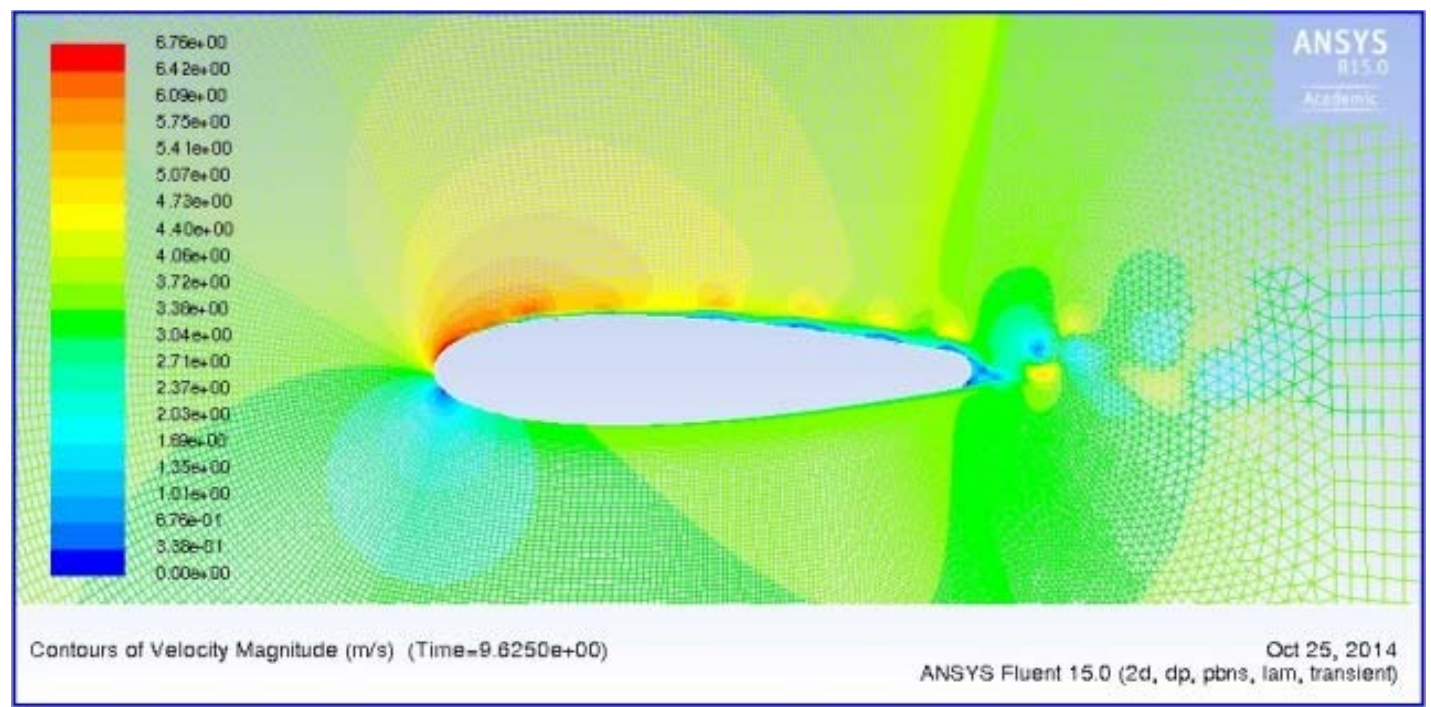

Figure 114. Velocity Magnitude of Baseline CCNACA0018: Laminar $\left(\operatorname{Re}=180 \times 10^{3}, \alpha=0^{\circ}, C_{\mu}=0, S t=0\right)$

In addition to time-history and averaged velocity magnitudes, long-time average $C_{p}$ values were monitored along both the upper and lower surfaces of the airfoil, along with the skin friction coefficient $\left(C_{\tau}\right)$ on the body of the airfoil $(0.000 \leq x / c \leq 0.969)$, as presented at the 2004 AIAA CFD Validation Workshop on Synthetic Jets and Turbulent Separation Control (155).

\subsection{Numerical Results: Pitching Airfoil}

\subsubsection{Turbulence Models \& Experimental Data Comparison}

A comparison of turbulence models was again completed for URANS simulations involving airfoil pitch. The laminar model and 1-equation SARC turbulence model were used to predict baseline CCNACA lift for $R e=180 \times 10^{3}$, pitching at $k=0.100$. Both models predicted similar lift hysteresis loops (see Figure 115), with comparable lift-curve slopes. 


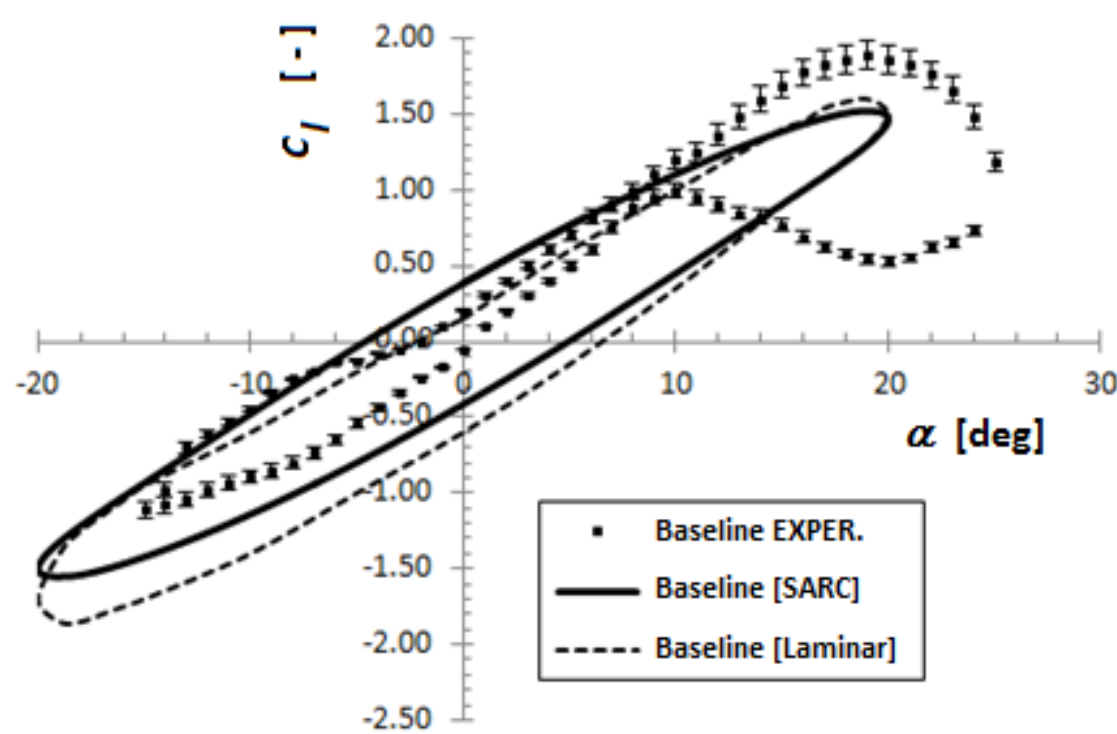

Figure 115. Numerical Analysis of Pitching Baseline CCNACA0018: Comparison to Experiment

A summary of numerical results for the baseline airfoil is included in Table 19, including lift-curve slope and maximum lift. The column with a delta symbol indicates a percent difference comparison to experiment for each numerical model. The laminar model showed better agreement to experimental data in terms of $\mathrm{d} C_{l} / \mathrm{d} \alpha$, while both models predicted matching $C_{l, \text { max }}$ values. The salient features of both numerical values agree well in terms of trends, but show $15-30 \%$ difference compared to measured data, revealing only qualitative agreement.

Table 19. Baseline Numerical Model Results Compared to Experimental CCNACA0018 Data

\begin{tabular}{|c|c|c|c|c|}
\hline Data Source & $\mathrm{d} C_{l} / \mathrm{d} \boldsymbol{\alpha}$ & $\Delta\left(\mathrm{d} C_{l} / \mathrm{d} \alpha\right)$ & $C_{l, \max }$ & $\Delta C_{l, \max }$ \\
\hline Theory & 0.11 & - & - & - \\
\hline Experiment & 0.097 & - & 1.882 & - \\
\hline Laminar CFD & 0.085 & $14 \%$ & 1.500 & $25 \%$ \\
\hline SARC CFD & 0.074 & $32 \%$ & 1.520 & $24 \%$ \\
\hline
\end{tabular}


A comparison of experimental and computed lift for a pitching CCNACA0018 airfoil with steady blowing $\left(C_{\mu}=005, S t=0.00\right)$ is included in Figure 116.

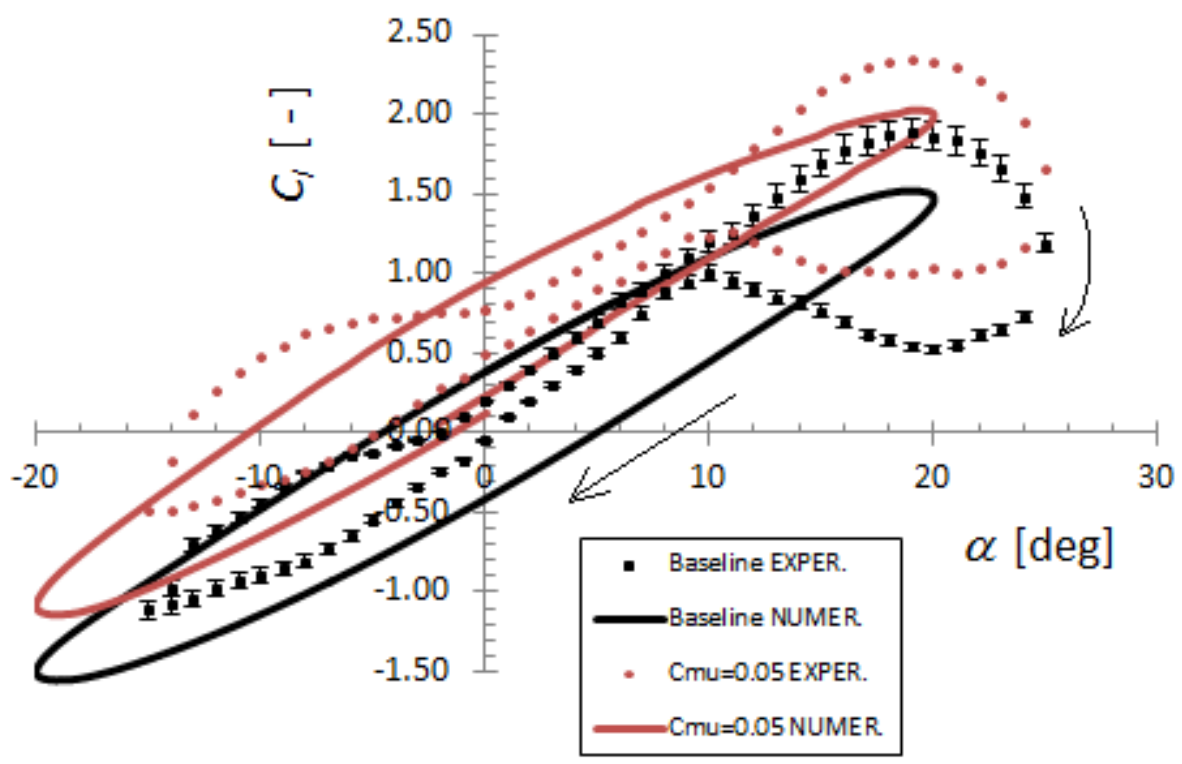

Figure 116. Pitching CCNACA0018 Lift $\left(C_{\mu}=0.05, S t=0.00\right)$ : CFD vs Experiment

\subsubsection{Reduced Frequency $(k)$}

For computational simulations, the reduced pitch frequency of the airfoil was varied $(k=0.075$, 0.100 ) to identify the role of $k$ on airfoil performance. A $C+$ code was written to define desired oscillation motions of the airfoil. The codes were implemented into FLUENT as UDFs to simulate a pitching airfoil. The position of the airfoil was plotted against time (Figure 117) for each pitching condition to verify the angular acceleration matched the desired pitch frequency.

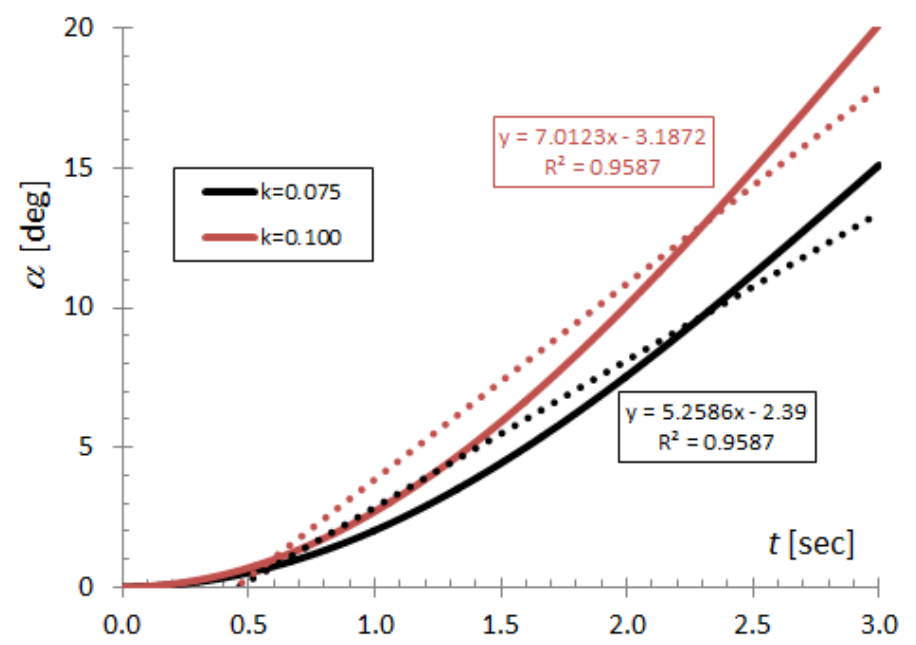

Figure 117. CFD Reduced Frequency Study: UDF Verification of $\mathrm{d} \alpha / \mathrm{d} t$ 
Based on the slope of airfoil position against time, the simulated pitch frequencies matched the desired rates (see key of Figure 117) well, with a maximum disagreement of less than $3 \%$.

Table 20. Influence of Reduced Frequency on CCNACA Total Lift: $\alpha(t)=0^{\circ}+20^{\circ} \sin (\omega t)\left[\operatorname{Re}=180 \times 10^{3}\right]$

\begin{tabular}{|c|c|c|c|c|}
\hline & $C_{\mu}=0.00$ & $C_{\mu}=0.01(S t=0.00)$ & $C_{\mu}=0.01(S t=1.00)$ & $C_{\mu}=0.05(S t=0.00)$ \\
\hline$k=0.075$ & $\Delta C_{I}=31.62$ & $\Delta C_{I}=33.60$ & $\Delta C_{I}=37.24$ & $\Delta C_{I}=53.48$ \\
\hline$k=0.100$ & $\Delta C_{I}=41.31$ & $\Delta C_{I}=27.86$ & $\Delta C_{I}=33.53$ & $\Delta C_{I}=46.20$ \\
\hline TREND: $k \uparrow$ & $\Delta C_{i(\text { Baseline })} \uparrow$ & $\Delta C_{i(S t=0)} \downarrow$ & $\Delta C_{i(S t \neq 0)} \downarrow$ & $\Delta C_{i(S t=0)} \downarrow$ \\
\hline
\end{tabular}

\subsubsection{Pitch Amplitude $\left(\alpha_{\max }\right)$}

The amplitude $\left(\alpha_{\max }=20^{\circ}, 30^{\circ}\right)$ of the pitching airfoil was varied to study the impact on CCNACA maximum lift $\left(C_{l, \text { max }}\right)$ with different CC actuation modes. The paths of airfoil travel were graphed and plotted to verify that the desired amplitudes were reached, as in the previous section to validate $k$, with comparable results. Table 21 includes the influence of maximum AoA on the maximum lift for baseline and steady CC jet conditions.

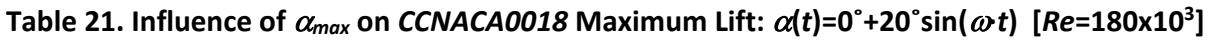

\begin{tabular}{|c|c|c|c|}
\hline & $C_{\mu}=0.00$ & $C_{\mu}=0.01(S t=0.0)$ & $C_{\mu}=0.05(S t=0.00)$ \\
\hline$\alpha_{\max }=20^{\circ}$ & $C_{i, \max }=1.06$ & $C_{i, \max }=2.18$ & $C_{i j \max }=2.82$ \\
\hline$\alpha_{\max }=30^{\circ}$ & $C_{l_{\max }}=1.90$ & $C_{i \max }=2.46$ & $C_{i \max }=3.75$ \\
\hline TREND: $\alpha_{\max } \uparrow$ & $C_{l, \max } \uparrow$ & $C_{i, \max } \uparrow$ & $C_{l, \max } \uparrow$ \\
\hline
\end{tabular}

\subsubsection{Pitching Airfoil: Dynamic Stall Vortex Convection Speed ( $\left.V_{D S V}\right)$}

The mass flow requirements for each simulated blowing condition are compiled in Table 22. It can be seen that a characteristic of the user defined functions to define the pulsed jet is an increase in $\dot{m}$ savings with increasing $f_{\text {jet }}$ based on the control function used. For $S t=4, \dot{m}$ is reduced by $19 \%$ and $31 \%$ for $C_{\mu}=0.01$ and $C_{\mu}=0.05$, respectively. 
Table 22. CCNACA0018 Mass Flow Requirements for Various CFD Blowing Conditions $\left(\operatorname{Re}=180 \times 10^{3}\right)$

\begin{tabular}{|c|c|c|c|}
\hline$C_{\mu}$ & $S t$ & & $\Delta$ \\
\hline$[-]$ & {$[-]$} & {$\left[\mathrm{lb}_{\mathrm{m}} / \mathrm{s}\right]$} & {$[\%]$} \\
\hline \multirow{4}{*}{0.01} & 0.00 & 0.00915 & - \\
\cline { 2 - 4 } & 0.50 & 0.00861 & $-6 \%$ \\
\cline { 2 - 4 } & 1.00 & 0.00848 & $-8 \%$ \\
\cline { 2 - 4 } & 2.00 & 0.00800 & $-14 \%$ \\
\cline { 2 - 4 } & 4.00 & 0.00768 & $-19 \%$ \\
\hline \multirow{4}{*}{0.05} & 0.00 & 0.01392 & - \\
\cline { 2 - 4 } & 0.50 & 0.01208 & $-15 \%$ \\
\cline { 2 - 4 } & 1.00 & 0.01073 & $-30 \%$ \\
\cline { 2 - 4 } & 2.00 & 0.01067 & $-30 \%$ \\
\cline { 2 - 4 } & 4.00 & 0.01066 & $-31 \%$ \\
\hline
\end{tabular}

Flow visualization from the numerical model was used to calculate the convection speed of the DSV speed $\left(V_{D S V}\right)$ along the suction surface of the CCNACA airfoil. An illustrative example of the estimation process is included in Figure 118, comparing the location of the DSV at two instances of time.
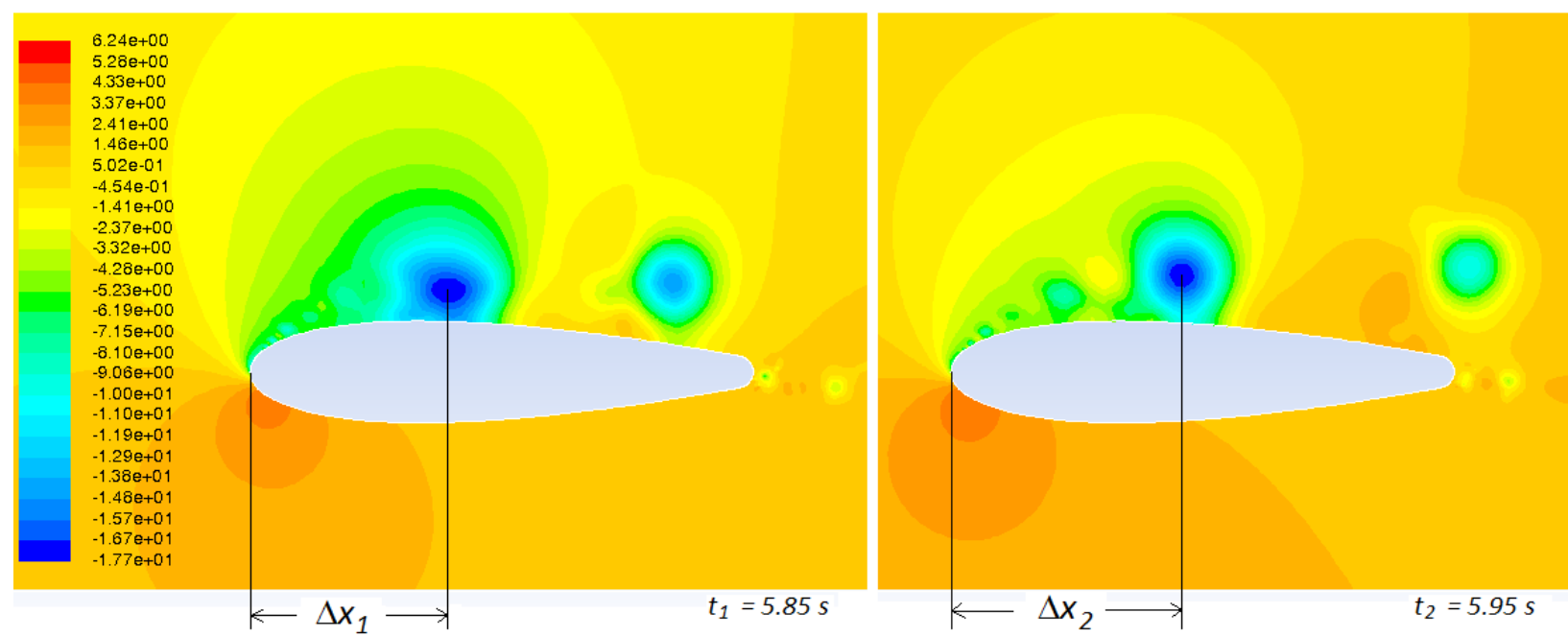

Figure 118. Static Pressure Contours for $V_{D S V}$ Calculation $\left(R e=180 \times 10^{3}, k=0.100, C_{\mu}=0.01(S t=0.00)\right)$

The computed results of $V_{D S V}$ are included in Table 23; the range of convection speeds for the baseline CCNACA airfoil $\left(V_{D S V} / V_{\infty}=0.295\right)$ agree well with pitching data of conventional airfoils from the literature. $V_{D S V} / V_{\infty}$ values of 0.24-0.30 were independently reported by Chandrasekhara, et al. (123), Tuncer (121), Lorber \& Carter (131), Green, et al. (132), and Shrewsbury (109). It should be noted that the current study was the first to report the influence of pulsed CC jet flow control on $V_{D S V}$, and thus, only baseline data can be compared to conventional airfoil performance reported in the literature. 
Table 23. Computed $V_{D S V}$ at Various Blowing Conditions: $R e=180 \times 10^{3}(k=0.100)$

\begin{tabular}{|c|c|c|}
\hline$C_{\mu}$ & $S t$ & $V_{\text {DSV }} / V_{\infty}$ \\
\hline$[-]$ & {$[-]$} & {$[-]$} \\
\hline Baseline & - & 0.295 \\
\hline 0.01 & 0.00 & 0.31 \\
\hline 0.01 & 1.00 & 0.357 \\
\hline 0.05 & 0.00 & 0.37 \\
\hline 0.05 & 1.00 & 0.383 \\
\hline
\end{tabular}

From Table 23, it can be seen that CC increases $V_{D S V}$, and thus $f_{\text {vortex, }}$ on the pitching CCNACA at $k=0.100$. Furthermore, for a given $C_{\mu}$ condition, increasing $S t$ of the jet also increases $V_{D S V}$. These results support separate experimental outcomes from Lorber \& Carta (156) and Green, et al. (132) that increasing camber increases the vortex convection speed.

An increase in $V_{D S V}$ results in an inherent growth in the frequency of large scale vorticity shedding $\left(f_{\text {vortex }}\right)$ from the airfoil surface just prior to stall. These results concur with previous experimental and computational work on CC airfoils by Shrewsbury (109). The static pressure contrours of Figure 119 highlight the influence of of flow control on LE vorticity prior to full DS.
(a.) Baseline Airfoil
(b.) $C_{\mu}=0.01(s t=0.00)$
(c.) $\mathrm{C}_{\mu}=0.01(S t=1.00)$
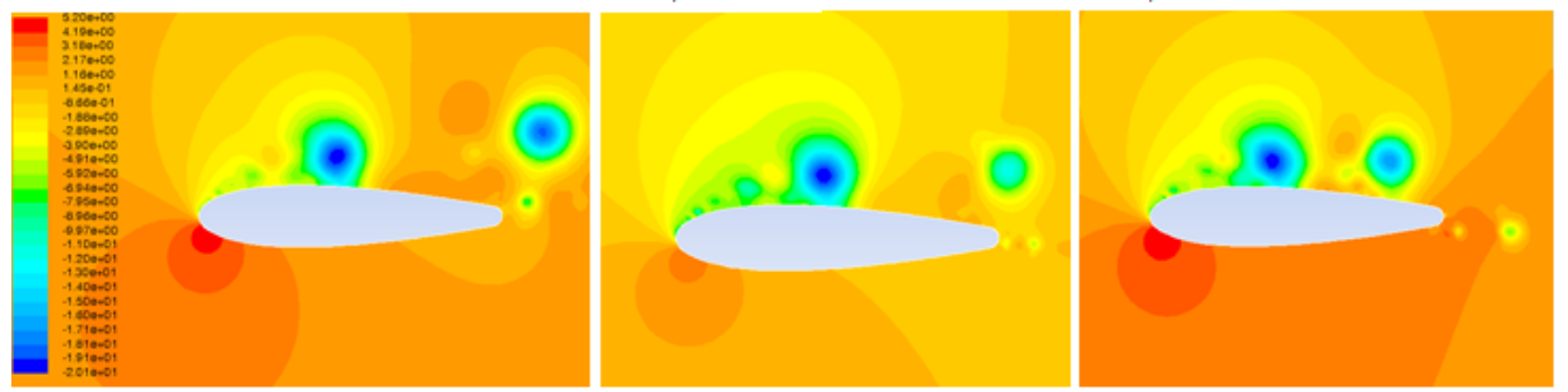

Figure 119. Influence of CC on Vortex Shedding $\left(R e=180 \times 10^{3}, k=0.100, \alpha=28^{\circ} \uparrow\right)$

The full progression of dynamic stall events, initiated by small-scale LE vorticity and finalized by the release of a large TE vortex, for the baseline airfoil is included in Figure 120. During pitch-up near maximum amplitude $\left(\alpha \approx 28^{\circ}\right)$, small scale vorticity forms and rapidly convects from the airfoil LE. Following this, upper surface flow reversal from the TE toward to LE causes a single vortex to build strength near the LE, forming the DSV. Full separation aft of $x / c=0.50$ occurs at this point. As the airfoil reaches peak motion $\left(\alpha_{\max }=30^{\circ}\right)$, the DSV begins to convect along the upper surface of the airfoil. Finally, near $x / c=0.75$ the DSV begins to shed from the airfoil into the freestream as the airfoil begins to pitchdown. 


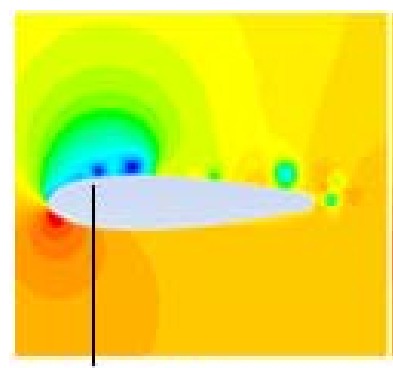

LE Vorticity

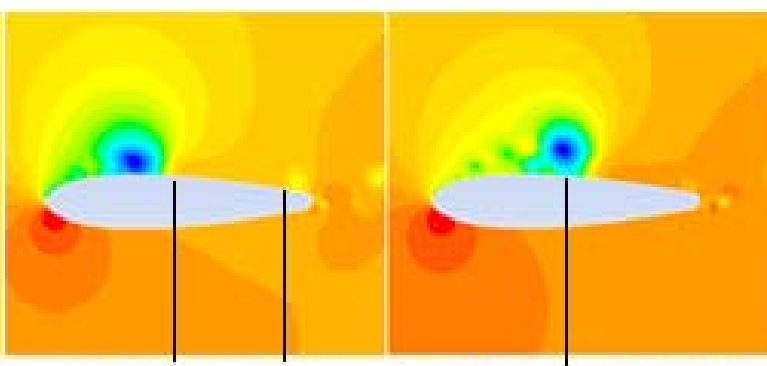

Full TE Separation

Aft Movement of DSV

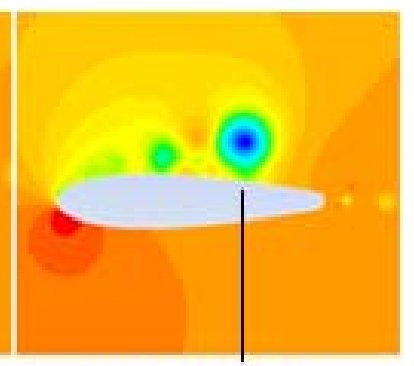

Shedding of DSV

Figure 120. Static Pressure Contours: Dynamic Stall Progression $\left(R e=180 \times 10^{3}, k=0.100, \alpha_{\max }=30^{\circ}\right)$

\subsubsection{Strouhal Number (St)}

The numerical frequency and jet cycle period values for each Strouhal condition are included in Table 24. Based on the freestream velocity $\left(V_{\infty}=2.659 \mathrm{~m} / \mathrm{s}\right)$ and airfoil chord $(c=1 \mathrm{~m})$, the convective time scale for all simulations was $T_{\text {conv }}=0.376$ seconds. Thus, all actuation frequencies of $S t \geq 1.0$ had jet cycle

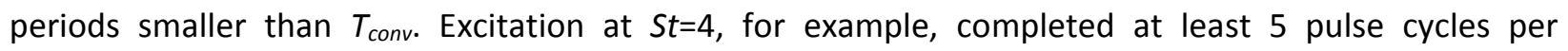
convective time period.

Table 24. Pulsed CC Jet Conditions from Numerical Analysis

\begin{tabular}{|c|c|c|}
\hline$S t$ & $f_{\text {jet }}$ & $T_{\text {jet }}$ \\
\hline$[-]$ & {$[\mathrm{Hz}]$} & {$[\mathrm{sec}]$} \\
\hline 0.25 & 0.84 & 1.19 \\
\hline 0.50 & 1.69 & 0.59 \\
\hline 1.00 & 3.36 & 0.30 \\
\hline 2.00 & 6.74 & 0.15 \\
\hline 4.00 & 13.48 & 0.07 \\
\hline
\end{tabular}

The influence of jet actuation frequency on CCNACA0018 total lift $\left(\Delta C_{l}\right)$ during pitch-up is included in Table 25. Two comparisons were made for this study. The first compares the lift enhancement of all flow

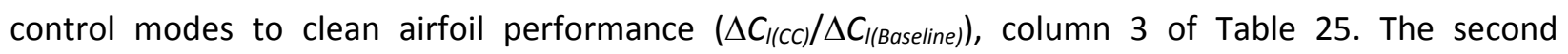
appraisal compares lift enhancement of pulsed CC jets relative to steady jet control $\left(\Delta C_{\mid(S t \neq 0) /} \Delta C_{\mid(S t=0))}\right.$. 
Table 25. Influence of Steady and Pulsed CC on Pitching CCNACA0018 Total Lift $\left(\Delta C_{l}\right)$

\begin{tabular}{|c|c|c|c|c|}
\hline$C_{\mu}$ & $S t$ & $\Delta C_{\text {I/Baseline) }} / \Delta C_{\text {l/(C) }}$ & $\Delta C_{\text {I/St } \pm 0)} / \Delta C_{\text {l/st }=0)}$ & $\Delta \dot{m}$ \\
\hline$[-]$ & {$[-]$} & {$[-]$} & {$[-]$} & {$[\%]$} \\
\hline Baseline & 0.00 & - & - & - \\
\hline & 0.00 & 1.6 & - & - \\
& 0.50 & 2.1 & 1.31 & $-6 \%$ \\
\hline \multirow{5}{*}{0.01} & 1.00 & 2.0 & 1.20 & $-8 \%$ \\
& 2.00 & 2.1 & 1.26 & $-19 \%$ \\
& 4.00 & 1.7 & 1.05 & $-19 \%$ \\
\hline \multirow{5}{*}{0.05} & 0.00 & 2.7 & - & - \\
& 0.50 & 2.4 & 1.13 & $-15 \%$ \\
& 1.00 & 2.4 & 1.14 & $-30 \%$ \\
& 2.00 & 2.5 & 1.09 & $-30 \%$ \\
& 4.00 & 2.9 & 0.95 & $-31 \%$ \\
\hline
\end{tabular}

The effectiveness of each frequency on pitching CCNACA0018 airfoil lift is better understood from comparing pulsed CC jet lift curves to those of baseline and steady CC jets. Figure 121 includes the lift performance of $C_{\mu}=0.01$ with a range of control frequencies $(0.50 \leq S t \leq 4)$, compared to steady blowing (solid blue line) and baseline performance (solid black line). The plots are for the time period $0 \mathrm{~s} \leq t \leq 3 \mathrm{~s}$ which corresponds to pitch-up between $\alpha=0^{\circ}$ and $\alpha=20^{\circ}$. Flow time was chosen to represent the $\mathrm{x}$-axis to show that $C_{1}$-curves had oscillating components matching $f_{\text {jet }}$ for some cases. Low jet frequency lift $(S t=0.50)$ (Figure 121a) shows large amplitude excursions past steady blowing lift. After each jet pulse, the lift oscillates about the steady jet lift value until the next jet actuation cycle, indicating airfoil circulation control authority is maintained between actuation cycles. Increasing actuation frequency to $S t=1.00$ generates smaller amplitude lift excursions above steady CC lift. For $S t=0.50$, the pneumatic valves are closed for twice as long, allowing supply pressure to build within the airfoil plenum. Thus, when the valves are opened, the higher plenum pressure generates a larger instantaneous jet velocity, resulting in larger lift jumps. For the CCNACA0018 model, as $f_{\text {jet }}$ increases, the RMS value of $\left\langle V_{\text {jet }}\right\rangle$ decreases. For the highest jet frequency (St=4: Figure $121 \mathrm{~d}$ ), the lift begins to mimic steady jet performance (improved stability), larger lift at low AoA (in this case, $t<1.5 \mathrm{~s}$ ), and $19 \%$ reduced mass flow. 
(a.) $S t=0.50$

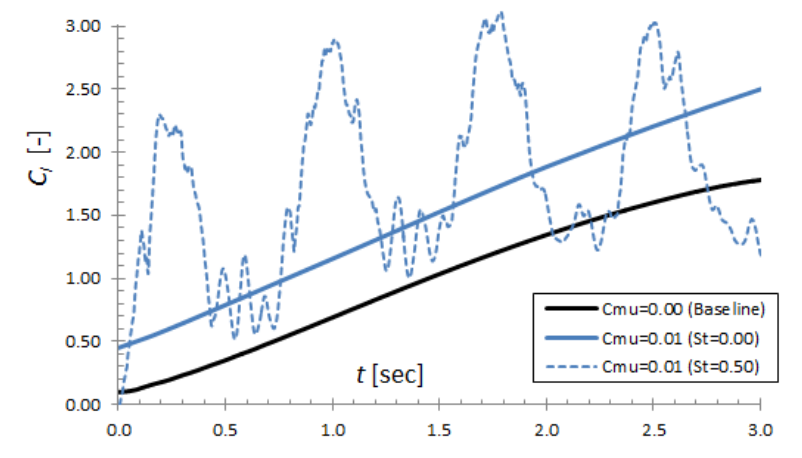

(c.) $S t=2.00$

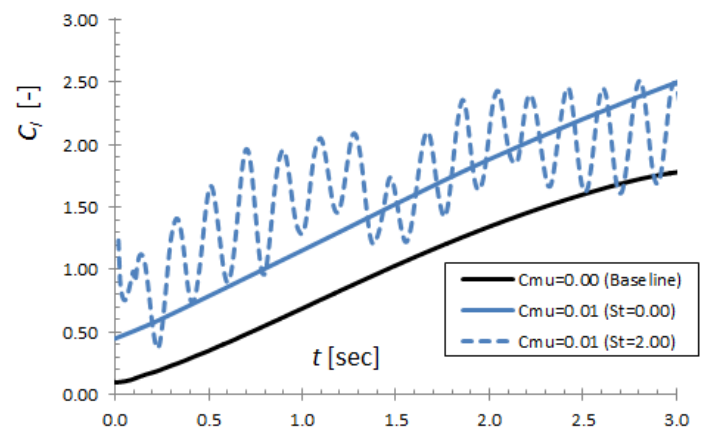

(b.) $S t=1.00$

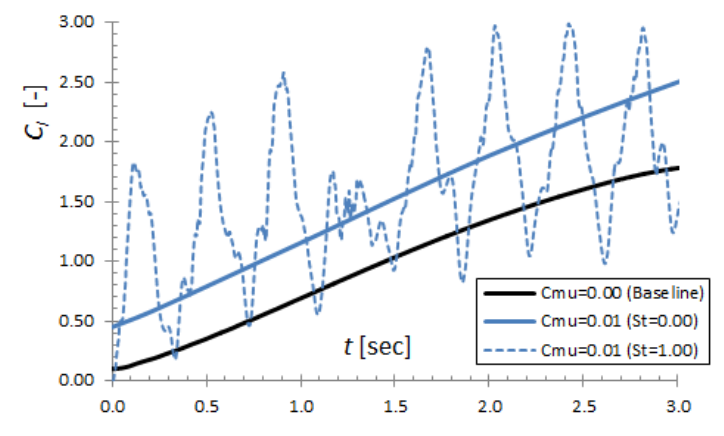

(d.) $S t=4.00$

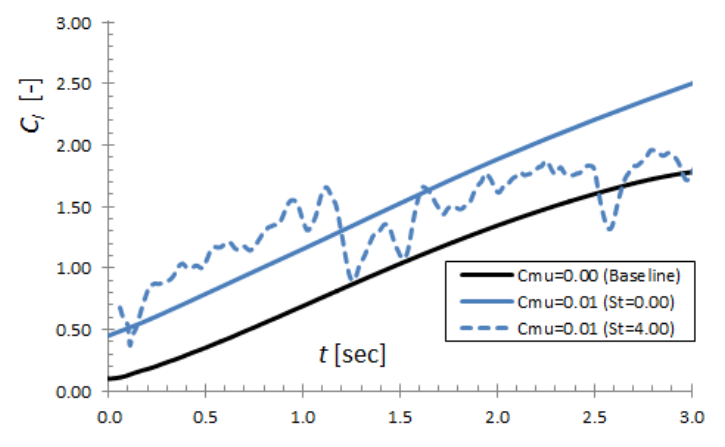

Figure 121. Influence of Various St on Pitching CCNACA0018 Lift $\left(C_{\mu}=0.01\right): \alpha(t)=0^{\circ}+20^{\circ} \sin (\omega t)$

Figure 122 overlays normalized versions of lift and mass flow rate curves representing case (a.) of Figure 122, highlighting the synchronicity between the two values and the control authority of the low frequency jet.

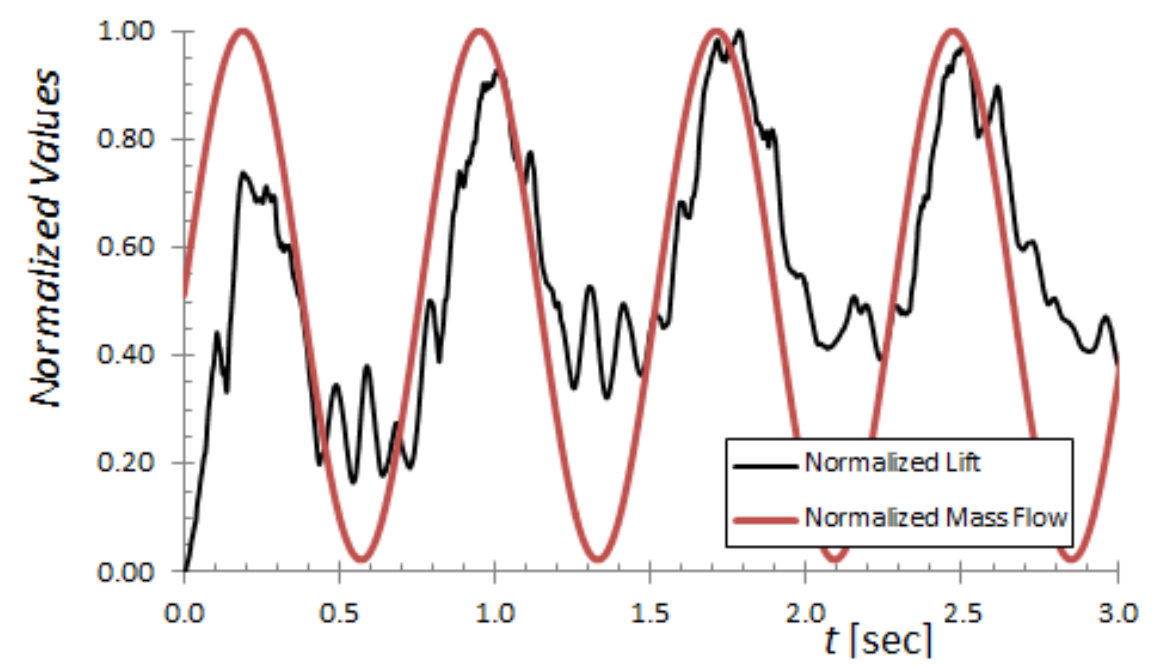

Figure 122. Overlay of Normalized Lift and Mass Flow Curves: $R e=180 \times 10^{3}, C_{\mu}=0.01(S t=0.50)$

The same analysis for various St of $C_{\mu}=0.05$ is included in Figure 123. The lowest actuation frequency $(S t=0.50)$ shows large lift excursions past steady $C_{\mu}$ lift that coincide with $f_{j e t}$. For this 
condition, the time between jet pulses is too large and $C_{I}$ returns to the baseline performance before the initiation of the following jet cycle. As $f_{j e t}$ is doubled $(S t=1.00)$, each pulse carries less momentum resulting in smaller excursions past steady- $C_{\mu}$ lift, and still returns to baseline values in between actuation phases. For $S t=2.00$ actuation, the time scale of the jet begins to exceed that of airfoil circulation loss (separation) as lift no longer returns to baseline airfoil performance in between jet pulses. Finally, the effectiveness of high frequency actuation, $S t=4.00\left(f_{\text {jet }}=13.5 \mathrm{~Hz}\right)$, (Figure 123d) is apparent as the lift-curve loses resemblance of a sinusoidal wave, indicating that the time scale of $f_{\text {jet }}$ now exceeds that of the separation process, and fully governs circulation in the same manner as the constant $C_{\mu}=0.05$ jet $(S t=0.00)$.

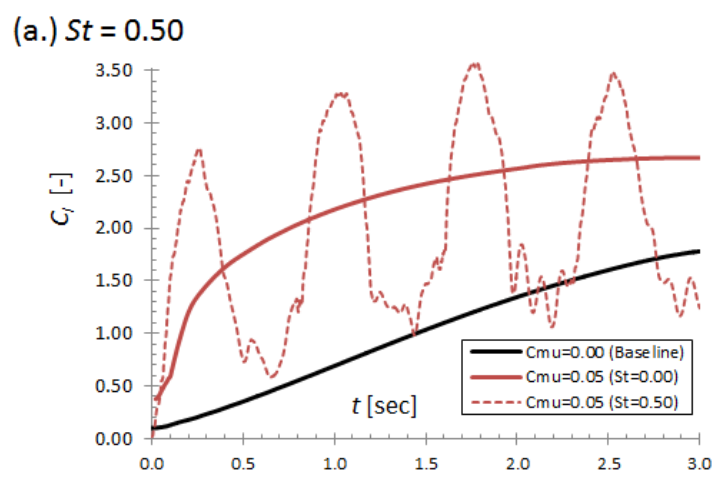

(c.) $S t=2.00$

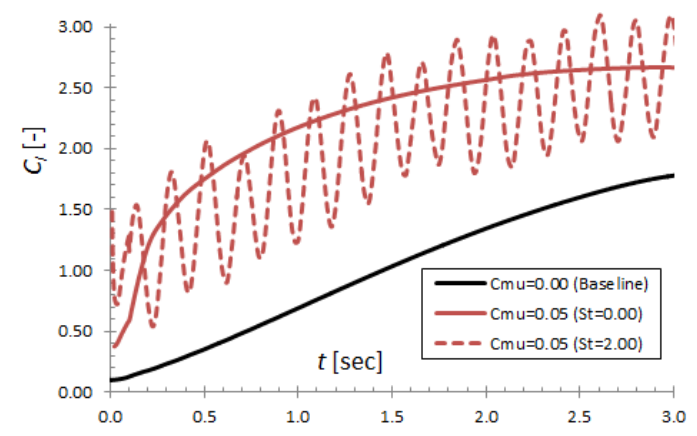

(b.) $S t=1.00$

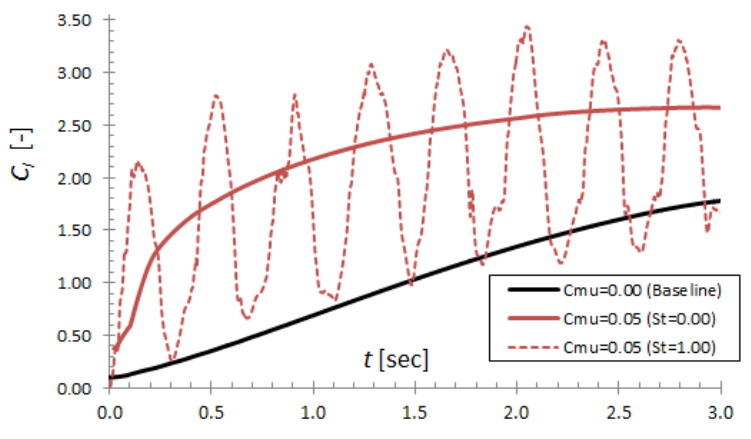

(d.) $S t=4.00$

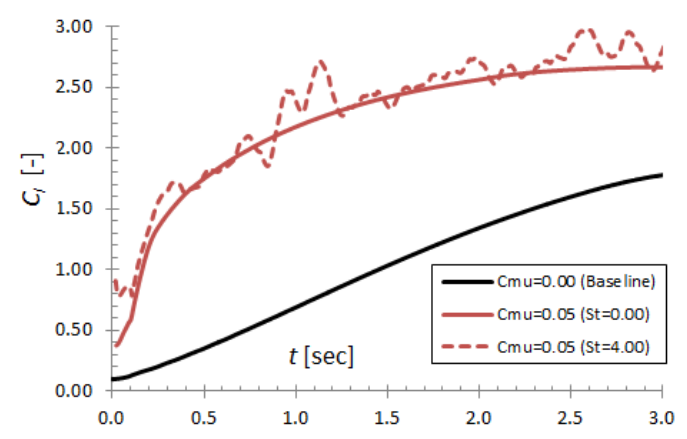

Figure 123. Influence of St on Pitching CCNACA0018 Lift $\left(C_{\mu}=0.05\right): \alpha(t)=0^{\circ}+20^{\circ} \sin (\omega t)$

Figure 124 compares the influence of actuation frequency $\left(C_{\mu}=0.01\right)$ on the lift of a CCNACA0018 airfoil following sinusoidal pitch motions between $\pm 20^{\circ}$. For low magnitudes of control, all pulsed jet frequency cases outperformed the steady jet, with required flow reductions of nearly $20 \%$ for St=4. For $\alpha \leq 12^{\circ}, S t=2$ produces the optimal lift performance, while $S t=0.50$ outperforms all other cases above this position. 


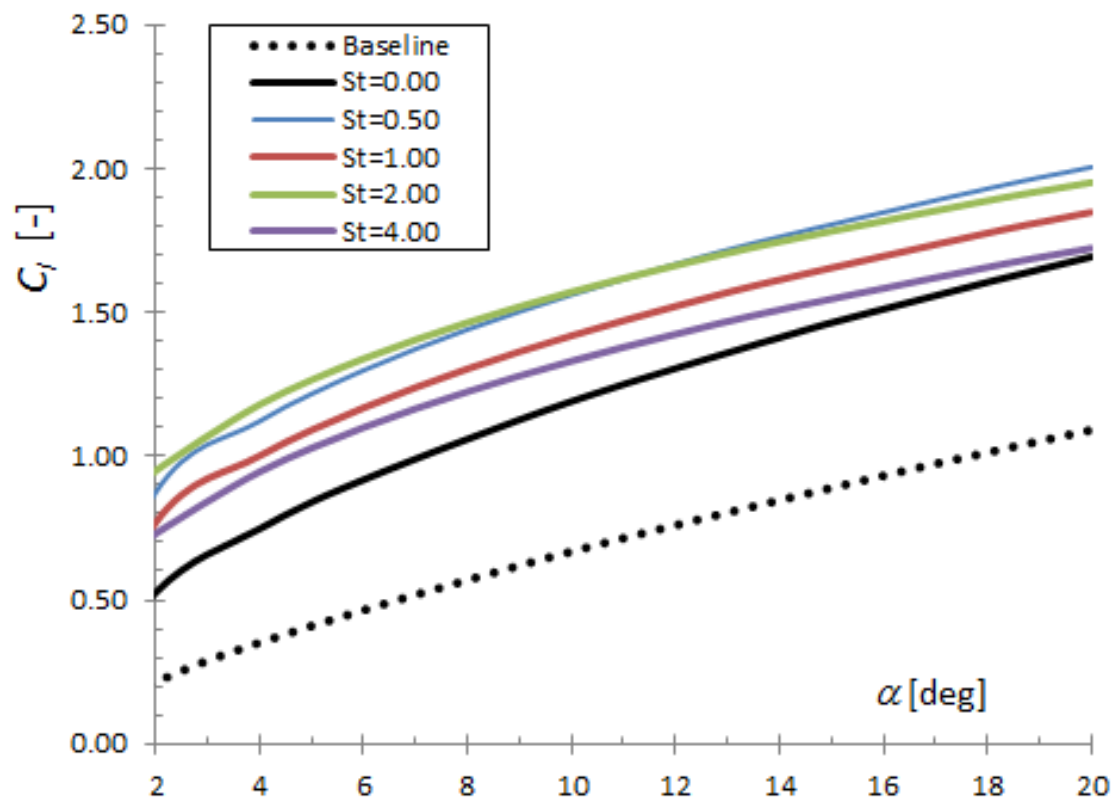

Figure 124. Influence of St on Pitching CCNACA0018 Lift $\left(C_{\mu}=0.01\right): \alpha(t)=0^{\circ}+20^{\circ} \sin (\omega t)$

Figure 125 compares the influence of actuation frequency for the same simulation conditions at a higher blowing coefficient of $5 \%$. High frequency actuation ( $S t=4)$ was the only pulsed CC case to exceed steady jet lift, with the benefit of $30 \%$ reduced mass flow.

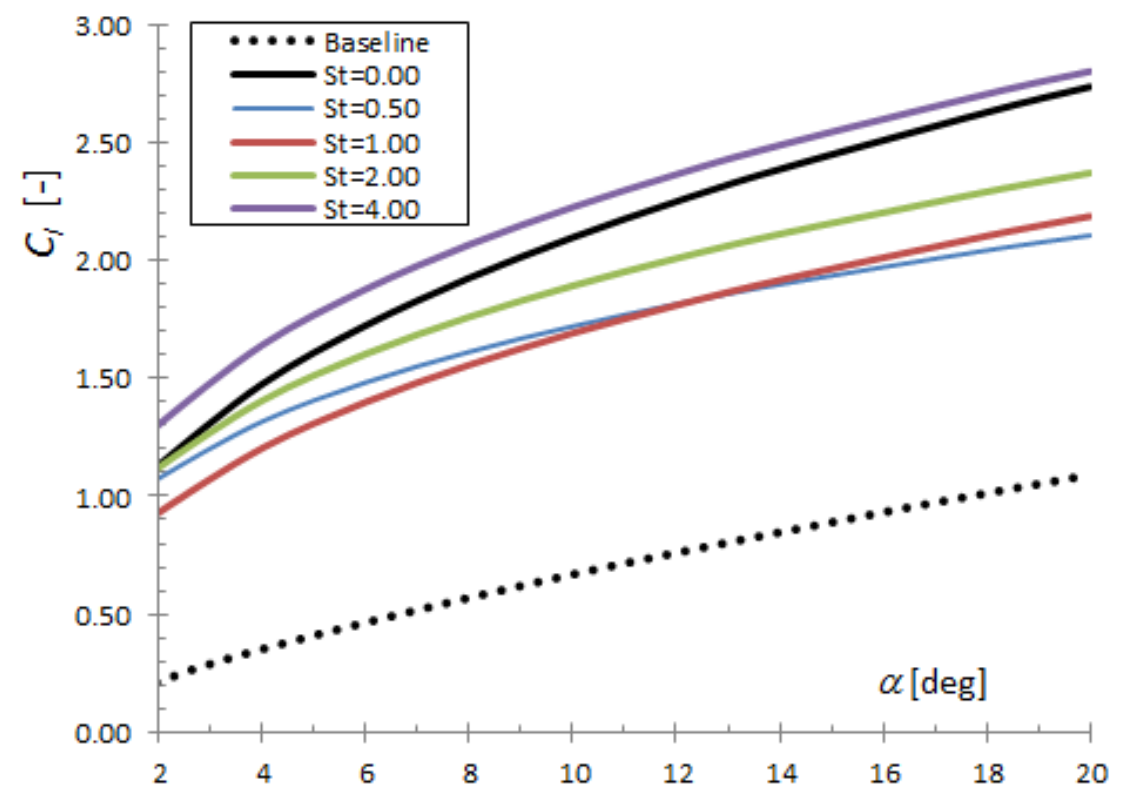

Figure 125. Influence of St on Pitching CCNACA0018 Lift $\left(C_{\mu}=0.05\right): \alpha(t)=0^{\circ}+20^{\circ} \sin (\omega t)$

Numerical results showed highest efficiency case of $5 \%$ blowing was high frequency actuation of St=4, reaching values above 25 at maximum AoA; see Figure 126. 


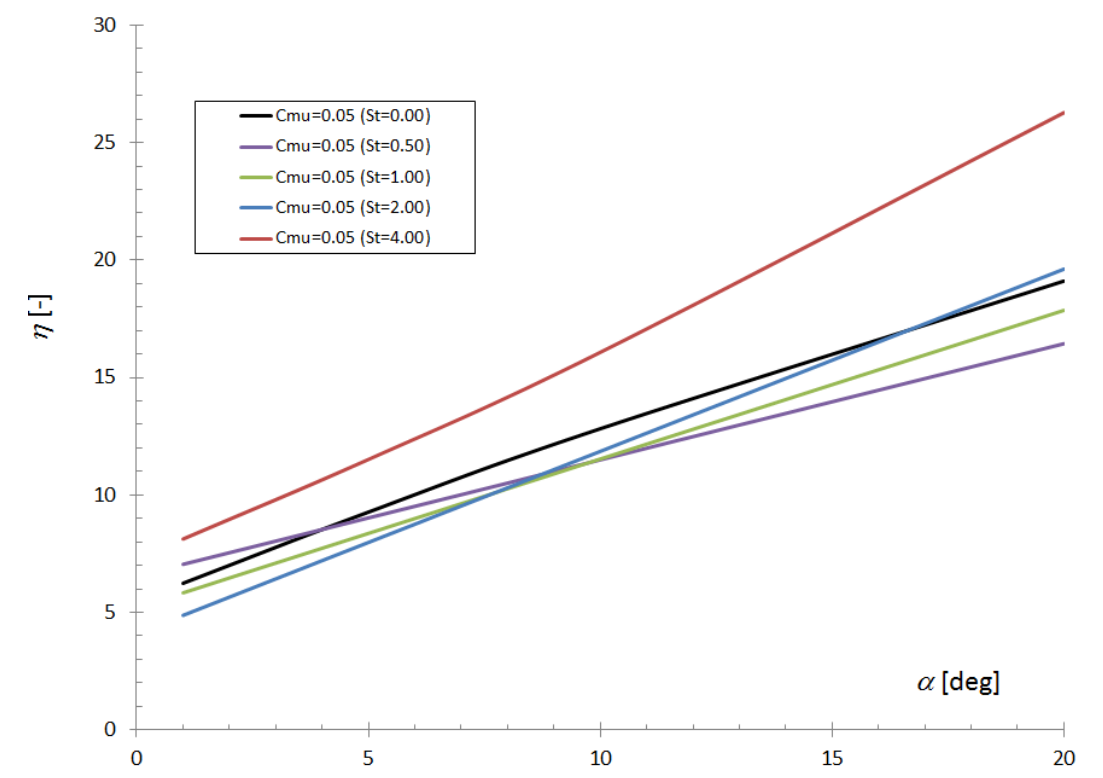

Figure 126. Influence of St on Pitching CCNACA0018 Efficiency $\left(C_{\mu}=0.05\right): \alpha(t)=0^{\circ}+20^{\circ} \sin (\omega t)$

\subsection{CCVAWT Control Strategy}

To effectively implement circulation control into VAWT blades within the built environment, an improved control actuation strategy must be created. Previous CCVAWT performance assessments were calculated with continuous steady blowing actuation during an entire blade revolution, due to limitations of experimental airfoil data. During certain conditions airfoil performance enhancement was minimal until moderate to high angle of attack $\left(\alpha \geq 10^{\circ}\right)$. Thus, mass flow could be significantly reduced by terminating flow control actuation during these portions of rotation, further reducing flow requirements by $30 \%$ per blade orbit. The response time for the CC jet to augment lift would be needed to allow lead time for actuation initiation during the orbital rotation. Shrewsbury (109) predicted a CC jet response time in the range $6.2 \mathrm{~ms} \leq t_{\text {response }} \leq 7.9 \mathrm{~ms}$, for the XW103 circulation controlled airfoil at $R e=3.06 \times 10^{6}$. Angle (85) measured a response interval of $56 \mathrm{~ms}$ for a $10 \%$ CC-Ellipse at $\alpha=0.68^{\circ}$, with numerical model validation predicting $t_{\text {response }}=55-60 \mathrm{~ms}$. The turbine rotational speed would then be used to determine the premature orbital position of CC actuation for a desired lift performance.

Plotting the influence of $C C$ on lift enhancement $\left[\delta C_{1}=C_{l(C C)}-C_{l(B a s e l i n e)}\right]$, relative to the baseline airfoil, throughout a pitch cycle helped reveal scenarios when flow control was not beneficial. Thus, strategic termination of flow control would further contribute to power savings on an operational CCVAWT. The value of this metric is realized in Figure 127, where it can be seen that steady blowing $\left(C_{\mu}=0.01\right)$ did not outperform the baseline airfoil during pitch-down between $6^{\circ} \leq \alpha \leq 25^{\circ}$ (filled triangular 
symbols); strategic termination of CC during this portion of the pitch cycle would reduce the power expenditure per pitch cycle by nearly $25 \%$ while still using constant blowing CC, for example.

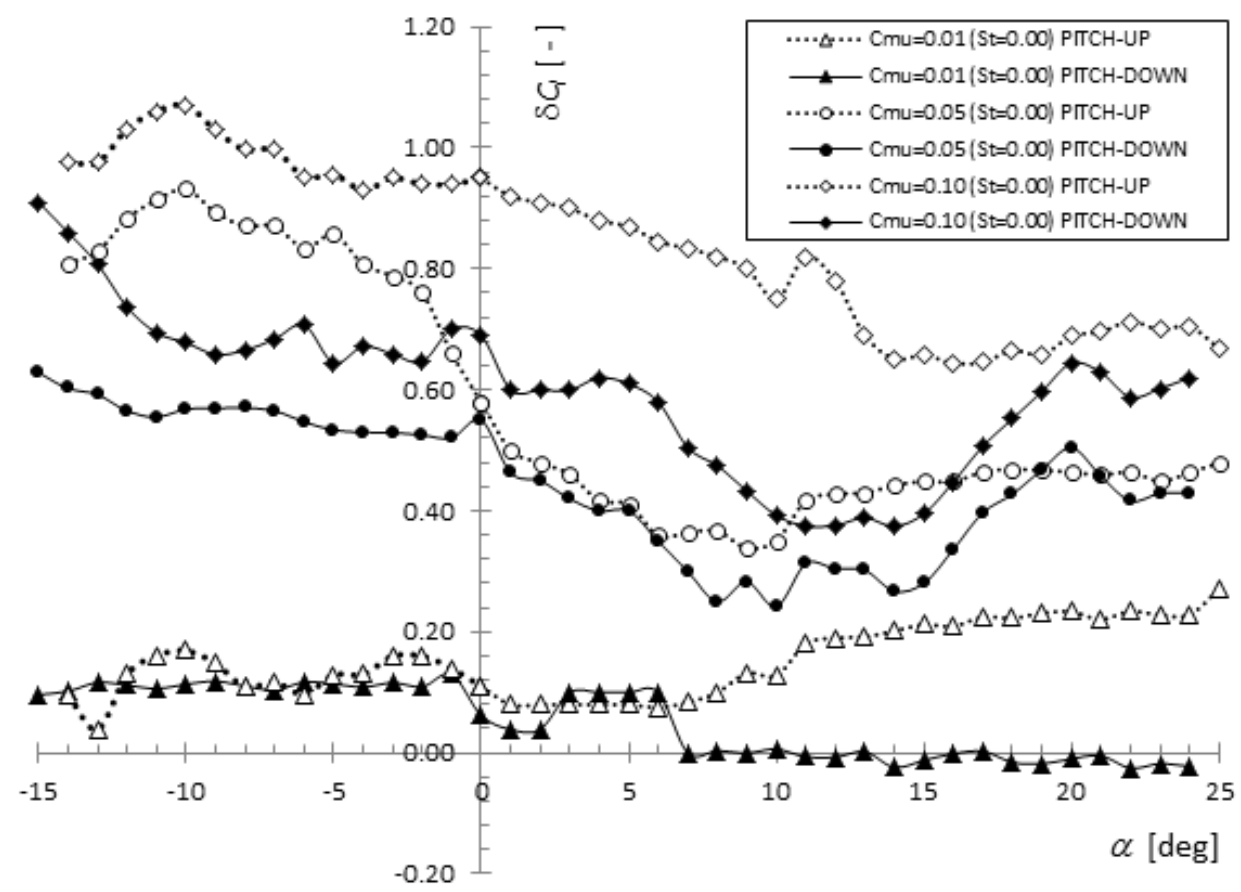

Figure 127. Baseline Lift Enhancement Pitching CCNACA0018 Airfoil: $R e=180 \times 10^{3}(k=0.100)$ 


\section{CHAPTER 6. DISCUSSION}

This section provides a comparison and summary of salient results from both experimental and numerical analysis. Topics of interest include pulsed CC airfoil performance enhancements and reduced flow requirements, along with the influence of dynamic pitch on CC airfoil aerodynamics. Pitching airfoil characteristics comprise of $R e, k$, and $\alpha_{\max }$ effects.

\subsection{Static Airfoil: Pulsed Jet Effects}

Experimental data showed overall good agreement to computed lift coefficients for static CCNACA0018 conditions at $\operatorname{Re}=180 \times 10^{3}\left(\alpha=0^{\circ}\right)$; see Figure 128 . The difference between experimental and computed $C_{l}$ increases with $C_{\mu}$, indicating that prediction of jet separation was the likely cause for discrepancy. Furthermore, $C_{1}$-differences increase with $S t$ for each magnitude of blowing. For $C_{\mu}=0.05$, disagreement between measured and calculated (CFD) lift for steady blowing was 6\%, 14\% for pulsed blowing of $S t=0.25$, and $18 \%$ difference for $S t=0.50$. A pulsed CC jet further increases the complexity of flow physics near the rounded TE, further supporting that simulations had difficulty predicting the jet separation location for unsteady actuation, since movement of the TE separation point directly influences lift production. For baseline and low blowing conditions, predicted lift values fell within the range of experimental uncertainty, proving excellent agreement between methods. For higher magnitudes of CC, numerical results fell just outside of experimental error.

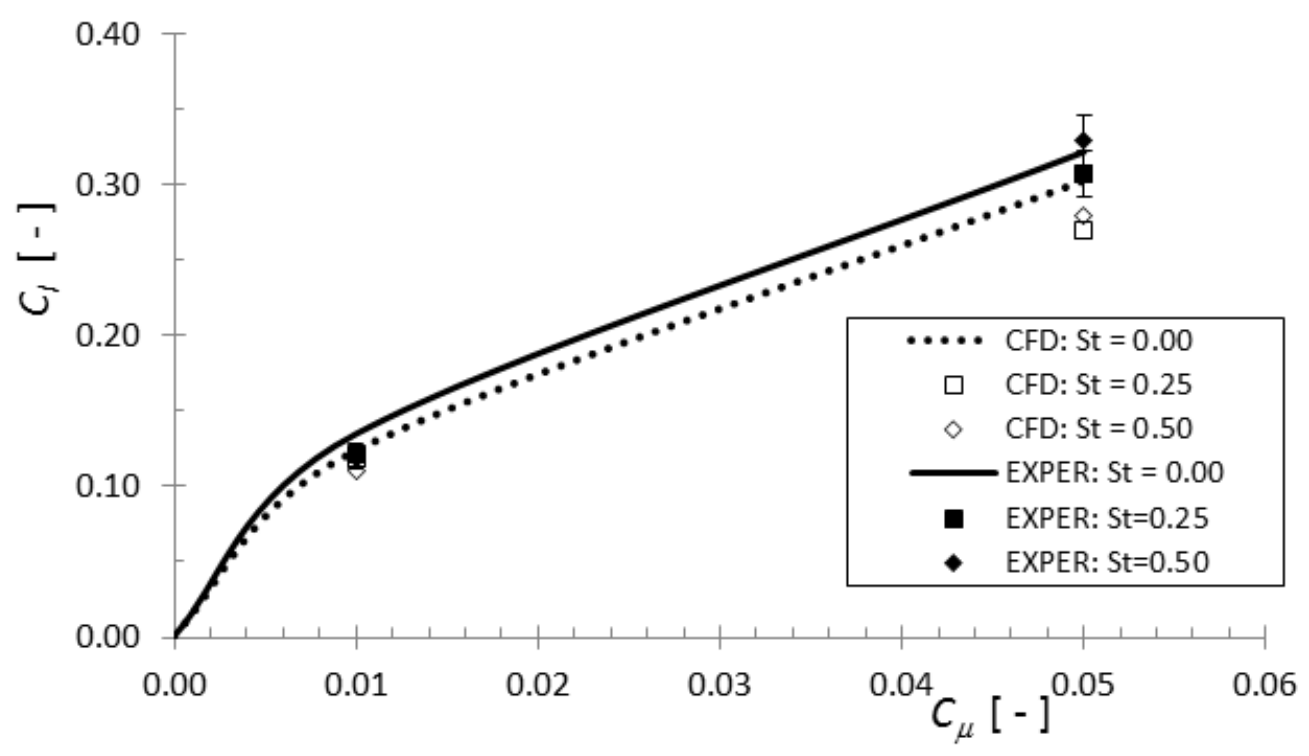

Figure 128. Influence of St on Static Lift: Experiment vs. CFD $\left(\operatorname{Re}=180 \times 10^{3}, \alpha=0^{\circ}\right)$ 


\subsection{Pitching Airfoil Motion}

Before comparing dynamic experimental and numerical results, the key test condition of pitching airfoil motion ( $\alpha$-traces) will first be compared. Figure 129 compares airfoil motion from experimental and numerical studies of $R e=180 \times 10^{3}$ and $k=0.100$; the curves are also compared to the analytical expression for VAWT blade AoA (Figure 129). Experimental and computational airfoil travel show excellent agreement during the pitch-up portion of the cycle, but are slightly out of phase during pitch-down. However, the most important dynamic features of $\mathrm{d} \alpha / \mathrm{d} t$ and $\alpha_{\max }$ agree and the small phase disagreement was considered negligible. All comparisons of experimental and numerical pitch conditions showed similar results; Figure 129 is included as an example.

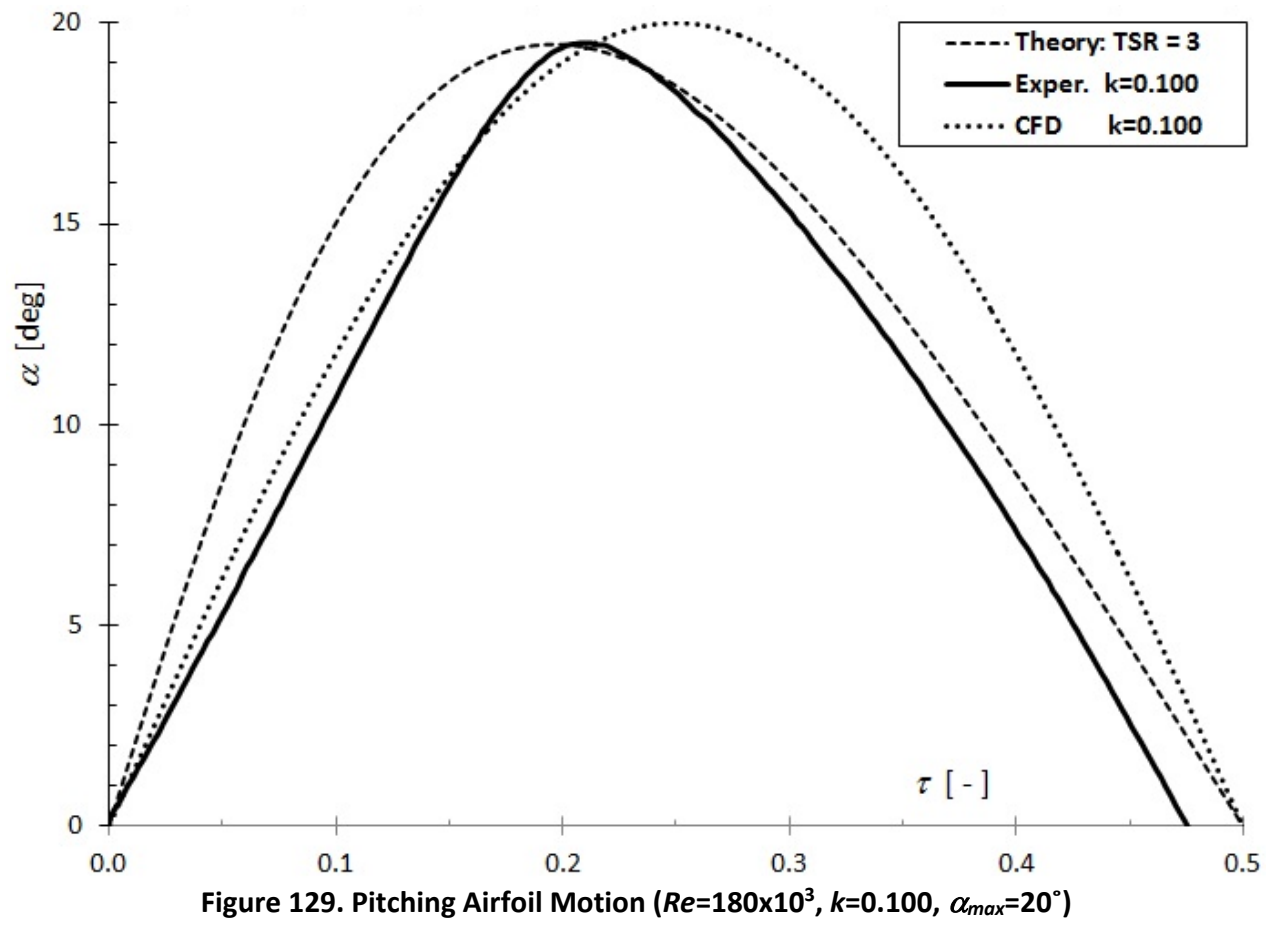




\subsection{Reynolds Number Effects (Re)}

The experimental trends for Re influence on pitch-up total lift for a pitching CCNACAO018 airfoil are included in Table 26.

Table 26. Experimental Trend Analysis: Influence of Re on Pitching CCNACA0018 Total Lift

\begin{tabular}{|c|c|c|c|c|c|}
\hline & $C_{\mu}=0.00$ & $C_{\mu}=0.01$ & $C_{\mu}=0.01(S t=0.50)$ & $C_{\mu}=0.05$ & $C_{\mu}=0.05(S t=0.50)$ \\
\hline$R e=180 \mathrm{~K}$ & $\Delta C_{I}=32.51$ & $\Delta C_{I}=36.58$ & $\Delta C_{i}=34.28$ & $\Delta C_{I}=36.90$ & $\Delta C_{i}=44.38$ \\
\hline$R e=300 \mathrm{~K}$ & $\Delta C_{i}=24.13$ & $\Delta C_{I}=30.84$ & $\Delta C_{I}=30.55$ & $\Delta C_{I}=34.29$ & $\Delta C_{I}=37.89$ \\
\hline TREND: $R e \uparrow$ & $\Delta C_{i \text { (Baseline) })} \downarrow$ & $\Delta C_{i(S t=0)} \downarrow$ & $\Delta C_{i(S t \neq 0)} \downarrow$ & $\Delta C_{i(S t=0)} \downarrow$ & $\Delta C_{i(S t \neq 0)} \downarrow$ \\
\hline
\end{tabular}

For the baseline airfoil, and all blowing cases, increasing Re resulted in a decrease in total lift for the first half of the pitch cycle. During pitch-up motion, Re did not have significant influence on lift performance. However, increasing Re resulted in delayed lift recovery after stall, resulting in larger hysteresis loops and lower total lift for positive AoA pitch. For computational analysis, $R e=180 \times 10^{3}$ was the only freestream condition simulated and thus, Re trends could not be established.

\subsection{Reduced Frequency Effects $(k)$}

The influence of reduced pitch frequency $(k)$ on measured total lift of a pitching CCNACAO018 airfoil with various modes of CC actuation is included in Table 27. To compare the effects of various blowing modes on airfoil performance, the net lift generated for each CC condition was calculated from the integral of the lift coefficient over angle of attack variation (i.e. the area enclosed by the $C_{l}(\alpha)$ curve for the range $0^{\circ} \leq \alpha \leq \alpha_{\max }$ ). For the baseline airfoil, total lift increased with increasing $k$, matching common trends from the literature. However, flow control cases showed a reverse trend, as both steady and oscillating jet cases generated more lift at lower pitch frequencies. In terms of VAWT power production, this result indicates that CC offers higher potential gains at low TSR $(\lambda)$, the operating regime currently plagued by poor performance due to dynamic stall.

Table 27. Experimental Trend Analysis: Influence of $k$ on Pitching CCNACA0018 Total Lift

\begin{tabular}{|c|c|c|c|c|}
\hline & $C_{\mu}=0.00$ & $C_{\mu}=0.01(S t=0.00)$ & $C_{\mu}=0.01(S t=0.25)$ & $C_{\mu}=0.05(S t=0.00)$ \\
\hline$k=0.050$ & $\Delta C_{I}=32.50$ & $\Delta C_{I}=36.58$ & $\Delta C_{i}=34.96$ & $\Delta C_{I}=44.38$ \\
\hline$k=0.100$ & $\Delta C_{l}=33.71$ & $\Delta C_{I}=35.67$ & $\Delta C_{l}=33.63$ & $\Delta C_{l}=42.43$ \\
\hline TREND: $k \uparrow$ & $\Delta C_{l(\text { Baseline })} \uparrow$ & $\Delta C_{i(S t=0)} \downarrow$ & $\Delta C_{i(S t \neq 0)} \downarrow$ & $\Delta C_{i(S t=0)} \downarrow$ \\
\hline
\end{tabular}

The trend analysis from numerical simulations for $k$ effects on total lift in summarized in Table 28. Computed trends for all conditions match experimental results. For each blowing condition, increasing $\mathrm{k}$ by a factor of 2 resulted in an average improvement of $18 \%$. Comparing individual case 
results of $\Delta C_{1}$, between experiment and CFD, shows an average of $11 \%$ difference for the 8 cases detailed.

Table 28. Numerical Trend Analysis: Influence of $k$ on Pitching CCNACA0018 Total Lift

\begin{tabular}{|c|c|c|c|c|}
\hline & $C_{\mu}=0.00$ & $C_{\mu}=0.01(S t=0.00)$ & $C_{\mu}=0.01(S t=1.00)$ & $C_{\mu}=0.05(S t=0.00)$ \\
\hline$k=0.075$ & $\Delta C_{I}=31.62$ & $\Delta C_{I}=33.60$ & $\Delta C_{I}=37.24$ & $\Delta C_{I}=53.48$ \\
\hline$k=0.100$ & $\Delta C_{I}=41.31$ & $\Delta C_{I}=27.86$ & $\Delta C_{I}=33.53$ & $\Delta C_{I}=46.20$ \\
\hline TREND: $k \uparrow$ & $\Delta C_{i(\text { (Baseline) }} \uparrow$ & $\Delta C_{i(S t=0)} \downarrow$ & $\Delta C_{i(S t \neq 0)} \downarrow$ & $\Delta C_{l(S t=0)} \downarrow$ \\
\hline
\end{tabular}

\subsection{Pitch Amplitude Effects $\left(\alpha_{\max }\right)$}

The experimental trends for pitch amplitude influence on pitch-up total lift for a pitching CCNACA0018 airfoil are included in Table 29. Increasing the pitch amplitude was shown to increase the maximum lift for all conditions, as expected. For the baseline airfoil, increasing $\alpha_{\max }$ by $5^{\circ}$ resulted in a $16 \%$ increase in $C_{l, \max }$; the amplitude increase resulted in maximum lift improvements of $12 \%$ and $14 \%$ for steady blowing of $C_{\mu}=0.01$, and $C_{\mu}=0.05$, respectively.

Table 29. Experimental Trend Analysis: Influence of $\alpha_{\max }$ on Pitching CCNACA0018 Total Lift

\begin{tabular}{|c|c|c|c|}
\hline & $C_{\mu}=0.00$ & $C_{\mu}=0.01(S t=0.0)$ & $C_{\mu}=0.05(S t=0.00)$ \\
\hline$\alpha_{\max }=25^{\circ}$ & $C_{i, \max }=1.88$ & $C_{i, \max }=2.11$ & $C_{i \max }=2.35$ \\
\hline$\alpha_{\max }=30^{\circ}$ & $C_{i, \max }=2.19$ & $C_{i, \max }=2.36$ & $C_{i \max }=2.69$ \\
\hline TREND: $\alpha_{\max } \uparrow$ & $C_{i, \max } \uparrow$ & $C_{i, \max } \uparrow$ & $C_{i \max } \uparrow$ \\
\hline
\end{tabular}

The numerical trends for pitch amplitude influence on pitch-up total lift for a pitching CCNACA0018 airfoil are included in Table 30. Computational trends matched experiment, but showed more dramatic increases in $C_{1, \max }$ for the same increase in amplitude. Comparing maximum lift for individual cases between measurement and calculation, an average difference between the six cases was 26\%; this range of discrepancy was too large to conclude quantitative agreement between methods.

Table 30. Numerical Trend Analysis: Influence of $\alpha_{\max }$ on Pitching CCNACA0018 Total Lift

\begin{tabular}{|c|c|c|c|}
\hline & $C_{\mu}=0.00$ & $C_{\mu}=0.01(S t=0.0)$ & $C_{\mu}=0.05(S t=0.00)$ \\
\hline$\alpha_{\max }=20^{\circ}$ & $C_{i \max }=1.06$ & $C_{i, \max }=2.18$ & $C_{l y \max }=2.82$ \\
\hline$\alpha_{\max }=30^{\circ}$ & $C_{i \max }=1.90$ & $C_{i \max }=2.46$ & $C_{l_{\max }}=3.75$ \\
\hline TREND: $\alpha_{\max } \uparrow$ & $C_{l, \max } \uparrow$ & $C_{i, \max } \uparrow$ & $C_{l, \max } \uparrow$ \\
\hline
\end{tabular}




\subsection{CCVAWT Control Strategy}

In this research, the aerodynamic benefits of circulation control (steady and pulsed) on dynamic stall were analyzed. In reality, implementing effective flow control strategies into an operational VAWT blade would require closed-loop control schemes. The feed-back system would need to monitor operating conditions (e.g. $\alpha, \theta, \lambda)$, and use logic to determine the azimuthal position of control $(\theta)$, the proper blowing slot (i.e. inner- or outer-surface jet), jet magnitude $\left(C_{\mu}\right)$, and pulsing frequency (St) to optimize the net power output of the CCVAWT. Thus, such a topic is beyond the scope of the current research, but will need to be considered before unsteady circulation control can be effectively implemented in the built environment.

All pitching CC-airfoil data included in this research was measured with continuous actuation (steady and pulsed jet) during the entire sinusoidal pitching motion. For higher magnitudes of blowing, whether steady or pulsed CC, is analogous to pitching a conventional airfoil with large camber. Past research has shown that symmetric airfoils generally possess improved stall recovery performance (small hysteresis loops) when compared to flapped and cambered profiles. Thus, the post-stall recovery of the CCNACA0018 would likely see improved performance (e.g. increases in $\mathrm{d} C_{I} / \mathrm{d} \alpha, C_{l, \text { max }}$, and $\alpha_{D S}$; decreases in post-stall $C_{d}$ and negative $C_{m}$ excursions) if control actuation was terminated, or reduced in magnitude, post stall. Furthermore, to better recreate the performance of a CCVAWT blade, the current slot (upper surface) could be actuated for the $\left(+10^{\circ} \leq \alpha \geq \alpha_{D S}\right)$ portion of the pitch-up cycle, while the currently unused slot (lower surface) could be actuated during the $\left(-\alpha_{D S} \leq \alpha \geq-10^{\circ}\right)$ section of the pitchdown cycle. This data would further enhance the performance predictions of the CCVAWT blade.

As previously mentioned, a VAWT blade experiences two dynamic stall processes for each rotation, with the airfoil surface housing the DSV dependent on upwind and downwind operation. Thus, a CCVAWT blade would need actuation capabilities from both upper and lower surface slots. The CCNACA0018 model tested in this study was only capable of upper slot CC. To approximate such performance, airfoil characteristics for positive AoA pitch could be mirrored about the $C_{1}$-axis (for example), to simulate a switch to lower slot blowing for negative AoA motion. Figure 130 includes an example of such curves (solid lines) for clean airfoil and steady blowing cases; for reference, the original data is included as symbols. The updated curves of Figure 130 would be more appropriate airfoil performance data for analytical predictions of CCVAWT power output. 


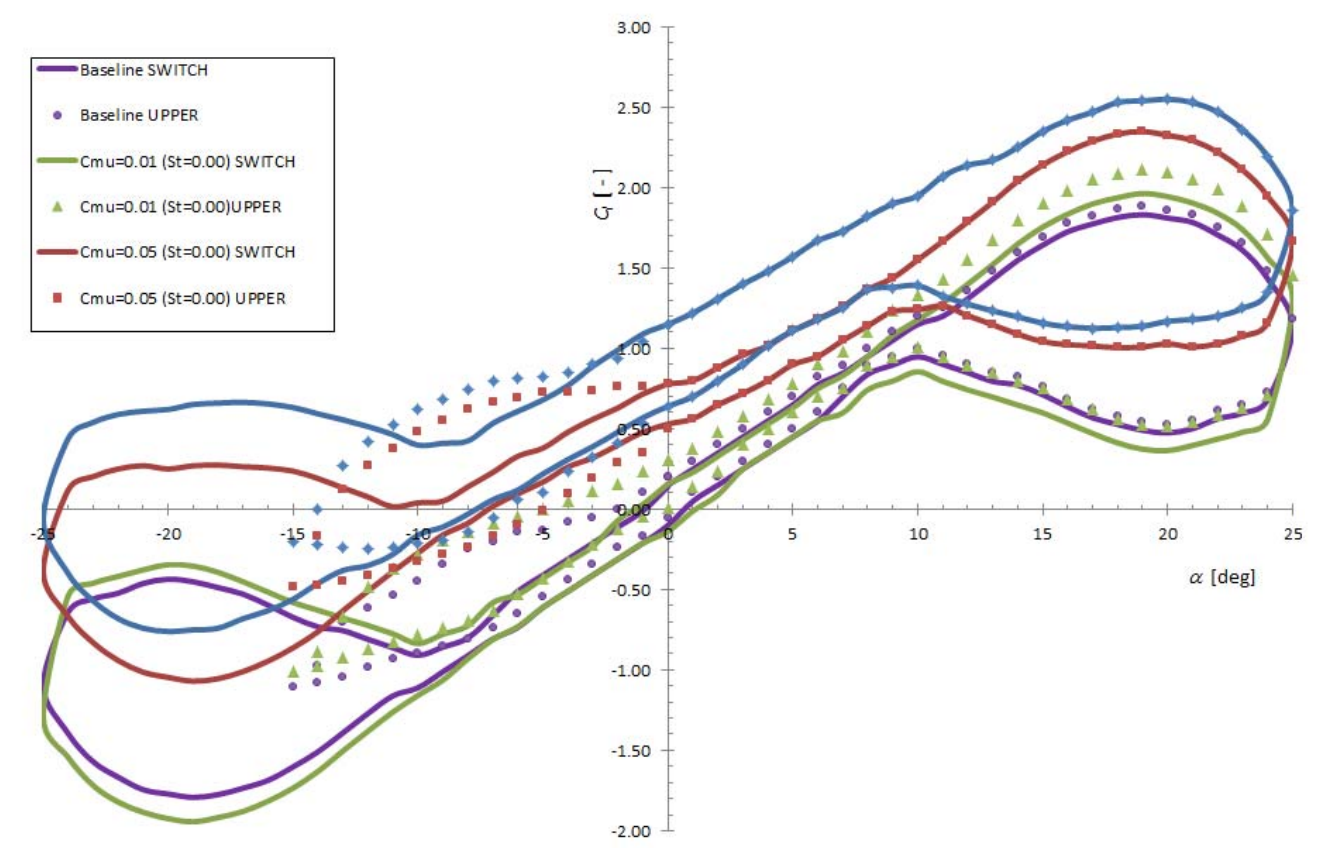

Figure 130. CCVAWT Lift Curves: Alternate Upper and Lower Slot Blowing: $\alpha(t)=0^{\circ}+25^{\circ} \sin (\omega t)$

\subsection{Summary of Results}

The experimental and computational results are summarized here, with respect to the research objectives of this study. The principal objective of this research was to examine the benefits of pulsed blowing to reduce mass flow requirements for desired CCVAWT blade performance. The objectives of this were met by answering the following questions:

I. Can experimental and CFD analyses prove a pulsed CC jet can match steady jet performance enhancements for a static CCNACA0018 airfoil, at reduced mass flow requirements?

Yes. Both wind tunnel and numerical results proved pulsed jet actuation could generate 90 to $100 \%$ of CCNACA0018 steady jet lift with up to $35 \%$ reduced mass flow requirements.

II. What is the dependency of flow control parameters $\left(C_{\mu}, S t\right)$ to achieve desired aerodynamic enhancements?

For the CCNACA0018 airfoil, under simulated VAWT conditions, it was found that the effective Strouhal number for jet actuation increased with the magnitude of momentum addition

[i.e. $\left(C_{\mu}\right) \uparrow:\left(\right.$ Effective $\left.\left.S t_{j e t}\right) \uparrow\right]$ 
III. How does dynamic pitch influence CCNACA0018 performance under simulated VAWT conditions?

Dynamic pitch was found to have a significant impact on baseline CCNACA0018 performance, with $k$ and $\alpha_{\max }$ showing dominating influences over $R e$. The influence of each dynamic pitch variable ( $\left.R e, k, \alpha_{\max }\right)$ on total lift, maximum lift, and stall AoA are summarized in Table 31; all trends for the baseline airfoil agree with the literature (see Chapter 2).

Table 31. Influence of Dynamic Pitch Variables on Baseline CCNACA0018 Performance

\begin{tabular}{|c|c|c|c|c|}
\hline \multicolumn{2}{|c|}{ Baseline } & $\int C_{l}$ & $C_{l, \max }$ & $\alpha_{\text {stall }}$ \\
\hline $\boldsymbol{R} e \uparrow$ & negligible & negligible & negligible \\
\hline $\boldsymbol{k}$ & $\uparrow$ & $\uparrow \mathbf{8 7 \%}$ & $\uparrow \mathbf{3 0 \%}$ & $\uparrow \mathbf{8}^{\circ}$ \\
\hline$\alpha_{\max } \uparrow$ & $\uparrow$ & $\uparrow$ & $\uparrow$ \\
\hline
\end{tabular}

IV. What is the relationship between effective flow control parameters $\left(C_{\mu}, S t\right)$ and flow conditions $\left(R e, k, \alpha_{\max }\right)$ ?

A relationship between effective flow control parameters and pitching airfoil conditions was established. The influence of all dynamic pitch variables on total and maximum lift were consistent for all CC cases, whether the CC jet was steady $(S t=0.00)$ or pulsed $(S t \neq 0.00)$. The influence of dynamic pitch variables on CC actuation performance are summarized in Table 32. The trends for increasing $\boldsymbol{k}$ were opposite that of the baseline airfoil, resulting in decreasing total lift.

Table 32. Influence of Dynamic Pitch Variables on Steady and Pulsed Jet CCNACA0018 Performance

\begin{tabular}{|c|c|c|c|c|}
\hline \multirow{2}{*}{$\begin{array}{c}\text { Circulation } \\
\text { Control }\end{array}$} & \multicolumn{2}{|c|}{$c_{\mu}=0.01$} & \multicolumn{2}{c|}{$c_{\mu}=0.05$} \\
\cline { 2 - 6 } & St $=0.00$ & St $\neq 0.00$ & St $=0.00$ & St $\neq 0.00$ \\
\hline$R e \quad \uparrow$ & negligible & negligible & negligible & negligible \\
\hline$k$ & $\int c_{l} \downarrow$ & $\int c_{l} \downarrow$ & $\int c_{l} \downarrow$ & $\int c_{l} \downarrow$ \\
\hline$\alpha_{\max } \uparrow$ & $c_{l, \max } \uparrow$ & $c_{l, \max } \uparrow$ & $c_{l, \max } \uparrow$ & $C_{l, \max } \uparrow$ \\
\hline
\end{tabular}




\section{CHAPTER 7. CONCLUSIONS}

The main objective of this research was to determine if pulsed circulation control would produce the same aerodynamic performance enhancements with the added benefit of reduced mass flow (power penalty) relative to steady actuation.

Both steady and pulsed circulation control proved potential as an instrument to prolong, or even prevent, dynamic stall on the blades of a VAWT. Pulsed blowing offers lift enhancement and extends $\alpha_{\text {stall, }}$ and shows potential for alleviation of unsteady load reductions. The efficiency of circulation control blowing on a VAWT blade could be further realized by varying $C_{\mu}$ as a function of orbital position. Using flow control on only a portion of a blade revolution could lead to more dramatic reductions of the blowing power penalty, making this a viable option to expand VAWT operational envelope to low TSR regimes. Variable blowing based on VAWT blade azimuth angle could also help mitigate the unsteady, cyclic loading experienced by a VAWT structure in certain conditions.

Typically, the unsteady aerodynamic loads on a pitching airfoil increase the static stall angle by $2^{\circ}-8^{\circ}$. A $+6^{\circ}$ increase to the $\alpha_{S S}$ was observed for dynamic testing of the baseline CCNACA0018 model at $k=0.100$. However, typical flow control cases showed lesser improvements of $4^{\circ}-5^{\circ}$, due to the increased airfoil camber imparted by the CC jet. $C_{l, \max }$ was drastically increased by as much as $30 \%$.

The aerodynamic performance of a pitching CCVAWT blade was measured and computed to update static force coefficients from previous work. The variance between integrated force coefficients between pitch-up and pitch-down airfoil motion proved the idealistic nature of static airfoil data. For example, at a pitch rate of $k=0.100$, the baseline airfoil showed an increase of $26 \%$ in $C_{l, \text { max }}$ and an $\alpha_{\text {stall }}$ extension of $6^{\circ}$, compared to static measurements. The updated pitch data, with realistic force hysteresis loops, will serve as more accurate input to analytical models for improved CCVAWT power predictions.Experimental and computational results both showed reduced force-hysteresis loops for increasing $k$ at each $R e$, as expected based on historical data from the literature.

The objectives of the study were successfully accomplished. A pulsed CC jet was proven to match steady jet lift enhancement with reduced flow requirements of $30 \%$. Lower magnitudes of pulsed blowing $\left(C_{\mu}=0.01\right)$ were more effective with higher actuation frequencies of $S t=4$, while increased amplitudes of control $\left(C_{\mu}=0.05\right)$ produced optimal results with lower actuation frequencies of $S t=0.5$. Trend studies of the influence dynamic pitch parameters on CCNACA performance were found to match those of conventional airfoils from the literature. 
Experimental data showed quantitative agreement to computed aerodynamic coefficients for static CCNACA0018 conditions at $\operatorname{Re}=180 \times 10^{3}\left(\alpha=0^{\circ}\right)$. The difference between experimental and computed $C_{l}$ increased with $C_{\mu}$ and $S t$, indicating that prediction of jet separation was the likely cause for discrepancy. For baseline and low blowing conditions, predicted lift values fell within the range of experimental uncertainty, while higher magnitudes of $C_{\mu}$ contained a maximum difference of $18 \%$ between measured and computed $C_{I}$ and $C_{d}$. Modulated CC jets were shown to match steady CC lift enhancements, with mass flow savings exceeding $30 \%$ for a static airfoil at low AoA. Low $C_{\mu}$ jets showed better $C_{1}$-performance with low frequency actuation, while the opposite was found for increased levels of CC.

Numerical results showed increasing $C_{\mu}$ (steady and pulsed modes) reduced the AoA of DSV formation and subsequent lift spike, an observation common to past studies of pitching airfoils with various camber. At high TSR, a VAWT blade operates in AoA ranges below static stall ( $\pm \alpha \leq \alpha$ ss). In such operating regimes, the dynamic stall conditions would be approached but not reached. It is thought that high $C_{\mu}$ blowing $(\geq 0.05)$, with actuation initiated $2^{\circ}-5^{\circ}$ degrees prior to $\alpha_{\max }$, could expedite DSV formation to produce significant lift gains of high $\lambda$ operation where the airfoil pitches between AoA near static stall.

The completion of this study has proven pulsed CC effective to improve CCVAWT efficiency. Optimal conditions of pulsed CC jets were show to match steady CC jet lift and reduce profile drag, while reducing required flow and power penalties by up to 35\%. Relative to a conventional VAWT with annual power output of $1 \mathrm{MW}$, previous work at $W V U$ proved that the addition of steady jet CC could improve total output to $1.25 \mathrm{MW}$. However, the pumping cost to generate the continuous jet reduced yearly CCVAWT net gains to $1.15 \mathrm{MW}$. The current study has shown that pulsed CC jets can recover $4 \%$ of the pumping demands due to reduced mass flow requirements, increasing annual CCVAWT net power production to $1.19 \mathrm{MW}$, a $19 \%$ improvement relative to the conventional turbine. 


\section{CHAPTER 8. RECOMMENDATIONS}

Based on the results of this study, pulsed CC was determined a viable method to improve the efficiency of a novel VAWT design. CCVAWT performance predictions can now be significantly improved with updated input data. Previous power outputs were calculated from static airfoil measurements, where "pitch-up" data unrealistically mirrors that of "pitch-down" motion. This study generated a new database of airfoil data that includes force overshoot and hysteresis effects from dynamic pitch. In addition, prior analytical modelling predicted turbine performance with the penalty of generating a steady blowing jet. This work proved the use of pulsed jets to improve steady CC lift enhancement at reduced mass flow rates. The new test data, including the effects due to unsteady dynamic pitch and pulsed CC effects on airfoil performance, should be used to update vortex model inputs for improved CCVAWT performance projections.

A few notes of recommendation regard the fabrication of an improved 2D CCNACA0018 wind tunnel model with improved internal pneumatic valving. The current model was originally designed to investigate the response time of the $\mathrm{CC}$ jet, where actuation frequency $>1 \mathrm{~Hz}$ was not needed. The current study was governed by the operating performance of the valves, where a maximum frequency of $150 \mathrm{~Hz}$ was attainable, limiting maximum St to 0.50 . The numerical results of this study indicated that jet frequencies up to $S t=4.0$ to be beneficial under various conditions. Thus, an experimental model with faster valve operation $(1.0 \leq S t \leq 4.0)$ is recommended to validate the computational model. Furthermore, the literature suggests that jet duty cycle $(D C)$ to influence pulsed jet performance. The current study completed all tests and simulations with $D C=50 \%$, but it is recommended to expand this range to $20 \% \leq D C \leq 80 \%$. Finally, it was realized during the current study that the model used in the current study contains a design flaw, namely an angular offset on the upper and lower airfoil surfaces in the region $0.905 \leq x / c \leq 0.969$, leading to the TE slots (see CCNACA profile in Figure 50). The location of this angular offset likely creates an unnecessary adverse pressure gradient upstream of the jet, reducing the effectiveness and control authority of CC of the model used in the current study. Removing the aft slope from the airfoil surface would improve the Coandă effect, entrain more freestream flow, and enhance lift for a given $\mathrm{Cm}$ condition. Thus, an experimental and/or numerical model should be created to quantify the effect of airfoil surface discontinuity.

Furthermore, during actual VAWT operation, each blade experiences a varying freestream velocity due to rotation, while the experimental and computational results included in this effort were completed within steady freestream velocity. To continue this research, 3D analysis of a scaled CCVAWT 
is necessary to investigate the influence of rotational motion on modulated CCVAWT performance. This study showed both steady and pulsed CC to influence the size and scale of TE vorticity on a pitching CCVAWT blade. Thus, the influence of shed wake vorticity on downwind airfoil performance for various modes of CC actuation should be investigated. Scale testing is needed to validate and improve control strategies that govern actuation based on blade azimuth position. It is known that flow separation (stall) occurs on the inner surface of the blade during upwind rotation and outboard surface during downwind rotation. Thus, effective control would require switching between inboard slot and outboard slot actuation during rotation. The scaled model will need airfoils with upper and lower slot actuation capabilities to properly analyze CCVAWT performance. Furthermore, the addition of internal valving will add weight to each blade, changing the moment of inertia. Thus, the influence of actuation components on the structural integrity and life of the turbine should be examined. An economic assessment factoring additional costs of internal valving components, fabrication, operations and maintenance for the augmented turbine blades will be necessary to determine annual cost per kWh.

Finally, evidence from the literature has proven closed-loop control to optimize airfoil efficiency by utilizing flow control during portions of a pitch cycle where effective. The development and integration of such technology into blades would further reduce mass flow and energy expenditure to improve CCVAWT net power gains. 


\section{WORKS CITED}

1. MacPhee, D., Beyene, A. Recent Advances in Rotor Design in Vertical Axis Wind Turbines. J. Wind Engineering, Vol. 36(6), 2012.

2. Carrigan, T. J. Aerodynamic Shape Optimization of a Vertical Axis Wind Turbine. Arlington, TX : University of Texas, MS Thesis, 2010.

3. Roh, S., Kang, S. Effects of Blade Profile, the Reynolds Number, and the Solidity on the Performance of a Straight Bladed Vertical Axis Wind Turbine. J. Mechanical Science \& Technology, Vol. 27(11), 2013.

4. Greenblatt, D., Mueller-Vahl, H. \& A. Ben-Harav. Mechanism of Dynamic Stall Control on a Vertical Axis Wind Turbine. Grapevine, TX : 51st AIAA Aerospace Sciences Meeting, January 7-10, 2013 (AIAA 2013-0851).

5. Spera, D. Wind Turbine Technology: Fundamental Concepts of Wind Turbine Engineering. New York, NY : ASME Press, ISBN: 0-7918-1205-7, 1998.

6. Paraschivoiu, I. Aerodynamic Loads and Rotor Performance For The Darrieus Turbines. Colorado Springs, CO : AIAA 2nd Terrestrial Energy Systems Conference, 1981 (AIAA-81-2582).

7. Carr, L. Progress in Analysis and Prediction of Dynamic Stall. J. of Aircraft, Vol. 25, 1988.

8. Troldborg, N. Computational Study of the Ris-B1-18 Airfoil with a Hinged Flap Providing Variable Trailing Edge Geometry. J. Wind Engineering, Vol. 29(2), 2005.

9. Kirke, B. Evaluation Of Self-Starting Vertical Axis Wind Turbines For Stand-Alone Applications. Brisbane, Australia : Griffith University, PhD Thesis, 1998.

10. Greenblatt, D., Shulman, M. \& A. Ben-Harav. Vertical Axis Wind Turbine Performance Enhancement using Plasma Actuators. J. Renewable Energy, Vol. 37, 2012.

11. Yen, J., Ahmed, N. Enhancing Vertical Axis Wind Turbine by Dynamic Stall Control using Synthetic Jets. J. Wind Engr. \& Ind. Aerodyn., Vol. 114, 2013.

12. Nakafuji, D., van Dam, C., Smith, R., \& S. Collins. Active Load Control for Airfoils using Microtabs. J. Solar Energy Engr., Vol. 123, 2001.

13. Lackner, M., van Kuik, G. The Performance of Wind Turbine Smart Rotor Control Approaches During Extreme Loads. J. Solar Energy Engr., Vol. 132(1), 2010.

14. Berg, D., Wilson, D., Resor, B., Berg, J., Barlas, J., Crowther, A., \& C. Halse. System ID Control Algorithms for Active Aerodynamic Load Control and Impact on Gearbox Loading. The European Wind Energy Association (EWEA), The Science of Making Torque from Wind, 2010 
15. Daniele, R., Ferreira, C., \& M. Barone. Experimental and Numerical Investigation of an Optimized Airfoil for Vertical Axis Wind Turbines. National Harbor, MD : AIAA 32nd ASME Wind Energy Symposium, 2014 (AIAA 2014-0171).

16. Muller-Vahl, H., Strangfeld, C., Nayeri, C., Paschereit, C., \& D. Greenblatt. Thick Airfoil Deep Dynamic Stall and Its Control. Grapevine, TX : 51st AIAA Aerospace Sciences Meeting, 2013 (AIAA 20130854).

17. Wickens, R. Wind Tunnel Investigation of Dynamic Stall of an NACA0018 Airfoil Oscillating in Pitch. Ottawa, Canada : National Aeronautical Establishment, 1985 (Aeronautical Note NAE-AN-27).

18. Pawsey, N. Development and Evaluation of Passive Variable-Pitch Vertical Axis Wind Turbines. South Wales, U.K. : The University of South Wales: School of Mechanical and Manufacturing Engineering, PhD Thesis, 2002.

19. Liiva, J., Davenport, F, \& I. Walton. Two-Dimensional Tests of Airfoils Oscillating Near Stall - Vol. 1. Tech. Report 68-13A : USAAVLABB , 1968.

20. Crimi, P., Yaggy, P. Dynamic Stall. AGARDograph No. 172 : North Atalantic Treaty Organization Advisory Group For Aerospace Research and Development, 1975.

21. Jee, S. Flow Control Simulation with Synthetic and Pulsed Jet Actuator. Austin : University of Texas, PhD Thesis, 2010.

22. Timmer, W. Two-Dimensional Low-Renolds Number Wind Tunnel Results for Airfoil NACA 0018. J. Wind Engineering, Vol. 32(6), 2008.

23. Raghunathan, S., Ombaka, O. A Thick Symmetric Aerofoil Oscillating about Zero Incidence Angle. Elsevier B.V. Amsterdam : J. Heat \& Fluid Flow, Vol. 7(2), 1986.

24. Danao, L. A. The Influence of Unsteady Wind on the Performance and Aerodynamics of Vertical Axis Wind Turbines. South Yorkshire, UK : The University of Sheffield, PhD Thesis, 2012.

25. Fiedler, A. J., Tullis, S. Blade Offset and Pitch Effects on a High Solidity Vertical Axis Wind Turbine. J. Wind Engineering, Vol. 33(3), 2009.

26. Danao, L., Edwards, J., Eboibi, O., \& R. Howell. The Performance of a Vertical Axis Wind Turbine in Fluctuating Wind - A Numerical Study. World Congress on Engineering (WCE), Vol. 3, 2013.

27. Claessens, M. The Design and Testing of Airfoils for Application in Small Vertical Axis Wind Turbines. Delft, Netherlands : Delft University of Technology, MS Thesis, 2006.

28. Oler, J., Strickland, J., Im, B., \& H. Graham. Dynamic Stall Regulation of the Darrieus Turbine. Lubbock, TX : SAND83-7029, 1983.

29. Holt, D. A Circulation Controlled Airfoil With Pulsed Flow. Morgantown, WV : West Virginia University, MS Thesis, 1972. 
30. Soraghan, C., Leithead, W., \& P. Jamieson. Influence of Lift to Drag Ratio on Optimal Aerodynamic Performance of Straight Bladed Vertical Axis Wid Turbines. Vienna, Austria : The European Wind Energy Association (EWEA), 2013.

31. Wilhelm, J. Power Envelope Expansion using a Solidity Matching Scheme for a Circulation Controlled Vertical Axis Wind Turbine. Morgantown, WV : West Virginia University, PhD Thesis, 2010.

32. McGrain, D., Angle, G., Wilhelm, J., Pertl, E., \& J. Smith. Circulation Control Applied to Wind Tubines. San Francisco, CA : ASME Energy Sustainability Conference, 2009 (ES2009-90076).

33. Graham, H., Panther, C., Hubbell, M., Wilhelm, J., Angle, G., \& J. Smith. Airfoil Selection For A Straight Bladed Circulation Controlled Vertical Axis Wind Turbine. San Francisco, CA : ASME Energy Sustainability Conference, 2009 (ES2009-90343).

34. Graham, H., Hubbell, M., Panther, C., Wilhelm, J., Angle, G., \& J. Smith. Circulation Controlled Airfoil Analysis Through 360 Degrees Angle of Attack. San Fransisco, CA : ASME Energy Sustainability Conference, 2009 (ES2009-90341).

35. Wilhelm, J., Panther, C., Pertl, F., \& J. Smith. Momentum Analytical Model Of A Circulation Controlled Vertical Axis Wind Turbine. San Francisco, CA : ASME Energy Sustainability Conference, 2009 (ES2009-90352).

36. Wilhelm, J., Panther, C., Pertl, F., \& J. Smith. Vortex Analytical Model Of A Circulation Controlled Vertical Axis Wind Turbine. San Francisco : ASME Energy Sustainability Conference, 2009 (ES200990348).

37. Wilhelm, J., Panther, C., \& J. Smith. Jet Usage Methodology for a Vertical Axis Wind Turbine using Circulation Control. J. Wind Energy, 2014 (submitted).

38. Panther, C., Williams, K., Wilhelm, J., \& J. Smith. Experimental Testing Of A Wind Tunnel Model For Use In A Vertical Axis Wind Turbine. Vancouver, B.C. : ASME, 2009 (IMECE2010-38816).

39. Dyachuk, E., Goude, A., \& H. Bernhoff. Dynamic Stall Modeling for the Conditions of Vertical Axis Wind Turbines. Reston, VA : AIAA Journal, Vol. 52(1), 2014.

40. Sheng, W., Galbraith, R., \& F. Coton. A New Stall Onset Criterion for Low Speed Dynamic Stall. New York, NY : J. Solar Energy, Vol. 128(4), 2006.

41. Sheldahl, R., Klimas, P. Aerodynamic Characteristics of Seven Symmetrical Airfoil Sections Through 180-Degree Angle of Attack for Use in Aerodynamic Analysis of Vertical Axis Wind Turbines.

Albuquerque, NM : Sandia National Laboratores, 1980 (SAND80-2114).

42. Jacobs, E., Ward, K., \& M. Pinkerton. The Characteristics of 78 Related Airfoils from Tests in the Variable-Density Wind Tunnel. Washington, D.C. : NACA Report 460, 1933. 
43. Abramson, J. Two-Dimensional Subsonic Wind Tunnel Evaluation Of A 20-Percent Thick Circulation Control Airfoil. Bethesda, MD : David W. Taylor Naval Ship Research And Development Center, 1975.

44. Wilhelm, J., Pertl, E., Pertl, F., \& J. Smith. Performance Predictions Of A Circulation ControlledVertical Axis Wind Turbine With Solidity Control. San Francisco : ASME Energy Sustainability Conference, 2009 (ES2009-90352).

45. Jones, G. Pneumatic Flap Performance for a 2D Circulation Control Airfoil, Steady and Pulsed. Langley, VA : NASA LaRC Report, 2005.

46. Jones, G., Englar, R. Advances In Pneumatic-Controlled High-Lift Systems Through Pulsed Blowing. Orlando, FL : AIAA 21st Applied Aerodynamics Conference, 2003 (AIAA 2003-3411).

47. Oyler, T., Palmer, W. Exploratory Investigation Of Pulse Blowing For Boundary Layer Control. Columbus, $\mathrm{OH}$ : Columbus Aircraft Division/North American Rockwell Corporation, 1972 (NR72H-12).

48. Sasson, B., Greenblatt, D. Effect of Leading-Edge Slot Blowing on a Vertical Axis Wind Turbine. AIAA Journal, Vol. 49(9), 2011.

49. Robinett III, R., Wilson, D. Maximizing the Performance of Wind Turbines with Nonlinear Aeroservoelastic Power Flow Control. Yokohama, Japan : IEEE Multi-Conference on Systems and Control, 2010.

50. Kostas, J., Foucaut, J., \& M. Stanislas. The Effects of Pulse Frequency and Duty Cycle on the Skin Friction Downstream of Pulsed Jet Vortex Generators in an Adverse Pressure Gradient Turbulent Boundary Layer. J. Aerospace Science \& Technology, Vol. 13, 2009.

51. Lundell, F. Pulse-Width Modulated Blowing/Suction As A Flow Control Actuator. J. Experiments in Fluids, Vol. 35, 2003.

52. Seifert, A., Pack, L. Oscillatory Control of Separation at High Reynolds Numbers. AIAA Journal, Vol. 37(9), 1999.

53. Magill, J., Bachmann, M., Rixon, G., \& K. McManus. Dynamic Stall Control Using A Model-Based Observer. Reno, NV : 39th AIAA Aerospace Sciences \& Exhibit, 2001 (AIAA 2001-0251).

54. Post, M. Plasma Actuators For Separation Control On Stationary And Oscillating Airfoils. Dept. of Aerospace and Mechanical Engineering, University of Notre Dame, PhD Thesis, 2004.

55. Griffin, C. Numerical and Experimental Study on the Ability of Dynamic Roughness to Alter the Development of a Leading Edge Vortex. Morgantown, WV : West Virginia University, PhD Thesis, 2013.

56. Anderson, Jr., J. Fundamentals of Aerodynamics (3 ${ }^{\text {rd }}$ Edition). New York, NY : McGraw-Hill, 2001.

57. Nelson, R., Corke, T., Othman, H., Patel, M., Vasudevan, \& T. Ng. A Smart Wind Turbine Blade Using Distributed Plasma Actuators for Improved Performance. Reno, NV : 46th AIAA Aerospace Sciences Meeting, 2008 (AIAA 2008-1312). 
58. Johnson, S., van Dam, C. , \& D. Berg. Active Load Control Techniques for Wind Turbines.

Albuquerque, NM : Sandia National Laboratories, 2008 (SAND2008-4809).

59. Wang, C., Lu, Y. Study on Performance Enhancement of Electrically Controlled Rotor Using 2/rev Flap Control. J. Aerospace Engr., Vol. 228(12), 2014.

60. Carr, L. W., McAlister, K., \& W. McCroskey. Analysis of The Development of Dynamic Stall Based on Oscillating Airfoil Experiments. Washington, DC : NASA TN D-8382, 1977.

61. Magill, J., McManus, K. Exploring The Feasibility Of Pulsed Jet Separation Control For Aircraft Configurations. J. of Aircraft, Vol. 38(1), 2001.

62. Sankar, L., Sahin, M., \& G. Naveen. Dynamic Stall Characteristics of Drooped Leading Edge Airfoils. Moffett Field, CA : NASA Ames Research Center Report, 2000.

63. Joo, W., Lee, B., Yee, K., \& D. Lee. Combining Passive Control Method for Dynamic Stall Control. J. of Aircraft, Vol. 43(4), 2006.

64. Beri, H., Yao, Y. Double Multiple Stream Tube Model and Numerical Analysis of Vertical Axis Wind Turbine. J. Energy \& Power Engr., Vol. 3, 2011.

65. Cattafesta, L., Sheplak, M. Actuators for Active Flow Control. Ann. Rev. Fluid Mech., Vol. 43., 2011.

66. KohIman, D. Introduction to V/STOL Airplanes. Ames, IA : lowa State Pr., 1981.

67. Hoerner, S., Borst, H. Fluid-Dynamic Lift (2 ${ }^{\text {nd }}$ Edition). New York, NY : Liselotte A. Hoerner, 1985.

68. Kweder, J., Panther, C., \& J. Smith. Applications of Circulation Control, Yesterday and Today. Int. J. Engr., Vol. 4(5), 2010

69. White, F. Viscous Fluid Flow. New York : McGraw-Hill, 2006.

70. Gibbs, E. Analysis of Circulation Controlled Airfoils. Morgantown, WV : West Virginia University, PhD Thesis, 1975.

71. Myer, D. An Experimental Investigation of a Circulation Controlled Cambered Elliptical Airfoil with a Rounded Trailing Edge. Morgantown, WV : West Virginia University, MS Thesis, 1972.

72. Verhoff, A. Steady and Pulsing Two-Dimensional, Turbulent Wall Jets in a Uniform Stream. Princeton, NJ : Princeton University Dept. of Aero. \& Mech. Sciences, 1970.

73. Grund, T., Nitsche, W. Active Flow Control on a S10 Glider. [book auth.] K. Rudibert. Active Flow Control II. Berlin : Springer Berlin Heidelberg, 2010.

74. Jones, G. Pneumatic Flap Performance for a Two-Dimensional Circulation Control Airfoil. [book auth.] F. Lu. Applications of Circulation Control Technology. Reston, VA : American Institute of Aeronautics and Astronautics, Inc., 2006. 
75. Seifert, A., Pack, L. Sweep and Compressibility Effects on Active Separation Control at High Reynolds Numbers. Reno, NV : 38th AIAA Aero. Sci. Meeting \& Exhibit, 2000 (AIAA 2000-0410).

76. Timor, I.,Ben-Hamou, E., Guy, Y., \& A. Seifert. Maneuvering Aspects and 3D Effects of Active Airfoil Flow Control. J. Flow Turbulence \& Combust, Vol. 78, 2007.

77. Post, M., Corke, T. Separation control using Plasma Actuators: Dynamic Stall Vortex Control on Oscillating Airfoil. AIAA Journal, Vol. 44(12), 2006.

78. Greenblatt, D., Wygnanski, I. The Control of Flow Separation by Periodic Excitation. Progress In Aerospace Sciences Journal, Vol. 37(7), 2000.

79. Liu, Y., Sankar, L., Englar, R., \& K. Ahuja. Numerical Simulations of the Steady and Unsteady Aerodynamic Characteristics of a Circulation Control Wing Airfoil. Reno, NV : American Institute of Aeronautics and Astronautics, 2001 (AIAA 2001-0704).

80. Zhou, M., Fernholtz, H., Ma, H., \& J. Wu. Vortex Capture By A Two-Dimensional Airfoil With A Small Oscillating Leading-Edge Flap. Orlando, FL : AIAA 3rd Shear Flow Conference, 1993.

81. Harness, G. An Experimental Investigation of a Circulation Controlled Cambered Elliptical Airfoil. Morgantown, WV : West Virginia University, PhD Thesis, 1970.

82. Williams, R., Howe, H. Two Dimensional Subsonic Wind Tunnel Tests on a 20 Percent Thick, 5 Percent Cambered Circulation Control Airfoil. Bethesda, MD : David W. Taylor Naval Ship R\&D Center, 1970 (Report \#AD0877764).

83. Walters, R., Myer, D. \& D. Holt. Circulation Control by Steady and Pulsed Blowing for a Cambered Elliptical Airfoil. Morgantown, WV : West Virginia University Dept. of Aero. Engr., 1972 (No. TR-32).

84. Loth, J., Boasson, M. Circulation Controlled STOL Wing Optimization. J. Aircraft, Vol. 21(2), 1984.

85. Angle II, G. Feasibility Study of Near Surface Actuated Circulation Control Blowing Slots for Rotorcraft. Morgantown, WV : West Virginia University, PhD Thesis, 2008.

86. Kweder, J. Effect of Circulation Control Blowing Slots on Stall Angles of a 10:1 Elliptical Airfoil. Morgantown, WV : West Virginia University, MS Thesis, 2008.

87. Weaver, D., McAlister, K., \& J. Tso. Control of VR-7 Dynamic Stall by Strong Steady Blowing. J. of Aircraft, Vol. 41(6), 2004.

88. McCroskey, W., McAlister, K., \& L. Carr. Dynamic Stall on Advanced Airfoil Sections. Moffett Field, CA : Ames Research Center AD085809, 1981.

89. McAlister, K., Tung, C. Suppression of Dynamic Stall with a Leading-Edge Slat on a VR-7 Airfoil. Moffett Field, CA : NASA Ames Research Center Tech. Paper 3357 [ATCOM TR 92-A-013], 1993.

90. McCroskey, W. Unsteady Airfoils. Ann. Rev. Fluid Mech., 1982, Vol. 14, 1982. 
91. Leishman, J. Principles of Helicopter Aerodynamics. New York : Cambridge University Press, 2000.

92. Sharma, D., Poddar, K. Effect of Reduce Frequency and Reynolds Number on Hysteresis Behavior of Flow Past an Oscillating Airfoil. Orland, FL : 47th AIAA Aerospace Sciences Meeting, 2009 (AIAA 2009330).

93. Soltani, M., Bakhshalipour, A. Effect of Amplitude and Mean Angle-of-Attack on the Boundary Layer of an Oscillating Aerofoil. The Aeronautical Journal, Vol. 112(1138), 2008.

94. Lee, T., Basu, S. Measurement of Unsteady Boundary Layer Developed on an Oscillating Airfoil using Multiple Hot-Film Sensors. J. Experiments in Fluids, Vol. 25, 1998.

95. Tsang, K., So, R., Leung, R., \& X. Wang. Dynamic Stall Behavior from Unsteady Force Measurements. J. Fluids \& Structures, Vol. 24, 2008.

96. McAlister, K., Carr, L., \& W. McCroskey. Dynamic Stall Experiments on the NACA0012 Airfoil. NASA Ames Research Center, Moffett Field, CA : NASA Technical Paper 1100, $1978 .$.

97. Griffin, C., Huebsch, W. Numerical and Experimental Study on the Ability of Dynamic Roughness to Alter the Development of a Leading Edge Vortex. West Virginia University : American Institute of Aeronautics and Astronautics (AIAA), 2013.

98. Lorber, P., Carta, F. Airfoil Stall Penetration at Constant Pitch Rate and High Reynolds Number. Hartford, CT : United Technologies Research Center, Report N89-19260, 1989.

99. Koochesfahani, M. Vortical Patterns in the Wake of an Oscillating Airfoil. AIAA Journal, Vol. 27(9), 1989.

100. OI, M., Dong, H., \& C. Webb. Motion Kinematics vs. Angle of Attack Effects in High-Frequency Airfoil Pitch/Plunge. Seattle, WA : 38th AIAA Fluid Dynamics Conference and Exhibit, 2008 (AIAA 2008-3822).

101. Amiralaei, M., Alighanbari, H., \& S. Hashemi. An Investigation into the Effects of Unsteady parameters on the Aerodynamics of a Low Reynolds Number Pitching Airfoil. J. of Fluids \& Structures, Vol. 26, 2010.

102. Mueller-Vahl, H., Strangfeld, C., Nayeri, C., Paschereit, C., \& D. Greenblatt. Thick Airfoil Deep Dynamic Stall. [book auth.] M. Hölling, J. Peinke and S. (Editors) Ivanfell. Wind Energy - Impact of Turbulence. Berlin : Springer-Verlag , 2014.

103. Piziali, R. 2D and 3D Oscillating Wing Aerodynamics for a Range of Angles of Attatck Including Stall. Moffett Field, CA : NASA Ames Research Center, Tech. Memo. 4632, 1994.

104. Lombardi, A. Closed-Loop Dynamic Stall Control Using a Plasma Actuator. Notre Dame, IN : University of Notre Dame, MS Thesis, 2011.

105. McCroskey, W., Pucci, L. Viscous-Inviscid Interaction on Oscillating Airfoils in Subsonic Flow. AIAA Journal, Vol. 20(2), 1982. 
106. Sharma, D., Poddar, K. Investigations on Quasi-Steady Characteristics for an Airfoil Oscillating at Low Reduced Frequencies. Int. J. of Aerospace Engineering, 2010.

107. Conger, R., Oshima, H., B. Ramaprian. Experimental Studies of the Unsteady Vortex Dynamics of a Two-Dimensional Pitching Airfoil. University of Tasmani, Hobart, Australia : 11th Australian Fluid Mechanics Conference, 1992.

108. Martin, J., Empey, R., McCroskey, W., \& F. Caradonna. An Experimental Analysis of Dynamic Stall on an Oscillating Airfoil. J. of the American Helicopter Society, Vol. 19(7), 1974.

109. Shrewsbury, G. Dynamic Stall of Circulation Control Airfoils. Atlanta, GA : Georgia Institute of Technology, PhD Thesis, 1990.

110. Shrewsbury, G., Sankar, L. Dynamic Stall of an Oscillating Circulation Control Airfoil. Toronto : Int. Symposium on Nonsteady Fluid Dynamics, 1990.

111. St. Hilaire, A., Carta, F. Analysis of Unswept and Swept Wing Chordwise Pressure Data from an Oscillating NACA0012 Airfoil Experiment. Washington, DC : Technical Report [NASA CR-3567], 1983.

112. Danao, L., Eboibi, O., \& R. Howell. An Experimental Investigation into the Influence of Unsteady Wind on the Performance of a Vertical Axis Wind Turbine. J. Applied Energy, Vol. 107, 2013.

113. Howell, R., Qin, N., Edwards, J., \& N. Durani. Wind Tunnel and Numerical Study of a Small Vertical Axis Wind Turbine. J. Renewable Energy, Vol. 35, 2010.

114. Lovato, J. Active Control of the Separation Region on a Two-Dimensional Airfoil. USAF Academy, CO : Frank J. Seiler Research Laboratory (FJSRL-TR-92-0001), 1992.

115. Akbari, M., Price, S. Simulation of Dynamic Stall for a NACA 0012 Airfoil using a Vortex Method. J. Fluids \& Structures, Vol. 17(6), 2003.

116. Bousman, W. Airfoil Dynamic Stall and Rotorcraft Manueverabiltiy. Moffett Field, CA : NASA Ames Research Center, TM-2000-209601, 2000.

117. Tchon, K., Halle, S. , \& I. Paraschivoiu. Dynamic Stall Simulation Applied to Vertical-Axis Wind Turbines. Advisory Group for Aerospace Research and Development (AGARD CP-552), 1994.

118. Greenblatt, D., Ben-Harav, A., \& H. Mueller-Vahl. Dynamic Stall Control on a Vertical-Axis Wind Turbine Using Plasma Actuators. AIAA Journal, Vol. 52(2), 2014.

119. Shrewsbury, G., Sankar, L. Dynamic Stall of an Oscillating Circulation Control Airfoil. Reno, NV : AIAA 28th Aerospace Sciences Meeting, 1990 (AIAA 90-0573).

120. Ligitt, N. Numerical Investigation of Static and Dynamic Stall of Single and Flapped Airfoils. Atlanta, GA : Georgia Institute of Technology, PhD Thesis, 2012. 
121. Tuncer, I. Unsteady Aerodynamics of Oscillating and Rapidly Pitching Airfoils. Atlanta, GA : Georgia Institute of Technology, 1988. PhD Thesis.

122. Chandrasekhara, M. Compressible Dynamic Stall Vorticity Flux Control Using A Dynamic Camber Airfoil. Bangalore, India : Sadhana Journal, Vol. 32(1), 2007.

123. Chandrasekhara, M., Platzer, M. Compressibility Effects on Dynamic Stall of Oscillating Airfoils. Monterey, CA : US Army Research Office, Final Report (ARO 23394-EG), 1990.

124. Rodriguez, D., Gennaro, E., \& P. Juniper. The Two Classes of Primary Modal Instability in Laminar Separation Bubbles. J. Fluid Mech., Vol. 734(4), 2013.

125. Aiken, E., Ormiston, R., \& L. Young. Future Directions in Rotorcraft Technology at NASA Ames Research Center. J. American Helicopter Society, 2000.

126. Woo, T., Glezer, A. Transitory Control of Dynamic Stall on a Pitching Airfoil. [book auth.] R. King. Active Flow Control II. Springer-Verlag Berlin Heidelberg : Springer, 2010.

127. Greenblatt, D., Nishri, B., Darabi, A., Wygnanski, I. Dynamic Stall Control by Periodic Excitation, Part 2: Mechanisms. J. Aircraft, Vol. 33(4), 2001.

128. Greenblatt, D., Wygnanski, I. Dynamic Stall Control by Periodic Excitation, Part 1: NACA0015 Parametric Study. J. Aircraft, Vol. 38(3), 2001.

129. Greenblatt, D., Schule, C., Romann, D., \& C. Paschereit. Dielectric Barrier Discharge Flow Control at Very Low Flight Reynolds Numbers. AIAA Journal, Vol. 46(6), 2008.

130. Lorber, P., McCormick, D., Anderson, T., Wake, B., MacMartin, D., Pollack, M., Corke, T., \& K. Breuer. Rotorcraft Retreating Blade Stall Control. Denver, CO : American Institute of Aeronautics and Astronautics, 2000 (AIAA 2000-2475).

131. Lorber, P., Carta, F. Unsteady Stall Penetration Experiments at High Reynolds Number. Hartford, CT : United Technologies Research Center, UTRC Report No R87-956939-3, 1987.

132. Green, R., Galbraith, R., \& A. Niven. The Convection Speed of the Dynamic Stall Vortex. Glasgow, Scotland : University of Glasgow, Technical Report No. 9202, 1992.

133. Jung, Y., Park, S. Vortex Shedding Characteristics in the Wake of an Oscillating Airfoil at Low Reynolds Numbers. J. Fluids \& Structures, Vol. 20(3), 2005.

134. Ganwani, S. Synthesized Airfoil Data Method for Prediction of Dynamic Stall and Unsteady Airloads. Langley, VA : NASA Langley Research Center, Report 3672, 1983.

135. Barlow, J., Rae, W., \& A. Pope. Low Speed Wind Tunnel Testing (3rd Edition). New York : John Wiley \& Sons, 1999. 
136. Lorber, P., McCormick, D., Wake, B., \& R. Florea. Separation Control for Rotorcraft. East Hartford, CT : United Technologies Research Center, Final Report (UTRC Report 2002-5.200.0015-5), 2002.

137. Whitmore, S., Moes, T. The Effects of Pressure Sensor Acoustics on Airdata Derived from a High Angle-of-Attack Flush Airdata Sensing (HI-FADS) System. Edwards, CA : NASA Ames Research Center, NASA TM-101736, 1991.

138. Bergh, H., Tijdeman, H. Theoretical and Experimental Results for the Dynamic Response of Pressure Measuring Systems. Amsterdam, Netherlands : The National Aerospace Laboratory of the Netherlands, Rep. NLR-TR F.238, 1965.

139. Hall, K., Kielb, R., \& J. Thomas. Unsteady Aerodynamics, Aeroacoustics and Aeroelasticity of Turbomachines. Berlin : Springer, 2006.

140. Rofail, A., Tonin, R., \& D. Hanafi. Sensitivity of Frequency Response to Type of Tubing. Darwin : Australian Wind Engineering Workshop, Windtech Consultants Pty Ltd, 2004.

141. Mousley, P. Tube Response Linearization. Victoria, Australia : Turbulent Flow Instrumentation (www.turbulentflow.com.au.), 2011.

142. American Institute of Aeronautics and Astronautics. AIAA Guide to Assessing Experimental Uncertainty - Supplement to S-071A-1999 (G-045-2003). Reston, VA : ISBN: 978-1-56347-663-1, 2003.

143. Stern, F., Muste, M., Beninati, L., \& B. Eichinger. Summary of Experimental Uncertainty Assessment Methodology with Example. lowa City, IA : lowa Institute of Hydraulic Research, University of lowa, IIHR Report No. 406, 1999.

144. McAlister, K., Pucci, S., McCroskey, W., \& L. Carr. An Experimental Study of Dynamic Stall on Advanced Airfoil Sections: Volume 2. Pressure and Force Data. Moffett Field, CA : NASA Ames Research Center, TM-84245, 1982.

145. Kline, S., McClintock, F. Describing Uncertainties in Single Sample Experiments. J. Mechanical Engineering, Vol. 75(1), 1953.

146. Zeiger, M. The Dynamic Character of the Flow over a 3.5 Caliber Tangent-Ogive Cylinder in Steayd and Maneuvering States at High Incidence. Blacksburg, VA : Virginia Polytechnic Institute and State University, PhD Thesis, 2013.

147. ANSYS, Inc. ANSYS FLUENT 15.0 User's Guide. [Electronic Manual] Release 15.0., 2013.

148. Rumsey, C., Nishino, T. Numerical Study Comparing RANS and LES Approaches on a Circulation Control Airfoil. Int. Journal of Heat \& Fluid Flow, Vol. 32, 2011.

149. Tongchitpakdee, C. Computational Study of the Effects of Active and Passive Circulation Enhancement Concepts on Wind Turbine Performance. Atlanta, GA : Georgia Institute of Technology, PhD Thesis, 2007. 
150. Tongchitpakdee, C., Benjanirat, S., \& L. Sankar. Numerical Studies of the Effects of Active and Passive Circulation Enhancement Concepts on Wind Turbine Performance. J. Solar Energy Eng., Vol. 128(4), 2006.

151. Towne, M. Numerical Simulation of Dynamic Stall Suppression by Tangential Blowing. WPAFB, OH : Air Force Institute of Technology, PhD Thesis, 1994.

152. Ghia, K., Yang, J., Osswald, G., \& U. Ghia. Study on the Role of Unsteady Separation in Formation of Dynamic Stall Vortex. Reno, NV : 30th Aerospace Sciences Meeting \& Exhibit, 1992 (AIAA 92-0196).

153. Smith, H., Schaefer, R. Aerodynamic Characteristics at Reynolds Numbers of $3 \times 10^{\wedge} 6$ and $6 \times 10^{\wedge} 6$ of Three Airfoil Sections Formed by Cutting Off Various Amounts from the Rear Portion of the NACA0012 Airfoil Section. Langley Aeronautical Laboratory, VA : National Advisory Committee for Aeronautics, NACA TN 2074, 1950.

154. Liu, Y. Numerical Simulations of the Aerodynamic Characteristics of Circulation Control Wing Sections. Atlanta, GA : Georgia Institute of Technology, PhD Thesis, 2003.

155. Rumsey, C., Gatski, T., Sellers, W., Vatsa, V., \& S. Viken. Summary of the 2004 CFD Validation Workshop on Synthetic Jets and Turbulent Separation Control. Portland, OR : American Institute of Aeronautics and Astronautics, 2004 (AIAA-2004-2217).

156. Lorber, P., Carta, F. Unsteady Stall Penetration Experiments at High Reynolds Number. East Hartford, CT : United Technologies Research Center, UTRC Report No. R87-956939-3, 1987.

157. Coleman, H., Steele, W. Engineering Application of Experimental Uncertainty Analysis. AIAA Journal, Vol. 33(10), 1995.

158. Moldover, M., Trusler, J., Edwards, T., Mehl, J., \& R. Davis. Measurement of the Universal Gas Constant R Using a Spherical Acoustic Resonator. Gaithersburg, MD : J. Research of. National Bureau of Standards, Vol. 93(2), 1988.

159. Tijdeman, H. On the Propagation of Sound Waves in Cylindrical Tubes. J. Sound \& Vibration, Vol. 39(1), 1975.

160. Chapin, W. Dynamic-Pressure Measurement Using an ESP Transducer Module. NASA Langley Research Center, Hampton, VA : NASA Application Note: \#A0034, 1984.

161. Scanivalve Corp. ZOC 33/64Px and ZOC 33/64PxX2 Electronic Pressure Scanning Module. Liberty Lake, WA : ZOC33 Instruction and Service Manual, 2010.

162. Blevins, R. Analysis of a Dynamic Pressure Measuring System. Blacksburg, VA : Virginia Polytechnic Institute, MS Thesis, 1970. 
163. Greenblatt, D., Kiedaisch, J., \& H. Nagib. Unsteady-Pressure Corrections in Highly Attenuated Measurements at Moderate Mach Numbers. Anaheim, CA : 31st AIAA Fluid Dynamics Conference \& Exhibit, 2001 (AIAA 2001-2983).

164. Tijdeman, H., Spiering, R. A System for Unsteady Pressure Measurements Revisited. Amsterdam, Netherlands : International Forum on Aeroelasticity and Structutral Dynamics , 2003.

165. DYNAMCO. DYNAMCO Catalog. Miniature High Speed Air Valves and Accesories. [Online] June 2010. [Cited: April 5, 2014.] http://www.valeader.co.uk/16-06/Dynamco Catalog Low Res (DVA 10).pdf. Form DVA-10 (Revised 6/10).

166. SparkFun Electronics. SparkFun MOSFET Power Control Kit. [Online] [Cited: February 9, 2015.] https://www.sparkfun.com/products/12959.

167. US Digital. MAE3 Absolute Encoder Kit. [Online] Revision 150122071900. [Cited: March 13, 2015.] http://cdn.usdigital.com/assets/datasheets/MAE3_datasheet.pdf.

168. KING Instrument Company. 7520 \& 7530 Series - Acrylic Tube Flowmeter. [Online] [Cited: December 3, 2013.] http://www.kinginstrumentco.com/pdf/King_7520_7530_Series_Flowmeter.pdf.

169. Badger Meter. Product Catalog: Variable Area Flow Meters and Flow Switches. [Online] [Cited: November 22, 2014.] http://www.badgermeter.com/Badger-Files/PDFs/Industrial-Products/VAM-CA00254-EN.pdf.

170. Ashcroft Inc. PTE-1 Handheld Pressure Calibrator. [Online] August (Rev. 10/08) 2008. Bulletin PTE1.

171. DWYER Instruments, Inc. DWYER Series 475 Mark III Digital Manometer. [Online] 2003 (Rev. 9). [Cited: June 12, 2014.] http://www.davis.com/Assets/manual_pdfs/68062-66-80.pdf. FR. 02-443041-00. 


\section{APPENDIX A: Experimental Error Analysis}

The methodology for quantifying the experimental uncertainty of pressure and aerodynamic force coefficients $\left(C_{p}, C_{l}, C_{d}\right.$, and $\left.C_{m}\right)$ from measured wind tunnel data follows procedures outlined by The lowa Institute of Hydraulic Research (IIHR, University of lowa) (143) and The American Institute of Aeronautics and Astronautics (AIAA) (157). The total uncertainty $\left(W_{R}\right)$ for a given quantity is determined considering the following terms: the individual instrument errors $\left(w_{i}\right)$ and the partial derivative of the resultant term $(R)$ with respect to each measured quantity $\left(x_{i}\right)$, as shown in (143). The data reduction and error estimation process for the pressure coefficient for a given static tap will be included to illustrate the methodology. The data reduction equation for pressure coefficient reveals the quantity to be a function of the variables included in Equation 70; $\Delta P, P_{\infty}$, and $T_{\infty}$ were all measured before each test run, while $V_{\infty}$ was calculated from observed values. Assuming air to be an ideal gas, the equation of state was used to calculate air density during wind tunnel testing. Thus, the universal gas constant $(R)$ used for data reduction was $8.314471 \pm 0.000014 \mathrm{~J} / \mathrm{kg} \cdot \mathrm{K}$, as measured by the National Bureau of Standards (158). The uncertainty of this measured value was several orders of magnitude smaller than all other dependent variables used to determine $C_{p}$, and was thus assumed negligible.

$C_{p}$ Dependent Variables $\quad C_{p}=f\left(\Delta P, \rho_{\infty}, V_{\infty}\right)=f\left(\Delta P, P_{\infty}, T_{\infty}, R, V_{\infty}\right)$

Equation 70

The total uncertainty for $C_{p}$, determined at each tap, is given by the relation in Equation 71 , where $B_{C p}$ and $P_{C p}$ represent the bias and precision limits of the data.

Total Uncertainty

$$
W_{C_{P}}^{2}=B_{C_{P}}^{2}+P_{C_{P}}^{2}
$$

Equation 71

$B_{C p}$ can be determined from Equation 72 .

Bias Limit

$$
B_{C_{P}}^{2}=\sum_{i=1}^{j} w_{i}^{2} \cdot B_{i}^{2}=w_{\Delta P}^{2} \cdot B_{\Delta P}^{2}
$$

Equation 72

Before calculating the bias limit, pertinent sensitivity coefficients must first be determined (Equation 73).

Sensitivity Coefficient

$$
w_{\Delta P}=\frac{\partial R}{\partial x_{i}}=\frac{\partial C_{p}}{\partial(\Delta P)}=\frac{2}{\rho_{\infty} \cdot V_{\infty}^{2}}=\frac{2}{\left(P_{\infty} / R \cdot T_{\infty}\right) \cdot V_{\infty}^{2}}
$$

Next, the precision limit $\left(P_{C p}\right)$ for each tap can be estimated using Equation 74 from (143), with $t=2$. 


\section{Precision Limit}

$$
P_{C_{P}}=\left(2 \cdot S_{C_{P}}\right) / \sqrt{M}
$$

Equation 74

Within the precision limit equation (Equation 74), $S_{C p}$ is the standard deviation of the time-averaged pressure coefficients at each tap; $M$ represents the number of cases considered when averaging data from multiple runs; see equation (22) of Stern, et al. (143) for further details. Thus, $(5 \leq M \leq 10)$ for both static and dynamic wind tunnel testing of the CCNACA0018 model. For steady cases, a "run" consisted of a large number of data samples collected at each port ( $N \geq 10 \mathrm{~K}$ samples/port); for dynamic cases, a "run" was at least one full pitch oscillation. For most cases, each dynamic run was a phase-average of 3-5 cycles. The DAQ program was set up to trigger the ScaniValve to begin pressure port \#1 measurements at $\alpha=0^{\circ}$ on the pitch-up maneuver, sample data for the desired number of pitch-cycles, switching to the next consecutive port, and repeating the process for all surface taps. Where available, the manufacturer designated specifications were used for the bias limit; for other instruments, half of the smallest scale division will be used as the uncertainty value. The above process was completed for the remaining function- variables $P_{\infty}, T_{\infty}$, and $V_{\infty}$, resulting in the total bias limit for $C_{P}$ at each tap (Equation 75).

$$
c_{p} \text { Bias Limit } \quad B_{c_{P}}=\left[\left(\frac{2 w_{\Delta P}}{\rho_{\infty} V_{\infty}^{2}}\right)^{2}+\left(\frac{2 w_{p_{\infty}}}{\rho_{\infty} V_{\infty}^{2}}\right)^{2}+\left(\frac{2(\Delta P) w_{p}}{\rho_{\infty} V_{\infty}^{2}}\right)^{2}+\left(\frac{4(\Delta P) w_{V_{\infty}}}{\rho_{\infty} V_{\infty}^{3}}\right)^{2}\right]^{1 / 2}
$$

Finally, the bias and precision limits can be summed to yield total uncertainty estimation $\left(W_{c p}\right)$ for each pressure port (Equation 76).

$$
\begin{aligned}
& C_{p} \text { Total } \\
& \text { Uncertainty }
\end{aligned} W_{c_{P}}=\left[\left(\frac{2 w_{\Delta P}}{\rho_{\infty} V_{\infty}^{2}}\right)^{2}+\left(\frac{2 w_{p_{\infty}}}{\rho_{\infty} V_{\infty}^{2}}\right)^{2}+\left(\frac{2(\Delta P) w_{p}}{\rho_{\infty} V_{\infty}^{2}}\right)^{2}+\left(\frac{4(\Delta P) w_{V_{\infty}}}{\rho_{\infty} V_{\infty}^{3}}\right)^{2}\right]^{1 / 2}+\left(\left(2 \cdot S_{C_{P}}\right) / \sqrt{M}\right)^{2}
$$

Past experimental work, completed at Virginia Tech (146), revealed uncertainty of wind tunnel model surface pressure measurements to be in the range $\left( \pm 0.02 \leq W_{c_{p}} \leq \pm 0.1\right)$, closely matching calculated error values from this study. The force terms for which the measured error was estimated were the coefficients of lift $\left(W_{C l}\right)$ and drag $\left(W_{C d}\right)$, as seen in Equation 77 and Equation 78.

$$
\begin{array}{ll}
\underset{\text { Uift }}{\text { Uncertainty }} & W_{C_{l}}=\left[\left(\frac{2 w_{L}}{\rho_{\infty} A_{b l} V_{\infty}^{2}}\right)^{2}+\left(\frac{2 L w_{\rho_{\infty}}}{\rho_{\infty}^{2} A_{b l} V_{\infty}^{2}}\right)^{2}+\left(\frac{4 L w_{V_{\infty}}}{\rho_{\infty} A_{b l} V_{\infty}^{3}}\right)^{2}+\left(\frac{2 L w_{A_{b l}}}{\rho_{\infty} A_{b l}^{2} V_{\infty}^{2}}\right)^{2}\right]^{1 / 2} \\
\begin{array}{c}
\text { Drag } \\
\text { Uncertainty }
\end{array} & W_{C_{d}}=\left[\left(\frac{2 w_{D}}{\rho_{\infty} A_{b l} V_{\infty}^{2}}\right)^{2}+\left(\frac{2 D w_{\rho_{\infty}}}{\rho_{\infty}^{2} A_{b l} V_{\infty}^{2}}\right)^{2}+\left(\frac{4 D w_{V_{\infty}}}{\rho_{\infty} A_{b l} V_{\infty}^{3}}\right)^{2}+\left(\frac{2 D w_{A_{b l}}}{\rho_{\infty} A_{b l}^{2} V_{\infty}^{2}}\right)^{2}\right]^{1 / 2}
\end{array}
$$


Recent wind tunnel tests conducted at the Ohio State Aeronautical and Astronautical Research Laboratory reported measured airfoil force coefficient uncertainties (force balance) of \pm 0.07 at $R e_{c}=0.86 \times 10^{6}$, and \pm 0.04 at $R e_{c}=1.72 \times 10^{6}(61)$. On the other hand, integration of surface pressure measurements for the same experimental set-up yielded $C_{l}$ values with uncertainties of \pm 0.005 $\left(R e_{c}=0.86 \times 10^{6}\right)$, due to high precision pressure transducers having an accuracy better than $0.0036 \mathrm{psi}$ (61).

The measurement error equation for the momentum coefficient $\left(W c_{\mu}\right)$ is listed in Equation 79. Test techniques for pressurized circulation control air supply, and subsequent methodology to calculate $C_{\mu}$, reflected previous work at WVU (38) (85). Thus, the momentum coefficient error equation from those studies was suitable for this work as well.

$$
\begin{aligned}
& \text { Momentum } \\
& \text { Coefficient } \\
& \text { Uncertainty } \\
& W_{C_{\mu}}=\left[\left(\frac{2 V_{j e t} w_{\dot{m}_{j e t}}}{\rho_{\infty} A_{b l} V_{\infty}^{2}}\right)^{2}+\left(\frac{2 \dot{m}_{j e t} w_{V_{j e t}}}{\rho_{\infty} A_{b l} V_{\infty}^{2}}\right)^{2}+\left(\frac{2 \dot{m}_{j e t} V_{j e t} w_{\rho}}{\rho_{\infty}^{2} A_{b l} V_{\infty}^{2}}\right)^{2}+\left(\frac{4 \dot{m}_{j e t} V_{j e t} w_{V_{\infty}}}{\rho_{\infty} A_{b l} V_{\infty}^{3}}\right)^{2}+\left(\frac{2 \dot{m}_{j e t} V_{j e t} w_{A_{b l}}}{\rho_{\infty} A_{b l}^{2} V_{\infty}^{2}}\right)^{2}\right]^{1 / 2}
\end{aligned}
$$

Additionally, the density $\left(\rho_{\infty}\right)$, free stream velocity $\left(V_{\infty}\right)$, and model wing area $(A)$ are also calculated values and therefore require uncertainty metrics. Equation 80 through Equation 82 show the uncertainty relationships for each of the aforementioned calculated terms.

Density Error

$$
w_{\rho}=\left[\left(\frac{w_{P_{\infty}}}{R T}\right)^{2}+\left(\frac{P_{\infty} w_{T}}{R T^{2}}\right)^{2}\right]^{1 / 2}
$$

$$
w_{V_{\infty}}=\left[\left(\frac{w_{\Delta P}}{2} \sqrt{\frac{2}{\rho(\Delta P)}}\right)^{2}+\left(\frac{w_{\rho}}{2} \sqrt{\frac{2(\Delta P)}{\rho^{3}}}\right)^{2}\right]^{1 / 2}
$$

$$
w_{A}=\left[\left(b w_{c}\right)^{2}+\left(c w_{b}\right)^{2}\right]^{1 / 2}
$$




\section{APPENDIX B: CCNACA0018 Jet Velocity Measurements and Analysis Plots}

This appendix includes plots of the measured data collected at the jet exit of the CCNACAO018 wind tunnel model; flow control conditions reflect all settings used during wind tunnel testing. It should be noted, however, that the data included herein was a benchtop style experiment in quiescent air. All pressure data was recorded via a high-frequency response ScaniValve transducer $( \pm 2.5 p s i d)$ in conjunction with a LabView express virtual data acquisition (.vi $D A Q$ ) environment.

Modes are commonly used as a means of characterizing dominant frequency components of oscillating signals such as the pulsed CC jet. Modal analysis can also help to identify resonant frequencies common when unsteady signals travel through enclosed volumes, with unique waveform interactions occurring for both open conduits and enclosures.

The Fast Fourier Transform (FFT) analysis of the raw jet data was completed with the following MATLAB code. The author would like to express a special thanks to Dr. Drew Lowery for his help constructing the included FFT script.

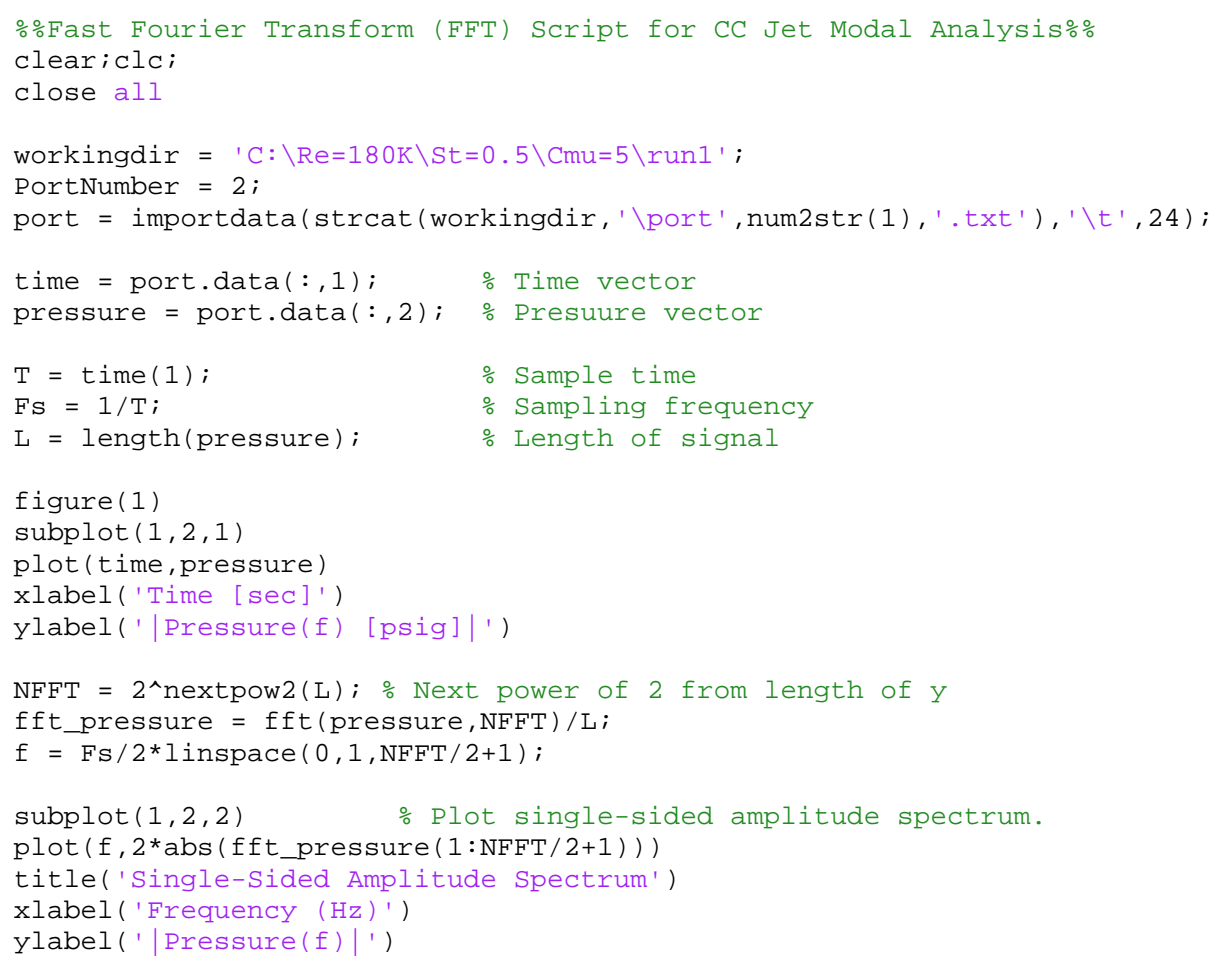




\section{Appendix B.1 $R e=180 \times 10^{3}$ Jet Velocity Measurements and Spectral Analysis Plots}
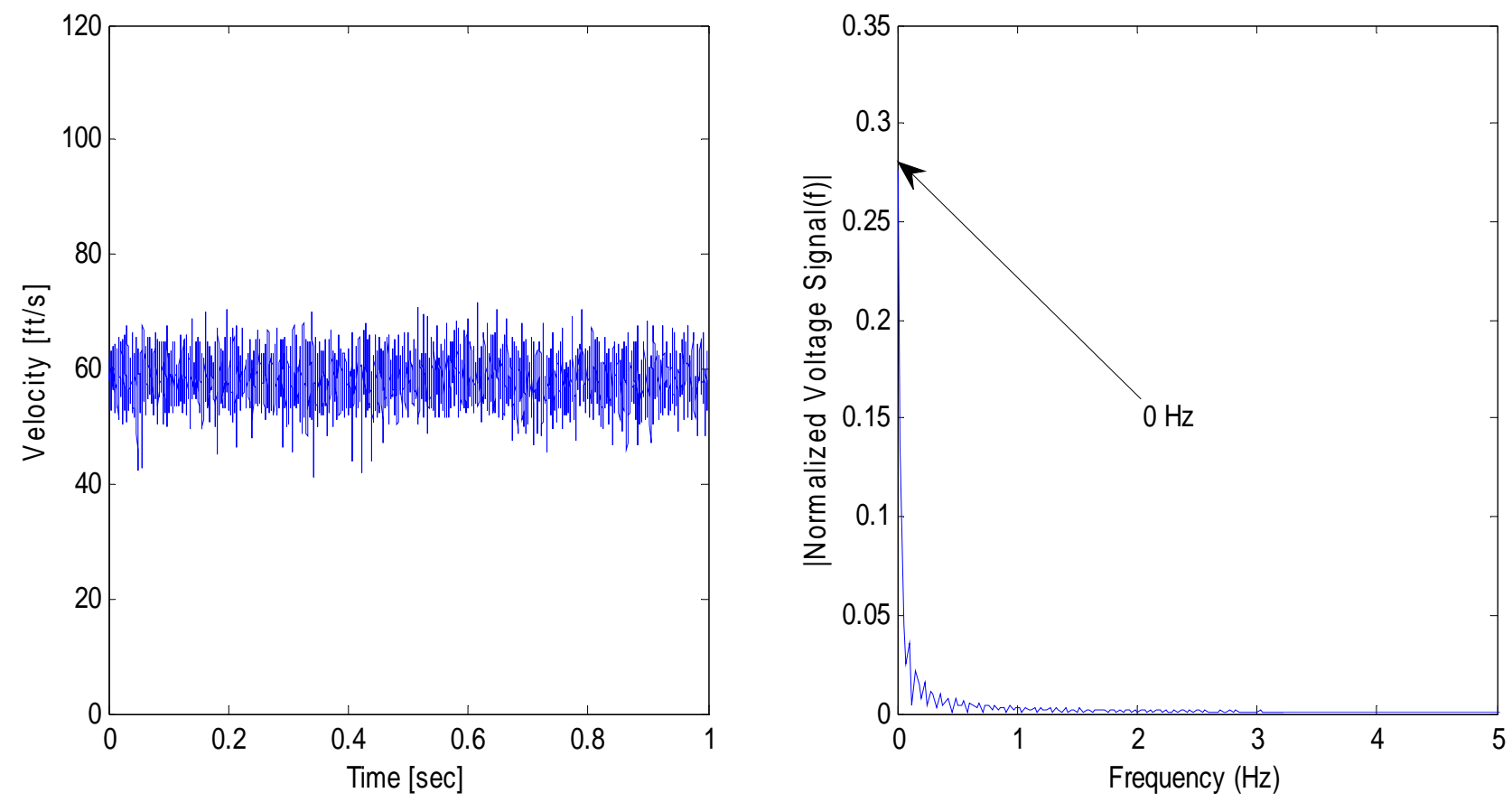

Figure 131. Measured CCNACA0018 Jet Velocity: $R e=180 \times 10^{3} C_{\mu}=0.01 \mathrm{St}=0.00\left(f_{\text {act }}=0 \mathrm{~Hz}\right)$
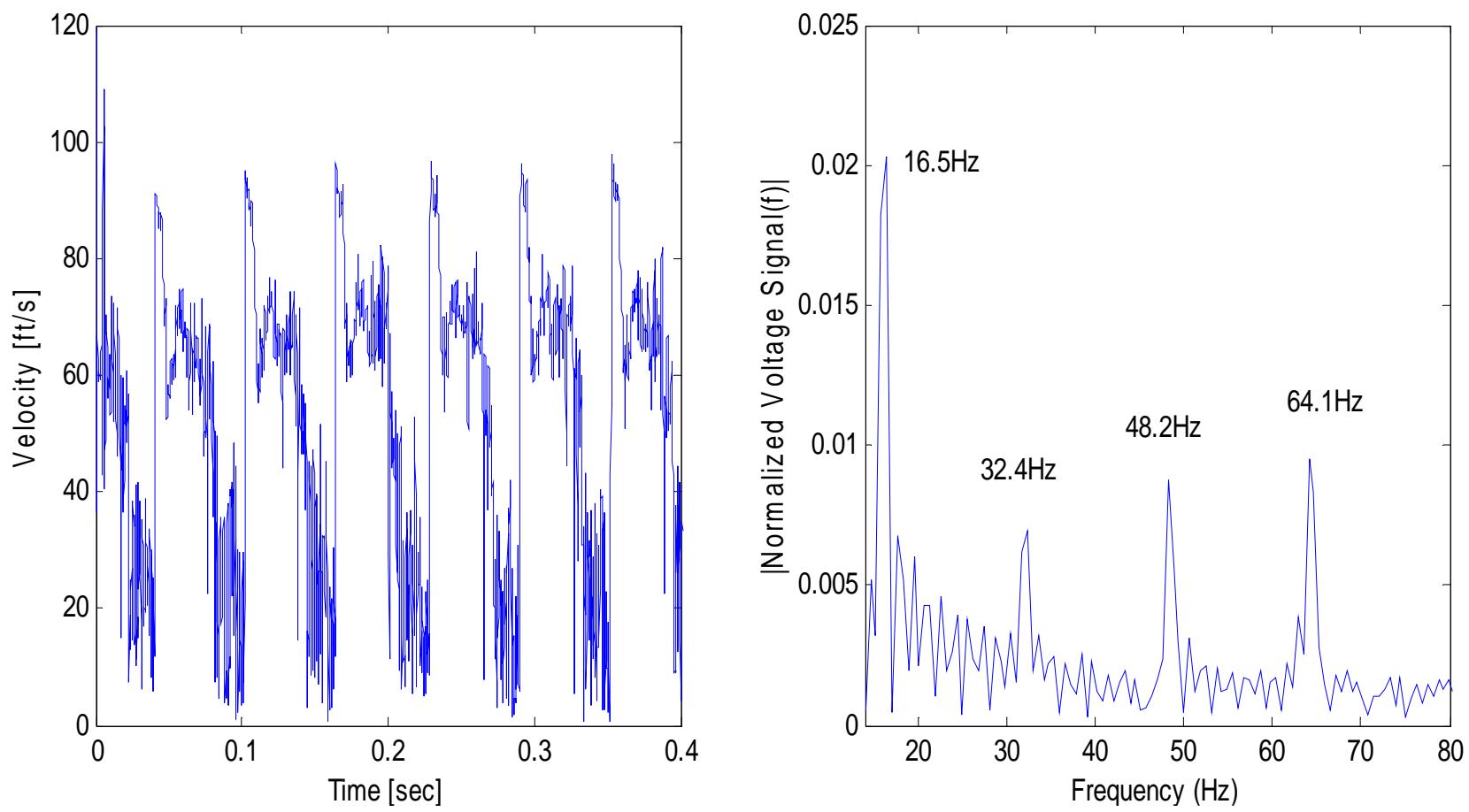

Figure 132. Measured CCNACA0018 Jet Velocity: $R e=180 \times 10^{3}\left\langle c_{\mu}\right\rangle=0.01 \mathrm{St}=0.25\left(f_{\text {jet }}=16.5 \mathrm{~Hz}\right)$ 

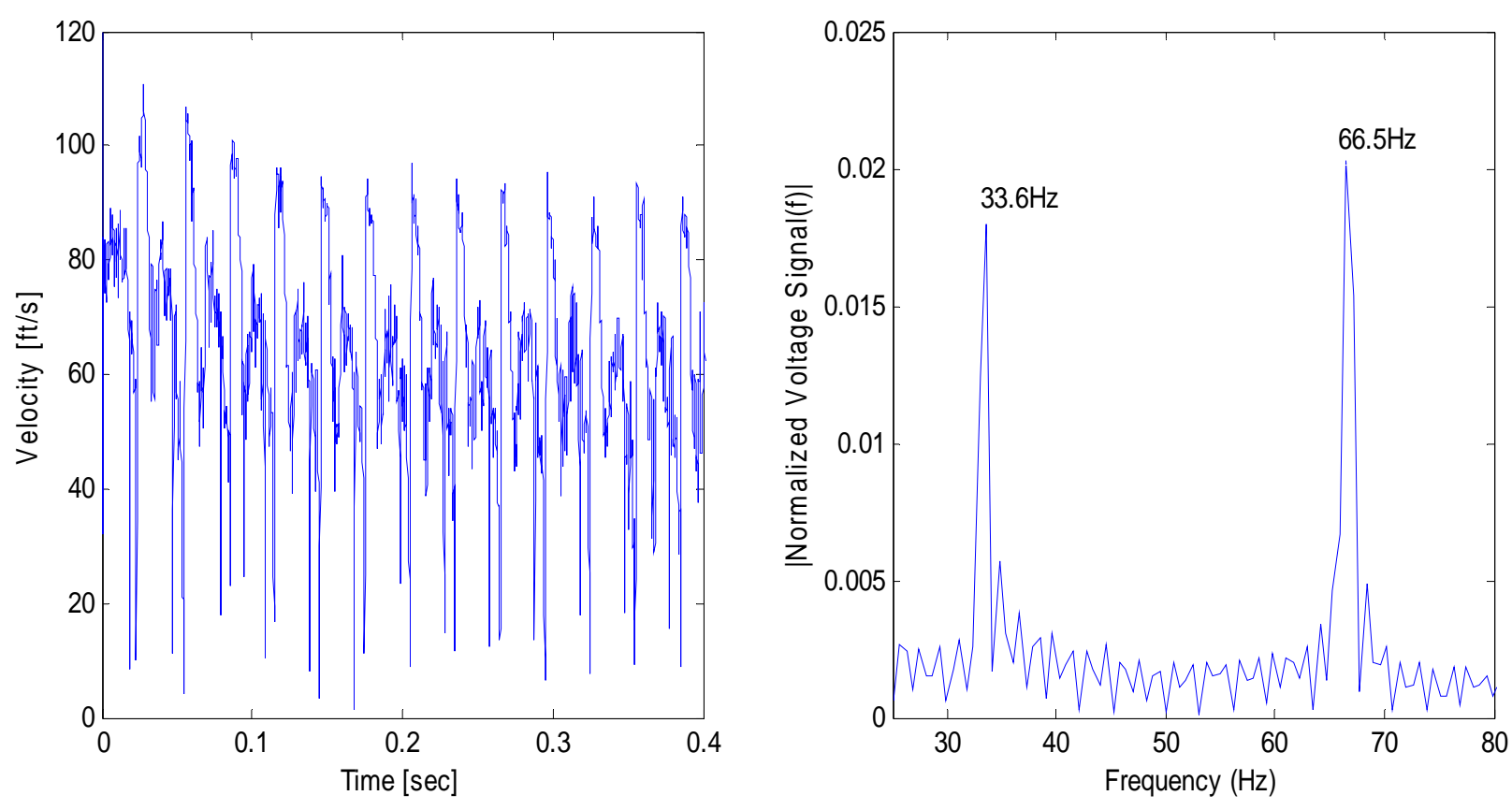

Figure 133. Measured CCNACA0018 Jet Velocity: $R e=180 \times 10^{3}<c_{\mu}>=0.01 S t=0.50\left(f_{\text {jet }}=34 \mathrm{~Hz}\right)$
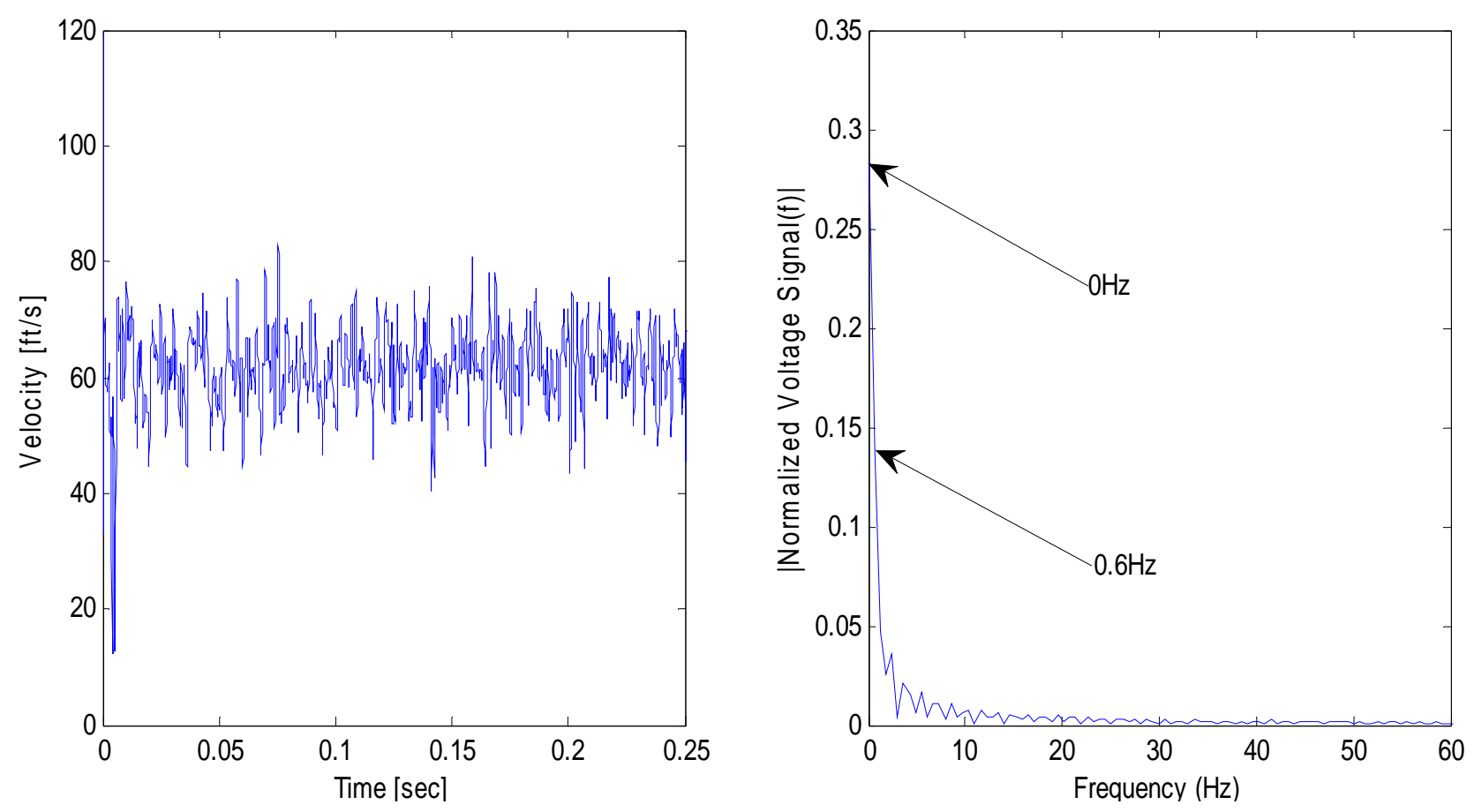

Figure 134. Measured CCNACA0018 Jet Velocity: $R e=180 \times 10^{3}\left\langle c_{\mu}>=0.01 S t=1.00\left(f_{j e t}=129 \mathrm{~Hz}\right)\right.$ 

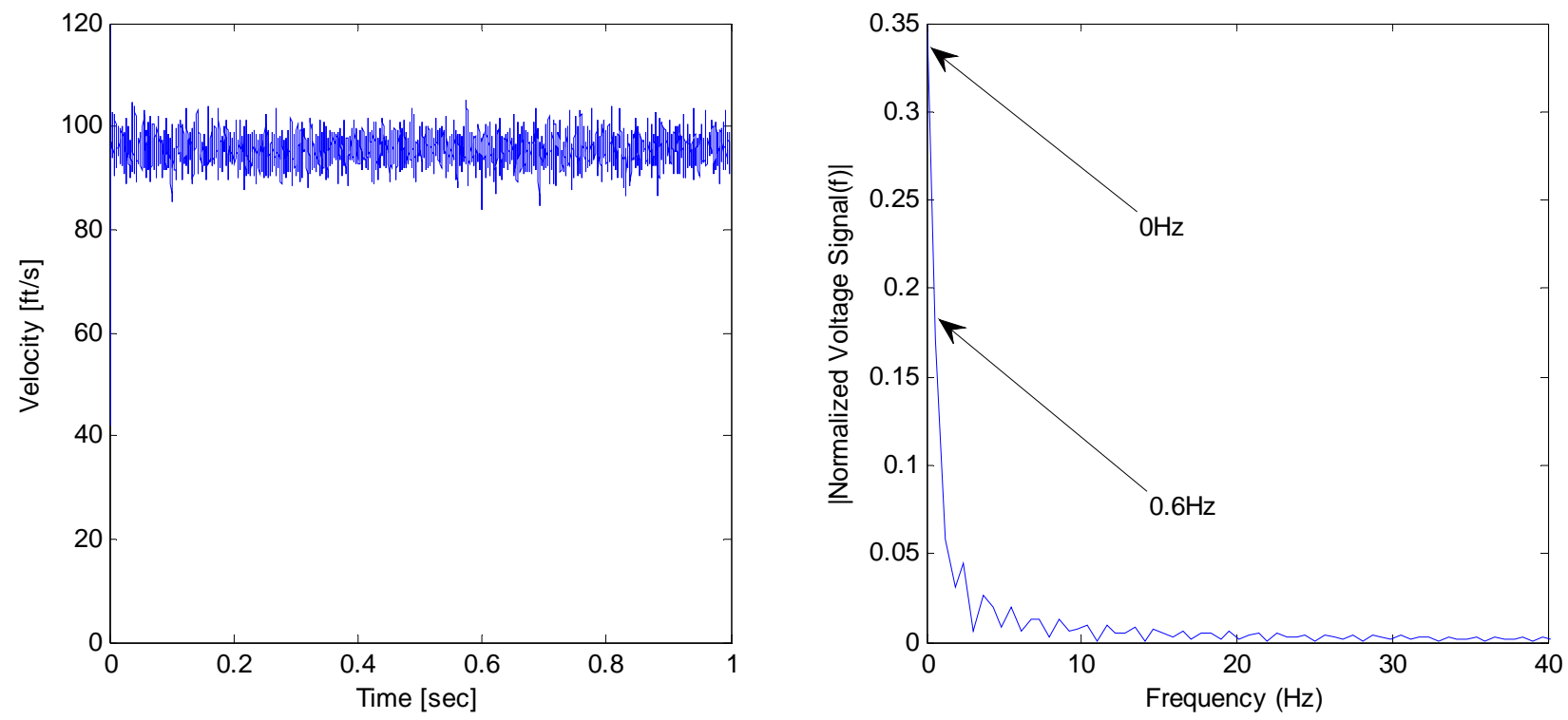

Figure 135. Measured CCNACA0018 Jet: $R e=180 \times 10^{3} C_{\mu}=0.05 \mathrm{St}=0.0\left(f_{\text {act }}=0 \mathrm{~Hz}\right)$
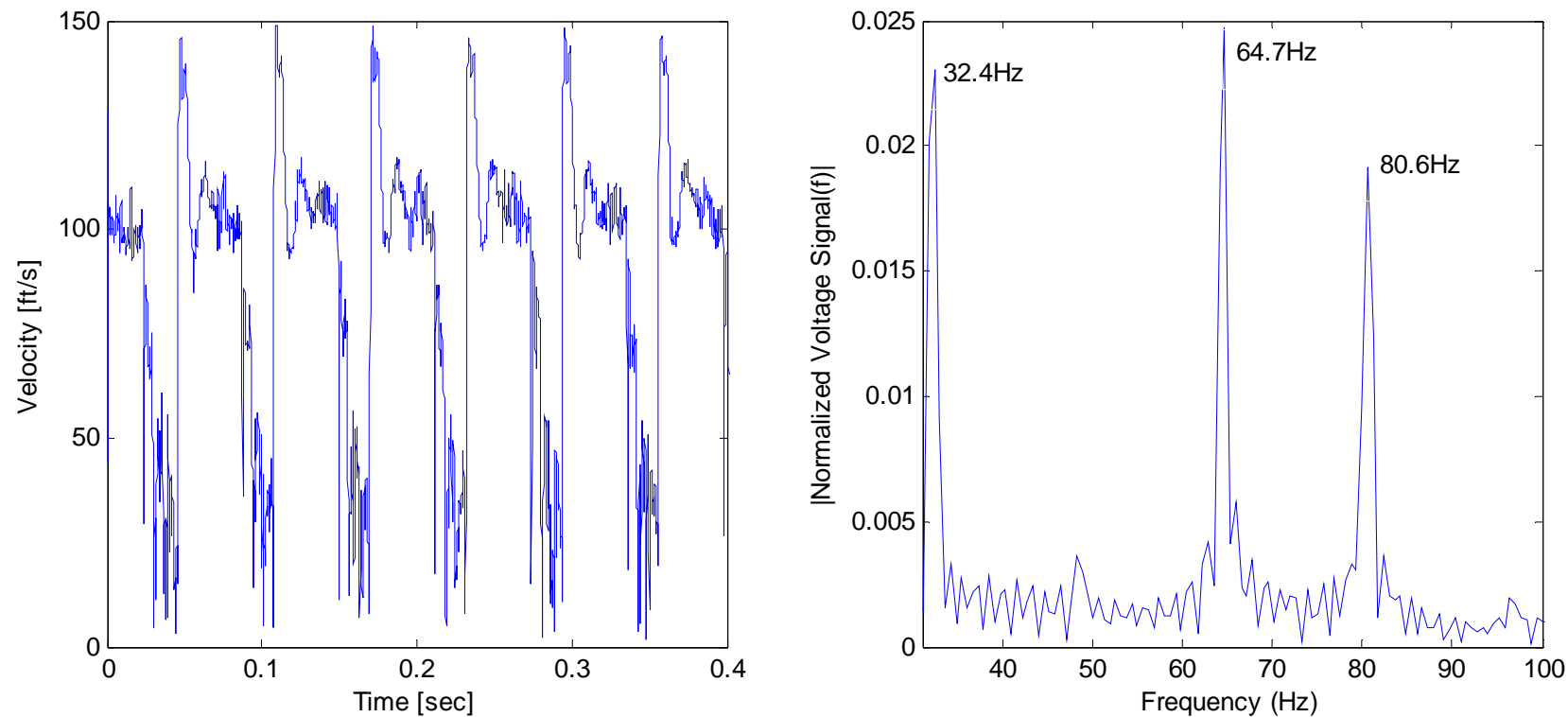

Figure 136. Measured CCNACA0018 Jet: $R e=180 \times 10^{3}\left\langle c_{\mu}>=0.05 S t=0.25\left(f_{\text {act }}=32 \mathrm{~Hz}\right)\right.$ 

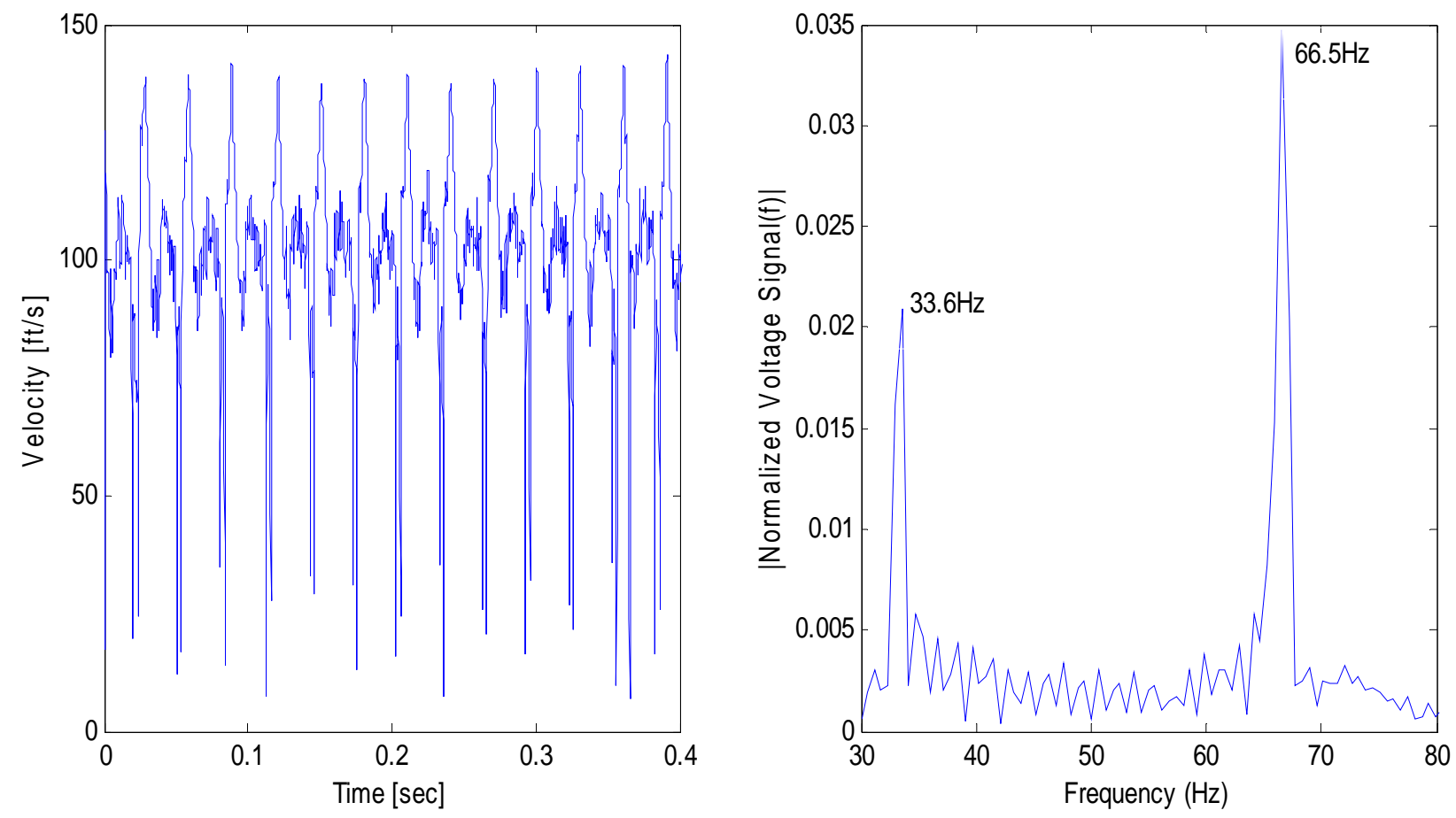

Figure 137. Measured CCNACA0018 Jet Velocity: $R e=180 \times 10^{3}\left\langle c_{\mu}>=0.05 \mathrm{St}=0.5\left(f_{\text {act }}=64 \mathrm{~Hz}\right)\right.$
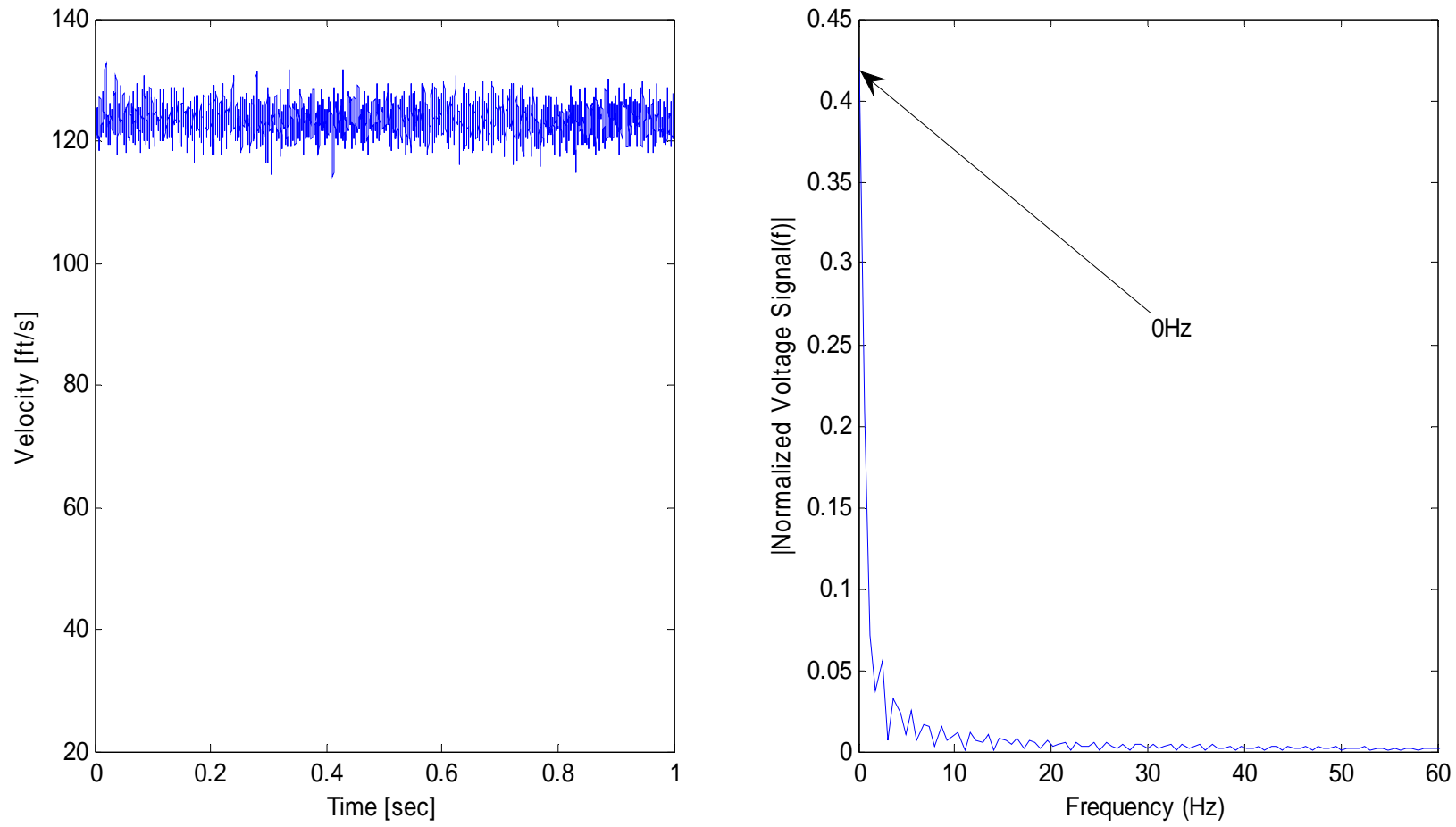

Figure 138. Measured CCNACA0018 Jet Velocity: $R e=180 \times 10^{3} C_{\mu}=0.10 \mathrm{St}=0.0\left(f_{\text {act }}=\mathrm{OHz}\right)$ 
Appendix B. $2 R e=300 \times 10^{3}$ Jet Velocity Measurements and Spectral Analysis Plots
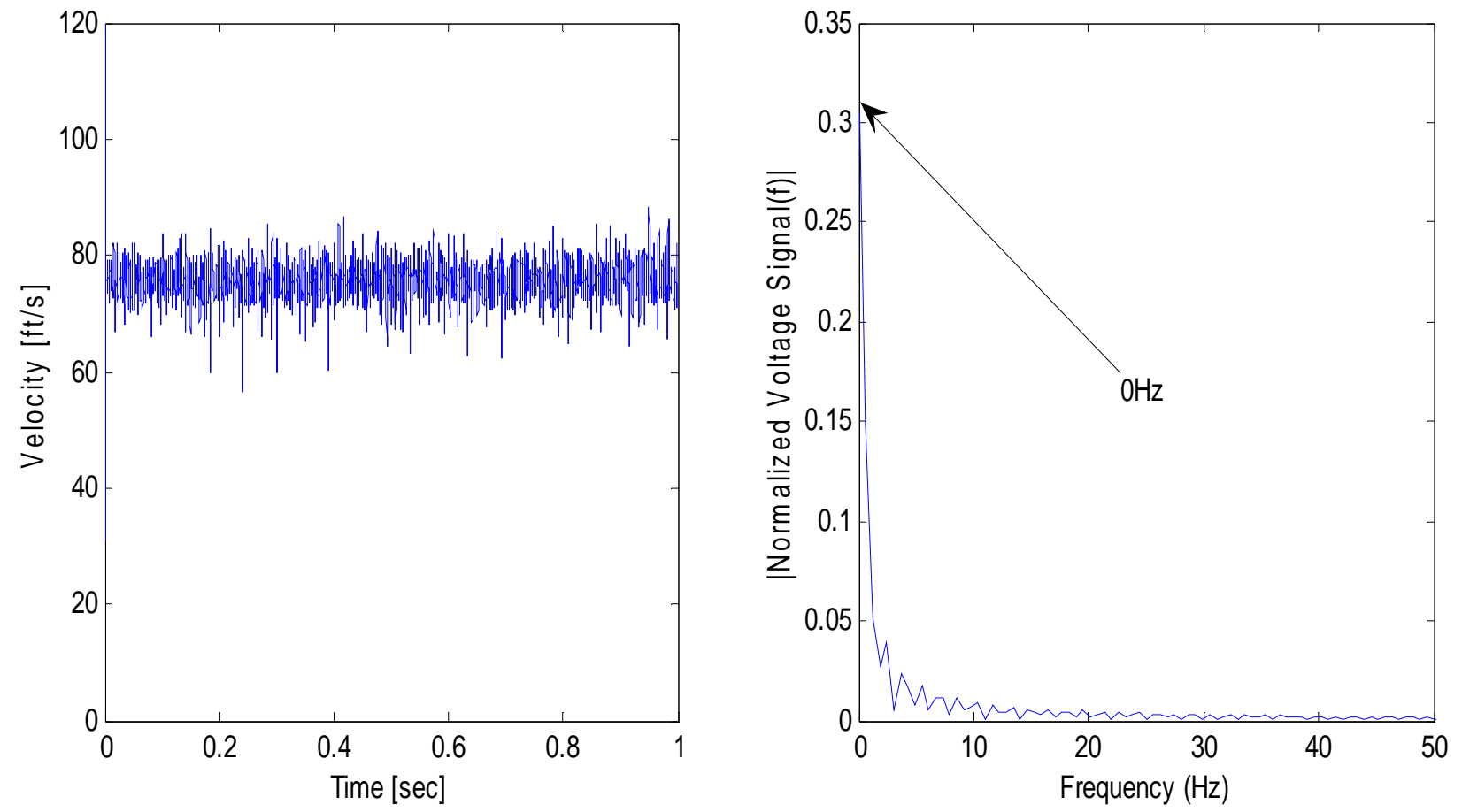

Figure 139. Measured CCNACAO018 Jet Velocity: $R e=300 \times 10^{3} C_{\mu}=0.01 \mathrm{St}=0.0\left(f_{\text {act }}=0 \mathrm{~Hz}\right)$
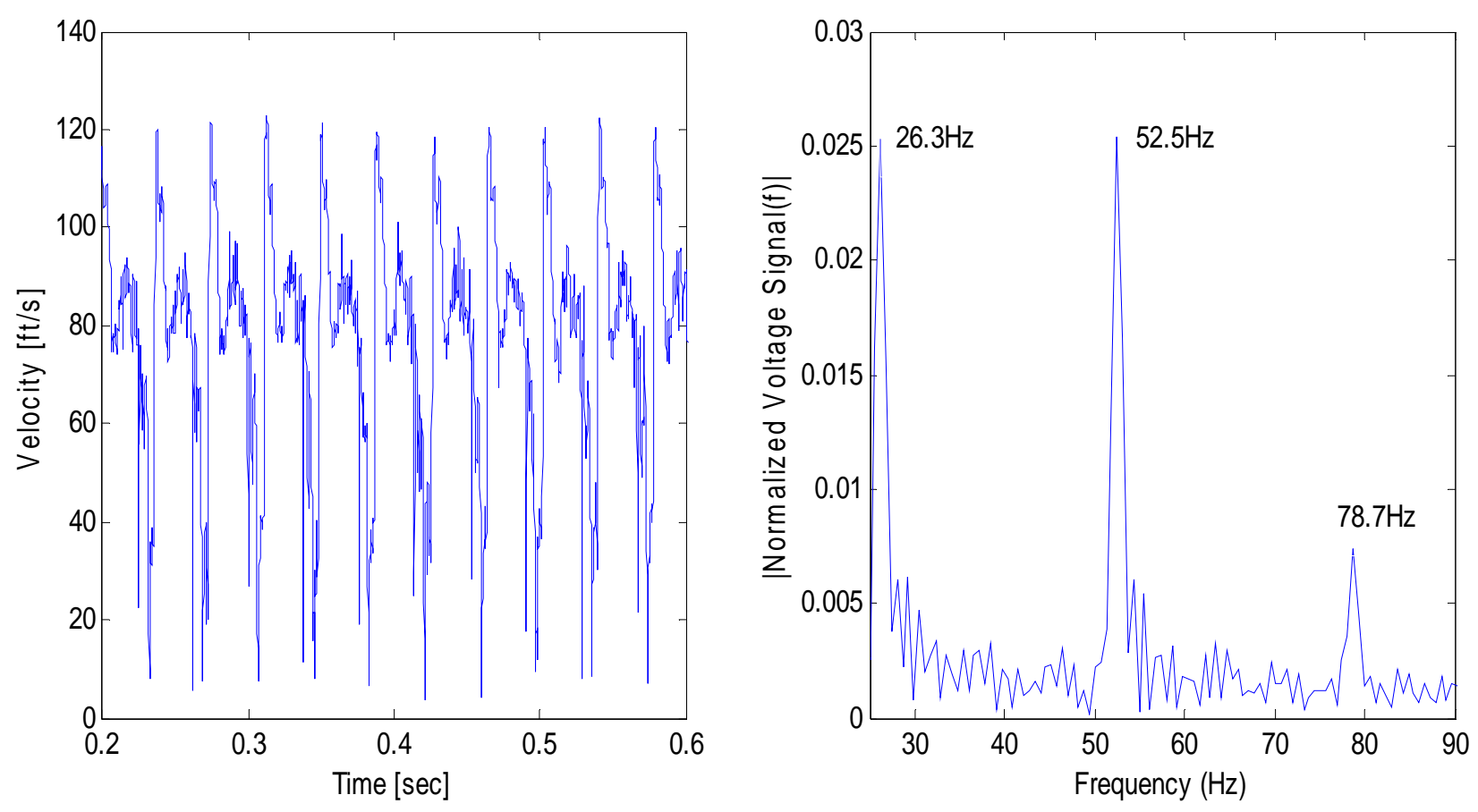

Figure 140. Measured CCNACA0018 Jet Velocity: $R e=300 \times 10^{3}<c_{\mu}>0.01$ St $=0.5\left(f_{\text {act }}=26 \mathrm{~Hz}\right)$ 

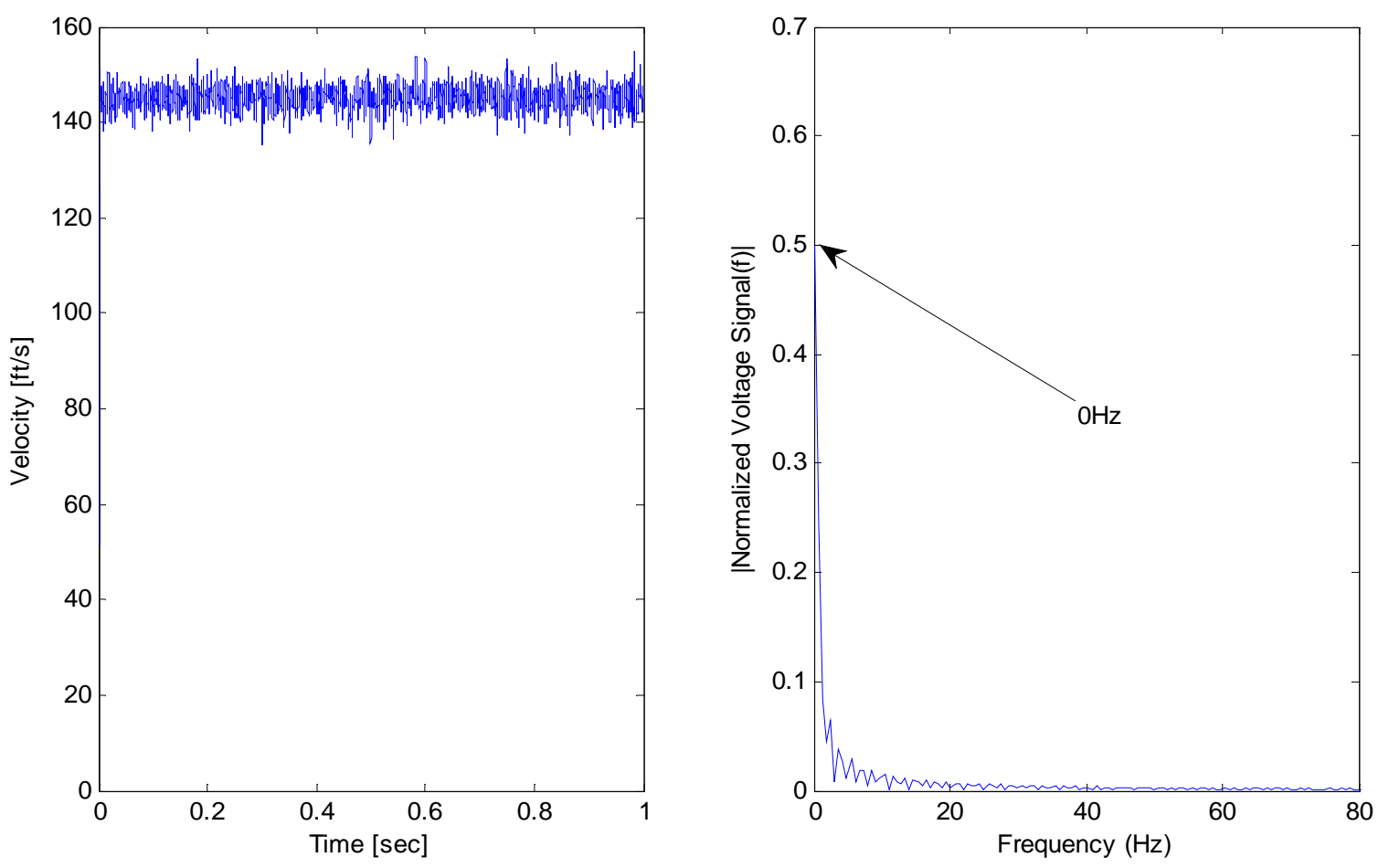

Figure 141. Measured CCNACA0018 Jet Velocity: $R e=300 \times 10^{3} \mathrm{C}_{\mu}=0.05 \mathrm{St}=0.0\left(\mathrm{fact}_{\mathrm{act}}=\mathrm{OHz}\right)$
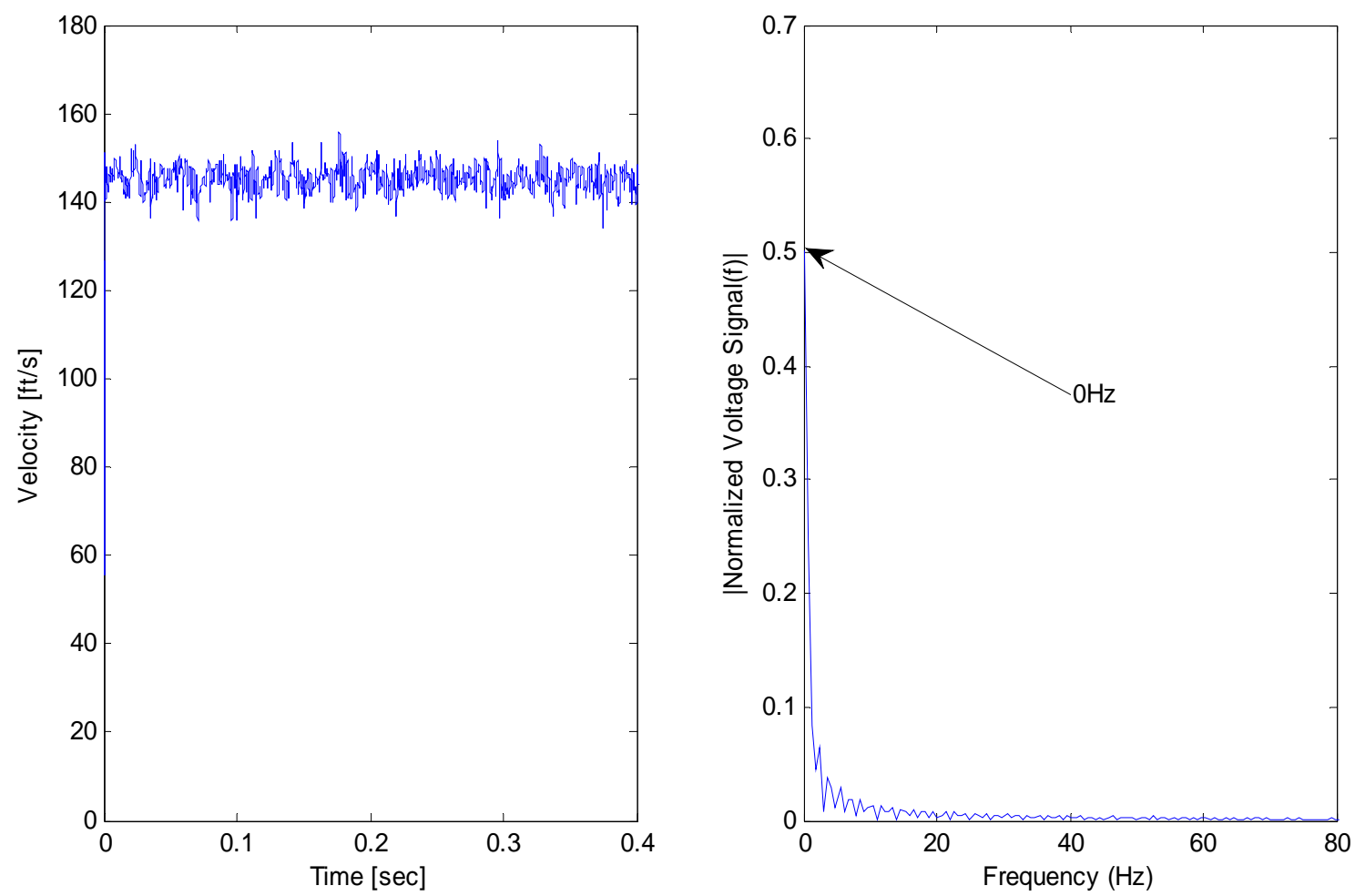

Figure 142. Measured CCNACA0018 Jet Velocity: $R e=300 \times 10^{3}\left\langle c_{\mu}\right\rangle=0.05 \mathrm{St}=0.5\left(f_{\text {act }}=54 \mathrm{~Hz}\right)$ 

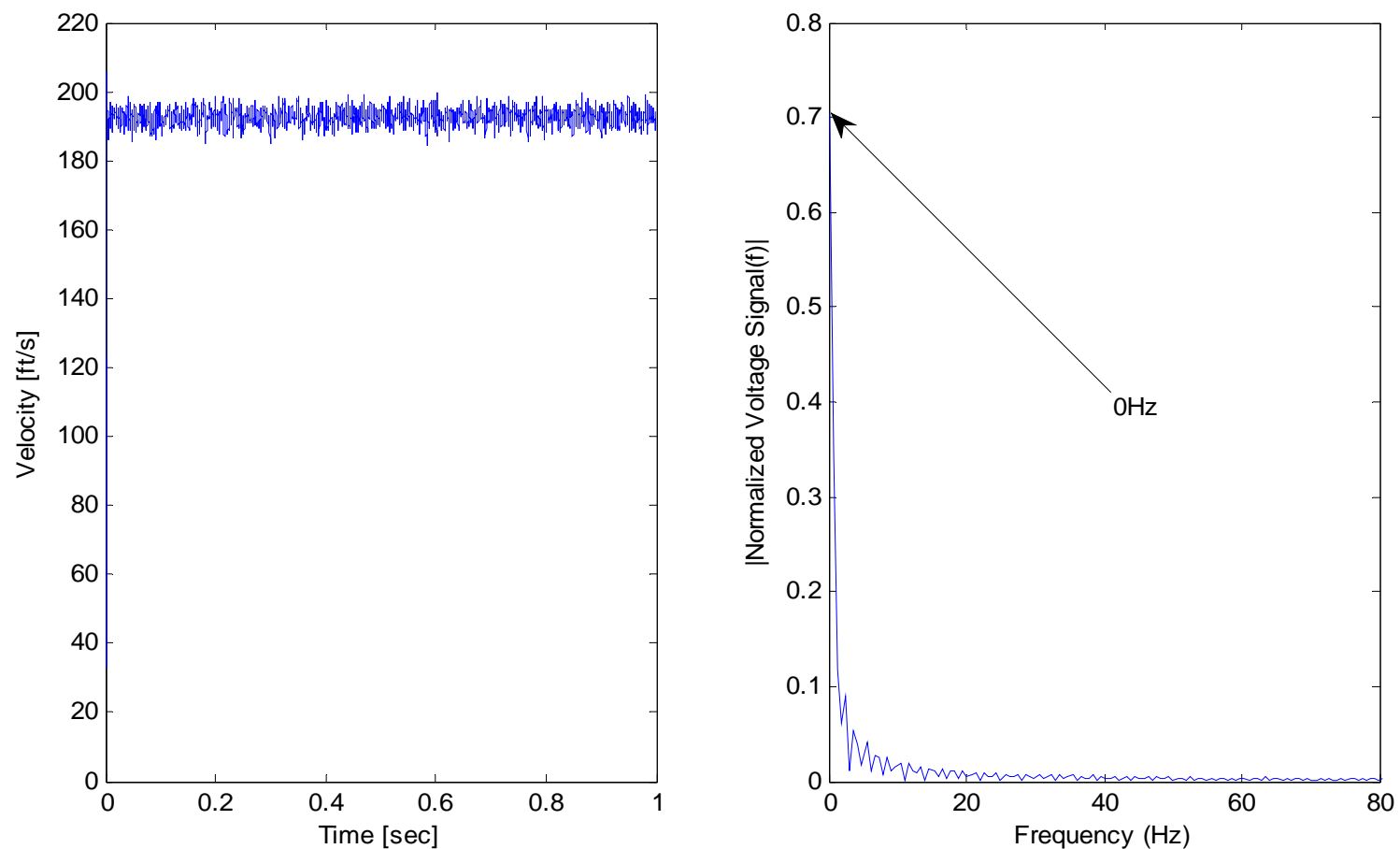

Figure 143. Measured CCNACA0018 Jet Velocity: $R e=300 \times 10^{3} C_{\mu}=0.10 \mathrm{St}=0.0\left(f_{\text {act }}=0 \mathrm{~Hz}\right)$ 


\section{APPENDIX C: ScaniValve Pressure Transducer Calibrations}

Included are the calibration curves for each of the 32 ports of the ScaniValve pressure transducer, recorded at the beginning of each testing day. If the temperature of the lab exceeded a change of $2^{\circ} \mathrm{F}$, the ScaniValve was recalibrated due to high thermal sensitivity of the piezoresistive transducers. The range of the transducer was 2.5 psid. At the beginning of each day, a 30+point calibration was completed with a Dwyer calibration pump (Model A-396A) and a HEISE Hand Held Calibrator (Model PTE-1) with a Quick Select pressure module (HQS-2) of range 0-15 psia $( \pm 0.05 \% \mathrm{FS}=$ $\pm 0.0075 \mathrm{psi}$ ). The Dwyer pump was capable of producing and holding a full range of $\pm 5 \mathrm{psi}$, exceeding the range of the ScaniValve. The minimum number of calibration points needed to assure adequate correction for non-linearity (for sensor accuracy of $0.05 \%$ ) is 20, with a 10 point upscale and 10 point downscale linearization check and hysteresis check,. The resulting equations to convert voltage to gauge pressure for each port are included above each subplot in Figure 144 through Figure 150. The MATLAB script to convert raw voltage data to respective pressure [psig] values is included in this appendix; the author would like to thank Dr. Jay Wilhelm for his assistance with coding to generate the calibration curves in an organized manner. 


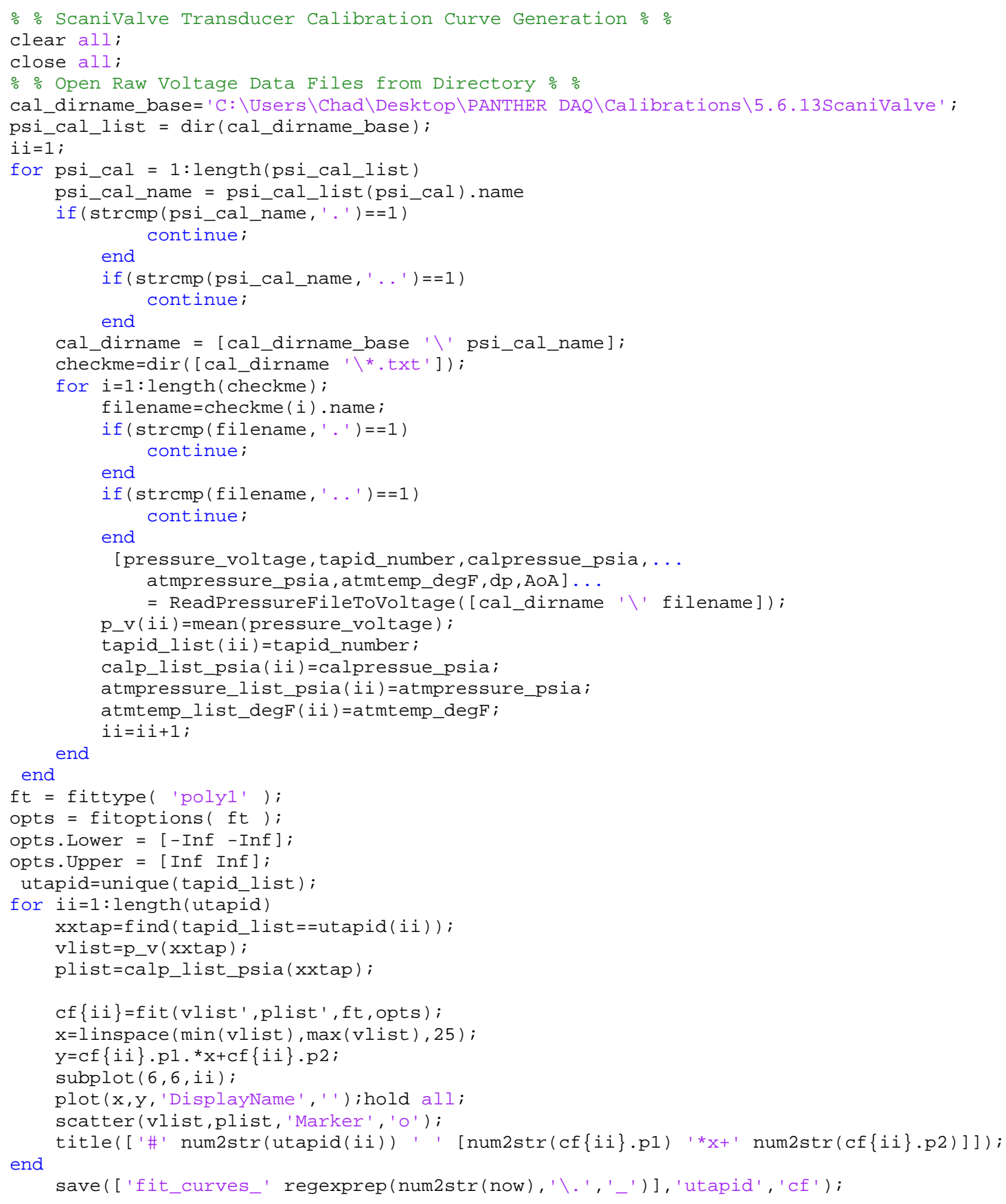



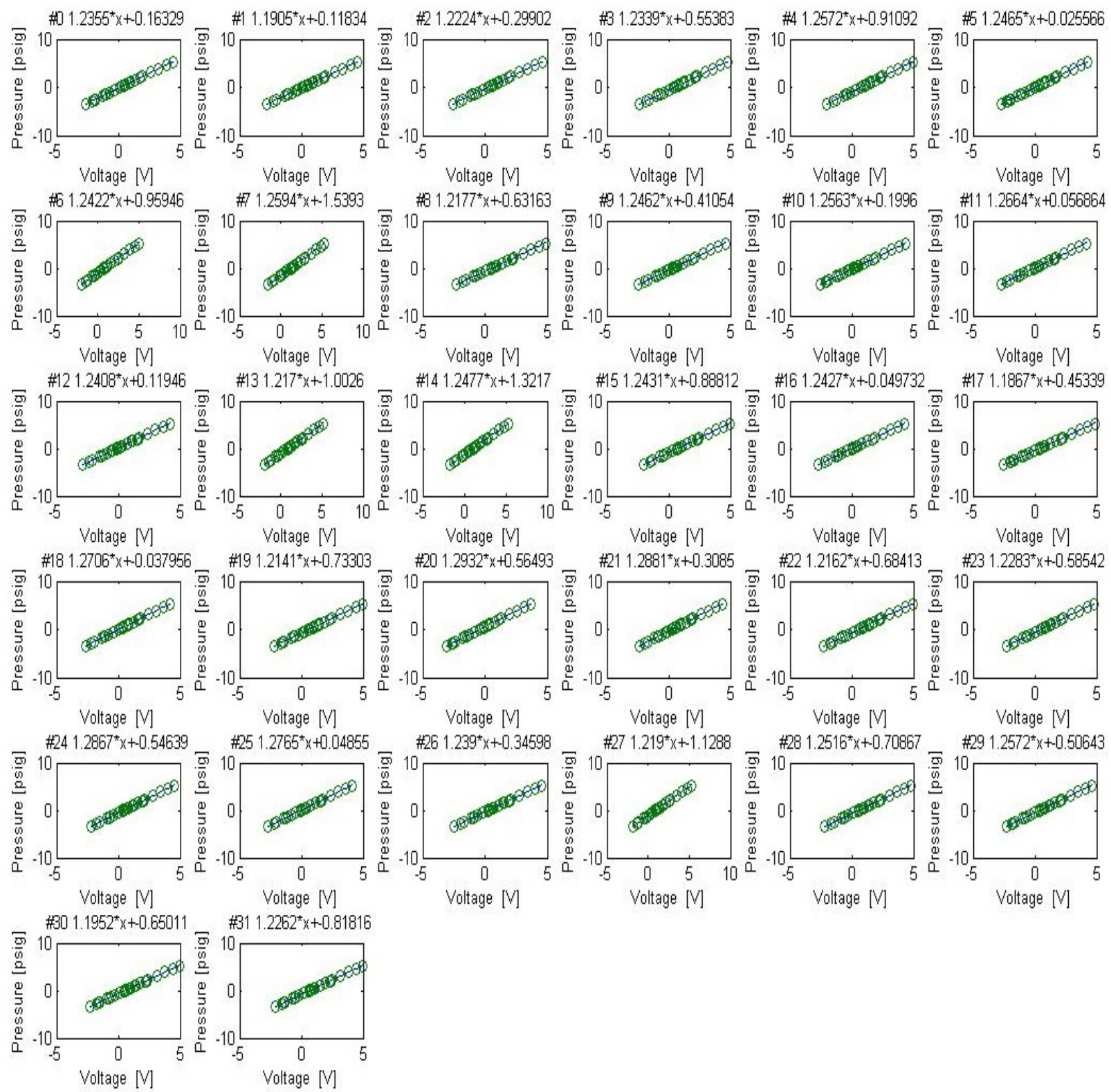

Figure 144. ScaniValve Pressure Port Calibration Curves (Date: 05.01.13) 

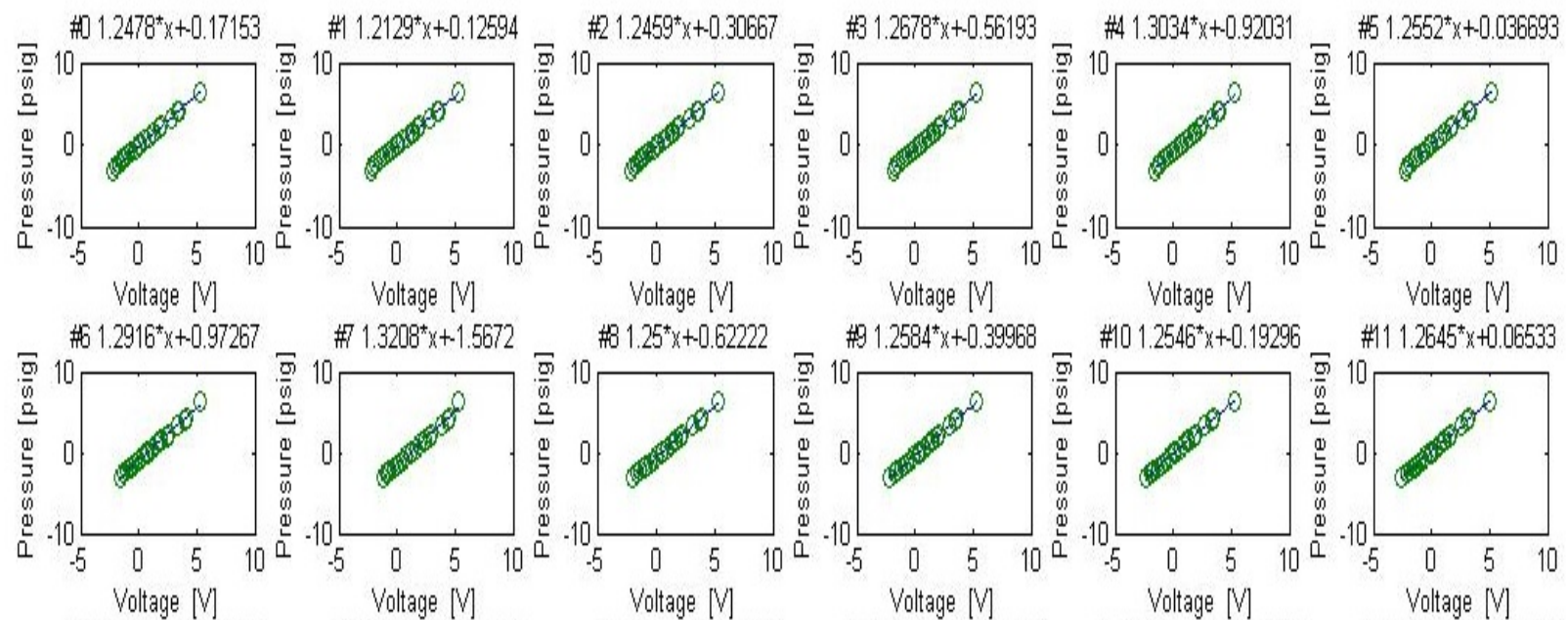

Voltage $[\mathrm{V}]$
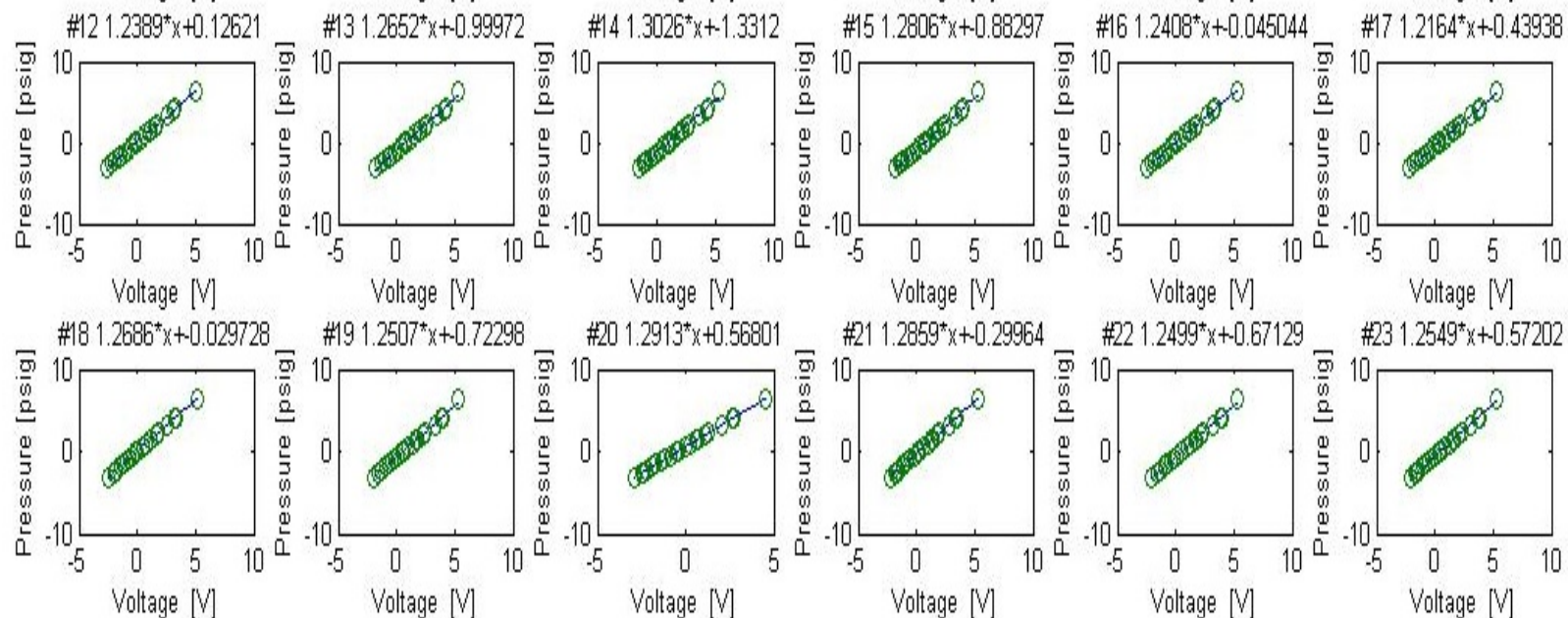

Voltage $[\mathrm{V}]$

Voltage $[\mathrm{V}$

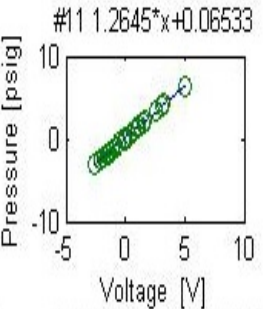

$41.301 * x+0.05456$
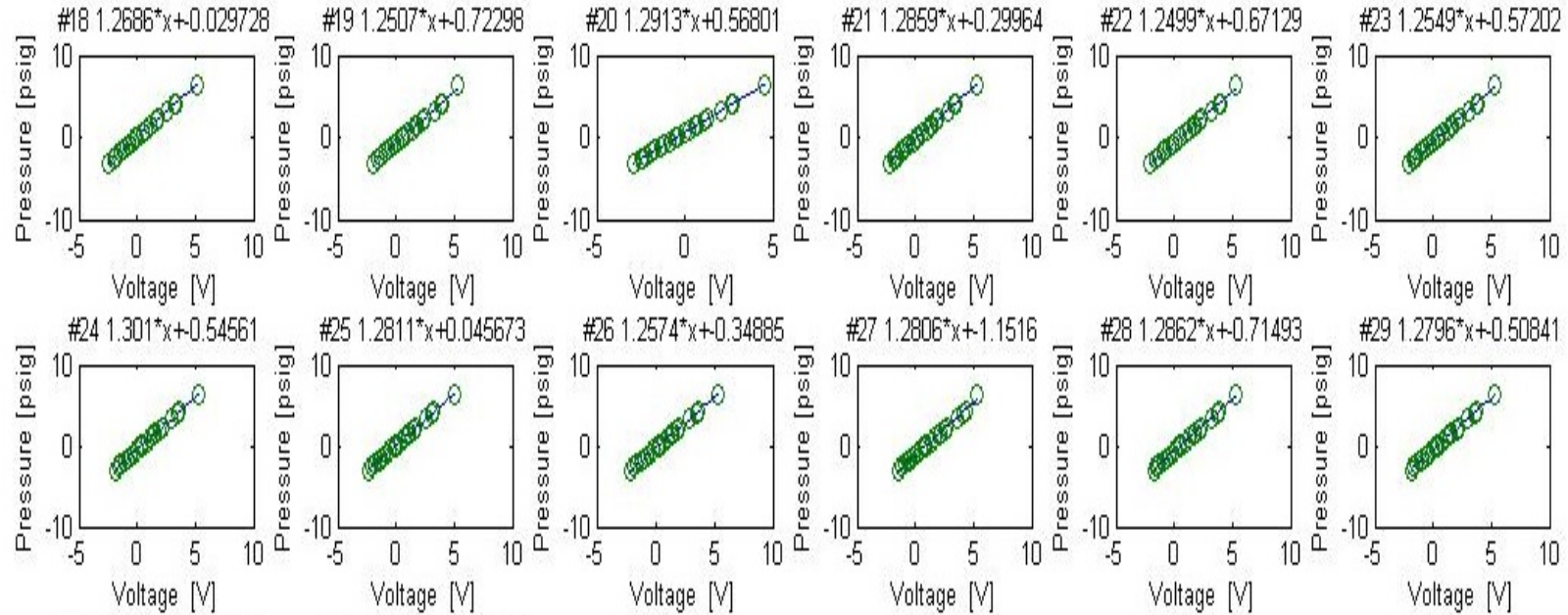

$\$ 301.2401 * x+0.0554$
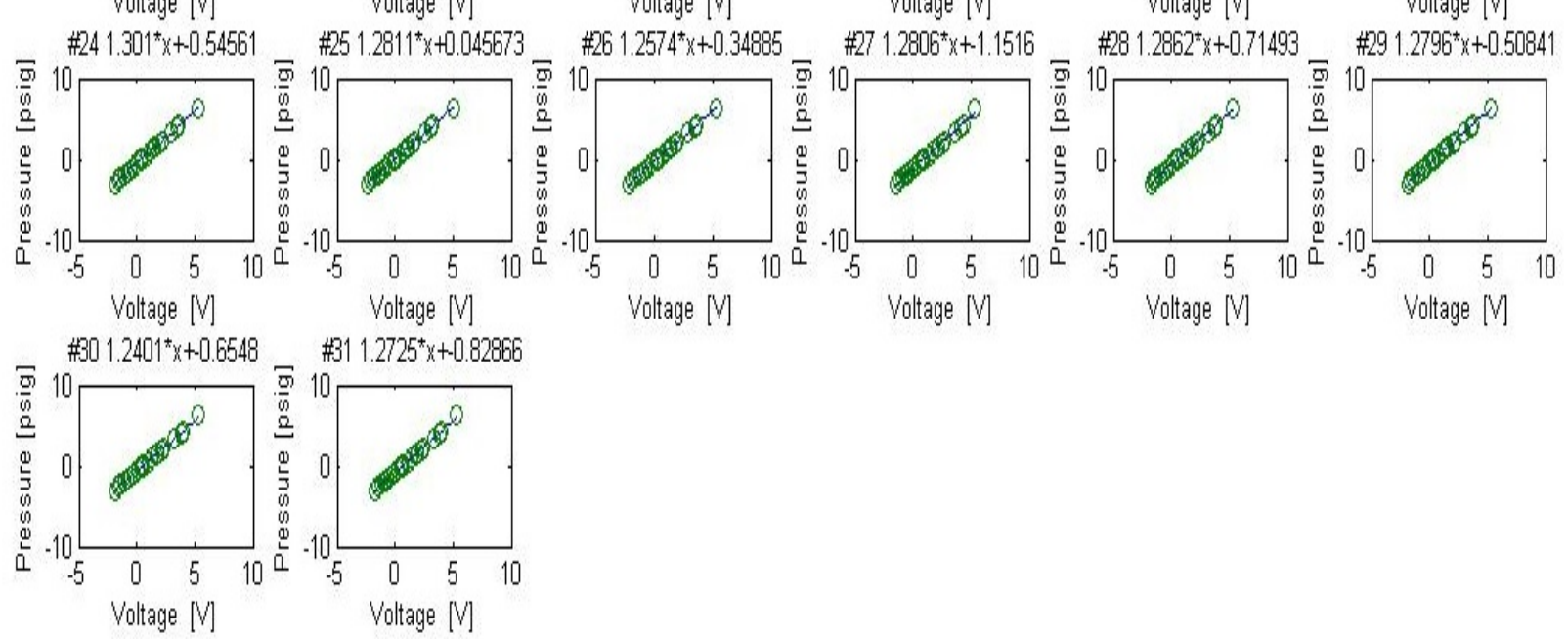

Figure 145. ScaniValve Pressure Port Calibration Curves (Date: 05.02.2013) 

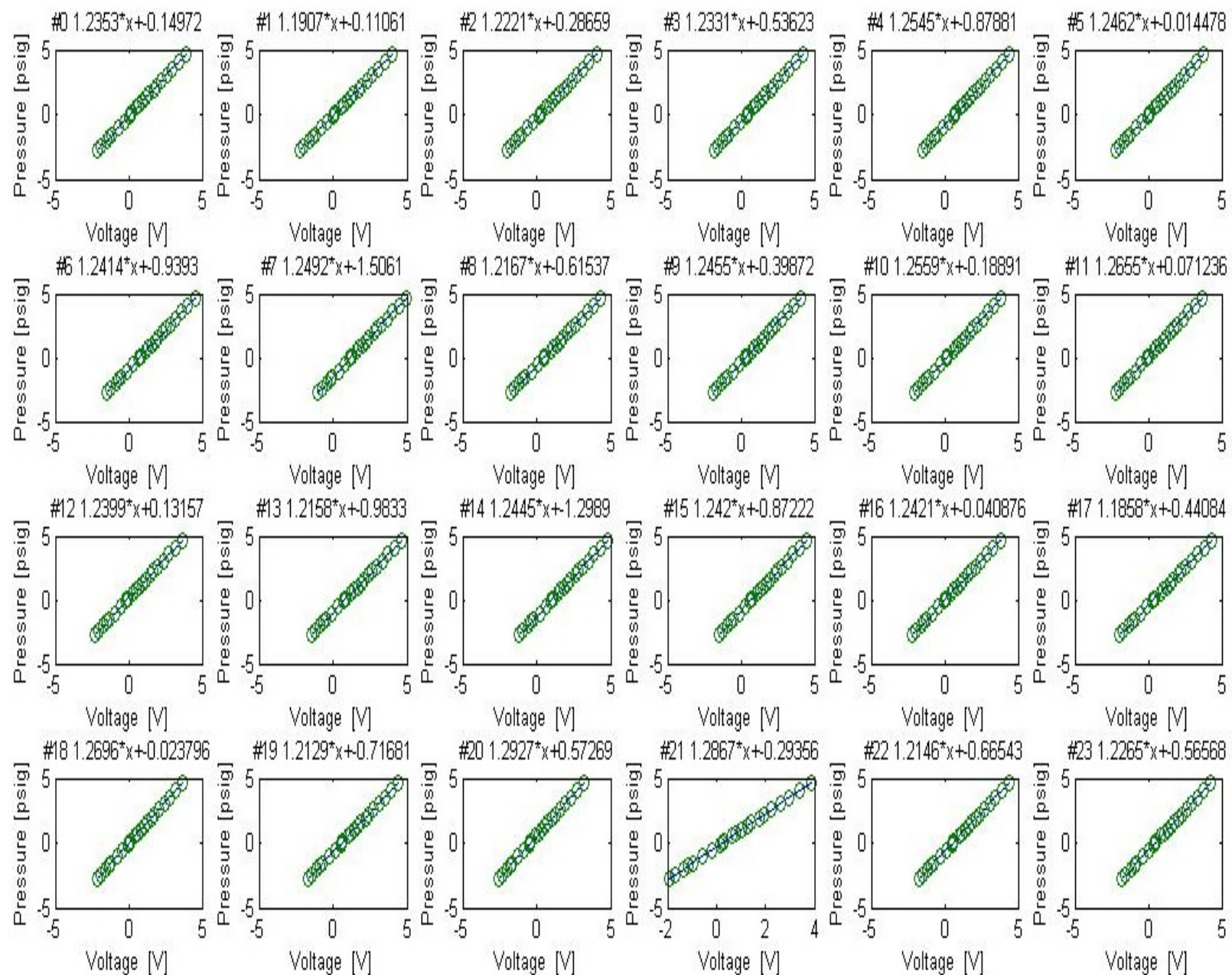

Voltage $[\mathrm{V}]$

Voltage $[\mathrm{V}]$

$1.2374^{*} x+-0.33258$
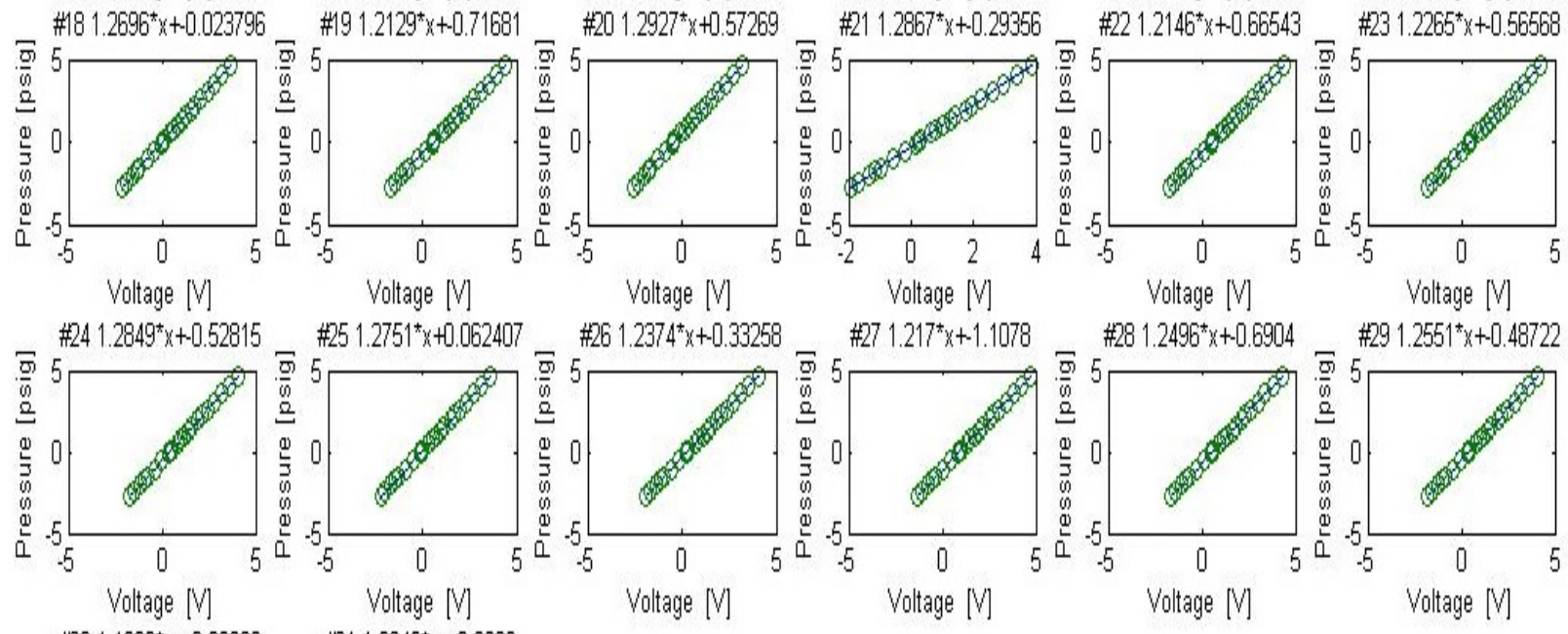

Voltage [V]

Voltage [V]
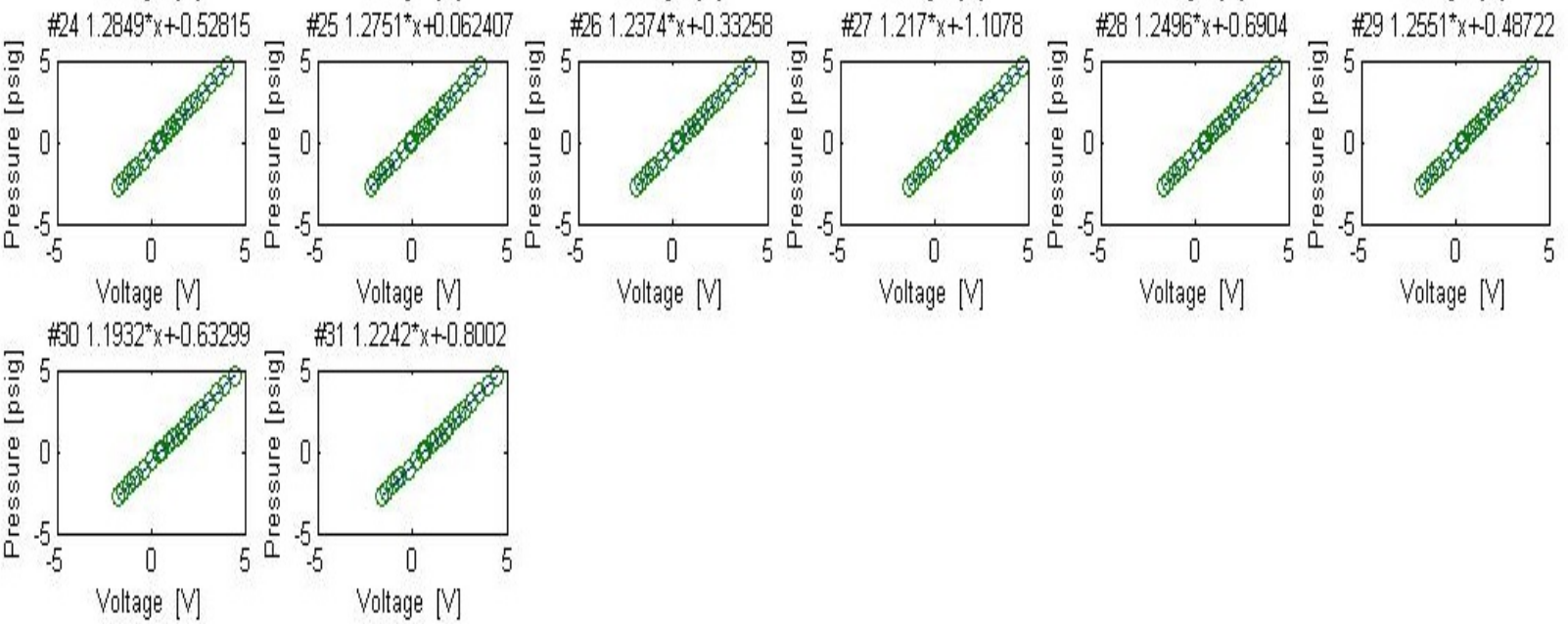

Figure 146. ScaniValve Pressure Port Calibration Curves (Date: 05.03.2013) 

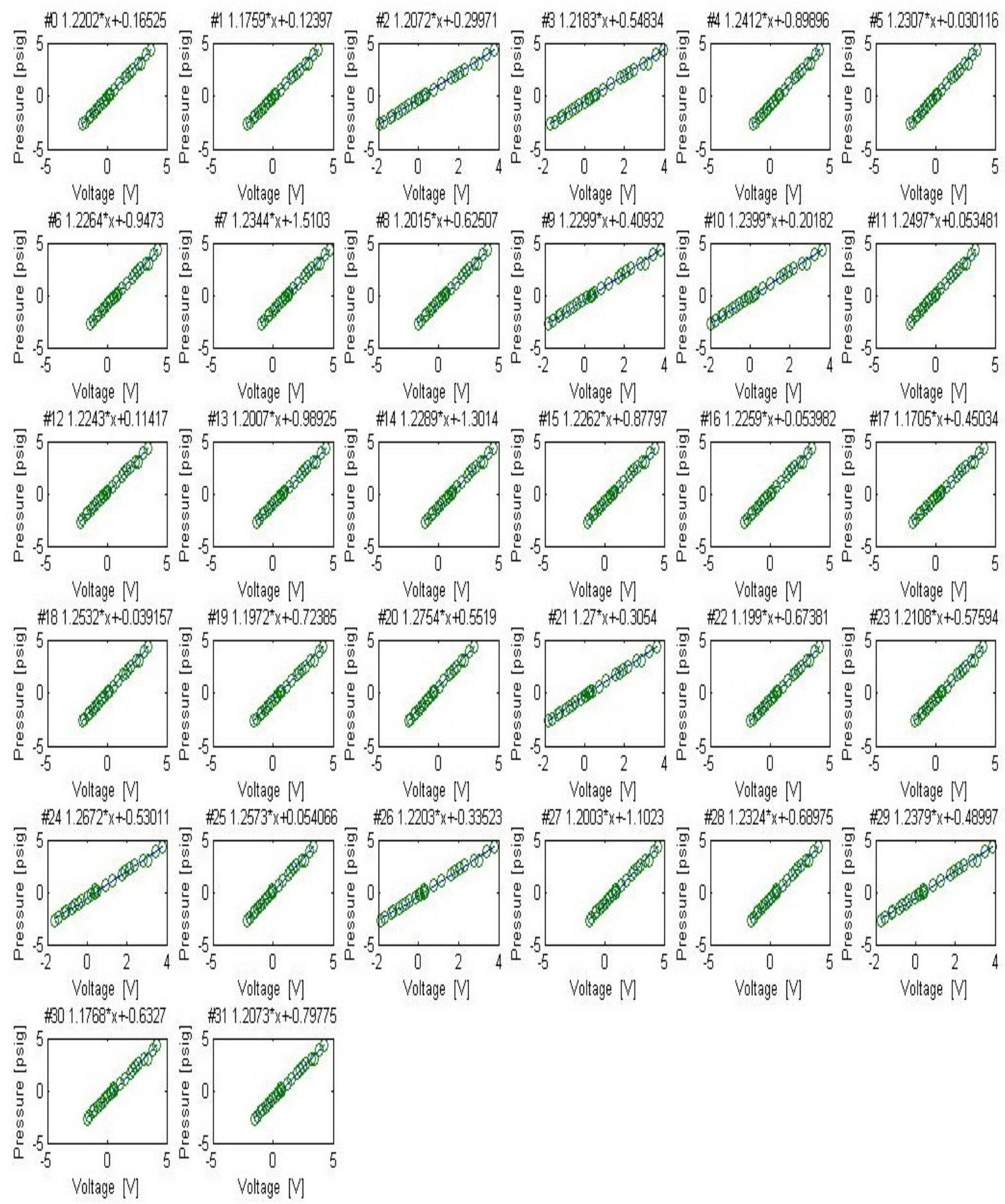

Figure 147. ScaniValve Pressure Port Calibration Curves (05.04.2013) 

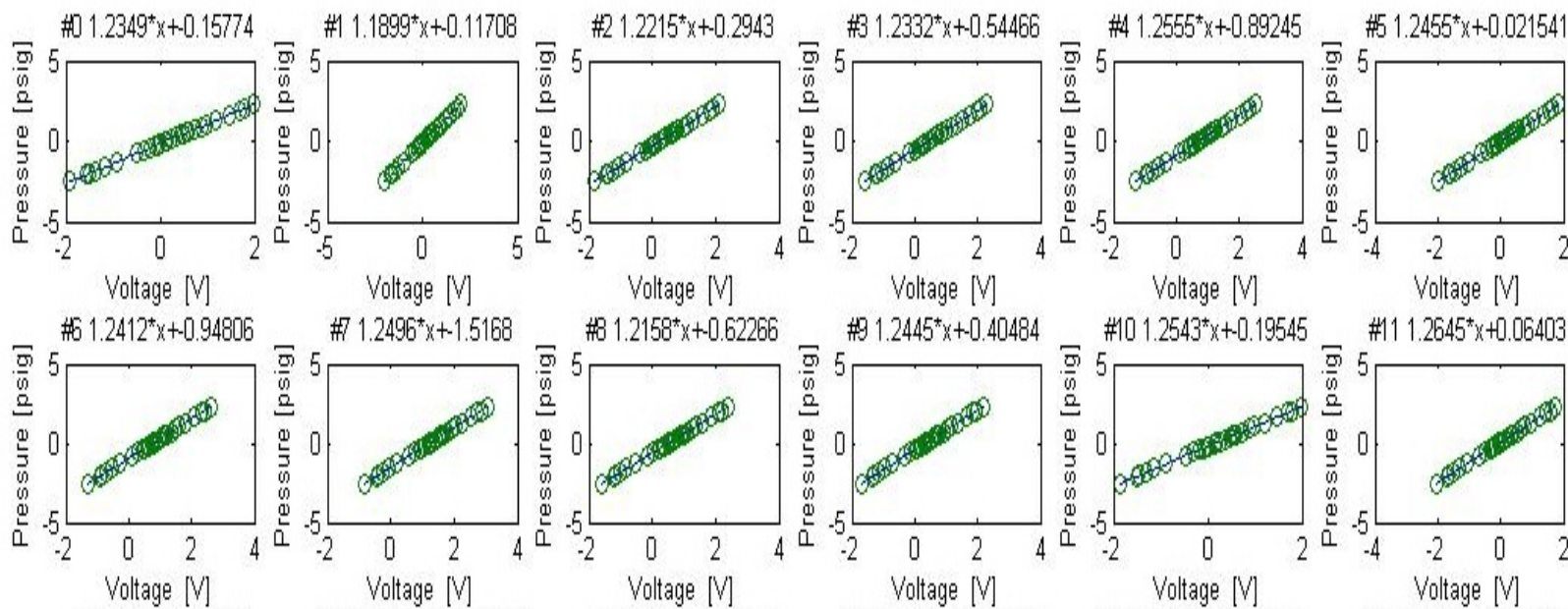

\#+121.2387* $x+0.125$
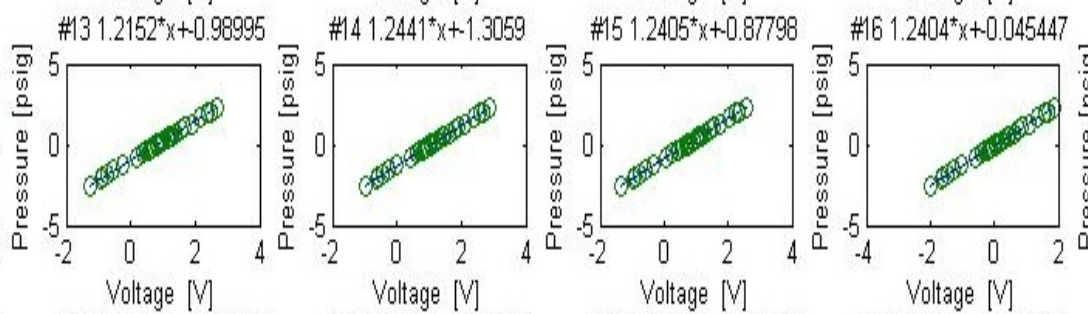

Voltage $[\mathrm{V}]$

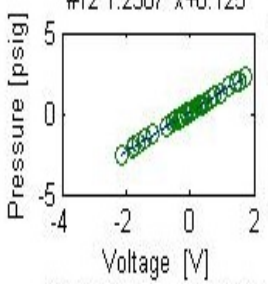

$91.2115^{*} \times+.0 .72206$
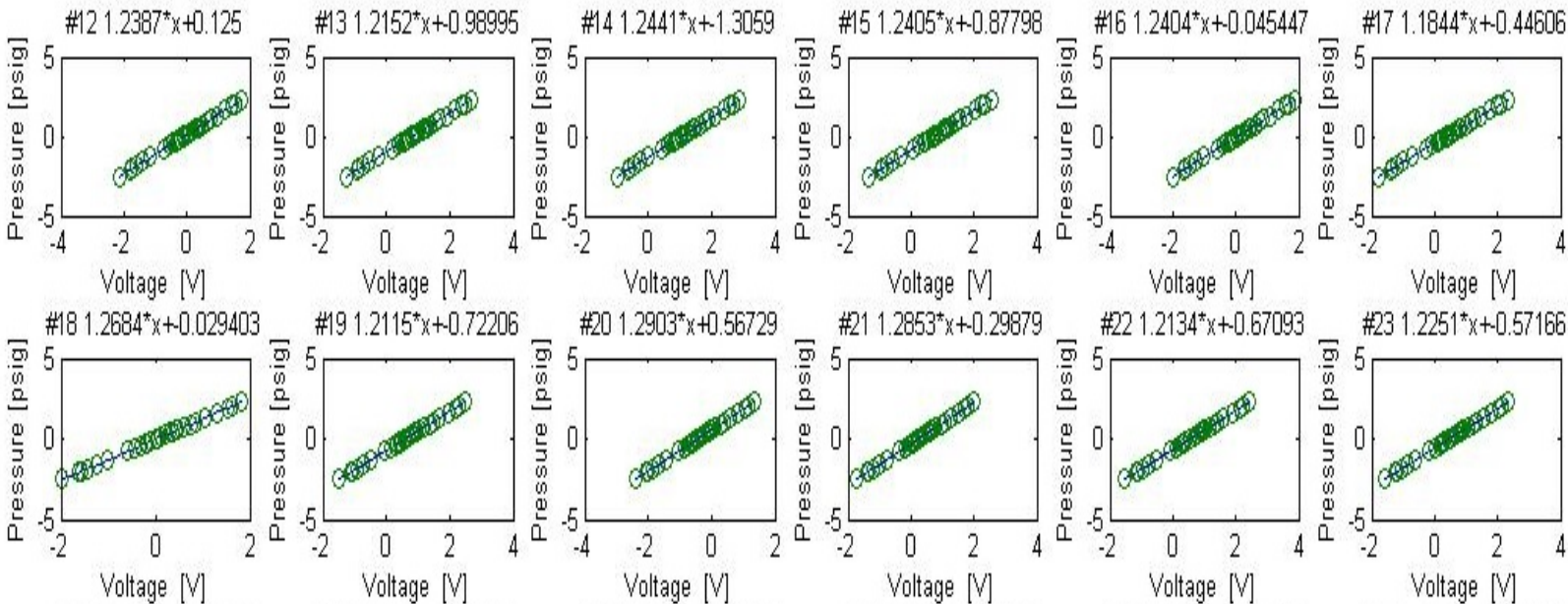

Voltage $[\mathrm{V}]$
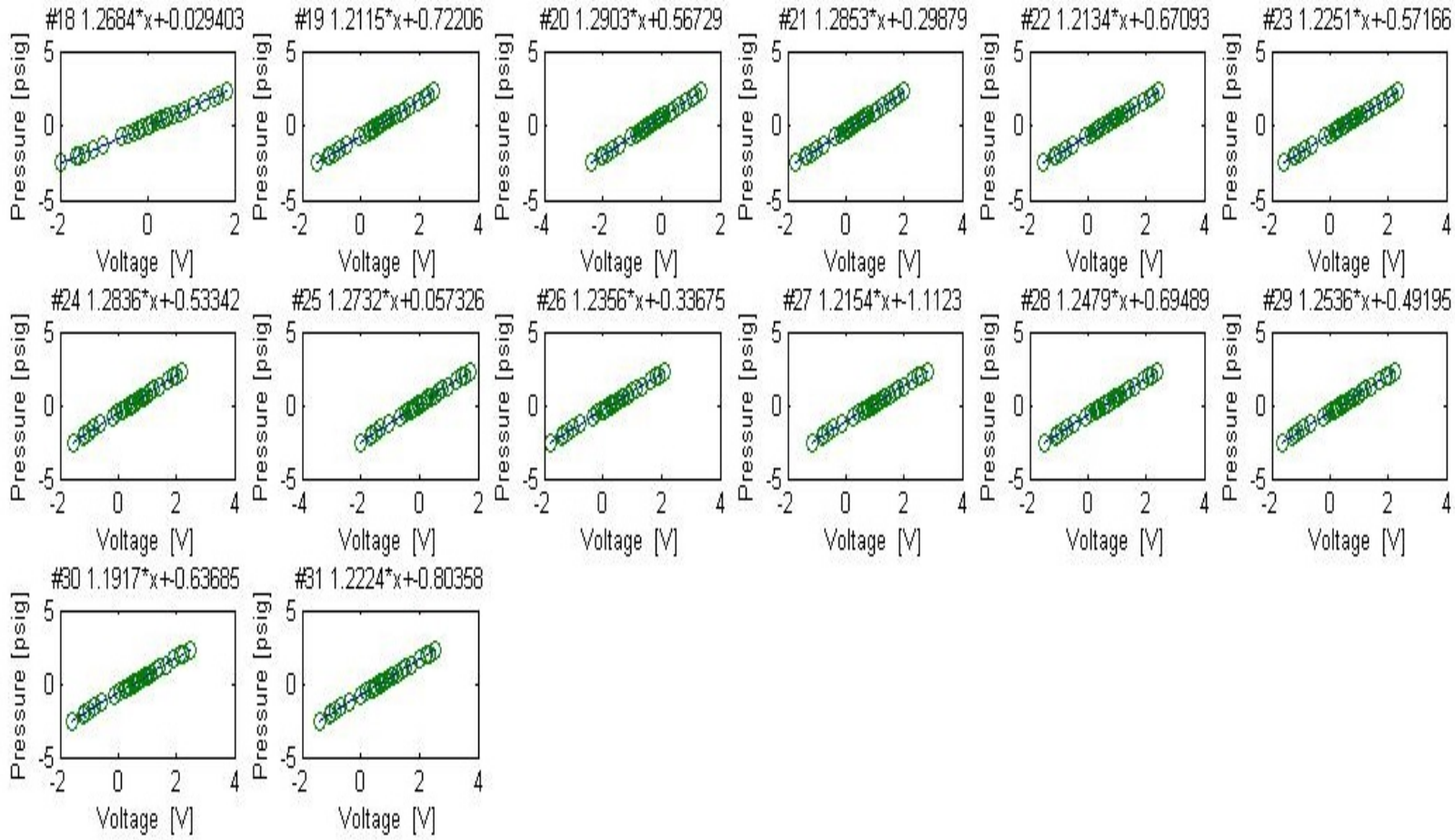

Figure 148. ScaniValve Pressure Port Calibration Curves (05.05.2013) 

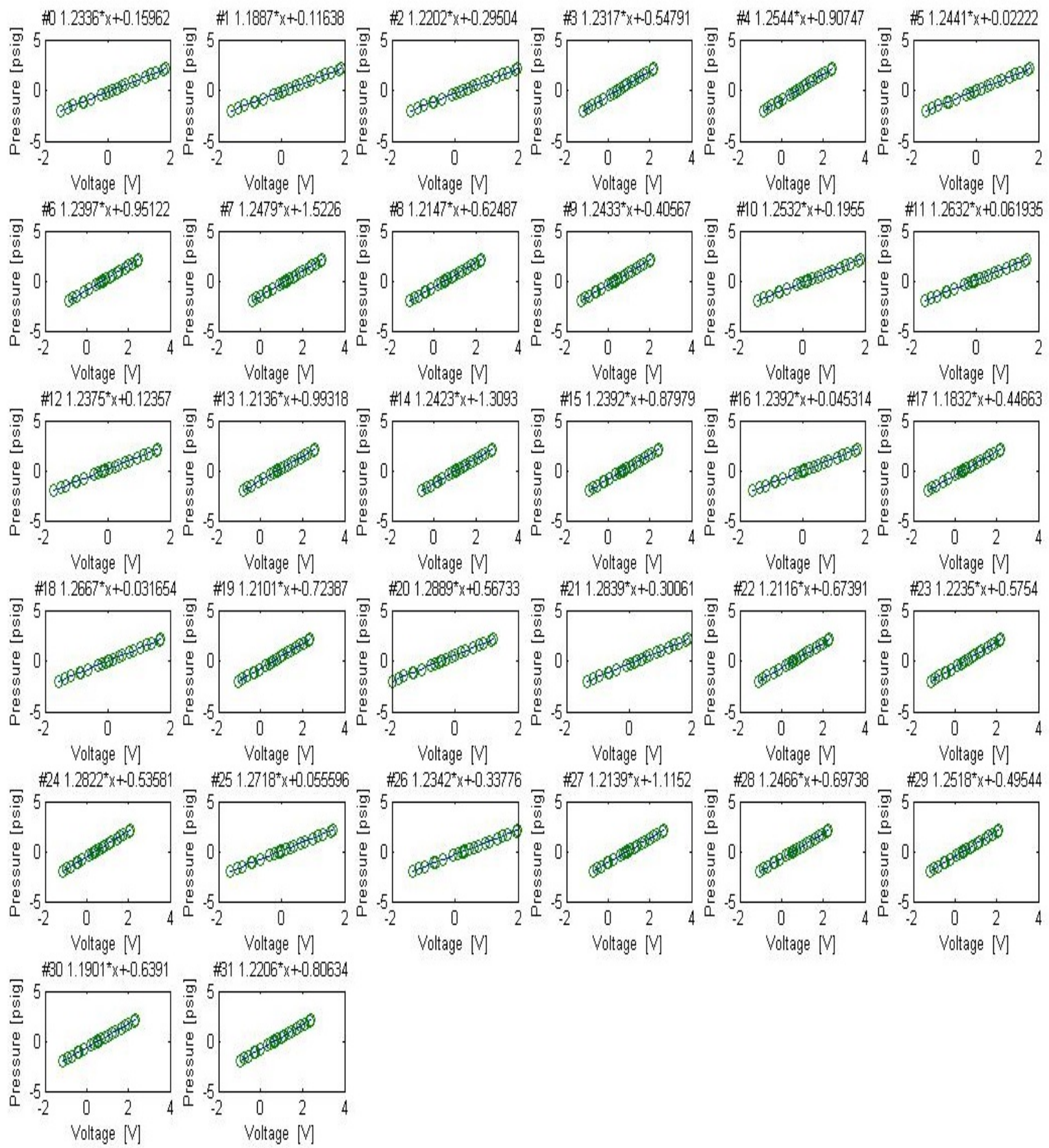

Figure 149. ScaniValve Pressure Port Calibration Curves (05.06.2013) 

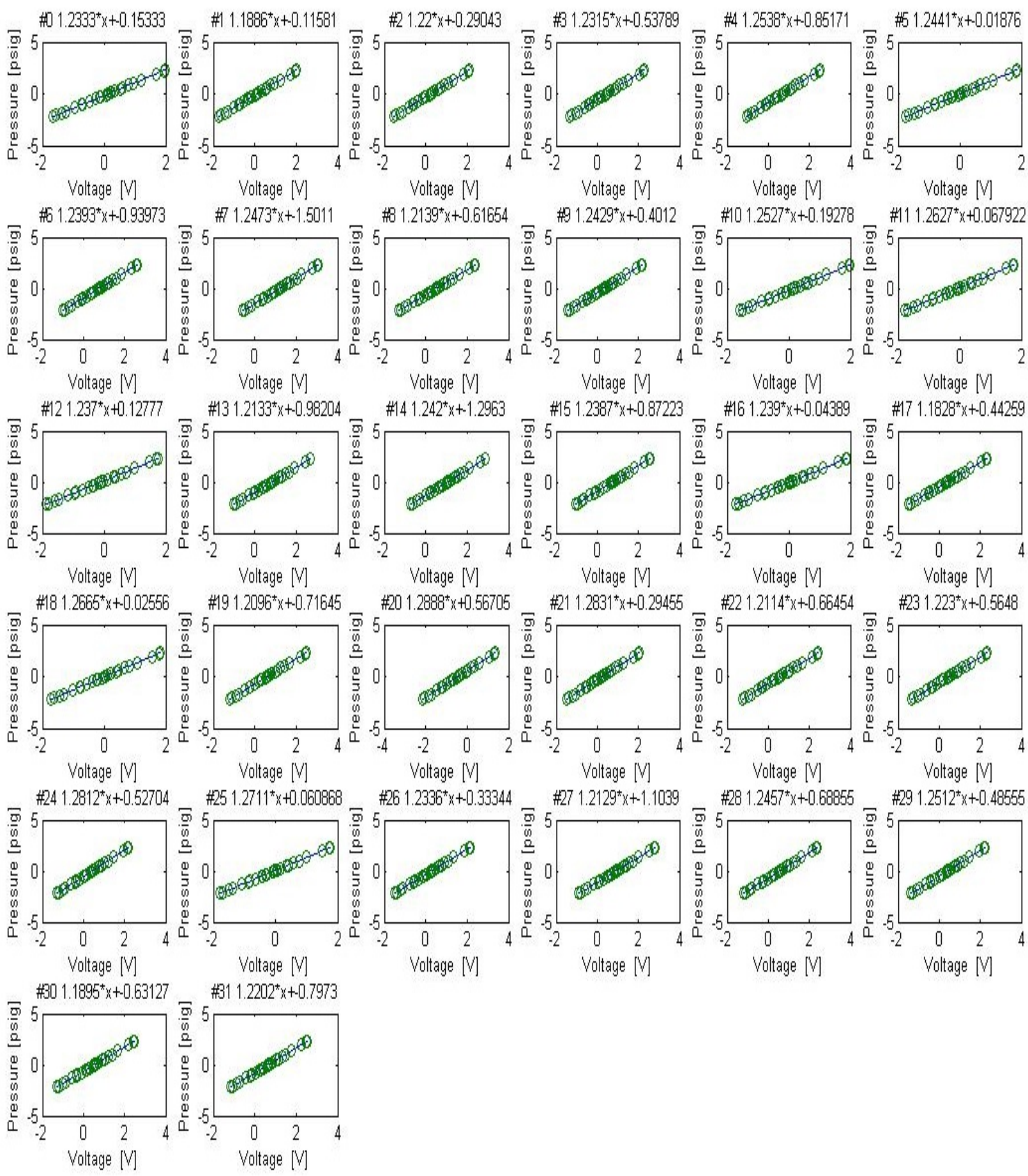

Voltage $[\mathrm{V}]$

Voltage [V]

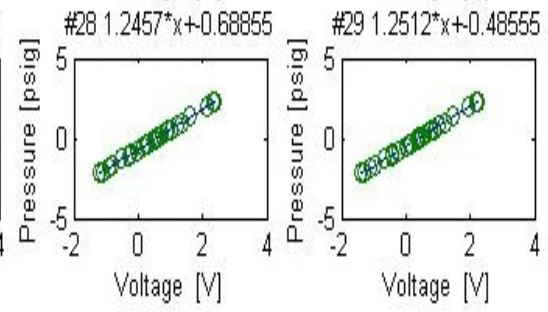

Figure 150. ScaniValve Pressure Port Calibration Curves (05.07.2013) 
The sensitivity of the ScaniValve calibration coefficients to sampling frequency (sampling rate, $\mathrm{SR}$ ) was checked for each of the 33 ports used during testing. Two sampling rates were examined, $5 \mathrm{kHz}$ and $80 \mathrm{kHz}$, with the lower value exceeding the standard double Nyquist frequency. A 25 point calibration was completed using each SR to complete a representative set of calibration coefficients for each port; individual port coefficients can be found above each calibration plot in Figure 144 through Figure 150. Next, constant and steady pressure was applied to each port and the resulting voltage measurements were recorded with the LabView DAQ program, converted to pressure values, and stored on the lab DAQ computer. The controlled supply pressure magnitudes covered the full range of the ScaniValve transducers ( $\pm 2.5 \mathrm{psi}$ ). An illustrative result is included in Equation 151; open circles represent $\mathrm{SR}=5 \mathrm{kHz}$ and solid line denotes $\mathrm{SR}=80 \mathrm{kHz}$.

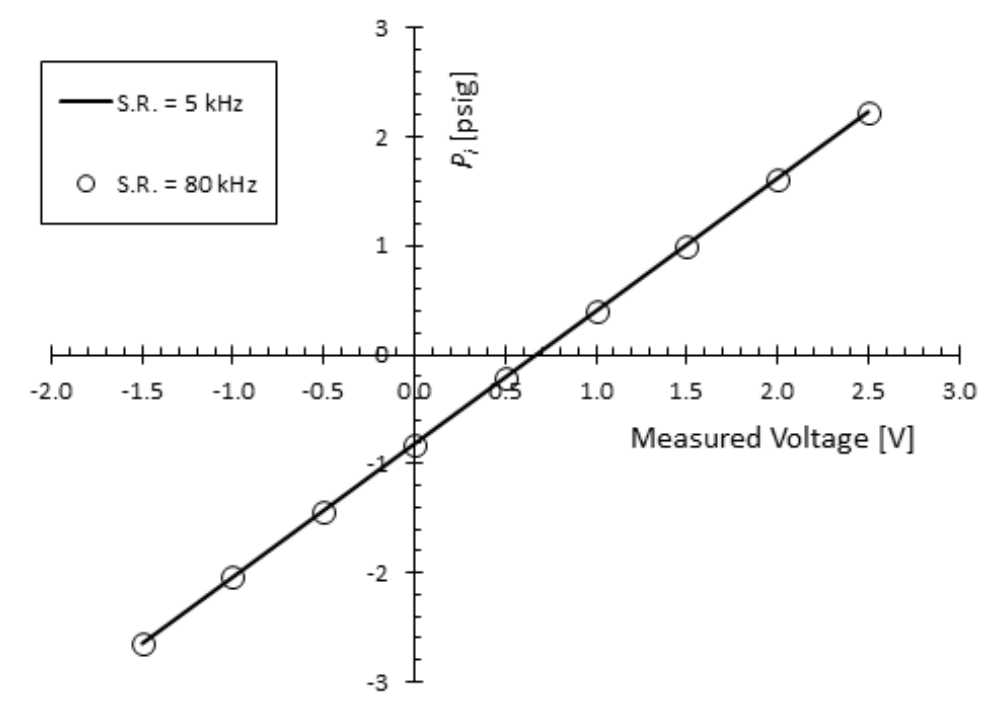

Figure 151. Influence of Sampling Frequency on ScaniValve Pressure Measurements

As shown in Figure 151, the SR used during calibration was found to have a very small influence on the resulting calibration coefficients. For the worst case scenario, discrepancy between resulting pressure magnitudes calculated with each SR was found to be $0.16 \% \mathrm{FS}(0.008 \mathrm{psi})$. The average disagreement between pressure values from each calibration was $\approx 0.05 \% \mathrm{FS}(0.003 \mathrm{psi})$. Considering the specified accuracy of the transducers to be $\pm 0.15 \% \mathrm{FS}(0.005 \mathrm{psi})$, this level of discrepancy is considered within the range of accepted measurement error. 


\section{APPENDIX D: Unsteady Pressure Signal Attenuation in Cylindrical Tubes}

The problem of the propagation of sound waves in gases contained in cylindrical tubes is a classical predicament, to which many studies dealing with the dynamic response of pressure transmission lines have been devoted (159). The two main parameters governing the transmission of pressure waves (through rigid cylindrical tubing) are the shear wave number $(S)$ and the reduced frequency ( $k$ ). $S$ is defined by Equation 83 , relating tube radius $(R[\mathrm{ft}])$, density of air $\left(\rho_{s}\left[\mathrm{slug} / \mathrm{ft}^{3}\right]\right)$, angular frequency of the signal ( $\omega[\mathrm{rad} / \mathrm{s}])$, and the kinematic viscosity of air $\left(\mu\left[\mathrm{lb}-\mathrm{s} / \mathrm{ft}^{2}\right]\right)$. Most of the analytical solutions are dependent on $S$.

Shear Wave Number

$$
S=R \sqrt{\rho_{s} \cdot \omega / \mu}
$$

Equation 83

The reduced wave frequency, Equation 84, includes the angular frequency of a travelling wave $(\omega$ $[\mathrm{rad} / \mathrm{s}])$ and the speed of sound in quiescent air $\left(a_{o}[\mathrm{ft} / \mathrm{s}]\right)$.

Wave Reduced Frequency

$$
k=\omega \cdot R / a_{o}
$$

Equation 84

The theoretical work by Bergh and Tijdeman (138) on the dynamic response of pressuremeasuring tube systems was first applied to a generalized tube-transducer system, included in Figure 152. The theoretical method factors the influence of changing volumes in signal transmission signals, due to connections and pressure transducers.

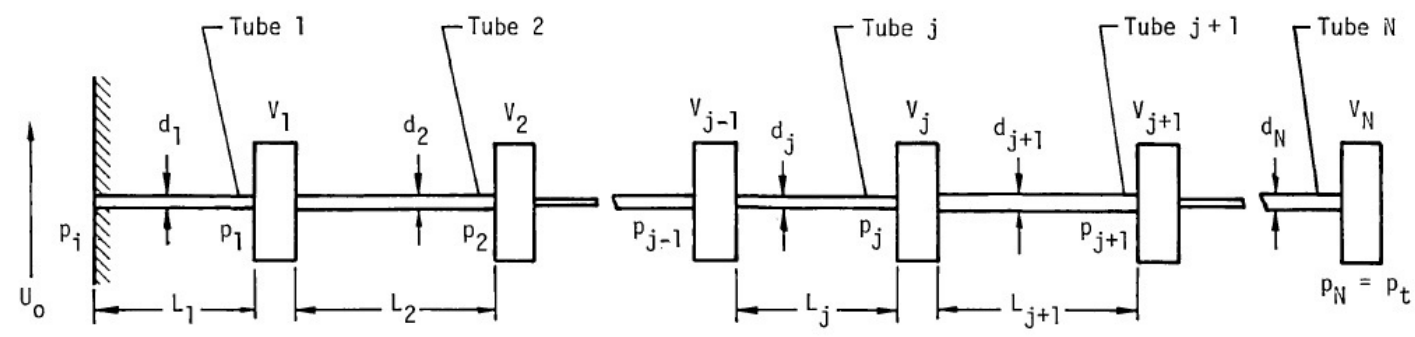

Figure 152. Generalized Pneumatic Tube-Transducer System (138)

Tijdeman (159) derived recursion formulas to calculate dynamic response of signal transmission lines with a sinusoidal input. The equation is long and involved and thus will not be explicitly detailed, only briefly summarized. The equations are derived from the Navier-Stokes and energy equations, along with the equation of state for an ideal gas. However, it expresses the complex ration $\left(P_{j} / P_{j-1}\right)$ in terms of $\left(P_{j+1} / P_{j}\right)$, volume $\left(V_{j}\right)$, geometry of tube $j$ and $j+1(I, I D)$, and the following environmental parameters: viscosity $\left(\mu_{\infty}\right)$, density $\left(\rho_{\infty}\right)$, average pressure $\left(P_{\text {avg }}\right)$, the square root of the Prandt number $(\operatorname{Pr})$, and the ratio of specific heat at constant pressure to that at constant volume (160). Considering the simplified system of the current work, involving an external tube and a pressure transducer with internal tube and volume, simplifying assumptions are made to model a three-tube system without any intermediate cavity volumes. For the ScaniValve ESP module, there is negligible cavity volume in front of the transducer, abridging the pertinent system to a 3-tube zero-volume configuration; see (160) for further detail of validation tests supporting the simplifying assumptions included in this appendix. 
The internal volume of the ScaniValve transducer is included in the schematic of Figure 153. Dimensions specified in the ScaniValve ZOC 33 Service Manual were used as inputs into the sinusoidalresponse model.

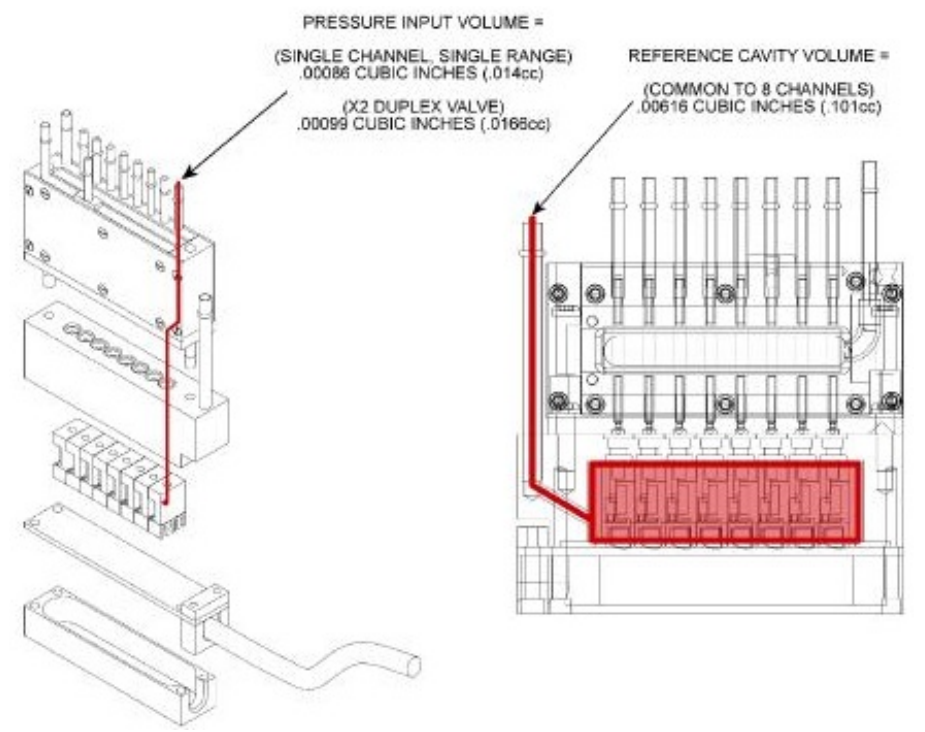

Figure 153. ScaniValve ZOC33 Pressure Transducer Internal Volume (161)

A series of assumptions results in a simplified edition of the aforementioned recursion formula (Equation 85).

$$
\frac{P_{j}}{P_{j-1}}=\left\{\cosh (\alpha+i \beta)_{j}+\frac{\left(d_{j}^{2}\right)+\left(1_{j}^{l}\right)+\left(1^{n}\right) \cdot(\alpha+i \beta)_{j} \cdot \sinh (\alpha+i \beta)_{j}}{\left(d_{j}^{2}\right)+\left(l_{j} \cdot n_{j+1}\right) \cdot(\alpha+i \beta)_{j+1} \cdot \sinh (\alpha+i \beta)_{j+1}} \cdot\left[\cosh (\alpha+i \beta)_{j+1}-\frac{P_{j+1}}{P_{j}}\right]\right\}^{-1}
$$

Equation 85

The model can be applied to a series of tubes of varying geometry in a piecewise manner. For example, to compute the net sinusoidal response $\left(P_{t} / P_{i}\right)$ of the system in Figure 152, the response of each section $\left(P_{j} / P_{j-1}\right)$ is first computed, starting with tube $N$ and working toward the source $\left(P_{i}\right)$. Other approaches to determine the dynamic frequency response of a variable-diameter tubing system is to first calculate an effective geometry $(I, I D)$ to represent the series as a single tube of constant geometry, based on weighted averaging techniques.

Equivalent Diameter (162)

$$
\begin{gathered}
d_{e q}=\left[\frac{\sum l_{i} \cdot\left(d_{i}\right)^{2}}{\sum l_{i}}\right]^{1 / 2} \\
d_{\text {eff }}=\left[\frac{\sum_{(i=1)}^{(n-1)} d_{i} \cdot l_{i, i+1}}{\sum_{(i=1)}^{n} l_{i, i+1}}\right]
\end{gathered}
$$

Equation 86

Equation 87

Applying Equation 86 and Equation 87 to the geometry of the test system of this study, results in values of $d_{e q}=0.068 \mathrm{in}$ and $d_{\text {eff }}=0.066 \mathrm{in}$. Input of either value into the frequency response model reveals negligible differences, as will be discussed in further detail. 
\% \% Frequency Response Model Pressure Transducer-Tube System \% \%

\% \% Transient Signal Attenuation through Cylindrical Tubes \% \%

$\% \%$ USER INPUTS (PRESSURE SYSTEM GEOMETRY) \% \%

$\% \%$ Give the Length (L) and Internal Diameter (D) of Tube [meters]

$\% \mathrm{~L}=79.491 \mathrm{in}=2.0191 \mathrm{~m}$

$\mathrm{L}=1.5$;

$\mathrm{D}=0.001425$;

$\%$ Give the Scanivalve Transducer Volume (Vt) [cubic meters]

Vsv $=4 \mathrm{E}-7$;

$\% \%$ Properties of Air at To [K] and Po [Pa]\% \%

$\mathrm{T} 0=294.28$;

$\%$ Pressure $[\mathrm{Pa}]$

$\mathrm{P} \odot=\odot .97866 \mathrm{e} 5$;

$\%$ Molar Mass $[\mathrm{kg} / \mathrm{mol}]$

$M=28.8 e-3$;

$\%$ Gas Constant [ J/(mol K $)]$

$\mathrm{R}=8.3143$;

$\%$ Density $\left[\mathrm{kg} / \mathrm{m}^{\wedge} 3\right]$

rho=1.185;

$\%$ rho=PO/ $\left(\mathrm{R}^{*} \mathrm{~T} \odot\right)$;

$\%$ Specific Heat $[\mathrm{J} /(\mathrm{kg} \mathrm{K})]$

$\mathrm{Cp}=1007$;

$\%$ Heat Conductivity Coefficient [W/(m K)]

lambda $=\odot .0261$;

$\%$ Dynamic Viscosity $[\mathrm{Pa} \mathrm{s}]$

$m u=1.85 e-5$;

$\%$ Specific Heat Ratio [-]

kappa=1.402;

$\%$ Velocity of Sound [m/s]

$\mathrm{C}=\operatorname{sqrt}\left(\mathrm{kappa}^{*} \mathrm{P} \odot / \mathrm{rho}\right)$;

$\%$ Internal Volume of TUBE [m^3]

\%Scanivalve INPUT Port

$\% \mathrm{Vp}=1.4093 \mathrm{e}-8$;

\%ScaniValve REF Port

$\% \mathrm{Vp}=1.0094 \mathrm{e}-7$

Vtube $=(\mathrm{pi} / 4) *\left(\mathrm{D}^{\wedge} 2{ }^{*} \mathrm{~L}\right)$;

$\%$ Polytropic Factor for Air in the Transducer Volume [-]

$\mathrm{k}=1.402$;

\% Dimensionless Increase in Transducer Volume [-]

sigma $=0$;

$\%$ Frequency vector $[\mathrm{Hz}]$

$\mathrm{f}=0$ : 200 ;

$\%$ Angular Frequency Vector $[\mathrm{rad} / \mathrm{s}] \%$

$w=f * 2 * p i$;

$\%$ Shear Wave \# [-] \%

alpha $=i^{*} \operatorname{sqrt}(i){ }^{*} \mathrm{D} / 2{ }^{*} \operatorname{sqrt}\left(\mathrm{rho}{ }^{*} \mathrm{~W} / \mathrm{mu}\right)$

$\%$ Prandtl \# [-]

$\mathrm{Pr}=\mathrm{mu}{ }^{*} \mathrm{Cp} / \mathrm{lambda}$

$\%$ \% BESSEL FUNCTIONS (POLYTROPIC FACTOR)

$[\mathrm{J} \odot \mathrm{a}$, acc1] $=$ besselj $(\odot$, alpha $)$;

[J2a, acc2] =besselj (2, alpha);

$[\mathrm{J} \odot \mathrm{p}$, acc 3$]=\operatorname{bessel} j(\odot$, alpha* $\operatorname{sqrt}(\operatorname{Pr}))$;

$[\mathrm{J} 2 \mathrm{p}$, acc 4$]=$ besselj $\left(2\right.$, alpha $\left.{ }^{*} \operatorname{sqrt}(\operatorname{Pr})\right)$;

$\mathrm{n}=\left(1+(\mathrm{kappa}-1) / \mathrm{kappa}^{*} \mathrm{~J} 2 \mathrm{p} . / \mathrm{J} \odot \mathrm{p}\right) \cdot \wedge(-1)$;

$\%$ Help Variable $[\mathrm{rad} / \mathrm{m}]$

phi=w./c. * $\operatorname{sqrt}(\mathrm{J} \odot \mathrm{a} . / \mathrm{J} 2 \mathrm{a}) \cdot{ }^{*} \operatorname{sqrt}(\mathrm{kappa} . / \mathrm{n})$;

\% Transfer Function [-] \% \% Frequency Response Function \% \%

$\mathrm{TF}=\left(\cosh \left(\right.\right.$ phi.$\left.{ }^{*} \mathrm{~L}\right)+\mathrm{Vsv} . /$ Vtube . $\left.{ }^{*}(\operatorname{sigma}+1 . / \mathrm{k}) .{ }^{*} \mathrm{n} .{ }^{*} \mathrm{phi} .{ }^{*} \mathrm{~L} .{ }^{*} \sinh \left(\operatorname{phi} .{ }^{*} \mathrm{~L}\right)\right) \cdot \wedge(-1) ;$

\% Magnitude [-] \% \% Amplitude Ratio \%

$M A G=a b s(T F)$;

MAG_dB $=20 * \log 10(\mathrm{Mag})$;

$\%$ PHASE SHIFT [rad]

PHASE_rad=unwrap (angle (TF)) ;

PHASE_deg=Phase_rad*57.29578;

$[\mathrm{b}, \mathrm{a}]=$ invfreqs $(\mathrm{TF}, \mathrm{W}, 2,3)$; 
The Bergh \& Tijdeman (138) (164) model results of the frequency response function for the CCNACA0018-pneumatic tubing-transducer system used in this study is included Figure 154. The geometric and environmental conditions are included as a title description in the figure.
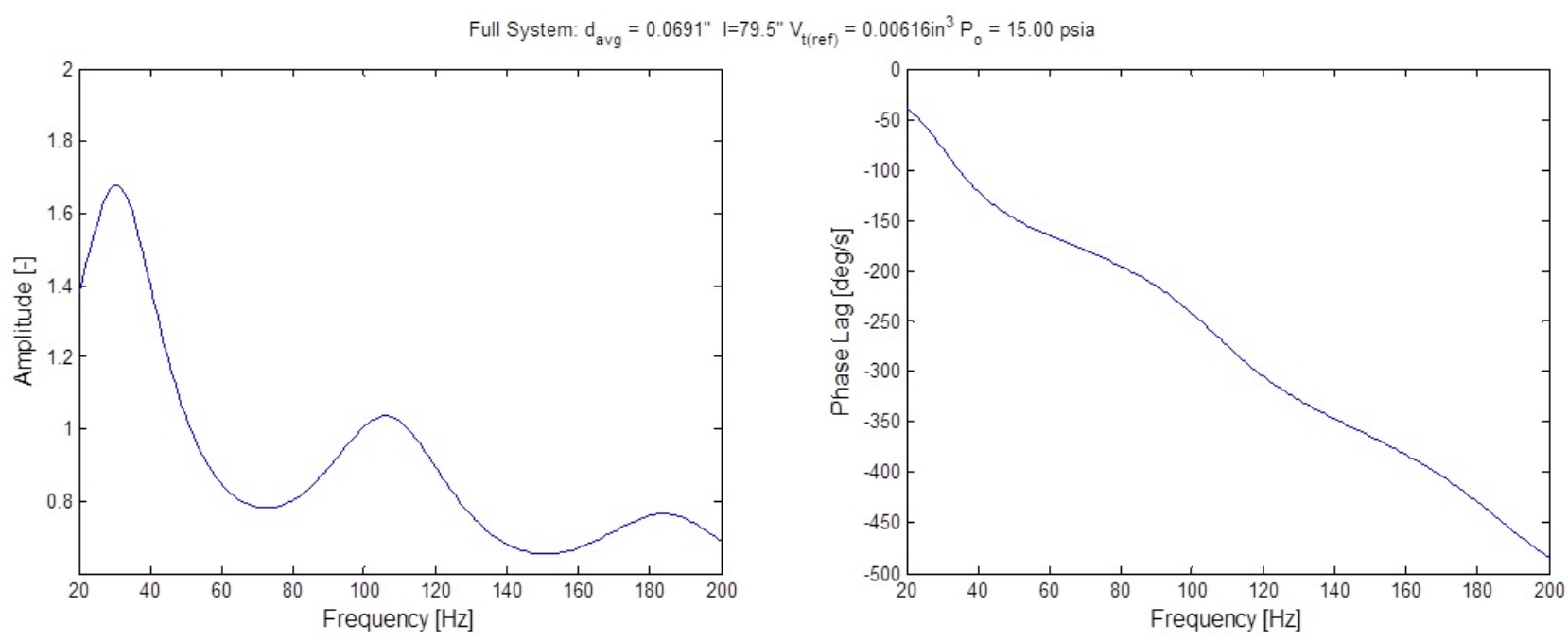

Figure 154. Analytical Frequency Response Model of CCNACA Pressure Port Tubing System

The LabVIEW program (TF Measurement.vi) used to monitor and measure the condenser microphone outputs during frequency response testing of the pressure measurement system are included in Figure 156 and Figure 157. Both figures show the preliminary calibration procedure completed before each set of mic measurements. During calibration, both mics were connected directly to the oscillating pressure source (speaker driven by function generator). The first plot, in the upper left hand corner of each .vi window was a real-time display of the sinusoidal input signal created by the function generator; the frequency, amplitude, and waveform could all be varied to create a range of oscillating signals. It should be noted that only sinusoidal inputs were used for frequency response testing, due to the fact that you can differentiate sine waves while maintaining frequency-fidelity. The block diagram of the DAQ program used for frequency response testing is included in Figure 155. 


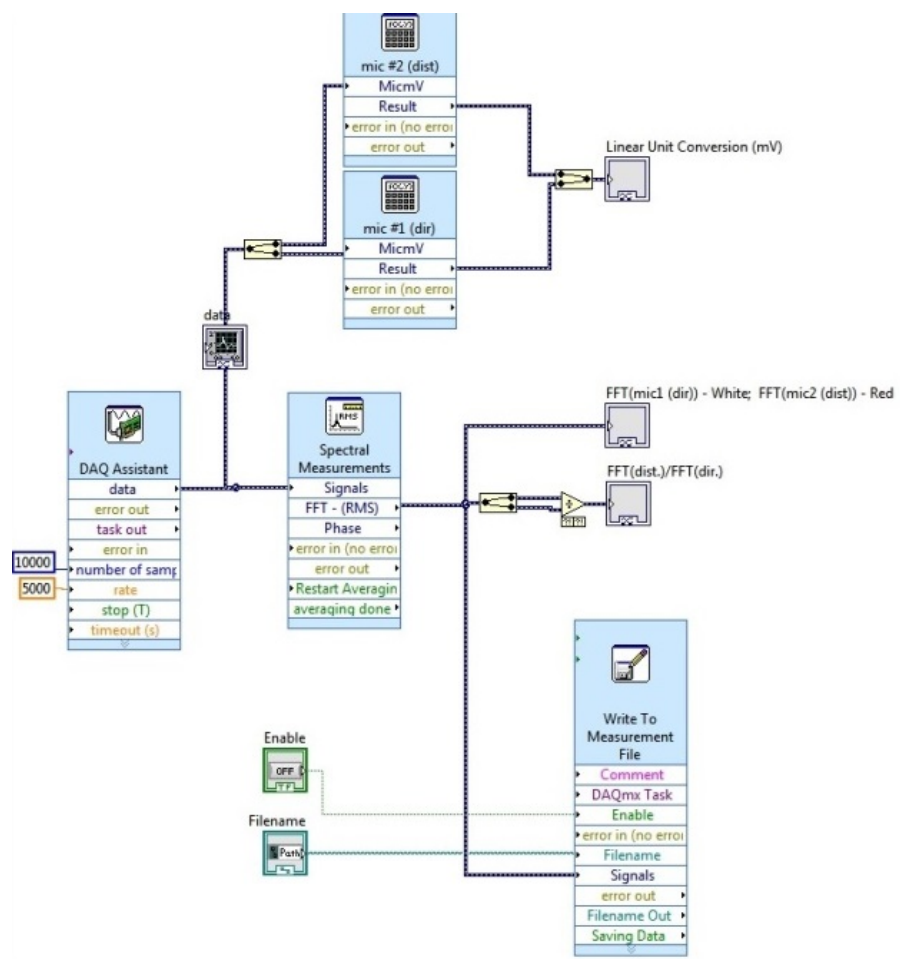

Figure 155. Pneumatic Tubing Transfer Function Measurement: 2 Condenser Mic Signal Analysis

Figure 156 shows the LabVIEW DAQ program measurements for an input signal of $f_{\text {sig }}=200 \mathrm{~Hz}$ The box labelled " $f(M 1)[\mathrm{Hz}]$ " represents the direct signal measurement of microphone 1 . The first "Amplitude" box calculates the ratio of RMS signal measurements from microphone 1 (M1) and microphone 2 (M2); the second "Amplitude" box shows the same calculation for peak-to-peak measurements for each mic signal. In this example, both mics are directly connected to the signal source, via a tee connection, and hence the amplitude reading of unity represents zero distortion between microphone signals (the phase lag of $4.22^{\circ}$ is considered negligible). A graphical Fourier analysis can be seen in the lower left of the LabVIEW GUI, plotting the FFT analysis of the 3 measured signals (source, mic 1, and mic2). The dominant peak at $200 \mathrm{~Hz}$ shows the agreement between measurements, while the secondary peak at $60 \mathrm{~Hz}$ reveals an influence of common noise in the wind tunnel lab. 


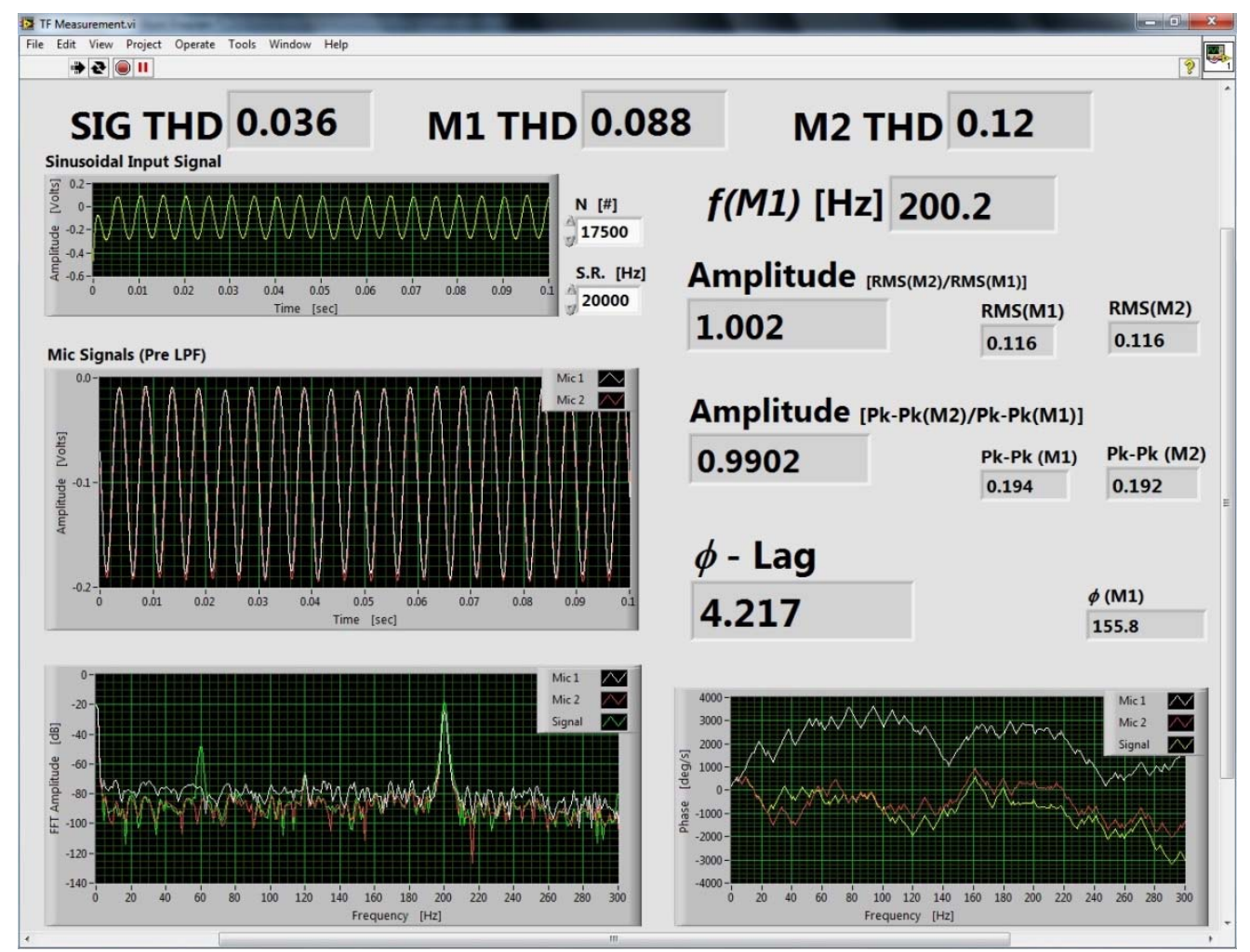

Figure 156. DAQ Program to Measure Frequency Response Tubing

Furthermore, the noise floor (Figure 157) of the mics during testing was found to be between $80-90 \mathrm{~dB}$ in WVU's Aero. Lab. This observation was made when all instrumentation involved in the testing was powered on; the function generator signal was set to $0 \mathrm{~Hz}$.

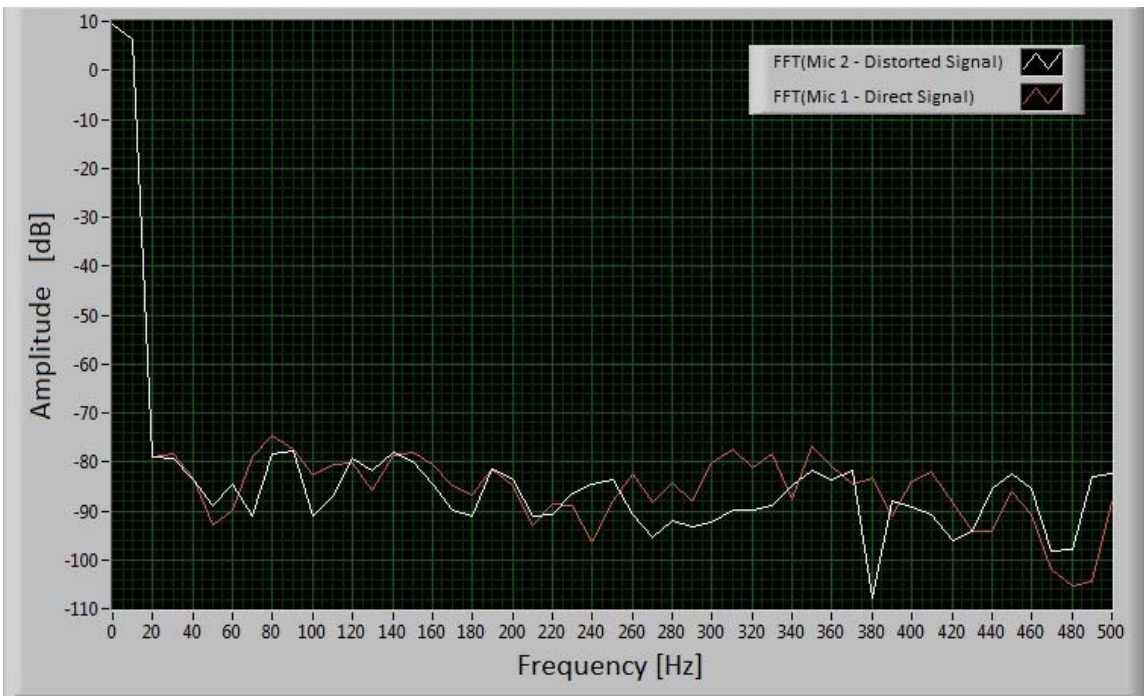

Figure 157. WVU Aero. Lab Noise Floor during Frequency Response Testing 
APPENDIX E: Static Wind Tunnel Test Matrices

Table 33. Static Wind Tunnel Test Matrix: $R e=180 \times 10^{3}$ (All $C_{\mu}$ Conditions)

\begin{tabular}{|c|c|c|c|c|c|c|c|c|c|c|c|c|c|}
\hline Actuation & $C_{\mu}[-]$ & St [ - ] & $\alpha\left[^{\circ}\right]$ & run \#1 & run \#2 & run \#3 & run \#4 & run \#5 & run \#6 & run \#7 & run \#8 & run \#9 & run \#10 \\
\hline \multirow{4}{*}{$\begin{array}{c}4 \\
0 \\
111 \\
v\end{array}$} & \multirow{4}{*}{$\begin{array}{l}0 \\
0 \\
11 \\
u^{7}\end{array}$} & \multirow{4}{*}{$\begin{array}{l}0 \\
0 \\
11 \\
\hbar\end{array}$} & $0^{\circ}$ & $\checkmark$ & $\checkmark$ & $\checkmark$ & $\checkmark$ & $\checkmark$ & $\checkmark$ & $\checkmark$ & $\checkmark$ & $\checkmark$ & $\checkmark$ \\
\hline & & & $5^{\circ}$ & $\checkmark$ & $\checkmark$ & $\checkmark$ & $\checkmark$ & $\checkmark$ & $x$ & $x$ & $x$ & $x$ & $x$ \\
\hline & & & $10^{\circ}$ & $\checkmark$ & $\checkmark$ & $\checkmark$ & $\checkmark$ & $\checkmark$ & $x$ & $x$ & $x$ & $x$ & $x$ \\
\hline & & & $15^{\circ}$ & $\checkmark$ & $\checkmark$ & $\checkmark$ & $\checkmark$ & $\checkmark$ & $\checkmark$ & $\checkmark$ & $\checkmark$ & $\checkmark$ & $\checkmark$ \\
\hline Actuation & $C_{\mu}[-]$ & St [ - ] & $\alpha\left[{ }^{\circ}\right]$ & run \#1 & run \#2 & run \#3 & run \#4 & run \#5 & run \#6 & run \#7 & run \#8 & run \#9 & run $\# 10$ \\
\hline \multirow{8}{*}{ 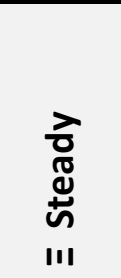 } & \multirow{4}{*}{$\begin{array}{l}5 \\
0 \\
11 \\
0 \\
v^{7}\end{array}$} & \multirow{4}{*}{$\begin{array}{l}0 \\
0 \\
11 \\
\hbar\end{array}$} & $0^{\circ}$ & $\checkmark$ & $\checkmark$ & $\checkmark$ & $\checkmark$ & $\checkmark$ & $\checkmark$ & $\checkmark$ & $\checkmark$ & $\checkmark$ & $\checkmark$ \\
\hline & & & $5^{\circ}$ & $\checkmark$ & $\checkmark$ & $\checkmark$ & $\checkmark$ & $\checkmark$ & $x$ & $x$ & $x$ & $x$ & $x$ \\
\hline & & & $10^{\circ}$ & $\checkmark$ & $\checkmark$ & $\checkmark$ & $\checkmark$ & $\checkmark$ & $x$ & $x$ & $x$ & $x$ & $x$ \\
\hline & & & $15^{\circ}$ & $\checkmark$ & $\checkmark$ & $\checkmark$ & $\checkmark$ & $\checkmark$ & $\checkmark$ & $\checkmark$ & $\checkmark$ & $\checkmark$ & $\checkmark$ \\
\hline & \multirow{4}{*}{$\begin{array}{l}n \\
0 \\
11 \\
v^{7}\end{array}$} & \multirow{4}{*}{$\begin{array}{l}0 \\
0 \\
11 \\
\hbar\end{array}$} & $0^{\circ}$ & $\checkmark$ & $\checkmark$ & $\checkmark$ & $\checkmark$ & $\checkmark$ & $\checkmark$ & $\checkmark$ & $\checkmark$ & $\checkmark$ & $\checkmark$ \\
\hline & & & $5^{\circ}$ & $\checkmark$ & $\checkmark$ & $\checkmark$ & $\checkmark$ & $\checkmark$ & $x$ & $x$ & $x$ & $x$ & $x$ \\
\hline & & & $10^{\circ}$ & $\checkmark$ & $\checkmark$ & $\checkmark$ & $\checkmark$ & $\checkmark$ & $x$ & $x$ & $x$ & $x$ & $x$ \\
\hline & & & $15^{\circ}$ & $\checkmark$ & $\checkmark$ & $\checkmark$ & $\checkmark$ & $\checkmark$ & $\checkmark$ & $\checkmark$ & $\checkmark$ & $\checkmark$ & $\checkmark$ \\
\hline \multirow{4}{*}{$v^{z}$} & \multirow{4}{*}{$\begin{array}{l}\text { 울 } \\
0 \\
11 \\
\text { c }^{7}\end{array}$} & \multirow{4}{*}{$\begin{array}{l}0 \\
0 \\
11 \\
\hbar\end{array}$} & $0^{\circ}$ & $\checkmark$ & $\checkmark$ & $\checkmark$ & $\checkmark$ & $\checkmark$ & $\checkmark$ & $\checkmark$ & $\checkmark$ & $\checkmark$ & $\checkmark$ \\
\hline & & & $5^{\circ}$ & $\checkmark$ & $\checkmark$ & $\checkmark$ & $\checkmark$ & $\checkmark$ & $x$ & $x$ & $x$ & $x$ & $x$ \\
\hline & & & $10^{\circ}$ & $\checkmark$ & $\checkmark$ & $\checkmark$ & $\checkmark$ & $\checkmark$ & $x$ & $x$ & $x$ & $x$ & $x$ \\
\hline & & & $15^{\circ}$ & $\checkmark$ & $\checkmark$ & $\checkmark$ & $\checkmark$ & $\checkmark$ & $\checkmark$ & $\checkmark$ & $\checkmark$ & $\checkmark$ & $\checkmark$ \\
\hline Actuation & $C_{\mu}[-]$ & St [ - ] & $\alpha\left[^{\circ}\right]$ & run \#1 & run \#2 & run \#3 & run \#4 & run \#5 & run \#6 & run \#7 & run \#8 & run \#9 & run $\# 10$ \\
\hline \multirow{36}{*}{$\begin{array}{l}\text { Dृ } \\
\frac{n}{5} \\
0 \\
111\end{array}$} & \multirow{12}{*}{$\begin{array}{l}\text { o' } \\
0 \\
11 \\
u^{2}\end{array}$} & \multirow{4}{*}{$\begin{array}{l}\text { L̊ } \\
\text { "I } \\
\hbar\end{array}$} & $0^{\circ}$ & $\checkmark$ & $\checkmark$ & $\checkmark$ & $\checkmark$ & $\checkmark$ & $\checkmark$ & $\checkmark$ & $\checkmark$ & $\checkmark$ & $\checkmark$ \\
\hline & & & $5^{\circ}$ & $\checkmark$ & $\checkmark$ & $\checkmark$ & $\checkmark$ & $\checkmark$ & $x$ & $x$ & $x$ & $x$ & $x$ \\
\hline & & & $10^{\circ}$ & $\checkmark$ & $\checkmark$ & $\checkmark$ & $\checkmark$ & $\checkmark$ & $x$ & $x$ & $x$ & $x$ & $x$ \\
\hline & & & $15^{\circ}$ & $\checkmark$ & $\checkmark$ & $\checkmark$ & $\checkmark$ & $\checkmark$ & $\checkmark$ & $\checkmark$ & $\checkmark$ & $\checkmark$ & $\checkmark$ \\
\hline & & \multirow{4}{*}{$\begin{array}{l}\stackrel{0}{i} \\
\stackrel{1}{11} \\
\hbar\end{array}$} & $0^{\circ}$ & $\checkmark$ & $\checkmark$ & $\checkmark$ & $\checkmark$ & $\checkmark$ & $\checkmark$ & $\checkmark$ & $\checkmark$ & $\checkmark$ & $\checkmark$ \\
\hline & & & $5^{\circ}$ & $\checkmark$ & $\checkmark$ & $\checkmark$ & $\checkmark$ & $\checkmark$ & $x$ & $x$ & $x$ & $x$ & $x$ \\
\hline & & & $10^{\circ}$ & $\checkmark$ & $\checkmark$ & $\checkmark$ & $\checkmark$ & $\checkmark$ & $x$ & $x$ & $x$ & $x$ & $x$ \\
\hline & & & $15^{\circ}$ & $\checkmark$ & $\checkmark$ & $\checkmark$ & $\checkmark$ & $\checkmark$ & $\checkmark$ & $\checkmark$ & $\checkmark$ & $\checkmark$ & $\checkmark$ \\
\hline & & & $0^{\circ}$ & $\checkmark$ & $\checkmark$ & $\checkmark$ & $\checkmark$ & $\checkmark$ & $\checkmark$ & $\checkmark$ & $\checkmark$ & $\checkmark$ & $\checkmark$ \\
\hline & & $\bar{i}$ & $5^{\circ}$ & $\checkmark$ & $\checkmark$ & $\checkmark$ & $\checkmark$ & $\checkmark$ & $x$ & $x$ & $x$ & $x$ & $x$ \\
\hline & & II & $10^{\circ}$ & $\checkmark$ & $\checkmark$ & $\checkmark$ & $\checkmark$ & $\checkmark$ & $x$ & $x$ & $x$ & $x$ & $x$ \\
\hline & & & $15^{\circ}$ & $\checkmark$ & $\checkmark$ & $\checkmark$ & $\checkmark$ & $\checkmark$ & $\checkmark$ & $\checkmark$ & $\checkmark$ & $\checkmark$ & $\checkmark$ \\
\hline & & & $0^{\circ}$ & $\checkmark$ & $\checkmark$ & $\checkmark$ & $\checkmark$ & $\checkmark$ & $\checkmark$ & $\checkmark$ & $\checkmark$ & $\checkmark$ & $\checkmark$ \\
\hline & & 0 & $5^{\circ}$ & $\checkmark$ & $\checkmark$ & $\checkmark$ & $\checkmark$ & $\checkmark$ & $x$ & $x$ & $x$ & $x$ & $x$ \\
\hline & & 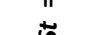 & $10^{\circ}$ & $\checkmark$ & $\checkmark$ & $\checkmark$ & $\checkmark$ & $\checkmark$ & $x$ & $x$ & $x$ & $x$ & $x$ \\
\hline & & & $15^{\circ}$ & $\checkmark$ & $\checkmark$ & $\checkmark$ & $\checkmark$ & $\checkmark$ & $\checkmark$ & $\checkmark$ & $\checkmark$ & $\checkmark$ & $\checkmark$ \\
\hline & & & $0^{\circ}$ & $\checkmark$ & $\checkmark$ & $\checkmark$ & $\checkmark$ & $\checkmark$ & $\checkmark$ & $\checkmark$ & $\checkmark$ & $\checkmark$ & $\checkmark$ \\
\hline & $0^{\circ}$ & $\stackrel{-}{-}$ & $5^{\circ}$ & $\checkmark$ & $\checkmark$ & $\checkmark$ & $\checkmark$ & $\checkmark$ & $x$ & $x$ & $x$ & $x$ & $x$ \\
\hline & II & 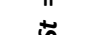 & $10^{\circ}$ & $\checkmark$ & $\checkmark$ & $\checkmark$ & $\checkmark$ & $\checkmark$ & $x$ & $x$ & $x$ & $x$ & $x$ \\
\hline & $u^{2}$ & & $15^{\circ}$ & $\checkmark$ & $\checkmark$ & $\checkmark$ & $\checkmark$ & $\checkmark$ & $\checkmark$ & $\checkmark$ & $\checkmark$ & $\checkmark$ & $\checkmark$ \\
\hline & & & $0^{\circ}$ & $\checkmark$ & $\checkmark$ & $\checkmark$ & $\checkmark$ & $\checkmark$ & $\checkmark$ & $\checkmark$ & $\checkmark$ & $\checkmark$ & $\checkmark$ \\
\hline & & $\dot{\sim}$ & $5^{\circ}$ & $\checkmark$ & $\checkmark$ & $\checkmark$ & $\checkmark$ & $\checkmark$ & $x$ & $x$ & $x$ & $x$ & $x$ \\
\hline & & $\ddot{n}$ & $10^{\circ}$ & $\checkmark$ & $\checkmark$ & $\checkmark$ & $\checkmark$ & $\checkmark$ & $x$ & $x$ & $x$ & $x$ & $x$ \\
\hline & & & $15^{\circ}$ & $\checkmark$ & $\checkmark$ & $\checkmark$ & $\checkmark$ & $\checkmark$ & $\checkmark$ & $\checkmark$ & $\checkmark$ & $\checkmark$ & $\checkmark$ \\
\hline & & & $0^{\circ}$ & $\bar{\checkmark}$ & $\bar{\checkmark}$ & $\bar{\checkmark}$ & $\checkmark$ & $\checkmark$ & $\checkmark$ & $\checkmark$ & $\bar{\checkmark}$ & $\bar{\checkmark}$ & $\checkmark$ \\
\hline & & 0 & $5^{\circ}$ & $\checkmark$ & $\checkmark$ & $\checkmark$ & $\checkmark$ & $\checkmark$ & $x$ & $x$ & $x$ & $x$ & $x$ \\
\hline & & 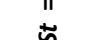 & $10^{\circ}$ & $\checkmark$ & $\checkmark$ & $\checkmark$ & $\checkmark$ & $\checkmark$ & $x$ & $x$ & $x$ & $x$ & $x$ \\
\hline & & & $15^{\circ}$ & $\checkmark$ & $\checkmark$ & $\checkmark$ & $\checkmark$ & $\checkmark$ & $\checkmark$ & $\checkmark$ & $\checkmark$ & $\checkmark$ & $\checkmark$ \\
\hline & 욱 & & $0^{\circ}$ & $\checkmark$ & $\checkmark$ & $\checkmark$ & $\checkmark$ & $\checkmark$ & $\checkmark$ & $\checkmark$ & $\checkmark$ & $\checkmark$ & $\checkmark$ \\
\hline & 0 & $-i$ & $5^{\circ}$ & $\checkmark$ & $\checkmark$ & $\checkmark$ & $\checkmark$ & $\checkmark$ & $x$ & $x$ & $x$ & $x$ & $x$ \\
\hline & II & 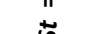 & $10^{\circ}$ & $\checkmark$ & $\checkmark$ & $\checkmark$ & $\checkmark$ & $\checkmark$ & $x$ & $x$ & $x$ & $x$ & $x$ \\
\hline & $v^{2}$ & & $15^{\circ}$ & $\checkmark$ & $\checkmark$ & $\checkmark$ & $\checkmark$ & $\checkmark$ & $\checkmark$ & $\checkmark$ & $\checkmark$ & $\checkmark$ & $\checkmark$ \\
\hline & & & $0^{\circ}$ & $\checkmark$ & $\checkmark$ & $\checkmark$ & $\checkmark$ & $\checkmark$ & $\checkmark$ & $\checkmark$ & $\checkmark$ & $\checkmark$ & $\checkmark$ \\
\hline & & $\dot{\mathrm{i}}$ & $5^{\circ}$ & $\checkmark$ & $\checkmark$ & $\checkmark$ & $\checkmark$ & $\checkmark$ & $x$ & $x$ & $x$ & $x$ & $x$ \\
\hline & & 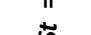 & $10^{\circ}$ & $\checkmark$ & $\checkmark$ & $\checkmark$ & $\checkmark$ & $\checkmark$ & $x$ & $x$ & $x$ & $x$ & $x$ \\
\hline & & & $15^{\circ}$ & $\checkmark$ & $\checkmark$ & $\checkmark$ & $\checkmark$ & $\checkmark$ & $\checkmark$ & $\checkmark$ & $\checkmark$ & $\checkmark$ & $\checkmark$ \\
\hline
\end{tabular}


Table 34. Static Wind Tunnel Test Matrix: $R e=300 \times 10^{3}$ (Baseline, $C_{\mu}=$ Steady Cases)

\begin{tabular}{|c|c|c|c|c|c|c|c|c|c|c|c|c|c|}
\hline Actuation & $C_{\mu}[-]$ & St [ - ] & $\alpha\left[^{\circ}\right]$ & run \#1 & run \#2 & run \#3 & run \#4 & run \#5 & run \#6 & run \#7 & run \#8 & run \#9 & run \#10 \\
\hline \multirow{9}{*}{$\begin{array}{c}4 \\
0 \\
111\end{array}$} & \multirow{9}{*}{$\begin{array}{l}0 \\
0 \\
11 \\
ن\end{array}$} & \multirow{9}{*}{ 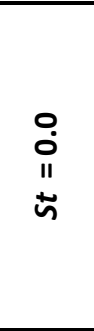 } & $0^{\circ}$ & $\checkmark$ & $\checkmark$ & $\checkmark$ & $\checkmark$ & $\checkmark$ & $x$ & $x$ & $x$ & $x$ & $x$ \\
\hline & & & $5^{\circ}$ & $\checkmark$ & $\checkmark$ & $\checkmark$ & $\checkmark$ & $\checkmark$ & $x$ & $x$ & $x$ & $x$ & $x$ \\
\hline & & & $10^{\circ}$ & $\checkmark$ & $\checkmark$ & $\checkmark$ & $\checkmark$ & $\checkmark$ & $x$ & $x$ & $x$ & $x$ & $x$ \\
\hline & & & $15^{\circ}$ & $\checkmark$ & $\checkmark$ & $\checkmark$ & $\checkmark$ & $\checkmark$ & $x$ & $x$ & $x$ & $x$ & $x$ \\
\hline & & & $16^{\circ}$ & $\checkmark$ & $\checkmark$ & $\checkmark$ & $\checkmark$ & $\checkmark$ & $\checkmark$ & $\checkmark$ & $\checkmark$ & $\checkmark$ & $\checkmark$ \\
\hline & & & $17^{\circ}$ & $\checkmark$ & $\checkmark$ & $\checkmark$ & $\checkmark$ & $\checkmark$ & $\checkmark$ & $\checkmark$ & $\checkmark$ & $\checkmark$ & $\checkmark$ \\
\hline & & & $18^{\circ}$ & $\checkmark$ & $\checkmark$ & $\checkmark$ & $\checkmark$ & $\checkmark$ & $\checkmark$ & $\checkmark$ & $\checkmark$ & $\checkmark$ & $\checkmark$ \\
\hline & & & $19^{\circ}$ & $\checkmark$ & $\checkmark$ & $\checkmark$ & $\checkmark$ & $\checkmark$ & $\checkmark$ & $\checkmark$ & $\checkmark$ & $\checkmark$ & $\checkmark$ \\
\hline & & & $20^{\circ}$ & $\checkmark$ & $\checkmark$ & $\checkmark$ & $\checkmark$ & $\checkmark$ & $\checkmark$ & $\checkmark$ & $\checkmark$ & $\checkmark$ & $\checkmark$ \\
\hline \multirow{27}{*}{ 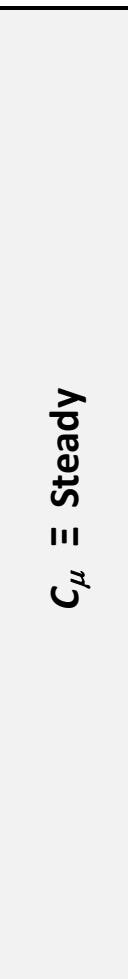 } & \multirow{9}{*}{$\begin{array}{l}-1 \\
0 \\
0 \\
11 \\
u^{2}\end{array}$} & \multirow{9}{*}{$\begin{array}{l}0 \\
0 \\
11 \\
\ddot{n}\end{array}$} & $0^{\circ}$ & $\checkmark$ & $\checkmark$ & $\checkmark$ & $\checkmark$ & $\checkmark$ & $x$ & $x$ & $x$ & $x$ & $x$ \\
\hline & & & $5^{\circ}$ & $\checkmark$ & $\checkmark$ & $\checkmark$ & $\checkmark$ & $\checkmark$ & $x$ & $x$ & $x$ & $x$ & $x$ \\
\hline & & & $10^{\circ}$ & $\checkmark$ & $\checkmark$ & $\checkmark$ & $\checkmark$ & $\checkmark$ & $x$ & $x$ & $x$ & $x$ & $x$ \\
\hline & & & $15^{\circ}$ & $\checkmark$ & $\checkmark$ & $\checkmark$ & $\checkmark$ & $\checkmark$ & $x$ & $x$ & $x$ & $x$ & $x$ \\
\hline & & & $16^{\circ}$ & $\checkmark$ & $\checkmark$ & $\checkmark$ & $\checkmark$ & $\checkmark$ & $\checkmark$ & $\checkmark$ & $\checkmark$ & $\checkmark$ & $\checkmark$ \\
\hline & & & $17^{\circ}$ & $\checkmark$ & $\checkmark$ & $\checkmark$ & $\checkmark$ & $\checkmark$ & $\checkmark$ & $\checkmark$ & $\checkmark$ & $\checkmark$ & $\checkmark$ \\
\hline & & & $18^{\circ}$ & $\checkmark$ & $\checkmark$ & $\checkmark$ & $\checkmark$ & $\checkmark$ & $\checkmark$ & $\checkmark$ & $\checkmark$ & $\checkmark$ & $\checkmark$ \\
\hline & & & $19^{\circ}$ & $\checkmark$ & $\checkmark$ & $\checkmark$ & $\checkmark$ & $\checkmark$ & $\checkmark$ & $\checkmark$ & $\checkmark$ & $\checkmark$ & $\checkmark$ \\
\hline & & & $20^{\circ}$ & $\checkmark$ & $\checkmark$ & $\checkmark$ & $\checkmark$ & $\checkmark$ & $\checkmark$ & $\checkmark$ & $\checkmark$ & $\checkmark$ & $\checkmark$ \\
\hline & \multirow{9}{*}{$\begin{array}{l}n \\
0 \\
0 \\
11 \\
ن\end{array}$} & \multirow{9}{*}{ 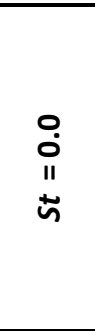 } & $0^{\circ}$ & $\checkmark$ & $\checkmark$ & $\checkmark$ & $\checkmark$ & $\checkmark$ & $x$ & $x$ & $x$ & $x$ & $x$ \\
\hline & & & $5^{\circ}$ & $\checkmark$ & $\checkmark$ & $\checkmark$ & $\checkmark$ & $\checkmark$ & $x$ & $x$ & $x$ & $x$ & $x$ \\
\hline & & & $10^{\circ}$ & $\checkmark$ & $\checkmark$ & $\checkmark$ & $\checkmark$ & $\checkmark$ & $x$ & $x$ & $x$ & $x$ & $x$ \\
\hline & & & $15^{\circ}$ & $\checkmark$ & $\checkmark$ & $\checkmark$ & $\checkmark$ & $\checkmark$ & $x$ & $x$ & $x$ & $x$ & $x$ \\
\hline & & & $16^{\circ}$ & $\checkmark$ & $\checkmark$ & $\checkmark$ & $\checkmark$ & $\checkmark$ & $\checkmark$ & $\checkmark$ & $\checkmark$ & $\checkmark$ & $\checkmark$ \\
\hline & & & $17^{\circ}$ & $\checkmark$ & $\checkmark$ & $\checkmark$ & $\checkmark$ & $\checkmark$ & $\checkmark$ & $\checkmark$ & $\checkmark$ & $\checkmark$ & $\checkmark$ \\
\hline & & & $18^{\circ}$ & $\checkmark$ & $\checkmark$ & $\checkmark$ & $\checkmark$ & $\checkmark$ & $\checkmark$ & $\checkmark$ & $\checkmark$ & $\checkmark$ & $\checkmark$ \\
\hline & & & $19^{\circ}$ & $\checkmark$ & $\checkmark$ & $\checkmark$ & $\checkmark$ & $\checkmark$ & $\checkmark$ & $\checkmark$ & $\checkmark$ & $\checkmark$ & $\checkmark$ \\
\hline & & & $20^{\circ}$ & $\checkmark$ & $\checkmark$ & $\checkmark$ & $\checkmark$ & $\checkmark$ & $\checkmark$ & $\checkmark$ & $\checkmark$ & $\checkmark$ & $\checkmark$ \\
\hline & \multirow{9}{*}{$\begin{array}{l}0 \\
\underset{1}{0} \\
0 \\
11 \\
ن\end{array}$} & \multirow{9}{*}{$\begin{array}{l}0 \\
0 \\
11 \\
艹\end{array}$} & $0^{\circ}$ & $\checkmark$ & $\checkmark$ & $\checkmark$ & $\checkmark$ & $\checkmark$ & $x$ & $x$ & $x$ & $x$ & $x$ \\
\hline & & & $5^{\circ}$ & $\checkmark$ & $\checkmark$ & $\checkmark$ & $\checkmark$ & $\checkmark$ & $x$ & $x$ & $x$ & $x$ & $x$ \\
\hline & & & $10^{\circ}$ & $\checkmark$ & $\checkmark$ & $\checkmark$ & $\checkmark$ & $\checkmark$ & $x$ & $x$ & $x$ & $x$ & $x$ \\
\hline & & & $15^{\circ}$ & $\checkmark$ & $\checkmark$ & $\checkmark$ & $\checkmark$ & $\checkmark$ & $x$ & $x$ & $x$ & $x$ & $x$ \\
\hline & & & $16^{\circ}$ & $\checkmark$ & $\checkmark$ & $\checkmark$ & $\checkmark$ & $\checkmark$ & $\checkmark$ & $\checkmark$ & $\checkmark$ & $\checkmark$ & $\checkmark$ \\
\hline & & & $17^{\circ}$ & $\checkmark$ & $\checkmark$ & $\checkmark$ & $\checkmark$ & $\checkmark$ & $\checkmark$ & $\checkmark$ & $\checkmark$ & $\checkmark$ & $\checkmark$ \\
\hline & & & $18^{\circ}$ & $\checkmark$ & $\checkmark$ & $\checkmark$ & $\checkmark$ & $\checkmark$ & $\checkmark$ & $\checkmark$ & $\checkmark$ & $\checkmark$ & $\checkmark$ \\
\hline & & & $19^{\circ}$ & $\checkmark$ & $\checkmark$ & $\checkmark$ & $\checkmark$ & $\checkmark$ & $\checkmark$ & $\checkmark$ & $\checkmark$ & $\checkmark$ & $\checkmark$ \\
\hline & & & $20^{\circ}$ & $\checkmark$ & $\checkmark$ & $\checkmark$ & $\checkmark$ & $\checkmark$ & $\checkmark$ & $\checkmark$ & $\checkmark$ & $\checkmark$ & $\checkmark$ \\
\hline
\end{tabular}


Table 35. Static Wind Tunnel Test Matrix: $R e=300 \times 10^{3}$ (Pulsed $C_{\mu}=0.01,0.05$ Cases)

\begin{tabular}{|c|c|c|c|c|c|c|c|c|c|c|c|c|c|}
\hline Actuation & $C_{\mu}[-]$ & St [ - ] & $\alpha\left[^{\circ}\right]$ & run \#1 & run \#2 & run \#3 & run \#4 & run \#5 & run \#6 & run \#7 & run \#8 & run \#9 & run \#10 \\
\hline \multirow{54}{*}{ 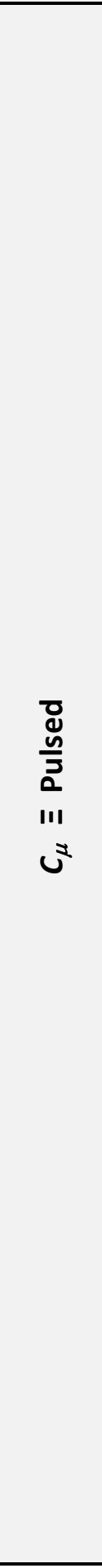 } & \multirow{27}{*}{$\begin{array}{c}\sigma_{0}^{\prime} \\
0 \\
11 \\
\dot{u}^{2}\end{array}$} & \multirow{9}{*}{ 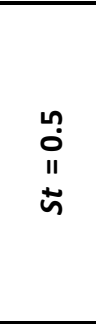 } & $0^{\circ}$ & $\checkmark$ & $\checkmark$ & $\checkmark$ & $\checkmark$ & $\checkmark$ & $x$ & $x$ & $x$ & $x$ & $x$ \\
\hline & & & $5^{\circ}$ & $\checkmark$ & $\checkmark$ & $\checkmark$ & $\checkmark$ & $\checkmark$ & $x$ & $x$ & $x$ & $x$ & $x$ \\
\hline & & & $10^{\circ}$ & $\checkmark$ & $\checkmark$ & $\checkmark$ & $\checkmark$ & $\checkmark$ & $x$ & $x$ & $x$ & $x$ & $x$ \\
\hline & & & $15^{\circ}$ & $\checkmark$ & $\checkmark$ & $\checkmark$ & $\checkmark$ & $\checkmark$ & $x$ & $x$ & $x$ & $x$ & $x$ \\
\hline & & & $16^{\circ}$ & $\checkmark$ & $\checkmark$ & $\checkmark$ & $\checkmark$ & $\checkmark$ & $\checkmark$ & $\checkmark$ & $\checkmark$ & $\checkmark$ & $\checkmark$ \\
\hline & & & $17^{\circ}$ & $\checkmark$ & $\checkmark$ & $\checkmark$ & $\checkmark$ & $\checkmark$ & $\checkmark$ & $\checkmark$ & $\checkmark$ & $\checkmark$ & $\checkmark$ \\
\hline & & & $18^{\circ}$ & $\checkmark$ & $\checkmark$ & $\checkmark$ & $\checkmark$ & $\checkmark$ & $\checkmark$ & $\checkmark$ & $\checkmark$ & $\checkmark$ & $\checkmark$ \\
\hline & & & $19^{\circ}$ & $\checkmark$ & $\checkmark$ & $\checkmark$ & $\checkmark$ & $\checkmark$ & $\checkmark$ & $\checkmark$ & $\checkmark$ & $\checkmark$ & $\checkmark$ \\
\hline & & & $20^{\circ}$ & $\checkmark$ & $\checkmark$ & $\checkmark$ & $\checkmark$ & $\checkmark$ & $\checkmark$ & $\checkmark$ & $\checkmark$ & $\checkmark$ & $\checkmark$ \\
\hline & & \multirow{9}{*}{$\begin{array}{l}0 \\
\dot{-1} \\
\text { II } \\
\ddot{n}\end{array}$} & $0^{\circ}$ & $\checkmark$ & $\checkmark$ & $\checkmark$ & $\checkmark$ & $\checkmark$ & $x$ & $x$ & $x$ & $x$ & $x$ \\
\hline & & & $5^{\circ}$ & $\checkmark$ & $\checkmark$ & $\checkmark$ & $\checkmark$ & $\checkmark$ & $x$ & $x$ & $x$ & $x$ & $x$ \\
\hline & & & $10^{\circ}$ & $\checkmark$ & $\checkmark$ & $\checkmark$ & $\checkmark$ & $\checkmark$ & $x$ & $x$ & $x$ & $x$ & $x$ \\
\hline & & & $15^{\circ}$ & $\checkmark$ & $\checkmark$ & $\checkmark$ & $\checkmark$ & $\checkmark$ & $x$ & $x$ & $x$ & $x$ & $x$ \\
\hline & & & $16^{\circ}$ & $\checkmark$ & $\checkmark$ & $\checkmark$ & $\checkmark$ & $\checkmark$ & $\checkmark$ & $\checkmark$ & $\checkmark$ & $\checkmark$ & $\checkmark$ \\
\hline & & & $17^{\circ}$ & $\checkmark$ & $\checkmark$ & $\checkmark$ & $\checkmark$ & $\checkmark$ & $\checkmark$ & $\checkmark$ & $\checkmark$ & $\checkmark$ & $\checkmark$ \\
\hline & & & $18^{\circ}$ & $\checkmark$ & $\checkmark$ & $\checkmark$ & $\checkmark$ & $\checkmark$ & $\checkmark$ & $\checkmark$ & $\checkmark$ & $\checkmark$ & $\checkmark$ \\
\hline & & & $19^{\circ}$ & $\checkmark$ & $\checkmark$ & $\checkmark$ & $\checkmark$ & $\checkmark$ & $\checkmark$ & $\checkmark$ & $\checkmark$ & $\checkmark$ & $\checkmark$ \\
\hline & & & $20^{\circ}$ & $\checkmark$ & $\checkmark$ & $\checkmark$ & $\checkmark$ & $\checkmark$ & $\checkmark$ & $\checkmark$ & $\checkmark$ & $\checkmark$ & $\checkmark$ \\
\hline & & \multirow{9}{*}{$\begin{array}{l}0 \\
\sim \\
I 1 \\
\ddot{n}\end{array}$} & $0^{\circ}$ & $\checkmark$ & $\checkmark$ & $\checkmark$ & $\checkmark$ & $\checkmark$ & $x$ & $x$ & $x$ & $x$ & $x$ \\
\hline & & & $5^{\circ}$ & $\checkmark$ & $\checkmark$ & $\checkmark$ & $\checkmark$ & $\checkmark$ & $x$ & $x$ & $x$ & $x$ & $x$ \\
\hline & & & $10^{\circ}$ & $\checkmark$ & $\checkmark$ & $\checkmark$ & $\checkmark$ & $\checkmark$ & $x$ & $x$ & $x$ & $x$ & $x$ \\
\hline & & & $15^{\circ}$ & $\checkmark$ & $\checkmark$ & $\checkmark$ & $\checkmark$ & $\checkmark$ & $x$ & $x$ & $x$ & $x$ & $x$ \\
\hline & & & $16^{\circ}$ & $\checkmark$ & $\checkmark$ & $\checkmark$ & $\checkmark$ & $\checkmark$ & $\checkmark$ & $\checkmark$ & $\checkmark$ & $\checkmark$ & $\checkmark$ \\
\hline & & & $17^{\circ}$ & $\checkmark$ & $\checkmark$ & $\checkmark$ & $\checkmark$ & $\checkmark$ & $\checkmark$ & $\checkmark$ & $\checkmark$ & $\checkmark$ & $\checkmark$ \\
\hline & & & $18^{\circ}$ & $\checkmark$ & $\checkmark$ & $\checkmark$ & $\checkmark$ & $\checkmark$ & $\checkmark$ & $\checkmark$ & $\checkmark$ & $\checkmark$ & $\checkmark$ \\
\hline & & & $19^{\circ}$ & $\checkmark$ & $\checkmark$ & $\checkmark$ & $\checkmark$ & $\checkmark$ & $\checkmark$ & $\checkmark$ & $\checkmark$ & $\checkmark$ & $\checkmark$ \\
\hline & & & $20^{\circ}$ & $\checkmark$ & $\checkmark$ & $\checkmark$ & $\checkmark$ & $\checkmark$ & $\checkmark$ & $\checkmark$ & $\checkmark$ & $\checkmark$ & $\checkmark$ \\
\hline & \multirow{27}{*}{ 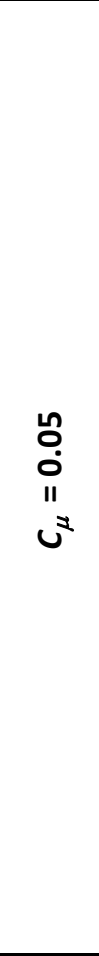 } & \multirow{9}{*}{$\begin{array}{l}\tilde{0} \\
0 \\
11 \\
\ddot{n}\end{array}$} & $0^{\circ}$ & $\checkmark$ & $\checkmark$ & $\checkmark$ & $\checkmark$ & $\checkmark$ & $x$ & $x$ & $x$ & $x$ & $x$ \\
\hline & & & $5^{\circ}$ & $\checkmark$ & $\checkmark$ & $\checkmark$ & $\checkmark$ & $\checkmark$ & $x$ & $x$ & $x$ & $x$ & $x$ \\
\hline & & & $10^{\circ}$ & $\checkmark$ & $\checkmark$ & $\checkmark$ & $\checkmark$ & $\checkmark$ & $x$ & $x$ & $x$ & $x$ & $x$ \\
\hline & & & $15^{\circ}$ & $\checkmark$ & $\checkmark$ & $\checkmark$ & $\checkmark$ & $\checkmark$ & $x$ & $x$ & $x$ & $x$ & $x$ \\
\hline & & & $16^{\circ}$ & $\checkmark$ & $\checkmark$ & $\checkmark$ & $\checkmark$ & $\checkmark$ & $\checkmark$ & $\checkmark$ & $\checkmark$ & $\checkmark$ & $\checkmark$ \\
\hline & & & $17^{\circ}$ & $\checkmark$ & $\checkmark$ & $\checkmark$ & $\checkmark$ & $\checkmark$ & $\checkmark$ & $\checkmark$ & $\checkmark$ & $\checkmark$ & $\checkmark$ \\
\hline & & & $18^{\circ}$ & $\checkmark$ & $\checkmark$ & $\checkmark$ & $\checkmark$ & $\checkmark$ & $\checkmark$ & $\checkmark$ & $\checkmark$ & $\checkmark$ & $\checkmark$ \\
\hline & & & $19^{\circ}$ & $\checkmark$ & $\checkmark$ & $\checkmark$ & $\checkmark$ & $\checkmark$ & $\checkmark$ & $\checkmark$ & $\checkmark$ & $\checkmark$ & $\checkmark$ \\
\hline & & & $20^{\circ}$ & $\checkmark$ & $\checkmark$ & $\checkmark$ & $\checkmark$ & $\checkmark$ & $\checkmark$ & $\checkmark$ & $\checkmark$ & $\checkmark$ & $\checkmark$ \\
\hline & & \multirow{9}{*}{$\begin{array}{l}0 \\
\dot{j} \\
\text { II } \\
\ddot{n}\end{array}$} & $0^{\circ}$ & $\checkmark$ & $\checkmark$ & $\checkmark$ & $\checkmark$ & $\checkmark$ & $x$ & $x$ & $x$ & $x$ & $x$ \\
\hline & & & $5^{\circ}$ & $\checkmark$ & $\checkmark$ & $\checkmark$ & $\checkmark$ & $\checkmark$ & $x$ & $x$ & $x$ & $x$ & $x$ \\
\hline & & & $10^{\circ}$ & $\checkmark$ & $\checkmark$ & $\checkmark$ & $\checkmark$ & $\checkmark$ & $x$ & $x$ & $x$ & $x$ & $x$ \\
\hline & & & $15^{\circ}$ & $\checkmark$ & $\checkmark$ & $\checkmark$ & $\checkmark$ & $\checkmark$ & $x$ & $x$ & $x$ & $x$ & $x$ \\
\hline & & & $16^{\circ}$ & $\checkmark$ & $\checkmark$ & $\checkmark$ & $\checkmark$ & $\checkmark$ & $\checkmark$ & $\checkmark$ & $\checkmark$ & $\checkmark$ & $\checkmark$ \\
\hline & & & $17^{\circ}$ & $\checkmark$ & $\checkmark$ & $\checkmark$ & $\checkmark$ & $\checkmark$ & $\checkmark$ & $\checkmark$ & $\checkmark$ & $\checkmark$ & $\checkmark$ \\
\hline & & & $18^{\circ}$ & $\checkmark$ & $\checkmark$ & $\checkmark$ & $\checkmark$ & $\checkmark$ & $\checkmark$ & $\checkmark$ & $\checkmark$ & $\checkmark$ & $\checkmark$ \\
\hline & & & $19^{\circ}$ & $\checkmark$ & $\checkmark$ & $\checkmark$ & $\checkmark$ & $\checkmark$ & $\checkmark$ & $\checkmark$ & $\checkmark$ & $\checkmark$ & $\checkmark$ \\
\hline & & & $20^{\circ}$ & $\checkmark$ & $\checkmark$ & $\checkmark$ & $\checkmark$ & $\checkmark$ & $\checkmark$ & $\checkmark$ & $\checkmark$ & $\checkmark$ & $\checkmark$ \\
\hline & & \multirow{9}{*}{$\begin{array}{l}0 \\
\dot{N} \\
I I \\
\ddot{n}\end{array}$} & $0^{\circ}$ & $\checkmark$ & $\checkmark$ & $\checkmark$ & $\checkmark$ & $\checkmark$ & $x$ & $x$ & $x$ & $x$ & $x$ \\
\hline & & & $5^{\circ}$ & $\checkmark$ & $\checkmark$ & $\checkmark$ & $\checkmark$ & $\checkmark$ & $x$ & $x$ & $x$ & $x$ & $x$ \\
\hline & & & $10^{\circ}$ & $\checkmark$ & $\checkmark$ & $\checkmark$ & $\checkmark$ & $\checkmark$ & $x$ & $x$ & $x$ & $x$ & $x$ \\
\hline & & & $15^{\circ}$ & $\checkmark$ & $\checkmark$ & $\checkmark$ & $\checkmark$ & $\checkmark$ & $x$ & $x$ & $x$ & $x$ & $x$ \\
\hline & & & $16^{\circ}$ & $\checkmark$ & $\checkmark$ & $\checkmark$ & $\checkmark$ & $\checkmark$ & $\checkmark$ & $\checkmark$ & $\checkmark$ & $\checkmark$ & $\checkmark$ \\
\hline & & & $17^{\circ}$ & $\checkmark$ & $\checkmark$ & $\checkmark$ & $\checkmark$ & $\checkmark$ & $\checkmark$ & $\checkmark$ & $\checkmark$ & $\checkmark$ & $\checkmark$ \\
\hline & & & $18^{\circ}$ & $\checkmark$ & $\checkmark$ & $\checkmark$ & $\checkmark$ & $\checkmark$ & $\checkmark$ & $\checkmark$ & $\checkmark$ & $\checkmark$ & $\checkmark$ \\
\hline & & & $19^{\circ}$ & $\checkmark$ & $\checkmark$ & $\checkmark$ & $\checkmark$ & $\checkmark$ & $\checkmark$ & $\checkmark$ & $\checkmark$ & $\checkmark$ & $\checkmark$ \\
\hline & & & $20^{\circ}$ & $\checkmark$ & $\checkmark$ & $\checkmark$ & $\checkmark$ & $\checkmark$ & $\checkmark$ & $\checkmark$ & $\checkmark$ & $\checkmark$ & $\checkmark$ \\
\hline
\end{tabular}


Table 36. Static Wind Tunnel Test Matrix: $R e=300 \times 10^{3}$ (Pulsed $C_{\mu}=0.10$ Pulsed Cases)

\begin{tabular}{|c|c|c|c|c|c|c|c|c|c|c|c|c|c|}
\hline Actuation & $C_{\mu}[-]$ & St [ - ] & $\alpha\left[^{\circ}\right]$ & run \#1 & run \#2 & run \#3 & run \#4 & run \#5 & run \#6 & run \#7 & run \#8 & run \#9 & run \#10 \\
\hline \multirow{27}{*}{ 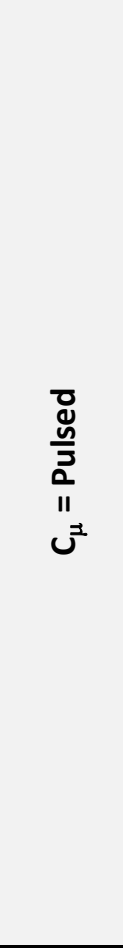 } & \multirow{27}{*}{$\begin{array}{l}\text { 울 } \\
0 \\
11 \\
v^{2}\end{array}$} & \multirow{9}{*}{$\begin{array}{l}n \\
0 \\
11 \\
\ddot{n}\end{array}$} & $0^{\circ}$ & $\checkmark$ & $\checkmark$ & $\checkmark$ & $\checkmark$ & $\checkmark$ & $x$ & $x$ & $x$ & $x$ & $x$ \\
\hline & & & $5^{\circ}$ & $\checkmark$ & $\checkmark$ & $\checkmark$ & $\checkmark$ & $\checkmark$ & $x$ & $x$ & $x$ & $x$ & $x$ \\
\hline & & & $10^{\circ}$ & $\checkmark$ & $\checkmark$ & $\checkmark$ & $\checkmark$ & $\checkmark$ & $x$ & $x$ & $x$ & $x$ & $x$ \\
\hline & & & $15^{\circ}$ & $\checkmark$ & $\checkmark$ & $\checkmark$ & $\checkmark$ & $\checkmark$ & $x$ & $x$ & $x$ & $x$ & $x$ \\
\hline & & & $16^{\circ}$ & $\checkmark$ & $\checkmark$ & $\checkmark$ & $\checkmark$ & $\checkmark$ & $\checkmark$ & $\checkmark$ & $\checkmark$ & $\checkmark$ & $\checkmark$ \\
\hline & & & $17^{\circ}$ & $\checkmark$ & $\checkmark$ & $\checkmark$ & $\checkmark$ & $\checkmark$ & $\checkmark$ & $\checkmark$ & $\checkmark$ & $\checkmark$ & $\checkmark$ \\
\hline & & & $18^{\circ}$ & $\checkmark$ & $\checkmark$ & $\checkmark$ & $\checkmark$ & $\checkmark$ & $\checkmark$ & $\checkmark$ & $\checkmark$ & $\checkmark$ & $\checkmark$ \\
\hline & & & $19^{\circ}$ & $\checkmark$ & $\checkmark$ & $\checkmark$ & $\checkmark$ & $\checkmark$ & $\checkmark$ & $\checkmark$ & $\checkmark$ & $\checkmark$ & $\checkmark$ \\
\hline & & & $20^{\circ}$ & $\checkmark$ & $\checkmark$ & $\checkmark$ & $\checkmark$ & $\checkmark$ & $\checkmark$ & $\checkmark$ & $\checkmark$ & $\checkmark$ & $\checkmark$ \\
\hline & & \multirow{9}{*}{$\begin{array}{l}0 \\
\text { i } \\
\text { II } \\
\dot{n}\end{array}$} & $0^{\circ}$ & $\checkmark$ & $\checkmark$ & $\checkmark$ & $\checkmark$ & $\checkmark$ & $x$ & $x$ & $x$ & $x$ & $x$ \\
\hline & & & $5^{\circ}$ & $\checkmark$ & $\checkmark$ & $\checkmark$ & $\checkmark$ & $\checkmark$ & $x$ & $x$ & $x$ & $x$ & $x$ \\
\hline & & & $10^{\circ}$ & $\checkmark$ & $\checkmark$ & $\checkmark$ & $\checkmark$ & $\checkmark$ & $x$ & $x$ & $x$ & $x$ & $x$ \\
\hline & & & $15^{\circ}$ & $\checkmark$ & $\checkmark$ & $\checkmark$ & $\checkmark$ & $\checkmark$ & $x$ & $x$ & $x$ & $x$ & $x$ \\
\hline & & & $16^{\circ}$ & $\checkmark$ & $\checkmark$ & $\checkmark$ & $\checkmark$ & $\checkmark$ & $\checkmark$ & $\checkmark$ & $\checkmark$ & $\checkmark$ & $\checkmark$ \\
\hline & & & $17^{\circ}$ & $\checkmark$ & $\checkmark$ & $\checkmark$ & $\checkmark$ & $\checkmark$ & $\checkmark$ & $\checkmark$ & $\checkmark$ & $\checkmark$ & $\checkmark$ \\
\hline & & & $18^{\circ}$ & $\checkmark$ & $\checkmark$ & $\checkmark$ & $\checkmark$ & $\checkmark$ & $\checkmark$ & $\checkmark$ & $\checkmark$ & $\checkmark$ & $\checkmark$ \\
\hline & & & $19^{\circ}$ & $\checkmark$ & $\checkmark$ & $\checkmark$ & $\checkmark$ & $\checkmark$ & $\checkmark$ & $\checkmark$ & $\checkmark$ & $\checkmark$ & $\checkmark$ \\
\hline & & & $20^{\circ}$ & $\checkmark$ & $\checkmark$ & $\checkmark$ & $\checkmark$ & $\checkmark$ & $\checkmark$ & $\checkmark$ & $\checkmark$ & $\checkmark$ & $\checkmark$ \\
\hline & & \multirow{9}{*}{$\begin{array}{l}0 \\
\dot{N} \\
\text { II } \\
\ddot{n}\end{array}$} & $0^{\circ}$ & $\checkmark$ & $\checkmark$ & $\checkmark$ & $\checkmark$ & $\checkmark$ & $x$ & $x$ & $x$ & $x$ & $x$ \\
\hline & & & $5^{\circ}$ & $\checkmark$ & $\checkmark$ & $\checkmark$ & $\checkmark$ & $\checkmark$ & $x$ & $x$ & $x$ & $x$ & $x$ \\
\hline & & & $10^{\circ}$ & $\checkmark$ & $\checkmark$ & $\checkmark$ & $\checkmark$ & $\checkmark$ & $x$ & $x$ & $x$ & $x$ & $x$ \\
\hline & & & $15^{\circ}$ & $\checkmark$ & $\checkmark$ & $\checkmark$ & $\checkmark$ & $\checkmark$ & $x$ & $x$ & $x$ & $x$ & $x$ \\
\hline & & & $16^{\circ}$ & $\checkmark$ & $\checkmark$ & $\checkmark$ & $\checkmark$ & $\checkmark$ & $\checkmark$ & $\checkmark$ & $\checkmark$ & $\checkmark$ & $\checkmark$ \\
\hline & & & $17^{\circ}$ & $\checkmark$ & $\checkmark$ & $\checkmark$ & $\checkmark$ & $\checkmark$ & $\checkmark$ & $\checkmark$ & $\checkmark$ & $\checkmark$ & $\checkmark$ \\
\hline & & & $18^{\circ}$ & $\checkmark$ & $\checkmark$ & $\checkmark$ & $\checkmark$ & $\checkmark$ & $\checkmark$ & $\checkmark$ & $\checkmark$ & $\checkmark$ & $\checkmark$ \\
\hline & & & $19^{\circ}$ & $\checkmark$ & $\checkmark$ & $\checkmark$ & $\checkmark$ & $\checkmark$ & $\checkmark$ & $\checkmark$ & $\checkmark$ & $\checkmark$ & $\checkmark$ \\
\hline & & & $20^{\circ}$ & $\checkmark$ & $\checkmark$ & $\checkmark$ & $\checkmark$ & $\checkmark$ & $\checkmark$ & $\checkmark$ & $\checkmark$ & $\checkmark$ & $\checkmark$ \\
\hline
\end{tabular}


Table 37. Static Wind Tunnel Test Matrix: $R e=450 \times 10^{3}$ (All $C_{\mu}$ Conditions)

\begin{tabular}{|c|c|c|c|c|c|c|c|c|c|c|c|c|c|}
\hline Actuation & $C_{\mu}[-]$ & St [ - ] & $\alpha\left[^{\circ}\right]$ & run \#1 & run \#2 & run \#3 & run \#4 & run \#5 & run \#6 & run \#7 & run \#8 & run \#9 & run \#10 \\
\hline \multirow{4}{*}{$\begin{array}{c}4 \\
0 \\
11 \\
4\end{array}$} & \multirow{4}{*}{$\begin{array}{l}0 \\
0 \\
11 \\
v^{2}\end{array}$} & \multirow{4}{*}{$\begin{array}{l}0 \\
0 \\
11 \\
\dot{n}\end{array}$} & $0^{\circ}$ & $\checkmark$ & $\checkmark$ & $\checkmark$ & $\checkmark$ & $\checkmark$ & $x$ & $x$ & $x$ & $x$ & $x$ \\
\hline & & & $5^{\circ}$ & $\checkmark$ & $\checkmark$ & $\checkmark$ & $\checkmark$ & $\checkmark$ & $x$ & $x$ & $x$ & $x$ & $x$ \\
\hline & & & $10^{\circ}$ & $\checkmark$ & $\checkmark$ & $\checkmark$ & $\checkmark$ & $\checkmark$ & $x$ & $x$ & $x$ & $x$ & $x$ \\
\hline & & & $15^{\circ}$ & $\checkmark$ & $\checkmark$ & $\checkmark$ & $\checkmark$ & $\checkmark$ & $\checkmark$ & $\checkmark$ & $\checkmark$ & $\checkmark$ & $\checkmark$ \\
\hline \multirow{8}{*}{ 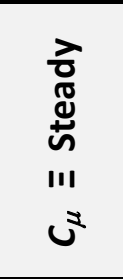 } & \multirow{4}{*}{ " } & \multirow{4}{*}{$\begin{array}{l}0 \\
0 \\
11 \\
\dot{n}\end{array}$} & $0^{\circ}$ & $\checkmark$ & $\checkmark$ & $\checkmark$ & $\checkmark$ & $\checkmark$ & $x$ & $x$ & $x$ & $x$ & $x$ \\
\hline & & & $5^{\circ}$ & $\checkmark$ & $\checkmark$ & $\checkmark$ & $\checkmark$ & $\checkmark$ & $x$ & $x$ & $x$ & $x$ & $x$ \\
\hline & & & $10^{\circ}$ & $\checkmark$ & $\checkmark$ & $\checkmark$ & $\checkmark$ & $\checkmark$ & $x$ & $x$ & $x$ & $x$ & $x$ \\
\hline & & & $15^{\circ}$ & $\checkmark$ & $\checkmark$ & $\checkmark$ & $\checkmark$ & $\checkmark$ & $\checkmark$ & $\checkmark$ & $\checkmark$ & $\checkmark$ & $\checkmark$ \\
\hline & \multirow{4}{*}{ " } & \multirow{4}{*}{$\begin{array}{l}0 \\
0 \\
11 \\
\dot{n}\end{array}$} & $0^{\circ}$ & $\checkmark$ & $\checkmark$ & $\checkmark$ & $\checkmark$ & $\checkmark$ & $x$ & $x$ & $x$ & $x$ & $x$ \\
\hline & & & $5^{\circ}$ & $\checkmark$ & $\checkmark$ & $\checkmark$ & $\checkmark$ & $\checkmark$ & $x$ & $x$ & $x$ & $x$ & $x$ \\
\hline & & & $10^{\circ}$ & $\checkmark$ & $\checkmark$ & $\checkmark$ & $\checkmark$ & $\checkmark$ & $x$ & $x$ & $x$ & $x$ & $x$ \\
\hline & & & $15^{\circ}$ & $\checkmark$ & $\checkmark$ & $\checkmark$ & $\checkmark$ & $\checkmark$ & $\checkmark$ & $\checkmark$ & $\checkmark$ & $\checkmark$ & $\checkmark$ \\
\hline \multirow{24}{*}{ 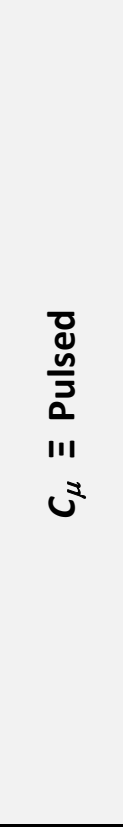 } & \multirow{12}{*}{$\begin{array}{l}-1 \\
0 \\
0 \\
11 \\
ن\end{array}$} & \multirow{4}{*}{$\begin{array}{l}\tilde{0} \\
0 \\
11 \\
\tilde{n}\end{array}$} & $0^{\circ}$ & $\checkmark$ & $\checkmark$ & $\checkmark$ & $\checkmark$ & $\checkmark$ & $x$ & $x$ & $x$ & $x$ & $x$ \\
\hline & & & $5^{\circ}$ & $\checkmark$ & $\checkmark$ & $\checkmark$ & $\checkmark$ & $\checkmark$ & $x$ & $x$ & $x$ & $x$ & $x$ \\
\hline & & & $10^{\circ}$ & $\checkmark$ & $\checkmark$ & $\checkmark$ & $\checkmark$ & $\checkmark$ & $x$ & $x$ & $x$ & $x$ & $x$ \\
\hline & & & $15^{\circ}$ & $\checkmark$ & $\checkmark$ & $\checkmark$ & $\checkmark$ & $\checkmark$ & $\checkmark$ & $\checkmark$ & $\checkmark$ & $\checkmark$ & $\checkmark$ \\
\hline & & \multirow{4}{*}{$\begin{array}{l}0 \\
\dot{-1} \\
\text { II } \\
\ddot{n}\end{array}$} & $0^{\circ}$ & $\checkmark$ & $\checkmark$ & $\checkmark$ & $\checkmark$ & $\checkmark$ & $x$ & $x$ & $x$ & $x$ & $x$ \\
\hline & & & $5^{\circ}$ & $\checkmark$ & $\checkmark$ & $\checkmark$ & $\checkmark$ & $\checkmark$ & $x$ & $x$ & $x$ & $x$ & $x$ \\
\hline & & & $10^{\circ}$ & $\checkmark$ & $\checkmark$ & $\checkmark$ & $\checkmark$ & $\checkmark$ & $x$ & $x$ & $x$ & $x$ & $x$ \\
\hline & & & $15^{\circ}$ & $\checkmark$ & $\checkmark$ & $\checkmark$ & $\checkmark$ & $\checkmark$ & $\checkmark$ & $\checkmark$ & $\checkmark$ & $\checkmark$ & $\checkmark$ \\
\hline & & \multirow{4}{*}{ 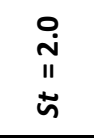 } & $0^{\circ}$ & $\checkmark$ & $\checkmark$ & $\checkmark$ & $\checkmark$ & $\checkmark$ & $x$ & $x$ & $x$ & $x$ & $x$ \\
\hline & & & $5^{\circ}$ & $\checkmark$ & $\checkmark$ & $\checkmark$ & $\checkmark$ & $\checkmark$ & $x$ & $x$ & $x$ & $x$ & $x$ \\
\hline & & & $10^{\circ}$ & $\checkmark$ & $\checkmark$ & $\checkmark$ & $\checkmark$ & $\checkmark$ & $x$ & $x$ & $x$ & $x$ & $x$ \\
\hline & & & $15^{\circ}$ & $\checkmark$ & $\checkmark$ & $\checkmark$ & $\checkmark$ & $\checkmark$ & $\checkmark$ & $\checkmark$ & $\checkmark$ & $\checkmark$ & $\checkmark$ \\
\hline & \multirow{12}{*}{$\begin{array}{l}\text { no } \\
0 \\
11 \\
v^{2}\end{array}$} & \multirow{4}{*}{$\begin{array}{l}n \\
0 \\
11 \\
\tilde{n}\end{array}$} & $0^{\circ}$ & $\checkmark$ & $\checkmark$ & $\checkmark$ & $\checkmark$ & $\checkmark$ & $x$ & $x$ & $x$ & $x$ & $x$ \\
\hline & & & $5^{\circ}$ & $\checkmark$ & $\checkmark$ & $\checkmark$ & $\checkmark$ & $\checkmark$ & $x$ & $x$ & $x$ & $x$ & $x$ \\
\hline & & & $10^{\circ}$ & $\checkmark$ & $\checkmark$ & $\checkmark$ & $\checkmark$ & $\checkmark$ & $x$ & $x$ & $x$ & $x$ & $x$ \\
\hline & & & $15^{\circ}$ & $\checkmark$ & $\checkmark$ & $\checkmark$ & $\checkmark$ & $\checkmark$ & $\checkmark$ & $\checkmark$ & $\checkmark$ & $\checkmark$ & $\checkmark$ \\
\hline & & \multirow{4}{*}{ 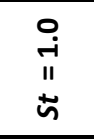 } & $0^{\circ}$ & $\checkmark$ & $\checkmark$ & $\checkmark$ & $\checkmark$ & $\checkmark$ & $x$ & $x$ & $x$ & $x$ & $x$ \\
\hline & & & $5^{\circ}$ & $\checkmark$ & $\checkmark$ & $\checkmark$ & $\checkmark$ & $\checkmark$ & $x$ & $x$ & $x$ & $x$ & $x$ \\
\hline & & & $10^{\circ}$ & $\checkmark$ & $\checkmark$ & $\checkmark$ & $\checkmark$ & $\checkmark$ & $x$ & $x$ & $x$ & $x$ & $x$ \\
\hline & & & $15^{\circ}$ & $\checkmark$ & $\checkmark$ & $\checkmark$ & $\checkmark$ & $\checkmark$ & $\checkmark$ & $\checkmark$ & $\checkmark$ & $\checkmark$ & $\checkmark$ \\
\hline & & \multirow{4}{*}{$\begin{array}{l}0 \\
\dot{N} \\
11 \\
\dot{n}\end{array}$} & $0^{\circ}$ & $\checkmark$ & $\checkmark$ & $\checkmark$ & $\checkmark$ & $\checkmark$ & $x$ & $x$ & $x$ & $x$ & $x$ \\
\hline & & & $5^{\circ}$ & $\checkmark$ & $\checkmark$ & $\checkmark$ & $\checkmark$ & $\checkmark$ & $x$ & $x$ & $x$ & $x$ & $x$ \\
\hline & & & $10^{\circ}$ & $\checkmark$ & $\checkmark$ & $\checkmark$ & $\checkmark$ & $\checkmark$ & $x$ & $x$ & $x$ & $x$ & $x$ \\
\hline & & & $15^{\circ}$ & $\checkmark$ & $\checkmark$ & $\checkmark$ & $\checkmark$ & $\checkmark$ & $\checkmark$ & $\checkmark$ & $\checkmark$ & $\checkmark$ & $\checkmark$ \\
\hline
\end{tabular}




\section{APPENDIX F: Dynamic Pitch Wind Tunnel Test Matrices}

The specific test cases recorded, including the number of repeated trials for each condition, are included for all pitching airfoil experiments completed in the WVU Subsonic Closed Loop Wind Tunnel. Check marks represent a completed case, including measurement, data reduction, and data validation. For all completed cases (denoted by " $\checkmark$ " symbol), a minimum of 10 pitch cycles were monitored and recorded; the reported results of aerodynamic coefficients were ensemble averaged for each test condition.

Table 38. Dynamic Pitch Wind Tunnel Test Matrix: $R e=180 \times 10^{3}, \alpha_{\max }=25^{\circ}$ (All $C_{\mu}$ Conditions)

\begin{tabular}{|c|c|c|c|c|c|c|c|c|}
\hline \multicolumn{3}{|c|}{$R e=180 \mathrm{~K}$} & \multicolumn{6}{|c|}{$\alpha(t)=5^{\circ}+20^{\circ} \sin (\omega \cdot t)$} \\
\hline$C_{\mu}=0.00$ & $k=0.010$ & $x$ & $k=0.025$ & $\checkmark$ & $k=0.050$ & $\bar{\checkmark}$ & $k=0.100$ & $\checkmark$ \\
\hline$C_{\mu}=0.01$ & $k=0.010$ & $x$ & $k=0.025$ & $\checkmark$ & $k=0.050$ & $\checkmark$ & $k=0.100$ & $\bar{\checkmark}$ \\
\hline$C_{\mu}=0.05$ & $k=0.010$ & $x$ & $k=0.025$ & $\checkmark$ & $k=0.050$ & $\checkmark$ & $k=0.100$ & $\checkmark$ \\
\hline$C_{\mu}=0.10$ & $k=0.010$ & $x$ & $k=0.025$ & $\checkmark$ & $k=0.050$ & $\bar{\checkmark}$ & $k=0.100$ & $\bar{\checkmark}$ \\
\hline$\left\langle c_{\mu}\right\rangle=0.01(S t=0.25)$ & $k=0.010$ & $x$ & $k=0.025$ & $\checkmark$ & $k=0.050$ & $\checkmark$ & $k=0.100$ & $\checkmark$ \\
\hline$\left\langle c_{\mu}\right\rangle=0.01(S t=0.50)$ & $k=0.010$ & $x$ & $k=0.025$ & $\checkmark$ & $k=0.050$ & $\checkmark$ & $k=0.100$ & $\checkmark$ \\
\hline$\left\langle c_{\mu}\right\rangle=0.01(S t=1.00)$ & $k=0.010$ & $x$ & $k=0.025$ & $x$ & $k=0.050$ & $\bar{x}$ & $k=0.100$ & $\bar{x}$ \\
\hline$\left\langle c_{\mu}\right\rangle=0.05(S t=0.25)$ & $k=0.010$ & $x$ & $k=0.025$ & $\checkmark$ & $k=0.050$ & $\checkmark$ & $k=0.100$ & $\checkmark$ \\
\hline$\left\langle c_{\mu}\right\rangle=0.05(S t=0.50)$ & $k=0.010$ & $\bar{x}$ & $k=0.025$ & $\checkmark$ & $k=0.050$ & $\checkmark$ & $k=0.100$ & $\sqrt{ }$ \\
\hline$\left\langle c_{\mu}\right\rangle=0.05(S t=1.00)$ & $k=0.010$ & $x$ & $k=0.025$ & $x$ & $k=0.050$ & $x$ & $k=0.100$ & $x$ \\
\hline$\left\langle c_{\mu}\right\rangle=0.10(S t=0.25)$ & $k=0.010$ & $x$ & $k=0.025$ & $x$ & $k=0.050$ & $x$ & $k=0.100$ & $x$ \\
\hline$\left\langle c_{\mu}\right\rangle=0.10(S t=0.50)$ & $k=0.010$ & $x$ & $k=0.025$ & $x$ & $k=0.050$ & $x$ & $k=0.100$ & $x$ \\
\hline$\left\langle c_{\mu}\right\rangle=0.10(S t=1.00)$ & $k=0.010$ & $x$ & $k=0.025$ & $x$ & $k=0.050$ & $x$ & $k=0.100$ & $x$ \\
\hline
\end{tabular}


Table 39. Dynamic Pitch Wind Tunnel Test Matrix: $R e=180 \times 10^{3}, \alpha_{\max }=30^{\circ}$ (All $C_{\mu}$ Conditions)

\begin{tabular}{|c|c|c|c|c|c|c|c|c|}
\hline \multicolumn{3}{|c|}{$R e=180 \mathrm{~K}$} & \multicolumn{6}{|c|}{$\alpha(t)=10^{\circ}+20^{\circ} \sin (\omega \cdot t)$} \\
\hline$C_{\mu}=0.00$ & $k=0.010$ & $\mathbf{x}$ & $k=0.025$ & $x$ & $k=0.050$ & $\checkmark$ & $k=0.100$ & $\checkmark$ \\
\hline$C_{\mu}=0.01$ & $k=0.010$ & $x$ & $k=0.025$ & x & $k=0.050$ & $x$ & $k=0.100$ & $x$ \\
\hline$C_{\mu}=0.05$ & $k=0.010$ & $x$ & $k=0.025$ & $x$ & $k=0.050$ & $\checkmark$ & $k=0.100$ & $\checkmark$ \\
\hline$C_{\mu}=0.10$ & $k=0.010$ & $x$ & $k=0.025$ & $x$ & $k=0.050$ & $\checkmark$ & $k=0.100$ & $x$ \\
\hline$\left\langle c_{\mu}\right\rangle=0.01(S t=0.25)$ & $k=0.010$ & $x$ & $k=0.025$ & $x$ & $k=0.050$ & $x$ & $k=0.100$ & $x$ \\
\hline$\left\langle c_{\mu}\right\rangle=0.01(S t=0.50)$ & $k=0.010$ & $x$ & $k=0.025$ & x & $k=0.050$ & $x$ & $k=0.100$ & $x$ \\
\hline$\left\langle c_{\mu}\right\rangle=0.01(S t=1.00)$ & $k=0.010$ & $x$ & $k=0.025$ & $x$ & $k=0.050$ & $x$ & $k=0.100$ & $x$ \\
\hline$\left\langle c_{\mu}\right\rangle=0.05(S t=0.25)$ & $k=0.010$ & $x$ & $k=0.025$ & $x$ & $k=0.050$ & $\checkmark$ & $k=0.100$ & $\checkmark$ \\
\hline$\left\langle c_{\mu}\right\rangle=0.05(S t=0.50)$ & $k=0.010$ & $x$ & $k=0.025$ & $x$ & $k=0.050$ & $\checkmark$ & $k=0.100$ & $\checkmark$ \\
\hline$\left\langle c_{\mu}\right\rangle=0.05(S t=1.00)$ & $k=0.010$ & $x$ & $k=0.025$ & $x$ & $k=0.050$ & $x$ & $k=0.100$ & $x$ \\
\hline$\left\langle c_{\mu}\right\rangle=0.10(S t=0.25)$ & $k=0.010$ & $x$ & $k=0.025$ & $x$ & $k=0.050$ & $x$ & $k=0.100$ & $x$ \\
\hline$\left\langle c_{\mu}\right\rangle=0.10(S t=0.50)$ & $k=0.010$ & $x$ & $k=0.025$ & $x$ & $k=0.050$ & $x$ & $k=0.100$ & $x$ \\
\hline$\left\langle c_{\mu}\right\rangle=0.10(S t=1.00)$ & $k=0.010$ & $x$ & $k=0.025$ & $x$ & $k=0.050$ & $x$ & $k=0.100$ & $x$ \\
\hline
\end{tabular}

Table 40. Dynamic Pitch Wind Tunnel Test Matrix: $R e=300 \times 10^{3}, \alpha_{\max }=25^{\circ}$ (All $C_{\mu}$ Conditions)

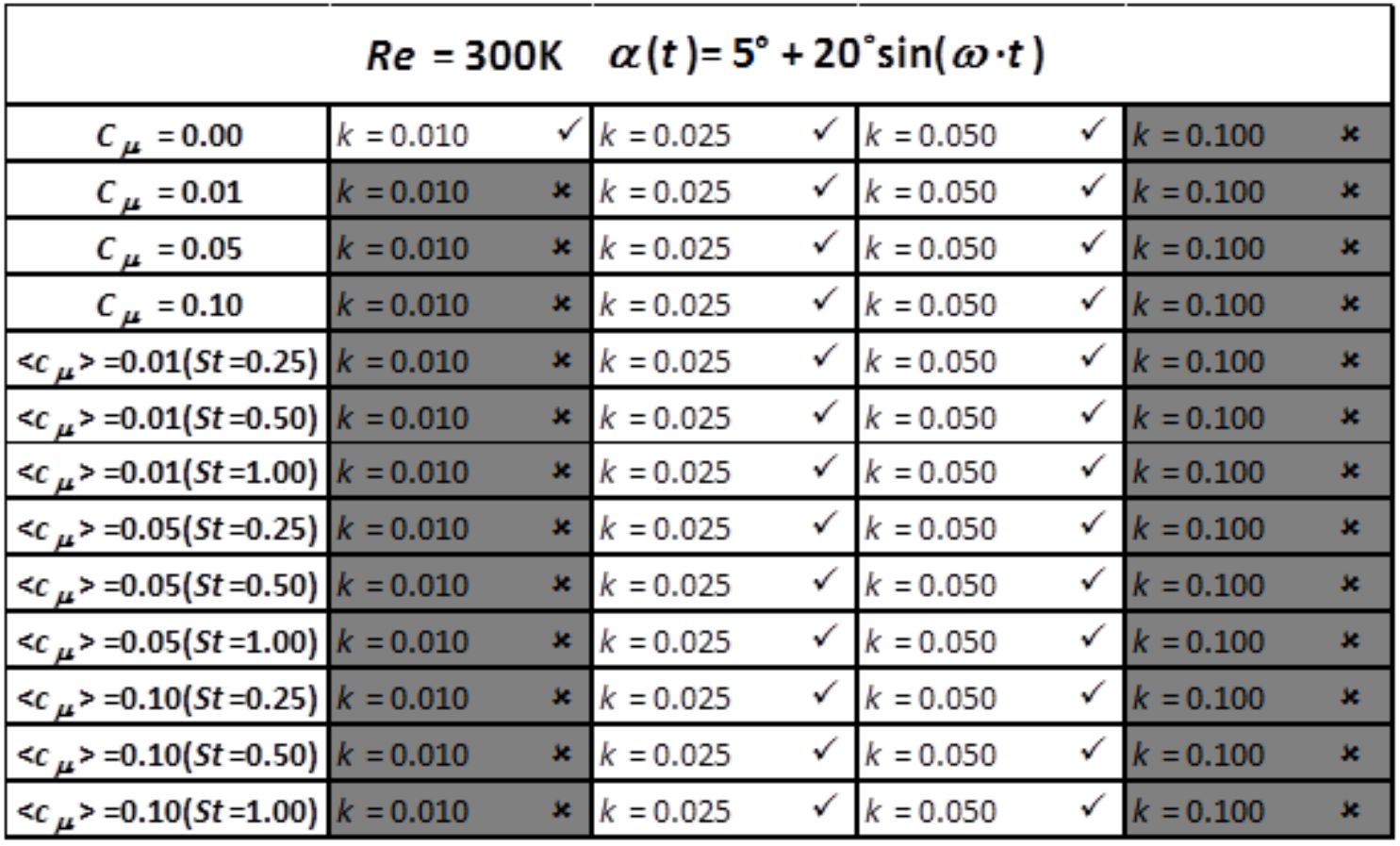


Table 41. Dynamic Pitch Wind Tunnel Test Matrix: $R e=450 \times 10^{3}, \alpha_{\max }=25^{\circ}$ (All $C_{\mu}$ Conditions)

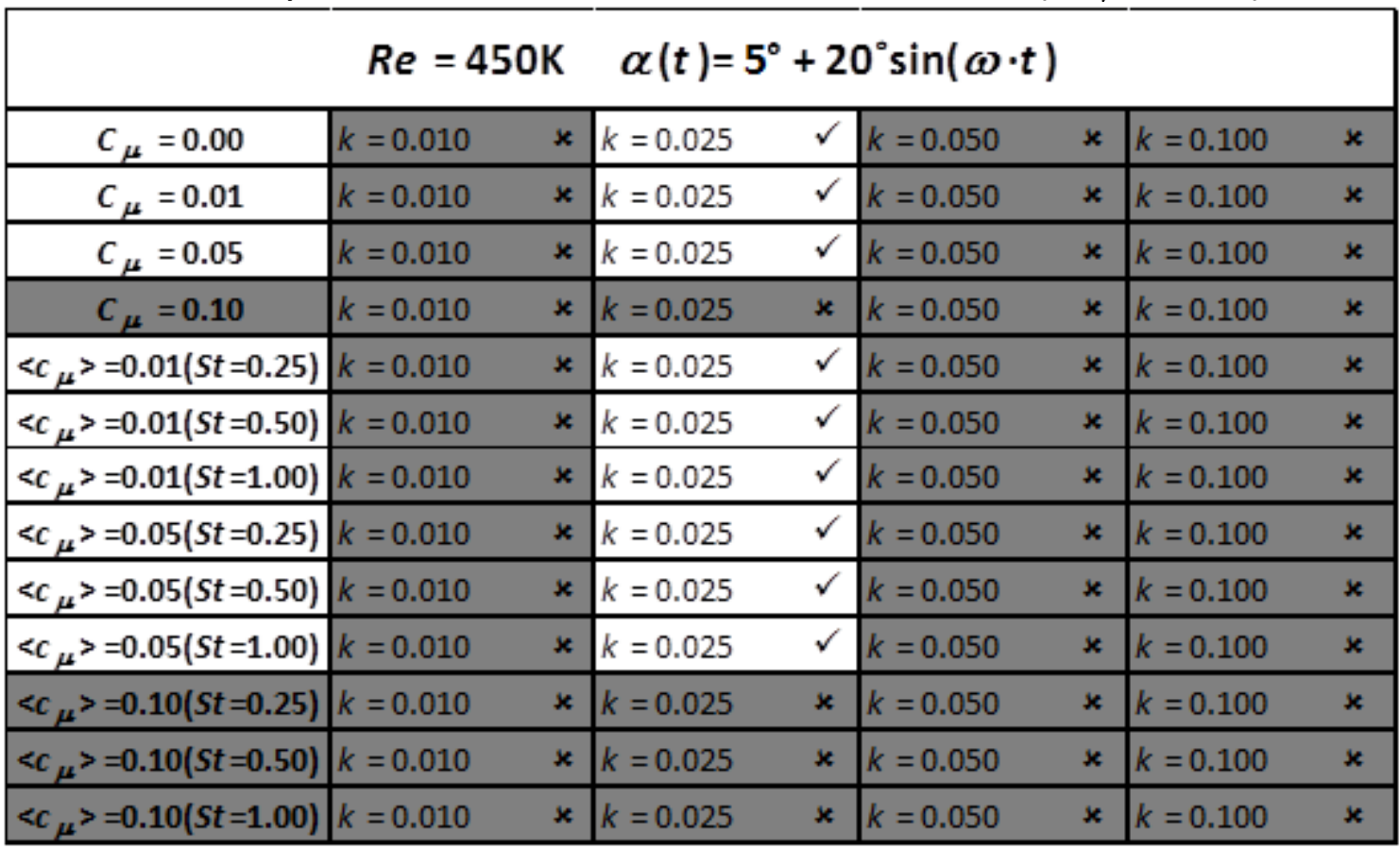

Table 42. Dynamic Pitch Wind Tunnel Test Matrix: $R e=450 \times 10^{3}, \alpha_{\max }=30^{\circ}$ (All $C_{\mu}$ Conditions)

\begin{tabular}{|c|c|c|c|c|c|c|c|c|}
\hline \multicolumn{3}{|c|}{$R e=450 \mathrm{~K}$} & \multicolumn{6}{|c|}{$\alpha(t)=10^{\circ}+20^{\circ} \sin (\omega \cdot t)$} \\
\hline$C_{\mu}=0.00$ & $k=0.010$ & $x$ & $k=0.025$ & $\checkmark$ & $k=0.050$ & $x$ & $k=0.100$ & $x$ \\
\hline$C_{\mu}=0.01$ & $k=0.010$ & $x$ & $k=0.025$ & $\checkmark$ & $k=0.050$ & $x$ & $k=0.100$ & $x$ \\
\hline$C_{\mu}=0.05$ & $k=0.010$ & $x$ & $k=0.025$ & $\checkmark$ & $k=0.050$ & $x$ & $k=0.100$ & $x$ \\
\hline$C_{\mu}=0.10$ & $k=0.010$ & $x$ & $k=0.025$ & $x$ & $k=0.050$ & $x$ & $k=0.100$ & $x$ \\
\hline$\left\langle c_{\mu}\right\rangle=0.01(S t=0.25)$ & $k=0.010$ & $x$ & $k=0.025$ & $\checkmark$ & $k=0.050$ & $x$ & $k=0.100$ & $x$ \\
\hline$\left\langle c_{\mu}\right\rangle=0.01(S t=0.50)$ & $k=0.010$ & $x$ & $k=0.025$ & $\checkmark$ & $k=0.050$ & $x$ & $k=0.100$ & $x$ \\
\hline$\left\langle c_{\mu}\right\rangle=0.01(S t=1.00)$ & $k=0.010$ & $x$ & $k=0.025$ & $x$ & $k=0.050$ & $x$ & $k=0.100$ & $x$ \\
\hline$\left\langle c_{\mu}\right\rangle=0.05(S t=0.25)$ & $k=0.010$ & $x$ & $k=0.025$ & $\checkmark$ & $k=0.050$ & $x$ & $k=0.100$ & $x$ \\
\hline$\left\langle c_{\mu}\right\rangle=0.05(S t=0.50)$ & $k=0.010$ & $x$ & $k=0.025$ & $\checkmark$ & $k=0.050$ & $x$ & $k=0.100$ & $x$ \\
\hline$\left\langle c_{\mu}\right\rangle=0.05(S t=1.00)$ & $k=0.010$ & $x$ & $k=0.025$ & $x$ & $k=0.050$ & $x$ & $k=0.100$ & $x$ \\
\hline$\left\langle c_{\mu}\right\rangle=0.10(S t=0.25)$ & $k=0.010$ & $x$ & $k=0.025$ & $x$ & $k=0.050$ & $x$ & $k=0.100$ & $x$ \\
\hline$\left\langle c_{\mu}\right\rangle=0.10(S t=0.50)$ & $k=0.010$ & $x$ & $k=0.025$ & $x$ & $k=0.050$ & $x$ & $k=0.100$ & $x$ \\
\hline$\left\langle c_{\mu}\right\rangle=0.10(S t=1.00)$ & $k=0.010$ & $x$ & $k=0.025$ & $x$ & $k=0.050$ & $x$ & $k=0.100$ & $x$ \\
\hline
\end{tabular}




\section{APPENDIX G: Surface Pressure Scans}

The evolution of pressure activity at each port was plotted for an entire pitch cycle as a visual aid to compare DS stall events for varying freestream and CC actuation conditions. The code used to organize data and create the pressure scan subplots is included below. The author would like to thank Dr. Drew Lowery for his assistance with coding to generate the surface pressure scans.

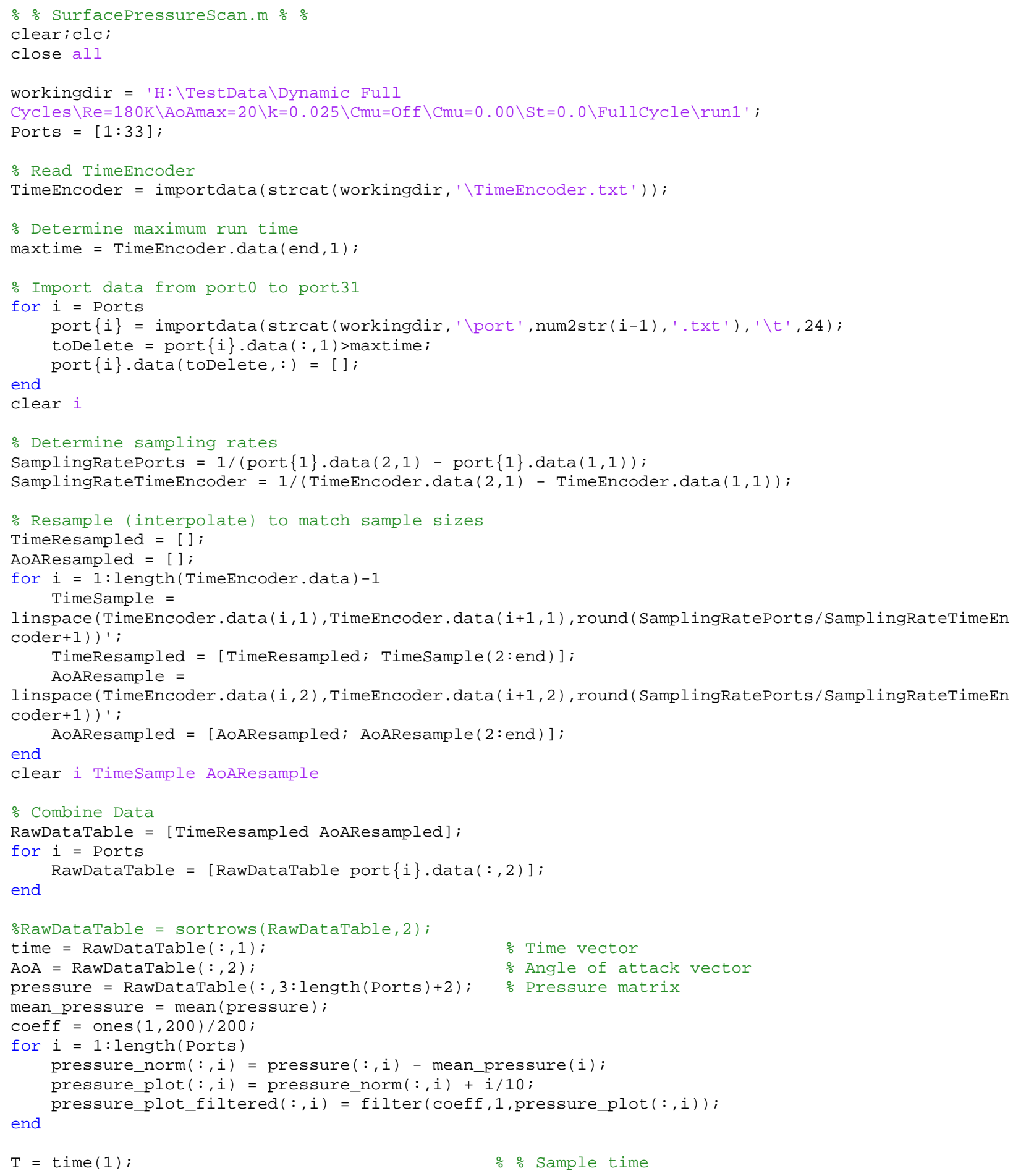




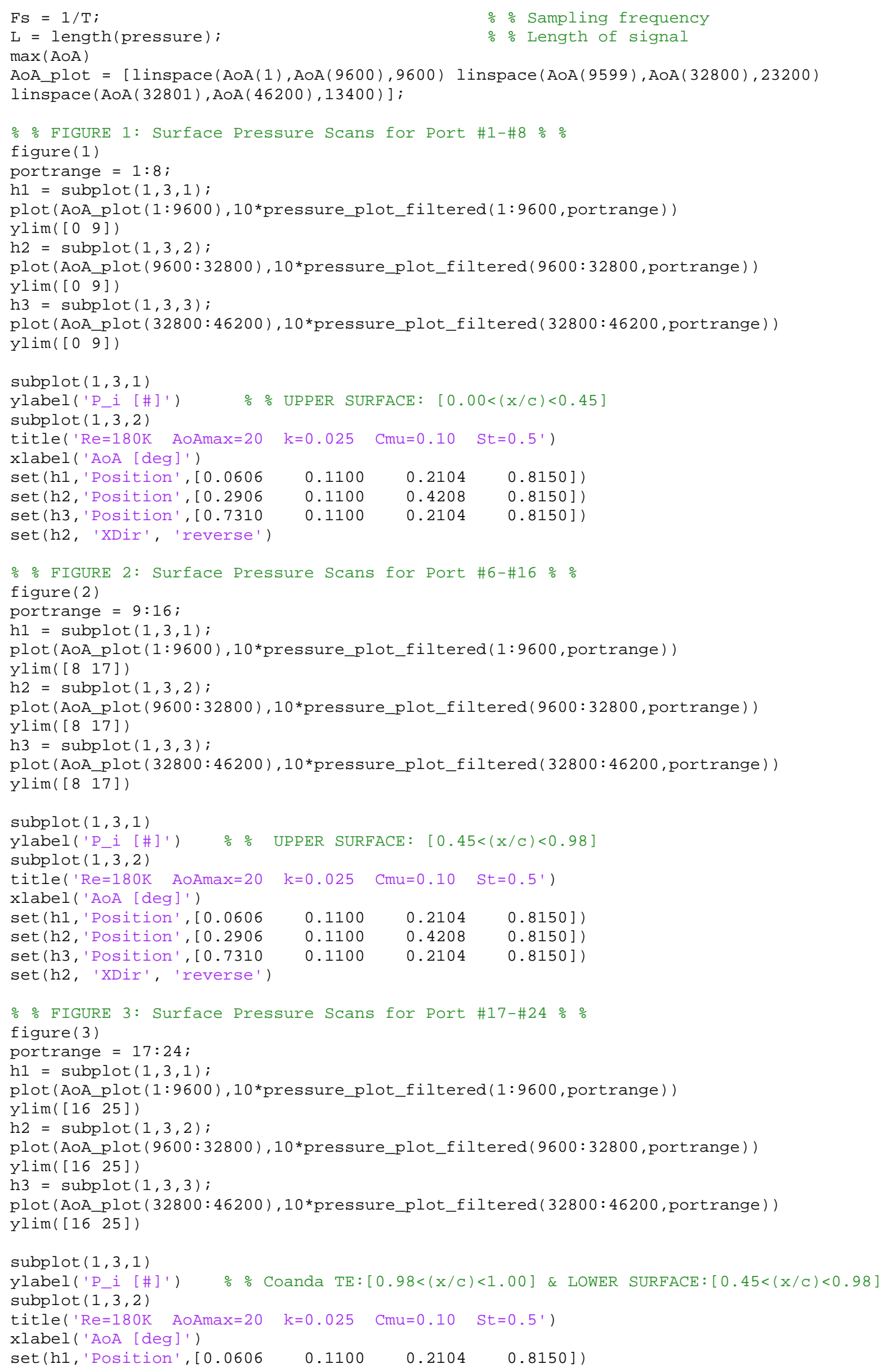




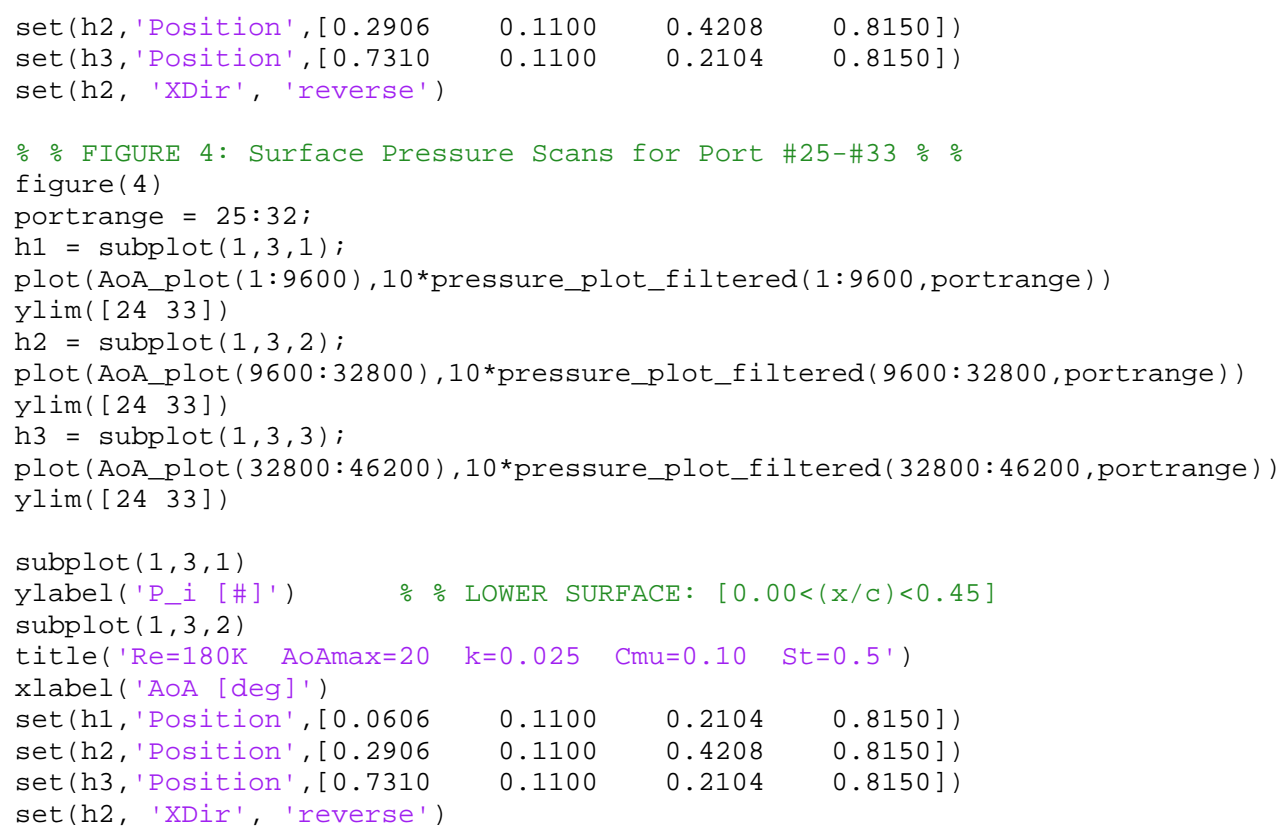


It should be noted that each trace represents recorded data at a single pressure port during the course of one full pitch cycle. Concerning the pressure recordings, the magnitude is not of interest for the study of upper surface vortex motion. Instead, noticeable deviations and trend inflection points are of importance, with regard to measurement location (i.e. $x / c$ position to pitch-cycle $\alpha$ ). As mentioned in Chapter 5, these points of interest were compared to correlate influential parameters of circulation control and pitch characteristics on VAWT blade aerodynamics. Also, each pressure scan was given a unique offset in order to plot all surface port evaluations in close proximity. In short, the $y$-axis of the surface scan plots is for qualitative comparison only, while the $x$-axis ( $\alpha$-position) is the quantity of interest.

The data plotting code included in this section (SurfacePressureScan.m) utilized a moving average command to filter the plotted data for improved visualization and presentation purposes. The main purpose of data smoothing was to dampen out the smaller local fluctuations, focusing instead on the significant global flowfield events (i.e. rapid transient events such as DSV movement). Green, et al. (132) give an excellent review of the methodology used here to analyze unsteady surface pressure traces, with particular emphasis on calculating the convection speed of the dynamic stall vortex (DSV). The accuracy of such methods and recommend techniques to minimize, or even eliminate, subjective influence on pressure scan analysis is included.

Figure 158 includes an example of time-accurate pressure port scans for a pitching CCNACA0018 with low magnitude of steady $\mathrm{CC}\left(C_{\mu}=0.01\right)$. Following the LE ports, the pressure spikes are initiated at port \#2 ( $\alpha=13^{\circ}$ during pitch-up), and gradually move toward the TE until $\alpha_{\max }=20^{\circ}$. This indicates that the CCNACA0018, with steady actuation, experiences a LE stall.

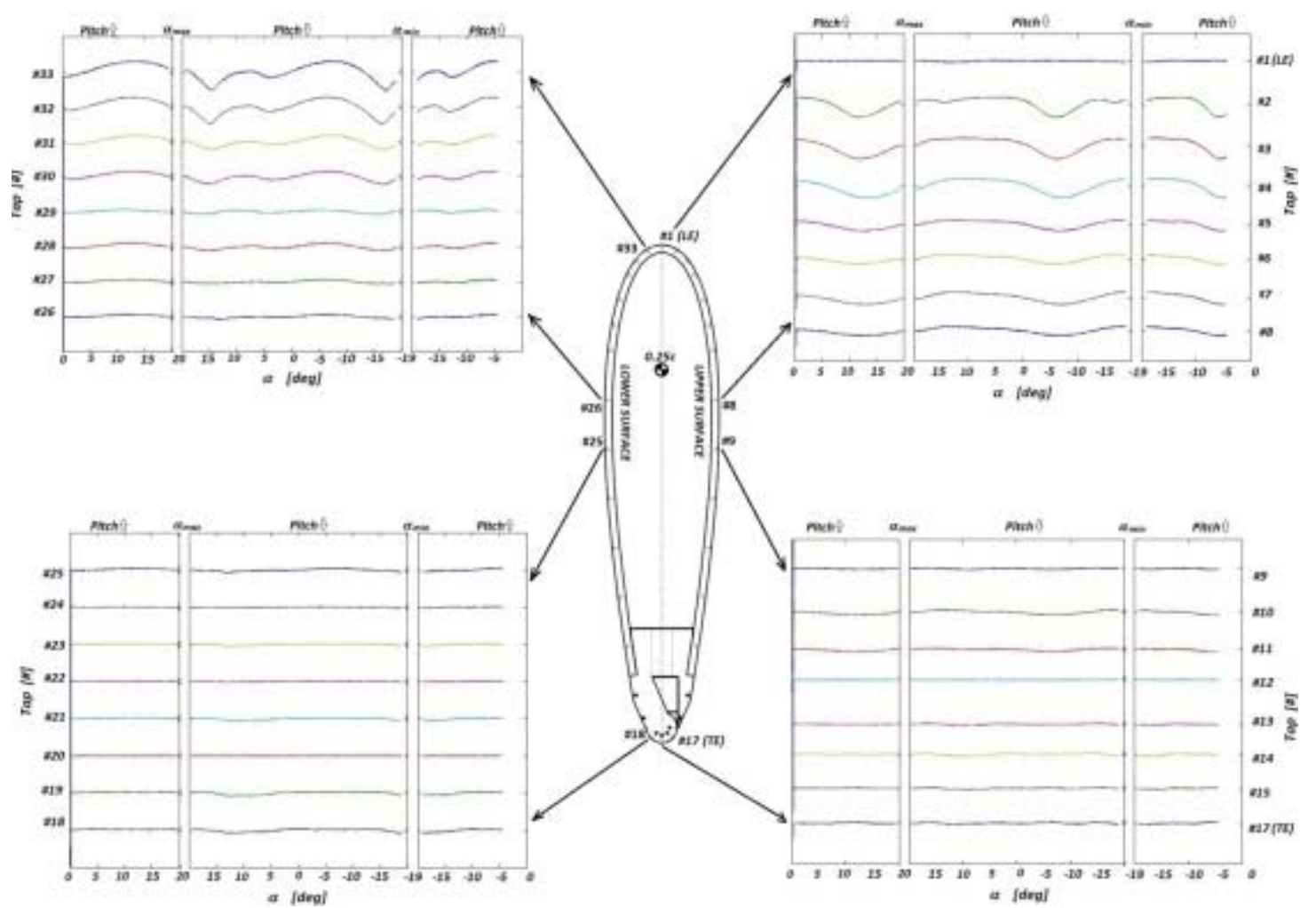

Figure 158. Pitching Airfoil $C_{p}$ Scan: $R e=180 \times 10^{3}, \alpha=0^{\circ} \pm 20^{\circ} \sin (\omega t), C_{\mu}=0.01(S t=0.0)$ 


\section{APPENDIX H: CCNACA0018 Pulsed CC Flow Actuation: Pneumatic Valves}

The actuator array used to control pulsed CC flow consisted of 14 DYNAMCO DASH-1 High-tech Direct Operating Mini Solenoid Valves. Each valve had a 0.089in with a volumetric flow $(Q)$ limitation of 3 SCFM. The DASH-1 family is based on a direct solenoid poppet, capable of either two-way (2/2) or three-way (3/2) operation. The valves have a typical response of $3 \mathrm{msec}$ but improved response of $1 \mathrm{msec}$ is reported achievable with enhanced electronics. The operating characteristics and specifications are included in Figure 159.

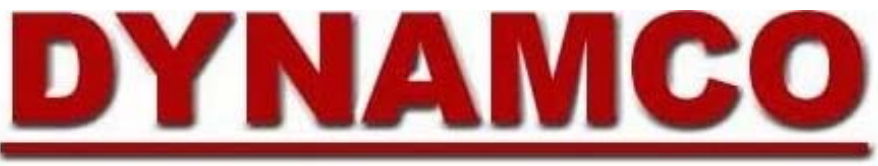

Dash 1 Model Numbers

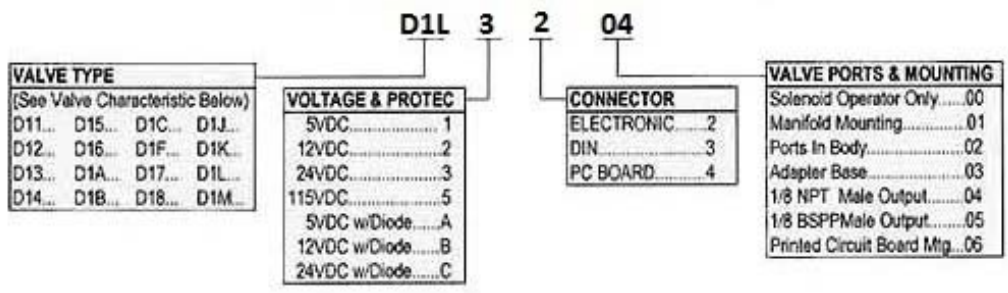

\section{Dash 1 Valve Characteristics}

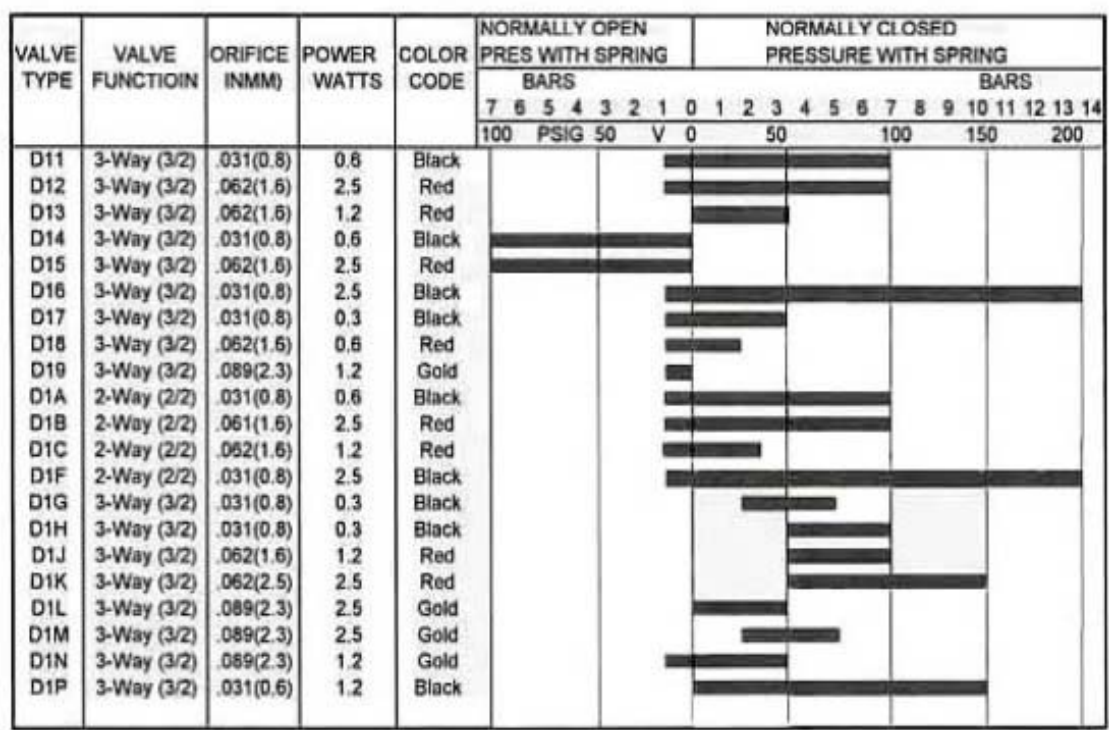

Figure 159. DYNAMCO DASH-1 Valve Specification Sheet (165)

The solenoid valves were current controlled devices. The 14 internal valves that actuated the pulsed jets were DYNAMCO Dash-1 solenoid valves. A CAD drawing of the internal components and various specs are included in Figure 160. 


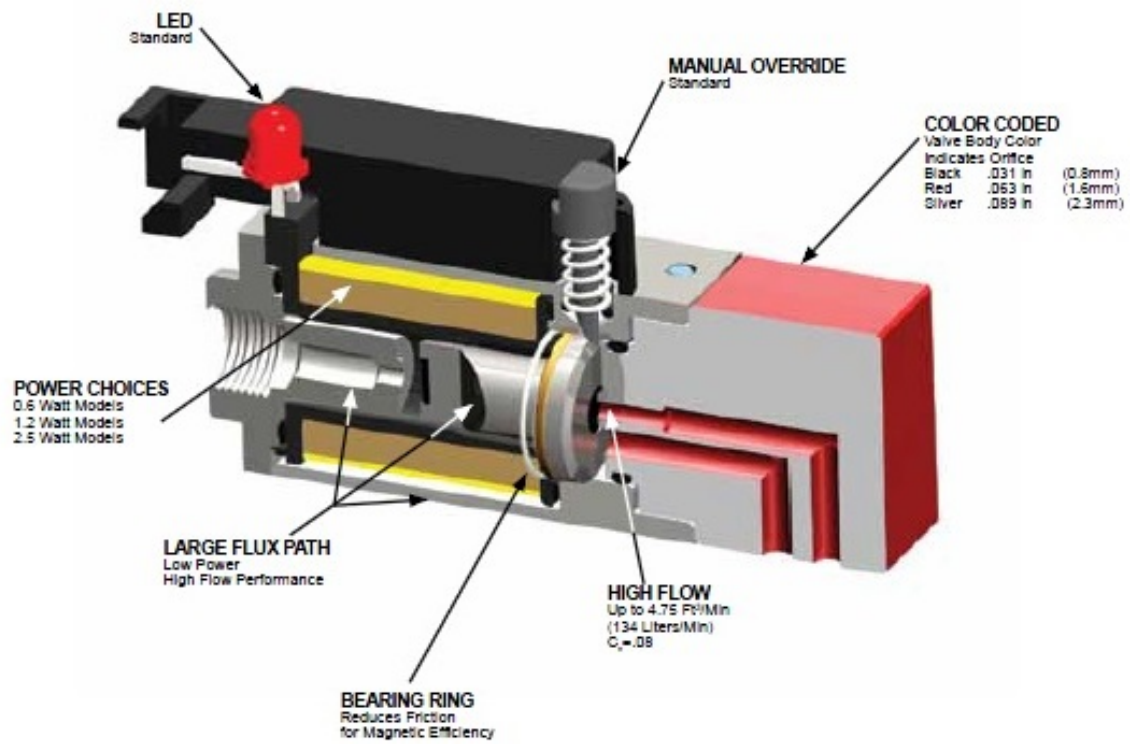

Figure 160. DYNAMCO Dash-1 Pneumatic Valve (CCNACA0018 Oscillatory Jet Driver) (165)

An array of integrated circuits was assembled, in conjunction with NI LabVIEW DAQ software, to manage the pulsed CC flow by a user input in the range $\left(\mathrm{OHz} \leq f_{\text {act }} \leq 200 \mathrm{~Hz}\right)$. The array of 14 DYNAMCO pneumatic valves was controlled by a MOSFET Power Control Kit (Figure 161) manufactured by Sparkfun. The basic kit is essentially a breakout for the RFP3ONO6LE MOSFET, including terminal blocks and a $10 \mathrm{k} \Omega$ resistor.

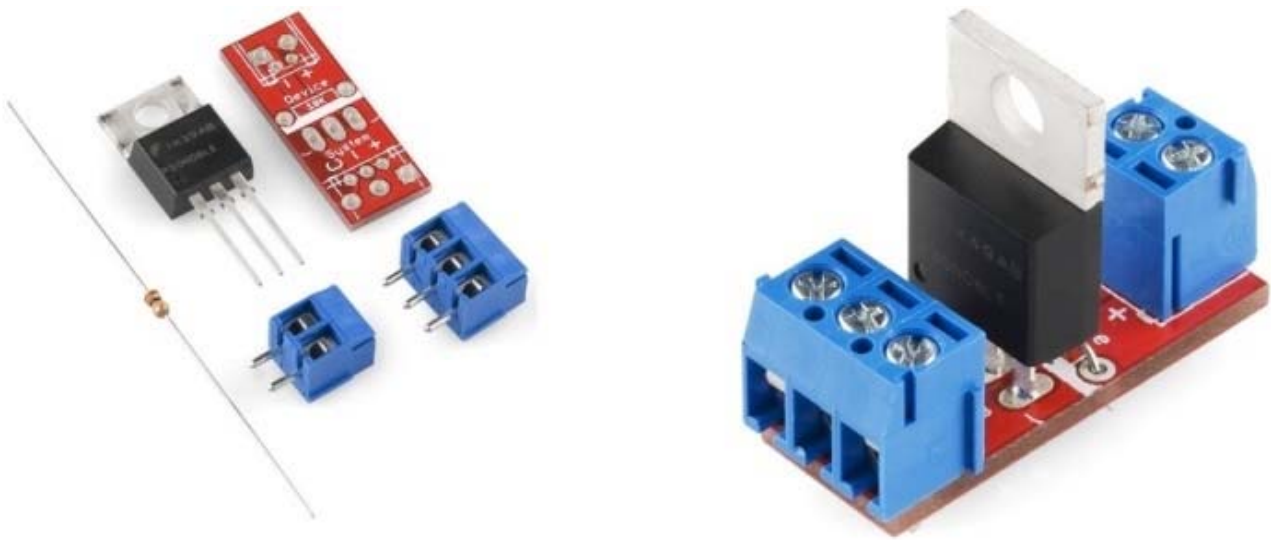

Figure 161. CCNACA0018 Model Plenum Controls: MOSFET Power Control IC for Jet Actuation (166) 


\section{APPENDIX I: Author's Publications}

Wilhelm, J., Panther, C., Smith, J., "Jet Usage Methodology for a Vertical Axis Wind Turbine using Circulation Control," Journal of Wind Energy. * Under Review: Submitted March 2015*

Kweder, J., Panther, C., Smith, J., "Applications of Circulation Control, Yesterday and Today," International Journal of Engineering (IJE): Vol. 4, Issue 4, September 2010.

Panther, C., Williams, K., Wilhelm, J., "Experimental Testing Of A Wind Tunnel Model For Use In A Vertical Axis Wind Turbine," ASME IMECE2010-38816, Vancouver, B.C. 2010.

Wilhelm, J., Panther, C., Pertl, F., Smith, J., "Vortex Analytical Model of A Circulation Controlled Vertical Axis Wind Turbine," SAE Technical Paper 2009-90348, 2009.

Graham, H., Panther, C., Hubbell, M., Wilhelm, J., Angle II, G., Smith, J., "Airfoil Selection for A Straight Bladed Circulation Controlled Vertical Axis Wind Turbine," ASME ES2009-90343, San Francisco, CA 2009.

Graham, H., Hubbell, M., Panther, C., Wilhelm, J., Angle II, G., Smith, J., "Circulation Controlled Airfoil Analysis Through 360 Degrees Angle Of Attack," ASME ES2009-90341, San Francisco, CA 2009.

Wilhelm, J., Panther, C., Pertl, F., Smith, J., "Vortex Analytical Model of A Circulation Controlled Vertical Axis Wind Turbine," ASME ES2009-90348, San Francisco, CA 2009.

Wilhelm, J., Panther, C., Pertl, F., Smith, J., "Momentum Analytical Model of A Circulation Controlled Vertical Axis Wind Turbine," ASME ES2009-90352, San Francisco, CA 2009.

Kweder, J., Wildfire, P., Panther, C., Nawrocki, A., "Design of a Flywheel Based Energy Storage and Distribution System for Rural Villages in China," SAE Int. J. Pass. Cars - Mech. Syst. 2(1): 703-713, 2009.

Kweder, J., Wildfire, P., Panther, C., Nawrocki, A., "Design of a Flywheel Based Energy Storage and Distribution System for Rural Villages in China," SAE World Congress \& Exhibition, Detroit, MI 2009.

Panther, C. Parasitic Drag Analysis of a High Inertia Flywheel Rotating within an Enclosure. MS Thesis, WVU, Morgantown, WV 2008. 


\section{APPENDIX J: Experimental Instrumentation Specifications}

Included in this appendix is manufacturer provided specifications for all measurement equipment used during wind tunnel testing, including both mechanical and electrical devices. All pertinent product specifications from devices contributing to measurement uncertainty are considered.

\section{Appendix J.1 Digital Encoder}

The $\alpha$-position of the airfoil, for both static and dynamic test conditions, was monitored by a US DIGITAL MAE3 Absolute Magnetic Encoder. The components (base, push-on magnetic hub, and encoder body) can be found in Figure 162 . The sensor provided digital feedback over $360^{\circ}$ of rotation, without stops or gaps. The encoder magnet was mounted directly on the central shaft of the model, aligned with the airfoil's $0.25 \mathrm{c}$ pitch axis; the sensor housing was hard mounted to a 0.5 in thick aluminum plate, attached to the independent, steel frame surrounding the test section. The encoder was governed by a maximum sampling rate of $268 \mathrm{~Hz}$. The sensor has an accuracy of $\delta \alpha \leq \pm 0.5^{\circ}$.
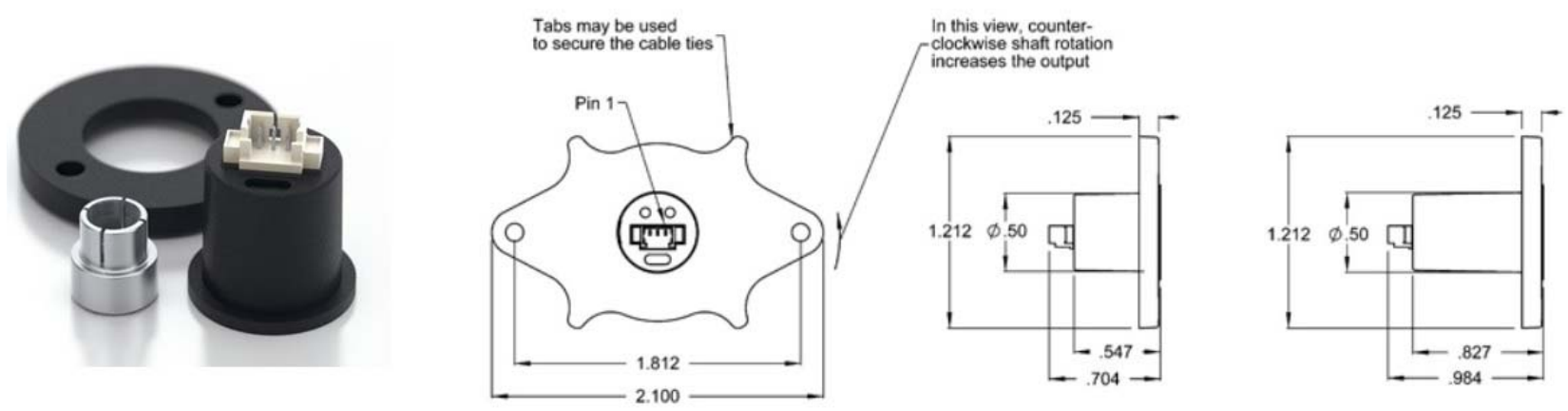

Figure 162. US DIGITAL MAE3 Absolute Encoder Geometry (167)

\section{Appendix J.2 KING Flow Meter}

The volumetric flow rate $(Q)$ of the supply line to the CCNACAO018 wind tunnel model was measured as a contributing value to calculate $C_{\mu}$. The pneumatic flow meter is a variable area type, with $1 \mathrm{scfm}$ increments. The manufacturer specified accuracy is $\pm 2 \% \mathrm{FS}$ for an operating range of 0-42 scfm. 


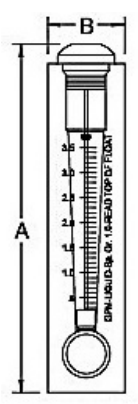

Front View

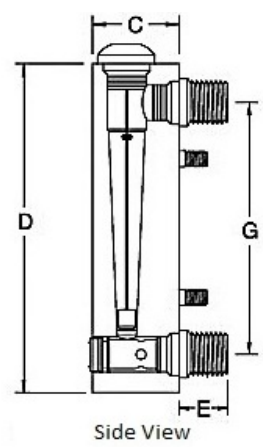

Side View

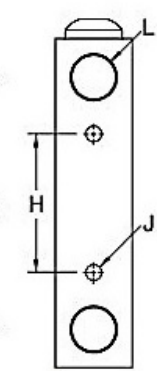

Rear View
Accuracy

$\pm 2 \%$ of Full Scale Flow, $250 \mathrm{~mm}$ scale

Repeatability

$1 / 2 \%, 250 \mathrm{~mm}$ scale

Max Temperature

Air $-100^{\circ} \mathrm{F}\left(38^{\circ} \mathrm{C}\right) \quad$ Water $-130^{\circ} \mathrm{F}\left(54^{\circ} \mathrm{C}\right)$

Max Pressure

Water-125 psig Air -100 psig

Ambient Temperature

$33^{\circ} \mathrm{F}$ to $125^{\circ} \mathrm{F}\left(1^{\circ} \mathrm{C}\right.$ to $\left.52^{\circ} \mathrm{C}\right)$

Specifications

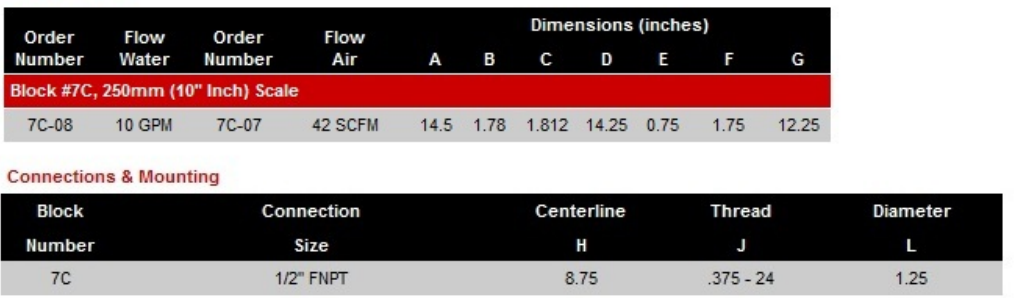

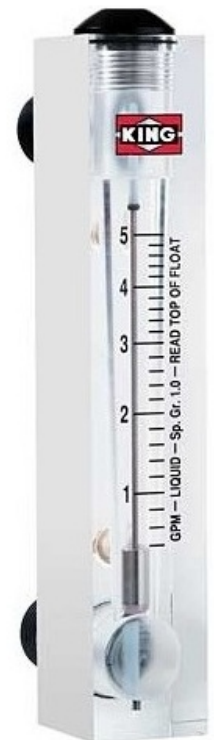

Figure 163.KING 7520 Series Pneumatic Flow Meter: 0-42scfm (168)

\section{Appendix J.3 HEDLAND Flow Meter}

For improved measurement resolution for small $C_{\mu}$ actuation, volumetric flow $(Q)$ to the CCNACA0018 wind tunnel model was measured with second flow with smaller scale $(\leq 5 \mathrm{cfm})$. A HEDLAND variable area flow meter (Figure 164) with a manufacturer specified standard operating range of 0.5 to $5 \mathrm{scfm}\left(-20^{\circ}\right.$ to $\left.240^{\circ} \mathrm{F}\right)$, accuracy of $\pm 2 \% \mathrm{FS}$, and repeatability of $\pm 1 \%$.
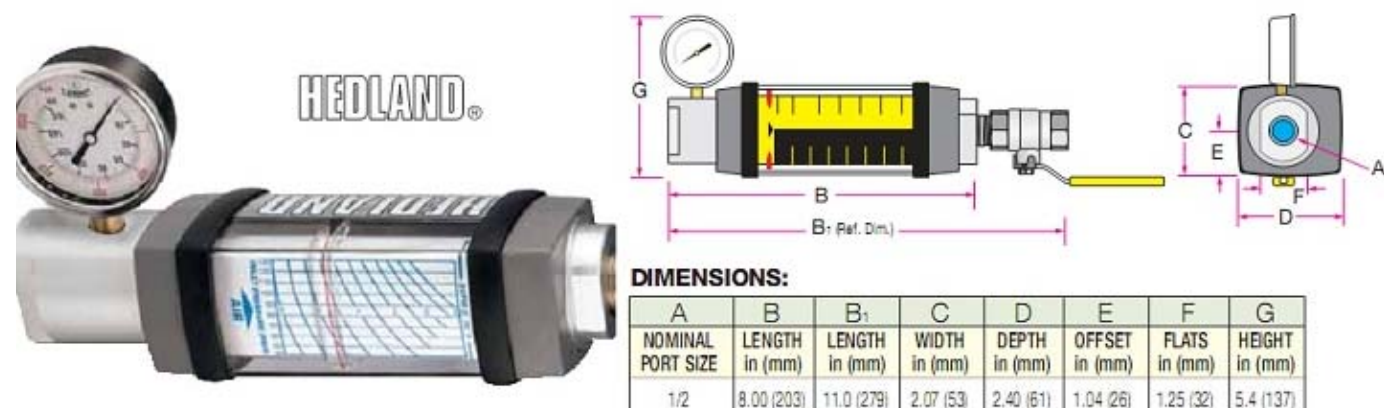

DIMENSIONS:

\begin{tabular}{|c|c|c|c|c|c|c|c|}
\hline A & B & B & C & D & E & F & G \\
\hline NOMINAL & LENGTH & LENGTH & WDDTH & DEPTH & OFFSET & FLATS & HEGGT \\
PORT SIZE & in (mm) & in (mm) & in (mm) & in (mm) & in (mm) & in (mm) & in $(\mathrm{mm})$ \\
\hline 1.2 & $8.00(2033)$ & $11.0(279)$ & $2.07(53)$ & $2.40(61)$ & $1.0426)$ & $1.25(32)$ & $5.4(137)$ \\
\hline
\end{tabular}

ACCURACY: $\pm 2 \%$ of full scale REPEATABILITY: $\pm 1 \%$

PRESSURE GAUGE: Gycerin dampened, 0 - 160 psi / 0 - 10 bar pressure

range avalable on all test kits.

Figure 164. HEDLAND Flow Meter with Pressure Gage: 0.5-5 scfm (169)

\section{Appendix J.4 DAQ Equipment}

A National Instruments (NI) PCleEX-6361 DAQ card was used to acquire all measured signals during wind tunnel testing. The $P C l e-6361$ is a multifunction analog, digital, and timing $\mathrm{I} / \mathrm{O}$ card with the following amenities: 16-bit ADC, (2) 16-bit DACs, (8) digital I/O lines, and (2) 24-bit counters for timing 
I/O. The card was interfaced to a NI PXI-1073 multifunction DAQ module system to simultaneously acquire digital signals from all instrumentation sensors, along with generating user-defined signals to control the pneumatic valve actuator array.

\section{Appendix J.5 Pressure Manometers}

A HEISE PTE-1 handheld pressure calibrator (Figure 165a) was used as a reference measurement of freestream dynamic pressure $\left(q_{\infty}\right)$ for various conditions during wind tunnel testing. The HEISE gage had interchangeable modules of various ranges all had specified accuracy of $\pm 0.10 \% \mathrm{FS}$. A DWYER Series 475 (Mark III) digital manometer was also used as a reference measurement for $q_{\infty}$.

(a.) HEISE PTE-1

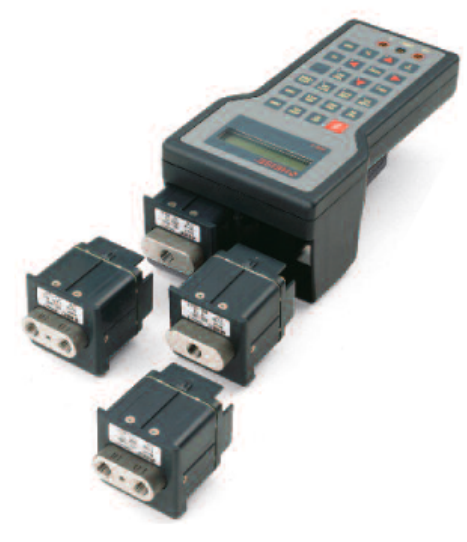

(b.) DWYER Series 475

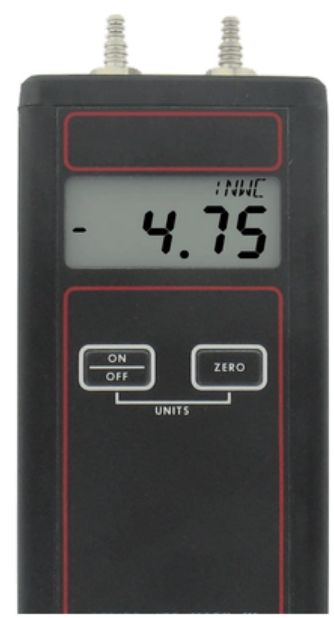

Figure 165. Pressure Instrumentation: (a.) HEISE PTE-1 (170), (b.) DWYER Series 475 (171)

The two HEISE modules used during wind tunnel testing (Module 1: (0-15) psig, Module II: (0-100) psig) were connected to a common pressure source to compare results (Figure 166). The results showed excellent agreement for the pressure range tested, with a maximum difference of $1.6 \%$ between measurements. A DWYER pump was used to create a constant pressure within a small chamber connected to both modules; the pump had a maximum holding limit of $5 \mathrm{psig}$.

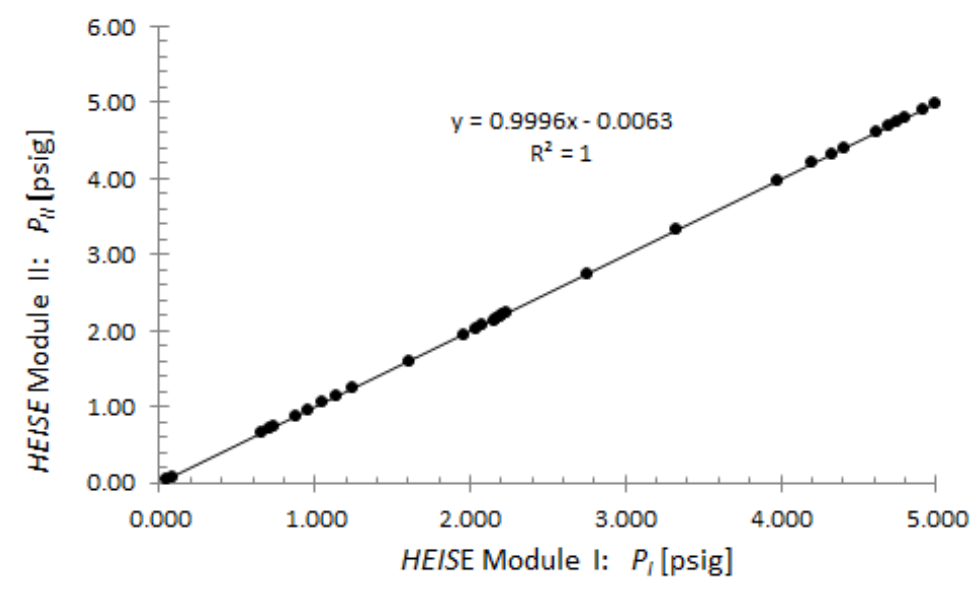

Figure 166. HEISE Pressure Gage Module Validation 


\section{Appendix J.6 Atmospheric Conditions}

A certified hygrometer and temperature indicator (Robert E. White Instruments, INC.: Model HTAB-176) was used to measure daily atmospheric conditions $\left(P_{\infty}, T_{\infty}\right)$ in the lab.

\section{Appendix J.7 CTA Hotwire: $\boldsymbol{V}_{\text {jet }}$ Characterization}

A TSI IFA-300 constant temperature anemometer was used to characterize CCNACA0018 jet velocity during benchtop experiments. A single element, Tungsten Platinum coated hotwire probe (TSI model 1210-T1.5, diameter $=0.000015$ in, frequency response $=260 \mathrm{kHz}$ ) in conjunction with a standard probe support (TSI model 1150) was used for all tests. Since the maximum operational frequency of the airfoil pneumatic vales was $200 \mathrm{~Hz}$, a low pass filter of $1,000 \mathrm{~Hz}$ was employed to remove chamber resonance effects and noise.

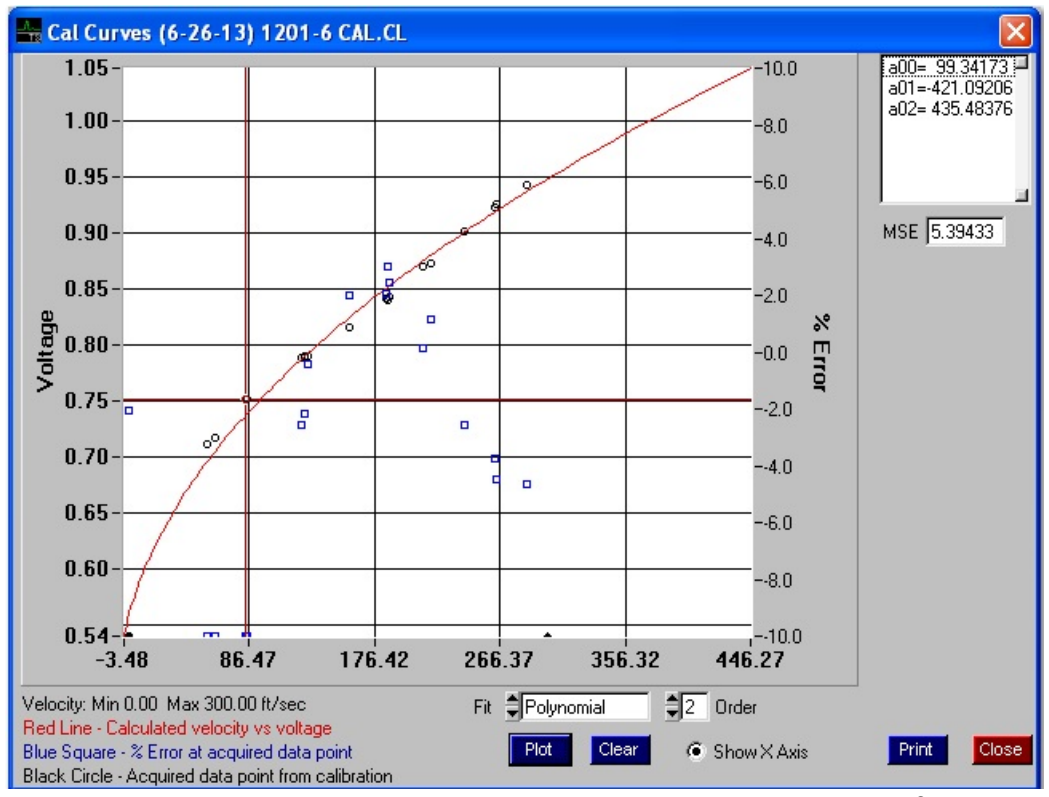

Figure 167. Hotwire Calibration generated by TSI THERMAL PRO Software

Atmospheric conditions and airflow $\triangle P$ were measured and entered into the THERMAL PRO calibration software, along with precise probe operating data (provided by manufacturer), prior to tests. The calibration curve in Figure 167 shows an example of air velocity [ft/s] versus bridge voltage; the calibration points (black circles), $2^{\text {nd }}$ order polynomial curve fit (red curve) with calibration coefficients (top right), and calculated \% error (blue squares) are all determined by the software (see Figure 167). The graph smoothly increases monotonically as desired. A maximum error of $\pm 5 \%$ for $\left(V_{j e t}=300 \mathrm{ft} / \mathrm{s}\right)$ was determined from THERMAL PRO software and instrumentation used for external measurements $\left(P_{\infty}, T_{\infty}\right.$, $\Delta P)$. 


\section{APPENDIX K: Additional CFD Flow Visualization}

This appendix includes additional contour plots from the numerical dynamic stall analysis of the CCNACA0018 airfoil pitching at $k=0.100\left(R e=180 \times 10^{3}\right)$. The plots highlight the influence of various modes of circulation control on the development and convection of the DSV during various phases of each pitch cycle. All pitch simulations were completed with the laminar model to better visualize DS events. Figure 168 displays the global flowfield around the CCNACA airfoil during pitch-up, approaching $\alpha_{\max }$. The $\alpha$ at which small scale vorticity forms at the LE is different for each actuation case. As the magnitude of $C_{\mu}$ increases, the AoA of such vorticity production decreases. Furthermore, pulsed actuation at $C_{\mu}=0.01$ and steady control of $C_{\mu}=0.05$ show a reduction in size and strength of LE vorticity, while the unsteady jet also reduces the size and scale of TE vorticity resulting less profile drag than all other cases. The LE stagnation point is also shifted further for the latter two cases of control.

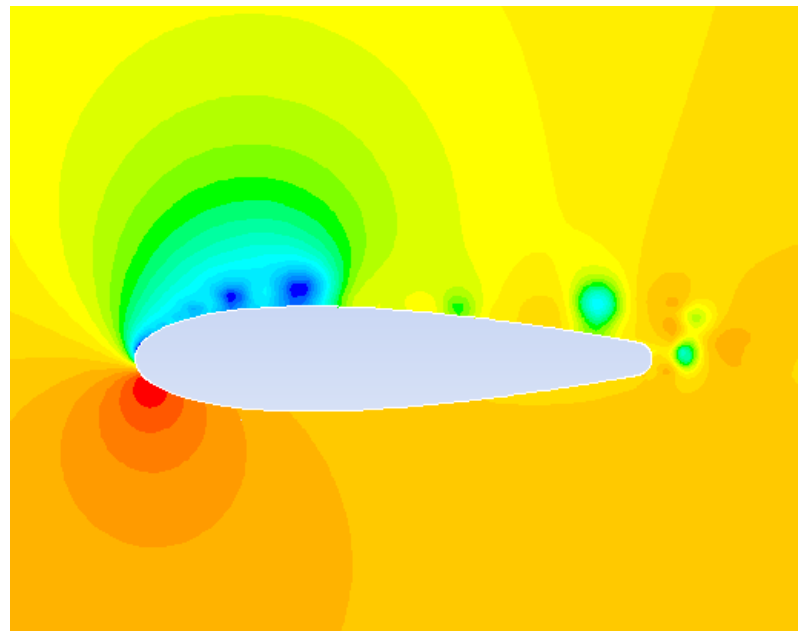

Baseline Airfoil: $\alpha=29.5^{\circ} \uparrow$

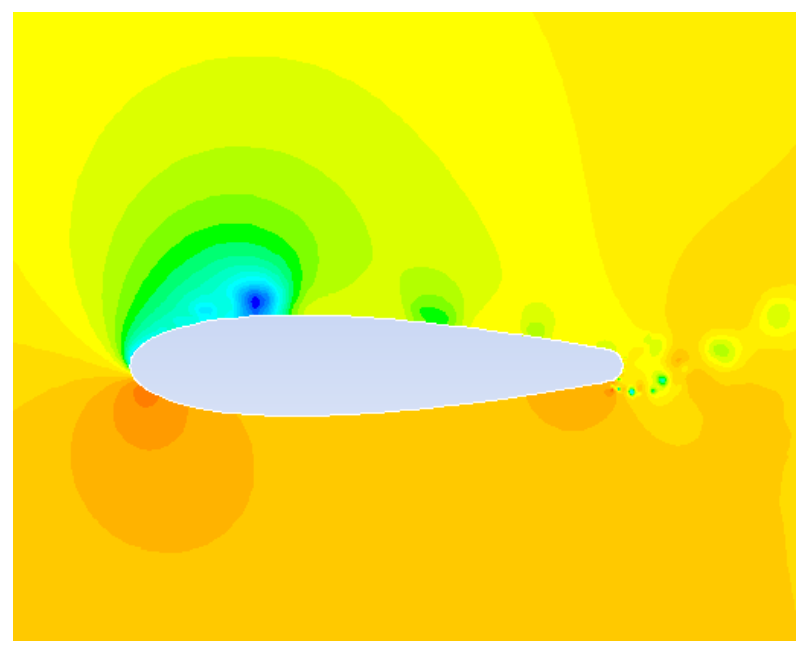

$C_{\mu}=0.01(S t=1.00): \alpha=28.5^{\circ} \uparrow$

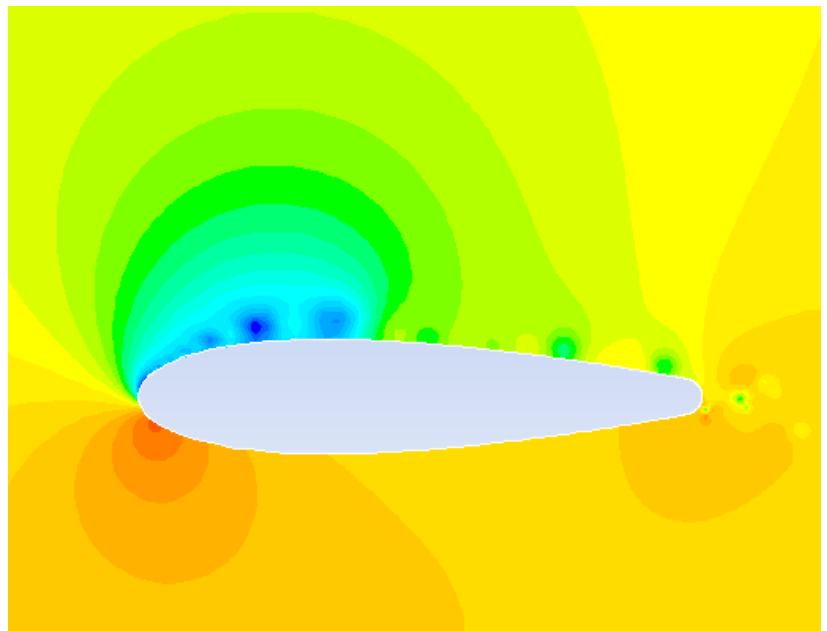

$C_{\mu}=0.01(S t=0.00): \alpha=28.6^{\circ} \uparrow$

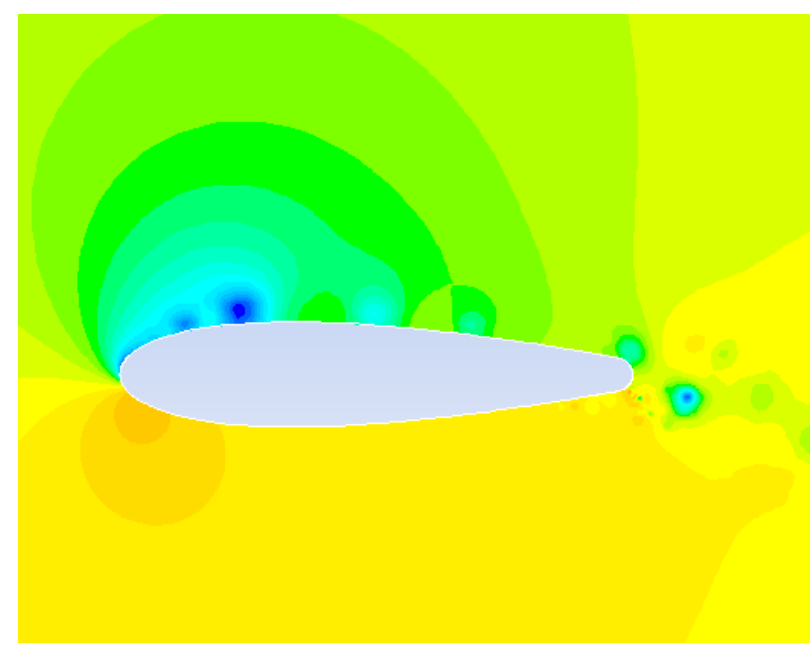

$C_{\mu}=0.05(S t=0.00): \alpha=26.5^{\circ} \uparrow$

Figure 168. Static Pressure Contours: Convection of Small Scale Vorticity for Various CC Modes 


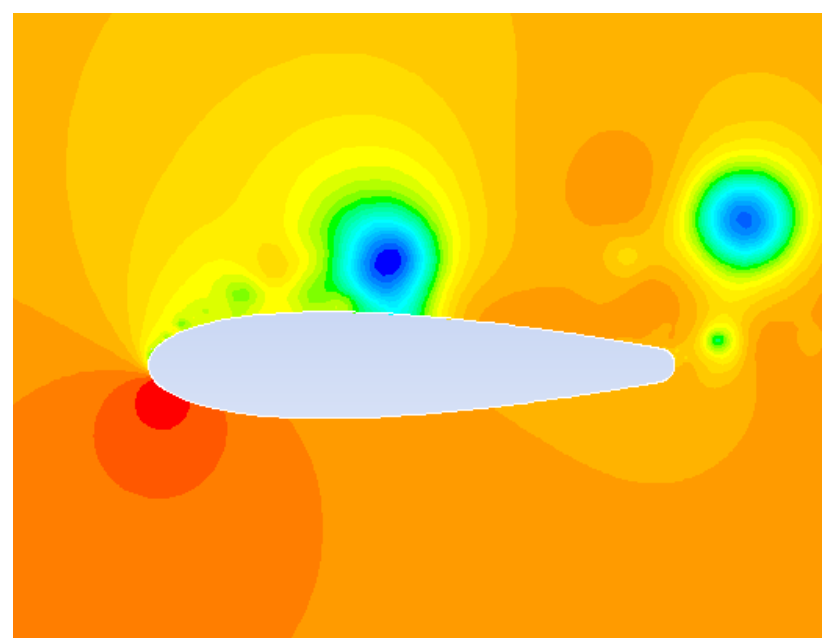

Baseline Airfoil: $\alpha=28.78 \downarrow$

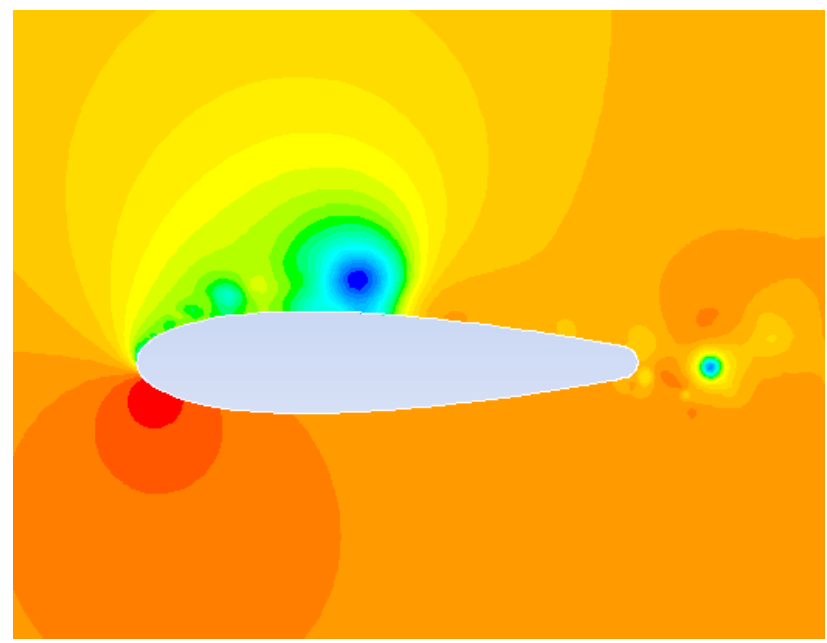

$C_{\mu}=0.01(S t=1.00): \alpha=29.71^{\circ} \uparrow$

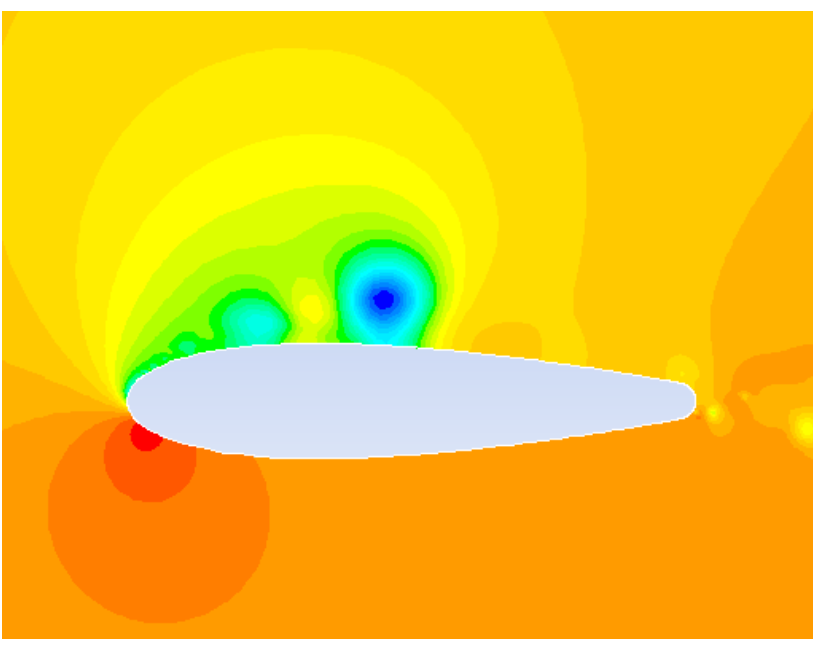

$C_{\mu}=0.01(S t=0.00): \alpha=29.54^{\circ} \uparrow$

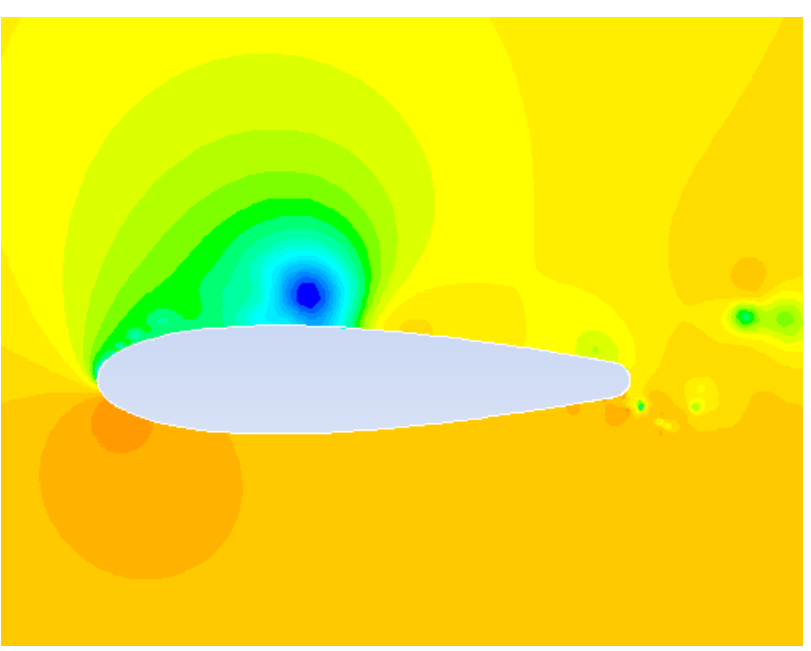

$C_{\mu}=0.05(S t=1.00): \alpha=28.45^{\circ} \uparrow$

Figure 169. Static Pressure Contours: Convection of DSV with Various CC Modes 


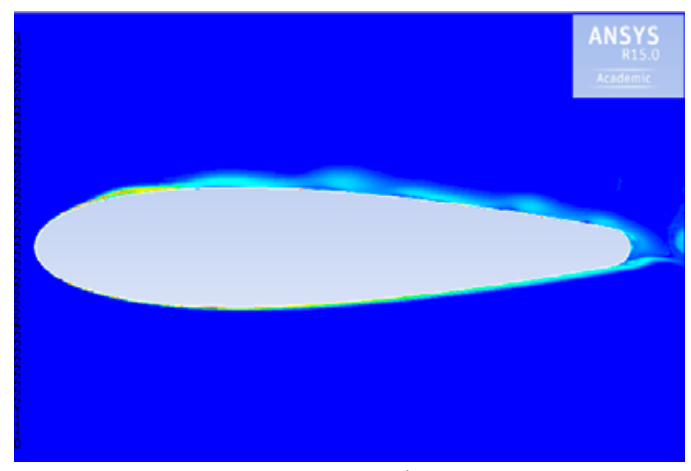

$\alpha=27.23^{\circ} \uparrow$

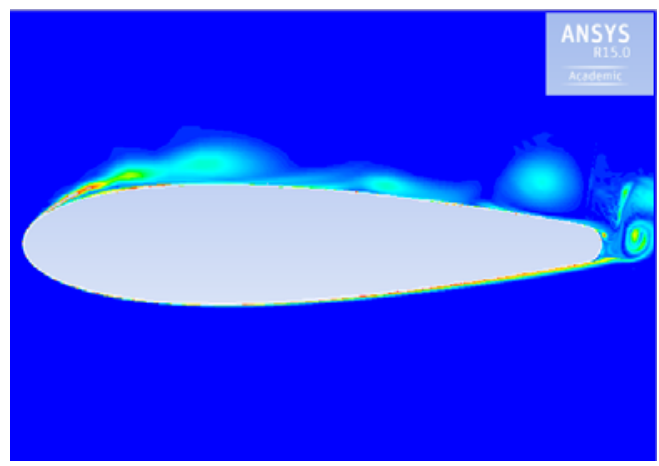

$\alpha=29.85^{\circ} \uparrow$

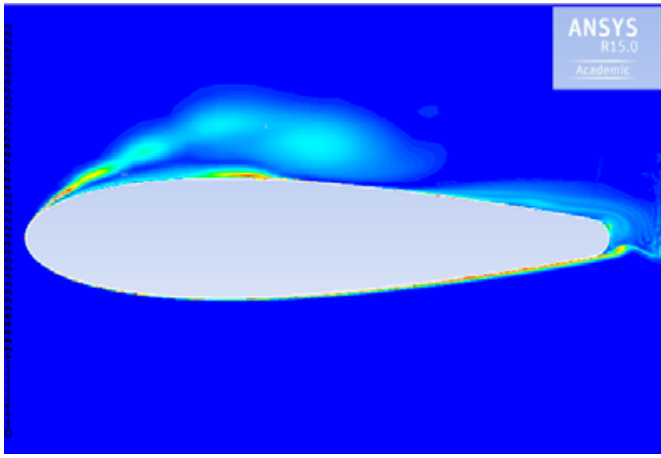

$\alpha=29.89^{\circ} \downarrow$

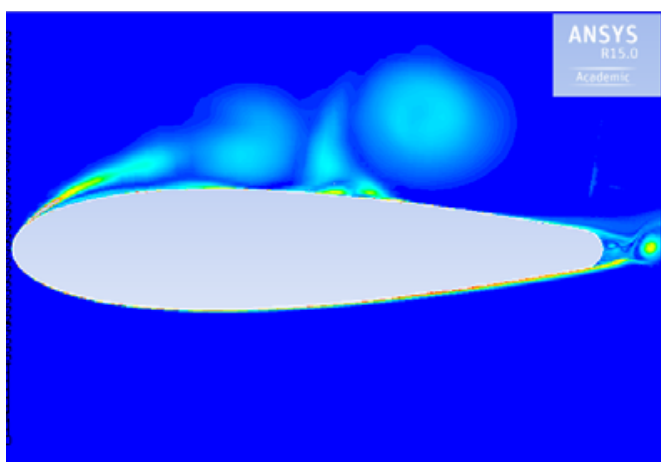

$\alpha=29.43^{\circ} \downarrow$

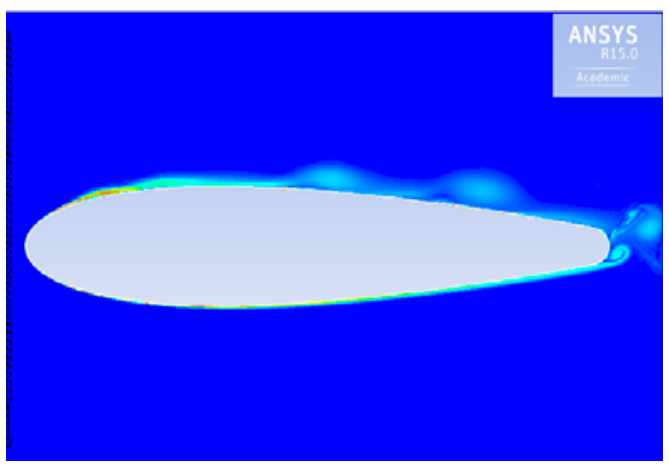

$\alpha=28.08^{\circ} \uparrow$

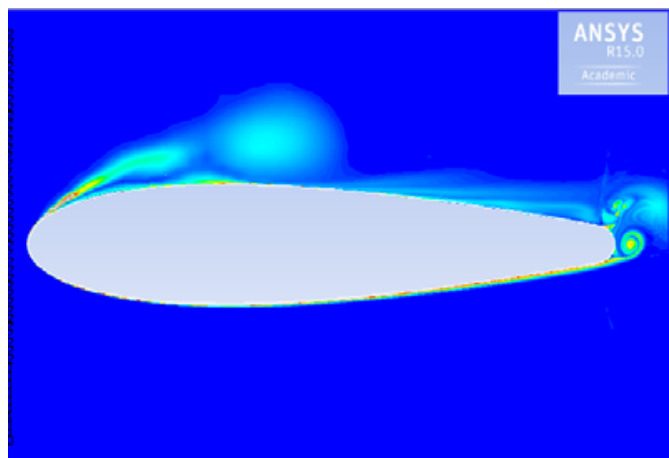

$\alpha=29.97^{\circ} \downarrow$

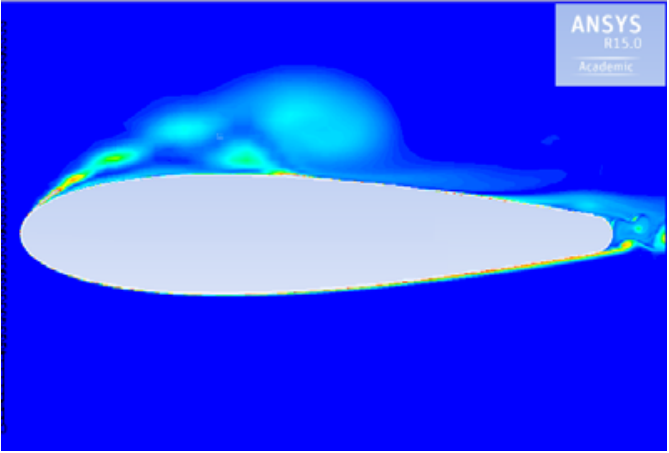

$\alpha=29.78^{\circ} \downarrow$

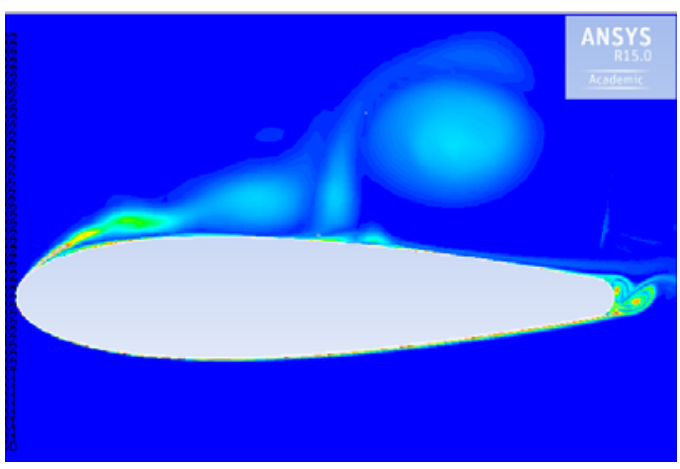

$\alpha=27.86^{\circ} \downarrow$

Figure 170. Vorticity Magnitude Contours: DS Events on Pitching CCNACA0018 (Baseline) 


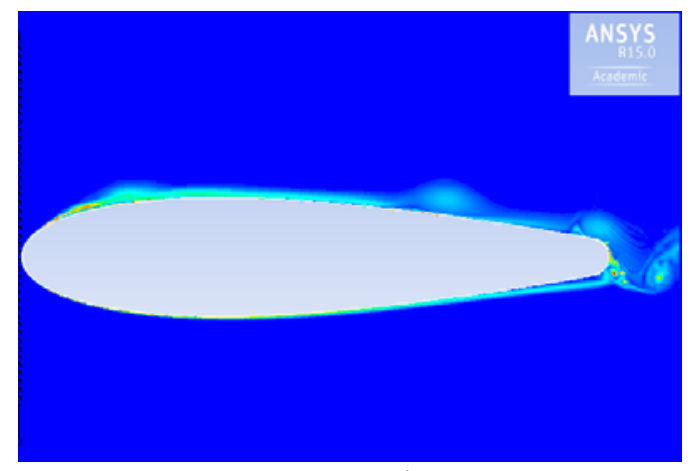

$\alpha=27.01^{\circ} \uparrow$

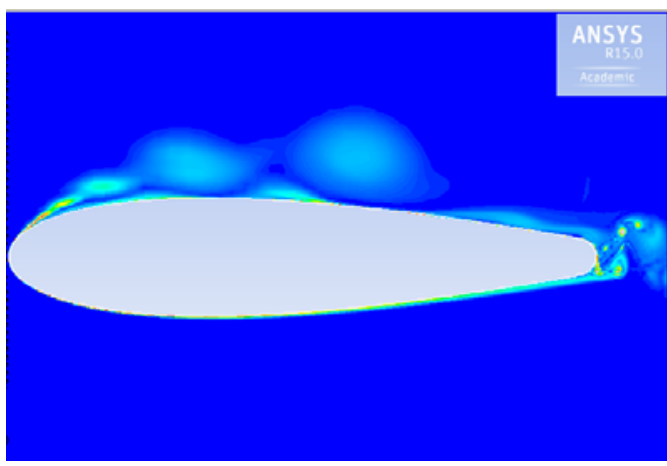

$\alpha=29.85^{\circ} \uparrow$

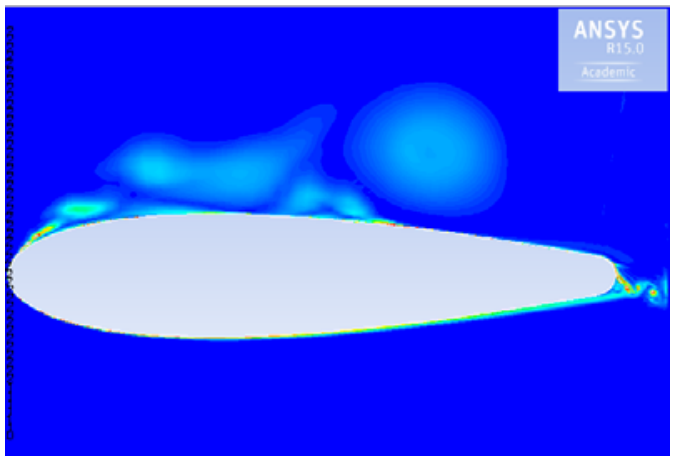

$\alpha=29.89^{\circ} \downarrow$

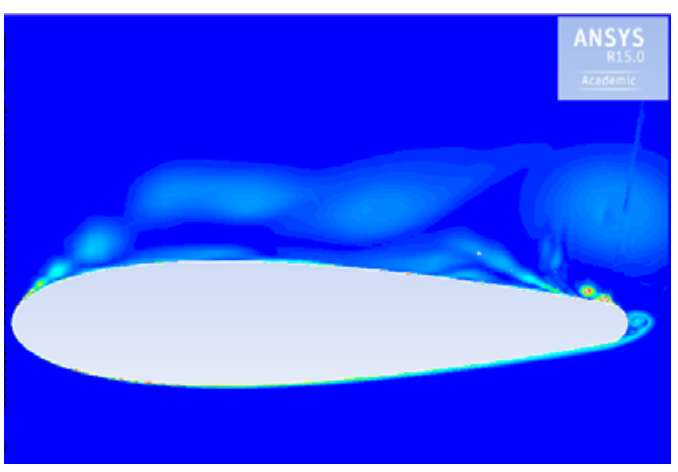

$\alpha=29.43^{\circ} \downarrow$

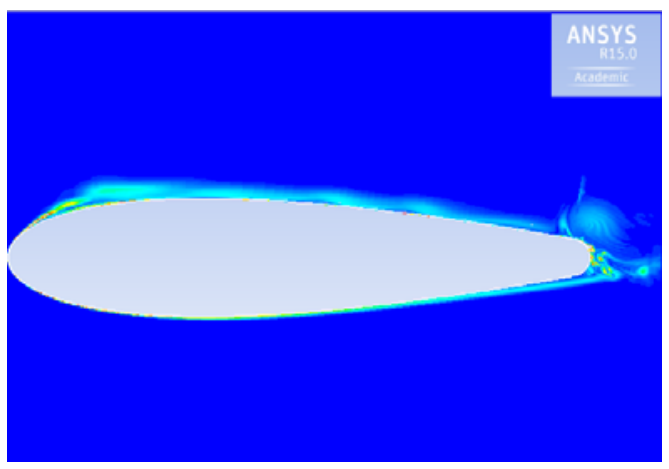

$\alpha=28.27^{\circ} \uparrow$

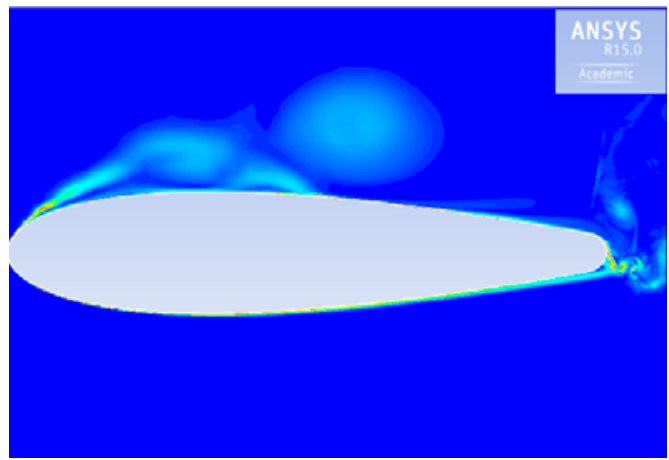

$\alpha=29.97^{\circ} \downarrow$

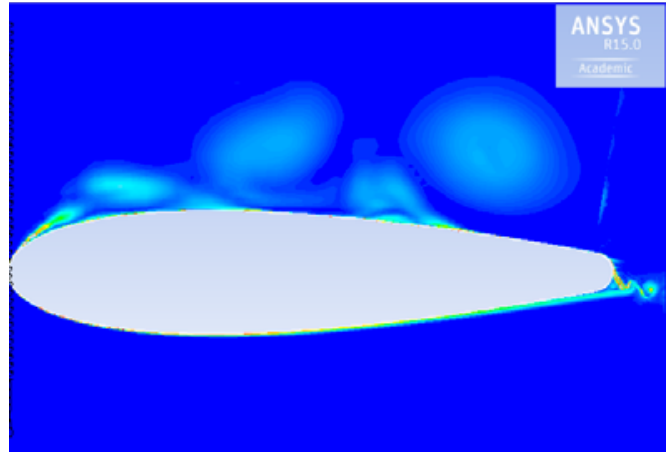

$\alpha=29.78^{\circ} \downarrow$

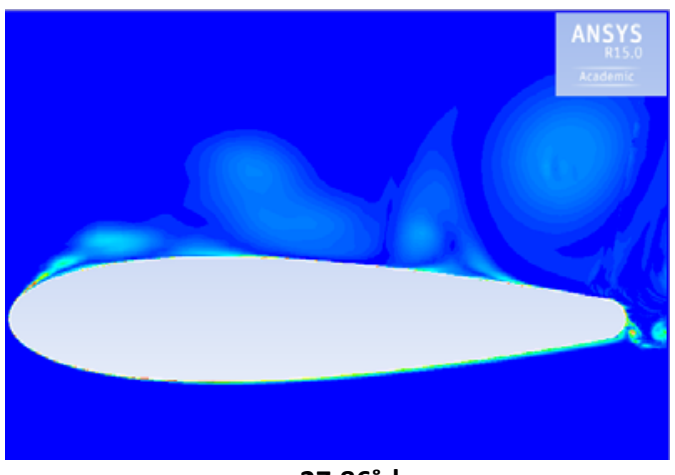

$\alpha=27.86^{\circ} \downarrow$

Figure 171. Vorticity Magnitude Contours: DS Events on Pitching CCNACA0018 $\left(C_{\mu}=0.01, S t=0.00\right)$ 


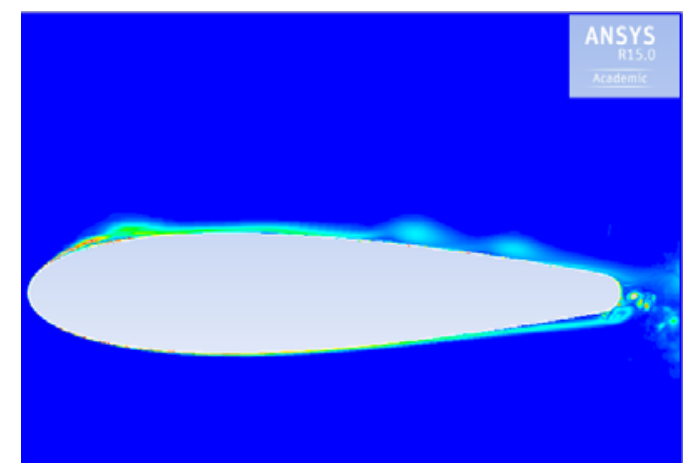

$\alpha=27.01^{\circ} \uparrow$

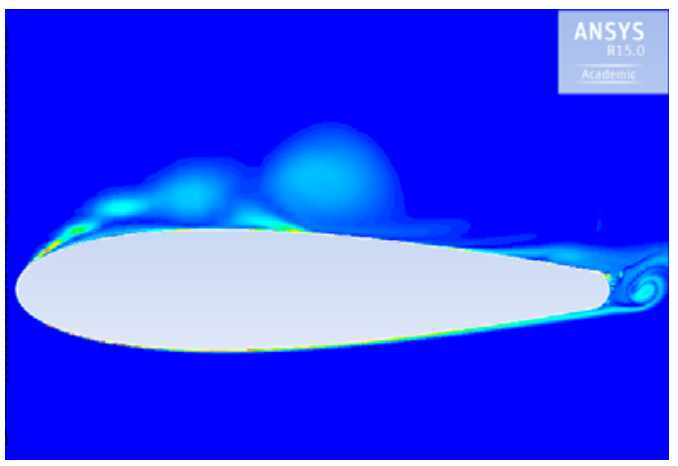

$\alpha=29.85^{\circ} \uparrow$

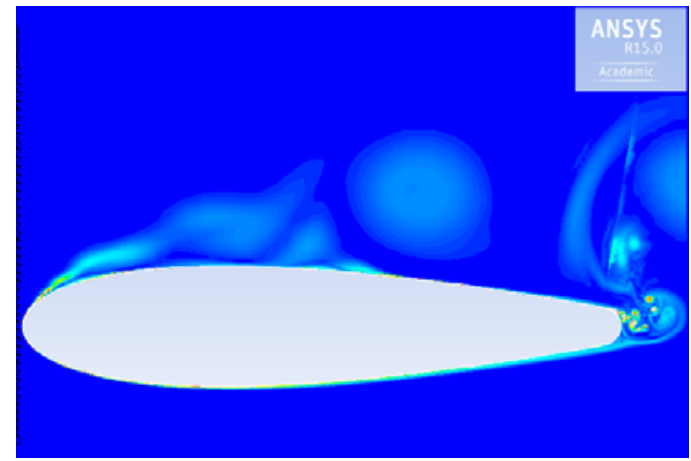

$\alpha=29.89^{\circ} \downarrow$

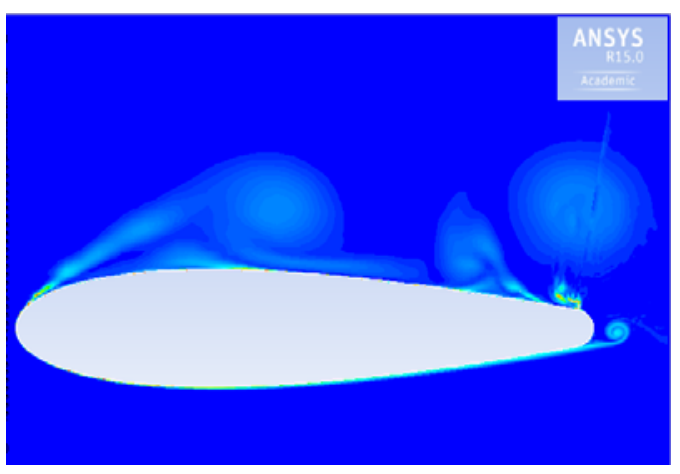

$\alpha=29.43^{\circ} \downarrow$

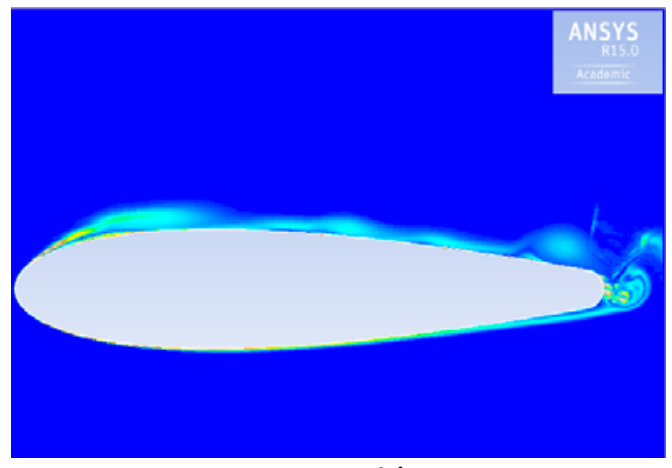

$\alpha=28.27^{\circ} \uparrow$

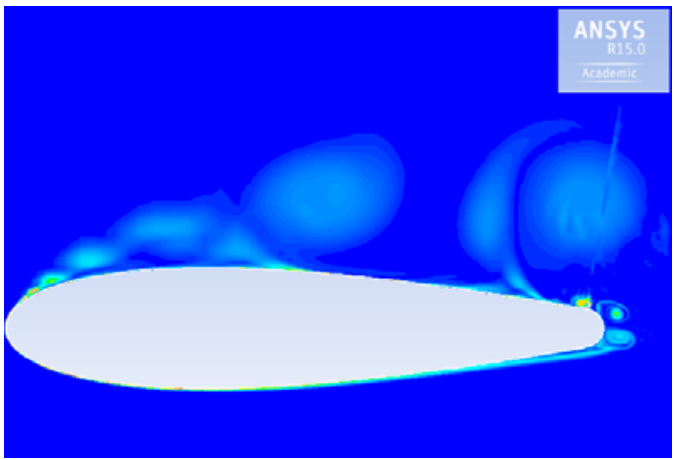

$\alpha=29.97^{\circ} \downarrow$

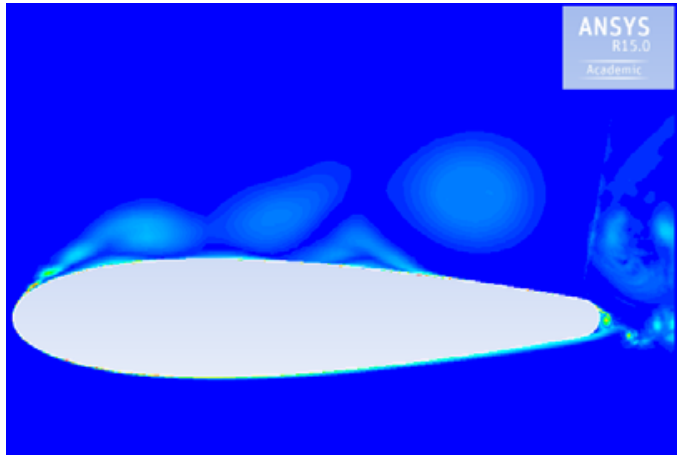

$\alpha=29.78^{\circ} \downarrow$

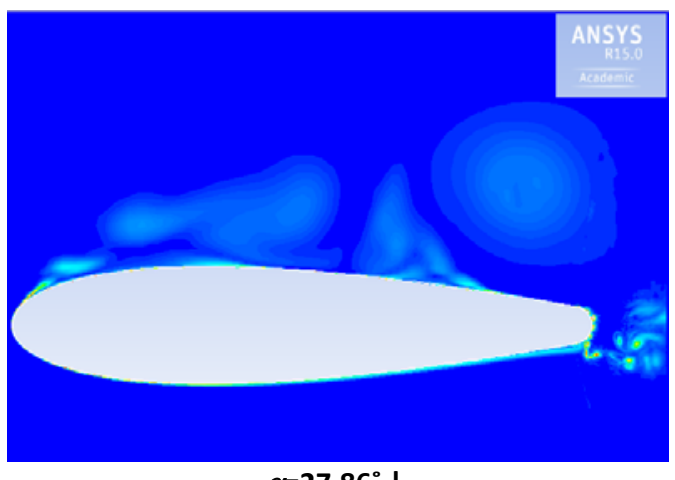

$\alpha=27.86^{\circ} \downarrow$

Figure 172. Vorticity Magnitude Contours: DS Events on Pitching CCNACA0018 $\left(C_{\mu}=0.01, S t=1.00\right)$ 


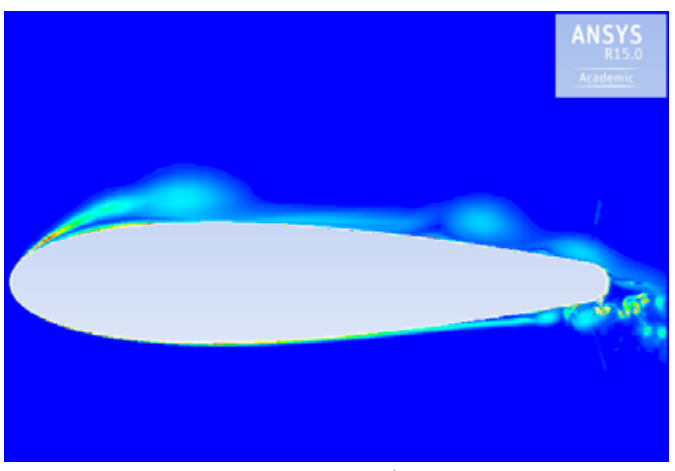

$\alpha=27.01^{\circ} \uparrow$

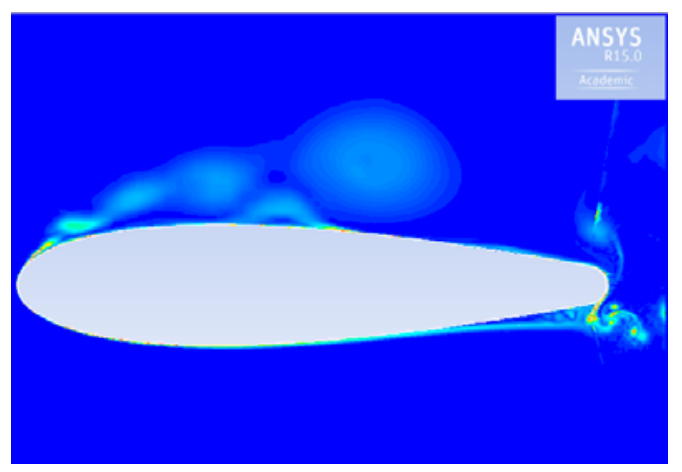

$\alpha=29.85^{\circ} \uparrow$

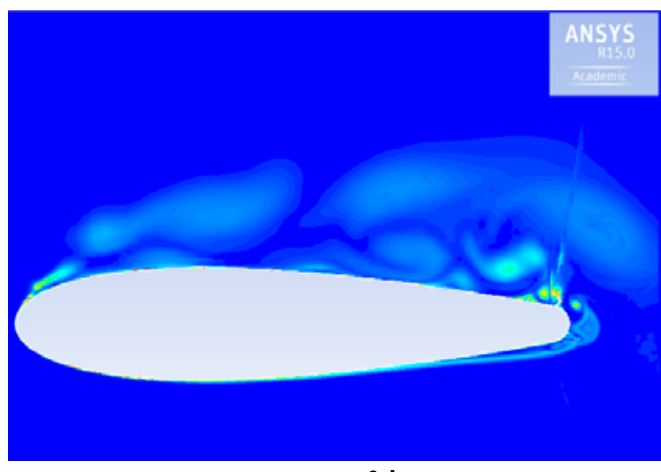

$\alpha=29.89^{\circ} \downarrow$

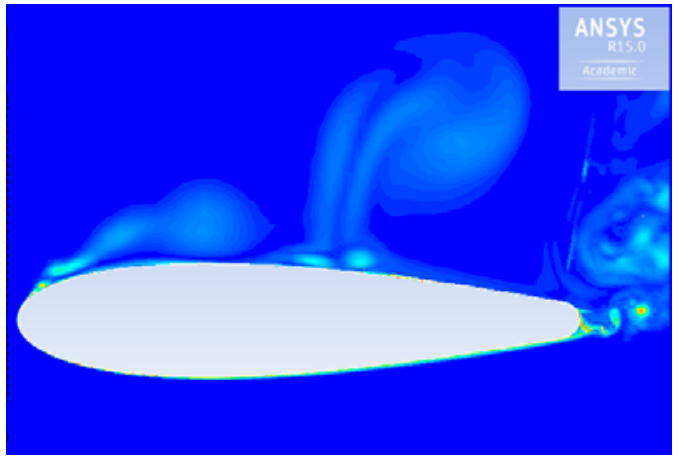

$\alpha=29.43^{\circ} \downarrow$

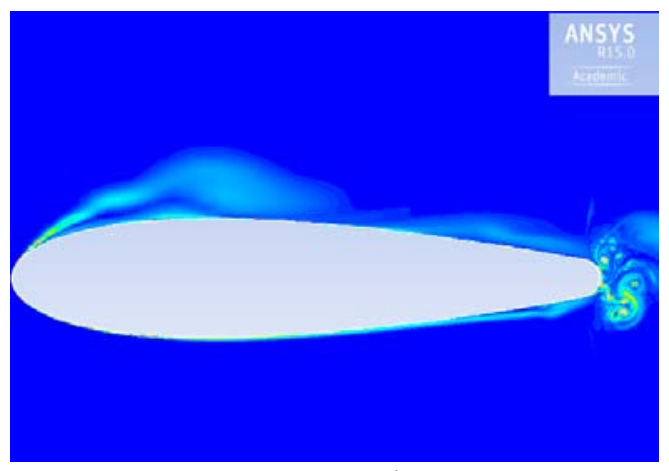

$\alpha=28.27^{\circ} \uparrow$

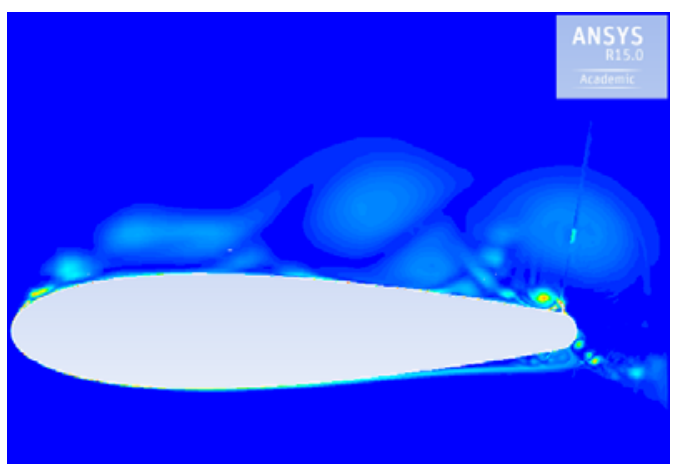

$\alpha=29.97^{\circ} \downarrow$

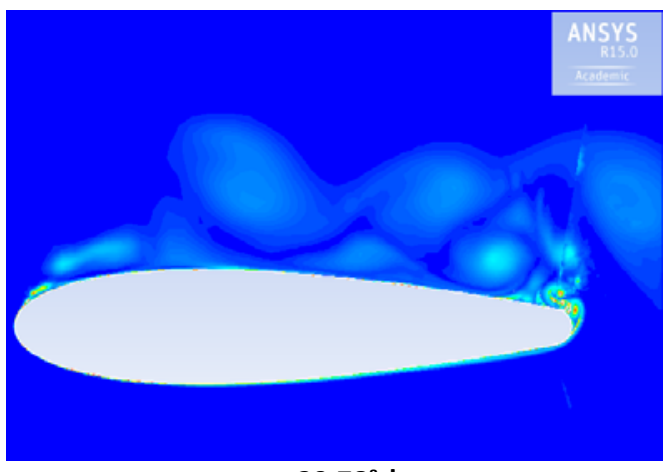

$\alpha=29.78^{\circ} \downarrow$

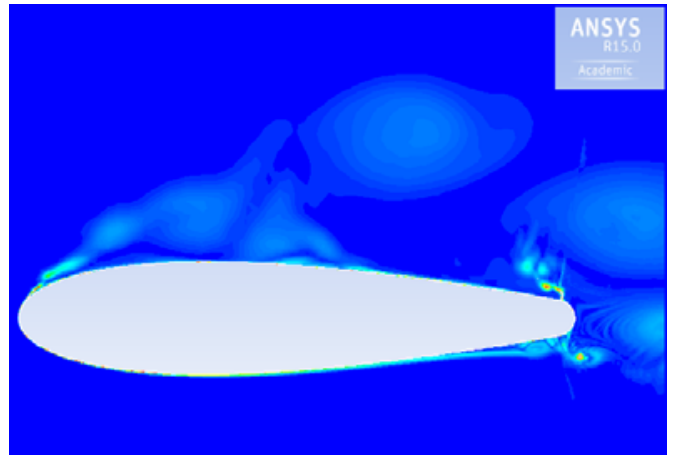

$\alpha=27.86^{\circ} \downarrow$

Figure 173. Vorticity Magnitude Contours: DS Events on Pitching CCNACA0018 $\left(C_{\mu}=0.05, S t=0.00\right)$ 


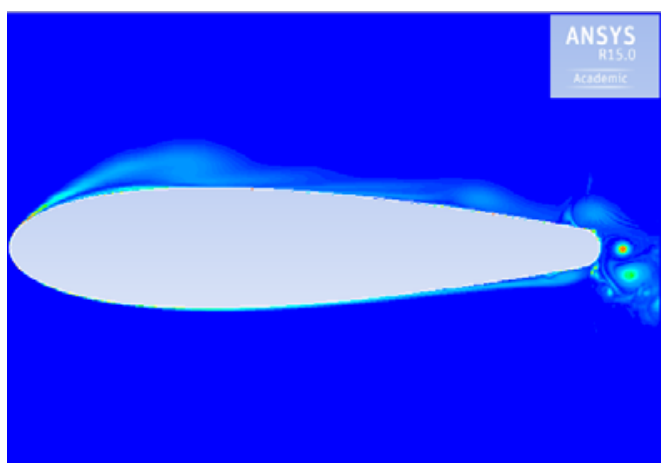

$\alpha=27.01^{\circ} \uparrow$

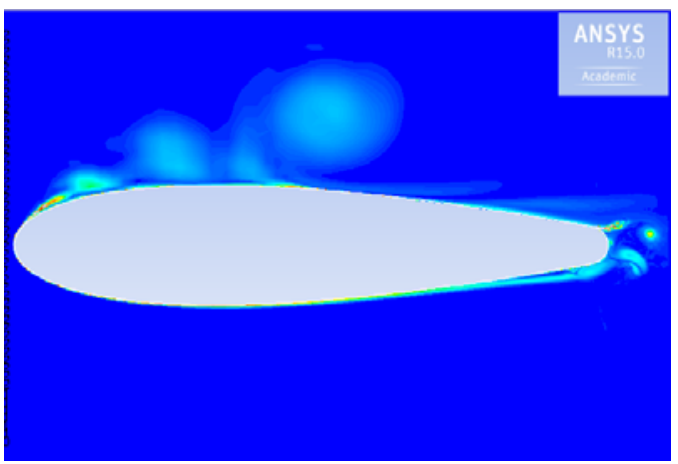

$\alpha=29.85^{\circ} \uparrow$

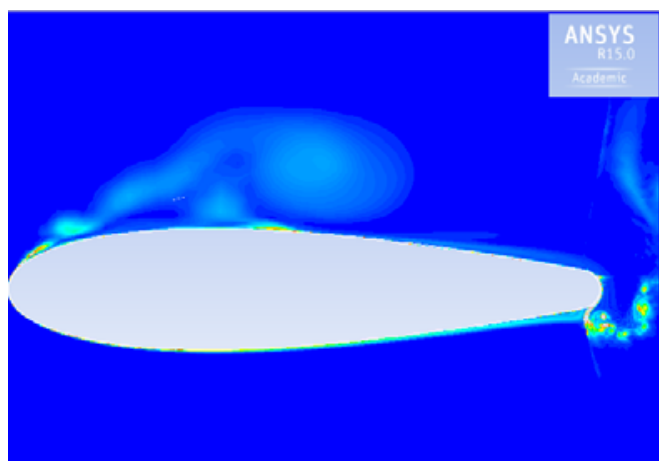

$\alpha=29.89^{\circ} \downarrow$

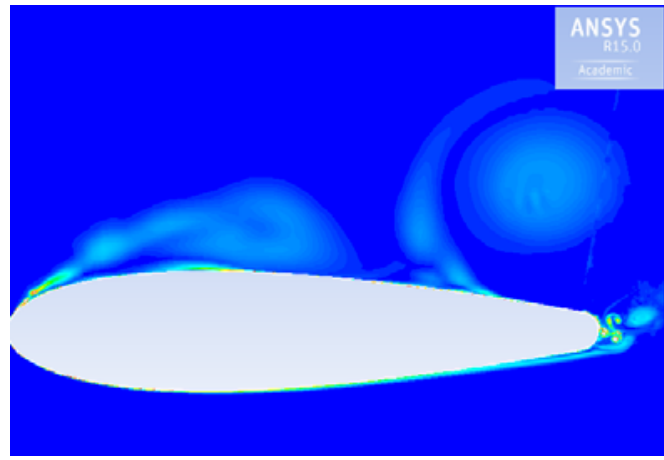

$\alpha=29.43^{\circ} \downarrow$

Figure 174. Vorticity Magnitude Contours: DS Events on Pitching CCNACA0018 $\left(C_{\mu}=0.05, S t=1.00\right)$

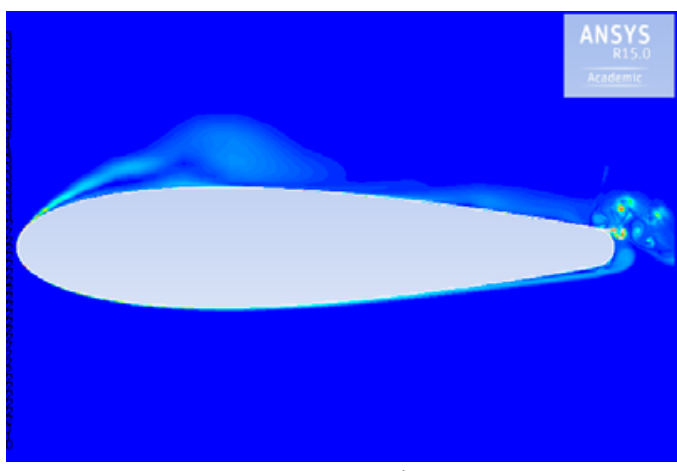

$\alpha=28.27^{\circ} \uparrow$

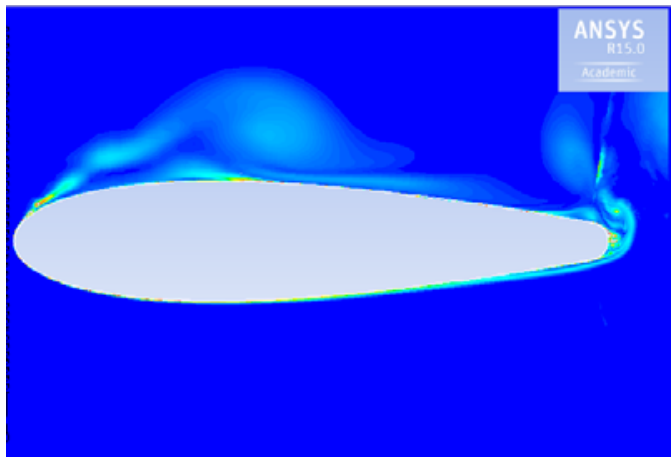

$\alpha=29.97^{\circ} \downarrow$

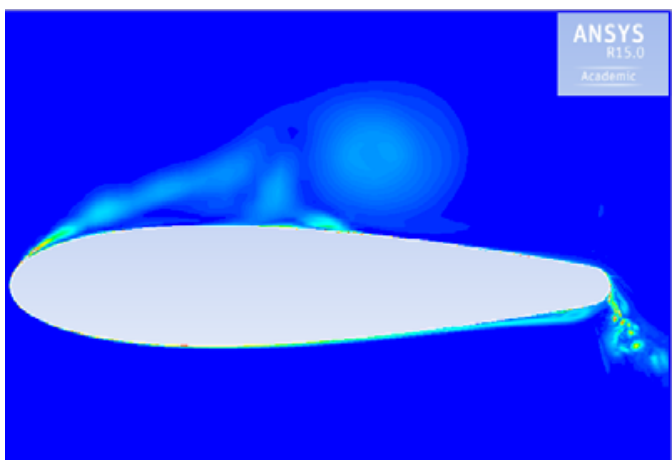

$\alpha=29.78^{\circ} \downarrow$

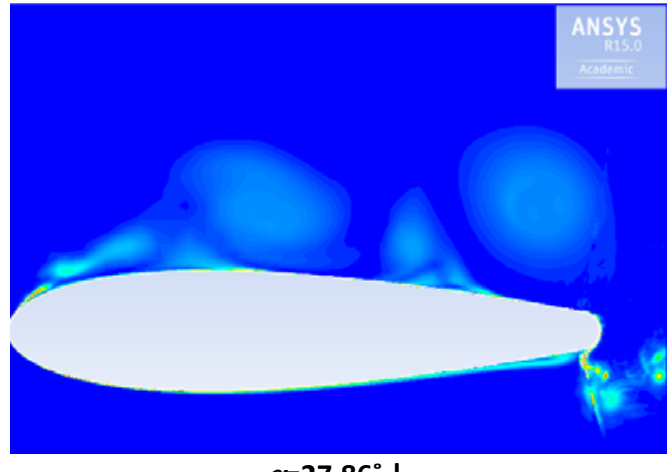

$\alpha=27.86^{\circ} \downarrow$ 